\title{
Testing of Enhanced Chemical Cleaning of SRS Actual Waste Tank 5F and Tank 12H Sludges
}

\author{
C. J. Martino \\ W. D. King
}

August 2011

Savannah River National Laboratory Savannah River Nuclear Solutions, LLC Aiken, SC 29808

Prepared for the U.S. Department of Energy under contract number DE-AC09-08SR22470.

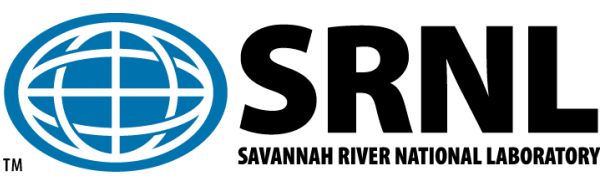


SRNL-STI-2011-00360

Revision 0

\section{DISCLAIMER}

This work was prepared under an agreement with and funded by the U.S. Government. Neither the U.S. Government or its employees, nor any of its contractors, subcontractors or their employees, makes any express or implied:

1. warranty or assumes any legal liability for the accuracy, completeness, or for the use or results of such use of any information, product, or process disclosed; or

2. representation that such use or results of such use would not infringe privately owned rights; or

3. endorsement or recommendation of any specifically identified commercial product, process, or service.

Any views and opinions of authors expressed in this work do not necessarily state or reflect those of the United States Government, or its contractors, or subcontractors.

\section{Printed in the United States of America \\ Prepared for \\ U.S. Department of Energy}




\title{
Testing of Enhanced Chemical Cleaning using Actual Waste Tank 5F and Tank 12H Sludges
}

\author{
C. J. Martino \\ W. D. King
}

August 2011

Savannah River National Laboratory

Savannah River Nuclear Solutions, LLC Aiken, SC 29808

Prepared for the U.S. Department of Energy under contract number DE-AC09-08SR22470.

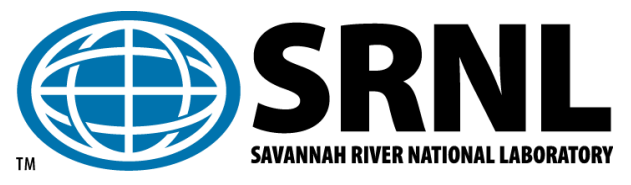




\section{REVIEWS AND APPROVALS}

AUTHORS:

C. J. Martino, Advanced Characterization and Processing Date

W. D. King, Advanced Characterization and Processing Date

TECHNICAL REVIEW:

M. S. Hay, Advanced Characterization and Processing Date

APPROVALS:

F. M. Pennebaker, Manager

Date

Advanced Characterization and Processing

S. L. Marra, Manager

Date

Environmental \& Chemical Process Technology Research Programs

E. T. Ketusky, SRR Closure Project Engineering

Date

P. E. Carroll, SRR Closure Project Engineering

Date

R. H. Spires, Manager

Date

SRR Closure Project Management 
SRNL-STI-2011-00360

Revision 0

\section{ACKNOWLEDGEMENTS}

The authors acknowledge the help and support of many others who contributed to the success of the ECC RWT. First and foremost are the technicians in the SRNL Shielded Cells, including Nan Stanley, Jane Howard, Jeff Mixon and Linda Bush, and the other SRNL technicians including Myra Pettis. This project was also supported by many Radiological Protection Department and SRNL Operations personnel.

Analytical support was primarily provided by SRNL Analytical Development Department. We thank David DiPrete and the rest of the radiochemistry leads and technicians. Other chemical preparation and analysis was provided by Boyd Wiedenman, Mark Jones, Curtis Johnson, Chuck Coleman, Amy Ekechukwu, and other Analytical Development personnel. We thank David Missimer for the particle size analysis of the high activity solids. Rheology measurement and interpretation support was provided by John Pareizs, Erich Hansen and Michael Poitier.

We acknowledge the tremendous support of Ron Blessing in preparing the equipment for use in the SRNL Shielded Cells. We thank the others in the Shielded Cells that helped with planning along the way, including Babb Attaway, Carolyn Conley and Steve Beard. We thank the other engineers and mechanics who helped with final equipment preparations, including Don Trapp, Montenius Collins, Gary Hall and Josh Perry. Members of the SRNL Engineering Development Laboratory, including Tim Steeper, Mark Fowley, Duane Adamson, Geoff Smoland and Doug Sumpter, were pivotal to this project during equipment design, hazards analysis, equipment receipt, and instrument calibration. We also acknowledge the support of Steven Snyder, Jim Ferguson and various SRNL subject matter experts who helped guide the project thorough the facility hazards analysis process.

We also acknowledge the helpful guidance from the SRR engineering and project personnel throughout the planning and testing process, including Ed Ketusky, Carol Sherburne, Christie Sudduth, Tim Punch and Renee Spires. We thank Dan McCabe for helping to set the foundation of this project. And of course we acknowledge and thank the many AREVA NP personnel who designed and fabricated the ECC RWT equipment, especially Daryl Gorton who troubleshot and reworked many of the system components. Other helpful AREVA NP and AREVA FS personnel that were instrumental to this project were Shan Peters, Ray Beaty Don Dalton and Dennis Jones. 


\section{EXECUTIVE SUMMARY}

In support of Savannah River Site (SRS) tank closure efforts, the Savannah River National Laboratory (SRNL) conducted Real Waste Testing (RWT) to evaluate an alternative to the baseline $8 \mathrm{wt} \%$ oxalic acid (OA) chemical cleaning technology for tank sludge heel removal. The large quantities of sodium oxalate and other metal oxalates formed impact downstream processes by requiring additional washing during sludge batch preparation and increase the amount of material that must be processed in the tank farm evaporator systems and the Saltstone Processing Facility. Enhanced Chemical Cleaning (ECC) was identified as a potential method for greatly reducing the impact of oxalate additions to the SRS Tank Farms without adding additional components to the waste that would extend processing or increase waste form volumes.

ECC is a promising alternative to bulk OA cleaning, which utilizes a more dilute OA and an oxalate destruction technology. Sludge is dissolved in a Treatment Tank by adding $2 \mathrm{wt} \%$ OA and mixing the tank contents. The mixture of dissolved sludge and suspended insoluble solids is transferred to the ECC reactor, where the OA and other oxalates are decomposed. Concentrated ozone gas is added to the reactor, resulting in soluble ozone and hydroxyl radicals that aid the decomposition of oxalate ion to carbon dioxide gas and raise the $\mathrm{pH}$ of the liquid. The change in chemistry inside the ECC reactor causes the dissolved sludge to precipitate, mostly in the form of metal oxides. Ultraviolet (UV) light can also be used to augment the decomposition of OA.

The primary goals for SRNL RWT are as follows:

- to confirm ECC performance with real tank sludge samples,

- to determine the impact of ECC on fate of actinides and the other sludge metals, and

- to determine changes, if any, in solids flow and settling behavior.

SRNL conducted two tests using actual SRS waste material from Tanks $5 \mathrm{~F}$ and $12 \mathrm{H}$. Testing involved sludge dissolution with an initial $2 \mathrm{wt} \% \mathrm{OA}$, the decomposition of the oxalates by ozonolysis, the evaporation of a portion of the material for water removal, and tracking the concentrations of key components in the Deposition Tank. The UV light was not utilized in the decomposition testing. The following are results of the ECC RWT:

- Using three OA batches per test, Test 1 showed an approximately $73 \%$ removal of Tank $12 \mathrm{H}$ sludge and Test 2 showed an approximately $80 \%$ removal of Tank $5 \mathrm{~F}$ sludge. Removal includes both dissolution of soluble components and partial transfer of insoluble solids. The dissolution process was constrained to eight hours per OA batch and thus did not precisely represent the in tank dissolution process.

- Residuals remaining in the dissolution vessel at the conclusion of Test 1 were primarily aluminum (accounts for over $90 \%$ of the mass assuming boehmite is the major form) with small amounts of iron and manganese. Residuals remaining in the dissolution vessel at the conclusion of Test 2 were primarily iron (accounts for $66 \%$ of the mass assuming hematite is the major form) with small amounts of manganese and nickel.

- Some minor metals were not removed effectively ( $<40 \%$ removal) from the dissolution vessel of the RWTE with $2 \mathrm{wt} \%$ OA. For Test 1, these metals included the lanthanides and actinides $\mathrm{Ce}, \mathrm{Gd}, \mathrm{La}, \mathrm{Th}, \mathrm{Eu}, \mathrm{Am}$, and possibly Np. For Test 2, OA was more effective at removing the minor metals and only thorium was removed at a level below $40 \%$. 
- Decomposition of $2 \mathrm{wt} \%$ OA to levels of $<100 \mathrm{ppm}$ using $2 \mathrm{~L} / \mathrm{min}$ of $5 \mathrm{wt} \%$ ozone at $70{ }^{\circ} \mathrm{C}$, a pressure of $8 \mathrm{psi}$, and a liquid recirculation rate of $1.8 \mathrm{gal} / \mathrm{min}$ required 8 to 14 hours for nominal 3.2L batches. The $\mathrm{pH}$ and oxidation/reduction potential (ORP) were tracked during decomposition testing.

- Sludge components were tracked during OA decomposition, showing that most components have the highest soluble levels in the initial dissolved sludge and early decomposition samples and exhibit lower soluble levels as OA decomposition progresses. Samples from the end of the decomposition process typically have the lowest soluble level of sludge components.

- Over the time period studied, the changes to the solubilities of important components in the Deposition Tank storage tests were insignificant. .

- Deposition Tank storage testing for Tank 12H ECC processing indicated higher solubilities for uranium and plutonium when compared with the control condition. Similar testing for Tank 5F indicated higher solubilities for uranium when compared with the control condition. The solubilities of uranium and plutonium remained within the levels expected for Tank Farm high $\mathrm{pH}$ supernatant liquids, which are relatively low compared to the levels in the sludge solids.

- Use of the ECC Evaporator generally led to higher soluble component concentrations in the Deposition Tank storage tests.

- Over the range of 25 to $70{ }^{\circ} \mathrm{C}$, the storage temperature was not a major factor affecting soluble concentrations during the Deposition Tank storage tests.

- Tank 5F sludge/OA slurries, believed to representative of ECC Treatment Tank materials, were not suitable for rheology measurements, due to the fact that most of the sludge particles settled rapidly. Chemical dissolution of these materials is likely necessary for the removal these materials from the treatment tank. Since iron oxide and hydroxide materials are primary chemical constituents for Tank $5 \mathrm{~F}$ sludge, chemical dissolution in OA should be effective.

- Tank 12H sludge/OA slurries, believed to representative of ECC Treatment Tank materials, formed suspensions which were suitable for rheology measurements. Stress versus strain curves observed for these materials revealed that the rheological properties were characteristic of Bingham Plastic materials. For sludge/acid slurries containing 10 weight percent total solids, the measured viscosity and yield stress values were $7 \mathrm{cP}$ and 6 $\mathrm{Pa}$, respectively. Only minor effects on the rheological properties were observed across the temperature range of 30 to $50^{\circ} \mathrm{C}$. Little difference was observed between the process midpoint and endpoint samples. Based on these results, the transfer of significant amounts of Tank $12 \mathrm{H}$ sludge materials from the treatment tank by suspension (rather than dissolution) appears possible.

- Rheology testing of the Tank 5F and $12 \mathrm{H}$ Deposition Tank samples revealed that these slurries were thin with little yield stress. Even the ECC product slurries that were evaporated to remove $\geq 85 \%$ of the water had low enough insoluble solids content as to not exhibit a significant yield stress. 
- Settling of the adjusted Deposition Tank solids was comparable to the control and is fast enough as to not likely delay processing. Only the Tank $5 \mathrm{~F}$ product without $\mathrm{pH}$ adjustment exhibited settling significantly slower than the control sample of sludge that did not go through the ECC process. 


\section{TABLE OF CONTENTS}

LIST OF TABLES xi

LIST OF FIGURES xiv

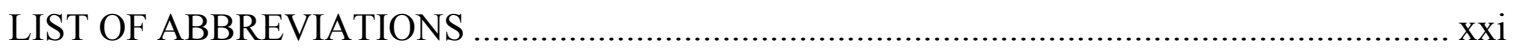

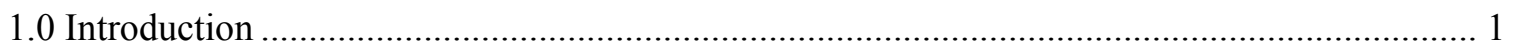

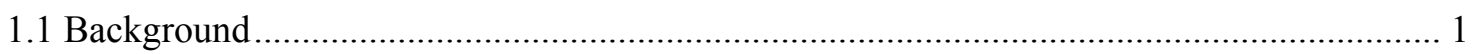

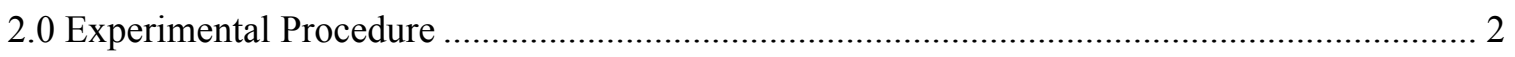

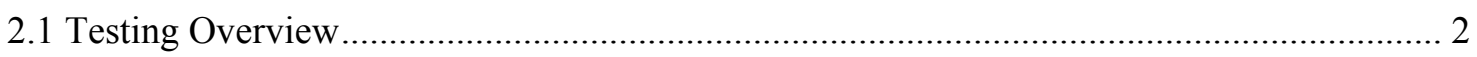

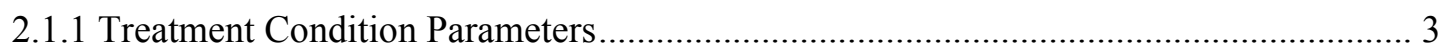

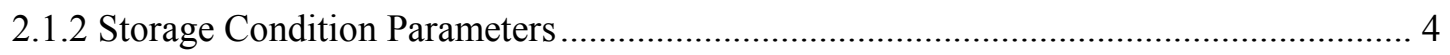

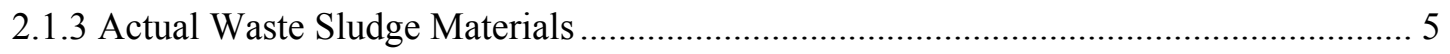

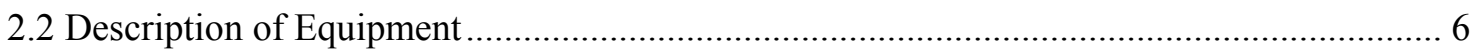

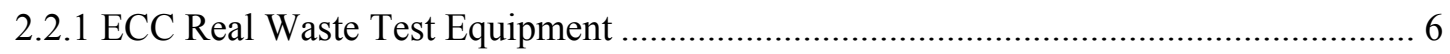

2.2.2 Treatment Tank Residual Sludge Testing for Rheology and Particle Size .................... 9

2.2.3 RWT Equipment and Measurement Calibrations ...................................................... 10

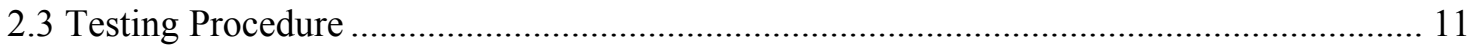

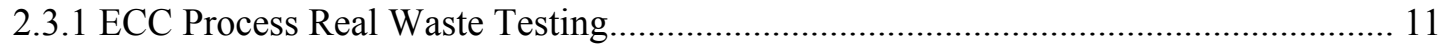

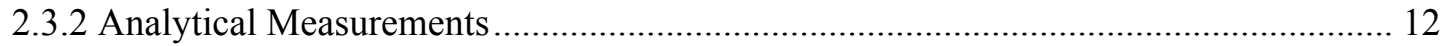

2.3.3 Rheology, Settling Rate, and Particle Size Measurements.......................................... 14

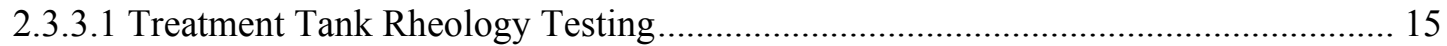

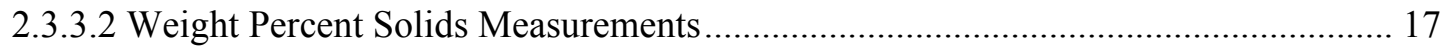

2.3.3.3 Rheology Measurement Equipment, Methodology, and Data Analysis .................... 17

2.3.4 Particle Size and Settling Rate Measurements ............................................................ 19

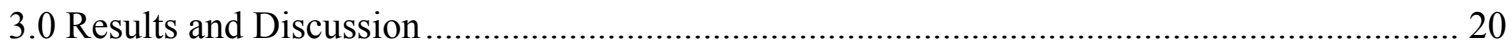

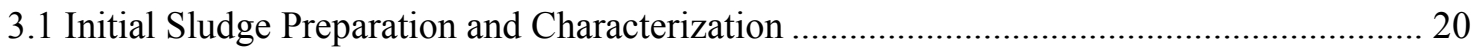

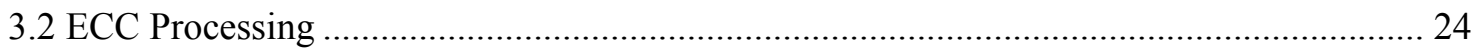

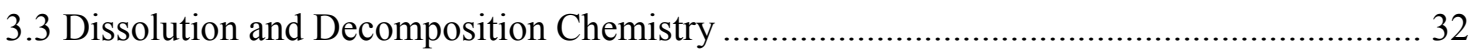

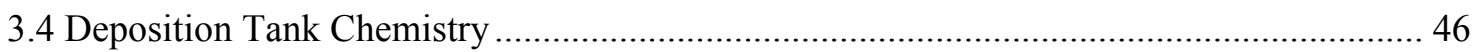

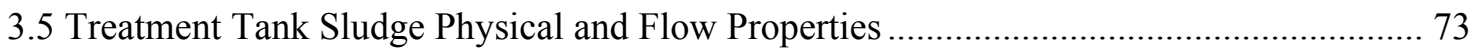

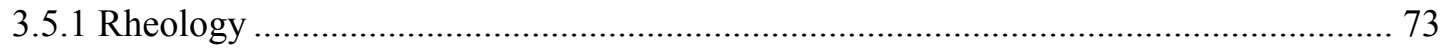

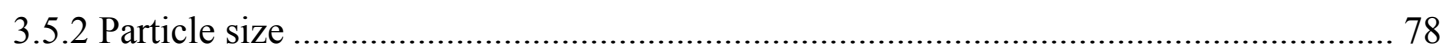

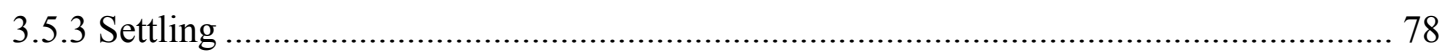

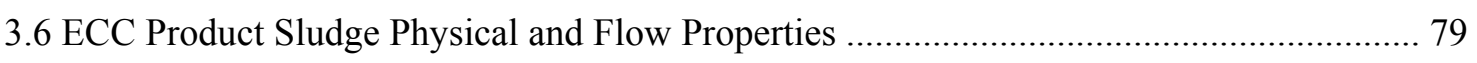

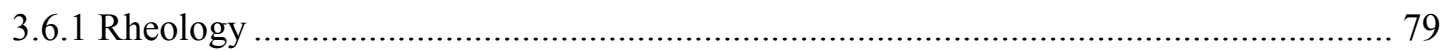




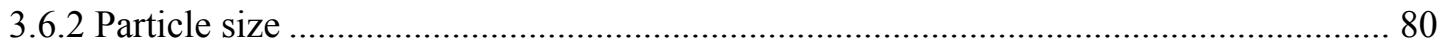

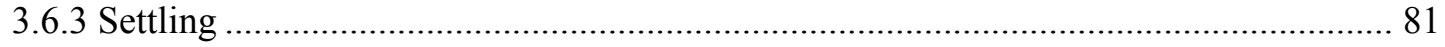

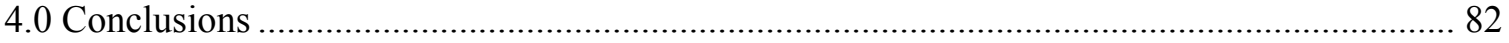

5.0 Recommendations, Path Forward or Future Work ….......................................................... 83

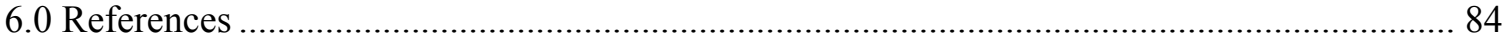

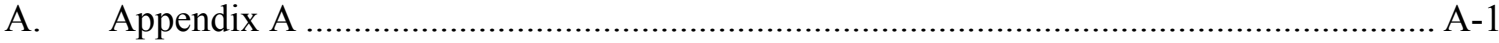

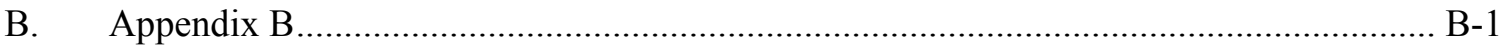




\section{LIST OF TABLES}

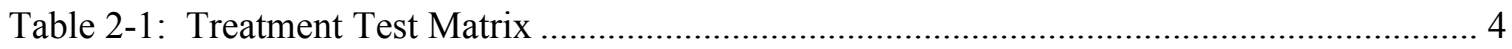

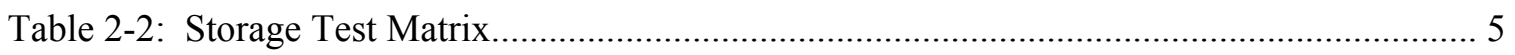

Table 2-3: Nominal Operating Parameters and Targets for ECC Real Waste Testing................. 11

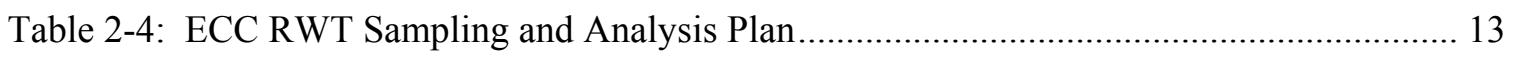

Table 2-5: MV I Rotor Specifications and Flow Curve Program............................................... 19

Table 3-1: Characterization of Tank 5F Sludge Sample Available for ECC Actual-Waste Testing

Table 3-2: Characterization of Tank 5F Sludge Sample Available for ECC Actual-Waste Testing,

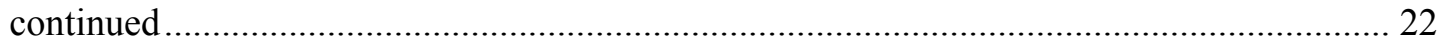

Table 3-3: Characterization of Tank 12H Sludge Sample Available for ECC Actual-Waste

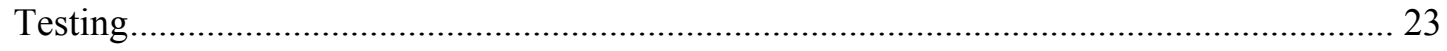

Table 3-4: Characterization of Tank 12H Sludge Sample Available for ECC Actual-Waste

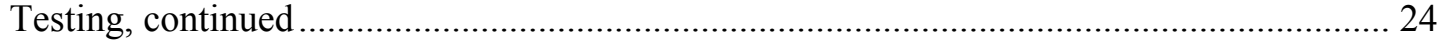

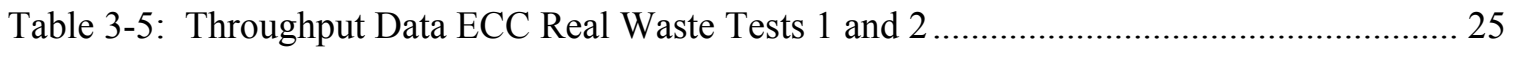

Table 3-6: Residual Sludge in Dissolution Module from Test 1 (Tank 12H) ............................. 33

Table 3-7: Residual Sludge in Dissolution Module from Test 1 (Tank 12H), continued............. 34

Table 3-8: Residual Sludge in Dissolution Module from Test 2 (Tank 5F) ................................ 35

Table 3-9: Residual Sludge in Dissolution Module from Test 2 (Tank 5F), continued ............... 36

Table 3-10: Summary of Soluble Components at Completion of Dissolution Step.................... 36

Table 3-11: Soluble Components throughout ECC Oxalate Decomposition: Test 1 (Tank 12H)

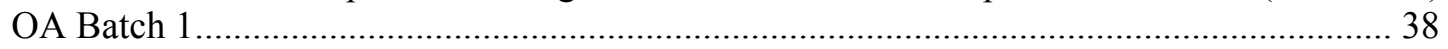

Table 3-12: Soluble Components throughout ECC Oxalate Decomposition: Test 1 (Tank 12H)

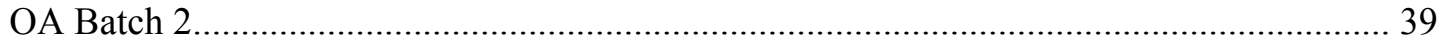

Table 3-13: Soluble Components throughout ECC Oxalate Decomposition: Test 1 (Tank 12H)

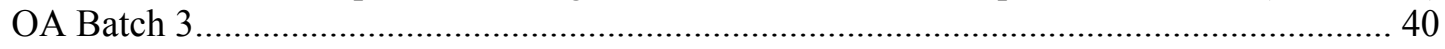

Table 3-14: Soluble Components throughout ECC Oxalate Decomposition: Test 2 (Tank 5F) OA Batch 1

Table 3-15: Soluble Components throughout ECC Oxalate Decomposition: Test 2 (Tank 5F) OA

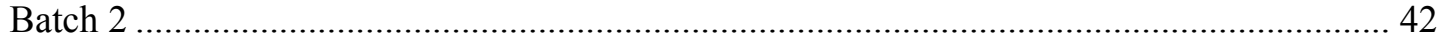

Table 3-16: Soluble Components throughout ECC Oxalate Decomposition: Test 2 (Tank 5F) OA Batch 3 
Table 3-17: Test 1 Dissolution and Decomposition Module Product Slurry Analysis 44

Table 3-18: Test 2 Dissolution and Decomposition Module Product Slurry Analysis 45

Table 3-19: Deposition Tank (Storage Bottle) Conditions for Test 1 47

Table 3-20: Deposition Tank (Storage Bottle) Conditions for Test 2 48

Table 3-21: Soluble Components in the Deposition Tank for the Tank 12H ECC No-Light Test, Storage Condition 1: Without Evaporation, Without Sludge Heel, With pH Adjustment, $\mathrm{T}=$ $50{ }^{\circ} \mathrm{C}$.

Table 3-22: Soluble Components in the Deposition Tank for the Tank 12H ECC No-Light Test, Storage Condition 2: Without Evaporation, With Sludge Heel, With $\mathrm{pH}$ Adjustment, $\mathrm{T}=$ $50{ }^{\circ} \mathrm{C}$.

Table 3-23: Soluble Components in the Deposition Tank for the Tank 12H ECC No-Light Test, Storage Condition 3: Without Evaporation, Without Sludge Heel, Without pH Adjustment, $\mathrm{T}=50^{\circ} \mathrm{C}$.

Table 3-24: Soluble Components in the Deposition Tank for the Tank 12H ECC No-Light Test, Storage Condition 4: With Evaporation, Without Sludge Heel, With $\mathrm{pH}$ Adjustment, $\mathrm{T}=$ $50{ }^{\circ} \mathrm{C}$.

Table 3-25: Soluble Components in the Deposition Tank for the Tank 12H ECC No-Light Test, Storage Condition 5: With Evaporation, With Sludge Heel, With $\mathrm{pH}$ Adjustment, $\mathrm{T}=50^{\circ} \mathrm{C}$.

Table 3-26: Soluble Components in the Deposition Tank for the Tank 12H ECC No-Light Test, Storage Condition 6: With Evaporation, Without Sludge Heel, Without pH Adjustment, $\mathrm{T}=$ $50{ }^{\circ} \mathrm{C}$.

Table 3-27: Soluble Components in the Deposition Tank for the Tank 12H ECC No-Light Test, Storage Condition 7: With Evaporation, With Sludge Heel, With $\mathrm{pH}$ Adjustment, $\mathrm{T}=$ $\sim 25^{\circ} \mathrm{C}$ (ambient).

Table 3-28: Soluble Components in the Deposition Tank for Test 1 (Tank 12H No-Light), Storage Condition 8: With Evaporation, With Sludge Heel, With $\mathrm{pH}$ Adjustment, $\mathrm{T}=70^{\circ} \mathrm{C}$.

Table 3-29: Soluble Components in the Deposition Tank for the Tank 12H Control (without ECC Processing): Without Evaporation, With Sludge Heel, With $\mathrm{pH}$ Adjustment, $\mathrm{T}=50^{\circ} \mathrm{C} \ldots . .59$

Table 3-30: Soluble Components in the Deposition Tank for Test 2 (Tank 5F No-Light), Storage Condition 1: Without Evaporation, Without Sludge Heel, With $\mathrm{pH}$ Adjustment, $\mathrm{T}=50{ }^{\circ} \mathrm{C}$.

Table 3-31: Soluble Components in the Deposition Tank for Test 2 (Tank 5F No-Light), Storage Condition 2: Without Evaporation, With Sludge Heel, With pH Adjustment, $\mathrm{T}=50^{\circ} \mathrm{C} \ldots 61$

Table 3-32: Soluble Components in the Deposition Tank for Test 2 (Tank 5F No-Light), Storage Condition 3: Without Evaporation, Without Sludge Heel, Without $\mathrm{pH}$ Adjustment, $\mathrm{T}=$ $50{ }^{\circ} \mathrm{C}$. 
Table 3-33: Soluble Components in the Deposition Tank for Test 2 (Tank 5F No-Light), Storage Condition 4: With Evaporation, Without Sludge Heel, With pH Adjustment, $\mathrm{T}=50^{\circ} \mathrm{C} \ldots 63$

Table 3-34: Soluble Components in the Deposition Tank for Test 2 (Tank 5F No-Light), Storage Condition 5: With Evaporation, With Sludge Heel, With $\mathrm{pH}$ Adjustment, $\mathrm{T}=50^{\circ} \mathrm{C}$.

Table 3-35: Soluble Components in the Deposition Tank for Test 2 (Tank 5F No-Light), Storage Condition 6: With Evaporation, Without Sludge Heel, Without $\mathrm{pH}$ Adjustment, $\mathrm{T}=50^{\circ} \mathrm{C}$.

Table 3-36: Soluble Components in the Deposition Tank for Test 2 (Tank 5F No-Light), Storage Condition 7: Without Evaporation, With Sludge Heel, With pH Adjustment, $\mathrm{T}=\sim 25{ }^{\circ} \mathrm{C}$ (ambient).

Table 3-37: Soluble Components in the Deposition Tank for Test 2 (Tank 5F No-Light), Storage Condition 8: Without Evaporation, With Sludge Heel, With pH Adjustment, $\mathrm{T}=70^{\circ} \mathrm{C} \ldots 67$

Table 3-38: Soluble Components in the Deposition Tank for the Tank 5F Control (without ECC Processing): Without Evaporation, With Sludge Heel, With $\mathrm{pH}$ Adjustment, $\mathrm{T}=50^{\circ} \mathrm{C} \ldots . .68$

Table 3-39: Deposition Tank Storage Condition Summary for Soluble U-238 (mg/L)..... 69

Table 3-40: Deposition Tank Storage Condition Summary for Soluble Pu-238 (dpm/mL) 69

Table 3-41: Deposition Tank Storage Condition Summary for Soluble Iron $(\mathrm{mg} / \mathrm{L})$................ 70

Table 3-42: Deposition Tank Storage Condition Summary for Soluble Aluminum (mg/L)........ 70

Table 3-43: Analysis of Test 1 Deposition Tank Solids, Wet Basis ............................................ 71

Table 3-44: Analysis of Test 2 Deposition Tank Solids, Wet Basis ......................................... 72

Table 3-45: Rheology Data for $2 \mathrm{wt} \%$ OA Solution at Various Temperatures............................ 75

Table 3-46: Rheology Data for Tank 5F Sludge/OA (2 wt\%) Slurry.......................................... 75

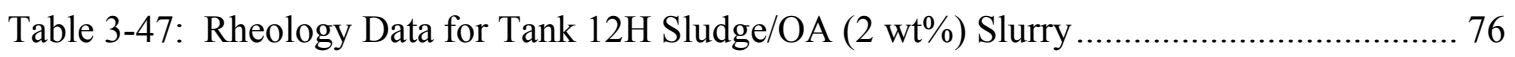

Table A-1: Oxalic Acid Decomposition (without actual tank waste sludge) ........................... A-1

Table A-2: Oxalic Acid Decomposition (without actual tank waste sludge), continued ........... A-2

Table A-3: Test 1 (Tank 12H) OA Batch 1 Dissolution Module Data..................................... A-3

Table A-4: Test 1 (Tank 12H Sludge) OA Batch 1 Decomposition Module Data...................... A-4

Table A-5: Test 1 (Tank 12H) OA Batch 1 Evaporator Module Data ...................................... A-5

Table A-6: Test 1 (Tank 12H) OA Batch 2 Dissolution Module Data...................................... A-5

Table A-7: Test 1 (Tank 12H Sludge) OA Batch 2 Decomposition Module Data..................... A-6

Table A-8: Test 1 (Tank 12H Sludge) OA Batch 2 Decomposition Module Data, continued... A-7

Table A-9: Test 1 (Tank 12H) OA Batch 2 Evaporator Module Data A-8 
Table A-10: Test 1 (Tank 12H) OA Batch 3 Dissolution Module Data................................... A-8

Table A-11 Test 1 (Tank 12H Sludge) OA Batch 3 Decomposition Module Data.

Table A-12: Test 1 (Tank12H Sludge) OA Batch 3 Decomposition Module Data, continued A-10

Table A-13: Test 1 (Tank12H) OA Batch 3 Evaporator Module Data A-11

Table A-14: Test 2 (Tank 5F) OA Batch 1 Dissolution Module Data A-11

Table A-15: Test 2 (Tank 5F Sludge) OA Batch 1 Decomposition Module Data A-12

Table A-16: Test 2 (Tank 5F Sludge) OA Batch 1 Decomposition Module Data, continued.. A-13

Table A-17: Test 2 (Tank 5F) OA Batch 1 Evaporator Module Data A-14

Table A-18: Test 2 (Tank 5F) OA Batch 2 Dissolution Module Data A-14

Table A-19: Test 2 (Tank 5F Sludge) OA Batch 2 Decomposition Module Data A-15

Table A-20: Test 2 (Tank 5F Sludge) OA Batch 2 Decomposition Module Data, continued.. A-16

Table A-21: Test 2 (Tank 5F) OA Batch 2 Evaporator Module Data A-17

Table A-22: Test 2 (Tank 5F) OA Batch 3 Dissolution Module Data A-17

Table A-23: Test 2 (Tank 5F Sludge) OA Batch 3 Decomposition Module Data A-18

Table A-24: Test 2 (Tank 5F Sludge) OA Batch 3 Decomposition Module Data, continued.. A-19 Table A-25: Test 2 (Tank 5F) OA Batch 3 Evaporator Module Data A-20

\section{LIST OF FIGURES}

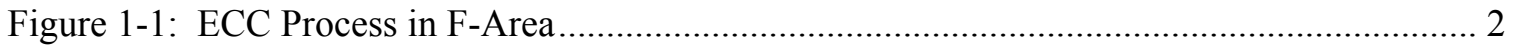

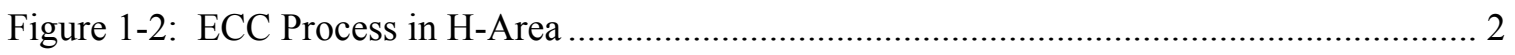

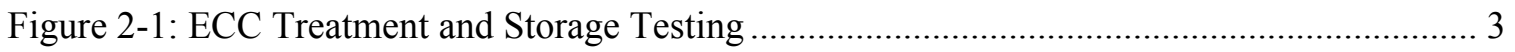

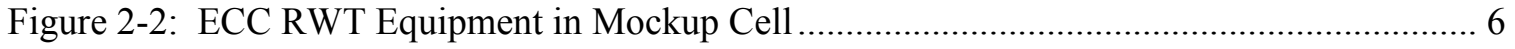

Figure 2-3: Schematic of ECC RWT Equipment Main Cells Skid (roughly related to the equipment elevation photograph of Figure 2-2) ................................................................... 7

Figure 2-4: ECC RWT Equipment in SRNL High Level Shielded Cells, B-Block Cell 11 .......... 8

Figure 2-5: ECC RWT Equipment in the Shielded Cells Operating Area (left) and Ozone Generator behind B-Block Cells (right).

Figure 2-6: Key process stages for rheology, particle size and/or settling behavior testing ........ 14

Figure 3-1: Sludge remaining in Dissolution Module basket after Test 1, OA Batch 1.............. 27 
Figure 3-2: Trends for $\mathrm{pH}$ and ORP during Decomposition of Oxalic Acid by Ozone ............... 28

Figure 3-3: Trends for $\mathrm{pH}$ and ORP during Decomposition Test 1 (Tank 12H) OA Batch 1...... 29

Figure 3-4: Trends for $\mathrm{pH}$ and ORP during Decomposition Test 1 (Tank 12H) OA Batch 2..... 29

Figure 3-5: Trends for $\mathrm{pH}$ and ORP during Decomposition Test 1 (Tank 12H) OA Batch 3..... 30

Figure 3-6: Trends for $\mathrm{pH}$ and ORP during Decomposition Test 2 (Tank 5F) OA Batch 1 ......... 30

Figure 3-7: Trends for $\mathrm{pH}$ and ORP during Decomposition Test 2 (Tank 5F) OA Batch 2 ........ 31

Figure 3-8: Trends for $\mathrm{pH}$ and ORP during Decomposition Test 2 (Tank 5F) OA Batch 3 ........ 31

Figure 3-9: Samples of Decomposition Module during Test 1, OA Batch 2. ............................. 37

Figure 3-10: Rheology Results for the Concentrated Tank 12H Slurry Midpoint Sample at $40{ }^{\circ} \mathrm{C}$. 77

Figure 3-11: Rheology Results for the Concentrated Tank 12H Slurry Endpoint Sample at $40{ }^{\circ} \mathrm{C}$.

Figure 3-12: Tank 5F Sludge Intermediate Dissolution Sample, a Graduated Cylinder with the Fluid Portion After 8 Minutes of Settling (left) and the Fast-Settling Portion Not Removed

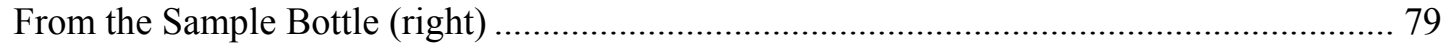

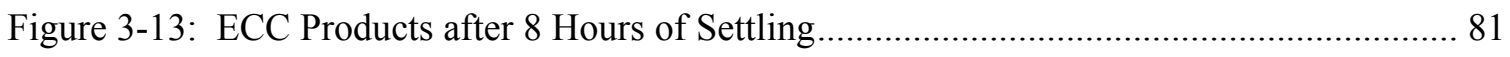

Figure B-1: Stress Versus Strain Curve for Tank 5F Baseline Dilute at $30^{\circ} \mathrm{C}$ (First Replicate). B2

Figure B-2: Stress Versus Strain Curve for Tank 5F Baseline Dilute at $30^{\circ} \mathrm{C}$ (Second Replicate). B-2

Figure B-3: Stress Versus Strain Curve for Tank 5F Baseline Dilute at $40^{\circ} \mathrm{C}$ (First Replicate). B3

Figure B-4: Stress Versus Strain Curve for Tank 5F Baseline Dilute at $40{ }^{\circ} \mathrm{C}$ (Second Replicate).

Figure B-5: Stress Versus Strain Curve for Tank 5F Baseline Dilute at $50^{\circ} \mathrm{C}$ (First Replicate). B4

Figure B-6: Stress Versus Strain Curve for Tank 5F Baseline Dilute at $50{ }^{\circ} \mathrm{C}$ (Second Replicate). B-4

Figure B-7: Stress Versus Strain Curve for Tank 5F Baseline Concentrate at $30{ }^{\circ} \mathrm{C}$ (First Replicate).

Figure B-8: Stress Versus Strain Curve for Tank 5F Baseline Concentrate at $30{ }^{\circ} \mathrm{C}$ (Second

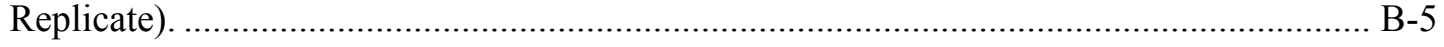

Figure B-9: Stress Versus Strain Curve for Tank 5F Baseline Concentrate at $40{ }^{\circ} \mathrm{C}$ (First Replicate) B-6 
Figure B-10: Stress Versus Strain Curve for Tank 5F Baseline Concentrate at $40{ }^{\circ} \mathrm{C}$ (Second Replicate). B-6

Figure B-11: Stress Versus Strain Curve for Tank 5F Baseline Concentrate at $50{ }^{\circ} \mathrm{C}$ (First Replicate). B-7

Figure B-12: Stress Versus Strain Curve for Tank 5F Baseline Concentrate at $50{ }^{\circ} \mathrm{C}$ (Second Replicate). B-7

Figure B-13: Stress Versus Strain Curve for Tank 5F Midpoint Concentrate at $30{ }^{\circ} \mathrm{C}$ (First Replicate). B-8

Figure B-14: Stress Versus Strain Curve for Tank 5F Midpoint Concentrate at $30{ }^{\circ} \mathrm{C}$ (Second Replicate). B-8

Figure B-15: Stress Versus Strain Curve for Tank 5F Endpoint Concentrate at $30{ }^{\circ} \mathrm{C}$ (First Replicate). B-9

Figure B-16: Stress Versus Strain Curve for Tank 5F Endpoint Concentrate at $30{ }^{\circ} \mathrm{C}$ (Second Replicate).

Figure B-17: Stress Versus Strain Curve for Tank 12H Baseline Dilute at $30{ }^{\circ} \mathrm{C}$ (First Replicate) B-10

Figure B-18: Stress Versus Strain Curve for Tank $12 \mathrm{H}$ Baseline Dilute at $30{ }^{\circ} \mathrm{C}$ (Second Replicate) B-10

Figure B-19: Stress Versus Strain Curve for Tank 12H Baseline Dilute at $40{ }^{\circ} \mathrm{C}$ (First Replicate) B-11

Figure B-20: Stress Versus Strain Curve for Tank $12 \mathrm{H}$ Baseline Dilute at $40{ }^{\circ} \mathrm{C}$ (Second Replicate) B-11

Figure B-21: Stress Versus Strain Curve for Tank 12H Baseline Dilute at $50{ }^{\circ} \mathrm{C}$ (First Replicate) B-12

Figure B-22: Stress Versus Strain Curve for Tank $12 \mathrm{H}$ Baseline Dilute at $50{ }^{\circ} \mathrm{C}$ (Second Replicate) B-12

Figure B-23: Stress Versus Strain Curve for Tank 12H Baseline Dilute at $30^{\circ} \mathrm{C}$ after 1 Day (First Replicate) B-13

Figure B-24: Stress Versus Strain Curve for Tank $12 \mathrm{H}$ Baseline Dilute at $30{ }^{\circ} \mathrm{C}$ after 1 Day (Second Replicate) B-13

Figure B-25: Stress Versus Strain Curve for Tank 12H Baseline Dilute at $40{ }^{\circ} \mathrm{C}$ after 1 Day (First Replicate) B-14

Figure B-26: Stress Versus Strain Curve for Tank $12 \mathrm{H}$ Baseline Dilute at $40{ }^{\circ} \mathrm{C}$ after 1 Day (Second Replicate) B-14

Figure B-27: Stress Versus Strain Curve for Tank 12H Baseline Concentrate at $30{ }^{\circ} \mathrm{C}$ (First Replicate) B-15 
Figure B-28: Stress Versus Strain Curve for Tank 12H Baseline Concentrate at $30{ }^{\circ} \mathrm{C}$ (Second Replicate) B-15

Figure B-29: Stress Versus Strain Curve for Tank 12H Baseline Concentrate at $40{ }^{\circ} \mathrm{C}$ (First Replicate) B-16

Figure B-30: Stress Versus Strain Curve for Tank 12H Baseline Concentrate at $40{ }^{\circ} \mathrm{C}$ (Second Replicate) B-16

Figure B-31: Stress Versus Strain Curve for Tank 12H Baseline Concentrate at $50{ }^{\circ} \mathrm{C}$ (First Replicate) B-17

Figure B-32: Stress Versus Strain Curve for Tank 12H Baseline Concentrate at $50{ }^{\circ} \mathrm{C}$ (Second Replicate) B-17

Figure B-33: Stress Versus Strain Curve for Tank $12 \mathrm{H}$ Baseline Concentrate at $30{ }^{\circ} \mathrm{C}$ after 4 Days (First Replicate) B-18

Figure B-34: Stress Versus Strain Curve for Tank 12H Baseline Concentrate at $30{ }^{\circ} \mathrm{C}$ after 4 Days (Second Replicate) B-18

Figure B-35: Stress Versus Strain Curve for Tank 12H Midpoint Dilute at $30^{\circ} \mathrm{C}$ (First Replicate) B-19

Figure B-36: Stress Versus Strain Curve for Tank $12 \mathrm{H}$ Midpoint Dilute at $30{ }^{\circ} \mathrm{C}$ (Second Replicate) B-19

Figure B-37: Stress Versus Strain Curve for Tank 12H Midpoint Dilute at $40{ }^{\circ} \mathrm{C}$ (First Replicate)

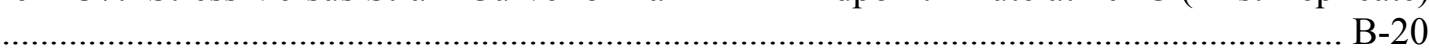

Figure B-38: Stress Versus Strain Curve for Tank 12H Midpoint Dilute at $40{ }^{\circ} \mathrm{C}$ (Second Replicate) B-20

Figure B-39: Stress Versus Strain Curve for Tank 12H Midpoint Dilute at $50{ }^{\circ} \mathrm{C}$ (First Replicate) B-21

Figure B-40: Stress Versus Strain Curve for Tank 12H Midpoint Dilute at $50{ }^{\circ} \mathrm{C}$ (Second Replicate) B-21

Figure B-41: Stress Versus Strain Curve for Tank 12H Midpoint Concentrate at $30{ }^{\circ} \mathrm{C}$ (First Replicate)

Figure B-42: Stress Versus Strain Curve for Tank 12H Midpoint Concentrate at $30{ }^{\circ} \mathrm{C}$ (Second Replicate) B-22

Figure B-43: Stress Versus Strain Curve for Tank 12H Midpoint Concentrate at $40{ }^{\circ} \mathrm{C}$ (First Replicate) B-23

Figure B-44: Stress Versus Strain Curve for Tank 12H Midpoint Concentrate at $40{ }^{\circ} \mathrm{C}$ (Second Replicate) B-23

Figure B-45: Stress Versus Strain Curve for Tank 12H Midpoint Concentrate at $50{ }^{\circ} \mathrm{C}$ (First Replicate) B-24 
Figure B-46: Stress Versus Strain Curve for Tank 12H Midpoint Concentrate at $50{ }^{\circ} \mathrm{C}$ (Second Replicate) B-24

Figure B-47: Stress Versus Strain Curve for Tank 12H Endpoint Dilute at $30{ }^{\circ} \mathrm{C}$ (First Replicate) B-25

Figure B-48: Stress Versus Strain Curve for Tank 12H Endpoint Dilute at $30{ }^{\circ} \mathrm{C}$ (Second Replicate) B-25

Figure B-49: Stress Versus Strain Curve for Tank 12H Endpoint Dilute at $40{ }^{\circ} \mathrm{C}$ (First Replicate) B-26

Figure B-50: Stress Versus Strain Curve for Tank 12H Endpoint Dilute at $40{ }^{\circ} \mathrm{C}$ (Second Replicate) B-26

Figure B-51: Stress Versus Strain Curve for Tank 12H Endpoint Dilute at $50{ }^{\circ} \mathrm{C}$ (First Replicate)

Figure B-52: Stress Versus Strain Curve for Tank 12H Endpoint Dilute at $50{ }^{\circ} \mathrm{C}$ (Second Replicate) B-27

Figure B-53: Stress Versus Strain Curve for Tank 12H Endpoint Concentrate at $30{ }^{\circ} \mathrm{C}$ (First Replicate) B-28

Figure B-54: Stress Versus Strain Curve for Tank 12H Endpoint Concentrate at $30{ }^{\circ} \mathrm{C}$ (Second Replicate) B-28

Figure B-55: Stress Versus Strain Curve for Tank 12H Endpoint Concentrate at $40{ }^{\circ} \mathrm{C}$ (First Replicate) B-29

Figure B-56: Stress Versus Strain Curve for Tank 12H Endpoint Concentrate at $40{ }^{\circ} \mathrm{C}$ (Second Replicate) B-29

Figure B-57: Stress Versus Strain Curve for Tank 12H Endpoint Concentrate at $50{ }^{\circ} \mathrm{C}$ (First Replicate) B-30

Figure B-58: Stress Versus Strain Curve for Tank 12H Endpoint Concentrate at $50{ }^{\circ} \mathrm{C}$ (Second Replicate) B-30

Figure B-59: Stress Versus Strain Curve for Tank 5F No Evaporation Deposition Sample at 30 ${ }^{\circ} \mathrm{C}$ (First Replicate). B-31

Figure B-60: Stress Versus Strain Curve for Tank 5F No Evaporation Deposition Sample at 30 ${ }^{\circ} \mathrm{C}$ (Second Replicate).... B-31

Figure B-61: Stress Versus Strain Curve for Tank 5F No Evaporation Deposition Sample at 45 ${ }^{\circ} \mathrm{C}$ (First Replicate). B-32

Figure B-62: Stress Versus Strain Curve for Tank 5F No Evaporation Deposition Sample at 45 ${ }^{\circ} \mathrm{C}$ (Second Replicate) B-32

Figure B-63: Stress Versus Strain Curve for Tank 5F Evaporated Deposition Sample at $30{ }^{\circ} \mathrm{C}$ (First Replicate) B-33 
Figure B-64: Stress Versus Strain Curve for Tank 5F Evaporated Deposition Sample at $30{ }^{\circ} \mathrm{C}$ (Second Replicate)

Figure B-65: Stress Versus Strain Curve for Tank 5F Evaporated Deposition Sample at $45^{\circ} \mathrm{C}$ (First Replicate)

Figure B-66: Stress Versus Strain Curve for Tank 5F Evaporated Deposition Sample at $45{ }^{\circ} \mathrm{C}$ (Second Replicate) B-34

Figure B-67: Stress Versus Strain Curve for Tank 12H No Evaporation Deposition Sample at 30 ${ }^{\circ} \mathrm{C}$ (First Replicate).

Figure B-68: Stress Versus Strain Curve for Tank 12H No Evaporation Deposition Sample at 30 ${ }^{\circ} \mathrm{C}$ (Second Replicate) B-35

Figure B-69: Stress Versus Strain Curve for Tank 12H No Evaporation Deposition Sample at 45 ${ }^{\circ} \mathrm{C}$ (First Replicate). B-36

Figure B-70: Stress Versus Strain Curve for Tank 12H No Evaporation Deposition Sample at 45 ${ }^{\circ} \mathrm{C}$ (Second Replicate) B-36

Figure B-71: Stress Versus Strain Curve for Tank 12H Evaporated Deposition Sample at $30{ }^{\circ} \mathrm{C}$ (First Replicate) B-37

Figure B-72: Stress Versus Strain Curve for Tank 12H Evaporated Deposition Sample at $30{ }^{\circ} \mathrm{C}$ (Second Replicate) B-37

Figure B-73: Stress Versus Strain Curve for Tank 12H Evaporated Deposition Sample at $45^{\circ} \mathrm{C}$ (First Replicate) B-38

Figure B-74: Stress Versus Strain Curve for Tank 12H Evaporated Deposition Sample at $45^{\circ} \mathrm{C}$ (Second Replicate) B-38

Figure B-75: Particle Size Analysis for Tank 5F Sludge Prior to Contact with OA..... B-39

Figure B-76: Particle Size Analysis for Tank 12H Sludge Prior to Contact with OA B-40

Figure B-77: Particle Size Analysis of Intermediate Treatment Tank Heel after Dissolution of Tank 5F Sludge with One OA Batch B-41

Figure B-78: Particle Size Analysis of Intermediate Treatment Tank Heel after Dissolution of Tank 12H Sludge with One OA Batch. B-42

Figure B-79: Particle Size Analysis of Final Treatment Tank Heel after Dissolution of Tank 5F Sludge with Two OA Batches..... B-43

Figure B-80: Particle Size Analysis of Final Treatment Tank Heel after Dissolution of Tank 12H Sludge with Two OA Batches. B-44

Figure B-81: Particle Size Analysis of Test 1 (Tank 12H) Storage Condition 3 (without evaporation, without $\mathrm{pH}$ adjustment, and without sludge heel), representing ECC product prior to $\mathrm{pH}$ adjustment and evaporation. B-45 
Figure B-82: Particle Size Analysis of Test 1 (Tank 12H) Storage Condition 5 (with evaporation, with $\mathrm{pH}$ adjustment, and with sludge heel), representing ECC product after evaporation (Harea baseline) and at storage tank conditions. B-46

Figure B-83: Particle Size Analysis of Test 1 (Tank 12H) Storage Condition 6 (with evaporation, without $\mathrm{pH}$ adjustment, and without sludge heel), representing ECC product just after evaporation and prior to $\mathrm{pH}$ adjustment. B-47

Figure B-84: Particle Size Analysis of Test 1 (Tank 12H) Control (Storage Condition 9) (without evaporation, with $\mathrm{pH}$ adjustment, and with sludge heel), representing the original Tank $12 \mathrm{H}$ sludge with $\mathrm{pH}$ adjustment and heel in deposition tank. B-48

Figure B-85: Particle Size Analysis of Test 2 (Tank 5F) Storage Condition 2 (without evaporation, with $\mathrm{pH}$ adjustment, and with sludge heel), representing ECC product without evaporation (F-area baseline) and at storage tank conditions. B-49

Figure B-86: Particle Size Analysis of Test 2 (Tank 5F) Storage Condition 3 (without evaporation, without $\mathrm{pH}$ adjustment, and without sludge heel), representing ECC product prior to $\mathrm{pH}$ adjustment and evaporation. B-50

Figure B-87: Particle Size Analysis of Test 2 (Tank 5F) Storage Condition 6 (with evaporation, without $\mathrm{pH}$ adjustment, and without sludge heel), representing ECC product just after evaporation and prior to $\mathrm{pH}$ adjustment. B-51

Figure B-88: Particle Size Analysis of Test 2 (Tank 5F) Control (Storage Condition 9) (without evaporation, with $\mathrm{pH}$ adjustment, and with sludge heel), representing the original Tank $12 \mathrm{H}$ sludge with $\mathrm{pH}$ adjustment and heel in deposition tank B-52

Figure B-89: ECC Product Settling (part 1 of 4) B-53

Figure B-90: ECC Product Settling (part 2 of 4).... B-54

Figure B-91: ECC Product Settling (part 3 of 4) B-55

Figure B-92: ECC Product Settling (part 4 of 4).... B-56 


\section{LIST OF ABBREVIATIONS}

$\begin{array}{ll}\text { CP } & \text { Valve prefix } \\ \text { CV AA } & \text { Cold Vapor Atomic Adsorption } \\ \text { ECC } & \text { Enhanced Chemical Cleaning } \\ \text { FM } & \text { Flow Measurement prefix } \\ \text { HLW } & \text { High-Level Waste } \\ \text { IC } & \text { Ion Chromatography } \\ \text { ICP-ES } & \text { Inductively Coupled Plasma -- Emission Spectroscopy } \\ \text { ICP-MS } & \text { Inductively Coupled Plasma -- Mass Spectrometry } \\ \text { M\&TE } & \text { Measurement and Test Equipment } \\ \text { NIST } & \text { National Institute for Standards and Technology } \\ \text { OA } & \text { oxalic acid } \\ \text { ODU } & \text { Ozone Destruction Unit } \\ \text { ORP } & \text { Oxidation / Reduction Potential } \\ \text { PI } & \text { Pressure Indicator prefix } \\ \text { PMP } & \text { Polymethylpentene } \\ \text { PTFE } & \text { Polytetrafluoroethalene } \\ \text { PuTTA } & \text { Plutonium separation by thenoyltrifluoracetone } \\ \text { RWT } & \text { Real Waste Testing } \\ \text { SC } & \text { Storage Condition } \\ \text { SRNL } & \text { Savannah River National Laboratory } \\ \text { SRR } & \text { Savannah River Remediation } \\ \text { SRS } & \text { Savannah River Site } \\ \text { TI } & \text { Temperature Indicator prefix } \\ \text { TOC } & \text { Total Organic Carbon } \\ \text { TTQAP } & \text { Task Technical and Quality Assurance Plan } \\ \text { TTR } & \text { Technical Task Request } \\ \text { UV } & \text { Ultraviolet } \\ & \end{array}$




\subsection{Introduction}

Forty three of the High Level Waste (HLW) tanks at the Savannah River Site (SRS) have internal structures that hinder removal of the last approximately five thousand gallons of waste sludge solely by mechanical means. Chemical cleaning can be utilized to dissolve the sludge heel with oxalic acid (OA) and pump the material to a separate waste tank in preparation for final disposition. ${ }^{1,2}$ This dissolved sludge material is $\mathrm{pH}$ adjusted downstream of the dissolution process, precipitating the sludge components along with sodium oxalate solids. The large quantities of sodium oxalate and other metal oxalates formed impact downstream processes by requiring additional washing during sludge batch preparation and increase the amount of material that must be processed in the tank farm evaporator systems and the Saltstone Processing Facility. Enhanced Chemical Cleaning (ECC) was identified as a potential method for greatly reducing the impact of oxalate additions to the SRS Tank Farms without adding additional components to the waste that would extend processing or increase waste form volumes. ${ }^{34}$

In support of Savannah River Site (SRS) tank closure efforts, the Savannah River National Laboratory (SRNL) conducted Real Waste Testing (RWT) to evaluate an alternative to the baseline 8 wt. \% OA chemical cleaning technology for tank sludge heel removal. The baseline OA technology results in the addition of significant volumes of oxalate salts to the SRS tank farm and there is insufficient space to accommodate the neutralized streams resulting from the treatment of the multiple remaining waste tanks requiring closure.

ECC is a promising alternative to bulk OA cleaning, which utilizes a more dilute OA (nominally 2 wt. $\%$ at a $\mathrm{pH}$ of around 2) and an oxalate destruction technology. The technology is being adapted by AREVA from their decontamination technology for Nuclear Power Plant secondary side scale removal.

This report contains results from the SRNL small scale testing of the ECC process using SRS sludge tank sample material. A Task Technical and Quality Assurance Plan (TTQAP) details the experimental plan as outlined by the Technical Task Request (TTR). ${ }^{6}$ The TTR identifies that the data produced by this testing and results included in this report will support the technical baseline with portions having a safety class functional classification. The primary goals for SRNL RWT are as follows:

- to confirm ECC performance with real tank sludge samples,

- to determine the impact of ECC on fate of actinides and the other sludge metals, and

- to determine changes, if any, in solids flow and settling behavior.

\subsection{Background}

The ECC process supports closure of non-compliant waste tanks at SRS by removing the residual sludge heels in the tanks. Sludge is dissolved in a Treatment Tank by adding $2 \mathrm{wt} \%$ OA and mixing the tank contents. This mixture of dissolved sludge and suspended insoluble solids is transferred to the ECC reactor, where the OA and other oxalates are decomposed. Concentrated ozone gas is added to the reactor, resulting in soluble ozone and hydroxyl radicals that aid the decomposition of oxalate ion to carbon dioxide gas and raise the $\mathrm{pH}$ of the liquid. The change in chemistry inside the ECC reactor causes the dissolved sludge to precipitate, mostly in the form of metal oxides/hydroxides. The supplemental use of UV light in the ECC reactor holds potential 
for increasing the oxalate decomposition but it is not included in the current baseline process design.

For F-Area tanks, the treated waste is transferred to a Deposition Tank where the solids will be allowed to settle, followed by water removal via the $2 \mathrm{~F}$ evaporator (Figure 1-1). The planned treatment of H-Area tanks includes a flash evaporator to recover and recycle water (Figure 1-2). Sodium hydroxide will be added in-line to both the F-Area and H-Area stream during the transfer to the Deposition Tank to ensure compliance with the Corrosion Control Program requirements.

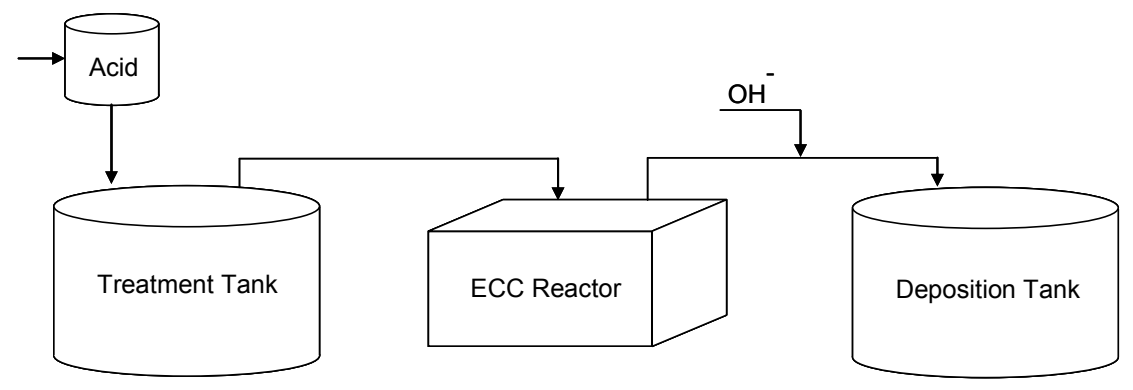

Figure 1-1: ECC Process in F-Area

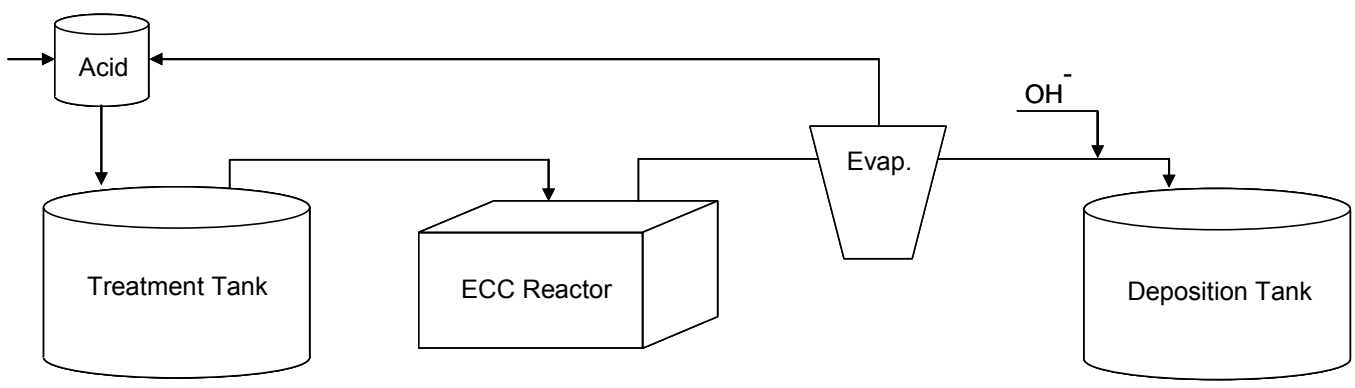

Figure 1-2: ECC Process in H-Area

\subsection{Experimental Procedure}

\subsection{Testing Overview}

The ECC RWT is designed to demonstrate the following:

- The use of ozone (with or without the use of UV light) will be effective for decomposing OA used for actual waste sludge dissolution.

- The solubility of some metals will change during the dissolution and the oxalate decomposition compared to nominal sludge storage conditions.

- The solubilities of metals and actinides in the ECC reactor product or evaporator product will return to levels comparable with the nominal sludge storage condition when brought back into Tank Farm corrosion control compliance (via contact with tank heel material and/or $\mathrm{pH}$ adjustment with sodium hydroxide) over a time period of hours to days. 


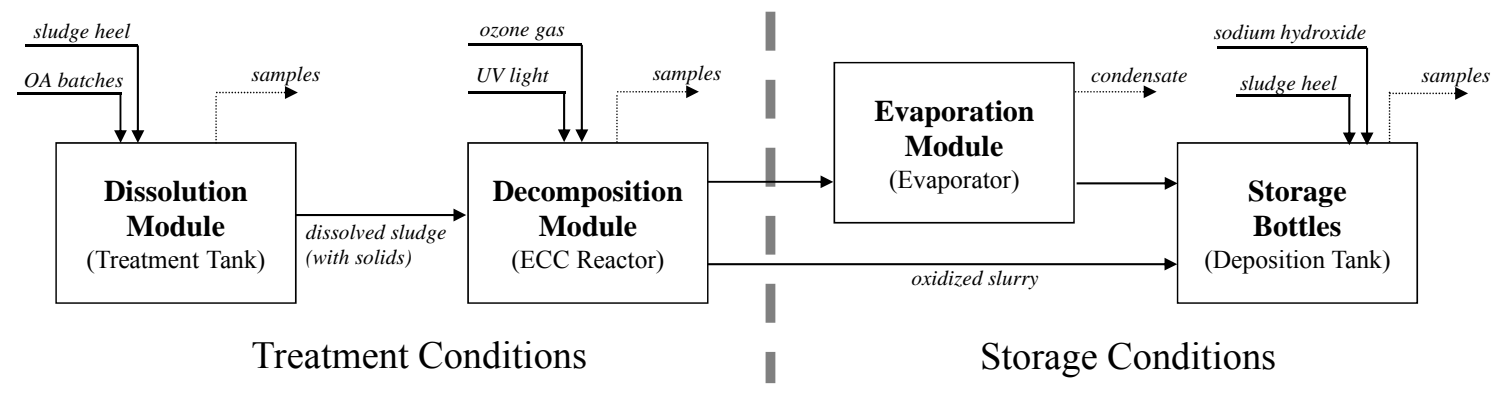

Figure 2-1: ECC Treatment and Storage Testing

The ECC RWT matrix is divided into two conceptual sections, treatment conditions and storage conditions. This distinction between the two types of testing is necessary because the tests will be performed concurrently, while involving two separate test matrices. Figure 2-1 illustrates the portions of the process affected by each matrix. The treatment conditions matrix pertains to the conditions and the samples from the Dissolution and Decomposition Modules. The storage conditions matrix pertains to the Deposition Tank conditions and related samples from the storage bottles.

\subsubsection{Treatment Condition Parameters}

Overall, four tests were planned at various treatment conditions, as shown in Table 2-1. Tests 1 and 2 are the process baseline cases, which do not use UV light to aid in the decomposition of oxalates. Tests 3 and 4, which use UV light to augment the decomposition of oxalate, are planned for a future report.

Each test was initiated by loading either 48.1 grams (Tank $12 \mathrm{H}$ ) or 65.1 grams (Tank 5F) of actual waste sludge solids into the Dissolution Module basket. A series of nominally $3.2 \mathrm{~L}$ batches of $2 \mathrm{wt} \%$ OA were added to the Dissolution Module and heated to the dissolution temperature. The nominal dissolution batch duration was eight hours at temperature. Temperature and $\mathrm{pH}$ were recorded periodically. At the end of eight hours, a sample was taken and the material was transferred forward to the Decomposition Module.

Three OA batches were used for each test with the goal of $95 \%$ sludge removal from the Dissolution Module. Based on the equipment design, it was only possible to quantify the amount of undissolved sludge remaining in the Dissolution Module basket at the end of each OA batch. Approximations of sludge removal were made from the amount of material collected from the Dissolution Module. The dissolution batches were expected to transfer some undissolved sludge solids forward to the Decomposition Module. Thus, removal of sludge material from the Dissolution Module does not directly equate to dissolution of sludge materials.

The Decomposition Module was operated using the methodology recommended by AREVA and adapted as necessary by SRNL. ${ }^{7}$ The flow of ozone into the system through the eductor was balanced to the recirculation of the dissolved sludge material. The Decomposition Module operating temperature was $70{ }^{\circ} \mathrm{C}$ and the expected decomposition duration was 6 to 20 hours. The Decomposition Module vessel included measurement of temperature, $\mathrm{pH}$, and ORP. Throughout Decomposition Module operations, several intermediate liquid or slurry samples were collected from the recirculation loop for preparation and analysis. 
SRNL-STI-2011-00360

Revision 0

Table 2-1: Treatment Test Matrix

\begin{tabular}{c|c|c|c|c}
\hline Test & $\begin{array}{c}\text { Sludge } \\
\text { Feed }\end{array}$ & Light? & $\begin{array}{c}\text { OA } \\
\text { Batches }\end{array}$ & $\begin{array}{c}\text { Included } \\
\text { in Report }\end{array}$ \\
\hline 1 & Tank 12H & No & 3 & Yes \\
\hline 2 & Tank 5F & No & 3 & Yes \\
\hline 3 & Tank 12H & Yes & 3 & No \\
\hline 4 & Tank 5F & Yes & 3 & No \\
\hline
\end{tabular}

The Decomposition Module was operated until the $\mathrm{pH}$ increased to above 7 and stabilized. At that point, a sample was tested by a permanganate method to confirm that the target endpoint of $100 \mathrm{ppm}$ oxalate was reached. For the RWT, the target for OA decomposition of less than 100 ppm corresponds to $99.5 \%$ oxalate decomposition.

As evident in the treatment test matrix, the following parameters were varied:

- The source of the actual waste sludge, from Tank $5 \mathrm{~F}$ or $12 \mathrm{H}$.

- The use or non-use of UV light to aid in OA decomposition (this report only contains results without the use of UV light)

\subsubsection{Storage Condition Parameters}

At the conclusion of Decomposition Module operation, the material was emptied into an auxiliary vessel and was split into portions. The largest portion of the material was transferred to the Evaporation Module for further processing. The other portion was split among several storage bottles representing possible Deposition Tank conditions without the use of an ECC Evaporator. The Evaporator Module was operated under vacuum to effect an $84 \%$ volume reduction of the slurry through water removal at approximately $70{ }^{\circ} \mathrm{C}$. At the conclusion of evaporation, the concentrated material was removed from the Evaporation Module and the concentrate slurry was split among several storage bottles representing possible Deposition Tank conditions after use of an ECC Evaporator.

The study of the interaction of components in the Deposition Tank was accomplished by monitoring the resultant ECC material in several storage bottles. Table 2-2 contains the storage test matrix. Each of the four tests utilized a separate set of nine storage bottles. The products from all three individual OA batches within each test utilized the same set of nine storage bottles. The storage bottles were held at the desired test conditions for 15 days (Tank 5F) or 35 days (Tank $12 \mathrm{H})$ and sampled periodically. Additionally, $\mathrm{pH}$ and ORP probes were used to gather this data on the storage bottle samples at the time of sampling. Deposition Bottles were manually mixed periodically (at the time of sampling) but were not mixed continuously. 
SRNL-STI-2011-00360

Revision 0

Table 2-2: Storage Test Matrix

\begin{tabular}{c|c|c|c|c}
\hline $\begin{array}{c}\text { Storage } \\
\text { Condition }\end{array}$ & $\begin{array}{c}\text { Temperature } \\
\left({ }^{\circ} \mathbf{C}\right)\end{array}$ & Evaporation? & $\begin{array}{c}\mathbf{p H} \\
\text { Adjust? }\end{array}$ & $\begin{array}{c}\text { Sludge } \\
\text { Heel? }\end{array}$ \\
\hline 1 & 50 & No & Yes & No \\
\hline 2 & 50 & No & Yes & Yes \\
\hline 3 & 50 & No & No & No \\
\hline 4 & 50 & Yes & Yes & No \\
\hline 5 & 50 & Yes & Yes & Yes \\
\hline 6 & 50 & Yes & No & No \\
\hline 7 & 70 & $*$ & Yes & Yes \\
\hline 8 & $\sim 25$ (ambient) & $*$ & Yes & Yes \\
\hline 9 (control) & 50 & No & Yes & Yes \\
\hline
\end{tabular}

* nominal condition, Yes for Tank $12 \mathrm{H}$, No for Tank $5 \mathrm{~F}$

For the planned F-area implementation of ECC, Storage Condition 2 (without evaporation, with $\mathrm{pH}$ adjustment and with tank heel material) is the nominal storage condition. For the planned $\mathrm{H}$ area implementation of ECC, Storage Condition 5 (with evaporation, with $\mathrm{pH}$ adjustment and with tank heel material) is the nominal storage condition.

A single storage condition control was filled and sampled in parallel with each test. The control used a small amount of feed tank sludge, sodium hydroxide, and heel material. Multiple batches of deionized water were added to the control sample in parallel with the batches of decomposed tank cleaning solution.

As evident in the storage test matrix, the following parameters were varied for material leaving the Decomposition Module:

- Material was either be sent to the Evaporation Module or directly to storage bottles. A larger fraction of the material was sent to the Evaporation Module so that the volume of all storage bottles was about the same.

- Most of the Deposition Bottles was held at $50^{\circ} \mathrm{C}$, with additional bottles from the nominal test condition held at ambient cell temperature and $70^{\circ} \mathrm{C}$.

- A subset of the storage bottles initially had a small heel of dilute sludge slurry (from Tank $51 \mathrm{H}$ ), while other storage bottles were initially be empty.

- A subset of the storage bottles were $\mathrm{pH}$ adjusted by addition of a small amount of $50 \mathrm{wt} \%$ $\mathrm{NaOH}$ targeting $1 \mathrm{M}$ free hydroxide.

\subsubsection{Actual Waste Sludge Materials}

Two actual-waste sludge feeds from previous tank sampling are utilized as feeds for the RWT with an additional sludge material utilized for Deposition Tank sludge heel. Tank 5F sludge material is used to represent F-Area PUREX sludges and Tank 12H sludge material are used to represent $\mathrm{H}$-Area HM sludges. Tank $51 \mathrm{H}$ sludge and supernate are used during testing as Deposition Tank heel material. Additional details and characterization of these materials are provided in Section 3.1. 
SRNL-STI-2011-00360

Revision 0

\subsection{Description of Equipment}

The majority of the ECC RWT was performed in SRNL Shielded Cells B-Block. The equipment simulating the Treatment Tank, ECC Reactor, and Evaporator for the RWT was designed and provided by AREVA. The Deposition Tank and intermediate storage was provided by SRNL. Due to equipment limitations, the treatment tank rheology portion of the testing was accomplished without the use of the AREVA-supplied equipment. The treatment tank rheology testing was performed in SRNL Shielded Cells A-Block in a location close to the rheometer.

\subsubsection{ECC Real Waste Test Equipment}

The primary ECC RWT equipment was designed and fabricated by AREVA per a contract with SRR and through consultation with SRNL. The equipment design is based on the SRR Statement of Work, ${ }^{8}$ the AREVA RWT technical requirements and equipment design report, ${ }^{9}$ and the AREVA RWT project plan ${ }^{10}$ and is reflected in the Piping and Instrumentation Diagram ${ }^{11}$. Configuration and operating conditions are outlined in the Process Flow Diagram. ${ }^{7}$

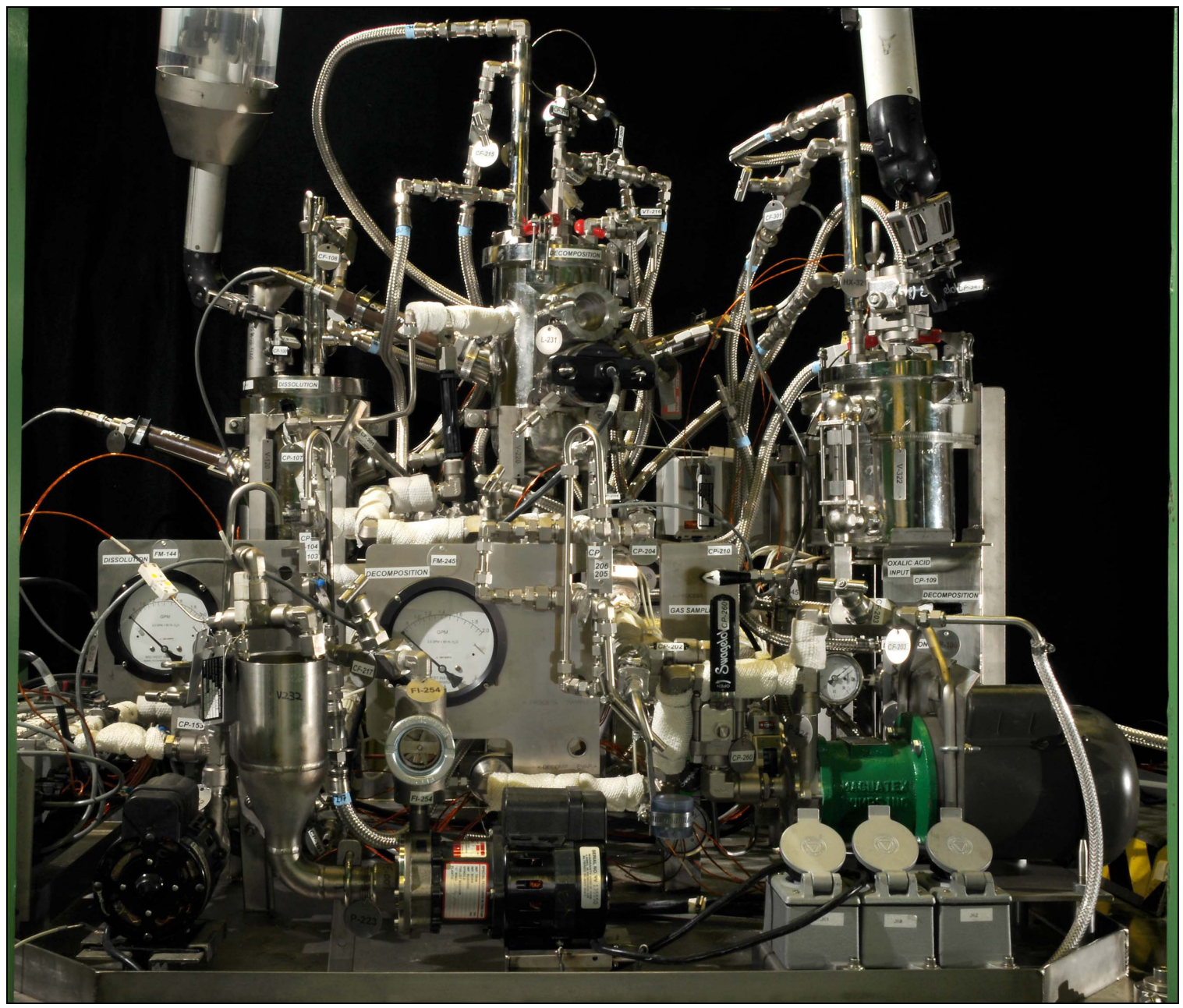

Figure 2-2: ECC RWT Equipment in Mockup Cell 
SRNL-STI-2011-00360

Revision 0

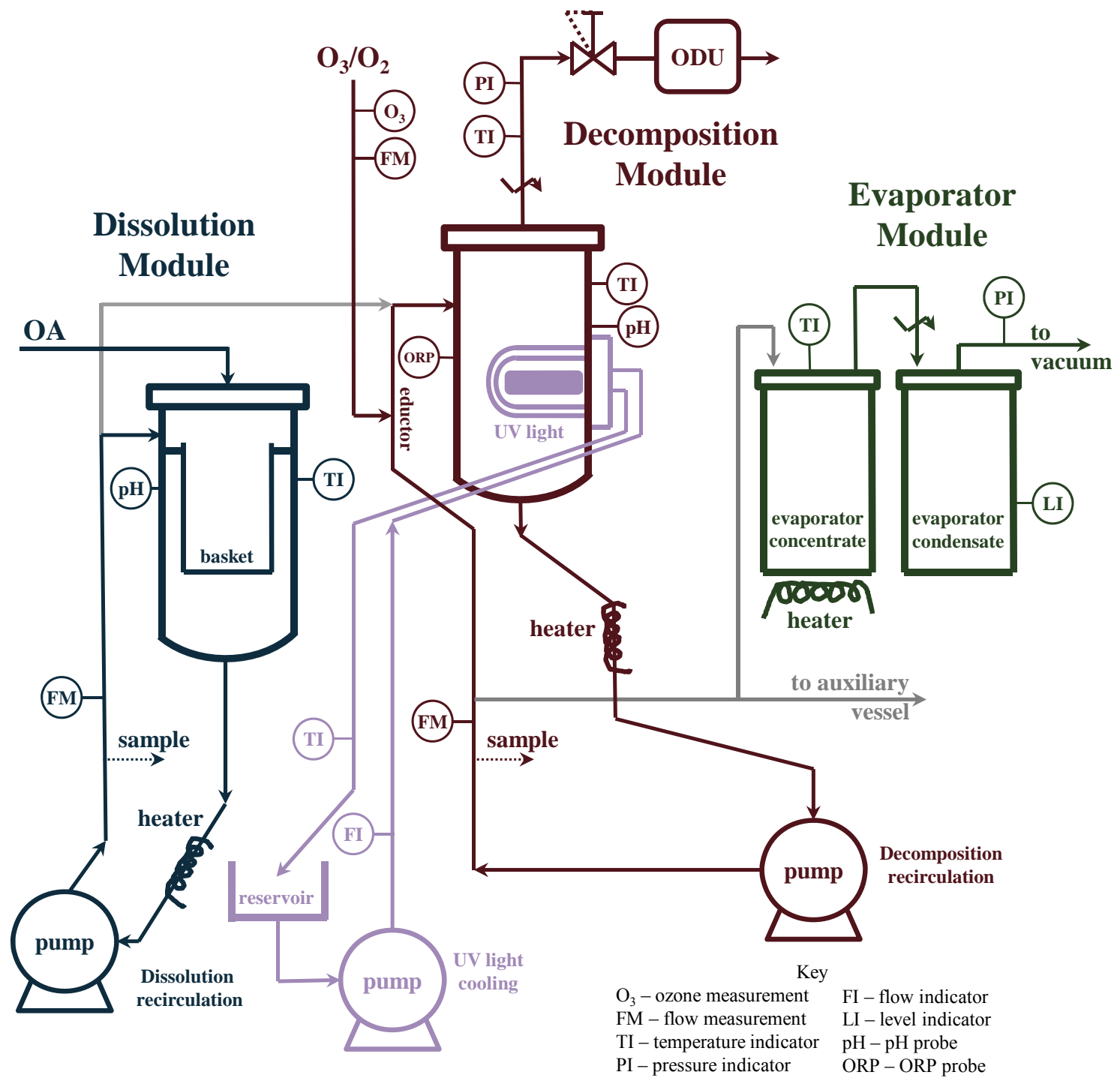

Figure 2-3: Schematic of ECC RWT Equipment Main Cells Skid (roughly related to the equipment elevation photograph of Figure 2-2)

The AREVA ECC RWT equipment (Figure 2-2, Figure 2-3 and Figure 2-4) contains three primary modules, one each for the dissolution of sludge from the treatment-tank (Dissolution Module), the ECC ozone/UV reactor for decomposition of the oxalate (Decomposition Module), and the removal of water for the recovery of dissolution fluid volume (Evaporator Module). The Dissolution Module contains a basket to hold the sludge, a heater to allow for dissolution at 70 ${ }^{\circ} \mathrm{C}$, and a recirculation loop with a pump to allow for mixing, sampling, and transfer. The Decomposition Module contains a reaction loop where ozone contacts the fluid through an eductor and a vessel that allows for gas/liquid separation and the application of UV light via a medium pressure source. Similar to the Dissolution Module, the Decomposition Module contains a heater to allow for reaction at $70{ }^{\circ} \mathrm{C}$ and a recirculation loop with a pump to allow for mixing, sampling, and transfer. The Decomposition Module has the ability to be operated both with and 
without the application of UV light to the fluid. The Decomposition Module includes a system for cleaning the UV light sheath with OA. The Evaporator is a vacuum evaporation system heated to $70^{\circ} \mathrm{C}$. The resultant evaporator concentrate is removed from the system manually via the removable evaporation chamber. Each module includes thermocouples to monitor the fluid temperature. The Dissolution Module additionally contains a probe to monitor the fluid $\mathrm{pH}$. The Decomposition Module includes probes to monitor both the fluid $\mathrm{pH}$ and the oxidation/reduction potential (ORP). Offgas is sent through an Ozone Destruction Unit (ODU) to decompose ozone to below $0.1 \mathrm{ppmv}$ before being vented to the Shielded Cell atmosphere.

An ozone generator was placed behind Shielded Cells B-Block for use in this study. Control modules for the ECC RWT equipment were accessible in the operations area in front of B-Block Cells 11 and 12. The modules outside of the cell are pictured in Figure 2-5.

Refer to the ECC RWT equipment Piping and Instrumentation Diagram for component labeling on the AREVA-supplied equipment. ${ }^{11}$

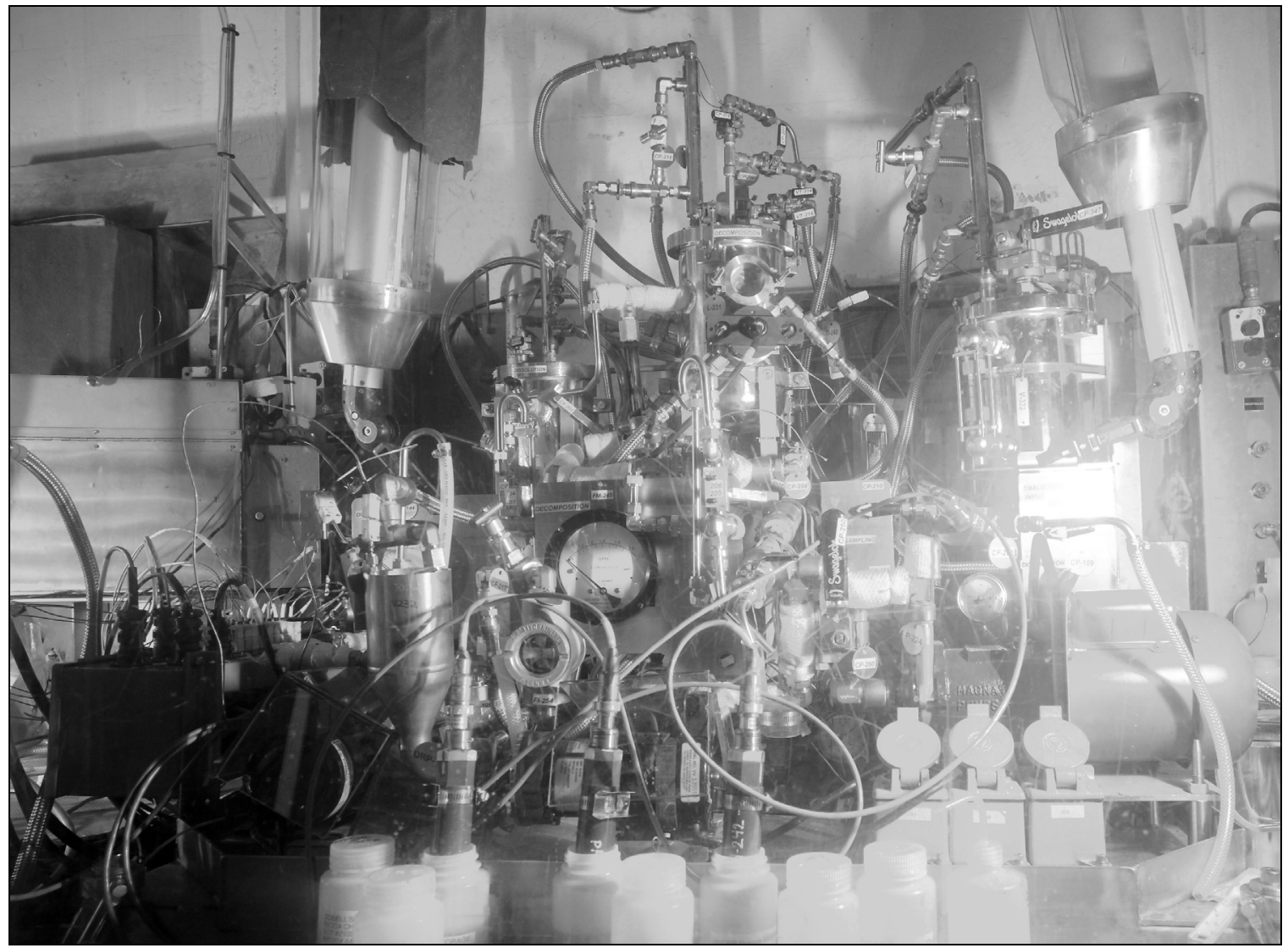

Figure 2-4: ECC RWT Equipment in SRNL High Level Shielded Cells, B-Block Cell 11 

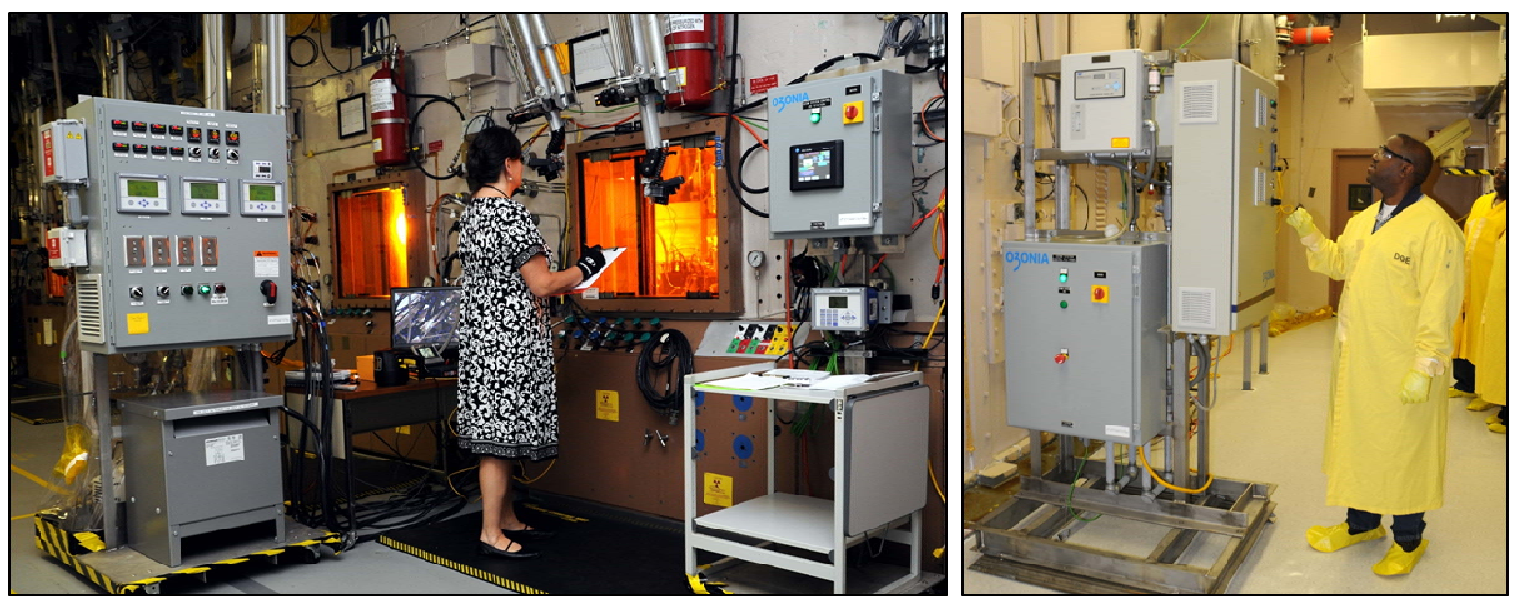

Figure 2-5: ECC RWT Equipment in the Shielded Cells Operating Area (left) and Ozone Generator behind B-Block Cells (right)

Additional ancillary equipment was provided by SRNL. Fabrication of the service plugs for shielded cell installation was performed by SRNL. SRNL used an auxiliary vessel (a 4L HDPE bottle with a magnetic stir bar on a stir plate) for splitting the material leaving the Decomposition Module so that representative portions could be stored in separate deposition bottles and other portions processed in the Evaporation Module. Fluids were able to be removed from the Dissolution and Decomposition Modules of the ECC RWT equipment using ports on the recirculation loops. A peristaltic pump with tygon tubing and stainless steel dip legs was used whenever material needed to be removed from the auxiliary vessels. SRNL also provided the deposition bottles, the equipment required for holding the bottles at deposition tank temperatures, and the equipment for sampling the deposition bottles. Portions of material prior to and after evaporation were stored at Deposition Tank conditions and sampled periodically. Storage bottles were PMP or Teflon for caustic storage conditions and glass for near neutral storage conditions. Bottles held at $50{ }^{\circ} \mathrm{C}$ were stored in a $20 \mathrm{~L}$ water bath. Bottles held at $70{ }^{\circ} \mathrm{C}$ were stored in a drying oven. Temperature, $\mathrm{pH}$, and ORP were periodically monitored in these Deposition Tank storage bottles using equipment procured by SRNL.

Based on an assumed 2000 gallon batch size for a field implementation of ECC, the scale of the Decomposition Module in the ECC RWT is between 1:2400 and 1:3000.

\subsubsection{Treatment Tank Residual Sludge Testing for Rheology and Particle Size}

Duplicate glass vessels were prepared by SRNL glassblowers for the purpose of conducting the OA dissolution batch contacts with both the Tank $5 \mathrm{~F}$ and $12 \mathrm{H}$ sludge samples. The vessels were $140 \mathrm{~mm}$ in diameter and approximately $330 \mathrm{~mm}$ tall. The vessels were designed to fit into freestanding, metal-jacketed heating mantles. The tops of the vessels were fitted with: 1) a sample loading and pour spout with a plastic cap, 2) a calibrated thermocouple attached through an o-ring seal, and 3) a ground glass joint with an attached air-cooled condenser. The samples were heated in the vessels without agitation using a dual temperature controller with over-temperature protection. The thermocouples extended to within a couple of inches of the vessel bottoms. After initial temperature stabilization, the sample temperatures were recorded once or twice per day and the temperature was confirmed to be within five degrees of the target temperature $\left(70 \pm 5^{\circ} \mathrm{C}\right)$ once 
per night during off-shift hours. Once the temperature stabilized, all recorded temperatures during the dissolution batch contacts were $70 \pm 1{ }^{\circ} \mathrm{C}$. During heater operation, water condensation was never observed at a height greater than one inch above the bottom of the condensers. At the conclusion of each sludge/OA batch contact, the materials were removed from the vessels for rheology measurements and characterization.

\subsubsection{RWT Equipment and Measurement Calibrations}

Many instruments involved with the ECC RWT were utilized inside of the SRNL shielded cells in radioactive service. For such equipment, it is often not practical to perform post-testing calibration in accordance with the Measurement and Test Equipment (M\&TE) program because the components will become too contaminated to be submitted to the SRS Standards Laboratory. For such equipment, pre-test calibration or calibration checking will be performed in accordance with the M\&TE program. Prior to removal from the shielded cells for end-of-use disposal, some M\&TE will be cross checked for measurement consistency.

Thermocouples that measure the temperatures inside of the three main process vessels (TI-140, TI-141, TI-240, TI-241, TI-340 and TI-341) underwent pre-test calibration checking and were placed in the M\&TE program. Thermocouples measuring the temperature of Deposition Bottle storage conditions and the Treatment Tank dissolution underwent pre-test calibration checking and were placed in the M\&TE program. Pressure gauges PI-247, PI-342 and PI-440 were calibrated and placed in the M\&TE program. Pressure Relief Valves CP-444 and CP-209 were bench tested as required by SRS Pressure Safety. In-cell balances and weight sets were already part of and maintained within the M\&TE program.

The $\mathrm{pH}$ meters used on the ECC RWT Equipment and used in the Deposition Bottles were checked using $\mathrm{pH}$ buffers over an appropriate range and were done in a manner consistent with program requirements for Analytical Measurement Systems. The ORP probes were checked by Light's Solution and Zobell's Solution in a manner consistent with program requirements for Analytical Measurement Systems. Calibration checks of $\mathrm{pH}$ and ORP probes contained in ECC RWT Equipment chambers were performed prior to and after each OA batch. The potential failure of probes during testing was known to be a risk. One $\mathrm{pH}$ probe failed during a run (Test 2, $\mathrm{OA}$ batch 1 Decomposition) and one $\mathrm{pH}$ probe failed during a storage period. Additionally, the behavior of the $\mathrm{pH}$ measurement during Test 2, OA batch 2 Dissolution was consistent with the probe not being submerged in the Dissolution Module fluid.

The liquid/slurry flow meters FM-144 and FM-245 provide important indications to the equipment operator so that the status of flow within the equipment is known but are not intended to provide useful measurement data during testing. Thus, FM-144 and FM-245 were not calibrated or placed in the M\&TE program. The process high concentration ozone monitor O3441 was not calibrated or placed in the M\&TE program and was used in the condition as provided by AREVA. The other ambient ozone monitors (O3-443 and O3-230) were not recalibrated by SRNL because they were not used as the primary Industrial Health monitor for ozone gas. 


\subsection{Testing Procedure}

\subsubsection{ECC Process Real Waste Testing}

Use of the ECC RWT equipment was governed by a Technical Reference procedure. ${ }^{12}$

Table 2-3 contains the ECC RWT system inputs. Each test used either 48.1 grams of Tank $12 \mathrm{H}$ sludge or 65.1 grams of Tank 5F sludge based on the design volume of the ECC RWT equipment vessels and sample availability. Each OA batch required the fill level of the Dissolution Module to be at least 3.2 L. Dissolution was accomplished with a $2 \mathrm{wt} \%$ OA feed heated to $70{ }^{\circ} \mathrm{C}$ for an 8 hour period with 1 gallon per minute recirculation. Decomposition of OA in the Decomposition Module was initiated by heating each batch of dissolved sludge to $70{ }^{\circ} \mathrm{C}$ with a recirculation of 1.8 gallons per minute (if attainable). Once at temperature, $2 \mathrm{~L}$ per minute of a $5 \mathrm{wt} \%$ ozone in oxygen stream was fed into the system with a back pressure of 8 psi. Decomposition was continued until, through a combination of $\mathrm{pH}$ measurement and permanganate end-of-process testing, it was determined that the soluble oxalate concentration was less than $100 \mathrm{ppm}$.

Table 2-3: Nominal Operating Parameters and Targets for ECC Real Waste Testing

Feed

\begin{tabular}{|c|c|}
\hline Sludge (Tank 12H) & $48.1 \mathrm{~g}$ \\
\hline Sludge (Tank 5F) & $65.1 \mathrm{~g}$ \\
\hline $\mathrm{OA}$ & $3.2 \mathrm{~L} / \mathrm{batch}$ \\
\hline \multicolumn{2}{|c|}{ Dissolution } \\
\hline Feed OA Conc. & $2 \mathrm{wt} \%$ \\
\hline Temperature & $70{ }^{\circ} \mathrm{C}$ \\
\hline Recirculation Rate & $1 \mathrm{gpm}$ \\
\hline Time & $8 \mathrm{hr}$ \\
\hline \multicolumn{2}{|c|}{ Decomposition } \\
\hline Temperature & $70{ }^{\circ} \mathrm{C}$ \\
\hline Pressure & 8 psig \\
\hline Recirculation Rate & $1.8 \mathrm{gpm}$ \\
\hline Feed Gas Flow & $2 \mathrm{~L} / \mathrm{min}$ \\
\hline Feed Gas $\mathrm{O}_{3}$ Conc. & $5 \mathrm{wt} \%$ \\
\hline Final Oxalate Conc. & $<100 \mathrm{mg} / \mathrm{L}$ \\
\hline \multicolumn{2}{|c|}{ Evaporation } \\
\hline Temperature & $70{ }^{\circ} \mathrm{C}$ \\
\hline Water Removal & $\geq 85 \%$ \\
\hline
\end{tabular}




\subsubsection{Analytical Measurements}

Table 2-4 lists the planned analyses for each type of sample taken during the ECC RWT. The primary analytes obtained by each method are also listed, although not all analytes were detectable in every sample and some additional analytes were attainable from some of the methods without additional effort. The different types of samples were taken on frequencies that have four different bases: per tank sludge sample, per sludge dissolution test, per OA batch, and per deposition bottle. The analysis plan was developed with the intent of providing enough information to provide a mass balance around key liquid and solid components throughout the process. However, complete closure of the mass balances remains unlikely due to holdup of material within the RWT equipment.

The initial feed materials were characterized for metals and radioactive components using two digestion methods and triplicate analysis. After each test, the residual insoluble solids in the dissolution module were removed and a portion of the solids were analyzed for metals and radioactive components.

Samples from the recirculation loops of the Dissolution and Decomposition Modules were either liquids or slurries depending on the stage at which sampling was performed. For some sample analysis the composition of the slurry was desired, but for most sample analysis the composition of the soluble components in the slurry was desired. To obtain analysis of the soluble components in the slurry, the slurry was filtered by passing the material through a 0.45 micron nylon syringe filter. Ideally, the smallest filter size possible should be used to minimize the impact of very small particles influencing the measurement. Initial attempts were made to use smaller pore size $(0.1$ micron) nylon filters for this testing. However, for the slurries encountered during the ECC RWT with Tank $5 \mathrm{~F}$ and $12 \mathrm{H}$ feed sludges, the 0.1 micron filters proved impractical to use due to the great resistance of the material to flow through the membrane.

At the completion of Dissolution Module operation, samples were taken from the recirculation loop prior to transfer of material to the Decomposition Module. Dissolution Module samples were filtered and the filtrate analyzed in duplicate for anions (including oxalate) by ion chromatography (IC), metals (including iron and aluminum) by inductively coupled plasmaemissions spectroscopy (ICP-ES), other radiochemical ( $\mathrm{Pu}$, gamma, $\mathrm{Sr}-90)$ and ionic components $\left(\mathrm{CO}_{3}\right)$. Single acid dissolutions of the slurry samples were prepared and analyzed for metals and radiochemical components. Residual solids isolated from the Dissolution Module at the conclusion of each test were digested by the aqua regia method and analyzed for metals and radiochemical components.

During Decomposition Module operation, 3 to 5 intermediate samples were taken from the recirculation loop at intervals during OA decomposition. The intermediate Decomposition Module samples were filtered and analyzed by IC, ICP-ES, ICP-MS, and radiochemistry (Pu). Final Decomposition Module samples were filtered and analyzed in duplicate for a large suite of soluble anions, metals, and radiochemical components. Single acid dissolutions of the Decomposition Module slurry samples were prepared and analyzed for a subset of the metal and radiochemical components.

No samples were taken directly from the evaporator pot, though Deposition Tank Storage Condition 6 corresponds to unadjusted sludge after evaporation. 
Table 2-4: ECC RWT Sampling and Analysis Plan

\begin{tabular}{|c|c|c|c|c|c|c|c|c|c|c|}
\hline Analysis & Primary Analytes & 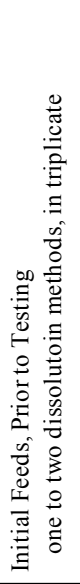 & 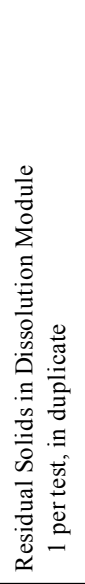 & 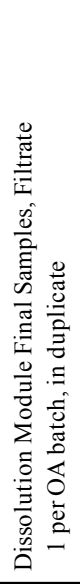 & 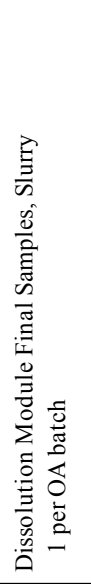 & 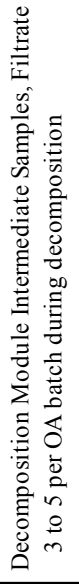 & 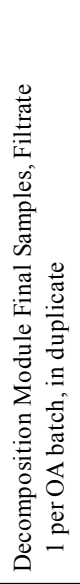 & 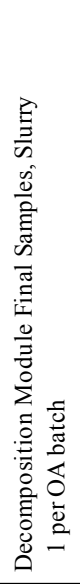 & 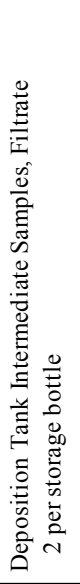 & 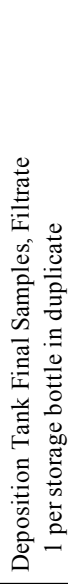 \\
\hline ICP-ES & $\begin{array}{l}\mathrm{Al}, \mathrm{Ca}, \mathrm{Cr}, \mathrm{Fe}, \mathrm{Gd}, \mathrm{Mn}, \\
\mathrm{Na}, \mathrm{Ni}, \mathrm{P}, \mathrm{S}, \mathrm{Si}, \mathrm{Sr}, \mathrm{Ti}\end{array}$ & $\mathbf{X}$ & $\mathbf{X}$ & $\mathrm{X}$ & $\mathbf{x}$ & $\mathbf{x}$ & $\mathbf{x}$ & $\mathbf{x}$ & $\mathbf{X}$ & $\mathrm{x}$ \\
\hline ICP-MS & $\begin{array}{l}\text { Th-232, U-235, } \\
\text { Np-237, U-238 }\end{array}$ & $\mathbf{X}$ & $\mathbf{x}$ & $\mathbf{X}$ & $\mathbf{X}$ & $\mathbf{x}$ & $\mathbf{X}$ & $\mathbf{x}$ & $\mathbf{X}$ & $\mathbf{x}$ \\
\hline PuTTA & $\mathrm{Pu}-238, \mathrm{Pu}-239 / 240$ & $\mathbf{x}$ & $\mathbf{X}$ & $\mathbf{x}$ & $\mathbf{X}$ & $\mathbf{X}$ & $\mathbf{X}$ & $\mathbf{X}$ & $\mathbf{X}$ & $\mathbf{x}$ \\
\hline CVAA & $\mathrm{Hg}$ & $\mathrm{x}$ & $\mathbf{X}$ & $\mathbf{X}$ & $\mathrm{X}$ & & $\mathbf{X}$ & $\mathbf{X}$ & & $\mathbf{x}$ \\
\hline Sr-90 & Sr-90 & $\mathbf{x}$ & $\mathbf{X}$ & $\mathrm{x}$ & $\mathbf{x}$ & & $\mathbf{X}$ & $\mathrm{X}$ & & $\mathrm{x}$ \\
\hline Gamma & Cs-137 & $\mathbf{x}$ & $\mathbf{X}$ & $\mathbf{x}$ & & & $\mathbf{X}$ & & & $\mathbf{x}$ \\
\hline $\mathrm{Am} / \mathrm{Cm}$ & $\begin{array}{l}\text { Am-241, Am-242m, } \\
\mathrm{Cm} 242, \mathrm{Cm}-244\end{array}$ & $\mathbf{X}$ & $\mathbf{X}$ & $\mathbf{x}$ & & & $\mathbf{X}$ & & & $\mathbf{x}$ \\
\hline IC Anions & $\begin{array}{l}\mathrm{C}_{2} \mathrm{O}_{4}, \mathrm{NO}_{3}, \mathrm{NO}_{2}, \\
\mathrm{SO}_{4}, \mathrm{Cl}, \mathrm{F}\end{array}$ & & & $\mathrm{X}$ & & $\mathrm{x}$ & $\mathbf{X}$ & & & $\mathrm{x}$ \\
\hline TIC/TOC & $\mathrm{CO}_{3}, \mathrm{TOC}$ & & & $\mathbf{x}$ & & & $\mathrm{x}$ & & & $\mathbf{x}$ \\
\hline wet chem & Free $\mathrm{OH}$ or Free $\mathrm{H}$ & & & $\mathbf{X}$ & & & $\mathbf{X}$ & & & $\mathbf{x}$ \\
\hline $\mathrm{Pu}-241$ & $\mathrm{Pu}-241$ & $\mathbf{X}$ & & & & & & & & \\
\hline Cs-rem gamma & Co-60, Sn-126, Eu-154 & $\mathbf{X}$ & & & & & & & & \\
\hline
\end{tabular}

Each test had nine corresponding Deposition Tank storage bottles. The storage bottles were mixed prior to sampling but were not mixed continuously. Three samples were taken from each of the storage bottles, filtered with a 0.45 micron filter, and the filtrate prepared for analysis. Intermediate samples were filtered, acidified, and analyzed by IC, ICP-ES, ICP-MS, and radiochemistry $(\mathrm{Pu})$. The sample taken after approximately two to three weeks of storage was filtered, acidified, and analyzed for the entire chemical and radiological suite. The bulk slurry was analyzed for a subset of the metal and radiochemical components. A subset of decomposition bottles were sampled after an additional two weeks of equilibration, with the filtrate analyzed by the suite used for the intermediate deposition bottle samples. 
All isotopes of uranium and plutonium were not necessarily tracked through all stages of the process because some isotopes of these elements were difficult and costly to determine in the soluble fraction due to the low concentrations. However, since the isotopic ratios have been established for the feed materials through the initial characterizations, estimates of the complete isotopics can be calculated from the results provided for other $\mathrm{Pu}$ and $\mathrm{U}$ isotopes.

For the majority of the analytical methods of Table 2-4, 1-sigma uncertainties are nominally $10 \%$ or less. Sample preparations were performed in a manner as not to add significantly to the overall $10 \%$ uncertainly of the analytical methods. Some radiochemical preparations followed by counting methods had 1-sigma uncertainties greater than $10 \%$ when activity was low (approaching the detection limit):

- $\mathrm{Am} / \mathrm{Cm}$ measurements in the Deposition Tank samples had uncertainties $>>10 \%$.

- Pu-238 and Pu-239/240 measurements in the Test 2 Deposition Tank samples had uncertainties $>10 \%$.

Calorimetric spot tests were conducted in the shielded cells near the end of each decomposition test to confirm that the oxalate anion levels in the spent acid solution had decreased to the target level of $100 \mathrm{ppm}$. Tests were conducted using small glass vials containing $150 \mathrm{mg}$ of $\mathrm{MnSO}_{4} \cdot \mathrm{H}_{2} \mathrm{O}$. A sample of solution was isolated from the decomposition module and a $5-\mathrm{mL}$ portion was filtered through a 0.45 um syringe filter directly into the vial. Then $1 \mathrm{~mL}$ of $50 \mathrm{wt} \%$ $(9.1 \mathrm{M})$ sulfuric acid was transferred into the vial and the vial was capped and shaken to promote dissolution of the manganese sulfate solids. A manual pipet was used to transfer $0.2 \mathrm{~mL}$ of $0.1 \mathrm{M}$ potassium permanganate solution into the vial and the vial was capped and shaken again. The color of the solution was observed and recorded after two minutes. A purple or pink colored solution was indication that the oxalate concentration was at or below the target concentration. Note that the amount of permanganate added to the solution was in excess of the amount required to react with $100 \mathrm{mg} / \mathrm{L}$ of oxalate anion, but previous spot tests had confirmed that this was the excess required to observe the color through the cell window.

\subsubsection{Rheology, Settling Rate, and Particle Size Measurements}

Rheology, particle size and/or settling behavior were investigated at key stages in the process. This information aids in the understanding of the removal of undissolved solids from the treatment tank, the flow of solids through the ECC process, and the effects of newly precipitated solids on downstream processes. The key process stages and the applicable test series are outlined in the schematic of Figure 2-6.

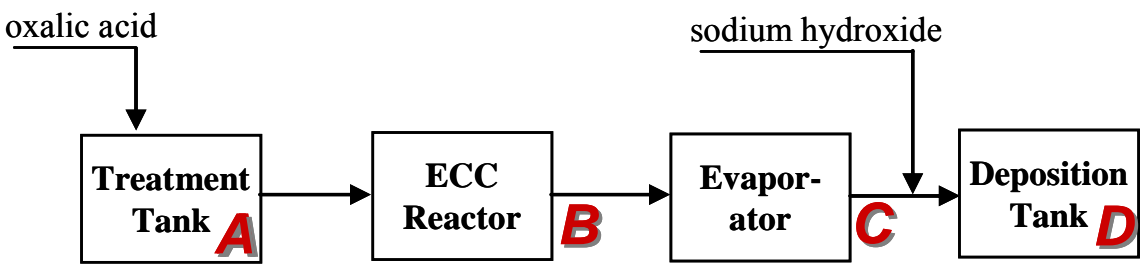

Figure 2-6: Key process stages for rheology, particle size and/or settling behavior testing 
A: In the Treatment Tank

Purpose: Provide input on the impact of sludge dissolution on the ability of the slurry pumps to adequately mix the tank contents. Provide evidence that the undissolved solids during and after ECC are easier to suspend and remain suspended as compared to the original sludge heel.

Measurements: Rheology of residual undissolved solids at concentrated solids levels, particle size distribution of residual undissolved solids.

Methodology: In order to determine the rheology of the residual Treatment Tank heel, separate sludge dissolution testing was performed outside of the AREVA-supplied ECC RWT Equipment dissolution module. The results are contained in this report.

B: After ECC Reactor, prior to Evaporator or $\mathrm{pH}$ adjustment

Purpose: Help determine the flow characteristics in the recirculation loop following oxalate decomposition.

Measurement: Rheology at process-related solids concentration, particle size distribution

Methodology: At the end of solubility testing, slurry from deposition bottles for Storage Condition 3 (see Table 2-2) were measured with the rheometer and submitted for Microtrac particle size analysis.

$\mathrm{C}$ : After Evaporator, prior to $\mathrm{pH}$ adjustment

Purpose: In the full scale ECC process, the concentrated material in the evaporator bottoms must be pumped to the deposition tank.

Measurement: rheology at process-related solids concentration, particle size distribution

Methodology: At the end of the solubility testing, slurries from deposition bottles for Storage Condition 6 (see Table 2-2) were measured with the rheometer and submitted for Microtrac particle size analysis.

D. In the Deposition Tank at nominal conditions

Purpose: Supernate will be decanted from the deposition tank to and processed in a HLW evaporator system. Settling information is needed to help justify the feasibility of decanting supernate from the deposition tank during periods of ECC processing.

Measurement: settling time, particle size distribution

Methodology. Storage Condition 2 is the applicable condition for F-Area deposition bottles and Storage Condition 5 is the applicable condition for H-Area deposition bottles (see Table 2-2). Small samples were collected from the applicable deposition bottles and submitted for Microtrac particle size analysis. At the completion of the solubility testing, the applicable storage bottles were removed from the $50{ }^{\circ} \mathrm{C}$ water bath, agitated, and held quiescent to allow the solids to settle. Periodic observations of solids settling (level, behavior, etc.) were made and recorded until the supernatant liquid had clarified.

\subsubsection{Treatment Tank Rheology Testing}

Rheology testing was conducted on samples of the Tank $5 \mathrm{~F}$ and $12 \mathrm{H}$ sludge which had previously been dried at $50{ }^{\circ} \mathrm{C}$. These samples are referred to as "Treatment Tank" samples, since they are believed to be representative of the materials in the front portion of the ECC process which are located in the tank to be treated for sludge heel removal. Sub-samples of each sludge sample were initially pre-wetted with water to produce 50 weight percent slurries and allowed to stand overnight to promote hydration of the solid phase. Sludge/OA slurries were then prepared for baseline rheology measurements at initial sludge solids loadings of 13 and 26 weight percent. 
Corrections were made to account for the initial water added for hydration to produce final slurries containing $0.224 \mathrm{M}$ oxalic acid ( $2 \mathrm{wt} \% \mathrm{OA})$.

Baseline rheology measurements were conducted on these slurries at two solids concentrations and three temperatures. It was observed during rheological evaluations that significant portions of the initial slurries settled quickly. This sample characteristic was particularly pronounced for the Tank 5F samples where it appeared that most of the sample settled within five minutes. The rapid settling rate prevented the measurement of the rheological properties of the fully suspended sample by standard methods. Therefore, the rheology data collected for the Tank 5F sample is representative of only the small fraction of the solid materials that remained suspended on the measurement timescale. The suspendable solids for these samples appeared to be so low that the rheological properties measured for the Tank $5 \mathrm{~F}$ samples were expected to be similar to those of pure OA or spent acid generated in the ECC process. Portions of the Tank $12 \mathrm{H}$ slurry used for baseline rheology measurements also settled during initial evaluations as exhibited by the presence of significant amounts of dark solids in the bottom of the rheology cup at the conclusion of each measurement. The Tank $12 \mathrm{H}$ rheology sample appeared to contain two types of solids with very different settling rates. The lighter colored solids in this sample remained well suspended over the rheology measurement timescale. The Tank $12 \mathrm{H}$ samples were observed to change over the first few days of acid contact. After 4 days, most of the concentrated Tank $12 \mathrm{H}$ slurry remained suspended throughout the analysis with few settled solids being observed.

Sub-samples of selected Tank $5 \mathrm{~F}$ and $12 \mathrm{H}$ slurries from the baseline rheology testing were collected to analyze the samples for weight percent solids and determine the amount of "suspendable" solids in the samples. Collection of approximately $3 \mathrm{~mL}$ slurry samples was accomplished using a disposable plastic pipet approximately 1 minute after agitating the samples in the same manner that the samples were agitated prior to rheology measurements.

After the completion of the baseline testing, the slurries used for rheology measurements were augmented to give a total of $62.9 \mathrm{~g}$ of Tank $5 \mathrm{~F}$ solids and $45.6 \mathrm{~g}$ of Tank $12 \mathrm{H}$ solids. The resulting slurries were transferred to the batch contact dissolution vessels. Additional $2 \mathrm{wt} \% \mathrm{OA}$ was added to the vessels to give a total of $2.9 \mathrm{~L}$ for the Tank $5 \mathrm{~F}$ slurry and $2.3 \mathrm{~L}$ for the Tank $12 \mathrm{H}$ slurry. The resulting acid:sludge mass ratios for each sample were approximately 50:1. The dissolution vessels were then heated to $70{ }^{\circ} \mathrm{C}$ and held at that temperature for seven days. At the conclusion of the first batch contact period, the solution in the Tank $5 \mathrm{~F}$ slurry was yellow-green in color while the solution in the Tank $12 \mathrm{H}$ slurry was slightly yellow. Most of the solution was subsequently removed from the settled solids in each vessel using a peristaltic pump. The solids were then suspended in the remaining solution and the resulting slurries were removed from the vessels for midpoint rheology measurements.

Samples were prepared for rheology measurements by further reducing the volume of spent OA in the slurries. As was observed for the baseline measurements, most of the Tank 5F solids settled quickly, such that meaningful rheology measurements were not practical. As a result, measurements were only conducted at one solids loading at $30^{\circ} \mathrm{C}$ for this sample. The Tank $12 \mathrm{~F}$ slurry was easily suspended with few rapidly settling particles being observed. Rheology measurements were conducted for this sample at two solids loadings and three temperatures. Sub-samples of the Tank 12H slurry were collected as described for the baseline samples with the goal of determining the "suspendable" weight percent solids levels. Samples of the filtered spent OA were also collected in order to determine the weight percent of dissolved solids. In this case, the samples were filtered through $0.45 \mu \mathrm{m}$ Nylon filter units. 
After the completion of the midpoint measurements and sampling, all of the remaining solids used for rheology measurements were returned to the dissolution vessels along with fresh $2 \mathrm{wt} \%$ OA totaling $2.9 \mathrm{~L}$ for the Tank $5 \mathrm{~F}$ sample and $2.3 \mathrm{~L}$ for the Tank $12 \mathrm{H}$ sample (approximately 50:1 acid:sludge based on the original sludge mass). The vessels were then heated to $70{ }^{\circ} \mathrm{C}$ and held at that temperature for three days. At the conclusion of the second batch contact period (endpoint), the solutions above the solids were lighter in color than the solutions isolated after the first OA contact (midpoint). Most of the solution was subsequently removed from the settled solids in each vessel using a peristaltic pump and the damp solids were isolated for endpoint rheology analysis.

As was observed for the baseline and midpoint rheology samples, most of the Tank $5 \mathrm{~F}$ solids settled within minutes. As a result, rheology testing was only conducted at one solids loading at $30{ }^{\circ} \mathrm{C}$. The Tank $12 \mathrm{H}$ slurry was easily suspended and suitable for analysis and rheology data was collected for this sample at two solids loadings and three temperatures. Limitations on the minimum volume of material needed for rheology measurements $(\sim 50 \mathrm{~mL})$ and the small amount of solids remaining for this sample prohibited the preparation of Tank $12 \mathrm{H}$ slurries containing greater than $5 \mathrm{wt} \%$ total solids. Slurry sub-samples (filtered and unfiltered) were collected as described for the baseline and midpoint samples. Single sub-samples of the baseline, midpoint, and endpoint rheology materials were also collected for particle size analysis. See Section 2.3.4 for details.

Rheology measurements were also conducted on four deposition tank samples generated during other ECC testing. The samples included Tank $5 \mathrm{~F}$ and $12 \mathrm{H}$ product materials generated during oxalate destruction tests. These samples are referred to as "Deposition" samples because they are believed to be representative of ECC process product materials deposited in the receipt tank. The samples included materials which had been evaporated and materials which were not exposed to evaporation. Rheology measurements were conducted on all four samples at the as-received solids loading levels and at two temperatures.

\subsubsection{Weight Percent Solids Measurements}

Weight percent solids measurements were conducted by drying samples in a drying oven at $112^{\circ} \mathrm{C}$ until no additional weight loss was observed. The weight percent of dissolved solids is defined as the weight of dissolved solids divided by the weight of initial filtered supernatant (x100). For these measurements, the supernatant was filtered using a $0.45 \mu \mathrm{m}$ Nylon filter. The weight percent of total solids is defined as the total weight of solids (dissolved and insoluble) divided by the weight of initial slurry $(\mathrm{x} 100)$. Weight percent dissolved and total solids measurements were conducted on single or on duplicate samples. The collection of replicate samples for analysis was prevented in some cases by sample size limitations.

\subsubsection{Rheology Measurement Equipment, Methodology, and Data Analysis}

The rheological properties of the samples were determined using a Haake M5/RV30 rotoviscometer. The M5/RV30 is a Searle sensor system, where the bob rotates and the cup is fixed. The torque and rotational speed of the bob are measured. Heating/cooling of the cup/sample/bob is accomplished through a jacket that surrounds the sample cup. The shear stress is determined from the torque measurement and is independent of the rheological properties. Conditions that impact the measured torque are: slip (material does not properly adhere to the 
rotor or cup), phase separation (buildup of liquid layer on rotor), sedimentation (particles settling out of the shearing zone), homogeneous sample (void of air), lack of sample (gap not filled), excess sample (primarily impacts rheologically thin fluids), completely filling up the void below the bob (air buffer that is now filled with fluid) and Taylor vortices. The first five items yield lower stresses and the last three add additional stresses. The shear rate is geometrically determined using the equations of change (continuity and motion) and is that for a Newtonian fluid. This approach assumes that the flow field is fully developed and the flow is laminar. The shear rate can be calculated for non-Newtonian fluid using the measured data by fitting this data to the rheological model or corrected as recommended by Darby ${ }^{8}$. In either case, for shear thinning, non-Newtonian fluids typical of SRS sludge wastes, the corrected shear rates are greater than their corresponding Newtonian shear rates, resulting in a thinner fluid. Flow curve corrections will not be performed in this task, which results in slightly high measurements of fluid viscosity.

The shape, dimensions, and geometric constants for the MV I rotor used for these measurements are provided in Table 1. Based on the expected particle size of the materials in this investigation, the MV 1 rotor was considered most appropriate. Prior to performing the measurements, the rotors and cups were inspected for physical damage. The torque/speed sensors and temperature bath were verified for functional operability using a bob/cup combination with a National Institute of Standards and Technology (NIST) traceable Newtonian oil standard, using the MV I rotor. The resulting flow curve was then fitted assuming a Newtonian fluid and the calculated viscosity was required to be within $\pm 10 \%$ of the reported NIST viscosity at a given temperature for the system to be considered functionally operable. A N10 oil standard was used to verify system operability prior to the sludge measurements.

The flow curves for the sludge were fitted to the down curves using the Bingham Plastic rheological model, Equation $\mathrm{q}$, where $\tau$ is the measured stress $(\mathrm{Pa}), \tau_{\mathrm{o}}$ is the Bingham Plastic yield stress $(\mathrm{Pa}), \mu_{\infty}$ is the plastic viscosity $(\mathrm{Pa} \cdot \mathrm{sec})$, and $\dot{\gamma}$ is the measured shear rate $\left(\mathrm{sec}^{-1}\right)$.

$$
\tau=\tau_{o}+\mu_{\infty} \dot{\gamma}
$$

All rheology measurements were conducted in duplicate. Between each rheology measurement, the sample cup was lowered, the sample was transferred to a bottle, and the sample cup was inspected to evaluate whether the solids remained suspended during the measurement. The sample bottle was then capped and shaken to promote agitation. The sample was immediately transferred back to the rheology cup and placed in the instrument for analysis. Loading the sample into the instrument typically took approximately four minutes and eleven minutes was required to complete each rheological measurement. Therefore, slurry samples for which the particles settled in $<15$ minutes were not considered suitable for rheology measurements.

The temperature of the samples was not measured directly during rheological measurements. Rather, the temperature was monitored and controlled using two thermocouples immersed within the heating fluid flow loop. One thermocouple was located in the flow loop at the heater location before the rotor and cup and the second thermocouple was located in the flow loop after the rotor and cup. This second thermocouple was believed to be most representative of the sample temperature and was used for temperature monitoring and control. During all analysis the temperature readout from the second thermocouple was typically within $\pm 2{ }^{\circ} \mathrm{C}$ of the target value, but occasionally the deviation from the target was $\pm 3{ }^{\circ} \mathrm{C}$. Due to the settling properties of the samples, it was not possible to allow as much time for temperature equilibration of the system as recommended. Rheology measurements were typically conducted within approximately one 
minute after the sample was loaded. Therefore, the actual sample temperatures may been slightly lower than the target values during the high temperature measurements. For each given sample and solids loading, all measurements were conducted in sequence and the time required for lowering the rotor and mixing the sample was kept short such that sample cooling during this time period was minimized. Tank $5 \mathrm{~F}$ and $12 \mathrm{H}$ treatment tank samples were analyzed at 30,40 , and $50{ }^{\circ} \mathrm{C}$, while the deposition tank samples were only analyzed at 30 and $45^{\circ} \mathrm{C}$.

All viscosity results are reported in centipoise (cP) and all yield stress values are reported in pascals $(\mathrm{Pa})$. Negative yield stress values were calculated for numerous samples analyzed due to difficulties in accurately adjusting the instrumental zero set point. This issue is apparent in the data plots for many thin samples in Appendix B, where the shear stress values on the down curve reached zero at a shear rate near $100 \mathrm{~s}^{-1}$. This is believed to be primarily an issue for the thin samples, since the shear stress values for thicker samples typically returned to the initial zero point.

Table 2-5: MV I Rotor Specifications and Flow Curve Program

\begin{tabular}{|c|c|c|}
\hline Rotor Design & \multicolumn{2}{|c|}{ Dimensions and Flow Curve Program } \\
\hline & Rotor Type & MV I \\
\hline & Rotor radius $-\mathrm{R}_{\mathrm{i}}(\mathrm{mm})$ & 20.04 \\
\hline & Cup Radius - $\mathrm{R}_{\mathrm{a}}(\mathrm{mm})$ & 21.0 \\
\hline & Height of rotor $-\mathrm{L}(\mathrm{mm})$ & 60 \\
\hline & $\begin{array}{c}\text { Sample Volume }\left(\mathrm{cm}^{3}\right) \\
\text { minimum }\end{array}$ & 40 \\
\hline & A factor $(\mathrm{Pa} / \%$ torque $)$ & 3.22 \\
\hline & $\mathrm{M}$ factor $\left(\mathrm{s}^{-1} / \% \mathrm{RPM}\right)$ & 11.7 \\
\hline & Shear rate range $\left(\mathrm{s}^{-1}\right)$ & $0-600$ \\
\hline & Ramp up time (min) & 5 \\
\hline & Hold time (min) & 1 \\
\hline & Ramp down time (min) & 5 \\
\hline
\end{tabular}

\subsubsection{Particle Size and Settling Rate Measurements}

Particle size distribution determination was accomplished by removing small amounts (approximately 0.03 grams) of solids from the shielded cells for measurement by a Microtrac particle size analyzer. The solid samples were suspended in liquids with similar characteristics to their slurry conditions. The dilution fluids were $0.00625 \mathrm{M}$ nitric acid for Treatment Tank samples; $0.02 \mathrm{M}$ sodium nitrate for post ECC Reactor fluids prior to $\mathrm{pH}$ adjustment; and $0.5 \mathrm{M}$ sodium hydroxide with $0.02 \mathrm{M}$ sodium nitrate for caustic Deposition Tank fluids.

Simple settling tests were also performed in the shielded cells. Applicable deposition bottles were agitated and then held quiescent. The appearance of the liquid and the sludge level were observed periodically until the supernatant liquid had clarified. 
SRNL-STI-2011-00360

Revision 0

\subsection{Results and Discussion}

\subsection{Initial Sludge Preparation and Characterization}

The ECC RWT used two actual-waste sludge materials as process feeds, Tank 5F sludge and Tank $12 \mathrm{H}$ sludge. Additional actual-waste sludge from Tank $51 \mathrm{H}$ was used as deposition tank heel material.

The sample used to represent F-Area PUREX sludge was a portion of Tank 5F sample FTF-0506-55. This sample was of the Tank $5 \mathrm{~F}$ sludge material prior to Tank $5 \mathrm{~F}$ chemical cleaning and had been characterized and used in previous testing. ${ }^{13}$ Prior to use, the sample material was washed with $0.01 \mathrm{M} \mathrm{NaOH}$ to remove salts and dried at $50{ }^{\circ} \mathrm{C}$. Subsamples of the washed and dried sludge were dissolved by aqua regia and peroxide fusion digestion methods and analyzed for chemical and radioactive components. Table 3-1 and Table 3-2 contain the analytical results for the Tank $5 \mathrm{~F}$ sample used in the testing. The Tank $5 \mathrm{~F}$ material was stored in its mostly dry state until used in ECC testing.

The sample used to represent H-Area HM sludge was a combination of several samples from Tank 12H taken from 2008 through 2010. Through waste removal activities, it was noted that the Tank 12H sludge was layered, with uranium enrichment and thorium content varying as layers were encountered. Because this Tank $12 \mathrm{H}$ material is a composite of several samples taken at different stages of waste removal, it is a blend of layers and does not represent any individual layer. Similar to the Tank 5F material, the Tank $12 \mathrm{H}$ samples were washed with $0.01 \mathrm{M} \mathrm{NaOH}$ to reduce the hydroxide concentration and remove other salts and dried at $50{ }^{\circ} \mathrm{C}$. Table $3-3$ and Table 3-4 contain the analytical results for Tank $12 \mathrm{H}$ sludge material prepared for ECC testing. The Tank $12 \mathrm{H}$ material was stored in its mostly dry state until used in ECC testing.

Additionally, a small amount of dilute sludge slurry was needed as a heel for the deposition bottles. For this heel material, we used sludge solids and sludge supernate from Tank $51 \mathrm{H}$ combined with additional simulated sludge supernate. The actual-waste sludge sample used was HTF-51-07-121. A previous characterization report exists for this sample and this material was not characterized again prior to use. ${ }^{14}$ The purpose of the solids in this material is to provide metal compounds to potentially be affected by the addition of ECC product material. The purpose of the liquid portion of this material is to maintain a $\mathrm{pH}$ appropriate to the Deposition Tank. As the sludge sample material was taken at the end of an aluminum dissolution campaign, the sludge supernate contained relatively high hydroxide content, more akin to a salt supernate. The deposition tank heel mixture contained $\sim 4 \%$ concentrated Tank $51 \mathrm{H}$ slurry, $\sim 25 \%$ clarified Tank $51 \mathrm{H}$ supernate, and the balance $(\sim 71 \%) 4 \mathrm{M} \mathrm{NaOH}$.

From the characterization, the primary component of the Tank $5 \mathrm{~F}$ sludge is iron while the primary component of the Tank $12 \mathrm{H}$ sludge is aluminum. Both sludge samples contain significant iron, aluminum, manganese, sodium, and uranium. Additionally, the Tank 5F sludge contains significant nickel while the Tank $12 \mathrm{H}$ sludge contains significant mercury and thorium. Both samples contain high activities of Sr-90, but there are differences in the radioactive isotope profiles between the two materials with respect to $\mathrm{Pu}-238, \mathrm{Pu}-239$, uranium enrichment, and other isotopes. 
SRNL-STI-2011-00360

Revision 0

Table 3-1: Characterization of Tank 5F Sludge Sample Available for ECC Actual-Waste Testing

\begin{tabular}{|c|c|c|c|c|c|c|c|c|}
\hline analyte & method & units & $\begin{array}{c}\text { aqua regia } \\
\text { average }\end{array}$ & $\begin{array}{c}\text { per. fusion } \\
\text { average }\end{array}$ & $\begin{array}{c}\text { overall } \\
\text { average }\end{array}$ & st. dev. & $\mathrm{g} / \mathrm{g} \mathrm{Fe}$ & g / g Al \\
\hline $\mathrm{Ag}$ & ICP-ES & $\mathrm{mg} / \mathrm{kg}$ & $<1.55 E+02$ & $<3.89 E+02$ & $<2.72 E+02$ & -- & -- & -- \\
\hline $\mathrm{Al}$ & ICP-ES & $\mathrm{mg} / \mathrm{kg}$ & $1.49 \mathrm{E}+04$ & $1.78 \mathrm{E}+04$ & $1.64 \mathrm{E}+04$ & $2.0 \mathrm{E}+03$ & 4.5E-02 & $1.0 \mathrm{E}+00$ \\
\hline B & ICP-ES & $\mathrm{mg} / \mathrm{kg}$ & $<8.02 E+01$ & $<3.40 E+02$ & $<2.10 E+02$ & -- & -- & -- \\
\hline $\mathrm{Ba}$ & ICP-ES & $\mathrm{mg} / \mathrm{kg}$ & $1.57 \mathrm{E}+03$ & $1.68 \mathrm{E}+03$ & $1.63 \mathrm{E}+03$ & $1.2 \mathrm{E}+02$ & 4.5E- 03 & 9.9E-02 \\
\hline $\mathrm{Be}$ & ICP-ES & $\mathrm{mg} / \mathrm{kg}$ & $<5.07 E+00$ & $<1.05 E+01$ & $<7.80 E+00$ & -- & -- & -- \\
\hline $\mathrm{Ca}$ & ICP-ES & $\mathrm{mg} / \mathrm{kg}$ & $3.12 \mathrm{E}+03$ & $6.01 \mathrm{E}+03$ & $4.57 \mathrm{E}+03$ & $1.60 \mathrm{E}+03$ & $1.3 \mathrm{E}-02$ & $2.8 \mathrm{E}-01$ \\
\hline $\mathrm{Cd}$ & ICP-ES & $\mathrm{mg} / \mathrm{kg}$ & $8.00 \mathrm{E}+01$ & $<1.92 E+02$ & $8.00 \mathrm{E}+01$ & $7.9 \mathrm{E}+00$ & 2.2E-04 & $4.9 \mathrm{E}-03$ \\
\hline $\mathrm{Ce}$ & ICP-ES & $\mathrm{mg} / \mathrm{kg}$ & $2.03 \mathrm{E}+03$ & $2.55 \mathrm{E}+03$ & $2.29 \mathrm{E}+03$ & $3.2 \mathrm{E}+02$ & $6.3 \mathrm{E}-03$ & $1.4 \mathrm{E}-01$ \\
\hline Co & ICP-ES & $\mathrm{mg} / \mathrm{kg}$ & $2.06 \mathrm{E}+02$ & $2.52 \mathrm{E}+02$ & $2.29 \mathrm{E}+02$ & $3.0 \mathrm{E}+01$ & $6.3 \mathrm{E}-04$ & $1.4 \mathrm{E}-02$ \\
\hline $\mathrm{Cr}$ & ICP-ES & $\mathrm{mg} / \mathrm{kg}$ & $4.98 \mathrm{E}+02$ & $4.56 \mathrm{E}+02$ & $4.77 \mathrm{E}+02$ & $5.6 \mathrm{E}+01$ & $1.3 \mathrm{E}-03$ & $2.9 \mathrm{E}-02$ \\
\hline $\mathrm{Cu}$ & ICP-ES & $\mathrm{mg} / \mathrm{kg}$ & $4.45 \mathrm{E}+02$ & $4.78 \mathrm{E}+02$ & $4.62 \mathrm{E}+02$ & $3.4 \mathrm{E}+01$ & $1.3 \mathrm{E}-03$ & $2.8 \mathrm{E}-02$ \\
\hline $\mathrm{Fe}$ & ICP-ES & $\mathrm{mg} / \mathrm{kg}$ & $3.65 \mathrm{E}+05$ & $3.59 \mathrm{E}+05$ & $3.62 \mathrm{E}+05$ & $2.1 \mathrm{E}+04$ & $1.0 \mathrm{E}+00$ & $2.2 \mathrm{E}+01$ \\
\hline $\mathrm{Gd}$ & ICP-ES & $\mathrm{mg} / \mathrm{kg}$ & $<2.00 E+02$ & $<1.93 E+02$ & $<1.96 E+02$ & -- & -- & -- \\
\hline $\mathrm{K}$ & ICP-ES & $\mathrm{mg} / \mathrm{kg}$ & $6.62 \mathrm{E}+02$ & $<3.71 E+03$ & $6.62 \mathrm{E}+02$ & $9.7 \mathrm{E}+01$ & $1.8 \mathrm{E}-03$ & 4.1E-02 \\
\hline $\mathrm{La}$ & ICP-ES & $\mathrm{mg} / \mathrm{kg}$ & $9.49 \mathrm{E}+02$ & $1.05 \mathrm{E}+03$ & $9.98 \mathrm{E}+02$ & $8.4 \mathrm{E}+01$ & $2.8 \mathrm{E}-03$ & $6.1 \mathrm{E}-02$ \\
\hline $\mathrm{Li}$ & ICP-ES & $\mathrm{mg} / \mathrm{kg}$ & $4.00 \mathrm{E}+02$ & $4.75 \mathrm{E}+02$ & $4.38 \mathrm{E}+02$ & $5.0 \mathrm{E}+01$ & $1.2 \mathrm{E}-03$ & 2.7E-02 \\
\hline $\mathrm{Mg}$ & ICP-ES & $\mathrm{mg} / \mathrm{kg}$ & $4.78 \mathrm{E}+02$ & $6.97 \mathrm{E}+02$ & $5.88 \mathrm{E}+02$ & $1.30 \mathrm{E}+02$ & $1.6 \mathrm{E}-03$ & 3.6E-02 \\
\hline $\mathrm{Mn}$ & ICP-ES & $\mathrm{mg} / \mathrm{kg}$ & $6.39 \mathrm{E}+04$ & $6.96 \mathrm{E}+04$ & $6.68 \mathrm{E}+04$ & $5.2 \mathrm{E}+03$ & $1.8 \mathrm{E}-01$ & $4.1 \mathrm{E}+00$ \\
\hline Mo & ICP-ES & $\mathrm{mg} / \mathrm{kg}$ & $7.22 \mathrm{E}+01$ & $<2.60 E+02$ & $1.66 \mathrm{E}+02$ & $1.04 \mathrm{E}+02$ & 4.6E-04 & $1.0 \mathrm{E}-02$ \\
\hline $\mathrm{Na}$ & ICP-ES & $\mathrm{mg} / \mathrm{kg}$ & $3.37 \mathrm{E}+04$ & -- & $3.37 \mathrm{E}+04$ & $3.6 \mathrm{E}+03$ & $9.3 \mathrm{E}-02$ & $2.1 \mathrm{E}+00$ \\
\hline $\mathrm{Ni}$ & ICP-ES & $\mathrm{mg} / \mathrm{kg}$ & $3.38 \mathrm{E}+04$ & $3.82 \mathrm{E}+04$ & $3.60 \mathrm{E}+04$ & $3.4 \mathrm{E}+03$ & $9.9 \mathrm{E}-02$ & $2.2 \mathrm{E}+00$ \\
\hline $\mathrm{P}$ & ICP-ES & $\mathrm{mg} / \mathrm{kg}$ & $<3.08 E+02$ & $<7.71 E+02$ & $<5.39 E+02$ & -- & -- & -- \\
\hline $\mathrm{Pb}$ & ICP-ES & $\mathrm{mg} / \mathrm{kg}$ & $3.89 \mathrm{E}+02$ & $<6.79 E+02$ & $3.89 \mathrm{E}+02$ & $1.98 \mathrm{E}+02$ & $1.1 \mathrm{E}-03$ & $2.4 \mathrm{E}-02$ \\
\hline $\mathrm{S}$ & ICP-ES & $\mathrm{mg} / \mathrm{kg}$ & $<2.17 E+03$ & $<6.81 E+03$ & $<4.49 E+03$ & -- & -- & -- \\
\hline $\mathrm{Sb}$ & ICP-ES & $\mathrm{mg} / \mathrm{kg}$ & $<4.99 E+02$ & $<6.25 E+02$ & $<5.62 E+02$ & -- & -- & -- \\
\hline $\mathrm{Si}$ & ICP-ES & $\mathrm{mg} / \mathrm{kg}$ & $5.30 \mathrm{E}+03$ & $1.08 \mathrm{E}+04$ & $8.07 \mathrm{E}+03$ & $3.05 \mathrm{E}+03$ & $2.2 \mathrm{E}-02$ & 4.9E-01 \\
\hline $\mathrm{Sn}$ & ICP-ES & $\mathrm{mg} / \mathrm{kg}$ & $<4.98 E+02$ & $<7.79 E+02$ & $<6.39 E+02$ & -- & -- & -- \\
\hline $\mathrm{Sr}$ & ICP-ES & $\mathrm{mg} / \mathrm{kg}$ & $7.48 \mathrm{E}+02$ & $8.12 \mathrm{E}+02$ & $7.80 \mathrm{E}+02$ & $6.0 \mathrm{E}+01$ & $2.2 \mathrm{E}-03$ & $4.8 \mathrm{E}-02$ \\
\hline Th & ICP-ES & $\mathrm{mg} / \mathrm{kg}$ & $<9.22 E+01$ & $<8.09 E+02$ & $<4.51 E+02$ & -- & $1.2 \mathrm{E}-03$ & $2.8 \mathrm{E}-02$ \\
\hline $\mathrm{Ti}$ & ICP-ES & $\mathrm{mg} / \mathrm{kg}$ & $1.86 \mathrm{E}+02$ & $2.11 \mathrm{E}+02$ & $1.99 \mathrm{E}+02$ & $1.9 \mathrm{E}+01$ & $5.5 \mathrm{E}-04$ & $1.2 \mathrm{E}-02$ \\
\hline $\mathrm{U}$ & ICP-ES & $\mathrm{mg} / \mathrm{kg}$ & $7.26 \mathrm{E}+04$ & $8.50 \mathrm{E}+04$ & $7.88 \mathrm{E}+04$ & $8.4 \mathrm{E}+03$ & 2.2E- 01 & $4.8 \mathrm{E}+00$ \\
\hline $\mathrm{V}$ & ICP-ES & $\mathrm{mg} / \mathrm{kg}$ & $<8.70 E+00$ & $<4.72 E+01$ & $<2.80 E+01$ & -- & -- & -- \\
\hline $\mathrm{Zn}$ & ICP-ES & $\mathrm{mg} / \mathrm{kg}$ & $2.26 \mathrm{E}+02$ & $2.64 \mathrm{E}+02$ & $2.45 \mathrm{E}+02$ & $2.6 \mathrm{E}+01$ & $6.8 \mathrm{E}-04$ & $1.5 \mathrm{E}-02$ \\
\hline $\mathrm{Zr}$ & ICP-ES & $\mathrm{mg} / \mathrm{kg}$ & $3.49 \mathrm{E}+03$ & -- & $3.49 \mathrm{E}+03$ & $3.5 \mathrm{E}+02$ & $9.6 \mathrm{E}-03$ & 2.1E-01 \\
\hline Co & ICP-MS & $\mathrm{mg} / \mathrm{kg}$ & $2.08 \mathrm{E}+02$ & $2.34 \mathrm{E}+02$ & $2.21 \mathrm{E}+02$ & $1.4 \mathrm{E}+01$ & $6.1 \mathrm{E}-04$ & $1.4 \mathrm{E}-02$ \\
\hline $\mathrm{Hg}$ & CVAA & $\mathrm{mg} / \mathrm{kg}$ & $6.95 \mathrm{E}+02$ & -- & $6.95 \mathrm{E}+02$ & $8.7 \mathrm{E}+01$ & $1.9 \mathrm{E}-03$ & 4.3E-02 \\
\hline $\mathrm{Pb}$ & ICP-MS & $\mathrm{mg} / \mathrm{kg}$ & $3.58 \mathrm{E}+02$ & $3.90 \mathrm{E}+02$ & $3.74 \mathrm{E}+02$ & $2.6 \mathrm{E}+01$ & $1.0 \mathrm{E}-03$ & 2.3E-02 \\
\hline Th-232 & ICP-MS & $\mathrm{mg} / \mathrm{kg}$ & $<3.19 E+01$ & $1.04 \mathrm{E}+01$ & $1.04 \mathrm{E}+01$ & $7 \mathrm{E}-01$ & $2.9 \mathrm{E}-05$ & 6.4E-04 \\
\hline U-234 & ICP-MS & $\mathrm{mg} / \mathrm{kg}$ & $<4.53 E+01$ & $6.06 \mathrm{E}+00$ & $6.06 \mathrm{E}+00$ & 4.2E-01 & $1.7 \mathrm{E}-05$ & 3.7E-04 \\
\hline U-235 & ICP-MS & $\mathrm{mg} / \mathrm{kg}$ & $4.42 \mathrm{E}+02$ & $5.39 \mathrm{E}+02$ & $4.90 \mathrm{E}+02$ & $6.1 \mathrm{E}+01$ & $1.4 \mathrm{E}-03$ & $3.0 \mathrm{E}-02$ \\
\hline U-236 & ICP-MS & $\mathrm{mg} / \mathrm{kg}$ & $2.53 \mathrm{E}+01$ & $2.54 \mathrm{E}+01$ & $2.54 \mathrm{E}+01$ & $3.9 \mathrm{E}+00$ & 7.0E-05 & $1.6 \mathrm{E}-03$ \\
\hline Np-237 & ICP-MS & $\mathrm{mg} / \mathrm{kg}$ & $4.90 \mathrm{E}+01$ & $5.32 \mathrm{E}+01$ & $5.11 \mathrm{E}+01$ & $3.0 \mathrm{E}+00$ & $1.4 \mathrm{E}-04$ & 3.1E-03 \\
\hline U-238 & ICP-MS & $\mathrm{mg} / \mathrm{kg}$ & $7.22 \mathrm{E}+04$ & $8.58 \mathrm{E}+04$ & $7.90 \mathrm{E}+04$ & $8.7 \mathrm{E}+03$ & $2.2 \mathrm{E}-01$ & $4.8 \mathrm{E}+00$ \\
\hline $\mathrm{Pu}-239$ & ICP-MS & $\mathrm{mg} / \mathrm{kg}$ & $9.81 \mathrm{E}+01$ & $1.07 \mathrm{E}+02$ & $1.03 \mathrm{E}+02$ & $1.2 \mathrm{E}+01$ & $2.8 \mathrm{E}-04$ & $6.3 \mathrm{E}-03$ \\
\hline $\mathrm{Pu}-240$ & ICP-MS & $\mathrm{mg} / \mathrm{kg}$ & $<2.72 E+01$ & $8.69 \mathrm{E}+00$ & $8.69 \mathrm{E}+00$ & $1.33 \mathrm{E}+00$ & 2.4E-05 & 5.3E-04 \\
\hline mass 241 & ICP-MS & $\mathrm{mg} / \mathrm{kg}$ & $<2.72 E+01$ & $1.41 \mathrm{E}+01$ & $1.41 \mathrm{E}+01$ & $1.9 \mathrm{E}+00$ & 3.9E-05 & 8.6E-04 \\
\hline
\end{tabular}


Table 3-2: Characterization of Tank 5F Sludge Sample Available for ECC Actual-Waste Testing, continued

\begin{tabular}{|c|c|c|c|c|c|c|}
\hline analyte & method & units & $\begin{array}{c}\text { per. fusion } \\
\text { average }\end{array}$ & st. dev. & $\mathrm{mCi} / \mathrm{g} \mathrm{Fe}$ & $\mathbf{m C i} / \mathbf{g ~ A l}$ \\
\hline $\mathrm{Pu}-238$ & PuTTA & $\mathrm{dpm} / \mathrm{g}$ & $4.04 \mathrm{E}+06$ & $2.3 \mathrm{E}+05$ & $5.0 \mathrm{E}-03$ & $1.1 \mathrm{E}-01$ \\
\hline $\mathrm{Pu}-239 / 240$ & PuTTA & $\mathrm{dpm} / \mathrm{g}$ & $2.04 \mathrm{E}+07$ & $1.5 \mathrm{E}+06$ & $2.5 \mathrm{E}-02$ & $5.6 \mathrm{E}-01$ \\
\hline $\mathrm{Pu}-241$ & $\mathrm{Pu}-241$ & $\mathrm{dpm} / \mathrm{g}$ & $<2.09 E+07$ & -- & -- & -- \\
\hline Co-60 & gamma & $\mathrm{dpm} / \mathrm{g}$ & $1.96 \mathrm{E}+07$ & $1.9 \mathrm{E}+06$ & $2.4 \mathrm{E}-02$ & 5.4E-01 \\
\hline Cs-137 & gamma & $\mathrm{dpm} / \mathrm{g}$ & $2.39 \mathrm{E}+09$ & $1.3 \mathrm{E}+08$ & $3.0 \mathrm{E}+00$ & $6.6 \mathrm{E}+01$ \\
\hline Eu-154 & gamma & $\mathrm{dpm} / \mathrm{g}$ & $4.52 \mathrm{E}+07$ & $2.4 \mathrm{E}+06$ & $5.6 \mathrm{E}-02$ & $1.2 \mathrm{E}+00$ \\
\hline $\mathrm{Eu}-155$ & gamma & $\mathrm{dpm} / \mathrm{g}$ & $<6.26 E+06$ & -- & -- & -- \\
\hline Np-237 & gamma & $\mathrm{dpm} / \mathrm{g}$ & $<1.04 E+07$ & -- & -- & -- \\
\hline Am-241 & gamma & $\mathrm{dpm} / \mathrm{g}$ & $9.69 \mathrm{E}+07$ & $5.5 \mathrm{E}+06$ & $1.2 \mathrm{E}-01$ & $2.7 \mathrm{E}+00$ \\
\hline Co-60 & Cs-rem $\gamma$ & $\mathrm{dpm} / \mathrm{g}$ & $1.74 \mathrm{E}+07$ & $1.0 \mathrm{E}+06$ & $2.2 \mathrm{E}-02$ & 4.8E-01 \\
\hline $\mathrm{Eu}-154$ & Cs-rem $\gamma$ & $\mathrm{dpm} / \mathrm{g}$ & $3.97 \mathrm{E}+07$ & $9 \mathrm{E}+05$ & 4.9E-02 & $1.1 \mathrm{E}+00$ \\
\hline $\mathrm{Eu}-155$ & Cs-rem $\gamma$ & $\mathrm{dpm} / \mathrm{g}$ & $<6.42 E+06$ & -- & -- & -- \\
\hline Am-241 & Cs-rem $\gamma$ & $\mathrm{dpm} / \mathrm{g}$ & $8.47 \mathrm{E}+07$ & $2.9 \mathrm{E}+06$ & $1.1 \mathrm{E}-01$ & $2.3 \mathrm{E}+00$ \\
\hline Sr-90 & Sr-90 & $\mathrm{dpm} / \mathrm{g}$ & $7.81 \mathrm{E}+10$ & $1.55 \mathrm{E}+10$ & $9.7 \mathrm{E}+01$ & $2.2 \mathrm{E}+03$ \\
\hline Am-241 & $\mathrm{Am} / \mathrm{Cm}$ & $\mathrm{dpm} / \mathrm{g}$ & $8.47 \mathrm{E}+07$ & $2.9 \mathrm{E}+06$ & $1.1 \mathrm{E}-01$ & $2.3 \mathrm{E}+00$ \\
\hline Am-243 & $\mathrm{Am} / \mathrm{Cm}$ & $\mathrm{dpm} / \mathrm{g}$ & $5.79 \mathrm{E}+05$ & $4.7 \mathrm{E}+04$ & $7.2 \mathrm{E}-04$ & $1.6 \mathrm{E}-02$ \\
\hline Am- $242 m$ & $\mathrm{Am} / \mathrm{Cm}$ & $\mathrm{dpm} / \mathrm{g}$ & $1.89 \mathrm{E}+05$ & $4.4 \mathrm{E}+04$ & $2.4 \mathrm{E}-04$ & $5.2 \mathrm{E}-03$ \\
\hline $\mathrm{Cm}-243$ & $\mathrm{Am} / \mathrm{Cm}$ & $\mathrm{dpm} / \mathrm{g}$ & $<1.50 E+06$ & -- & -- & -- \\
\hline $\mathrm{Cm}-245$ & $\mathrm{Am} / \mathrm{Cm}$ & $\mathrm{dpm} / \mathrm{g}$ & $<6.30 E+05$ & -- & -- & -- \\
\hline $\mathrm{Cm}-247$ & $\mathrm{Am} / \mathrm{Cm}$ & $\mathrm{dpm} / \mathrm{g}$ & $<3.11 E+05$ & -- & -- & -- \\
\hline Cf-249 & $\mathrm{Am} / \mathrm{Cm}$ & $\mathrm{dpm} / \mathrm{g}$ & $<3.18 E+05$ & -- & -- & -- \\
\hline Cf-251 & $\mathrm{Am} / \mathrm{Cm}$ & $\mathrm{dpm} / \mathrm{g}$ & $<2.29 E+05$ & -- & -- & -- \\
\hline $\mathrm{Cm}-242$ & $\mathrm{Am} / \mathrm{Cm}$ & $\mathrm{dpm} / \mathrm{g}$ & $1.57 \mathrm{E}+05$ & $3.7 \mathrm{E}+04$ & $1.9 \mathrm{E}-04$ & $4.3 \mathrm{E}-03$ \\
\hline $\mathrm{Cm}-244$ & $\mathrm{Am} / \mathrm{Cm}$ & $\mathrm{dpm} / \mathrm{g}$ & $5.29 \mathrm{E}+06$ & $4.1 \mathrm{E}+05$ & $6.6 \mathrm{E}-03$ & $1.5 \mathrm{E}-01$ \\
\hline
\end{tabular}


SRNL-STI-2011-00360

Revision 0

Table 3-3: Characterization of Tank 12H Sludge Sample Available for ECC Actual-Waste Testing

\begin{tabular}{|c|c|c|c|c|c|c|c|c|}
\hline analyte & method & units & $\begin{array}{l}\text { aqua regia } \\
\text { average }\end{array}$ & $\begin{array}{l}\text { per. fusion } \\
\text { average }\end{array}$ & $\begin{array}{c}\text { overall } \\
\text { average }\end{array}$ & st. dev. & $\mathrm{g} / \mathrm{g} \mathrm{Fe}$ & g / g Al \\
\hline $\mathrm{Ag}$ & ICP-ES & $\mathrm{mg} / \mathrm{kg}$ & $8.96 \mathrm{E}+01$ & $<2.05 E+02$ & $8.96 \mathrm{E}+01$ & $5.4 \mathrm{E}+00$ & $2.0 \mathrm{E}-03$ & 3.4E-04 \\
\hline $\mathrm{Al}$ & ICP-ES & $\mathrm{mg} / \mathrm{kg}$ & $2.68 \mathrm{E}+05$ & $2.56 \mathrm{E}+05$ & $2.62 \mathrm{E}+05$ & $1.2 \mathrm{E}+04$ & $5.8 \mathrm{E}+00$ & $1.0 \mathrm{E}+00$ \\
\hline B & ICP-ES & $\mathrm{mg} / \mathrm{kg}$ & $<1.31 E+02$ & $<1.68 E+02$ & $<1.50 E+02$ & -- & -- & -- \\
\hline $\mathrm{Ba}$ & ICP-ES & $\mathrm{mg} / \mathrm{kg}$ & $5.24 \mathrm{E}+02$ & 4.94E+02 & $5.09 \mathrm{E}+02$ & $2.6 \mathrm{E}+01$ & $1.1 \mathrm{E}-02$ & $1.9 \mathrm{E}-03$ \\
\hline $\mathrm{Be}$ & ICP-ES & $\mathrm{mg} / \mathrm{kg}$ & $<8.93 E+00$ & $<2.10 E+01$ & $<1.50 E+01$ & -- & -- & -- \\
\hline $\mathrm{Ca}$ & ICP-ES & $\mathrm{mg} / \mathrm{kg}$ & $2.46 \mathrm{E}+03$ & $4.12 \mathrm{E}+03$ & $3.29 \mathrm{E}+03$ & $9.44 \mathrm{E}+02$ & 7.3E-02 & 1.3E-02 \\
\hline $\mathrm{Cd}$ & ICP-ES & $\mathrm{mg} / \mathrm{kg}$ & $<6.25 E+01$ & $<1.22 E+02$ & $<9.24 E+01$ & -- & -- & -- \\
\hline $\mathrm{Ce}$ & ICP-ES & $\mathrm{mg} / \mathrm{kg}$ & $8.39 \mathrm{E}+02$ & $<1.25 E+03$ & $8.39 \mathrm{E}+02$ & $2.0 \mathrm{E}+01$ & $1.9 \mathrm{E}-02$ & $3.2 \mathrm{E}-03$ \\
\hline Co & ICP-ES & $\mathrm{mg} / \mathrm{kg}$ & $<6.32 E+01$ & $<8.83 E+01$ & $<7.58 E+01$ & -- & -- & -- \\
\hline $\mathrm{Cr}$ & ICP-ES & $\mathrm{mg} / \mathrm{kg}$ & $2.29 \mathrm{E}+02$ & $2.32 \mathrm{E}+02$ & $2.31 \mathrm{E}+02$ & $9 \mathrm{E}+00$ & $5.1 \mathrm{E}-03$ & 8.8E-04 \\
\hline $\mathrm{Cu}$ & ICP-ES & $\mathrm{mg} / \mathrm{kg}$ & $5.50 \mathrm{E}+02$ & $5.52 \mathrm{E}+02$ & $5.51 \mathrm{E}+02$ & $2.5 \mathrm{E}+01$ & $1.2 \mathrm{E}-02$ & 2.1E-03 \\
\hline $\mathrm{Fe}$ & ICP-ES & $\mathrm{mg} / \mathrm{kg}$ & $4.55 \mathrm{E}+04$ & $4.49 \mathrm{E}+04$ & $4.52 \mathrm{E}+04$ & $1.8 \mathrm{E}+03$ & $1.0 \mathrm{E}+00$ & $1.7 \mathrm{E}-01$ \\
\hline $\mathrm{Gd}$ & ICP-ES & $\mathrm{mg} / \mathrm{kg}$ & $1.65 \mathrm{E}+02$ & $<2.02 E+02$ & $1.65 \mathrm{E}+02$ & $2 \mathrm{E}+00$ & $3.6 \mathrm{E}-03$ & $6.3 \mathrm{E}-04$ \\
\hline $\mathrm{K}$ & ICP-ES & $\mathrm{mg} / \mathrm{kg}$ & $<1.49 E+03$ & $3.27 \mathrm{E}+03$ & $3.27 \mathrm{E}+03$ & $1.3 \mathrm{E}+02$ & 7.2E-02 & $1.2 \mathrm{E}-02$ \\
\hline $\mathrm{La}$ & ICP-ES & $\mathrm{mg} / \mathrm{kg}$ & $4.60 \mathrm{E}+02$ & $3.40 \mathrm{E}+02$ & $4.00 \mathrm{E}+02$ & $7.6 \mathrm{E}+01$ & $8.9 \mathrm{E}-03$ & $1.5 \mathrm{E}-03$ \\
\hline $\mathrm{Li}$ & ICP-ES & $\mathrm{mg} / \mathrm{kg}$ & $1.12 \mathrm{E}+02$ & $<1.65 E+02$ & $1.12 \mathrm{E}+02$ & $7 \mathrm{E}+00$ & $2.5 \mathrm{E}-03$ & 4.3E-04 \\
\hline $\mathrm{Mg}$ & ICP-ES & $\mathrm{mg} / \mathrm{kg}$ & $1.18 \mathrm{E}+03$ & $1.09 \mathrm{E}+03$ & $1.14 \mathrm{E}+03$ & $8 \mathrm{E}+01$ & $2.5 \mathrm{E}-02$ & 4.3E-03 \\
\hline $\mathrm{Mn}$ & ICP-ES & $\mathrm{mg} / \mathrm{kg}$ & $2.94 \mathrm{E}+04$ & $2.69 \mathrm{E}+04$ & $2.81 \mathrm{E}+04$ & $1.6 \mathrm{E}+03$ & $6.2 \mathrm{E}-01$ & $1.1 \mathrm{E}-01$ \\
\hline Mo & ICP-ES & $\mathrm{mg} / \mathrm{kg}$ & $<9.31 E+01$ & $<2.73 E+02$ & $<1.83 E+02$ & -- & -- & -- \\
\hline $\mathrm{Na}$ & ICP-ES & $\mathrm{mg} / \mathrm{kg}$ & $4.23 \mathrm{E}+04$ & -- & $4.23 \mathrm{E}+04$ & $3.1 \mathrm{E}+03$ & 9.4E-01 & $1.6 \mathrm{E}-01$ \\
\hline $\mathrm{Ni}$ & ICP-ES & $\mathrm{mg} / \mathrm{kg}$ & $4.62 \mathrm{E}+03$ & $4.62 \mathrm{E}+03$ & $4.62 \mathrm{E}+03$ & $1.6 \mathrm{E}+02$ & $1.0 \mathrm{E}-01$ & $1.8 \mathrm{E}-02$ \\
\hline $\mathrm{P}$ & ICP-ES & $\mathrm{mg} / \mathrm{kg}$ & $7.58 \mathrm{E}+02$ & $<8.11 E+02$ & $7.58 \mathrm{E}+02$ & $5.2 \mathrm{E}+01$ & $1.7 \mathrm{E}-02$ & $2.9 \mathrm{E}-03$ \\
\hline $\mathrm{Pb}$ & ICP-ES & $\mathrm{mg} / \mathrm{kg}$ & $<2.72 E+02$ & $<6.98 E+02$ & $<4.85 E+02$ & -- & -- & -- \\
\hline $\mathrm{S}$ & ICP-ES & $\mathrm{mg} / \mathrm{kg}$ & $<1.39 E+03$ & $<1.43 E+04$ & $<7.86 E+03$ & -- & -- & -- \\
\hline $\mathrm{Sb}$ & ICP-ES & $\mathrm{mg} / \mathrm{kg}$ & $<1.54 E+02$ & $<6.57 E+02$ & $<4.06 E+02$ & -- & -- & -- \\
\hline $\mathrm{Si}$ & ICP-ES & $\mathrm{mg} / \mathrm{kg}$ & $2.59 \mathrm{E}+03$ & $2.72 \mathrm{E}+03$ & $2.66 \mathrm{E}+03$ & $1.08 \mathrm{E}+02$ & $5.9 \mathrm{E}-02$ & $1.0 \mathrm{E}-02$ \\
\hline $\mathrm{Sn}$ & ICP-ES & $\mathrm{mg} / \mathrm{kg}$ & $<3.19 E+02$ & $<8.19 E+02$ & $<5.69 E+02$ & -- & -- & -- \\
\hline $\mathrm{Sr}$ & ICP-ES & $\mathrm{mg} / \mathrm{kg}$ & $1.97 \mathrm{E}+02$ & $2.35 \mathrm{E}+02$ & $2.16 \mathrm{E}+02$ & $2.5 \mathrm{E}+01$ & $4.8 \mathrm{E}-03$ & $8.2 \mathrm{E}-04$ \\
\hline Th & ICP-ES & $\mathrm{mg} / \mathrm{kg}$ & $1.73 E+04$ & $1.36 \mathrm{E}+04$ & $1.55 \mathrm{E}+04$ & $2.3 \mathrm{E}+03$ & $3.4 \mathrm{E}-01$ & $5.9 \mathrm{E}-02$ \\
\hline $\mathrm{Ti}$ & ICP-ES & $\mathrm{mg} / \mathrm{kg}$ & $1.03 \mathrm{E}+02$ & $1.13 \mathrm{E}+02$ & $1.08 \mathrm{E}+02$ & $7 \mathrm{E}+00$ & 2.4E-03 & 4.1E-04 \\
\hline $\mathrm{U}$ & ICP-ES & $\mathrm{mg} / \mathrm{kg}$ & $6.00 \mathrm{E}+03$ & $<7.17 E+03$ & $6.00 \mathrm{E}+03$ & $2.0 \mathrm{E}+02$ & $1.3 \mathrm{E}-01$ & $2.3 \mathrm{E}-02$ \\
\hline $\mathrm{V}$ & ICP-ES & $\mathrm{mg} / \mathrm{kg}$ & $<4.46 E+01$ & $<9.87 E+01$ & $<7.17 E+01$ & -- & -- & -- \\
\hline $\mathrm{Zn}$ & ICP-ES & $\mathrm{mg} / \mathrm{kg}$ & $3.56 \mathrm{E}+02$ & $3.30 \mathrm{E}+02$ & $3.43 \mathrm{E}+02$ & $2.0 \mathrm{E}+01$ & 7.6E-03 & $1.3 \mathrm{E}-03$ \\
\hline $\mathrm{Zr}$ & ICP-ES & $\mathrm{mg} / \mathrm{kg}$ & $1.46 \mathrm{E}+03$ & -- & $1.46 \mathrm{E}+03$ & $5 \mathrm{E}+01$ & $3.2 \mathrm{E}-02$ & $5.6 \mathrm{E}-03$ \\
\hline Co & ICP-MS & $\mathrm{mg} / \mathrm{kg}$ & $2.61 \mathrm{E}+01$ & $2.85 \mathrm{E}+01$ & $2.73 \mathrm{E}+01$ & $3.3 \mathrm{E}+00$ & $6.0 \mathrm{E}-04$ & $1.0 \mathrm{E}-04$ \\
\hline $\mathrm{Pb}$ & ICP-MS & $\mathrm{mg} / \mathrm{kg}$ & $2.98 \mathrm{E}+01$ & $3.15 \mathrm{E}+01$ & $3.07 \mathrm{E}+01$ & $8.2 \mathrm{E}+00$ & $6.8 \mathrm{E}-04$ & $1.2 \mathrm{E}-04$ \\
\hline $\mathrm{Hg}$ & CVAA & $\mathrm{mg} / \mathrm{kg}$ & $1.85 \mathrm{E}+04$ & -- & $1.85 \mathrm{E}+04$ & $1.2 \mathrm{E}+03$ & 4.1E-01 & 7.0E-02 \\
\hline Th-232 & ICP-MS & $\mathrm{mg} / \mathrm{kg}$ & $1.67 \mathrm{E}+04$ & $1.38 \mathrm{E}+04$ & $1.52 \mathrm{E}+04$ & $1.8 \mathrm{E}+03$ & 3.4E-01 & $5.8 \mathrm{E}-02$ \\
\hline U-233 & ICP-MS & $\mathrm{mg} / \mathrm{kg}$ & $1.30 \mathrm{E}+01$ & $9.29 \mathrm{E}+00$ & $1.11 \mathrm{E}+01$ & $2.8 \mathrm{E}+00$ & $2.5 \mathrm{E}-04$ & 4.2E-05 \\
\hline U-234 & ICP-MS & $\mathrm{mg} / \mathrm{kg}$ & $1.00 \mathrm{E}+01$ & $1.06 \mathrm{E}+01$ & $1.03 \mathrm{E}+01$ & $1.2 \mathrm{E}+00$ & $2.3 \mathrm{E}-04$ & 3.9E-05 \\
\hline U-235 & ICP-MS & $\mathrm{mg} / \mathrm{kg}$ & $1.01 \mathrm{E}+02$ & $9.16 \mathrm{E}+01$ & $9.63 \mathrm{E}+01$ & 7.4E+00 & $2.1 \mathrm{E}-03$ & 3.7E-04 \\
\hline U-236 & ICP-MS & $\mathrm{mg} / \mathrm{kg}$ & $1.29 \mathrm{E}+01$ & $1.37 \mathrm{E}+01$ & $1.33 \mathrm{E}+01$ & $1.9 \mathrm{E}+00$ & $2.9 \mathrm{E}-04$ & $5.1 \mathrm{E}-05$ \\
\hline Np-237 & ICP-MS & $\mathrm{mg} / \mathrm{kg}$ & $1.35 \mathrm{E}+01$ & $1.35 \mathrm{E}+01$ & $1.35 \mathrm{E}+01$ & $1.9 \mathrm{E}+00$ & $3.0 \mathrm{E}-04$ & $5.2 \mathrm{E}-05$ \\
\hline U-238 & ICP-MS & $\mathrm{mg} / \mathrm{kg}$ & $5.66 \mathrm{E}+03$ & $4.83 \mathrm{E}+03$ & $5.25 \mathrm{E}+03$ & $5.5 \mathrm{E}+02$ & $1.2 \mathrm{E}-01$ & $2.0 \mathrm{E}-02$ \\
\hline $\mathrm{Pu}-239$ & ICP-MS & $\mathrm{mg} / \mathrm{kg}$ & $2.15 \mathrm{E}+02$ & $1.88 \mathrm{E}+02$ & $2.02 \mathrm{E}+02$ & $2.3 \mathrm{E}+01$ & $4.5 \mathrm{E}-03$ & 7.7E-04 \\
\hline $\mathrm{Pu}-240$ & ICP-MS & $\mathrm{mg} / \mathrm{kg}$ & $2.26 \mathrm{E}+01$ & $2.05 \mathrm{E}+01$ & $2.16 \mathrm{E}+01$ & $2.2 \mathrm{E}+00$ & 4.8E-04 & 8.2E-05 \\
\hline
\end{tabular}


Table 3-4: Characterization of Tank 12H Sludge Sample Available for ECC Actual-Waste Testing, continued

\begin{tabular}{ccc|c|c|c|c}
\hline analyte & method & units & $\begin{array}{c}\text { overall } \\
\text { average }\end{array}$ & st. dev. & mCi / g Fe & mCi / g Al \\
\hline $\mathrm{Pu}-238$ & PuTTA & $\mathrm{dpm} / \mathrm{g}$ & $7.18 \mathrm{E}+08$ & $5.2 \mathrm{E}+07$ & $7.2 \mathrm{E}+00$ & $1.2 \mathrm{E}+00$ \\
\hline $\mathrm{Pu}-239 / 240$ & $\mathrm{PuTTA}$ & $\mathrm{dpm} / \mathrm{g}$ & $3.06 \mathrm{E}+07$ & $2.4 \mathrm{E}+06$ & $3.0 \mathrm{E}-01$ & $5.3 \mathrm{E}-02$ \\
\hline $\mathrm{Pu}-241$ & $\mathrm{Pu}-241$ & $\mathrm{dpm} / \mathrm{g}$ & $1.74 \mathrm{E}+08$ & $1.3 \mathrm{E}+07$ & $1.7 \mathrm{E}+00$ & $3.0 \mathrm{E}-01$ \\
\hline $\mathrm{Cs}-137$ & gamma & $\mathrm{dpm} / \mathrm{g}$ & $2.48 \mathrm{E}+08$ & $2.1 \mathrm{E}+07$ & $2.5 \mathrm{E}+00$ & $4.3 \mathrm{E}-01$ \\
\hline $\mathrm{Co}-60$ & $\mathrm{Cs}-\mathrm{rem} \mathrm{g}$ & $\mathrm{dpm} / \mathrm{g}$ & $4.42 \mathrm{E}+05$ & $3.5 \mathrm{E}+04$ & $4.4 \mathrm{E}-03$ & $7.6 \mathrm{E}-04$ \\
\hline $\mathrm{Eu}-152$ & $\mathrm{Cs}-\mathrm{rem} \mathrm{g}$ & $\mathrm{dpm} / \mathrm{g}$ & $2.43 \mathrm{E}+06$ & $1.3 \mathrm{E}+05$ & $2.4 \mathrm{E}-02$ & $4.2 \mathrm{E}-03$ \\
\hline $\mathrm{Eu}-154$ & $\mathrm{Cs}-\mathrm{remg}$ & $\mathrm{dpm} / \mathrm{g}$ & $3.15 \mathrm{E}+07$ & $2.6 \mathrm{E}+06$ & $3.1 \mathrm{E}-01$ & $5.4 \mathrm{E}-02$ \\
\hline $\mathrm{Eu}-155$ & $\mathrm{Cs}-\mathrm{rem} \mathrm{g}$ & $\mathrm{dpm} / \mathrm{g}$ & $2.10 \mathrm{E}+06$ & $4 \mathrm{E}+04$ & $2.1 \mathrm{E}-02$ & $3.6 \mathrm{E}-03$ \\
\hline $\mathrm{Am}-241$ & $\mathrm{Cs}-\mathrm{rem} \mathrm{g}$ & $\mathrm{dpm} / \mathrm{g}$ & $3.90 \mathrm{E}+07$ & $3.5 \mathrm{E}+06$ & $3.9 \mathrm{E}-01$ & $6.7 \mathrm{E}-02$ \\
\hline $\mathrm{Sr}-90$ & $\mathrm{Sr}-90$ & $\mathrm{dpm} / \mathrm{g}$ & $2.04 \mathrm{E}+10$ & $2.0 \mathrm{E}+09$ & $2.0 \mathrm{E}+02$ & $3.5 \mathrm{E}+01$ \\
\hline $\mathrm{Am}-241$ & $\mathrm{Am} / \mathrm{Cm}$ & $\mathrm{dpm} / \mathrm{g}$ & $3.90 \mathrm{E}+07$ & $3.5 \mathrm{E}+06$ & $3.9 \mathrm{E}-01$ & $6.7 \mathrm{E}-02$ \\
\hline $\mathrm{Am}-243$ & $\mathrm{Am} / \mathrm{Cm}$ & $\mathrm{dpm} / \mathrm{g}$ & $3.53 \mathrm{E}+04$ & $3.0 \mathrm{E}+03$ & $3.5 \mathrm{E}-04$ & $6.1 \mathrm{E}-05$ \\
\hline $\mathrm{Am}-242 \mathrm{~m}$ & $\mathrm{Am} / \mathrm{Cm}$ & $\mathrm{dpm} / \mathrm{g}$ & $2.83 \mathrm{E}+04$ & $4.63 \mathrm{E}+04$ & $2.8 \mathrm{E}-04$ & $4.9 \mathrm{E}-05$ \\
\hline $\mathrm{Cm}-243$ & $\mathrm{Am} / \mathrm{Cm}$ & $\mathrm{dpm} / \mathrm{g}$ & $<1.52 \mathrm{E}+05$ & -- & -- & -- \\
\hline $\mathrm{Cm}-245$ & $\mathrm{Am} / \mathrm{Cm}$ & $\mathrm{dpm} / \mathrm{g}$ & $<1.25 \mathrm{E}+05$ & -- & -- & -- \\
\hline $\mathrm{Cm}-247$ & $\mathrm{Am} / \mathrm{Cm}$ & $\mathrm{dpm} / \mathrm{g}$ & $<1.18 \mathrm{E}+05$ & -- & -- & - \\
\hline $\mathrm{Cf}-249$ & $\mathrm{Am} / \mathrm{Cm}$ & $\mathrm{dpm} / \mathrm{g}$ & $<1.18 E+05$ & -- & -- & -- \\
\hline $\mathrm{Cf}-251$ & $\mathrm{Am} / \mathrm{Cm}$ & $\mathrm{dpm} / \mathrm{g}$ & $<9.78 \mathrm{E}+04$ & -- & -- & - \\
\hline $\mathrm{Cm}-242$ & $\mathrm{Am} / \mathrm{Cm}$ & $\mathrm{dpm} / \mathrm{g}$ & $2.34 \mathrm{E}+04$ & $3.83 \mathrm{E}+04$ & $2.3 \mathrm{E}-04$ & $4.0 \mathrm{E}-05$ \\
\hline $\mathrm{Cm}-244$ & $\mathrm{Am} / \mathrm{Cm}$ & $\mathrm{dpm} / \mathrm{g}$ & $1.03 \mathrm{E}+06$ & $1.3 \mathrm{E}+05$ & $1.0 \mathrm{E}-02$ & $1.8 \mathrm{E}-03$ \\
\hline & & & & & &
\end{tabular}

\subsection{ECC Processing}

The primary ECC process testing involved the dissolution of actual waste sludge using $2 \mathrm{wt} \% \mathrm{OA}$ (Dissolution), the decomposition of OA and the precipitation of sludge by oxidation initiated with dissolved ozone in the ECC reactor (Decomposition), and liquid removal by a vacuum evaporator (Evaporation). This section contains the conditions and real-time observations made during the primary ECC process testing. The observations related to solubilities and sample analytical results for the dissolution and decomposition processes are discussed in the next section (Section 3.3). Outside of the primary ECC process, the storage of materials at different conditions for investigation of solubility effects is discussed in a separate section (Section 3.4).

Previous Table 2-1 contains a description of the ECC tests that were initially scheduled to be performed. This report contains the results of the testing performed without use of the UV light, Test 1 using Tank 12H sludge feed material and Test 2 using Tank 5F sludge feed material. Prior to initiating testing with actual-waste sludge, a test was performed that only involved the decomposition of $2 \mathrm{wt} \%$ OA (without running the other modules, without sludge feed, and without chemical and radiological analysis). 
Table 3-5 contains information on the material balance for the two ECC real waste tests. We determined that the dissolution module must contain at least $3.2 \mathrm{~L}$ of material in order for the $\mathrm{pH}$ probe to be in contact with the solution. However, when the RWT equipment transfer system is used between the Dissolution and Decomposition Module, there is significant holdup in the lines $(600 \mathrm{~mL}$ to $1 \mathrm{~L})$. The total volume in the Dissolution Module was 3.2L or greater for each test even though the volume of fresh $2 \mathrm{wt} \%$ OA was often less than $3.2 \mathrm{~L}$. The Decomposition Module product volume estimate is a more appropriate indication of the amount of material that was reacted in the ECC Decomposition Module than is the Dissolution Module OA volume feed.

\section{Table 3-5: Throughput Data ECC Real Waste Tests 1 and 2}

Test 1

\begin{tabular}{|c|c|}
\hline Initial Tank 12H Sludge Feed (dry) & $48.1 \mathrm{~g}$ \\
\hline Residual After OA Batch 3 (dry) & $13.1 \mathrm{~g}$ \\
\hline Sludge Removal (dry basis) & $73 \%$ \\
\hline \multicolumn{2}{|l|}{ OA Batch 1} \\
\hline Oxalic Acid Feed & $3.2 \mathrm{~L}$ \\
\hline Dis solution Time & $10.5 \mathrm{hr}$ \\
\hline Decomposition Module Product & $2.2 \mathrm{~L}$ \\
\hline Evaporator Module Feed & $1.75 \mathrm{~L}$ \\
\hline Evaporator Module Output (as slurry) & $189 \mathrm{~g}$ \\
\hline \multicolumn{2}{|l|}{ OA Batch 2} \\
\hline Oxalic Acid Feed & $3.0 \mathrm{~L}$ \\
\hline Dis solution Time & $8 \mathrm{hr}$ \\
\hline Decomposition Module Product & $2.8 \mathrm{~L}$ \\
\hline Evaporator Module Feed & $1.95 \mathrm{~L}$ \\
\hline Evaporator Module Output (as slurry) & $351 \mathrm{~g}$ \\
\hline \multicolumn{2}{|l|}{ OA Batch 3} \\
\hline Oxalic Acid Feed & $3.0 \mathrm{~L}$ \\
\hline Dis solution Time & $8 \mathrm{hr}$ \\
\hline Decomposition Module Product & $3.0 \mathrm{~L}$ \\
\hline Evaporator Module Feed & $1.95 \mathrm{~L}$ \\
\hline Evaporator Module Output (as slurry) & $276 \mathrm{~g}$ \\
\hline
\end{tabular}

Test 2

\begin{tabular}{lrl}
\hline Initial Tank 5F Sludge Feed (dry) & $65.1 \mathrm{~g}$ \\
Residual After OA Batch 3 (dry) & $12.7 \mathrm{~g}$ \\
Sludge Removal (dry basis) & $80 \%$ \\
\hline \multicolumn{1}{c}{ OA Batch 1 } & \\
\hline Oxalic Acid Feed & $3.2 \mathrm{~L}$ \\
Dissolution Time & $2.8 \mathrm{hr}$ \\
Decomposition Module Product & $2.0 \mathrm{~L}$ \\
Evaporator Module Feed & $310 \mathrm{~g}$ \\
Evaporator Module Output (as slurry)
\end{tabular}

OA Batch 2

\begin{tabular}{|c|c|}
\hline Oxalic Acid Feed & $2.8 \mathrm{~L}$ \\
\hline Dis solution Time & $8 \mathrm{hr}$ \\
\hline Decomposition Module Product & $2.3 \mathrm{~L}$ \\
\hline Evaporator Module Feed & $1.5 \mathrm{~L}$ \\
\hline Evaporator Module Output (as slurry) & $123 \mathrm{~g}$ \\
\hline \multicolumn{2}{|l|}{ OA Batch 3} \\
\hline Oxalic Acid Feed & $2.8 \mathrm{~L}$ \\
\hline Dis solution Time & $8 \mathrm{hr}$ \\
\hline Decomposition Module Product & $2.85 \mathrm{~L}$ \\
\hline Evaporator Module Feed & $2.2 \mathrm{~L}$ \\
\hline Evaporator Module Output (as slurry) & $187 \mathrm{~g}$ \\
\hline
\end{tabular}


During the two tests, the sludge removal did not meet the target of $95 \%$ removal by a combination of dissolution with $\mathrm{OA}$ and transfer of insoluble solids. Test 1 showed an approximately $73 \%$ removal of Tank $12 \mathrm{H}$ sludge and Test 2 showed an approximately $80 \%$ removal of Tank $5 \mathrm{~F}$ sludge. Although the dissolution in this testing did not meet the stated goal, the dissolution time (or cycle) in this testing was not representative of the ECC flowsheet as the dissolution time was limited to only 8 hours. The $1 \mathrm{gpm}$ circulation rate, with the discharge of the fluid onto the surface of the dissolution module fluid, results in a level of mixing at the bottom of the dissolution basket that is conservatively low. Visual observation of the sludge in the dissolution basket (made between OA batches) suggested that sludge suspension was not fully achieved during testing. Figure 3-1 contains a photograph of the dissolution basket made after the first OA batch for Test 1 . The tank $12 \mathrm{H}$ sludge was wetter and noticeably lighter in shade after the first OA batch. Even though this sludge had a particle size that would allow for it to be swept through the mesh, a significant portion of the undissolved solids remained in the dissolution basket.

Appendix A contains a series of tables (Table A-1 through Table A-25) that list the process conditions as a function of time for each of the dissolution, decomposition, and evaporation runs performed. Dissolution and evaporation data were recorded hourly. Decomposition data was recorded on a 15 minute interval except for periods where $\mathrm{pH}$ readings were changing rapidly enough to warrant more frequent observation.

Figure 3-2 through Figure 3-8 contain plots of the in-process $\mathrm{pH}$ and ORP measurement data during the ECC decomposition testing. During decomposition testing, $\mathrm{pH}$ was used as the indicator of OA decomposition. The $\mathrm{pH}$ generally started low, near the final $\mathrm{pH}$ of the OA and dissolved sludge mixture measured at the end of the dissolution process. As the ECC decomposition proceeded, the $\mathrm{pH}$ gradually increased, accelerating as $\mathrm{pH}$ approached 7 . The $\mathrm{pH}$ then leveled off, usually above $\mathrm{pH}$ 8. Overall, the ORP measurements were less regular than the $\mathrm{pH}$ measurements. ORP remained constant for the most part during the initial periods where $\mathrm{pH}$ was changing slowly. Then a change in the ORP measurement was usually noted during periods when $\mathrm{pH}$ was changing more rapidly. This period was usually followed by another period of nearly constant ORP.

Figure 3-2 contains the $\mathrm{pH}$ and ORP trends for the case where no sludge feed was added and 2 wt $\%$ OA was decomposed. Because magnetite and hematite dissolution testing had occurred previously using the ECC RWT equipment and cleaning of the equipment was not perfect, we consider this testing to also contain small amounts of iron. The appearance of the decomposition module effluent supported our assumption that the OA decomposition test contained iron. The OA decomposition test without sludge feed was the only decomposition test in this set that was split into two parts due to the long run time. The first 10.5 hours of run time was performed continuously, followed by a system shutdown overnight, with the additional run time resuming when reaction conditions were reestablished 13.5 hours later. The shutdown period is evident in Figure 3-2 by a slight discontinuity in the $\mathrm{pH}$ measurement, which indicates that some decomposition reaction may have continued to occur during the shutdown and restart period. The OA decomposition test without added sludge is distinguished by the relatively long reaction time required (greater than 13 hours) and by the relatively fast $\mathrm{pH}$ change from 2 to 7 , which took less than 1.5 hours. The ORP was steady throughout the first portion of processing, at 650 to 700 $\mathrm{mV}$. When the $\mathrm{pH}$ started changing rapidly, the ORP measurement increased to above $1000 \mathrm{mV}$ and then settled to around $900 \mathrm{mV}$ at the completion of the decomposition reaction. 
Figure 3-3, Figure 3-4, and Figure 3-5 contain the $\mathrm{pH}$ and ORP trends for Test 1 (Tank 12H sludge) OA decomposition for the first, second, and third batches, respectively. The three batches show similar trends in $\mathrm{pH}$ during OA decomposition, with the major differences being the initial $\mathrm{pH}$ and the amount of time required for OA decomposition. In all cases, once the $\mathrm{pH}$ had risen to 3 , it took an additional three hours for the $\mathrm{pH}$ to raise to 7 and above. However, since the starting $\mathrm{pH}$ was higher for the earlier batches than for the later batches, the amount of time to get to $\mathrm{pH} 3$ increased with subsequent batches. The threshold of $\mathrm{pH} 3$ was crossed at 30 minutes, five hours, and eight hours for the first, second and third OA batches, respectively. The change in $\mathrm{pH}$ temporarily slowed around $\mathrm{pH} 4$, possibly indicating that solids were precipitating or some other factor was contributing to $\mathrm{pH}$ buffering. Once again, the ORP measurement fluctuated during periods of more rapid $\mathrm{pH}$ change.

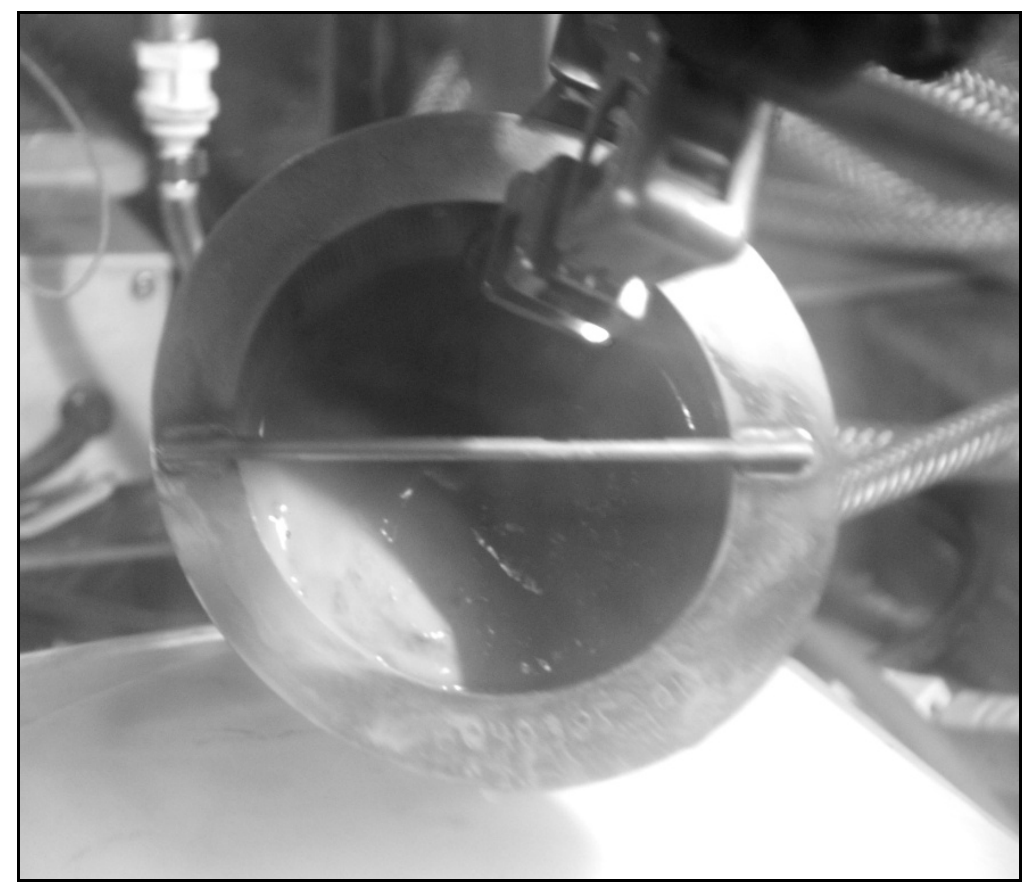

Figure 3-1: Sludge remaining in Dissolution Module basket after Test 1, OA Batch 1 
Figure 3-6, Figure 3-7, and Figure 3-8 contain the $\mathrm{pH}$ and ORP trends for Test 2 (Tank 5F sludge) $\mathrm{OA}$ decomposition for the first, second and third batches, respectively. In Figure 3-6, the $\mathrm{pH}$ measurements during the first OA decomposition batch are not correct but are shown to illustrate the real-time information available at the time of the experiment. Inspection of the $\mathrm{pH}$ probe after the first OA decomposition batch revealed that the probe was damaged either prior to or during the decomposition batch. Because the $\mathrm{pH}$ measurement is the primary indication of the progress of the decomposition reaction, decomposition of the first OA batch was performed for much longer than necessary. Based on the steadiness of the ORP measurement after about five hours of the decomposition reaction, it is likely that the target oxalate decomposition was attained in about six hours. Accounting for the $\mathrm{pH}$ probe problem with the first OA batch, similar trends in $\mathrm{pH}$ were noted for Test 2 as were seen in Test 1. Each subsequent OA decomposition batch started at a lower $\mathrm{pH}$, had a higher soluble oxalate concentration, and took a longer time to show large increases in the $\mathrm{pH}$ measurement, resulting in overall longer decomposition times required for the later batches. The $\mathrm{pH}$ and ORP trends for Test 2 are internally consistent but did not show the same exact shape as the trends for Test 1, possibly indicating slightly different chemistry. Overall, Test 2 OA decomposition was accomplished in less time than for Test 1, possibly due to the larger amount of iron in the Tank 5F sludge than in the Tank $12 \mathrm{H}$ sludge. The differences in the time required for decomposition may be due to the differences in concentrations of species like iron, which may catalyze the OA decomposition reactions.

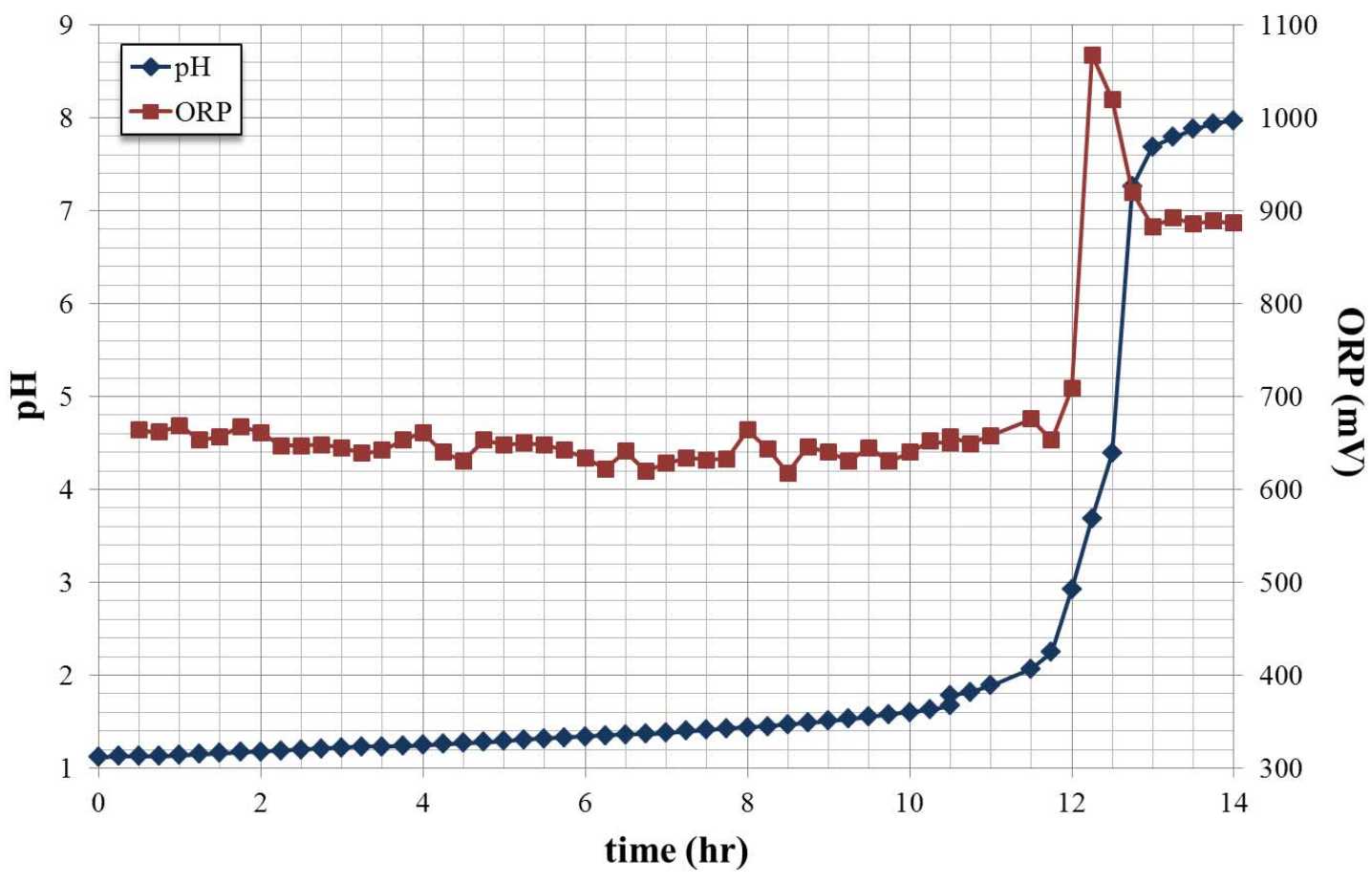

Figure 3-2: Trends for $\mathrm{pH}$ and ORP during Decomposition of Oxalic Acid by Ozone 


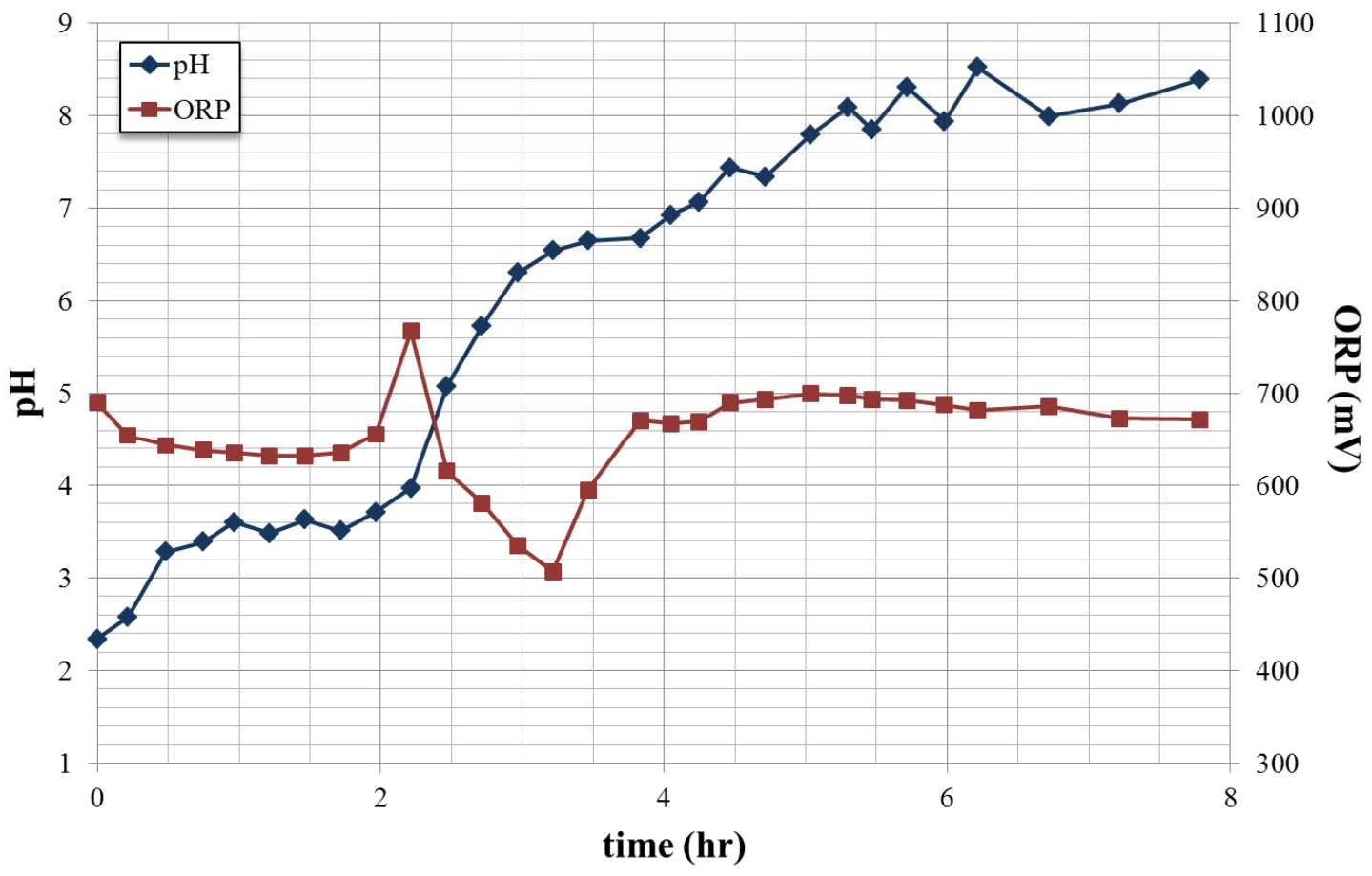

Figure 3-3: Trends for $\mathrm{pH}$ and ORP during Decomposition Test 1 (Tank 12H) OA Batch 1

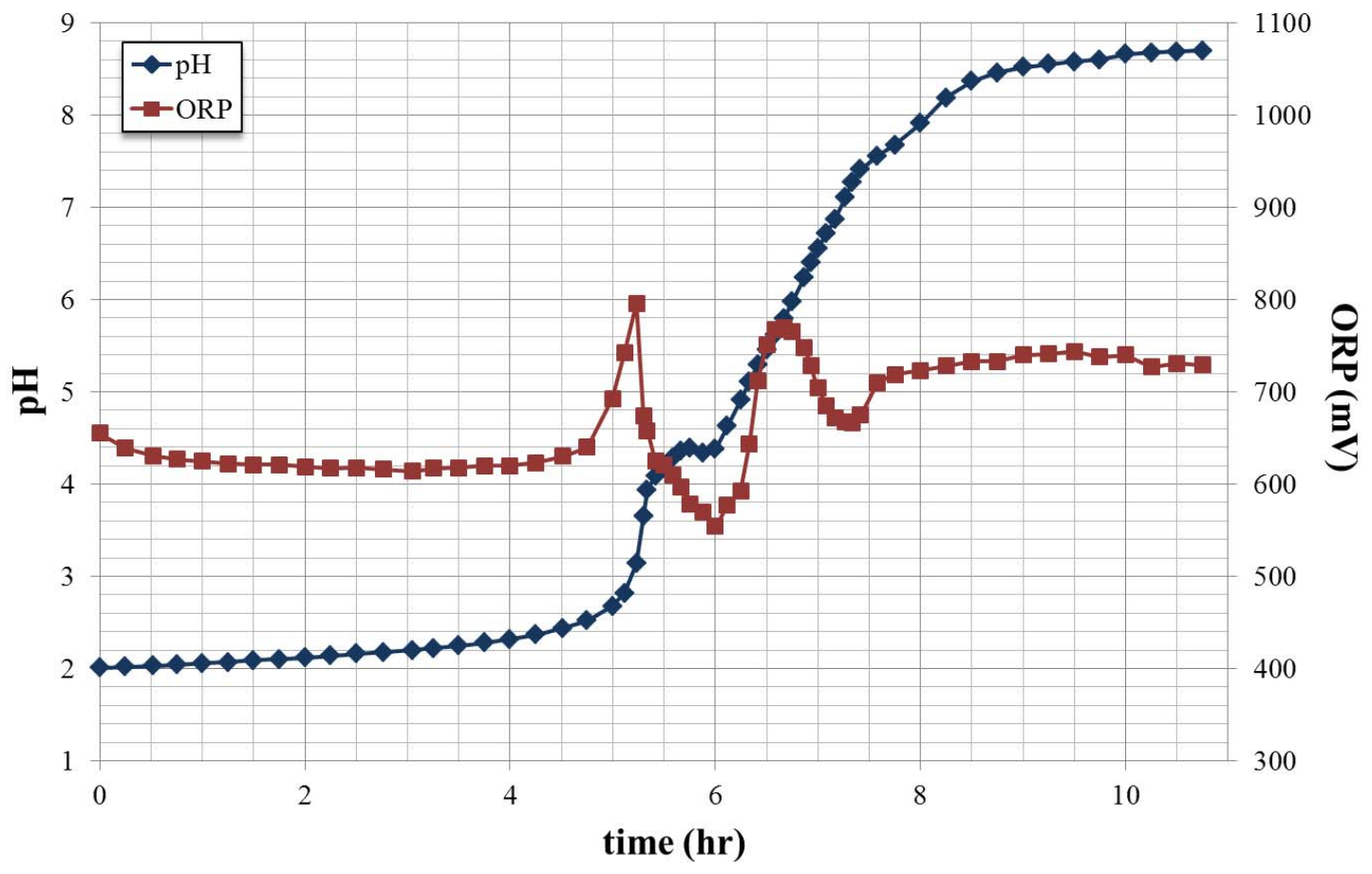

Figure 3-4: Trends for pH and ORP during Decomposition Test 1 (Tank 12H) OA Batch 2 


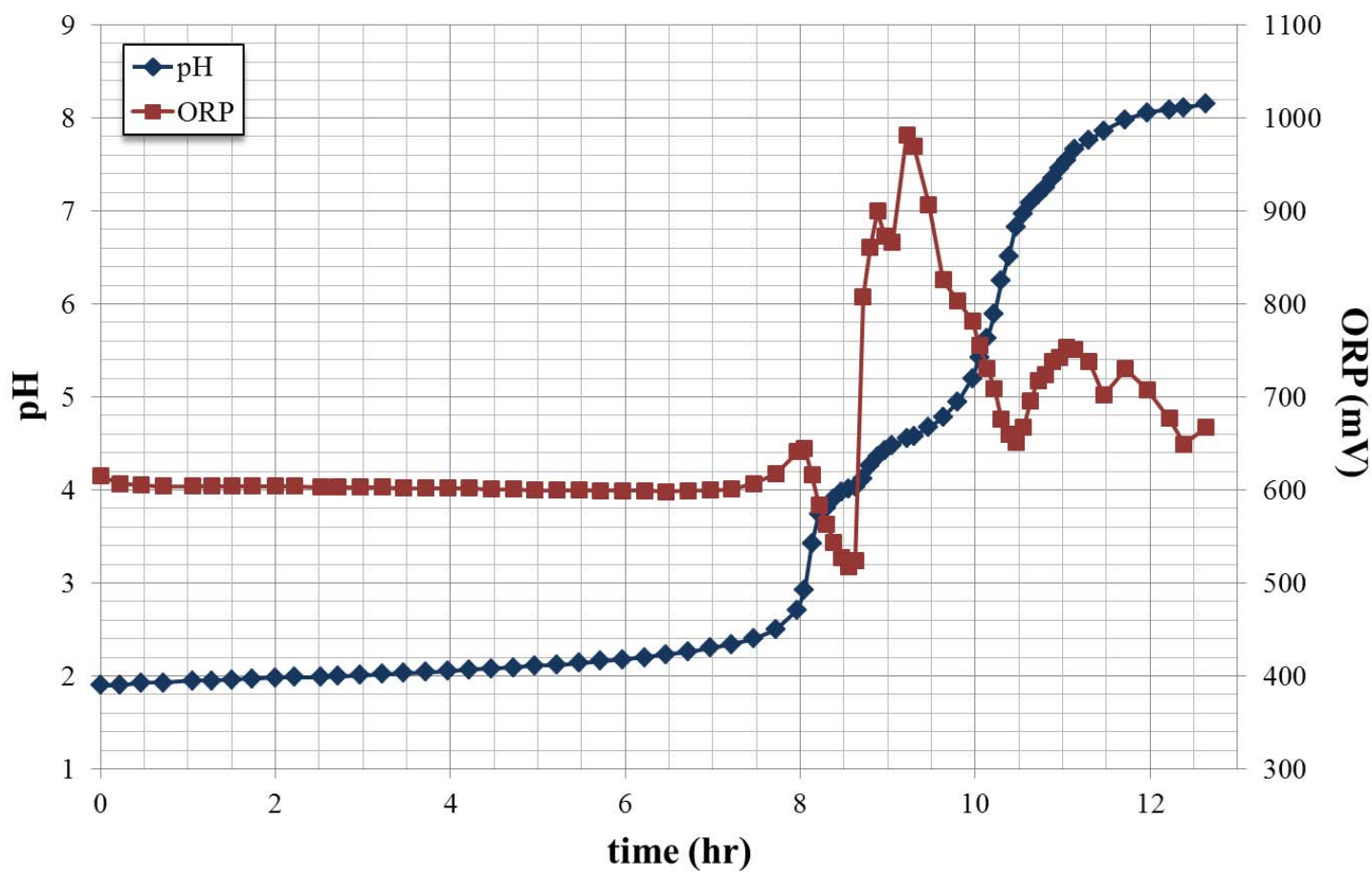

Figure 3-5: Trends for pH and ORP during Decomposition Test 1 (Tank 12H) OA Batch 3

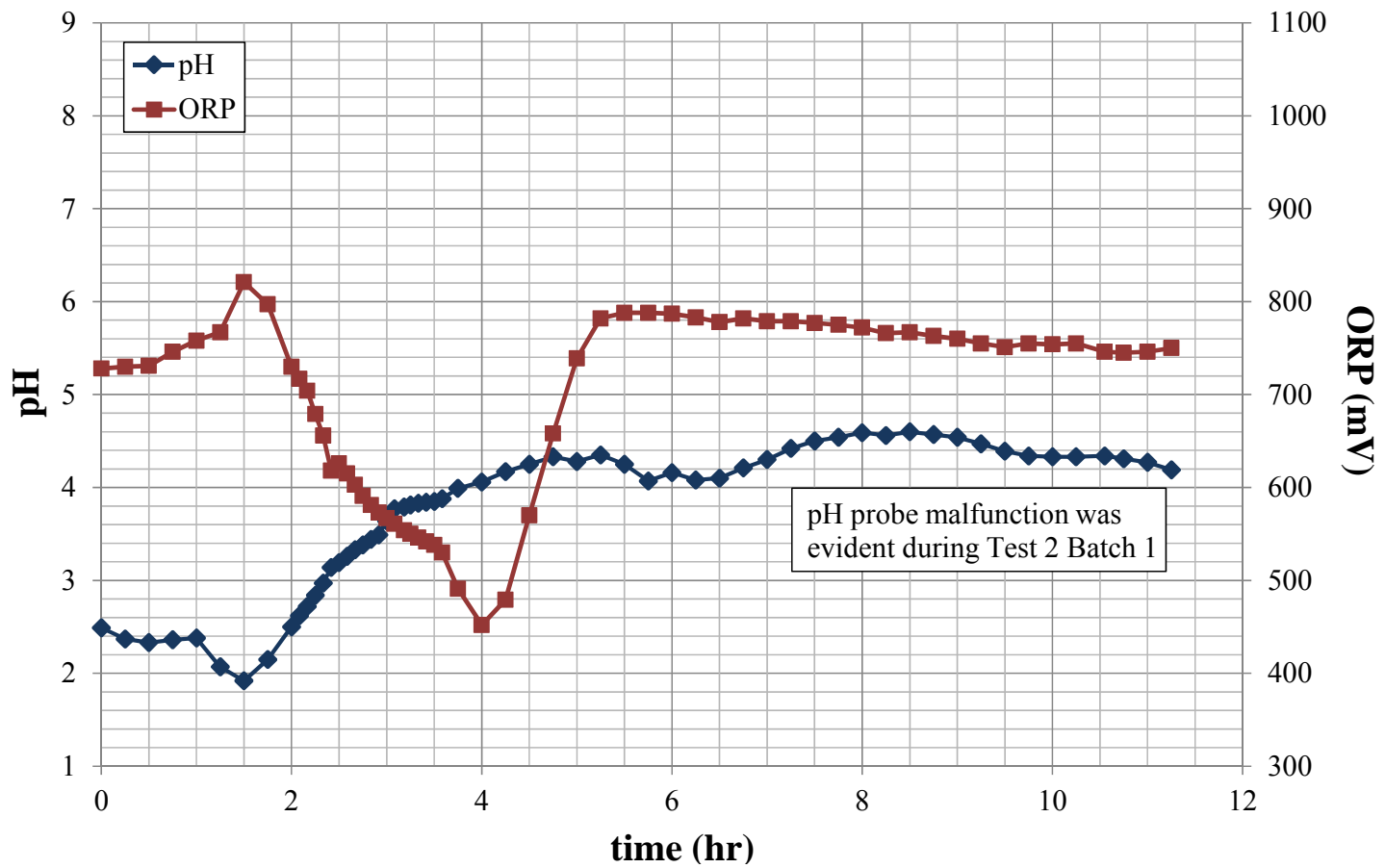

Figure 3-6: Trends for $\mathrm{pH}$ and ORP during Decomposition Test 2 (Tank 5F) OA Batch 1 


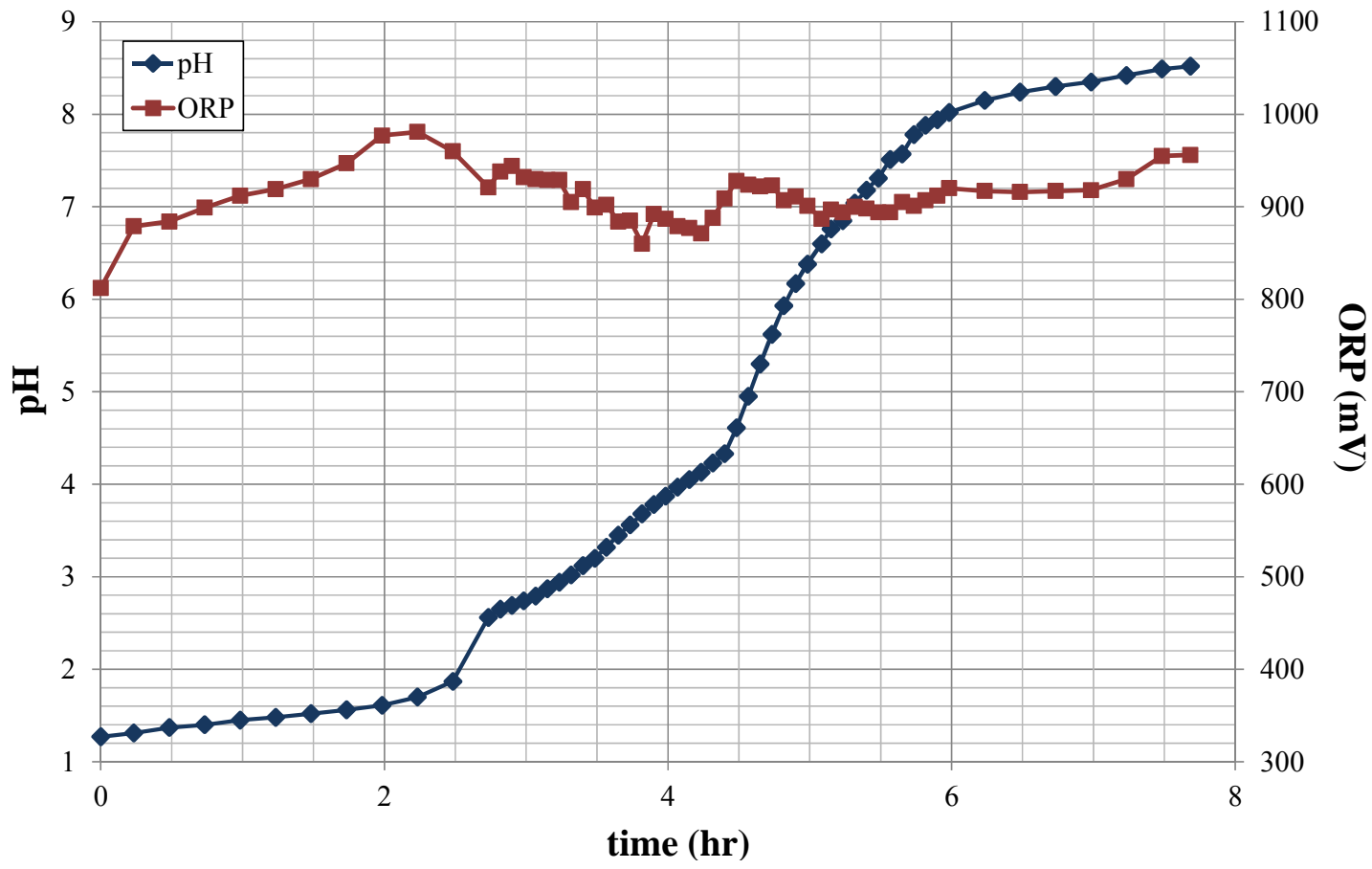

Figure 3-7: Trends for $\mathrm{pH}$ and ORP during Decomposition Test 2 (Tank 5F) OA Batch 2

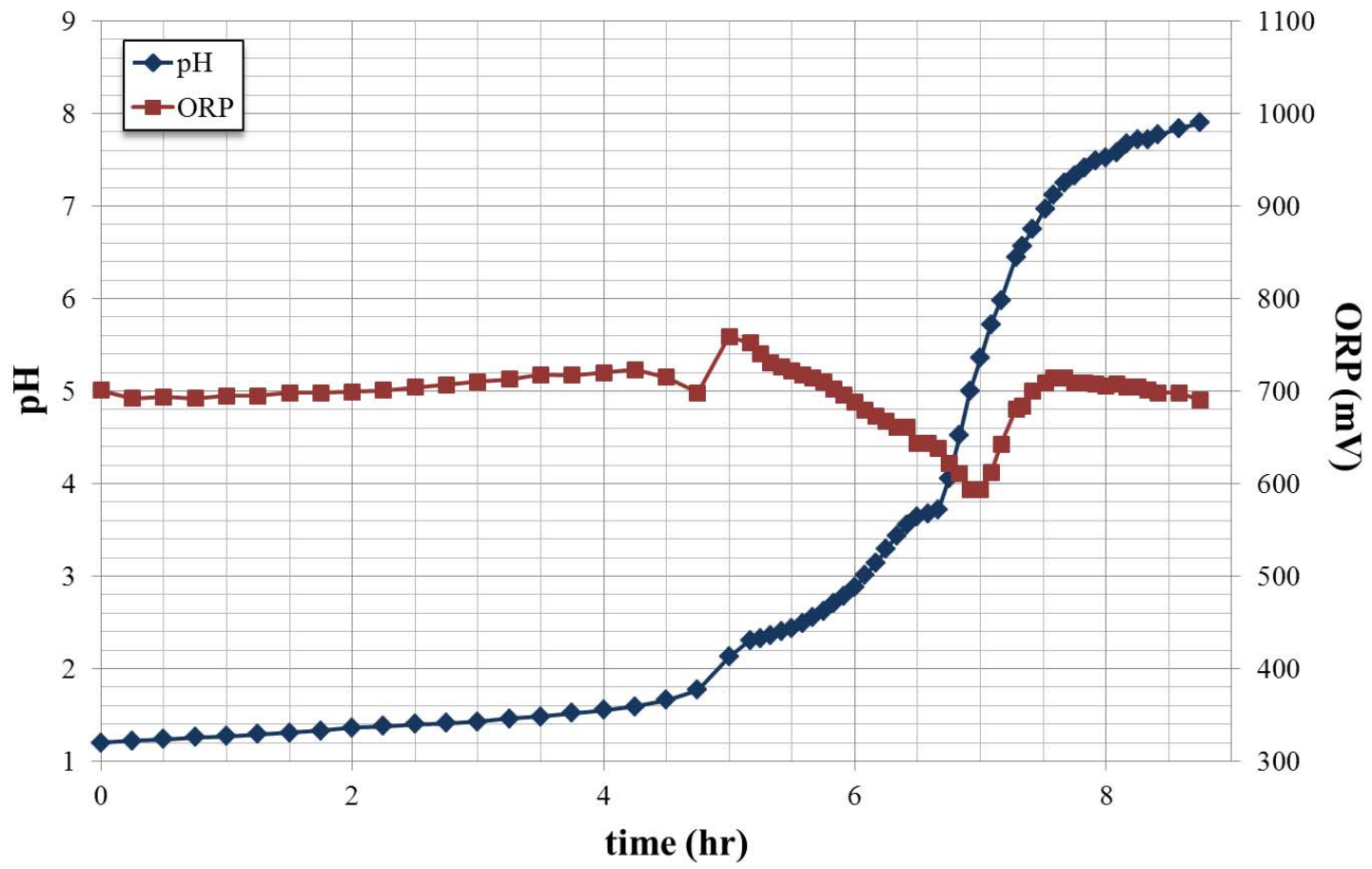

Figure 3-8: Trends for $\mathrm{pH}$ and ORP during Decomposition Test 2 (Tank 5F) OA Batch 3 


\subsection{Dissolution and Decomposition Chemistry}

In this section, data is presented for the soluble and insoluble portions analyzed after the sludge dissolution process and during and after the OA decomposition process.

An indicator of the effectiveness of the sludge dissolution with $2 \mathrm{wt} \% \mathrm{OA}$ is the analysis of the residual undissolved sludge and comparison of the components in the residual sludge to the components in the initial sludge feed. Table 3-6 and Table 3-7 contain the analytical results for the residual undissolved sludge from Test 1 and Table 3-8 and Table 3-9 contain the analogous results for Test 2. The results are given on a dry basis. The average and standard deviation of duplicate analyses are given.

By comparison of the species concentrations in the feed and the residue and considering the mass of residue recovered, a mass balance is used to calculate the "\% removal" for each species. Note that these percent removal values are sensitive to the ability to recover sludge from the Dissolution Module manually and are thus biased high. This bias is insignificant for high \% removal results (i.e. 99\%) but is very significant for moderate to low \% removal results (i.e., $30 \%$ ). Thus, this bias may cause low removal results to be overestimated.

The ratios of various species to iron and aluminum in the residual sludge are provided in Table 3-6 through Table 3-9. The "\% change" values for the relative amount of a component in the residual sludge are calculated as indicators of relative dissolution effectiveness of each component as compared with iron and aluminum. A "\% change" value of zero indicates that the ratio of the species to iron or aluminum did not change when comparing the residual sludge to the feed sludge. Negative "\% change" values (approaching -100\%) indicate reduced concentrations of the species in the residual sludge in comparison with iron or aluminum and signify relatively favorable dissolution and removal of those species. Positive "\% change" values indicate larger concentrations of the species in the residual sludge in comparison with iron or aluminum and signify relatively unfavorable dissolution and removal of those species.

For each test, the major component in the sludge (aluminum for Test 1 and iron for Test 2) remained the major component in the residual sludge. Comparison of the two tests reveals that the major component of the sludge tends to be amongst the most resistant to dissolution when based on the quantity in the residual material. For example, the high-aluminum Tank $12 \mathrm{H}$ sludge had a larger percentage removal of iron than aluminum while the high-iron Tank $5 \mathrm{~F}$ sludge had a larger percentage removal of aluminum than iron. Residuals remaining in the dissolution vessel at the conclusion of Test 1 were primarily aluminum (accounts for over $90 \%$ of the mass assuming boehmite is the major form) with small amounts of iron and manganese. Residuals remaining in the dissolution vessel at the conclusion of Test 2 were primarily iron (accounts for $66 \%$ of the mass assuming hematite is the major form) with small amounts of manganese and nickel.

Removal of uranium from the sludge by dissolution with $2 \mathrm{wt} \%$ OA was very effective for both the Tank $12 \mathrm{H}$ and Tank 5F sludge. The dissolution and removal of $\mathrm{Pu}$ was not as effective, but the percent removal was on the same order and typically intermediate to that of iron and aluminum. The strontium removal for Tank $5 \mathrm{~F}$ was 92 to $96 \%$ and for Tank $12 \mathrm{H}$ was 77 to $79 \%$. Some minor metals were not removed effectively ( $<40 \%$ removal) from the dissolution vessel of the RWT equipment with $2 \mathrm{wt} \%$ OA. For Test 1 , these metals included the lanthanides and actinides $\mathrm{Ce}, \mathrm{Gd}, \mathrm{La}, \mathrm{Th}, \mathrm{Eu}, \mathrm{Am}$, and possibly $\mathrm{Np}$. For Test 2, OA was more effective at removing the minor metals and only thorium was removed at a level below $40 \%$. 
Table 3-6: Residual Sludge in Dissolution Module from Test 1 (Tank 12H)

\begin{tabular}{|c|c|c|c|c|c|c|c|c|c|c|c|}
\hline \multirow{2}{*}{ analyte } & \multirow{2}{*}{ units } & \multirow{2}{*}{$\begin{array}{l}\text { residue } \\
\text { average }\end{array}$} & \multirow{2}{*}{ st. dev. } & \multirow{2}{*}{$\begin{array}{c}\text { feed } \\
\text { average }\end{array}$} & \multirow{2}{*}{$\begin{array}{c}\% \\
\text { removal }\end{array}$} & \multicolumn{3}{|c|}{$\mathrm{g} / \mathrm{g}$ Fe } & \multicolumn{3}{|c|}{ g / g Al } \\
\hline & & & & & & residue & feed & $\%$ change & residue & feed & \% change \\
\hline $\mathrm{Ag}$ & $\mathrm{mg} / \mathrm{kg}$ & $<2.69 E+01$ & -- & $8.96 \mathrm{E}+01$ & $>92 \%$ & -- & $2.0 \mathrm{E}-03$ & - & -- & $3.4 \mathrm{E}-04$ & -- \\
\hline $\mathrm{Al}$ & $\mathrm{mg} / \mathrm{kg}$ & $5.17 \mathrm{E}+05$ & $4.4 \mathrm{E}+04$ & $2.62 \mathrm{E}+05$ & $46 \%$ & $1.4 \mathrm{E}+01$ & $5.8 \mathrm{E}+00$ & $140 \%$ & $1.0 \mathrm{E}+00$ & $1.0 \mathrm{E}+00$ & $0 \%$ \\
\hline $\mathrm{Ba}$ & $\mathrm{mg} / \mathrm{kg}$ & $3.43 \mathrm{E}+02$ & $4.1 \mathrm{E}+01$ & $5.09 \mathrm{E}+02$ & $82 \%$ & $9.3 \mathrm{E}-03$ & $1.1 \mathrm{E}-02$ & $-18 \%$ & $6.6 \mathrm{E}-04$ & $1.9 \mathrm{E}-03$ & $-66 \%$ \\
\hline $\mathrm{Be}$ & $\mathrm{mg} / \mathrm{kg}$ & $1.82 \mathrm{E}+00$ & $3.65 \mathrm{E}-01$ & $<1.50 \mathrm{E}+01$ & -- & 4.9E-05 & -- & -- & $3.5 \mathrm{E}-06$ & -- & -- \\
\hline $\mathrm{Ca}$ & $\mathrm{mg} / \mathrm{kg}$ & $6.93 \mathrm{E}+02$ & $4.8 \mathrm{E}+01$ & $3.29 \mathrm{E}+03$ & $94 \%$ & $1.9 \mathrm{E}-02$ & 7.3E-02 & $-74 \%$ & $1.3 \mathrm{E}-03$ & $1.3 \mathrm{E}-02$ & $-89 \%$ \\
\hline $\mathrm{Ce}$ & $\mathrm{mg} / \mathrm{kg}$ & $1.75 \mathrm{E}+03$ & $3.03 \mathrm{E}+02$ & $8.39 \mathrm{E}+02$ & $43 \%$ & 4.7E-02 & $1.9 \mathrm{E}-02$ & $154 \%$ & $3.4 \mathrm{E}-03$ & $3.2 \mathrm{E}-03$ & $6 \%$ \\
\hline Co & $\mathrm{mg} / \mathrm{kg}$ & $2.87 \mathrm{E}+01$ & $2.4 \mathrm{E}+00$ & $<7.58 \mathrm{E}+01$ & -- & 7.7E-04 & -- & -- & $5.6 \mathrm{E}-05$ & -- & -- \\
\hline $\mathrm{Cr}$ & $\mathrm{mg} / \mathrm{kg}$ & $8.76 \mathrm{E}+01$ & $2.3 \mathrm{E}+01$ & $2.31 \mathrm{E}+02$ & $90 \%$ & $2.4 \mathrm{E}-03$ & $5.1 \mathrm{E}-03$ & $-54 \%$ & $1.7 \mathrm{E}-04$ & $8.8 \mathrm{E}-04$ & $-81 \%$ \\
\hline $\mathrm{Cu}$ & $\mathrm{mg} / \mathrm{kg}$ & $4.86 \mathrm{E}+02$ & $7.3 \mathrm{E}+01$ & $5.51 \mathrm{E}+02$ & $76 \%$ & $1.3 \mathrm{E}-02$ & $1.2 \mathrm{E}-02$ & $8 \%$ & $9.4 \mathrm{E}-04$ & $2.1 \mathrm{E}-03$ & $-55 \%$ \\
\hline $\mathrm{Fe}$ & $\mathrm{mg} / \mathrm{kg}$ & $3.71 \mathrm{E}+04$ & $1 \mathrm{E}+03$ & $4.52 \mathrm{E}+04$ & $78 \%$ & $1.0 \mathrm{E}+00$ & $1.0 \mathrm{E}+00$ & $0 \%$ & $7.2 \mathrm{E}-02$ & $1.7 \mathrm{E}-01$ & $-58 \%$ \\
\hline Gd & $\mathrm{mg} / \mathrm{kg}$ & $3.75 \mathrm{E}+02$ & $6.3 \mathrm{E}+01$ & $1.65 \mathrm{E}+02$ & $38 \%$ & $1.0 \mathrm{E}-02$ & $3.6 \mathrm{E}-03$ & $177 \%$ & 7.3E-04 & $6.3 \mathrm{E}-04$ & $15 \%$ \\
\hline $\mathrm{K}$ & $\mathrm{mg} / \mathrm{kg}$ & $<5.54 E+02$ & -- & $3.27 \mathrm{E}+03$ & $>95 \%$ & -- & $7.2 \mathrm{E}-02$ & -- & -- & $1.2 \mathrm{E}-02$ & -- \\
\hline $\mathrm{La}$ & $\mathrm{mg} / \mathrm{kg}$ & $9.02 \mathrm{E}+02$ & $1.5 \mathrm{E}+02$ & $4.00 \mathrm{E}+02$ & $39 \%$ & $2.4 \mathrm{E}-02$ & 8.9E-03 & $175 \%$ & $1.7 \mathrm{E}-03$ & $1.5 \mathrm{E}-03$ & $14 \%$ \\
\hline $\mathrm{Li}$ & $\mathrm{mg} / \mathrm{kg}$ & $1.41 \mathrm{E}+01$ & $1.6 \mathrm{E}+00$ & $1.12 \mathrm{E}+02$ & $97 \%$ & $3.8 \mathrm{E}-04$ & $2.5 \mathrm{E}-03$ & $-85 \%$ & 2.7E-05 & 4.3E-04 & $-94 \%$ \\
\hline $\mathrm{Mg}$ & $\mathrm{mg} / \mathrm{kg}$ & $4.03 \mathrm{E}+02$ & $2.8 \mathrm{E}+01$ & $1.14 \mathrm{E}+03$ & $90 \%$ & $1.1 \mathrm{E}-02$ & $2.5 \mathrm{E}-02$ & $-57 \%$ & $7.8 \mathrm{E}-04$ & 4.3E-03 & $-82 \%$ \\
\hline $\mathrm{Mn}$ & $\mathrm{mg} / \mathrm{kg}$ & $5.10 \mathrm{E}+03$ & $6.8 \mathrm{E}+02$ & $2.81 \mathrm{E}+04$ & $95 \%$ & $1.4 \mathrm{E}-01$ & $6.2 \mathrm{E}-01$ & $-78 \%$ & $9.9 \mathrm{E}-03$ & $1.1 \mathrm{E}-01$ & $-91 \%$ \\
\hline $\mathrm{Na}$ & $\mathrm{mg} / \mathrm{kg}$ & $6.28 \mathrm{E}+02$ & $2.6 \mathrm{E}+01$ & $4.23 \mathrm{E}+04$ & $99.6 \%$ & 1.7E-02 & $9.4 \mathrm{E}-01$ & $-98 \%$ & $1.2 \mathrm{E}-03$ & $1.6 \mathrm{E}-01$ & $-99 \%$ \\
\hline $\mathrm{Ni}$ & $\mathrm{mg} / \mathrm{kg}$ & $5.42 \mathrm{E}+03$ & $6.3 \mathrm{E}+02$ & $4.62 \mathrm{E}+03$ & $68 \%$ & $1.5 \mathrm{E}-01$ & $1.0 \mathrm{E}-01$ & $43 \%$ & $1.0 \mathrm{E}-02$ & $1.8 \mathrm{E}-02$ & $-40 \%$ \\
\hline $\mathrm{P}$ & $\mathrm{mg} / \mathrm{kg}$ & $<3.02 E+02$ & -- & $7.58 \mathrm{E}+02$ & $>89 \%$ & -- & $1.7 \mathrm{E}-02$ & -- & -- & $2.9 \mathrm{E}-03$ & -- \\
\hline $\mathrm{Si}$ & $\mathrm{mg} / \mathrm{kg}$ & $1.97 \mathrm{E}+02$ & $3.80 \mathrm{E}+01$ & $2.66 \mathrm{E}+03$ & $98 \%$ & 5.3E-03 & $5.9 \mathrm{E}-02$ & $-91 \%$ & $3.8 \mathrm{E}-04$ & $1.0 \mathrm{E}-02$ & $-96 \%$ \\
\hline $\mathrm{Sr}$ & $\mathrm{mg} / \mathrm{kg}$ & $1.82 \mathrm{E}+02$ & $1.1 \mathrm{E}+01$ & $2.16 \mathrm{E}+02$ & $77 \%$ & 4.9E-03 & $4.8 \mathrm{E}-03$ & $3 \%$ & $3.5 \mathrm{E}-04$ & $8.2 \mathrm{E}-04$ & $-57 \%$ \\
\hline $\mathrm{Th}$ & $\mathrm{mg} / \mathrm{kg}$ & $3.76 \mathrm{E}+04$ & $3.0 \mathrm{E}+03$ & $1.55 \mathrm{E}+04$ & $34 \%$ & $1.0 \mathrm{E}+00$ & $3.4 \mathrm{E}-01$ & $196 \%$ & 7.3E-02 & $5.9 \mathrm{E}-02$ & $23 \%$ \\
\hline $\mathrm{Ti}$ & $\mathrm{mg} / \mathrm{kg}$ & $1.10 \mathrm{E}+02$ & $1.0 \mathrm{E}+01$ & $1.08 \mathrm{E}+02$ & $72 \%$ & $3.0 \mathrm{E}-03$ & $2.4 \mathrm{E}-03$ & $24 \%$ & 2.1E-04 & 4.1E-04 & $-48 \%$ \\
\hline $\mathrm{U}$ & $\mathrm{mg} / \mathrm{kg}$ & $<5.52 E+02$ & - & $6.00 \mathrm{E}+03$ & $>97 \%$ & -- & $1.3 \mathrm{E}-01$ & - & -- & $2.3 \mathrm{E}-02$ & -- \\
\hline $\mathrm{V}$ & $\mathrm{mg} / \mathrm{kg}$ & $1.10 \mathrm{E}+02$ & $2.2 \mathrm{E}+01$ & $<7.17 \mathrm{E}+01$ & -- & $3.0 \mathrm{E}-03$ & -- & - & $2.1 \mathrm{E}-04$ & -- & - \\
\hline $\mathrm{Zn}$ & $\mathrm{mg} / \mathrm{kg}$ & $3.75 \mathrm{E}+02$ & $4.8 \mathrm{E}+01$ & $3.43 \mathrm{E}+02$ & $70 \%$ & $1.0 \mathrm{E}-02$ & $7.6 \mathrm{E}-03$ & $33 \%$ & 7.3E-04 & $1.3 \mathrm{E}-03$ & $-45 \%$ \\
\hline $\mathrm{Zr}$ & $\mathrm{mg} / \mathrm{kg}$ & $3.53 \mathrm{E}+02$ & $6.4 \mathrm{E}+01$ & $1.46 \mathrm{E}+03$ & $93 \%$ & $9.5 \mathrm{E}-03$ & $3.2 \mathrm{E}-02$ & $-70 \%$ & $6.8 \mathrm{E}-04$ & $5.6 \mathrm{E}-03$ & $-88 \%$ \\
\hline Co & $\mathrm{mg} / \mathrm{kg}$ & $2.08 \mathrm{E}+01$ & $1 \mathrm{E}+00$ & $2.73 \mathrm{E}+01$ & $79 \%$ & 5.6E-04 & $6.0 \mathrm{E}-04$ & $-7 \%$ & $4.0 \mathrm{E}-05$ & $1.0 \mathrm{E}-04$ & $-61 \%$ \\
\hline $\mathrm{Hg}$ & $\mathrm{mg} / \mathrm{kg}$ & $3.93 \mathrm{E}+02$ & $2.85 \mathrm{E}+00$ & $1.85 \mathrm{E}+04$ & $99 \%$ & $1.1 \mathrm{E}-02$ & 4.1E-01 & $-97 \%$ & 7.6E-04 & $7.0 \mathrm{E}-02$ & $-99 \%$ \\
\hline $\mathrm{Pb}$ & $\mathrm{mg} / \mathrm{kg}$ & $<6.28 E+01$ & -- & $3.07 \mathrm{E}+01$ & $>44 \%$ & -- & $6.8 \mathrm{E}-04$ & -- & -- & $1.2 \mathrm{E}-04$ & -- \\
\hline Th-232 & $\mathrm{mg} / \mathrm{kg}$ & $3.49 \mathrm{E}+04$ & $3.28 \mathrm{E}+03$ & $1.52 \mathrm{E}+04$ & $37 \%$ & $9.4 \mathrm{E}-01$ & $3.4 \mathrm{E}-01$ & $180 \%$ & $6.8 \mathrm{E}-02$ & $5.8 \mathrm{E}-02$ & $17 \%$ \\
\hline $\mathrm{Np}-237$ & $\mathrm{mg} / \mathrm{kg}$ & $<3.39 E+01$ & -- & $1.35 \mathrm{E}+01$ & $>32 \%$ & -- & $3.0 \mathrm{E}-04$ & -- & -- & $5.2 \mathrm{E}-05$ & -- \\
\hline $\mathrm{U}-238$ & $\mathrm{mg} / \mathrm{kg}$ & $1.05 \mathrm{E}+02$ & $2.21 \mathrm{E}+01$ & $5.25 \mathrm{E}+03$ & $99 \%$ & $2.8 \mathrm{E}-03$ & $1.2 \mathrm{E}-01$ & $-98 \%$ & $2.0 \mathrm{E}-04$ & $2.0 \mathrm{E}-02$ & $-99 \%$ \\
\hline $\mathrm{Pu}-239$ & $\mathrm{mg} / \mathrm{kg}$ & $2.03 \mathrm{E}+02$ & $3.47 \mathrm{E}+01$ & $2.02 \mathrm{E}+02$ & $73 \%$ & $5.5 \mathrm{E}-03$ & $4.5 \mathrm{E}-03$ & $23 \%$ & $3.9 \mathrm{E}-04$ & 7.7E-04 & $-49 \%$ \\
\hline $\mathrm{Pu}-240$ & $\mathrm{mg} / \mathrm{kg}$ & $2.44 \mathrm{E}+01$ & $4.56 \mathrm{E}+00$ & $2.16 \mathrm{E}+01$ & $69 \%$ & 6.6E-04 & 4.8E-04 & $38 \%$ & 4.7E-05 & $8.2 \mathrm{E}-05$ & $-43 \%$ \\
\hline
\end{tabular}


Table 3-7: Residual Sludge in Dissolution Module from Test 1 (Tank 12H), continued

\begin{tabular}{|c|c|c|c|c|c|c|c|c|c|c|c|}
\hline \multirow{2}{*}{ analyte } & \multirow{2}{*}{ units } & \multirow{2}{*}{$\begin{array}{l}\text { residue } \\
\text { average }\end{array}$} & \multirow{2}{*}{ st. dev. } & \multirow{2}{*}{$\begin{array}{c}\text { feed } \\
\text { average }\end{array}$} & \multirow{2}{*}{$\begin{array}{c}\% \\
\text { removal }\end{array}$} & \multicolumn{3}{|c|}{$\mathrm{mCi} / \mathrm{g} \mathrm{Fe}$} & \multicolumn{3}{|c|}{$\mathbf{m C i} / \mathbf{g ~ A l}$} \\
\hline & & & & & & residue & feed & \% change & residue & feed & $\%$ change \\
\hline $\mathrm{Pu}-238$ & $\mathrm{dpm} / \mathrm{g}$ & $1.01 \mathrm{E}+09$ & $2.25 \mathrm{E}+08$ & $7.18 \mathrm{E}+08$ & $62 \%$ & $1.2 \mathrm{E}+01$ & $7.2 \mathrm{E}+00$ & $71 \%$ & $8.8 \mathrm{E}-01$ & $1.2 \mathrm{E}+00$ & $-29 \%$ \\
\hline $\mathrm{Pu}-239 / 240$ & $\mathrm{dpm} / \mathrm{g}$ & $4.52 \mathrm{E}+07$ & $1.1 \mathrm{E}+07$ & $3.06 \mathrm{E}+07$ & $60 \%$ & $5.5 \mathrm{E}-01$ & $3.0 \mathrm{E}-01$ & $80 \%$ & 3.9E-02 & 5.3E-02 & $-25 \%$ \\
\hline Co-60 & $\mathrm{dpm} / \mathrm{g}$ & $<7.48 E+05$ & -- & $4.42 \mathrm{E}+05$ & $>54 \%$ & -- & 4.4E-03 & -- & -- & 7.6E-04 & -- \\
\hline Cs-137 & $\mathrm{dpm} / \mathrm{g}$ & $7.05 E+06$ & $1.0 \mathrm{E}+06$ & $2.48 \mathrm{E}+08$ & $99 \%$ & 8.6E-02 & $2.5 \mathrm{E}+00$ & $-97 \%$ & $6.1 \mathrm{E}-03$ & 4.3E-01 & $-99 \%$ \\
\hline $\mathrm{Eu}-154$ & $\mathrm{dpm} / \mathrm{g}$ & $6.62 \mathrm{E}+07$ & $6.1 \mathrm{E}+06$ & $3.15 \mathrm{E}+07$ & $43 \%$ & $8.0 \mathrm{E}-01$ & 3.1E-01 & $156 \%$ & $5.8 \mathrm{E}-02$ & $5.4 \mathrm{E}-02$ & $7 \%$ \\
\hline $\mathrm{Eu}-155$ & $\mathrm{dpm} / \mathrm{g}$ & $<4.41 E+06$ & -- & $2.10 \mathrm{E}+06$ & $>43 \%$ & -- & 2.1E-02 & - & -- & 3.6E-03 & -- \\
\hline Sr-90 & $\mathrm{dpm} / \mathrm{g}$ & $1.54 \mathrm{E}+10$ & $7.1 \mathrm{E}+08$ & $2.04 \mathrm{E}+10$ & $79 \%$ & $1.87 \mathrm{E}+02$ & $2.0 \mathrm{E}+02$ & $-8 \%$ & $1.34 \mathrm{E}+01$ & $3.5 \mathrm{E}+01$ & $-62 \%$ \\
\hline Am-241 & $\mathrm{dpm} / \mathrm{g}$ & $1.06 \mathrm{E}+08$ & $1.3 \mathrm{E}+07$ & $3.90 \mathrm{E}+07$ & $26 \%$ & $1.29 \mathrm{E}+00$ & $3.9 \mathrm{E}-01$ & $232 \%$ & $9.27 \mathrm{E}-02$ & 6.7E-02 & $38 \%$ \\
\hline Am-243 & $\mathrm{dpm} / \mathrm{g}$ & $1.10 \mathrm{E}+05$ & $5.1 \mathrm{E}+03$ & $3.53 \mathrm{E}+04$ & -- & $1.34 \mathrm{E}-03$ & $3.5 \mathrm{E}-04$ & -- & $9.61 \mathrm{E}-05$ & $6.1 \mathrm{E}-05$ & -- \\
\hline Am-242m & $\mathrm{dpm} / \mathrm{g}$ & $2.19 \mathrm{E}+04$ & $2.4 \mathrm{E}+03$ & $2.83 \mathrm{E}+04$ & -- & $2.66 \mathrm{E}-04$ & $2.8 \mathrm{E}-04$ & -- & 1.91E-05 & 4.9E-05 & -- \\
\hline $\mathrm{Cm}-242$ & $\mathrm{dpm} / \mathrm{g}$ & $1.80 \mathrm{E}+04$ & $2.2 \mathrm{E}+05$ & $2.34 \mathrm{E}+04$ & -- & 2.19E-04 & 2.3E-04 & -- & $1.57 \mathrm{E}-05$ & 4.0E-05 & -- \\
\hline $\mathrm{Cm}-244$ & $\mathrm{dpm} / \mathrm{g}$ & $3.12 \mathrm{E}+06$ & $8.8 \mathrm{E}+04$ & $1.03 \mathrm{E}+06$ & $17 \%$ & 3.79E-02 & $1.0 \mathrm{E}-02$ & $271 \%$ & $2.72 \mathrm{E}-03$ & $1.8 \mathrm{E}-03$ & $54 \%$ \\
\hline
\end{tabular}

The final $\mathrm{pH}$ values and the molar concentrations of selected species for each dissolution batch are provided in Table 3-10. For each test, the highest $\mathrm{pH}$ was observed for Batch 1 and the lowest for Batch 3, indicating that the sludge was less effective at acid neutralization with repeated contacts. Lower $\mathrm{pH}$ values were observed for the Tank $5 \mathrm{~F}$ material, where the final $\mathrm{pH}$ for batches 2 and 3 were near that of $2 \mathrm{wt} \%$ OA. The Tank $12 \mathrm{H}$ material contains more base equivalents due to the presence of more residual basic salts or basic metal oxides and hydroxides such as boehmite $(\mathrm{AlOOH})$, which is the primary aluminum phase in this sludge. ${ }^{15}$

The liquid phase concentrations of the primary sludge components ( $\mathrm{Al}$ and $\mathrm{Fe}$ ) indicate the effectiveness of $\mathrm{OA}$ for dissolution over the 8 hour test period. Boehmite is not highly soluble in oxalic acid. An aluminum concentration of $0.09 \mathrm{M}$ was observed in previous testing with pure boehmite in $1.1 \mathrm{wt} \% \mathrm{OA}$ at $70{ }^{\circ} \mathrm{C}(\mathrm{pH} 1.3,3$ week contact) when nitric acid was added for $\mathrm{pH}$ control. ${ }^{15}$ The aluminum concentration for Test 1 was near $0.008 \mathrm{M}$ for all three batches and significant residual aluminum remained (Table 3-6) after Batch 3. The low aluminum concentrations may be the result of slow boehmite dissolution kinetics in oxalic acid. For Test 2, nearly all of the aluminum had dissolved by the completion of Batch 3. Opposite trends were observed in the iron concentrations for Tests 1 and 2, where nearly all of the iron was dissolved from the Test 1 material by the completion of Batch 3, while significant iron remained in the Test 2 residue (Table 3-8). In previous testing with pure hematite $\left(\mathrm{Fe}_{2} \mathrm{O}_{3}\right)$, the iron solubility in $1 \mathrm{wt} \%$ OA was approximately $0.06 \mathrm{M}$ at $50{ }^{\circ} \mathrm{C}\left(\mathrm{pH} 1.5,6\right.$ week contact). ${ }^{16}$ In $2 \mathrm{wt} \% \mathrm{OA}$, the iron concentration would be expected to approach $0.12 \mathrm{M}$ under these same conditions, however, other metals in sludge compete for the added oxalate anion, resulting in a lower iron solubility. In addition, dissolution times for Tests 1 and 2 ranged from 8-11 hours (Table 3-5) and previous testing indicated that this timescale is insufficient to reach equilibrium for hematite dissolution in OA. ${ }^{16}$ Successively lower iron concentrations were observed for each Test 2 batch, despite the fact that $25 \%$ of the iron remained at the conclusion of Batch 3. The soluble iron concentration for Batch 3 was nearly $70 \%$ lower than that observed for Batch 1 . This is an indication that the iron particles remaining after the initial OA batches dissolve more slowly and longer OA contact times might be needed to increase the dissolution efficiency for F Area tanks. 
Trends in the oxalate concentrations in Table 3-10 are not fully understood. For Tests 1 and 2, the Batch 1 oxalate concentrations were only 19 and $41 \%$, respectively, of the initial OA concentration ( $2 \mathrm{wt} \%$ OA corresponds to $0.224 \mathrm{M}$ oxalate). The measured oxalate concentrations increased during successive batches for each test. The Batch 3 oxalate concentrations were $93 \%$ for Test 1 and $82 \%$ for Test 2, respectively, of the initial value. The low measured oxalate concentrations for early process batches could be due to: 1) analytical errors for highly complexed oxalate anion, 2) precipitation of oxalate compounds (including formation of films), or 3 ) decomposition of oxalate anion during dissolution.

Table 3-8: Residual Sludge in Dissolution Module from Test 2 (Tank 5F)

\begin{tabular}{|c|c|c|c|c|c|c|c|c|c|c|c|}
\hline \multirow{2}{*}{ analyte } & \multirow{2}{*}{ units } & \multirow{2}{*}{$\begin{array}{l}\text { residue } \\
\text { average }\end{array}$} & \multirow{2}{*}{ st. dev. } & \multirow{2}{*}{$\begin{array}{c}\text { feed } \\
\text { average }\end{array}$} & \multirow{2}{*}{$\begin{array}{c}\% \\
\text { removal }\end{array}$} & \multicolumn{3}{|c|}{ g / g Fe } & \multicolumn{3}{|c|}{ g / g Al } \\
\hline & & & & & & residue & feed & $\%$ change & residue & feed & $\%$ change \\
\hline $\mathrm{Al}$ & $\mathrm{mg} / \mathrm{kg}$ & $3.37 \mathrm{E}+03$ & $1 \mathrm{E}+02$ & $1.64 \mathrm{E}+04$ & $96 \%$ & 7.3E-03 & 4.5E-02 & $-84 \%$ & $1.0 \mathrm{E}+00$ & $1.0 \mathrm{E}+00$ & $0 \%$ \\
\hline $\mathrm{Ba}$ & $\mathrm{mg} / \mathrm{kg}$ & $6.04 \mathrm{E}+02$ & $4.5 \mathrm{E}+01$ & $1.63 \mathrm{E}+03$ & $93 \%$ & $1.3 \mathrm{E}-03$ & $4.5 \mathrm{E}-03$ & $-71 \%$ & $1.8 \mathrm{E}-01$ & 9.9E-02 & $80 \%$ \\
\hline $\mathrm{Be}$ & $\mathrm{mg} / \mathrm{kg}$ & $1.03 \mathrm{E}+01$ & $6.7 \mathrm{E}-01$ & $<7.80 E+00$ & -- & $2.2 \mathrm{E}-05$ & -- & -- & $3.1 \mathrm{E}-03$ & -- & -- \\
\hline $\mathrm{Ca}$ & $\mathrm{mg} / \mathrm{kg}$ & $8.51 \mathrm{E}+02$ & $5.4 \mathrm{E}+01$ & $4.57 \mathrm{E}+03$ & $96 \%$ & $1.8 \mathrm{E}-03$ & $1.3 \mathrm{E}-02$ & $-85 \%$ & $2.5 \mathrm{E}-01$ & $2.8 \mathrm{E}-01$ & $-10 \%$ \\
\hline $\mathrm{Cd}$ & $\mathrm{mg} / \mathrm{kg}$ & $6.31 \mathrm{E}+01$ & $1.9 \mathrm{E}+00$ & $8.00 \mathrm{E}+01$ & $85 \%$ & 1.4E-04 & 2.2E-04 & $-38 \%$ & $1.9 \mathrm{E}-02$ & 4.9E-03 & $282 \%$ \\
\hline $\mathrm{Ce}$ & $\mathrm{mg} / \mathrm{kg}$ & $2.64 \mathrm{E}+03$ & $4.1 \mathrm{E}+02$ & $2.29 \mathrm{E}+03$ & $78 \%$ & $5.7 \mathrm{E}-03$ & $6.3 \mathrm{E}-03$ & $-9 \%$ & $7.8 \mathrm{E}-01$ & $1.4 \mathrm{E}-01$ & $459 \%$ \\
\hline Co & $\mathrm{mg} / \mathrm{kg}$ & $3.17 \mathrm{E}+02$ & $5.4 \mathrm{E}+01$ & $2.29 \mathrm{E}+02$ & $73 \%$ & $6.9 \mathrm{E}-04$ & $6.3 \mathrm{E}-04$ & $9 \%$ & $9.4 \mathrm{E}-02$ & $1.4 \mathrm{E}-02$ & $572 \%$ \\
\hline $\mathrm{Cr}$ & $\mathrm{mg} / \mathrm{kg}$ & $5.18 \mathrm{E}+01$ & $5.7 \mathrm{E}+00$ & $4.77 \mathrm{E}+02$ & $98 \%$ & $1.1 \mathrm{E}-04$ & $1.3 \mathrm{E}-03$ & $-91 \%$ & $1.5 \mathrm{E}-02$ & $2.9 \mathrm{E}-02$ & $-47 \%$ \\
\hline $\mathrm{Cu}$ & $\mathrm{mg} / \mathrm{kg}$ & $9.56 \mathrm{E}+02$ & $8.2 \mathrm{E}+01$ & $4.62 \mathrm{E}+02$ & $60 \%$ & $2.1 \mathrm{E}-03$ & $1.3 \mathrm{E}-03$ & $63 \%$ & $2.8 \mathrm{E}-01$ & $2.8 \mathrm{E}-02$ & $903 \%$ \\
\hline $\mathrm{Fe}$ & $\mathrm{mg} / \mathrm{kg}$ & $4.61 \mathrm{E}+05$ & $3.9 \mathrm{E}+04$ & $3.62 \mathrm{E}+05$ & $75 \%$ & $1.0 \mathrm{E}+00$ & $1.0 \mathrm{E}+00$ & $0 \%$ & $1.4 \mathrm{E}+02$ & $2.2 \mathrm{E}+01$ & $516 \%$ \\
\hline Gd & $\mathrm{mg} / \mathrm{kg}$ & $1.80 \mathrm{E}+02$ & $2 \mathrm{E}+00$ & $<1.96 E+02$ & -- & $3.9 \mathrm{E}-04$ & -- & -- & 5.3E-02 & -- & -- \\
\hline $\mathrm{K}$ & $\mathrm{mg} / \mathrm{kg}$ & $<3.43 E+02$ & -- & $6.62 \mathrm{E}+02$ & $>90 \%$ & -- & $1.8 \mathrm{E}-03$ & -- & -- & 4.1E-02 & -- \\
\hline $\mathrm{La}$ & $\mathrm{mg} / \mathrm{kg}$ & $1.45 \mathrm{E}+03$ & $1.7 \mathrm{E}+02$ & $9.98 \mathrm{E}+02$ & $72 \%$ & $3.1 \mathrm{E}-03$ & $2.8 \mathrm{E}-03$ & $14 \%$ & 4.3E-01 & $6.1 \mathrm{E}-02$ & $603 \%$ \\
\hline $\mathrm{Li}$ & $\mathrm{mg} / \mathrm{kg}$ & $2.90 \mathrm{E}+01$ & $4.5 \mathrm{E}+00$ & $4.38 \mathrm{E}+02$ & $99 \%$ & $6.3 \mathrm{E}-05$ & $1.2 \mathrm{E}-03$ & $-95 \%$ & $8.6 \mathrm{E}-03$ & $2.7 \mathrm{E}-02$ & $-68 \%$ \\
\hline $\mathrm{Mg}$ & $\mathrm{mg} / \mathrm{kg}$ & $2.90 \mathrm{E}+02$ & $1 \mathrm{E}+01$ & $5.88 \mathrm{E}+02$ & $90 \%$ & $6.3 \mathrm{E}-04$ & $1.6 \mathrm{E}-03$ & $-61 \%$ & $8.6 \mathrm{E}-02$ & $3.6 \mathrm{E}-02$ & $139 \%$ \\
\hline $\mathrm{Mn}$ & $\mathrm{mg} / \mathrm{kg}$ & $3.45 \mathrm{E}+04$ & $4.9 \mathrm{E}+03$ & $6.68 \mathrm{E}+04$ & $90 \%$ & $7.5 \mathrm{E}-02$ & $1.8 \mathrm{E}-01$ & $-59 \%$ & $1.0 \mathrm{E}+01$ & $4.1 \mathrm{E}+00$ & $150 \%$ \\
\hline Mo & $\mathrm{mg} / \mathrm{kg}$ & $<3.06 E+01$ & -- & $1.66 \mathrm{E}+02$ & $>96 \%$ & - & 4.6E-04 & - & - & $1.0 \mathrm{E}-02$ & -- \\
\hline $\mathrm{Na}$ & $\mathrm{mg} / \mathrm{kg}$ & $2.26 \mathrm{E}+03$ & $1.6 \mathrm{E}+02$ & $3.37 \mathrm{E}+04$ & $99 \%$ & 4.9E-03 & $9.3 \mathrm{E}-02$ & $-95 \%$ & $6.7 \mathrm{E}-01$ & $2.1 \mathrm{E}+00$ & $-68 \%$ \\
\hline $\mathrm{Ni}$ & $\mathrm{mg} / \mathrm{kg}$ & $5.73 \mathrm{E}+04$ & $9.6 \mathrm{E}+03$ & $3.60 \mathrm{E}+04$ & $69 \%$ & $1.2 \mathrm{E}-01$ & $9.9 \mathrm{E}-02$ & $25 \%$ & $1.7 \mathrm{E}+01$ & $2.2 \mathrm{E}+00$ & $672 \%$ \\
\hline $\mathrm{P}$ & $\mathrm{mg} / \mathrm{kg}$ & $2.29 \mathrm{E}+02$ & $3.0 \mathrm{E}+01$ & $<5.39 E+02$ & - & $5.0 \mathrm{E}-04$ & -- & - & $6.8 \mathrm{E}-02$ & -- & -- \\
\hline $\mathrm{Pb}$ & $\mathrm{mg} / \mathrm{kg}$ & $3.17 \mathrm{E}+02$ & $2.4 \mathrm{E}+01$ & $3.89 \mathrm{E}+02$ & $84 \%$ & $6.9 \mathrm{E}-04$ & $1.1 \mathrm{E}-03$ & $-36 \%$ & $9.4 \mathrm{E}-02$ & $2.4 \mathrm{E}-02$ & $295 \%$ \\
\hline $\mathrm{Si}$ & $\mathrm{mg} / \mathrm{kg}$ & $1.23 \mathrm{E}+03$ & $2.6 \mathrm{E}+02$ & $8.07 \mathrm{E}+03$ & $97 \%$ & $2.7 \mathrm{E}-03$ & $2.2 \mathrm{E}-02$ & $-88 \%$ & $3.6 \mathrm{E}-01$ & 4.9E-01 & $-26 \%$ \\
\hline $\mathrm{Sr}$ & $\mathrm{mg} / \mathrm{kg}$ & $3.39 \mathrm{E}+02$ & $2.4 \mathrm{E}+01$ & $7.80 \mathrm{E}+02$ & $92 \%$ & 7.4E-04 & $2.2 \mathrm{E}-03$ & $-66 \%$ & $1.0 \mathrm{E}-01$ & 4.8E-02 & $111 \%$ \\
\hline Th & $\mathrm{mg} / \mathrm{kg}$ & $<3.06 E+02$ & -- & $<4.51 E+02$ & -- & -- & $1.2 \mathrm{E}-03$ & -- & -- & $2.8 \mathrm{E}-02$ & -- \\
\hline $\mathrm{Ti}$ & $\mathrm{mg} / \mathrm{kg}$ & $6.67 \mathrm{E}+01$ & $6.2 \mathrm{E}+00$ & $1.99 \mathrm{E}+02$ & $93 \%$ & $1.4 \mathrm{E}-04$ & $5.5 \mathrm{E}-04$ & $-74 \%$ & $2.0 \mathrm{E}-02$ & $1.2 \mathrm{E}-02$ & $63 \%$ \\
\hline $\mathrm{Zn}$ & $\mathrm{mg} / \mathrm{kg}$ & $5.28 \mathrm{E}+02$ & $6.6 \mathrm{E}+01$ & $2.45 \mathrm{E}+02$ & $58 \%$ & $1.1 \mathrm{E}-03$ & $6.8 \mathrm{E}-04$ & $69 \%$ & $1.6 \mathrm{E}-01$ & $1.5 \mathrm{E}-02$ & $943 \%$ \\
\hline $\mathrm{Zr}$ & $\mathrm{mg} / \mathrm{kg}$ & $9.00 \mathrm{E}+02$ & $1.8 \mathrm{E}+01$ & $3.49 \mathrm{E}+03$ & $95 \%$ & $2.0 \mathrm{E}-03$ & $9.6 \mathrm{E}-03$ & $-80 \%$ & 2.7E-01 & 2.1E-01 & $25 \%$ \\
\hline $\mathrm{Co}$ & $\mathrm{mg} / \mathrm{kg}$ & $2.88 \mathrm{E}+02$ & $5.4 \mathrm{E}+01$ & $2.21 \mathrm{E}+02$ & $75 \%$ & $6.2 \mathrm{E}-04$ & $6.1 \mathrm{E}-04$ & $2 \%$ & $8.5 \mathrm{E}-02$ & $1.4 \mathrm{E}-02$ & $531 \%$ \\
\hline $\mathrm{Hg}$ & $\mathrm{mg} / \mathrm{kg}$ & $9.10 \mathrm{E}+02$ & $2.30 \mathrm{E}+02$ & $6.95 \mathrm{E}+02$ & $74 \%$ & $2.0 \mathrm{E}-03$ & $1.9 \mathrm{E}-03$ & $3 \%$ & 2.7E-01 & 4.3E-02 & $535 \%$ \\
\hline $\mathrm{Pb}$ & $\mathrm{mg} / \mathrm{kg}$ & $2.03 \mathrm{E}+02$ & 1.7E-01 & $3.74 \mathrm{E}+02$ & $89 \%$ & 4.4E-04 & $1.0 \mathrm{E}-03$ & $-57 \%$ & $6.0 \mathrm{E}-02$ & $2.3 \mathrm{E}-02$ & $163 \%$ \\
\hline Th-232 & $\mathrm{mg} / \mathrm{kg}$ & $4.50 \mathrm{E}+01$ & $7.0 \mathrm{E}+00$ & $1.04 \mathrm{E}+01$ & $16 \%$ & $9.77 \mathrm{E}-05$ & 2.9E-05 & $240 \%$ & $1.3 \mathrm{E}-02$ & $6.4 \mathrm{E}-04$ & $1997 \%$ \\
\hline $\mathrm{Np}-237$ & $\mathrm{mg} / \mathrm{kg}$ & $2.55 \mathrm{E}+01$ & $3.5 \mathrm{E}+00$ & $5.11 \mathrm{E}+01$ & $90 \%$ & $5.53 \mathrm{E}-05$ & $1.4 \mathrm{E}-04$ & $-61 \%$ & $7.54 \mathrm{E}-03$ & $3.1 \mathrm{E}-03$ & $141 \%$ \\
\hline $\mathrm{U}-238$ & $\mathrm{mg} / \mathrm{kg}$ & $5.35 \mathrm{E}+02$ & $3.1 \mathrm{E}+01$ & $7.90 \mathrm{E}+04$ & $99.9 \%$ & $1.16 \mathrm{E}-03$ & $2.2 \mathrm{E}-01$ & $-99 \%$ & $1.59 \mathrm{E}-01$ & $4.8 \mathrm{E}+00$ & $-97 \%$ \\
\hline $\mathrm{Pu}-239$ & $\mathrm{mg} / \mathrm{kg}$ & $6.76 \mathrm{E}+01$ & $1.17 \mathrm{E}+01$ & $1.03 \mathrm{E}+02$ & $87 \%$ & $1.47 \mathrm{E}-04$ & $2.8 \mathrm{E}-04$ & $-48 \%$ & $2.00 \mathrm{E}-02$ & $6.3 \mathrm{E}-03$ & $219 \%$ \\
\hline Pu-240 & $\mathrm{mg} / \mathrm{kg}$ & $6.28 \mathrm{E}+00$ & $2.10 \mathrm{E}+00$ & $8.69 \mathrm{E}+00$ & $86 \%$ & $1.36 \mathrm{E}-05$ & $2.4 \mathrm{E}-05$ & $-43 \%$ & $1.86 \mathrm{E}-03$ & $5.3 \mathrm{E}-04$ & $250 \%$ \\
\hline m-241(Am,Pu) & $\mathrm{mg} / \mathrm{kg}$ & $1.98 \mathrm{E}+01$ & $6.7 \mathrm{E}+00$ & $1.41 \mathrm{E}+01$ & $73 \%$ & $4.30 \mathrm{E}-05$ & $3.9 \mathrm{E}-05$ & $10 \%$ & 5.87E-03 & $8.6 \mathrm{E}-04$ & $580 \%$ \\
\hline
\end{tabular}


Table 3-9: Residual Sludge in Dissolution Module from Test 2 (Tank 5F), continued

\begin{tabular}{|c|c|c|c|c|c|c|c|c|c|c|c|}
\hline \multirow{2}{*}{ analyte } & \multirow{2}{*}{ units } & \multirow{2}{*}{$\begin{array}{l}\text { residue } \\
\text { average }\end{array}$} & \multirow{2}{*}{ st. dev. } & \multirow{2}{*}{$\begin{array}{c}\text { feed } \\
\text { average }\end{array}$} & \multirow{2}{*}{$\begin{array}{c}\% \\
\text { removal }\end{array}$} & \multicolumn{3}{|c|}{$\mathrm{mCi} / \mathrm{g} \mathrm{Fe}$} & \multicolumn{3}{|c|}{$\mathbf{m C i} / \mathrm{g} \mathrm{Al}$} \\
\hline & & & & & & residue & feed & $\%$ change & residue & feed & $\%$ change \\
\hline $\mathrm{Pu}-238$ & $\mathrm{dpm} / \mathrm{g}$ & $3.71 \mathrm{E}+06$ & $2.6 \mathrm{E}+05$ & $4.04 \mathrm{E}+06$ & $82 \%$ & 3.63E-03 & $5.0 \mathrm{E}-03$ & $-28 \%$ & 4.96E-01 & $1.1 \mathrm{E}-01$ & $345 \%$ \\
\hline $\mathrm{Pu}-239 / 240$ & $\mathrm{dpm} / \mathrm{g}$ & $1.39 \mathrm{E}+07$ & $8 \mathrm{E}+05$ & $2.04 \mathrm{E}+07$ & $87 \%$ & $1.36 \mathrm{E}-02$ & $2.5 \mathrm{E}-02$ & $-46 \%$ & $1.85 \mathrm{E}+00$ & $5.6 \mathrm{E}-01$ & $231 \%$ \\
\hline Cs -137 & $\mathrm{dpm} / \mathrm{g}$ & $1.04 \mathrm{E}+07$ & $6.1 \mathrm{E}+05$ & $2.39 \mathrm{E}+09$ & $99.9 \%$ & $1.02 \mathrm{E}-02$ & $3.0 \mathrm{E}+00$ & $-100 \%$ & $1.39 \mathrm{E}+00$ & $6.6 \mathrm{E}+01$ & $-98 \%$ \\
\hline $\mathrm{Co}-60$ & $\mathrm{dpm} / \mathrm{g}$ & $2.00 \mathrm{E}+07$ & $2.3 \mathrm{E}+06$ & $1.96 \mathrm{E}+07$ & $80 \%$ & $1.95 \mathrm{E}-02$ & 2.4E-02 & $-20 \%$ & $2.67 \mathrm{E}+00$ & 5.4E-01 & $394 \%$ \\
\hline Eu-154 & $\mathrm{dpm} / \mathrm{g}$ & $5.54 \mathrm{E}+07$ & $5.0 \mathrm{E}+06$ & $4.52 \mathrm{E}+07$ & $76 \%$ & $5.42 \mathrm{E}-02$ & $5.6 \mathrm{E}-02$ & $-4 \%$ & $7.40 \mathrm{E}+00$ & $1.2 \mathrm{E}+00$ & $494 \%$ \\
\hline Sr-90 & $\mathrm{dpm} / \mathrm{g}$ & $1.74 \mathrm{E}+10$ & $1.5 \mathrm{E}+09$ & $7.81 \mathrm{E}+10$ & $96 \%$ & $1.70 \mathrm{E}+01$ & $9.7 \mathrm{E}+01$ & $-82 \%$ & $2.32 \mathrm{E}+03$ & $2.2 \mathrm{E}+03$ & $8 \%$ \\
\hline Am-241 & $\mathrm{dpm} / \mathrm{g}$ & $1.17 \mathrm{E}+08$ & $3.9 \mathrm{E}+07$ & $8.47 \mathrm{E}+07$ & $73 \%$ & $1.15 \mathrm{E}-01$ & $1.1 \mathrm{E}-01$ & $9 \%$ & $1.57 \mathrm{E}+01$ & $2.3 \mathrm{E}+00$ & $571 \%$ \\
\hline Am-243 & $\mathrm{dpm} / \mathrm{g}$ & $7.91 \mathrm{E}+05$ & $2.49 \mathrm{E}+05$ & $5.79 \mathrm{E}+05$ & $73 \%$ & 7.74E-04 & 7.2E-04 & $7 \%$ & $1.06 \mathrm{E}-01$ & $1.6 \mathrm{E}-02$ & $562 \%$ \\
\hline $\mathrm{Am}-242 \mathrm{~m}$ & $\mathrm{dpm} / \mathrm{g}$ & $2.55 \mathrm{E}+05$ & $1.23 \mathrm{E}+05$ & $1.89 \mathrm{E}+05$ & $74 \%$ & $2.49 \mathrm{E}-04$ & 2.4E-04 & $6 \%$ & $3.41 \mathrm{E}-02$ & $5.2 \mathrm{E}-03$ & $553 \%$ \\
\hline $\mathrm{Cm}-242$ & $\mathrm{dpm} / \mathrm{g}$ & $2.11 \mathrm{E}+05$ & $1.02 \mathrm{E}+05$ & $1.57 \mathrm{E}+05$ & $74 \%$ & $2.06 \mathrm{E}-04$ & $1.9 \mathrm{E}-04$ & $6 \%$ & $2.81 \mathrm{E}-02$ & 4.3E-03 & $552 \%$ \\
\hline $\mathrm{Cm}-244$ & $\mathrm{dpm} / \mathrm{g}$ & $5.39 \mathrm{E}+06$ & $1.90 \mathrm{E}+06$ & $5.29 \mathrm{E}+06$ & $80 \%$ & $5.28 \mathrm{E}-03$ & $6.6 \mathrm{E}-03$ & $-20 \%$ & $7.20 \mathrm{E}-01$ & $1.5 \mathrm{E}-01$ & $394 \%$ \\
\hline
\end{tabular}

Table 3-10: Summary of Soluble Components at Completion of Dissolution Step

\begin{tabular}{c|c|c|c|c|c|c}
\hline Test & OA Batch & $\mathrm{pH}$ & Oxalate $(\mathrm{M})$ & $\mathrm{Al}(\mathrm{M})$ & $\mathrm{Fe}(\mathrm{M})$ & $\mathrm{Na}(\mathrm{M})$ \\
\hline \multirow{3}{*}{$\begin{array}{c}1 \\
\text { (Tank 12H) }\end{array}$} & 1 & 2.34 & 0.091 & 0.009 & 0.008 & 0.030 \\
\cline { 2 - 7 } & 2 & 2.01 & 0.170 & 0.008 & 0.004 & 0.008 \\
\cline { 2 - 7 } & 3 & 1.90 & 0.209 & 0.007 & 0.001 & 0.002 \\
\hline \multirow{2}{*}{\begin{tabular}{c} 
(Tank 5F) \\
\cline { 2 - 7 }
\end{tabular}} & 2 & 2.20 & 0.043 & 0.011 & 0.049 & 0.009 \\
\cline { 2 - 7 } & 3 & 1.27 & 0.118 & 0.003 & 0.025 & 0.005 \\
\hline
\end{tabular}

Table 3-11 through Table 3-16 contain the results for the soluble components during OA decomposition by application of ozone at $70{ }^{\circ} \mathrm{C}$. Each table includes several columns of data that represent the composition of a series of samples taken as a function of time as the OA decomposition progressed during each batch. The column labeled "initial" shows an average of the duplicate analysis of the filtered samples taken from the Dissolution Module at the conclusion of the dissolution period. The columns labeled "1st sample," "2nd sample," etc. show the single analyses of the intermediate Decomposition Module samples. The "final sample" is an average of the duplicate analysis of the filtered samples taken from the Decomposition Module after the $\mathrm{pH}$ and permanganate end-of-process test indicated that OA decomposition was complete. Included for reference in the Decomposition chemistry tables are the run time and the $\mathrm{pH}$ and ORP readings as the time of sampling.

Two anomalies are evident in these Decomposition Module sample results. These anomalies occurred for the third sample of Test $1 \mathrm{OA}$ batch 1 and the second sample of Test $1 \mathrm{OA}$ batch 2 . Inconsistent high levels of many insoluble components indicate that solids likely bypassed the syringe filters for these samples and are included in the sample results. 
In general, most sludge components show the highest soluble concentrations in the initial dissolved sludge and early decomposition samples. As the sludge components drop out of solution, subsequent decomposition samples show lower concentrations of soluble sludge components until the end of the decomposition process that typically has the lowest concentration of soluble sludge components. This is true for Fe, Al, Mn, Sr-90 and is typically true for $\mathrm{U}$ and $\mathrm{Pu}$. This behavior does not hold for typical supernate components such as sodium and Cs-137, which remain largely soluble throughout the OA decomposition. Figure 3-9 contains a photograph of the intermediate samples and final sample from the decomposition portion of Test 1 OA Batch 2. This figure, which is representative of the observations of Decomposition Module samples from all other OA Batches during ECC RWT, illustrates the increase in precipitated solids as the OA decomposition progresses. This observation is also consistent with Decomposition Module samples taken during ECC simulant testing. ${ }^{17}$

During the ECC testing, approximately 5 moles of ozone was fed to the Decomposition Module for every mole of oxalate decomposed. This molar ratio was lower than the baseline 3:1 ozone to oxalate ratio that was easily attained during simulant testing. ${ }^{17}$ The relatively low utilization of ozone in the ECC RWT may be due to several factors.

- Production of ozone was at a lower concentration ( $5 \mathrm{wt} \%$ versus the baseline $10 \mathrm{wt} \%$ ).

- The ozone generator was located away from the RWT equipment, with the feed gas having to travel through over 20 feet of tubing between the ozone generator and the Decomposition Module. This extended distance may allow a portion of the ozone to decompose prior to introduction to the decomposition module.

- The ECC RWT Decomposition Module pressure was nominally 8 psig, which is lower than the baseline pressure. This lower pressure would result in a lower soluble ozone concentration and result in a higher fraction of the ozone exiting the Decomposition Module in the offgas stream.

- The Decomposition Module recirculation rate was not optimized and was maintained at the maximum capability of pump P-224, which was often less than $1.5 \mathrm{gpm}$.

Table 3-17 and Table 3-18 contain the results for the bulk analysis of the slurries at the end of the dissolution and decomposition processes for Tests 1 and 2, respectively. These results indicate the composition of the slurries within the process and are provided for reference.

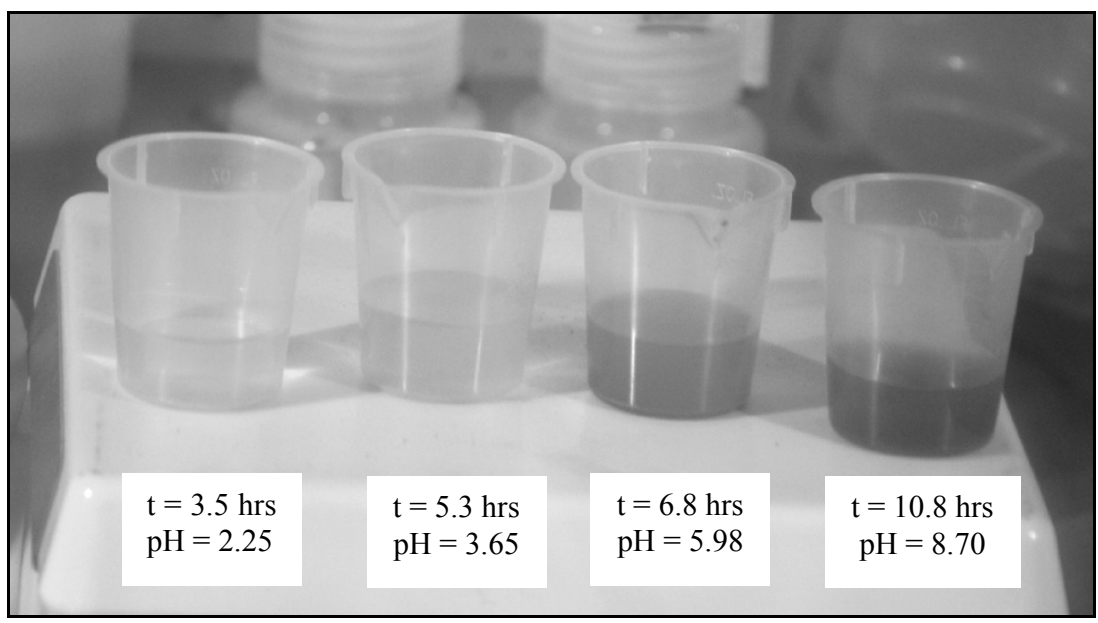

Figure 3-9: Samples of Decomposition Module during Test 1, OA Batch 2. 
Table 3-11: Soluble Components throughout ECC Oxalate Decomposition: Test 1 (Tank 12H) OA Batch 1

\begin{tabular}{|c|c|c|c|c|c|c|}
\hline analyte & units & initial & 1st sample & 2nd sample & 3rd sample* & final sample \\
\hline time & $\mathrm{hr}$ & 0.0 & 1.1 & 4.6 & 5.1 & 7.6 \\
\hline $\mathrm{pH}$ & -- & 2.34 & 3.48 & 7.34 & 7.79 & 8.39 \\
\hline ORP & $\mathrm{mV}$ & 690 & 632 & 694 & 700 & 672 \\
\hline Oxalate & $\mathrm{mg} / \mathrm{L}$ & $8.05 \mathrm{E}+03$ & $1.49 \mathrm{E}+03$ & $4.00 \mathrm{E}+02$ & $7.84 \mathrm{E}+03^{*}$ & $<4.79 E+01$ \\
\hline TOC & $\mathrm{mg} \mathrm{C} / \mathrm{L}$ & $3.88 \mathrm{E}+03$ & -- & -- & -- & $<2.97 E+02$ \\
\hline Nitrate & $\mathrm{mg} / \mathrm{L}$ & $3.21 \mathrm{E}+02$ & -- & -- & -- & $2.71 \mathrm{E}+02$ \\
\hline $\mathrm{Ag}$ & $\mathrm{mg} / \mathrm{L}$ & $6.64 \mathrm{E}-01$ & $<3.72 E-01$ & $<2.32 E-01$ & $<2.25 E-01$ & $<2.77 E-01$ \\
\hline $\mathrm{Al}$ & $\mathrm{mg} / \mathrm{L}$ & $2.35 \mathrm{E}+02$ & $1.32 \mathrm{E}+02$ & $3.51 \mathrm{E}+00$ & $3.68 \mathrm{E}+02 *$ & $2.93 \mathrm{E}+01$ \\
\hline $\mathrm{Ba}$ & $\mathrm{mg} / \mathrm{L}$ & $4.42 \mathrm{E}+00$ & $1.28 \mathrm{E}-01$ & $1.28 \mathrm{E}-02$ & $5.16 \mathrm{E}+00^{*}$ & $<9.56 E-03$ \\
\hline $\mathrm{Ca}$ & $\mathrm{mg} / \mathrm{L}$ & $2.90 \mathrm{E}+01$ & $9.08 \mathrm{E}+00$ & $2.62 \mathrm{E}+00$ & $3.32 \mathrm{E}+01 *$ & $1.65 \mathrm{E}+00$ \\
\hline $\mathrm{Cd}$ & $\mathrm{mg} / \mathrm{L}$ & $2.72 \mathrm{E}-01$ & $<7.70 E-02$ & $<4.80 E-02$ & $3.43 \mathrm{E}-01^{*}$ & $<5.73 E-02$ \\
\hline $\mathrm{Cr}$ & $\mathrm{mg} / \mathrm{L}$ & $4.17 \mathrm{E}+00$ & $6.72 \mathrm{E}+00$ & $5.66 \mathrm{E}+00$ & $5.27 \mathrm{E}+00^{*}$ & $6.61 \mathrm{E}+00$ \\
\hline $\mathrm{Cu}$ & $\mathrm{mg} / \mathrm{L}$ & $3.86 \mathrm{E}+00$ & $2.13 \mathrm{E}+00$ & 3.87E-01 & $1.65 \mathrm{E}+00^{*}$ & $<1.24 E-01$ \\
\hline $\mathrm{Fe}$ & $\mathrm{mg} / \mathrm{L}$ & $4.43 \mathrm{E}+02$ & $3.62 \mathrm{E}+00$ & 3.39E-01 & $4.53 \mathrm{E}+02 *$ & 4.97E-01 \\
\hline $\mathrm{K}$ & $\mathrm{mg} / \mathrm{L}$ & $1.47 \mathrm{E}+01$ & $1.10 \mathrm{E}+01$ & $8.32 \mathrm{E}+00$ & $8.39 \mathrm{E}+00^{*}$ & $<5.83 E+00$ \\
\hline $\mathrm{La}$ & $\mathrm{mg} / \mathrm{L}$ & $2.29 \mathrm{E}-01$ & $<1.67 E-01$ & $<1.04 E-01$ & $<1.01 E-01$ & $<1.24 E-01$ \\
\hline $\mathrm{Li}$ & $\mathrm{mg} / \mathrm{L}$ & $<2.84 E-01$ & $<3.72 E-01$ & $<2.32 E-01$ & $3.25 \mathrm{E}-01^{*}$ & $<2.77 E-01$ \\
\hline $\mathrm{Mg}$ & $\mathrm{mg} / \mathrm{L}$ & $1.42 \mathrm{E}+01$ & $1.44 \mathrm{E}+01$ & $6.73 \mathrm{E}+00$ & $1.56 \mathrm{E}+01 *$ & $1.97 \mathrm{E}-01$ \\
\hline $\mathrm{Mn}$ & $\mathrm{mg} / \mathrm{L}$ & $3.21 \mathrm{E}+02$ & $3.90 \mathrm{E}-01$ & $9.92 \mathrm{E}-01$ & $3.00 \mathrm{E}+02 *$ & $3.20 \mathrm{E}+00$ \\
\hline $\mathrm{Na}$ & $\mathrm{mg} / \mathrm{L}$ & $6.79 \mathrm{E}+02$ & $6.75 \mathrm{E}+02$ & $5.33 \mathrm{E}+02$ & $7.45 \mathrm{E}+02$ & $5.27 \mathrm{E}+02$ \\
\hline $\mathrm{Ni}$ & $\mathrm{mg} / \mathrm{L}$ & $1.39 \mathrm{E}+01$ & $9.41 \mathrm{E}+00$ & $2.42 \mathrm{E}+00$ & $3.13 \mathrm{E}+00^{*}$ & $<3.06 E-01$ \\
\hline $\mathrm{P}$ & $\mathrm{mg} / \mathrm{L}$ & $8.92 \mathrm{E}+00$ & $<4.21 E+00$ & $<2.62 E+00$ & $1.12 \mathrm{E}+01 *$ & $<3.13 E+00$ \\
\hline $\mathrm{S}$ & $\mathrm{mg} / \mathrm{L}$ & $1.96 \mathrm{E}+01$ & $<1.93 E+01$ & $1.56 \mathrm{E}+01$ & $1.82 \mathrm{E}+01 *$ & $<1.75 E+01$ \\
\hline $\mathrm{Si}$ & $\mathrm{mg} / \mathrm{L}$ & $3.82 \mathrm{E}+01$ & $1.86 \mathrm{E}+01$ & $2.55 \mathrm{E}+00$ & $4.45 \mathrm{E}+01^{*}$ & $<1.86 E+00$ \\
\hline $\mathrm{Sr}$ & $\mathrm{mg} / \mathrm{L}$ & $2.19 \mathrm{E}+00$ & $5.55 \mathrm{E}-01$ & $1.38 \mathrm{E}-01$ & $2.53 \mathrm{E}+00^{*}$ & $1.72 \mathrm{E}-02$ \\
\hline $\mathrm{Th}$ & $\mathrm{mg} / \mathrm{L}$ & $1.66 \mathrm{E}+01$ & $<1.41 E+00$ & $<8.80 E-01$ & $1.16 \mathrm{E}+01 *$ & $<1.05 E+00$ \\
\hline $\mathrm{Ti}$ & $\mathrm{mg} / \mathrm{L}$ & $9.79 \mathrm{E}-01$ & $<1.03 E-01$ & $<6.40 E-02$ & $1.31 \mathrm{E}+00^{*}$ & $<7.65 E-02$ \\
\hline $\mathrm{U}$ & $\mathrm{mg} / \mathrm{L}$ & $8.73 \mathrm{E}+01$ & $6.59 \mathrm{E}+01$ & $2.56 \mathrm{E}+01$ & $9.86 \mathrm{E}+01^{*}$ & $3.52 \mathrm{E}+01$ \\
\hline $\mathrm{Zn}$ & $\mathrm{mg} / \mathrm{L}$ & $1.26 \mathrm{E}+00$ & $2.95 \mathrm{E}-01$ & $<1.44 E-01$ & $2.53 \mathrm{E}-01^{*}$ & $<1.72 E-01$ \\
\hline $\mathrm{Zr}$ & $\mathrm{mg} / \mathrm{L}$ & $1.64 \mathrm{E}+01$ & $<6.42 E-02$ & $<4.00 E-02$ & $2.00 \mathrm{E}+01 *$ & $<4.78 E-02$ \\
\hline $\mathrm{Hg}$ & $\mathrm{mg} / \mathrm{L}$ & $1.18 \mathrm{E}+01$ & -- & -- & -- & $<3.08 E+00$ \\
\hline Co & $\mathrm{mg} / \mathrm{L}$ & $9.89 \mathrm{E}-02$ & $<2.57 E-02$ & $<1.60 E-02$ & $3.22 \mathrm{E}-02 *$ & $<1.91 E-02$ \\
\hline $\mathrm{Pb}$ & $\mathrm{mg} / \mathrm{L}$ & $3.96 \mathrm{E}-01$ & $<7.70 E-02$ & $<4.80 E-02$ & $3.77 \mathrm{E}-01^{*}$ & $<5.73 E-02$ \\
\hline $\mathrm{m}-232(\mathrm{Th})$ & $\mathrm{mg} / \mathrm{L}$ & $1.55 \mathrm{E}+01$ & $3.34 \mathrm{E}-01$ & $1.63 \mathrm{E}-02$ & $1.02 \mathrm{E}+01 *$ & $<1.91 E-02$ \\
\hline $\mathrm{m}-233(\mathrm{U})$ & $\mathrm{mg} / \mathrm{L}$ & $1.03 \mathrm{E}-01$ & $9.75 \mathrm{E}-02$ & $3.33 \mathrm{E}-02$ & $1.11 \mathrm{E}-01^{*}$ & $5.38 \mathrm{E}-02$ \\
\hline $\mathrm{m}-234(\mathrm{U})$ & $\mathrm{mg} / \mathrm{L}$ & $1.18 \mathrm{E}-01$ & $1.09 \mathrm{E}-01$ & 4.99E-02 & $1.25 \mathrm{E}-01 *$ & $5.76 \mathrm{E}-02$ \\
\hline $\mathrm{m}-235(\mathrm{U})$ & $\mathrm{mg} / \mathrm{L}$ & $1.43 \mathrm{E}+00$ & $1.20 \mathrm{E}+00$ & 4.81E-01 & $1.76 \mathrm{E}+00^{*}$ & $6.30 \mathrm{E}-01$ \\
\hline $\mathrm{m}-236(\mathrm{U})$ & $\mathrm{mg} / \mathrm{L}$ & $1.79 \mathrm{E}-01$ & $1.43 \mathrm{E}-01$ & $4.88 \mathrm{E}-02$ & $1.91 \mathrm{E}-01^{*}$ & $6.39 \mathrm{E}-02$ \\
\hline $\mathrm{m}-237(\mathrm{~Np})$ & $\mathrm{mg} / \mathrm{L}$ & $1.21 \mathrm{E}-01$ & $1.03 \mathrm{E}-01$ & 4.53E-02 & $1.20 \mathrm{E}-01^{*}$ & $4.05 \mathrm{E}-02$ \\
\hline $\mathrm{m}-238(\mathrm{U}, \mathrm{Pu})$ & $\mathrm{mg} / \mathrm{L}$ & $8.08 \mathrm{E}+01$ & $6.37 \mathrm{E}+01$ & $2.54 \mathrm{E}+01$ & $9.48 \mathrm{E}+01 *$ & $3.38 \mathrm{E}+01$ \\
\hline $\mathrm{m}-239(\mathrm{Pu})$ & $\mathrm{mg} / \mathrm{L}$ & $1.79 \mathrm{E}+00$ & $1.10 \mathrm{E}+00$ & $5.30 \mathrm{E}-01$ & $1.12 \mathrm{E}+00^{*}$ & $6.14 \mathrm{E}-01$ \\
\hline $\mathrm{m}-240(\mathrm{Pu})$ & $\mathrm{mg} / \mathrm{L}$ & $1.69 \mathrm{E}-01$ & $1.04 \mathrm{E}-01$ & $6.25 \mathrm{E}-02$ & $1.13 \mathrm{E}-01^{*}$ & $6.15 \mathrm{E}-02$ \\
\hline $\mathrm{Pu}-238$ & $\mathrm{dpm} / \mathrm{mL}$ & $6.46 \mathrm{E}+06$ & $4.21 \mathrm{E}+06$ & $2.06 \mathrm{E}+06$ & $4.19 \mathrm{E}+06^{*}$ & $2.20 \mathrm{E}+06$ \\
\hline $\mathrm{Pu}-239 / 240$ & $\mathrm{dpm} / \mathrm{mL}$ & $3.46 \mathrm{E}+05$ & $2.20 \mathrm{E}+05$ & $1.04 \mathrm{E}+05$ & $2.20 \mathrm{E}+05^{*}$ & $1.13 \mathrm{E}+05$ \\
\hline Sr-90 & $\mathrm{dpm} / \mathrm{mL}$ & $1.28 \mathrm{E}+08$ & -- & -- & -- & $8.17 \mathrm{E}+05$ \\
\hline Cs-137 & $\mathrm{dpm} / \mathrm{mL}$ & $3.55 \mathrm{E}+06$ & -- & -- & -- & $2.64 \mathrm{E}+06$ \\
\hline Am-241 & $\mathrm{dpm} / \mathrm{mL}$ & $5.84 \mathrm{E}+03$ & -- & -- & -- & $6.74 \mathrm{E}+03$ \\
\hline $\mathrm{Cm}-244$ & $\mathrm{dpm} / \mathrm{mL}$ & $6.42 \mathrm{E}+02$ & -- & -- & -- & $5.14 \mathrm{E}+01$ \\
\hline
\end{tabular}

* high result is possibly due to filter breakthrough or misidentified sample 
Table 3-12: Soluble Components throughout ECC Oxalate Decomposition: Test 1 (Tank 12H) OA Batch 2

\begin{tabular}{|c|c|c|c|c|c|c|}
\hline analyte & units & initial & 1st sample & 2nd sample* & 3rd sample & final sample \\
\hline time & $\mathrm{hr}$ & 0.0 & 3.5 & 5.3 & 6.8 & 10.8 \\
\hline $\mathrm{pH}$ & -- & 2.01 & 2.25 & 3.65 & 5.98 & 8.70 \\
\hline ORP & $\mathrm{mV}$ & 655 & 618 & 674 & 765 & 7.29 \\
\hline Oxalate & $\mathrm{mg} / \mathrm{L}$ & $1.50 \mathrm{E}+04$ & $2.88 \mathrm{E}+03$ & $1.27 \mathrm{E}+03$ & $7.12 \mathrm{E}+02$ & $<4.98 E+01$ \\
\hline TOC & $\mathrm{mg} \mathrm{C} / \mathrm{L}$ & $4.65 \mathrm{E}+03$ & -- & -- & -- & $<3.09 E+02$ \\
\hline Nitrate & $\mathrm{mg} / \mathrm{L}$ & $7.64 \mathrm{E}+01$ & -- & -- & -- & $8.71 \mathrm{E}+01$ \\
\hline $\mathrm{Ag}$ & $\mathrm{mg} / \mathrm{L}$ & $<2.69 E-01$ & 3.33E-01 & $<2.75 E-01$ & $<2.95 E-01$ & $<2.63 E-01$ \\
\hline Al & $\mathrm{mg} / \mathrm{L}$ & $2.19 \mathrm{E}+02$ & $2.71 \mathrm{E}+02$ & $3.19 \mathrm{E}+02 *$ & $9.09 \mathrm{E}+01$ & $1.51 \mathrm{E}+01$ \\
\hline $\mathrm{Ba}$ & $\mathrm{mg} / \mathrm{L}$ & $1.42 \mathrm{E}+00$ & $2.03 \mathrm{E}+00$ & $5.79 \mathrm{E}+00^{*}$ & $4.80 \mathrm{E}-01$ & $<9.08 E-03$ \\
\hline $\mathrm{Ca}$ & $\mathrm{mg} / \mathrm{L}$ & $9.84 \mathrm{E}+00$ & $1.48 \mathrm{E}+01$ & $2.70 \mathrm{E}+01 *$ & $1.24 \mathrm{E}+01$ & 7.59E-01 \\
\hline $\mathrm{Cd}$ & $\mathrm{mg} / \mathrm{L}$ & $8.82 \mathrm{E}-02$ & $1.34 \mathrm{E}-01$ & $6.39 \mathrm{E}-01 *$ & $<6.10 E-02$ & $<5.45 E-02$ \\
\hline $\mathrm{Cr}$ & $\mathrm{mg} / \mathrm{L}$ & $8.75 \mathrm{E}+00$ & $9.44 \mathrm{E}+00$ & $8.98 \mathrm{E}+01 *$ & $8.62 \mathrm{E}+00$ & $1.01 \mathrm{E}+01$ \\
\hline $\mathrm{Cu}$ & $\mathrm{mg} / \mathrm{L}$ & $1.59 \mathrm{E}+00$ & $2.27 \mathrm{E}+00$ & $3.59 \mathrm{E}+00^{*}$ & $1.14 \mathrm{E}+00$ & $<1.18 E-01$ \\
\hline $\mathrm{Fe}$ & $\mathrm{mg} / \mathrm{L}$ & $1.97 \mathrm{E}+02$ & $2.64 \mathrm{E}+02$ & $7.33 \mathrm{E}+02 *$ & $1.12 \mathrm{E}+01$ & $5.51 \mathrm{E}-01$ \\
\hline $\mathrm{K}$ & $\mathrm{mg} / \mathrm{L}$ & $1.90 \mathrm{E}+01$ & $1.17 \mathrm{E}+01$ & $1.09 \mathrm{E}+01 *$ & $1.06 \mathrm{E}+01$ & $6.71 \mathrm{E}+00$ \\
\hline $\mathrm{La}$ & $\mathrm{mg} / \mathrm{L}$ & $3.31 \mathrm{E}-01$ & 4.04E-01 & $4.11 \mathrm{E}+00^{*}$ & $<1.32 E-01$ & $<1.18 E-01$ \\
\hline $\mathrm{Li}$ & $\mathrm{mg} / \mathrm{L}$ & $<2.69 E-01$ & $<2.86 E-01$ & $5.49 \mathrm{E}+00^{*}$ & $<2.95 E-01$ & $<2.63 E-01$ \\
\hline $\mathrm{Mg}$ & $\mathrm{mg} / \mathrm{L}$ & $4.42 \mathrm{E}+00$ & $6.31 \mathrm{E}+00$ & $9.76 \mathrm{E}+00^{*}$ & $6.48 \mathrm{E}+00$ & $1.11 \mathrm{E}-01$ \\
\hline $\mathrm{Mn}$ & $\mathrm{mg} / \mathrm{L}$ & $1.00 \mathrm{E}+02$ & $1.48 \mathrm{E}+02$ & $1.53 \mathrm{E}+02 *$ & $3.27 \mathrm{E}+00$ & $2.09 \mathrm{E}+00$ \\
\hline $\mathrm{Na}$ & $\mathrm{mg} / \mathrm{L}$ & $1.94 \mathrm{E}+02$ & $2.08 \mathrm{E}+02$ & $2.73 \mathrm{E}+02$ & $2.01 \mathrm{E}+02$ & $1.88 \mathrm{E}+02$ \\
\hline $\mathrm{Ni}$ & $\mathrm{mg} / \mathrm{L}$ & $8.35 \mathrm{E}+00$ & $1.35 \mathrm{E}+01$ & $1.95 \mathrm{E}+01 *$ & $1.19 \mathrm{E}+01$ & $<2.90 E-01$ \\
\hline $\mathrm{P}$ & $\mathrm{mg} / \mathrm{L}$ & $3.54 \mathrm{E}+00$ & $4.01 \mathrm{E}+00$ & $2.15 \mathrm{E}+01 *$ & $<3.34 E+00$ & $<2.98 E+00$ \\
\hline $\mathrm{S}$ & $\mathrm{mg} / \mathrm{L}$ & $<1.39 E+01$ & $<1.48 E+01$ & $2.37 \mathrm{E}+01 *$ & $<1.53 E+01$ & $<1.36 E+01$ \\
\hline $\mathrm{Si}$ & $\mathrm{mg} / \mathrm{L}$ & $1.09 \mathrm{E}+01$ & $1.75 \mathrm{E}+01$ & $8.31 \mathrm{E}+01 *$ & $1.12 \mathrm{E}+01$ & $<1.77 E+00$ \\
\hline $\mathrm{Sr}$ & $\mathrm{mg} / \mathrm{L}$ & 7.22E-01 & $1.03 \mathrm{E}+00$ & $2.42 \mathrm{E}+00^{*}$ & $6.97 \mathrm{E}-01$ & 1.09E-02 \\
\hline Th & $\mathrm{mg} / \mathrm{L}$ & $1.05 \mathrm{E}+01$ & $1.28 \mathrm{E}+01$ & $8.80 \mathrm{E}+00^{*}$ & $<1.12 E+00$ & $<9.98 E-01$ \\
\hline $\mathrm{Ti}$ & $\mathrm{mg} / \mathrm{L}$ & $5.17 \mathrm{E}-01$ & 7.39E-01 & $1.67 \mathrm{E}+00^{*}$ & $<8.14 E-02$ & $<7.26 E-02$ \\
\hline $\mathrm{U}$ & $\mathrm{mg} / \mathrm{L}$ & $2.50 \mathrm{E}+01$ & $3.26 \mathrm{E}+01$ & $3.49 \mathrm{E}+01 *$ & $2.32 \mathrm{E}+01$ & $<1.47 E+01$ \\
\hline $\mathrm{Zn}$ & $\mathrm{mg} / \mathrm{L}$ & $6.71 \mathrm{E}-01$ & $1.10 \mathrm{E}+00$ & $4.17 \mathrm{E}+00^{*}$ & $2.43 \mathrm{E}-01$ & $<1.63 E-01$ \\
\hline $\mathrm{Zr}$ & $\mathrm{mg} / \mathrm{L}$ & $5.77 \mathrm{E}+00$ & $8.04 \mathrm{E}+00$ & $8.83 \mathrm{E}+00^{*}$ & $<5.09 E-02$ & $<4.54 E-02$ \\
\hline $\mathrm{Hg}$ & $\mathrm{mg} / \mathrm{L}$ & $2.70 \mathrm{E}+00$ & -- & -- & -- & $2.54 \mathrm{E}+00$ \\
\hline Co & $\mathrm{mg} / \mathrm{L}$ & $8.98 \mathrm{E}-02$ & $1.44 \mathrm{E}-01$ & $2.09 \mathrm{E}-01^{*}$ & $2.15 \mathrm{E}-02$ & $<9.08 E-03$ \\
\hline $\mathrm{Pb}$ & $\mathrm{mg} / \mathrm{L}$ & $1.33 \mathrm{E}-01$ & 2.11E-01 & $1.48 \mathrm{E}+00^{*}$ & $<6.10 E-02$ & $3.70 \mathrm{E}-02$ \\
\hline m-232 (Th) & $\mathrm{mg} / \mathrm{L}$ & $1.09 \mathrm{E}+01$ & $1.24 \mathrm{E}+01$ & $8.05 \mathrm{E}+00^{*}$ & $1.11 \mathrm{E}-01$ & $<9.08 E-03$ \\
\hline m-233 (U) & $\mathrm{mg} / \mathrm{L}$ & 4.03E-02 & $<3.94 E-02$ & $4.58 \mathrm{E}-02 *$ & $<4.07 E-02$ & $<1.82 E-02$ \\
\hline $\mathrm{m}-234(\mathrm{U})$ & $\mathrm{mg} / \mathrm{L}$ & $3.75 \mathrm{E}-02$ & 4.22E-02 & $5.27 \mathrm{E}-02 *$ & 4.09E-02 & $<9.08 E-03$ \\
\hline $\mathrm{m}-235(\mathrm{U})$ & $\mathrm{mg} / \mathrm{L}$ & $4.25 \mathrm{E}-01$ & $5.67 \mathrm{E}-01$ & $5.94 \mathrm{E}-01^{*}$ & $3.89 \mathrm{E}-01$ & $<5.34 E-02$ \\
\hline m-236(U) & $\mathrm{mg} / \mathrm{L}$ & 4.73E-02 & $7.30 \mathrm{E}-02$ & $8.12 \mathrm{E}-02 *$ & $4.78 \mathrm{E}-02$ & $<1.82 E-02$ \\
\hline m-237 (Np) & $\mathrm{mg} / \mathrm{L}$ & $3.05 \mathrm{E}-02$ & $4.57 \mathrm{E}-02$ & $6.75 \mathrm{E}-02 *$ & $3.87 \mathrm{E}-02$ & $<9.08 E-03$ \\
\hline m-238 (U,Pu) & $\mathrm{mg} / \mathrm{L}$ & $2.33 \mathrm{E}+01$ & $2.85 \mathrm{E}+01$ & $3.18 \mathrm{E}+01 *$ & $2.09 \mathrm{E}+01$ & $2.64 \mathrm{E}+00$ \\
\hline $\mathrm{m}-239(\mathrm{Pu})$ & $\mathrm{mg} / \mathrm{L}$ & $4.80 \mathrm{E}-01$ & $6.98 \mathrm{E}-01$ & $5.38 \mathrm{E}+00^{*}$ & $4.62 \mathrm{E}-01$ & $7.00 \mathrm{E}-02$ \\
\hline $\mathrm{m}-240(\mathrm{Pu})$ & $\mathrm{mg} / \mathrm{L}$ & 4.73E-02 & 7.14E-02 & $4.11 \mathrm{E}-01^{*}$ & $4.92 \mathrm{E}-02$ & $<9.58 E-03$ \\
\hline $\mathrm{Pu}-238$ & $\mathrm{dpm} / \mathrm{mL}$ & $1.73 \mathrm{E}+06$ & $2.33 \mathrm{E}+06$ & $2.51 \mathrm{E}+06^{*}$ & $1.72 \mathrm{E}+06$ & $2.48 \mathrm{E}+05$ \\
\hline $\mathrm{Pu}-239 / 240$ & $\mathrm{dpm} / \mathrm{mL}$ & $8.53 \mathrm{E}+04$ & $1.20 \mathrm{E}+05$ & $1.27 \mathrm{E}+05^{*}$ & $8.94 \mathrm{E}+04$ & $1.25 \mathrm{E}+04$ \\
\hline $\mathrm{Sr}-90$ & $\mathrm{dpm} / \mathrm{mL}$ & $5.21 \mathrm{E}+07$ & -- & -- & -- & $1.68 \mathrm{E}+05$ \\
\hline Cs-137 & $\mathrm{dpm} / \mathrm{mL}$ & $1.03 \mathrm{E}+06$ & -- & -- & -- & $9.85 \mathrm{E}+05$ \\
\hline Am-241 & $\mathrm{dpm} / \mathrm{mL}$ & $1.96 \mathrm{E}+04$ & -- & -- & -- & $2.21 \mathrm{E}+03$ \\
\hline $\mathrm{Cm}-244$ & $\mathrm{dpm} / \mathrm{mL}$ & $9.15 \mathrm{E}+02$ & -- & -- & -- & $7.08 \mathrm{E}+00$ \\
\hline
\end{tabular}

* high result is possibly due to filter breakthrough 
Table 3-13: Soluble Components throughout ECC Oxalate Decomposition: Test 1 (Tank 12H) OA Batch 3

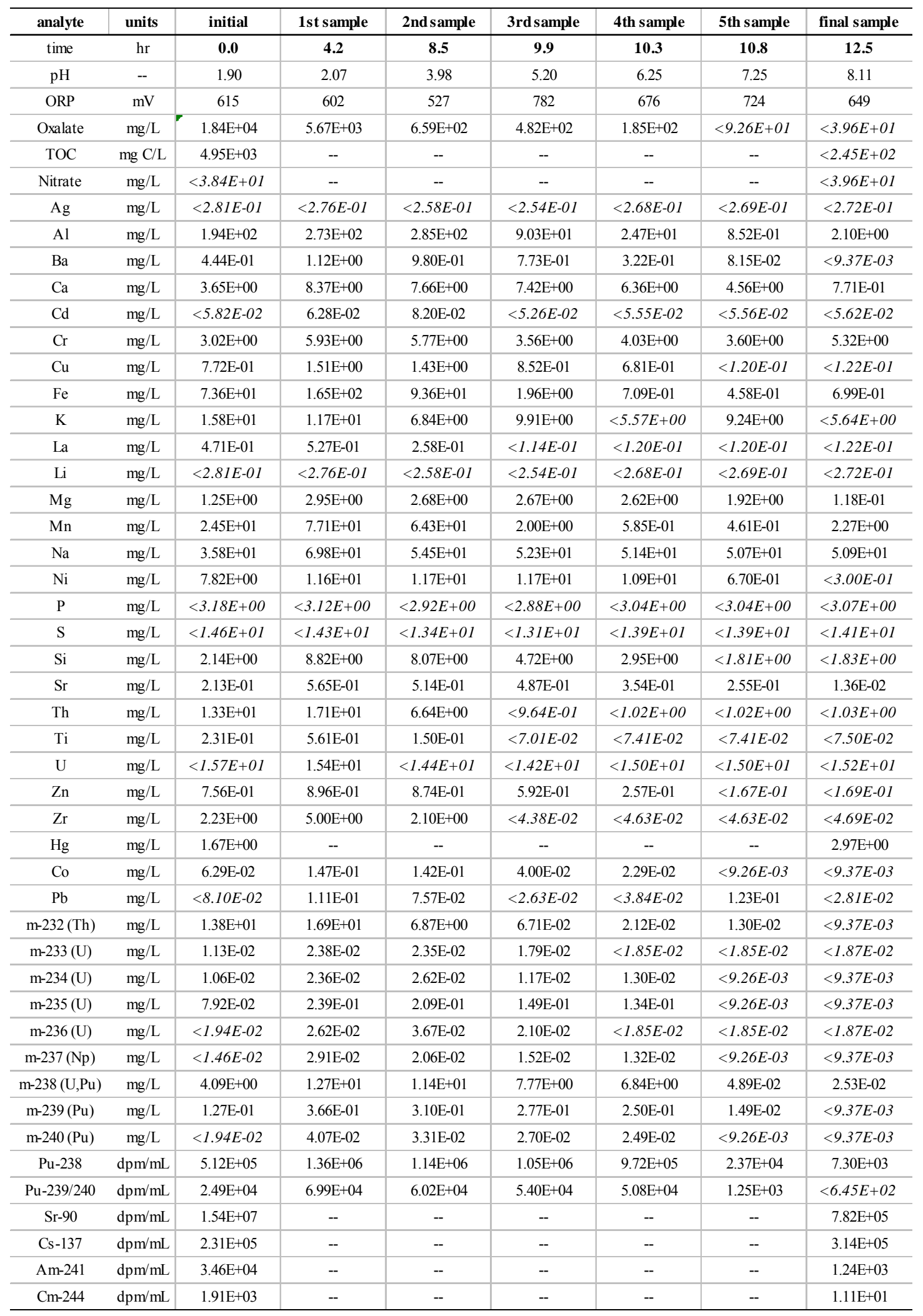


Table 3-14: Soluble Components throughout ECC Oxalate Decomposition: Test 2 (Tank 5F) OA Batch 1

\begin{tabular}{|c|c|c|c|c|c|c|}
\hline analyte & units & initial & 1st sample & 2nd sample & 3rd sample & final sample \\
\hline time & hrs & 0 & 2.5 & 5.1 & 9.7 & 10.9 \\
\hline $\mathrm{pH}$ & -- & 2.20 & -- & -- & -- & -- \\
\hline ORP & $\mathrm{mV}$ & 728 & 626 & 739 & 755 & 746 \\
\hline Oxalate & $\mathrm{mg} / \mathrm{L}$ & $3.75 \mathrm{E}+03$ & $3.13 \mathrm{E}+02$ & $6.51 \mathrm{E}+02$ & $<8.93 E+01$ & $<7.82 E+01$ \\
\hline TOC & $\mathrm{mg} \mathrm{C} / \mathrm{L}$ & $1.30 \mathrm{E}+03$ & -- & -- & -- & $<7.82 E+01$ \\
\hline Nitrate & $\mathrm{mg} / \mathrm{L}$ & $1.15 \mathrm{E}+02$ & -- & -- & -- & $1.03 \mathrm{E}+02$ \\
\hline Carbonate & $\mathrm{mg} / \mathrm{L}$ & $4.15 \mathrm{E}+02$ & -- & -- & - & $1.07 \mathrm{E}+03$ \\
\hline $\mathrm{Ag}$ & $\mathrm{mg} / \mathrm{L}$ & $9.59 \mathrm{E}-01$ & $<6.62 E-01$ & $<7.21 E-01$ & $<6.43 E-01$ & $<6.80 E-01$ \\
\hline $\mathrm{Al}$ & $\mathrm{mg} / \mathrm{L}$ & $2.95 \mathrm{E}+02$ & $2.70 \mathrm{E}+02$ & $3.87 \mathrm{E}+01$ & $2.82 \mathrm{E}+01$ & $2.81 \mathrm{E}+01$ \\
\hline $\mathrm{Ba}$ & $\mathrm{mg} / \mathrm{L}$ & $2.58 \mathrm{E}+01$ & $9.75 \mathrm{E}+00$ & $3.76 \mathrm{E}-01$ & $5.80 \mathrm{E}-02$ & $6.61 \mathrm{E}-02$ \\
\hline $\mathrm{Ca}$ & $\mathrm{mg} / \mathrm{L}$ & $5.94 \mathrm{E}+01$ & $4.44 \mathrm{E}+01$ & $1.50 \mathrm{E}+01$ & $4.53 \mathrm{E}+00$ & $3.75 \mathrm{E}+00$ \\
\hline $\mathrm{Cd}$ & $\mathrm{mg} / \mathrm{L}$ & $9.04 \mathrm{E}-01$ & $6.35 \mathrm{E}-01$ & $<1.60 E-01$ & $<1.43 E-01$ & $<1.51 E-01$ \\
\hline $\mathrm{Cr}$ & $\mathrm{mg} / \mathrm{L}$ & $1.40 \mathrm{E}+01$ & $1.21 \mathrm{E}+01$ & $1.05 \mathrm{E}+01$ & $1.04 \mathrm{E}+01$ & $9.54 \mathrm{E}+00$ \\
\hline $\mathrm{Cu}$ & $\mathrm{mg} / \mathrm{L}$ & $7.13 \mathrm{E}+00$ & $6.30 \mathrm{E}+00$ & $4.07 \mathrm{E}+00$ & $3.79 \mathrm{E}+00$ & $3.44 \mathrm{E}+00$ \\
\hline $\mathrm{Fe}$ & $\mathrm{mg} / \mathrm{L}$ & $2.72 \mathrm{E}+03$ & $8.47 \mathrm{E}+02$ & $5.46 \mathrm{E}+01$ & $5.48 \mathrm{E}+00$ & $5.08 \mathrm{E}+00$ \\
\hline $\mathrm{Gd}$ & $\mathrm{mg} / \mathrm{L}$ & $5.47 \mathrm{E}+00$ & $4.83 \mathrm{E}+00$ & $3.17 \mathrm{E}+00$ & $2.90 \mathrm{E}+00$ & $2.78 \mathrm{E}+00$ \\
\hline $\mathrm{K}$ & $\mathrm{mg} / \mathrm{L}$ & $2.00 \mathrm{E}+01$ & $1.88 \mathrm{E}+01$ & $1.51 \mathrm{E}+01$ & $1.35 \mathrm{E}+01$ & $<1.42 E+01$ \\
\hline $\mathrm{La}$ & $\mathrm{mg} / \mathrm{L}$ & $4.16 \mathrm{E}+00$ & $8.49 \mathrm{E}-01$ & $<4.31 E-01$ & $<3.84 E-01$ & $<4.06 E-01$ \\
\hline $\mathrm{Li}$ & $\mathrm{mg} / \mathrm{L}$ & $6.64 \mathrm{E}+00$ & $6.23 \mathrm{E}+00$ & $5.51 \mathrm{E}+00$ & $4.53 \mathrm{E}+00$ & $3.94 \mathrm{E}+00$ \\
\hline $\mathrm{Mg}$ & $\mathrm{mg} / \mathrm{L}$ & $6.92 \mathrm{E}+00$ & $6.41 \mathrm{E}+00$ & $3.03 \mathrm{E}+00$ & $1.96 \mathrm{E}-01$ & $1.75 \mathrm{E}-01$ \\
\hline $\mathrm{Mn}$ & $\mathrm{mg} / \mathrm{L}$ & $8.77 \mathrm{E}+02$ & $7.24 \mathrm{E}+02$ & $1.80 \mathrm{E}+01$ & $4.61 \mathrm{E}+00$ & $4.11 \mathrm{E}+00$ \\
\hline $\mathrm{Na}$ & $\mathrm{mg} / \mathrm{L}$ & $7.97 \mathrm{E}+02$ & $7.10 \mathrm{E}+02$ & $7.07 \mathrm{E}+02$ & $7.03 \mathrm{E}+02$ & $6.52 \mathrm{E}+02$ \\
\hline $\mathrm{Ni}$ & $\mathrm{mg} / \mathrm{L}$ & $4.37 \mathrm{E}+01$ & $3.31 \mathrm{E}+01$ & $4.74 \mathrm{E}+00$ & $<7.14 E-01$ & $<7.56 E-01$ \\
\hline $\mathrm{Si}$ & $\mathrm{mg} / \mathrm{L}$ & $1.76 \mathrm{E}+02$ & $1.25 \mathrm{E}+02$ & $1.81 \mathrm{E}+01$ & $7.53 \mathrm{E}+00$ & $6.62 \mathrm{E}+00$ \\
\hline $\mathrm{Sr}$ & $\mathrm{mg} / \mathrm{L}$ & $1.29 \mathrm{E}+01$ & $8.35 \mathrm{E}+00$ & $1.54 \mathrm{E}+00$ & $2.90 \mathrm{E}-01$ & $2.18 \mathrm{E}-01$ \\
\hline $\mathrm{Th}$ & $\mathrm{mg} / \mathrm{L}$ & $5.30 \mathrm{E}+00$ & $4.16 \mathrm{E}+00$ & $2.78 \mathrm{E}+00$ & $<2.45 E+00$ & $<2.59 E+00$ \\
\hline $\mathrm{Ti}$ & $\mathrm{mg} / \mathrm{L}$ & $2.37 \mathrm{E}+00$ & $6.07 \mathrm{E}-01$ & $3.76 \mathrm{E}-01$ & 3.57E-01 & $3.28 \mathrm{E}-01$ \\
\hline $\mathrm{U}$ & $\mathrm{mg} / \mathrm{L}$ & $1.66 \mathrm{E}+03$ & $1.45 \mathrm{E}+03$ & $8.62 \mathrm{E}+02$ & $7.95 \mathrm{E}+02$ & $7.28 \mathrm{E}+02$ \\
\hline $\mathrm{Zn}$ & $\mathrm{mg} / \mathrm{L}$ & $6.63 \mathrm{E}-01$ & $5.43 \mathrm{E}-01$ & $3.56 \mathrm{E}-01$ & $3.17 \mathrm{E}-01$ & $3.57 \mathrm{E}-01$ \\
\hline $\mathrm{Zr}$ & $\mathrm{mg} / \mathrm{L}$ & $6.49 \mathrm{E}+01$ & 7.17E-01 & $<2.81 E-01$ & $<2.50 E-01$ & $<2.64 E-01$ \\
\hline $\mathrm{Hg}$ & $\mathrm{mg} / \mathrm{L}$ & $7.29 \mathrm{E}+00$ & -- & -- & -- & $<2.83 E-01$ \\
\hline $\mathrm{Pb}$ & $\mathrm{mg} / \mathrm{L}$ & $4.68 \mathrm{E}+00$ & $<2.53 E+00$ & $<1.38 E+00$ & $<1.23 E+00$ & $<1.65 E+00$ \\
\hline $\mathrm{m}-232(\mathrm{Th})$ & $\mathrm{mg} / \mathrm{L}$ & $<9.64 E-01$ & $<9.20 E-01$ & $<5.01 E-01$ & $<4.46 E-01$ & $<4.72 E-01$ \\
\hline $\mathrm{m}-235(\mathrm{U})$ & $\mathrm{mg} / \mathrm{L}$ & $1.17 \mathrm{E}+01$ & $9.93 \mathrm{E}+00$ & $6.05 \mathrm{E}+00$ & $5.36 \mathrm{E}+00$ & $4.58 \mathrm{E}+00$ \\
\hline $\mathrm{m}-236(\mathrm{U})$ & $\mathrm{mg} / \mathrm{L}$ & $<9.64 E-01$ & $<6.90 E-01$ & $4.23 \mathrm{E}-01$ & $3.53 \mathrm{E}-01$ & $<4.72 E-01$ \\
\hline $\mathrm{m}-237(\mathrm{~Np})$ & $\mathrm{mg} / \mathrm{L}$ & $<9.64 E-01$ & $6.12 \mathrm{E}-01$ & $<2.51 E-01$ & $<2.23 E-01$ & $1.02 \mathrm{E}+00$ \\
\hline $\mathrm{m}-238(\mathrm{U}, \mathrm{Pu})$ & $\mathrm{mg} / \mathrm{L}$ & $1.72 \mathrm{E}+03$ & $1.45 \mathrm{E}+03$ & $8.84 \mathrm{E}+02$ & $7.38 \mathrm{E}+02$ & $6.73 \mathrm{E}+02$ \\
\hline $\mathrm{m}-239(\mathrm{Pu})$ & $\mathrm{mg} / \mathrm{L}$ & $1.28 \mathrm{E}+00$ & $<6.90 E-01$ & $<3.76 E-01$ & $<3.35 E-01$ & $3.16 \mathrm{E}+01$ \\
\hline $\mathrm{m}-240(\mathrm{Pu})$ & $\mathrm{mg} / \mathrm{L}$ & $<1.45 E+00$ & $<6.90 E-01$ & $<3.76 E-01$ & $<3.35 E-01$ & $1.82 \mathrm{E}+00$ \\
\hline $\mathrm{Pu}-238$ & $\mathrm{dpm} / \mathrm{mL}$ & $4.13 \mathrm{E}+04$ & $3.58 \mathrm{E}+04$ & $1.94 \mathrm{E}+04$ & $7.40 \mathrm{E}+03$ & $7.37 \mathrm{E}+03$ \\
\hline $\mathrm{Pu}-239 / 240$ & $\mathrm{dpm} / \mathrm{mL}$ & $1.27 \mathrm{E}+05$ & $3.68 \mathrm{E}+04$ & $8.36 \mathrm{E}+03$ & $5.46 \mathrm{E}+03$ & $4.88 \mathrm{E}+03$ \\
\hline Sr-90 & $\mathrm{dpm} / \mathrm{mL}$ & $1.15 \mathrm{E}+09$ & -- & -- & -- & $1.56 \mathrm{E}+07$ \\
\hline Cs-137 & $\mathrm{dpm} / \mathrm{mL}$ & $4.91 \mathrm{E}+07$ & -- & -- & -- & $2.46 \mathrm{E}+07$ \\
\hline Am-241 & $\mathrm{dpm} / \mathrm{mL}$ & $2.25 \mathrm{E}+04$ & -- & -- & -- & $6.50 \mathrm{E}+02$ \\
\hline Am-243 & $\mathrm{dpm} / \mathrm{mL}$ & $1.56 \mathrm{E}+02$ & -- & -- & -- & $<4.92 E+02$ \\
\hline $\mathrm{Am}-242 \mathrm{~m}$ & $\mathrm{dpm} / \mathrm{mL}$ & $4.67 \mathrm{E}+01$ & -- & - & -- & $<7.65 E+01$ \\
\hline $\mathrm{Cm}-242$ & $\mathrm{dpm} / \mathrm{mL}$ & $3.86 \mathrm{E}+01$ & -- & -- & -- & $<6.32 E+01$ \\
\hline $\mathrm{Cm}-244$ & $\mathrm{dpm} / \mathrm{mL}$ & $1.17 \mathrm{E}+03$ & -- & -- & -- & $2.36 \mathrm{E}+02$ \\
\hline
\end{tabular}


Table 3-15: Soluble Components throughout ECC Oxalate Decomposition: Test 2 (Tank 5F) OA Batch 2

\begin{tabular}{|c|c|c|c|c|c|}
\hline analyte & units & initial & 1st sample & 2nd sample & final sample \\
\hline time & hrs & 0 & 3.5 & 5.0 & 7.6 \\
\hline $\mathrm{pH}$ & -- & 1.27 & 3.20 & 6.38 & 8.52 \\
\hline ORP & $\mathrm{mV}$ & 812 & 899 & 901 & 956 \\
\hline Oxalate & $\mathrm{mg} / \mathrm{L}$ & $1.04 \mathrm{E}+04$ & $<8.94 E+01$ & $4.04 \mathrm{E}+02$ & $<8.20 E+01$ \\
\hline TOC & $\mathrm{mg} \mathrm{C} / \mathrm{L}$ & $3.73 \mathrm{E}+03$ & -- & -- & $<1.67 E+02$ \\
\hline Nitrate & $\mathrm{mg} / \mathrm{L}$ & $<6.22 E+01$ & -- & -- & $<8.20 E+01$ \\
\hline Carbonate & $\mathrm{mg} / \mathrm{L}$ & $<6.53 E+02$ & -- & -- & $<4.10 E+02$ \\
\hline $\mathrm{Ag}$ & $\mathrm{mg} / \mathrm{L}$ & $<6.79 E-01$ & $<6.43 E-01$ & $<5.81 E-01$ & $<7.06 E-01$ \\
\hline $\mathrm{Al}$ & $\mathrm{mg} / \mathrm{L}$ & $7.04 \mathrm{E}+01$ & $1.56 \mathrm{E}+02$ & $2.59 \mathrm{E}+01$ & $5.69 \mathrm{E}+00$ \\
\hline $\mathrm{Ba}$ & $\mathrm{mg} / \mathrm{L}$ & $7.71 \mathrm{E}+00$ & $7.74 \mathrm{E}+00$ & $7.35 \mathrm{E}-01$ & $8.97 \mathrm{E}-01$ \\
\hline $\mathrm{Ca}$ & $\mathrm{mg} / \mathrm{L}$ & $1.32 \mathrm{E}+01$ & $2.79 \mathrm{E}+01$ & $9.29 \mathrm{E}+00$ & $1.48 \mathrm{E}+00$ \\
\hline $\mathrm{Cd}$ & $\mathrm{mg} / \mathrm{L}$ & 4.93E-01 & 7.64E-01 & $3.51 \mathrm{E}-01$ & $4.51 \mathrm{E}-01$ \\
\hline $\mathrm{Cr}$ & $\mathrm{mg} / \mathrm{L}$ & $4.80 \mathrm{E}+00$ & $6.80 \mathrm{E}+00$ & $4.09 \mathrm{E}+00$ & $5.23 \mathrm{E}+00$ \\
\hline $\mathrm{Cu}$ & $\mathrm{mg} / \mathrm{L}$ & $1.95 \mathrm{E}+00$ & $3.19 \mathrm{E}+00$ & $1.59 \mathrm{E}+00$ & $<7.45 E-01$ \\
\hline $\mathrm{Fe}$ & $\mathrm{mg} / \mathrm{L}$ & $1.42 \mathrm{E}+03$ & $8.29 \mathrm{E}+02$ & $3.60 \mathrm{E}+00$ & $4.59 \mathrm{E}+00$ \\
\hline $\mathrm{Gd}$ & $\mathrm{mg} / \mathrm{L}$ & $<1.02 E+00$ & $2.25 \mathrm{E}+00$ & $1.56 \mathrm{E}+00$ & $<1.06 E+00$ \\
\hline $\mathrm{K}$ & $\mathrm{mg} / \mathrm{L}$ & $<1.42 E+01$ & $1.42 \mathrm{E}+01$ & $1.26 \mathrm{E}+01$ & $<1.47 E+01$ \\
\hline $\mathrm{La}$ & $\mathrm{mg} / \mathrm{L}$ & $1.72 \mathrm{E}+00$ & $6.19 \mathrm{E}-01$ & $<3.47 E-01$ & $<4.22 E-01$ \\
\hline $\mathrm{Li}$ & $\mathrm{mg} / \mathrm{L}$ & $2.39 \mathrm{E}+00$ & $3.28 \mathrm{E}+00$ & $2.81 \mathrm{E}+00$ & $2.60 \mathrm{E}+00$ \\
\hline $\mathrm{Mg}$ & $\mathrm{mg} / \mathrm{L}$ & $2.22 \mathrm{E}+00$ & $4.23 \mathrm{E}+00$ & $3.49 \mathrm{E}+00$ & $1.32 \mathrm{E}-01$ \\
\hline $\mathrm{Mn}$ & $\mathrm{mg} / \mathrm{L}$ & $1.75 \mathrm{E}+02$ & $2.88 \mathrm{E}+02$ & $4.81 \mathrm{E}-01$ & $1.74 \mathrm{E}+00$ \\
\hline $\mathrm{Na}$ & $\mathrm{mg} / \mathrm{L}$ & $1.07 \mathrm{E}+02$ & $2.03 \mathrm{E}+02$ & $1.74 \mathrm{E}+02$ & $1.70 \mathrm{E}+02$ \\
\hline $\mathrm{Ni}$ & $\mathrm{mg} / \mathrm{L}$ & $1.57 \mathrm{E}+01$ & $3.70 \mathrm{E}+01$ & $1.66 \mathrm{E}+01$ & $<7.85 E-01$ \\
\hline $\mathrm{Si}$ & $\mathrm{mg} / \mathrm{L}$ & $3.43 \mathrm{E}+01$ & $8.94 \mathrm{E}+01$ & $2.51 \mathrm{E}+01$ & $<2.16 E+00$ \\
\hline $\mathrm{Sr}$ & $\mathrm{mg} / \mathrm{L}$ & $2.59 \mathrm{E}+00$ & $4.92 \mathrm{E}+00$ & $8.80 \mathrm{E}-01$ & $3.07 \mathrm{E}-02$ \\
\hline $\mathrm{Th}$ & $\mathrm{mg} / \mathrm{L}$ & $<2.65 E+00$ & $<2.45 E+00$ & $<2.21 E+00$ & $<2.69 E+00$ \\
\hline $\mathrm{Ti}$ & $\mathrm{mg} / \mathrm{L}$ & $1.13 \mathrm{E}+00$ & $2.68 \mathrm{E}-01$ & 1.74E-01 & $<1.86 E-01$ \\
\hline $\mathrm{U}$ & $\mathrm{mg} / \mathrm{L}$ & $2.05 \mathrm{E}+02$ & $5.88 \mathrm{E}+02$ & $3.63 \mathrm{E}+02$ & $5.19 \mathrm{E}+01$ \\
\hline $\mathrm{Zn}$ & $\mathrm{mg} / \mathrm{L}$ & $<3.46 E-01$ & $4.51 \mathrm{E}-01$ & $<2.67 E-01$ & $<3.24 E-01$ \\
\hline $\mathrm{Zr}$ & $\mathrm{mg} / \mathrm{L}$ & $1.45 \mathrm{E}+01$ & $4.38 \mathrm{E}-01$ & $<2.26 E-01$ & $<2.75 E-01$ \\
\hline $\mathrm{Hg}$ & $\mathrm{mg} / \mathrm{L}$ & $3.74 \mathrm{E}+00$ & -- & -- & $<2.94 E-01$ \\
\hline $\mathrm{Pb}$ & $\mathrm{mg} / \mathrm{L}$ & $2.08 \mathrm{E}+00$ & $<1.23 E+00$ & $<8.88 E-01$ & $<6.89 E-02$ \\
\hline $\mathrm{m}-232(\mathrm{Th})$ & $\mathrm{mg} / \mathrm{L}$ & $1.32 \mathrm{E}+00$ & $<4.47 E-01$ & $<3.23 E-01$ & $<1.96 E-02$ \\
\hline $\mathrm{m}-235(\mathrm{U})$ & $\mathrm{mg} / \mathrm{L}$ & $1.33 \mathrm{E}+00$ & $3.78 \mathrm{E}+00$ & $2.33 \mathrm{E}+00$ & $3.30 \mathrm{E}-01$ \\
\hline $\mathrm{m}-236(\mathrm{U})$ & $\mathrm{mg} / \mathrm{L}$ & $<4.72 E-01$ & $<3.35 E-01$ & $<2.42 E-01$ & $2.42 \mathrm{E}-02$ \\
\hline $\mathrm{m}-237(\mathrm{~Np})$ & $\mathrm{mg} / \mathrm{L}$ & $1.07 \mathrm{E}+00$ & $2.80 \mathrm{E}-01$ & $1.67 \mathrm{E}-01$ & $<1.96 E-02$ \\
\hline m-238 (U,Pu) & $\mathrm{mg} / \mathrm{L}$ & $1.85 \mathrm{E}+02$ & $5.79 \mathrm{E}+02$ & $3.64 \mathrm{E}+02$ & $4.99 \mathrm{E}+01$ \\
\hline $\mathrm{m}-239(\mathrm{Pu})$ & $\mathrm{mg} / \mathrm{L}$ & $3.21 \mathrm{E}+01$ & $<3.35 E-01$ & $<2.42 E-01$ & $2.33 \mathrm{E}-02$ \\
\hline $\mathrm{m}-240(\mathrm{Pu})$ & $\mathrm{mg} / \mathrm{L}$ & $1.94 \mathrm{E}+00$ & $<3.35 E-01$ & $<2.42 E-01$ & $<2.94 E-02$ \\
\hline $\mathrm{Pu}-238$ & $\mathrm{dpm} / \mathrm{mL}$ & $4.10 \mathrm{E}+04$ & $7.16 \mathrm{E}+04$ & $3.15 \mathrm{E}+04$ & $4.42 \mathrm{E}+03$ \\
\hline $\mathrm{Pu}-239 / 240$ & $\mathrm{dpm} / \mathrm{mL}$ & $3.89 \mathrm{E}+04$ & $4.80 \mathrm{E}+04$ & $1.83 \mathrm{E}+04$ & $2.79 \mathrm{E}+03$ \\
\hline $\mathrm{Sr}-90$ & $\mathrm{dpm} / \mathrm{mL}$ & $1.83 \mathrm{E}+08$ & -- & -- & $2.58 \mathrm{E}+06$ \\
\hline Cs-137 & $\mathrm{dpm} / \mathrm{mL}$ & $7.52 \mathrm{E}+06$ & -- & -- & $7.33 \mathrm{E}+06$ \\
\hline Am-241 & $\mathrm{dpm} / \mathrm{mL}$ & $1.55 \mathrm{E}+04$ & -- & -- & $1.97 \mathrm{E}+03$ \\
\hline Am-243 & $\mathrm{dpm} / \mathrm{mL}$ & $<8.21 E+01$ & -- & -- & $<4.46 E+01$ \\
\hline Am-242m & $\mathrm{dpm} / \mathrm{mL}$ & $3.18 \mathrm{E}+01$ & -- & - & $<2.58 E+00$ \\
\hline $\mathrm{Cm}-242$ & $\mathrm{dpm} / \mathrm{mL}$ & $2.64 \mathrm{E}+01$ & -- & -- & $<2.14 E+00$ \\
\hline $\mathrm{Cm}-244$ & $\mathrm{dpm} / \mathrm{mL}$ & $1.19 \mathrm{E}+03$ & -- & -- & $4.44 \mathrm{E}+02$ \\
\hline
\end{tabular}


Table 3-16: Soluble Components throughout ECC Oxalate Decomposition: Test 2 (Tank 5F) OA Batch 3

\begin{tabular}{|c|c|c|c|c|c|c|}
\hline analyte & units & initial & 1st sample & 2nd sample & 3rd sample & final sample \\
\hline time & hrs & 0 & 3.3 & 5.8 & 7.3 & 8.6 \\
\hline $\mathrm{pH}$ & -- & 1.20 & 1.46 & 2.71 & 6.45 & 7.84 \\
\hline ORP & $\mathrm{mV}$ & 701 & 713 & 702 & 681 & 698 \\
\hline Oxalate & $\mathrm{mg} / \mathrm{L}$ & $1.61 \mathrm{E}+04$ & $<9.57 E+01$ & $<9.29 E+01$ & $<9.50 E+01$ & $<7.98 E+01$ \\
\hline TOC & $\mathrm{mg} \mathrm{C} / \mathrm{L}$ & $3.95 \mathrm{E}+03$ & -- & -- & -- & $<7.98 E+01$ \\
\hline Nitrate & $\mathrm{mg} / \mathrm{L}$ & $<6.70 E+01$ & -- & -- & -- & $<7.98 E+01$ \\
\hline Carbonate & $\mathrm{mg} / \mathrm{L}$ & $<7.37 E+02$ & -- & -- & -- & $<3.99 E+02$ \\
\hline $\mathrm{Ag}$ & $\mathrm{mg} / \mathrm{L}$ & $<6.61 E-01$ & $<6.89 E-01$ & $<6.69 E-01$ & $<6.84 E-01$ & $<6.67 E-01$ \\
\hline $\mathrm{Al}$ & $\mathrm{mg} / \mathrm{L}$ & $2.56 \mathrm{E}+01$ & $6.91 \mathrm{E}+01$ & $7.18 \mathrm{E}+01$ & $1.88 \mathrm{E}+00$ & $<1.54 E+00$ \\
\hline $\mathrm{Ba}$ & $\mathrm{mg} / \mathrm{L}$ & $3.29 \mathrm{E}+00$ & $6.11 \mathrm{E}+00$ & $5.35 \mathrm{E}+00$ & $8.12 \mathrm{E}-01$ & $7.46 \mathrm{E}-01$ \\
\hline $\mathrm{Ca}$ & $\mathrm{mg} / \mathrm{L}$ & $4.89 \mathrm{E}+00$ & $1.38 \mathrm{E}+01$ & $1.29 \mathrm{E}+01$ & $4.49 \mathrm{E}+00$ & $1.07 \mathrm{E}+00$ \\
\hline $\mathrm{Cd}$ & $\mathrm{mg} / \mathrm{L}$ & 5.09E-01 & $6.99 \mathrm{E}-01$ & $6.32 \mathrm{E}-01$ & $3.90 \mathrm{E}-01$ & 3.62E-01 \\
\hline $\mathrm{Cr}$ & $\mathrm{mg} / \mathrm{L}$ & $2.85 \mathrm{E}+00$ & $5.26 \mathrm{E}+00$ & $4.70 \mathrm{E}+00$ & $2.14 \mathrm{E}+00$ & $2.97 \mathrm{E}+00$ \\
\hline $\mathrm{Cu}$ & $\mathrm{mg} / \mathrm{L}$ & $1.04 \mathrm{E}+00$ & $1.99 \mathrm{E}+00$ & $2.02 \mathrm{E}+00$ & $<7.22 E-01$ & $<7.04 E-01$ \\
\hline $\mathrm{Fe}$ & $\mathrm{mg} / \mathrm{L}$ & $8.17 \mathrm{E}+02$ & $1.45 \mathrm{E}+03$ & $7.42 \mathrm{E}+02$ & $4.49 \mathrm{E}+00$ & $1.72 \mathrm{E}+00$ \\
\hline Gd & $\mathrm{mg} / \mathrm{L}$ & $<9.91 E-01$ & $<1.03 E+00$ & $<1.00 E+00$ & $<1.03 E+00$ & $<1.00 E+00$ \\
\hline $\mathrm{K}$ & $\mathrm{mg} / \mathrm{L}$ & $<1.38 E+01$ & $<1.44 E+01$ & $<1.39 E+01$ & $<1.43 E+01$ & $<1.39 E+01$ \\
\hline $\mathrm{La}$ & $\mathrm{mg} / \mathrm{L}$ & $1.23 \mathrm{E}+00$ & $2.16 \mathrm{E}+00$ & $7.18 \mathrm{E}-01$ & $<4.09 E-01$ & $<3.98 E-01$ \\
\hline $\mathrm{Li}$ & $\mathrm{mg} / \mathrm{L}$ & $<7.32 E-01$ & $1.28 \mathrm{E}+00$ & 9.94E-01 & 8.98E-01 & $<6.76 E-01$ \\
\hline $\mathrm{Mg}$ & $\mathrm{mg} / \mathrm{L}$ & $9.18 \mathrm{E}-01$ & $1.93 \mathrm{E}+00$ & $1.92 \mathrm{E}+00$ & $1.33 \mathrm{E}+00$ & $1.09 \mathrm{E}-01$ \\
\hline $\mathrm{Mn}$ & $\mathrm{mg} / \mathrm{L}$ & $8.14 \mathrm{E}+01$ & $1.56 \mathrm{E}+02$ & $1.57 \mathrm{E}+02$ & 2.33E-01 & $3.61 \mathrm{E}-01$ \\
\hline $\mathrm{Na}$ & $\mathrm{mg} / \mathrm{L}$ & $2.68 \mathrm{E}+01$ & $4.92 \mathrm{E}+01$ & $4.07 \mathrm{E}+01$ & $3.89 \mathrm{E}+01$ & $3.74 \mathrm{E}+01$ \\
\hline $\mathrm{Ni}$ & $\mathrm{mg} / \mathrm{L}$ & $1.01 \mathrm{E}+01$ & $2.02 \mathrm{E}+01$ & $3.42 \mathrm{E}+01$ & $6.88 \mathrm{E}+00$ & $<7.41 E-01$ \\
\hline $\mathrm{Si}$ & $\mathrm{mg} / \mathrm{L}$ & $1.06 \mathrm{E}+01$ & $4.04 \mathrm{E}+01$ & $3.91 \mathrm{E}+01$ & $7.86 \mathrm{E}+00$ & $<2.40 E+00$ \\
\hline $\mathrm{Sr}$ & $\mathrm{mg} / \mathrm{L}$ & $9.96 \mathrm{E}-01$ & $2.61 \mathrm{E}+00$ & $2.41 \mathrm{E}+00$ & $3.59 \mathrm{E}-01$ & $<2.78 E-02$ \\
\hline $\mathrm{Th}$ & $\mathrm{mg} / \mathrm{L}$ & $<2.51 E+00$ & $4.35 \mathrm{E}+00$ & $<2.54 E+00$ & $<2.60 E+00$ & $<2.54 E+00$ \\
\hline $\mathrm{Ti}$ & $\mathrm{mg} / \mathrm{L}$ & $5.35 \mathrm{E}-01$ & $1.36 \mathrm{E}+00$ & $<1.76 E-01$ & $<1.81 E-01$ & $<1.76 E-01$ \\
\hline $\mathrm{U}$ & $\mathrm{mg} / \mathrm{L}$ & $4.05 \mathrm{E}+01$ & $2.19 \mathrm{E}+02$ & $2.14 \mathrm{E}+02$ & $2.25 \mathrm{E}+01$ & $<1.51 E+01$ \\
\hline $\mathrm{Zn}$ & $\mathrm{mg} / \mathrm{L}$ & $<3.13 E-01$ & $3.49 \mathrm{E}-01$ & $4.50 \mathrm{E}-01$ & $<3.14 E-01$ & $<3.06 E-01$ \\
\hline $\mathrm{Zr}$ & $\mathrm{mg} / \mathrm{L}$ & $4.41 \mathrm{E}+00$ & $1.81 \mathrm{E}+01$ & $2.15 \mathrm{E}+00$ & $<2.66 E-01$ & $<2.59 E-01$ \\
\hline $\mathrm{Hg}$ & $\mathrm{mg} / \mathrm{L}$ & $1.11 \mathrm{E}+00$ & -- & -- & -- & $<2.78 E-01$ \\
\hline $\mathrm{Pb}$ & $\mathrm{mg} / \mathrm{L}$ & $9.38 \mathrm{E}-01$ & $1.90 \mathrm{E}+00$ & $<5.54 E-01$ & $<6.62 E-02$ & $<6.49 E-02$ \\
\hline m-232 (Th) & $\mathrm{mg} / \mathrm{L}$ & $9.17 \mathrm{E}-01$ & $3.00 \mathrm{E}+00$ & $8.06 \mathrm{E}-01$ & $<1.90 E-02$ & $<1.85 E-02$ \\
\hline $\mathrm{m}-235(\mathrm{U})$ & $\mathrm{mg} / \mathrm{L}$ & $2.82 \mathrm{E}-01$ & $1.27 \mathrm{E}+00$ & $1.50 \mathrm{E}+00$ & $1.53 \mathrm{E}-01$ & $<1.85 E-02$ \\
\hline $\mathrm{m}-236(\mathrm{U})$ & $\mathrm{mg} / \mathrm{L}$ & $<1.87 E-02$ & $<1.44 E-01$ & $<1.39 E-01$ & $<1.43 E-02$ & $<1.85 E-02$ \\
\hline m-237 (Np) & $\mathrm{mg} / \mathrm{L}$ & $5.72 \mathrm{E}-02$ & $1.51 \mathrm{E}-01$ & $1.70 \mathrm{E}-01$ & $3.52 \mathrm{E}-02$ & $<1.85 E-02$ \\
\hline m-238 (U,Pu) & $\mathrm{mg} / \mathrm{L}$ & $4.38 \mathrm{E}+01$ & $2.01 \mathrm{E}+02$ & $2.06 \mathrm{E}+02$ & $2.44 \mathrm{E}+01$ & $1.24 \mathrm{E}+00$ \\
\hline $\mathrm{m}-239(\mathrm{Pu})$ & $\mathrm{mg} / \mathrm{L}$ & $1.15 \mathrm{E}-01$ & $4.50 \mathrm{E}-01$ & $2.93 \mathrm{E}-01$ & $5.79 \mathrm{E}-02$ & $<1.85 E-02$ \\
\hline $\mathrm{m}-240(\mathrm{Pu})$ & $\mathrm{mg} / \mathrm{L}$ & $<2.75 E-02$ & $<1.44 E-01$ & $<1.39 E-01$ & $<1.43 E-02$ & $<2.78 E-02$ \\
\hline $\mathrm{Pu}-238$ & $\mathrm{dpm} / \mathrm{mL}$ & $3.11 \mathrm{E}+04$ & $8.61 \mathrm{E}+04$ & $6.23 \mathrm{E}+04$ & $2.01 \mathrm{E}+04$ & $6.43 \mathrm{E}+02$ \\
\hline $\mathrm{Pu}-239 / 240$ & $\mathrm{dpm} / \mathrm{mL}$ & $2.24 \mathrm{E}+04$ & $4.79 \mathrm{E}+04$ & $3.00 \mathrm{E}+04$ & $1.01 \mathrm{E}+04$ & $<3.46 E+02$ \\
\hline $\mathrm{Sr}-90$ & $\mathrm{dpm} / \mathrm{mL}$ & $7.78 \mathrm{E}+07$ & -- & - & -- & $7.65 \mathrm{E}+05$ \\
\hline Cs -137 & $\mathrm{dpm} / \mathrm{mL}$ & $1.78 \mathrm{E}+06$ & -- & -- & -- & $3.05 \mathrm{E}+06$ \\
\hline Am-241 & $\mathrm{dpm} / \mathrm{mL}$ & $2.74 \mathrm{E}+04$ & -- & -- & -- & $9.16 \mathrm{E}+02$ \\
\hline Am-243 & $\mathrm{dpm} / \mathrm{mL}$ & $1.96 \mathrm{E}+02$ & -- & -- & -- & $<3.00 E+01$ \\
\hline Am- $242 \mathrm{~m}$ & $\mathrm{dpm} / \mathrm{mL}$ & $6.37 \mathrm{E}+01$ & -- & -- & -- & $<3.38 E+00$ \\
\hline $\mathrm{Cm}-242$ & $\mathrm{dpm} / \mathrm{mL}$ & $5.27 \mathrm{E}+01$ & -- & -- & -- & $<2.80 E+00$ \\
\hline $\mathrm{Cm}-244$ & $\mathrm{dpm} / \mathrm{mL}$ & $1.58 \mathrm{E}+03$ & -- & -- & -- & $1.04 \mathrm{E}+02$ \\
\hline
\end{tabular}


Table 3-17: Test 1 Dissolution and Decomposition Module Product Slurry Analysis

\begin{tabular}{|c|c|c|c|c|c|c|c|}
\hline \multirow{2}{*}{ analyte } & \multirow{2}{*}{ units } & \multicolumn{3}{|c|}{ dissolution slurry } & \multicolumn{3}{|c|}{ decomposition slurry } \\
\hline & & OA batch 1 & OA batch 2 & OA batch 3 & OA batch 1 & OA batch 2 & OA batch 3 \\
\hline $\mathrm{Ag}$ & $\mathrm{mg} / \mathrm{kg}$ & $<2.46 E+00$ & $<2.23 E+00$ & $<2.49 E+00$ & $<2.37 E+00$ & $<2.31 E+00$ & $<2.39 E+00$ \\
\hline $\mathrm{Al}$ & $\mathrm{mg} / \mathrm{kg}$ & $9.93 \mathrm{E}+02$ & $3.23 \mathrm{E}+02$ & $3.46 \mathrm{E}+02$ & $3.64 \mathrm{E}+02$ & $1.99 \mathrm{E}+02$ & $1.47 \mathrm{E}+02$ \\
\hline $\mathrm{Ba}$ & $\mathrm{mg} / \mathrm{kg}$ & $5.47 \mathrm{E}+00$ & $1.33 \mathrm{E}+00$ & $5.46 \mathrm{E}-01$ & $2.24 \mathrm{E}+00$ & $9.83 \mathrm{E}-01$ & 4.09E-01 \\
\hline $\mathrm{Be}$ & $\mathrm{mg} / \mathrm{kg}$ & $<1.35 E-01$ & $<1.22 E-01$ & $<1.36 E-01$ & $<1.30 E-01$ & $<1.27 E-01$ & $<1.31 E-01$ \\
\hline $\mathrm{Ca}$ & $\mathrm{mg} / \mathrm{kg}$ & $3.55 \mathrm{E}+01$ & $1.08 \mathrm{E}+01$ & $4.98 \mathrm{E}+00$ & $1.66 \mathrm{E}+01$ & $8.37 \mathrm{E}+00$ & $5.18 \mathrm{E}+00$ \\
\hline $\mathrm{Cd}$ & $\mathrm{mg} / \mathrm{kg}$ & 5.89E-01 & $<4.73 E-01$ & $<5.29 E-01$ & $<5.03 E-01$ & $<4.92 E-01$ & $<5.07 E-01$ \\
\hline $\mathrm{Cr}$ & $\mathrm{mg} / \mathrm{kg}$ & $5.82 \mathrm{E}+00$ & $9.15 \mathrm{E}+00$ & $3.98 \mathrm{E}+00$ & $6.52 \mathrm{E}+00$ & $1.09 \mathrm{E}+01$ & $5.85 \mathrm{E}+00$ \\
\hline $\mathrm{Cu}$ & $\mathrm{mg} / \mathrm{kg}$ & $4.93 \mathrm{E}+00$ & $1.72 \mathrm{E}+00$ & $<1.07 E+00$ & $2.48 \mathrm{E}+00$ & $1.01 \mathrm{E}+00$ & $<1.03 E+00$ \\
\hline $\mathrm{Fe}$ & $\mathrm{mg} / \mathrm{kg}$ & $5.80 \mathrm{E}+02$ & $1.87 \mathrm{E}+02$ & $1.05 \mathrm{E}+02$ & $2.69 \mathrm{E}+02$ & $1.32 \mathrm{E}+02$ & $5.96 \mathrm{E}+01$ \\
\hline $\mathrm{Gd}$ & $\mathrm{mg} / \mathrm{kg}$ & $<1.31 E+00$ & $<1.19 E+00$ & $<1.33 E+00$ & $<1.27 E+00$ & $<1.24 E+00$ & $<1.28 E+00$ \\
\hline $\mathrm{K}$ & $\mathrm{mg} / \mathrm{kg}$ & $<5.06 E+01$ & $<4.59 E+01$ & $<5.13 E+01$ & $<4.88 E+01$ & $<4.77 E+01$ & $<4.92 E+01$ \\
\hline $\mathrm{La}$ & $\mathrm{mg} / \mathrm{kg}$ & $1.82 \mathrm{E}+00$ & $<1.31 E+00$ & $<1.47 E+00$ & $<1.39 E+00$ & $<1.36 E+00$ & $<1.41 E+00$ \\
\hline $\mathrm{Li}$ & $\mathrm{mg} / \mathrm{kg}$ & $<2.46 E+00$ & $<2.23 E+00$ & $<2.49 E+00$ & $<2.37 E+00$ & $<2.31 E+00$ & $<2.39 E+00$ \\
\hline $\mathrm{Mg}$ & $\mathrm{mg} / \mathrm{kg}$ & $1.64 \mathrm{E}+01$ & $4.24 \mathrm{E}+00$ & $1.85 \mathrm{E}+00$ & $7.61 \mathrm{E}+00$ & $3.51 \mathrm{E}+00$ & $1.76 \mathrm{E}+00$ \\
\hline $\mathrm{Mn}$ & $\mathrm{mg} / \mathrm{kg}$ & $3.76 \mathrm{E}+02$ & $9.32 \mathrm{E}+01$ & $3.10 \mathrm{E}+01$ & $1.61 \mathrm{E}+02$ & $6.98 \mathrm{E}+01$ & $2.43 \mathrm{E}+01$ \\
\hline $\mathrm{Na}$ & $\mathrm{mg} / \mathrm{kg}$ & $7.13 \mathrm{E}+02$ & $1.69 \mathrm{E}+02$ & $6.16 \mathrm{E}+01$ & $5.15 \mathrm{E}+02$ & $2.09 \mathrm{E}+02$ & $6.28 \mathrm{E}+01$ \\
\hline $\mathrm{Ni}$ & $\mathrm{mg} / \mathrm{kg}$ & $3.34 \mathrm{E}+01$ & $1.16 \mathrm{E}+01$ & $1.05 \mathrm{E}+01$ & $1.64 \mathrm{E}+01$ & $8.69 \mathrm{E}+00$ & $7.52 \mathrm{E}+00$ \\
\hline $\mathrm{Si}$ & $\mathrm{mg} / \mathrm{kg}$ & $4.59 \mathrm{E}+01$ & $1.45 \mathrm{E}+01$ & $<7.49 E+00$ & $2.65 \mathrm{E}+01$ & $1.05 \mathrm{E}+01$ & $<7.18 E+00$ \\
\hline $\mathrm{Sr}$ & $\mathrm{mg} / \mathrm{kg}$ & $2.64 \mathrm{E}+00$ & $6.64 \mathrm{E}-01$ & $2.65 \mathrm{E}-01$ & $1.08 \mathrm{E}+00$ & $5.00 \mathrm{E}-01$ & 2.37E-01 \\
\hline Th & $\mathrm{mg} / \mathrm{kg}$ & $7.33 \mathrm{E}+01$ & $1.65 \mathrm{E}+01$ & $1.92 \mathrm{E}+01$ & $3.29 \mathrm{E}+01$ & $1.35 \mathrm{E}+01$ & $<8.96 E+00$ \\
\hline $\mathrm{Ti}$ & $\mathrm{mg} / \mathrm{kg}$ & $1.56 \mathrm{E}+00$ & $5.18 \mathrm{E}-01$ & 4.44E-01 & $9.08 \mathrm{E}-01$ & $3.49 \mathrm{E}-01$ & $2.78 \mathrm{E}-01$ \\
\hline $\mathrm{U}$ & $\mathrm{mg} / \mathrm{kg}$ & $7.88 \mathrm{E}+01$ & $<4.57 E+01$ & $<5.11 E+01$ & $<4.86 E+01$ & $<4.75 E+01$ & $<4.90 E+01$ \\
\hline $\mathrm{Zn}$ & $\mathrm{mg} / \mathrm{kg}$ & $2.73 \mathrm{E}+00$ & $1.15 \mathrm{E}+00$ & $<1.13 E+00$ & $1.39 \mathrm{E}+00$ & $<1.05 E+00$ & $<1.08 E+00$ \\
\hline $\mathrm{Zr}$ & $\mathrm{mg} / \mathrm{kg}$ & $1.80 \mathrm{E}+01$ & $4.74 \mathrm{E}+00$ & $2.35 \mathrm{E}+00$ & $7.61 \mathrm{E}+00$ & $3.12 \mathrm{E}+00$ & $1.36 \mathrm{E}+00$ \\
\hline $\mathrm{Co}$ & $\mathrm{mg} / \mathrm{kg}$ & $3.44 \mathrm{E}-01$ & $1.32 \mathrm{E}-01$ & $8.80 \mathrm{E}-02$ & $9.90 \mathrm{E}-02$ & $7.20 \mathrm{E}-02$ & $6.30 \mathrm{E}-02$ \\
\hline Th-232 & $\mathrm{mg} / \mathrm{kg}$ & $6.75 \mathrm{E}+01$ & $1.55 \mathrm{E}+01$ & $1.83 \mathrm{E}+01$ & $3.10 \mathrm{E}+01$ & $1.22 \mathrm{E}+01$ & $7.32 \mathrm{E}+00$ \\
\hline U-233 & $\mathrm{mg} / \mathrm{kg}$ & $<2.52 E-01$ & $5.60 \mathrm{E}-02$ & $<5.12 E-02$ & $1.15 \mathrm{E}-01$ & $<4.76 E-02$ & $<4.91 E-02$ \\
\hline U-234 & $\mathrm{mg} / \mathrm{kg}$ & $<1.68 E-01$ & $3.50 \mathrm{E}-02$ & $<3.41 E-02$ & $9.00 \mathrm{E}-02$ & $3.80 \mathrm{E}-02$ & $<3.27 E-02$ \\
\hline $\mathrm{U}-235$ & $\mathrm{mg} / \mathrm{kg}$ & $1.28 \mathrm{E}+00$ & $3.46 \mathrm{E}-01$ & $1.06 \mathrm{E}-01$ & $8.14 \mathrm{E}-01$ & $2.10 \mathrm{E}-01$ & $7.50 \mathrm{E}-02$ \\
\hline U-236 & $\mathrm{mg} / \mathrm{kg}$ & 1.94E-01 & $3.80 \mathrm{E}-02$ & $<3.41 E-02$ & 1.62E-01 & 4.00E-02 & $<3.27 E-02$ \\
\hline $\mathrm{Np}-237$ & $\mathrm{mg} / \mathrm{kg}$ & $<2.52 E-01$ & $<4.57 E-02$ & $<5.12 E-02$ & $<9.73 E-02$ & $<4.76 E-02$ & $<4.91 E-02$ \\
\hline $\mathrm{U}-238$ & $\mathrm{mg} / \mathrm{kg}$ & $7.84 \mathrm{E}+01$ & $1.81 \mathrm{E}+01$ & $4.57 \mathrm{E}+00$ & $4.46 \mathrm{E}+01$ & $1.28 \mathrm{E}+01$ & $4.39 \mathrm{E}+00$ \\
\hline $\mathrm{Pu}-239$ & $\mathrm{mg} / \mathrm{kg}$ & $1.84 \mathrm{E}+00$ & 3.93E-01 & $1.45 \mathrm{E}-01$ & $9.47 \mathrm{E}-01$ & 2.73E-01 & $1.58 \mathrm{E}-01$ \\
\hline $\mathrm{Pu}-240$ & $\mathrm{mg} / \mathrm{kg}$ & $<1.68 E-01$ & $4.20 \mathrm{E}-02$ & $<3.41 E-02$ & $1.35 \mathrm{E}-01$ & $<3.17 E-02$ & $<3.27 E-02$ \\
\hline $\mathrm{Hg}$ & $\mathrm{mg} / \mathrm{kg}$ & $6.50 \mathrm{E}+01$ & $9.12 \mathrm{E}+00$ & $6.72 \mathrm{E}+00$ & $2.04 \mathrm{E}+01$ & $1.76 \mathrm{E}+01$ & $9.46 \mathrm{E}+00$ \\
\hline $\mathrm{Pb}$ & $\mathrm{mg} / \mathrm{kg}$ & n.d. & n.d. & n.d. & n.d. & n.d. & n.d. \\
\hline $\mathrm{Pu}-238$ & $\mathrm{dpm} / \mathrm{g}$ & $7.01 \mathrm{E}+06$ & $1.58 \mathrm{E}+06$ & $5.10 \mathrm{E}+05$ & $3.79 \mathrm{E}+06$ & $1.31 \mathrm{E}+06$ & $4.99 \mathrm{E}+05$ \\
\hline $\mathrm{Pu}-239 / 240$ & $\mathrm{dpm} / \mathrm{g}$ & $3.45 \mathrm{E}+05$ & $8.27 \mathrm{E}+04$ & $2.08 \mathrm{E}+04$ & $1.99 \mathrm{E}+05$ & $6.29 \mathrm{E}+04$ & $2.62 \mathrm{E}+04$ \\
\hline Sr-90 & $\mathrm{dpm} / \mathrm{g}$ & $1.75 \mathrm{E}+08$ & $4.53 \mathrm{E}+07$ & $2.89 \mathrm{E}+07$ & $8.51 \mathrm{E}+07$ & $3.84 \mathrm{E}+07$ & $1.78 \mathrm{E}+07$ \\
\hline
\end{tabular}


Table 3-18: Test 2 Dissolution and Decomposition Module Product Slurry Analysis

\begin{tabular}{|c|c|c|c|c|c|c|c|}
\hline \multirow{2}{*}{ analyte } & \multirow{2}{*}{ units } & \multicolumn{3}{|c|}{ dissolution slurry } & \multicolumn{3}{|c|}{ decomposition slurry } \\
\hline & & OA batch 1 & OA batch 2 & OA batch 3 & OA batch 1 & OA batch 2 & OA batch 3 \\
\hline $\mathrm{Al}$ & $\mathrm{mg} / \mathrm{kg}$ & $3.02 \mathrm{E}+02$ & $7.22 \mathrm{E}+01$ & $3.15 \mathrm{E}+01$ & $1.00 \mathrm{E}+02$ & $6.19 \mathrm{E}+01$ & $2.49 \mathrm{E}+01$ \\
\hline $\mathrm{Ba}$ & $\mathrm{mg} / \mathrm{kg}$ & $1.92 \mathrm{E}+01$ & $7.35 \mathrm{E}+00$ & $2.73 \mathrm{E}+00$ & $6.32 \mathrm{E}+00$ & $4.42 \mathrm{E}+00$ & $1.56 \mathrm{E}+00$ \\
\hline $\mathrm{Be}$ & $\mathrm{mg} / \mathrm{kg}$ & $<1.31 E-01$ & $<1.27 E-01$ & $<1.33 E-01$ & $<1.31 E-01$ & $<1.32 E-01$ & $<1.29 E-01$ \\
\hline $\mathrm{Ca}$ & $\mathrm{mg} / \mathrm{kg}$ & $6.05 \mathrm{E}+01$ & $1.46 \mathrm{E}+01$ & $6.54 \mathrm{E}+00$ & $1.77 \mathrm{E}+01$ & $1.28 \mathrm{E}+01$ & $6.20 \mathrm{E}+00$ \\
\hline $\mathrm{Cd}$ & $\mathrm{mg} / \mathrm{kg}$ & $<1.15 E+00$ & $<1.11 E+00$ & $<1.16 E+00$ & $<1.15 E+00$ & $<1.16 E+00$ & $<1.13 E+00$ \\
\hline $\mathrm{Ce}$ & $\mathrm{mg} / \mathrm{kg}$ & $<1.16 E+01$ & $<1.12 E+01$ & $<1.18 E+01$ & $<1.16 E+01$ & $<1.17 E+01$ & $<1.14 E+01$ \\
\hline Co & $\mathrm{mg} / \mathrm{kg}$ & $<1.59 E+00$ & $<1.54 E+00$ & $<1.61 E+00$ & $<1.59 E+00$ & $<1.60 E+00$ & $<1.56 E+00$ \\
\hline $\mathrm{Cr}$ & $\mathrm{mg} / \mathrm{kg}$ & $1.13 \mathrm{E}+01$ & $3.91 \mathrm{E}+00$ & $2.15 \mathrm{E}+00$ & $9.78 \mathrm{E}+00$ & $5.74 \mathrm{E}+00$ & $3.25 \mathrm{E}+00$ \\
\hline $\mathrm{Cu}$ & $\mathrm{mg} / \mathrm{kg}$ & $<1.04 E+00$ & $<9.98 E-01$ & $<1.05 E+00$ & $<1.03 E+00$ & $<1.04 E+00$ & $<1.01 E+00$ \\
\hline $\mathrm{Fe}$ & $\mathrm{mg} / \mathrm{kg}$ & $5.20 \mathrm{E}+02$ & $1.36 \mathrm{E}+03$ & $8.17 \mathrm{E}+02$ & $2.53 \mathrm{E}+02$ & $5.16 \mathrm{E}+02$ & $3.17 \mathrm{E}+02$ \\
\hline $\mathrm{Gd}$ & $\mathrm{mg} / \mathrm{kg}$ & $5.77 \mathrm{E}+00$ & $<3.41 E+00$ & $<3.58 E+00$ & $3.72 \mathrm{E}+00$ & $<3.56 E+00$ & $<3.46 E+00$ \\
\hline $\mathrm{K}$ & $\mathrm{mg} / \mathrm{kg}$ & $<4.94 E+01$ & $<4.76 E+01$ & $<5.01 E+01$ & $<4.93 E+01$ & $<4.98 E+01$ & $<4.84 E+01$ \\
\hline $\mathrm{La}$ & $\mathrm{mg} / \mathrm{kg}$ & $2.27 \mathrm{E}+00$ & $2.11 \mathrm{E}+00$ & $1.28 \mathrm{E}+00$ & $1.13 \mathrm{E}+00$ & $<8.93 E-01$ & $<8.69 E-01$ \\
\hline $\mathrm{Li}$ & $\mathrm{mg} / \mathrm{kg}$ & $8.23 \mathrm{E}+00$ & $3.10 \mathrm{E}+00$ & $1.93 \mathrm{E}+00$ & $4.86 \mathrm{E}+00$ & $2.95 \mathrm{E}+00$ & $1.64 \mathrm{E}+00$ \\
\hline $\mathrm{Mg}$ & $\mathrm{mg} / \mathrm{kg}$ & $4.97 \mathrm{E}+00$ & $2.33 \mathrm{E}+00$ & $9.98 \mathrm{E}-01$ & $2.68 \mathrm{E}+00$ & $1.78 \mathrm{E}+00$ & $9.18 \mathrm{E}-01$ \\
\hline $\mathrm{Mn}$ & $\mathrm{mg} / \mathrm{kg}$ & $2.07 \mathrm{E}+02$ & $1.73 E+02$ & $9.31 \mathrm{E}+01$ & $2.58 \mathrm{E}+02$ & $1.38 \mathrm{E}+02$ & $5.35 \mathrm{E}+01$ \\
\hline Mo & $\mathrm{mg} / \mathrm{kg}$ & $<4.40 E+00$ & $<4.25 E+00$ & $<4.46 E+00$ & $<4.40 E+00$ & $<4.43 E+00$ & $<4.31 E+00$ \\
\hline $\mathrm{Na}$ & $\mathrm{mg} / \mathrm{kg}$ & $7.88 \mathrm{E}+02$ & $1.01 \mathrm{E}+02$ & $2.64 \mathrm{E}+01$ & $6.30 \mathrm{E}+02$ & $1.57 \mathrm{E}+02$ & $4.36 \mathrm{E}+01$ \\
\hline $\mathrm{Ni}$ & $\mathrm{mg} / \mathrm{kg}$ & $<7.48 E+00$ & $1.84 \mathrm{E}+01$ & $3.04 \mathrm{E}+01$ & $1.57 \mathrm{E}+01$ & $1.53 \mathrm{E}+01$ & $1.46 \mathrm{E}+01$ \\
\hline $\mathrm{P}$ & $\mathrm{mg} / \mathrm{kg}$ & $<2.70 E+01$ & $<2.60 E+01$ & $<2.73 E+01$ & $<2.69 E+01$ & $<2.71 E+01$ & $<2.64 E+01$ \\
\hline $\mathrm{Si}$ & $\mathrm{mg} / \mathrm{kg}$ & $1.49 \mathrm{E}+02$ & $2.94 \mathrm{E}+01$ & $<1.63 E+01$ & $2.84 \mathrm{E}+01$ & $2.82 \mathrm{E}+01$ & $<1.57 E+01$ \\
\hline $\mathrm{Sr}$ & $\mathrm{mg} / \mathrm{kg}$ & $1.19 \mathrm{E}+01$ & $2.45 \mathrm{E}+00$ & $1.02 \mathrm{E}+00$ & $3.18 \mathrm{E}+00$ & $1.98 \mathrm{E}+00$ & 7.41E-01 \\
\hline Th & $\mathrm{mg} / \mathrm{kg}$ & $<9.01 E+00$ & $<8.68 E+00$ & $<9.12 E+00$ & $<8.99 E+00$ & $<9.07 E+00$ & $<8.82 E+00$ \\
\hline $\mathrm{Ti}$ & $\mathrm{mg} / \mathrm{kg}$ & $1.96 \mathrm{E}+00$ & $1.04 \mathrm{E}+00$ & $<6.32 E-01$ & $<6.23 E-01$ & $<6.29 E-01$ & $<6.12 E-01$ \\
\hline $\mathrm{U}$ & $\mathrm{mg} / \mathrm{kg}$ & $1.65 \mathrm{E}+03$ & $2.00 \mathrm{E}+02$ & $<4.99 E+01$ & $8.76 \mathrm{E}+02$ & $2.28 \mathrm{E}+02$ & $6.42 \mathrm{E}+01$ \\
\hline $\mathrm{Zn}$ & $\mathrm{mg} / \mathrm{kg}$ & $<1.08 E+00$ & $<1.05 E+00$ & $<1.10 E+00$ & $<1.08 E+00$ & $<1.09 E+00$ & $<1.06 E+00$ \\
\hline $\mathrm{Zr}$ & $\mathrm{mg} / \mathrm{kg}$ & $6.31 \mathrm{E}+01$ & $1.43 \mathrm{E}+01$ & $4.21 \mathrm{E}+00$ & $9.02 \mathrm{E}-01$ & $5.56 \mathrm{E}+00$ & $2.72 \mathrm{E}+00$ \\
\hline Co & $\mathrm{mg} / \mathrm{kg}$ & $<6.57 E-01$ & $<1.58 E-01$ & $2.06 \mathrm{E}-01$ & $<3.28 E-01$ & $<1.65 E-01$ & $1.34 \mathrm{E}-01$ \\
\hline $\mathrm{Hg}$ & $\mathrm{mg} / \mathrm{kg}$ & $<3.62 E+00$ & $<3.48 E+00$ & $<3.66 E+00$ & $<3.61 E+00$ & $3.75 \mathrm{E}+00$ & $<3.54 E+00$ \\
\hline $\mathrm{Pb}$ & $\mathrm{mg} / \mathrm{kg}$ & $2.92 \mathrm{E}+00$ & $3.12 \mathrm{E}+00$ & $9.59 \mathrm{E}-01$ & $<1.41 E+00$ & $1.08 \mathrm{E}+00$ & 5.04E-01 \\
\hline Th-232 & $\mathrm{mg} / \mathrm{kg}$ & $<6.57 E-01$ & $1.63 \mathrm{E}+00$ & $9.71 \mathrm{E}-01$ & 4.29E-01 & $1.38 \mathrm{E}+00$ & $6.46 \mathrm{E}-01$ \\
\hline U-235 & $\mathrm{mg} / \mathrm{kg}$ & $1.13 \mathrm{E}+01$ & $1.27 \mathrm{E}+00$ & $2.83 \mathrm{E}-01$ & $5.92 \mathrm{E}+00$ & $1.63 \mathrm{E}+00$ & 4.12E-01 \\
\hline U-238 & $\mathrm{mg} / \mathrm{kg}$ & $1.64 \mathrm{E}+03$ & $1.97 \mathrm{E}+02$ & $4.13 \mathrm{E}+01$ & $9.11 \mathrm{E}+02$ & $2.32 \mathrm{E}+02$ & $6.00 \mathrm{E}+01$ \\
\hline $\mathrm{Pu}-239$ & $\mathrm{mg} / \mathrm{kg}$ & $<1.31 E+00$ & 4.39E-01 & $1.18 \mathrm{E}-01$ & $<6.56 E-01$ & $<3.31 E-01$ & $<6.44 E-02$ \\
\hline $\mathrm{Pu}-238$ & $\mathrm{dpm} / \mathrm{g}$ & $2.11 \mathrm{E}+04$ & $5.15 E+04$ & $2.70 \mathrm{E}+04$ & $1.86 \mathrm{E}+04$ & $3.74 \mathrm{E}+04$ & $1.67 \mathrm{E}+04$ \\
\hline $\mathrm{Pu}-239 / 240$ & $\mathrm{dpm} / \mathrm{g}$ & $5.48 \mathrm{E}+04$ & $4.95 \mathrm{E}+04$ & $2.19 \mathrm{E}+04$ & $9.56 \mathrm{E}+03$ & $1.80 \mathrm{E}+04$ & $1.21 \mathrm{E}+04$ \\
\hline
\end{tabular}


SRNL-STI-2011-00360

Revision 0

\subsection{Deposition Tank Chemistry}

For each test, the product slurry from each OA batch was combined in a series of nine storage bottles (eight conditions plus one control) to simulate different potential adjustment scenarios after the dissolution and decomposition processes. Different parameters varied include Deposition Tank storage temperature, whether or not the slurry was evaporated prior to storage, whether or not sodium hydroxide was added to the slurry for $\mathrm{pH}$ adjustment, and whether or not dilute Tank $51 \mathrm{H}$ slurry was stored with the product slurry to simulate a Deposition Tank heel. An additional control was performed for each test by using dilute slurry of the original feed sludge rather than the ECC process effluent. Table 2-2 contains a description of the eight storage conditions plus the control. Table 3-19 and Table 3-20 contain details of the makeup of each storage condition, plus storage bottle supernatant liquid analysis for Test 1 and 2, respectively. Note that the ECC Batch additions listed in the table are slurry additions. The ECC Batch additions for Deposition Tank conditions with evaporation are more concentrated slurries than the additions for the conditions without evaporation.

Of note in Table 3-19 and Table 3-20 are the analytical results showing supernate concentrations of nitrate, oxalate, and carbonate. For Test 1, the oxalate concentrations for the storage conditions without evaporation were less than the detection limit (approximately $<115 \mathrm{mg} / \mathrm{L}$ ) while the oxalate concentrations for the storage conditions with evaporation were higher (500 to $800 \mathrm{mg} / \mathrm{L}$ ) due to the concentration factor. For Test 2, the oxalate concentrations for the storage conditions receiving ECC effluent without evaporation were greater than $100 \mathrm{mg} / \mathrm{L}$ (265 to 300 $\mathrm{mg} / \mathrm{L}$ ). The higher oxalate concentration in Test 2 Deposition Tank storage bottles may be due to any of several factors: 1) there may have been incomplete oxalate decomposition in one or more batches, 2) there may have been holdup in an area of the transfer system with material that did not receive treatment in the Decomposition Module, and 3) there may have been cross contamination with a heel of $2 \mathrm{wt} \% \mathrm{OA}$ in the Auxiliary Vessel used for transfers to the Evaporator Module. Also note for Test 2 Storage Condition 4, the oxalate and TOC measurements are inconsistent, with the likely true oxalate concentration in Storage Condition 4 being similar to that for Storage Conditions 5 and 6 . 
Table 3-19: Deposition Tank (Storage Bottle) Conditions for Test 1

\begin{tabular}{l|c|c|c|c|c|c|c|c|c}
\hline Storage Condition & $\mathbf{1}$ & $\mathbf{2}$ & $\mathbf{3}$ & $\mathbf{4}$ & $\mathbf{5}$ & $\mathbf{6}$ & $\mathbf{7}$ & $\mathbf{8}$ & $\mathbf{9}$ \\
\hline Temperature $\left({ }^{\circ} \mathrm{C}\right)$ & 50 & 50 & 50 & 50 & 50 & 50 & $25^{* *}$ & 70 & 50 \\
\hline Evaporation? & No & No & No & Yes & Yes & Yes & Yes & Yes & No \\
\hline Container Material & PTFE & PTFE & Glass & PMP & PMP & Glass & PMP & PMP & PMP \\
\hline
\end{tabular}

\section{Additions :}

\begin{tabular}{l|c|c|c|c|c|c|c|c|c}
\hline Tank 51H Heel $(\mathrm{mL})$ & 0 & 10 & 0 & 0 & 10 & 0 & 10 & 10 & 10 \\
\hline $50 \mathrm{wt} \% \mathrm{NaOH}(\mathrm{mL})$ & 25 & 25 & 0 & 12.5 & 12.5 & 0 & 12.5 & 12.5 & 12.5 \\
\hline ECC Batch 1 $(\mathrm{g})$ & 144 & 156 & 133 & 24 & 27 & 72 & 28 & 30 & $71^{*}$ \\
\hline ECC Batch 2 $(\mathrm{g})$ & 137 & 134 & 139 & 63 & 64 & 81 & 61 & 72 & $73 *$ \\
\hline ECC Batch 3 $(\mathrm{g})$ & 134 & 132 & 135 & 34 & 63 & 36 & 66 & 64 & 0 \\
\hline
\end{tabular}

\section{Analysis:}

\begin{tabular}{l|c|c|c|c|c|c|c|c|c}
\hline $\mathrm{pH}$ & $>12$ & $>12$ & 8.69 & $>12$ & $>12$ & 9.73 & $>12$ & $>12$ & $>12$ \\
\hline Free Hydroxide (M) & 0.77 & 0.85 & $<0.12$ & 1.15 & 1.26 & $<0.11$ & 1.27 & 1.29 & 1.03 \\
\hline Fluoride (mg/L) & $<113$ & $<116$ & $<116$ & $<106$ & $<109$ & $<112$ & $<110$ & $<102$ & $<112$ \\
\hline Formate (mg/L) & $<113$ & $<116$ & $<116$ & $<106$ & $<109$ & $<112$ & $<110$ & $<102$ & $<112$ \\
\hline Chloride (mg/L) & $<113$ & $<116$ & $<116$ & $<106$ & $<109$ & $<112$ & $<110$ & $<102$ & $<112$ \\
\hline Nitrite (mg/L) & $<113$ & $<116$ & $<116$ & $<106$ & 164 & $<112$ & 154 & 234 & $<212$ \\
\hline Bromide (mg/L) & $<113$ & $<116$ & $<116$ & $<106$ & $<109$ & $<112$ & $<110$ & $<102$ & $<112$ \\
\hline Nitrate (mg/L) & 181 & 186 & 128 & 745 & 810 & 1230 & 837 & 1140 & 212 \\
\hline Phosphate (mg/L) & $<113$ & $<116$ & $<116$ & $<106$ & $<109$ & $<112$ & $<110$ & 122 & $<112$ \\
\hline Sulfate (mg/L) & $<113$ & $<116$ & $<116$ & $<106$ & $<109$ & $<112$ & $<110$ & $<102$ & $<112$ \\
\hline Oxalate (mg/L) & $<113$ & $<116$ & $<116$ & 585 & 525 & 781 & 463 & 764 & $<112$ \\
\hline Carbonate (mg/L) & 136 & 139 & $<116$ & 362 & 307 & 502 & 308 & 438 & $<112$ \\
\hline TOC (mg C/L) & $<113$ & $<116$ & $<116$ & 192 & 197 & 223 & 165 & 275 & 816 \\
\hline
\end{tabular}

* deionized water added to control sample

** ambient temperature, approximately $25^{\circ} \mathrm{C}$ 
Table 3-20: Deposition Tank (Storage Bottle) Conditions for Test 2

\begin{tabular}{l|c|c|c|c|c|c|c|c|c}
\hline Storage Condition & $\mathbf{1}$ & $\mathbf{2}$ & $\mathbf{3}$ & $\mathbf{4}$ & $\mathbf{5}$ & $\mathbf{6}$ & $\mathbf{7}$ & $\mathbf{8}$ & $\mathbf{9}$ \\
\hline Temperature $\left({ }^{\circ} \mathrm{C}\right)$ & 50 & 50 & 50 & 50 & 50 & 50 & $25^{* *}$ & 70 & 50 \\
\hline Evaporation? & No & No & No & Yes & Yes & Yes & No & No & No \\
\hline Container Material & PMP & PMP & Glass & PMP & PMP & Glass & PMP & PMP & PMP \\
\hline
\end{tabular}

Additions:

\begin{tabular}{l|c|c|c|c|c|c|c|c|c}
\hline Tank 51H Heel $(\mathrm{mL})$ & 0 & 10 & 0 & 0 & 10 & 0 & 10 & 10 & 10 \\
\hline $50 \mathrm{wt} \% \mathrm{NaOH}(\mathrm{mL})$ & 25 & 25 & 0 & 12.5 & 12.5 & 0 & 12.5 & 12.5 & 12.5 \\
\hline ECC Batch 1 $(\mathrm{g})$ & 155 & 167 & 151 & 70 & 75 & 163 & 160 & 164 & $66^{*}$ \\
\hline ECC Batch 2 $(\mathrm{g})$ & 155 & 159 & 152 & 36 & 42 & 38 & 152 & 156 & 0 \\
\hline ECC Batch 3 $(\mathrm{g})$ & 134 & 121 & 147 & 63 & 61 & 55 & 121 & 134 & $55^{*}$ \\
\hline
\end{tabular}

\begin{tabular}{l|c|c|c|c|c|c|c|c|c}
\hline \multicolumn{1}{c}{ Analysis: } & $>12$ & $>12$ & 7.52 & $>12$ & $>12$ & 9.15 & $>12$ & $>12$ & $>12$ \\
\hline $\mathrm{pH}$ & 0.59 & 0.63 & $<0.03$ & 0.98 & 1.14 & $<0.04$ & 0.86 & 0.87 & 1.46 \\
\hline Free Hydroxide (M) & $<115$ & $<110$ & $<67$ & $<78$ & 163 & 283 & $<114$ & $<108$ & $<109$ \\
\hline Fluoride (mg/L) & $<115$ & $<110$ & $<67$ & $<78$ & $<109$ & $<71$ & $<114$ & $<108$ & $<109$ \\
\hline Formate (mg/L) & $<115$ & $<110$ & $<67$ & $<78$ & $<109$ & $<71$ & $<114$ & $<108$ & $<109$ \\
\hline Chloride (mg/L) & $<115$ & $<110$ & $<67$ & $<78$ & 283 & 205 & $<114$ & 108 & 305 \\
\hline Nitrite $(\mathrm{mg} / \mathrm{L})$ & $<115$ & $<110$ & $<67$ & $<78$ & $<109$ & $<71$ & $<114$ & $<108$ & $<109$ \\
\hline Bromide (mg/L) & $<115$ & $<110$ & $<67$ & 85 & 479 & 354 & 126 & 108 & 305 \\
\hline Nitrate $(\mathrm{mg} / \mathrm{L})$ & $<115$ & $<110$ & $<67$ & $<78$ & $<109$ & $<71$ & $<114$ & $<108$ & $<109$ \\
\hline Phosphate (mg/L) & $<115$ & $<110$ & $<67$ & $<78$ & $<109$ & $<71$ & $<114$ & $<108$ & $<109$ \\
\hline Sulfate $(\mathrm{mg} / \mathrm{L})$ & 264 & 265 & 300 & 171 & 1882 & 1840 & 252 & 271 & $<109$ \\
\hline Oxalate (mg/L) & 149 & 155 & 73 & 411 & 413 & 510 & 149 & 152 & 131 \\
\hline Carbonate (mg/L) & $<115$ & $<110$ & 87 & 466 & 446 & 453 & $<114$ & $<108$ & $<109$ \\
\hline TOC (mg C/L) & $<109$
\end{tabular}

* deionized water added to control sample

** ambient temperature, approximately $25^{\circ} \mathrm{C}$

Table 3-21 through Table 3-38 contain data and measurements for the soluble components in the storage bottles. Table 3-21 through Table 3-29 are the eight storage bottles plus the control for Test 1 and Table 3-30 through Table 3-38 are for the eight storage bottles plus control for Test 2 . Table 3-39 through Table 3-42 contain summaries of the Deposition Tank storage bottle solubility measurements for four of the key species, uranium-238, plutonium-238, iron and aluminum. The summary tables may prove more useful in comparing results for different storage conditions.

The time scale at which the sampling of the Deposition Tank conditions occurred differed for the two tests. The storage bottles for Test 1 were sampled on a longer time basis in order to span storage times of over one month $(7,12$, and 35 days). The storage bottles for Test 2 were sampled on a shorter time basis with some overlap of the Test 1 time scale (1, 8, and 15 days). 
Note that for Table 3-21 through Table 3-38, the Deposition Tank holding time is calculated from the time of the last process effluent addition (OA Batch 3 ) to the storage bottles. For Test 1, the time between the batch 1 addition and the batch 3 addition was 2 to 3 days. For Test 2, the time between the batch 1 addition and the batch 3 addition was 7 to 9 days.

The information provided by storage bottle analysis can be used to determine how the following factors influence the solubilities of the species of interest in the Deposition Tank:

- storage time

- processing in ECC reactor

- processing in ECC evaporator

- storage temperature

- $\mathrm{pH}$ adjustment

- sludge heel contact

The effect of storage time is indicated by changes in soluble components in each storage bottle sampled at three different time periods (7,21 and 35 days for Test 1 and 1,8 and 15 days for Test 2 ). In general, solubilities of the components of interest did not significantly change as a function of time when compared with the changes in the control storage condition. Uranium in the Test 2 conditions with $\mathrm{pH}$ adjustment is an exception to this, where the concentration tends to decrease with time. The soluble plutonium in Test 2 was overall at low levels, but did show an increase with time. This increase, however, only brought the soluble plutonium in line with the levels noted in the control storage sample. The plutonium solubility remained relatively constant during test 1, although it was at higher levels and storage testing was performed over a longer time period.

The effect of processing in the ECC reactor is indicated by differences in the soluble components between Storage Condition 2 and Storage Condition 9. The concentrations of soluble uranium in the baseline adjusted ECC effluent (Storage Condition 2) was higher than that in the control (Storage Condition 9) for Tests 1 and 2. Similarly, the concentration of soluble $\mathrm{Pu}$ in the baseline adjusted ECC effluent is higher than the control for Test 1, but is not distinguishably different for Test 2. A similar trend is noted for uranium in Tests 1 and 2 and plutonium in Test 1 when comparing the solubilities at other storage conditions with the control, with the control condition having lower soluble concentrations of these components. Clear trends cannot be noted for iron and aluminum differences between the baseline ECC effluent and the control.

The effect of processing ECC reactor effluent through the ECC evaporator is indicated by differences in the soluble components between Storage Conditions 1 and 4, between Storage Conditions 2 and 5, and between Storage Conditions 3 and 6. The soluble concentrations of many components are higher for the storage conditions that received evaporation than for the storage conditions that did not receive evaporation. This phenomenon is not likely unique to ECC, as soluble concentrations of uranium in Tank Farm evaporator systems have been seen to be high compared to unconcentrated feeds. This can be due to chemistry changes of the solution as the evaporator concentrates ionic components, possible subsaturation of components in the evaporator feed, or possible supersaturation of components in the evaporator product. For example, soluble aluminum concentrations are 4 to 6 times higher in the evaporated and $\mathrm{pH}$ adjusted cases than for the only $\mathrm{pH}$ adjusted cases. For the similar storage conditions with added heel, the aluminum concentration is 2 to 3 times higher for the evaporated than for the nonevaporated. One extreme case is that of soluble uranium concentration in the unadjusted cases, where the evaporated concentrations were on average 28-times higher than the non-evaporated concentration. For the $\mathrm{pH}$ adjusted cases with and without sludge heel, the soluble uranium 
concentrations for the evaporated cases averaged less than 2-times higher than for the nonevaporated cases.

The effect of storage temperature on ECC product is indicated by comparing the soluble components for Storage Conditions 7 and 8 with Storage Condition 5 (for Test 1) and Storage Condition 2 (for Test 2). Overall the effect of storage temperature was minor. For iron and aluminum, higher temperatures led to slightly higher soluble concentrations in most cases. Uranium showed a slight trend in the opposite direction, with higher storage temperatures leading to slightly lower soluble concentrations. The trend for soluble plutonium concentration with storage temperature was not clear. There is no evidence that a phenomenon specific to the ECC process is influencing the behavior of these components with respect to storage temperature.

The effect of $\mathrm{pH}$ adjustment on ECC product is indicated by differences in the soluble components between Storage Conditions 1 and 3 and between Storage Conditions 4 and 6 . Adjustment of the $\mathrm{pH}$ tended to increase the soluble concentrations of uranium and plutonium for cases where evaporation was not performed and tended to decrease the concentrations of uranium and plutonium for cases where evaporation was performed. The $\mathrm{pH}$ adjustment increased the soluble concentrations of iron and aluminum regardless of whether evaporation was used. In general, the cases with $\mathrm{pH}$ adjustment tended to bring the soluble uranium and plutonium concentrations to consistent levels when compared to the unadjusted cases.

The effect of the contact of $\mathrm{pH}$ adjusted ECC product with a sludge heel is indicated by differences in the soluble components between Storage Conditions 1 and 2 and between Storage Conditions 4 and 5. Although differences were seen between soluble concentrations of some individual components depending on contact with the sludge heel, no universal trends were noted as a function of contact with the sludge heel.

Table 3-43 and Table 3-44 contain the analyses of the wet solids in the Deposition Tanks for Tests 1 and 2, respectively. These tables show the components of the sludge transferred forward from the ECC process with and without mixing with a sludge heel. Some variation is caused by the incomplete separation of Deposition Tank supernate from the settled sludge. 
Table 3-21: Soluble Components in the Deposition Tank for the Tank 12H ECC No-Light Test, Storage Condition 1: Without Evaporation, Without Sludge Heel, With pH Adjustment, $\mathrm{T}=50^{\circ} \mathrm{C}$.

\begin{tabular}{|c|c|c|c|c|}
\hline \multirow{2}{*}{ analyte } & \multirow{2}{*}{ units } & \multicolumn{3}{|c|}{ deposition tank holding time } \\
\hline & & 7 days & 21 days & 35 days \\
\hline $\mathrm{pH}$ & -- & -- & $>12$ & $>12$ \\
\hline ORP & $\mathrm{mV}$ & -- & 255 & 87 \\
\hline $\mathrm{Al}$ & $\mathrm{mg} / \mathrm{L}$ & $2.16 \mathrm{E}+02$ & $2.99 \mathrm{E}+02$ & $3.33 \mathrm{E}+02$ \\
\hline $\mathrm{Ba}$ & $\mathrm{mg} / \mathrm{L}$ & $<5.44 E-02$ & 8.77E-01 & $8.13 \mathrm{E}-01$ \\
\hline $\mathrm{Ca}$ & $\mathrm{mg} / \mathrm{L}$ & $7.16 \mathrm{E}-01$ & $6.75 \mathrm{E}-01$ & $1.13 \mathrm{E}+00$ \\
\hline $\mathrm{Cd}$ & $\mathrm{mg} / \mathrm{L}$ & $<2.81 E-01$ & 4.45E-01 & $3.80 \mathrm{E}-01$ \\
\hline $\mathrm{Cr}$ & $\mathrm{mg} / \mathrm{L}$ & $6.91 \mathrm{E}+00$ & $7.01 \mathrm{E}+00$ & $6.98 \mathrm{E}+00$ \\
\hline $\mathrm{Cu}$ & $\mathrm{mg} / \mathrm{L}$ & $<5.71 E-01$ & $<3.00 E-01$ & $<3.04 E-01$ \\
\hline $\mathrm{Fe}$ & $\mathrm{mg} / \mathrm{L}$ & $1.08 \mathrm{E}+00$ & $1.42 \mathrm{E}+00$ & $1.50 \mathrm{E}+00$ \\
\hline $\mathrm{K}$ & $\mathrm{mg} / \mathrm{L}$ & $8.37 \mathrm{E}+01$ & $<1.20 E+01$ & $<1.43 E+01$ \\
\hline $\mathrm{Mg}$ & $\mathrm{mg} / \mathrm{L}$ & $<1.36 E-01$ & $<7.50 E-02$ & 8.08E-02 \\
\hline Mo & $\mathrm{mg} / \mathrm{L}$ & $<2.80 E+00$ & $<1.26 E+00$ & $<1.27 E+00$ \\
\hline $\mathrm{Na}$ & $\mathrm{mg} / \mathrm{L}$ & $2.55 \mathrm{E}+04$ & -- & $2.69 \mathrm{E}+04$ \\
\hline $\mathrm{Ni}$ & $\mathrm{mg} / \mathrm{L}$ & $<1.45 E+00$ & $<7.50 E-01$ & $<7.61 E-01$ \\
\hline $\mathrm{P}$ & $\mathrm{mg} / \mathrm{L}$ & $<7.43 E+00$ & $<7.69 E+00$ & $<7.80 E+00$ \\
\hline $\mathrm{S}$ & $\mathrm{mg} / \mathrm{L}$ & $<3.40 E+01$ & $<3.52 E+01$ & $4.11 \mathrm{E}+01$ \\
\hline $\mathrm{Si}$ & $\mathrm{mg} / \mathrm{L}$ & $1.28 \mathrm{E}+01$ & $1.42 \mathrm{E}+01$ & $1.27 \mathrm{E}+01$ \\
\hline $\mathrm{U}$ & $\mathrm{mg} / \mathrm{L}$ & $<3.66 E+01$ & $3.90 \mathrm{E}+01$ & $2.67 \mathrm{E}+01$ \\
\hline $\mathrm{Zn}$ & $\mathrm{mg} / \mathrm{L}$ & 7.30E-01 & $5.72 \mathrm{E}-01$ & $5.28 \mathrm{E}-01$ \\
\hline $\mathrm{Zr}$ & $\mathrm{mg} / \mathrm{L}$ & $2.27 \mathrm{E}-01$ & $<1.22 E-01$ & $<1.50 E+01$ \\
\hline $\mathrm{Hg}$ & $\mathrm{mg} / \mathrm{L}$ & -- & $1.25 \mathrm{E}+01$ & -- \\
\hline m-232 (Th) & $\mathrm{mg} / \mathrm{L}$ & $<4.53 E-02$ & $<3.75 E-02$ & $<7.61 E-02$ \\
\hline $\mathrm{m}-233(\mathrm{U})$ & $\mathrm{mg} / \mathrm{L}$ & $<4.53 E-02$ & 4.40E-02 & $6.21 \mathrm{E}-02$ \\
\hline $\mathrm{m}-234(\mathrm{U})$ & $\mathrm{mg} / \mathrm{L}$ & $5.60 \mathrm{E}-02$ & $<3.75 E-02$ & $<5.71 E-02$ \\
\hline $\mathrm{m}-235(\mathrm{U})$ & $\mathrm{mg} / \mathrm{L}$ & 4.49E-01 & $5.08 \mathrm{E}-01$ & $5.34 \mathrm{E}-01$ \\
\hline $\mathrm{m}-236(\mathrm{U})$ & $\mathrm{mg} / \mathrm{L}$ & $6.15 \mathrm{E}-02$ & 7.14E-02 & $<7.61 E-02$ \\
\hline m-237 (Np) & $\mathrm{mg} / \mathrm{L}$ & $<6.80 E-02$ & $3.98 \mathrm{E}-02$ & $<1.33 E-01$ \\
\hline m-238 (U,Pu) & $\mathrm{mg} / \mathrm{L}$ & $2.59 \mathrm{E}+01$ & $2.53 \mathrm{E}+01$ & $2.56 \mathrm{E}+01$ \\
\hline $\mathrm{m}-239(\mathrm{Pu})$ & $\mathrm{mg} / \mathrm{L}$ & $<9.06 E-02$ & $8.09 \mathrm{E}-02$ & $1.06 \mathrm{E}-01$ \\
\hline $\mathrm{Pu}-238$ & $\mathrm{dpm} / \mathrm{mL}$ & $2.24 \mathrm{E}+05$ & $2.33 \mathrm{E}+05$ & $2.78 \mathrm{E}+05$ \\
\hline $\mathrm{Pu}-239 / 240$ & $\mathrm{dpm} / \mathrm{mL}$ & $1.23 \mathrm{E}+04$ & $1.40 \mathrm{E}+04$ & $1.45 \mathrm{E}+04$ \\
\hline $\mathrm{Sr}-90$ & $\mathrm{dpm} / \mathrm{mL}$ & -- & $6.13 E+04$ & -- \\
\hline Cs-137 & $\mathrm{dpm} / \mathrm{mL}$ & -- & $1.24 \mathrm{E}+06$ & -- \\
\hline Am-241 & $\mathrm{dpm} / \mathrm{mL}$ & -- & $<1.05 E+02$ & -- \\
\hline $\mathrm{Cm}-244$ & $\mathrm{dpm} / \mathrm{mL}$ & -- & $<2.87 E+00$ & -- \\
\hline
\end{tabular}


Table 3-22: Soluble Components in the Deposition Tank for the Tank 12H ECC No-Light Test, Storage Condition 2: Without Evaporation, With Sludge Heel, With pH Adjustment, $\mathbf{T}=50^{\circ} \mathbf{C}$.

\begin{tabular}{|c|c|c|c|c|}
\hline \multirow{2}{*}{ analyte } & \multirow{2}{*}{ units } & \multicolumn{3}{|c|}{ deposition tank holding time } \\
\hline & & 7 days & 21 days & 35 days \\
\hline $\mathrm{pH}$ & -- & -- & $>12$ & $>12$ \\
\hline ORP & $\mathrm{mV}$ & -- & 47.9 & 98 \\
\hline $\mathrm{Al}$ & $\mathrm{mg} / \mathrm{L}$ & $4.67 \mathrm{E}+02$ & $5.62 \mathrm{E}+02$ & $7.05 \mathrm{E}+02$ \\
\hline $\mathrm{Ba}$ & $\mathrm{mg} / \mathrm{L}$ & $<5.65 E-02$ & $7.56 \mathrm{E}-01$ & 7.87E-01 \\
\hline $\mathrm{Ca}$ & $\mathrm{mg} / \mathrm{L}$ & $1.17 \mathrm{E}+00$ & $5.38 \mathrm{E}-01$ & $6.35 \mathrm{E}-01$ \\
\hline $\mathrm{Cd}$ & $\mathrm{mg} / \mathrm{L}$ & $<2.92 E-01$ & 3.65E-01 & $4.46 \mathrm{E}-01$ \\
\hline $\mathrm{Cr}$ & $\mathrm{mg} / \mathrm{L}$ & $6.94 \mathrm{E}+00$ & $6.38 \mathrm{E}+00$ & $7.11 \mathrm{E}+00$ \\
\hline $\mathrm{Cu}$ & $\mathrm{mg} / \mathrm{L}$ & $<5.93 E-01$ & $<3.00 E-01$ & 3.73E-01 \\
\hline $\mathrm{Fe}$ & $\mathrm{mg} / \mathrm{L}$ & $3.82 \mathrm{E}+00$ & $1.56 \mathrm{E}+00$ & $4.62 \mathrm{E}+00$ \\
\hline $\mathrm{K}$ & $\mathrm{mg} / \mathrm{L}$ & $8.05 \mathrm{E}+01$ & $<1.20 E+01$ & $<1.38 E+01$ \\
\hline $\mathrm{Mg}$ & $\mathrm{mg} / \mathrm{L}$ & $<1.41 E-01$ & $<7.49 E-02$ & $<5.52 E-02$ \\
\hline Mo & $\mathrm{mg} / \mathrm{L}$ & $<2.91 E+00$ & $<1.25 E+00$ & $1.33 \mathrm{E}+00$ \\
\hline $\mathrm{Na}$ & $\mathrm{mg} / \mathrm{L}$ & $2.64 \mathrm{E}+04$ & -- & $2.55 \mathrm{E}+04$ \\
\hline $\mathrm{Ni}$ & $\mathrm{mg} / \mathrm{L}$ & $<1.51 E+00$ & $<7.49 E-01$ & $<7.36 E-01$ \\
\hline $\mathrm{P}$ & $\mathrm{mg} / \mathrm{L}$ & $9.05 \mathrm{E}+00$ & $8.86 \mathrm{E}+00$ & $9.01 \mathrm{E}+00$ \\
\hline $\mathrm{S}$ & $\mathrm{mg} / \mathrm{L}$ & $<3.53 E+01$ & $<3.51 E+01$ & $<3.45 E+01$ \\
\hline $\mathrm{Si}$ & $\mathrm{mg} / \mathrm{L}$ & $2.51 \mathrm{E}+01$ & $2.45 \mathrm{E}+01$ & $2.57 \mathrm{E}+01$ \\
\hline $\mathrm{U}$ & $\mathrm{mg} / \mathrm{L}$ & $3.81 \mathrm{E}+01$ & $4.79 \mathrm{E}+01$ & $4.09 \mathrm{E}+01$ \\
\hline $\mathrm{Zn}$ & $\mathrm{mg} / \mathrm{L}$ & $9.98 \mathrm{E}-01$ & $7.35 \mathrm{E}-01$ & $8.88 \mathrm{E}-01$ \\
\hline $\mathrm{Zr}$ & $\mathrm{mg} / \mathrm{L}$ & $1.46 \mathrm{E}-01$ & $2.29 \mathrm{E}-01$ & $<1.45 E+01$ \\
\hline $\mathrm{Hg}$ & $\mathrm{mg} / \mathrm{L}$ & -- & $2.79 \mathrm{E}+01$ & -- \\
\hline m-232 (Th) & $\mathrm{mg} / \mathrm{L}$ & $<4.71 E-02$ & $<3.74 E-02$ & $<7.36 E-02$ \\
\hline $\mathrm{m}-233(\mathrm{U})$ & $\mathrm{mg} / \mathrm{L}$ & $5.81 \mathrm{E}-02$ & 5.13E-02 & $5.69 \mathrm{E}-02$ \\
\hline $\mathrm{m}-234(\mathrm{U})$ & $\mathrm{mg} / \mathrm{L}$ & $7.38 \mathrm{E}-02$ & 7.72E-02 & $7.21 \mathrm{E}-02$ \\
\hline $\mathrm{m}-235(\mathrm{U})$ & $\mathrm{mg} / \mathrm{L}$ & $6.68 \mathrm{E}-01$ & $8.25 \mathrm{E}-01$ & 7.49E-01 \\
\hline m-236 (U) & $\mathrm{mg} / \mathrm{L}$ & $1.18 \mathrm{E}-01$ & $1.17 \mathrm{E}-01$ & 9.11E-02 \\
\hline $\mathrm{m}-237(\mathrm{~Np})$ & $\mathrm{mg} / \mathrm{L}$ & $<7.06 E-02$ & $5.27 \mathrm{E}-02$ & $<1.29 E-01$ \\
\hline m-238 (U,Pu) & $\mathrm{mg} / \mathrm{L}$ & $3.95 \mathrm{E}+01$ & $3.88 \mathrm{E}+01$ & $3.85 \mathrm{E}+01$ \\
\hline $\mathrm{m}-239(\mathrm{Pu})$ & $\mathrm{mg} / \mathrm{L}$ & $1.64 \mathrm{E}-01$ & $1.61 \mathrm{E}-01$ & 1.44E-01 \\
\hline $\mathrm{Pu}-238$ & $\mathrm{dpm} / \mathrm{mL}$ & $4.62 \mathrm{E}+05$ & $4.97 \mathrm{E}+05$ & $5.34 \mathrm{E}+05$ \\
\hline $\mathrm{Pu}-239 / 240$ & $\mathrm{dpm} / \mathrm{mL}$ & $2.27 \mathrm{E}+04$ & $2.36 \mathrm{E}+04$ & $2.78 \mathrm{E}+04$ \\
\hline Sr-90 & $\mathrm{dpm} / \mathrm{mL}$ & -- & $4.60 \mathrm{E}+04$ & -- \\
\hline Cs-137 & $\mathrm{dpm} / \mathrm{mL}$ & -- & $1.69 \mathrm{E}+06$ & -- \\
\hline Am-241 & $\mathrm{dpm} / \mathrm{mL}$ & -- & $<2.60 E+02$ & -- \\
\hline $\mathrm{Cm}-244$ & $\mathrm{dpm} / \mathrm{mL}$ & -- & $1.08 \mathrm{E}+02$ & -- \\
\hline
\end{tabular}


Table 3-23: Soluble Components in the Deposition Tank for the Tank 12H ECC No-Light Test, Storage Condition 3: Without Evaporation, Without Sludge Heel, Without pH Adjustment, $\mathrm{T}=50^{\circ} \mathrm{C}$.

\begin{tabular}{|c|c|c|c|c|}
\hline \multirow{2}{*}{ analyte } & \multirow{2}{*}{ units } & \multicolumn{3}{|c|}{ deposition tank holding time } \\
\hline & & 7 days & 21 days & 35 days \\
\hline $\mathrm{pH}$ & -- & -- & 8.67 & 8.71 \\
\hline ORP & $\mathrm{mV}$ & -- & 184 & 247 \\
\hline $\mathrm{Al}$ & $\mathrm{mg} / \mathrm{L}$ & $<3.11 E+00$ & $<1.58 E+00$ & $1.08 \mathrm{E}+00$ \\
\hline $\mathrm{Ba}$ & $\mathrm{mg} / \mathrm{L}$ & $<5.63 E-02$ & 7.98E-01 & 5.85E-02 \\
\hline $\mathrm{Ca}$ & $\mathrm{mg} / \mathrm{L}$ & $2.61 \mathrm{E}+00$ & $1.82 \mathrm{E}+00$ & $2.03 \mathrm{E}+00$ \\
\hline $\mathrm{Cd}$ & $\mathrm{mg} / \mathrm{L}$ & $<2.91 E-01$ & 3.88E-01 & $<6.05 E-02$ \\
\hline $\mathrm{Cr}$ & $\mathrm{mg} / \mathrm{L}$ & $7.39 \mathrm{E}+00$ & $7.21 \mathrm{E}+00$ & $7.86 \mathrm{E}+00$ \\
\hline $\mathrm{Cu}$ & $\mathrm{mg} / \mathrm{L}$ & $<5.91 E-01$ & $<3.04 E-01$ & $<1.31 E-01$ \\
\hline $\mathrm{Fe}$ & $\mathrm{mg} / \mathrm{L}$ & $<9.00 E-01$ & 8.65E-01 & 2.72E-01 \\
\hline K & $\mathrm{mg} / \mathrm{L}$ & $7.34 \mathrm{E}+01$ & $<1.22 E+01$ & $8.23 \mathrm{E}+00$ \\
\hline $\mathrm{Mg}$ & $\mathrm{mg} / \mathrm{L}$ & 4.41E-01 & 3.75E-01 & 3.85E-01 \\
\hline Mo & $\mathrm{mg} / \mathrm{L}$ & $<2.90 E+00$ & $<1.27 E+00$ & $<5.45 E-01$ \\
\hline $\mathrm{Na}$ & $\mathrm{mg} / \mathrm{L}$ & $2.60 \mathrm{E}+02$ & -- & $<7.45 E+02$ \\
\hline $\mathrm{Ni}$ & $\mathrm{mg} / \mathrm{L}$ & $<1.50 E+00$ & $<7.60 E-01$ & $<3.23 E-01$ \\
\hline $\mathrm{P}$ & $\mathrm{mg} / \mathrm{L}$ & $<7.69 E+00$ & $<7.79 E+00$ & $<3.31 E+00$ \\
\hline $\mathrm{S}$ & $\mathrm{mg} / \mathrm{L}$ & $<3.52 E+01$ & $<3.56 E+01$ & $1.72 \mathrm{E}+01$ \\
\hline $\mathrm{Si}$ & $\mathrm{mg} / \mathrm{L}$ & $<4.59 E+00$ & $<2.09 E+00$ & $1.41 \mathrm{E}+00$ \\
\hline $\mathrm{U}$ & $\mathrm{mg} / \mathrm{L}$ & $<3.79 E+01$ & $<1.55 E+01$ & $6.64 \mathrm{E}+00$ \\
\hline $\mathrm{Zn}$ & $\mathrm{mg} / \mathrm{L}$ & $<4.31 E-01$ & $<3.14 E-01$ & $<1.82 E-01$ \\
\hline $\mathrm{Zr}$ & $\mathrm{mg} / \mathrm{L}$ & $<1.22 E-01$ & $<1.24 E-01$ & $<6.40 E+00$ \\
\hline $\mathrm{Hg}$ & $\mathrm{mg} / \mathrm{L}$ & -- & $2.47 \mathrm{E}+00$ & -- \\
\hline $\mathrm{m}-232(\mathrm{Th})$ & $\mathrm{mg} / \mathrm{L}$ & $<4.69 E-02$ & $<3.80 E-02$ & $<8.07 E-02$ \\
\hline $\mathrm{m}-233(\mathrm{U})$ & $\mathrm{mg} / \mathrm{L}$ & $<4.69 E-02$ & $<3.80 E-02$ & $<4.04 E-02$ \\
\hline $\mathrm{m}-234(\mathrm{U})$ & $\mathrm{mg} / \mathrm{L}$ & $<4.69 E-02$ & $<3.80 E-02$ & $<6.05 E-02$ \\
\hline $\mathrm{m}-235(\mathrm{U})$ & $\mathrm{mg} / \mathrm{L}$ & $1.33 \mathrm{E}-01$ & $<1.33 E-01$ & $1.31 \mathrm{E}-01$ \\
\hline $\mathrm{m}-236(\mathrm{U})$ & $\mathrm{mg} / \mathrm{L}$ & $<4.69 E-02$ & $<5.70 E-02$ & $<8.07 E-02$ \\
\hline $\mathrm{m}-237(\mathrm{~Np})$ & $\mathrm{mg} / \mathrm{L}$ & $<7.03 E-02$ & $<3.80 E-02$ & $<1.41 E-01$ \\
\hline $\mathrm{m}-238(\mathrm{U}, \mathrm{Pu})$ & $\mathrm{mg} / \mathrm{L}$ & $5.19 \mathrm{E}+00$ & $5.32 \mathrm{E}+00$ & $5.31 \mathrm{E}+00$ \\
\hline $\mathrm{m}-239(\mathrm{Pu})$ & $\mathrm{mg} / \mathrm{L}$ & $<9.38 E-02$ & $<3.80 E-02$ & $<6.05 E-02$ \\
\hline $\mathrm{Pu}-238$ & $\mathrm{dpm} / \mathrm{mL}$ & $1.87 \mathrm{E}+05$ & $4.05 \mathrm{E}+04$ & $1.93 \mathrm{E}+04$ \\
\hline $\mathrm{Pu}-239 / 240$ & $\mathrm{dpm} / \mathrm{mL}$ & $8.41 \mathrm{E}+03$ & $<2.23 E+03$ & $9.38 \mathrm{E}+02$ \\
\hline Sr-90 & $\mathrm{dpm} / \mathrm{mL}$ & -- & $1.05 \mathrm{E}+06$ & -- \\
\hline Cs -137 & $\mathrm{dpm} / \mathrm{mL}$ & -- & $1.33 \mathrm{E}+06$ & -- \\
\hline Am-241 & $\mathrm{dpm} / \mathrm{mL}$ & -- & $<6.78 E+02$ & -- \\
\hline $\mathrm{Cm}-244$ & $\mathrm{dpm} / \mathrm{mL}$ & -- & $2.42 \mathrm{E}+01$ & -- \\
\hline
\end{tabular}


Table 3-24: Soluble Components in the Deposition Tank for the Tank 12H ECC No-Light Test, Storage Condition 4: With Evaporation, Without Sludge Heel, With pH Adjustment, $\mathbf{T}=50^{\circ} \mathrm{C}$.

\begin{tabular}{|c|c|c|c|c|}
\hline \multirow{2}{*}{ analyte } & \multirow{2}{*}{ units } & \multicolumn{3}{|c|}{ deposition tank holding time } \\
\hline & & 7 days & 21 days & 35 days \\
\hline $\mathrm{pH}$ & -- & -- & $>12$ & $>12$ \\
\hline ORP & $\mathrm{mV}$ & -- & 55 & 66.1 \\
\hline Al & $\mathrm{mg} / \mathrm{L}$ & $1.25 \mathrm{E}+03$ & $1.46 \mathrm{E}+03$ & $1.58 \mathrm{E}+03$ \\
\hline $\mathrm{Ba}$ & $\mathrm{mg} / \mathrm{L}$ & $<5.55 E-02$ & $8.56 \mathrm{E}-01$ & 5.77E-02 \\
\hline $\mathrm{Ca}$ & $\mathrm{mg} / \mathrm{L}$ & $1.43 \mathrm{E}+00$ & $6.56 \mathrm{E}-01$ & $7.45 \mathrm{E}-01$ \\
\hline $\mathrm{Cd}$ & $\mathrm{mg} / \mathrm{L}$ & $<2.87 E-01$ & 4.70E-01 & $<1.42 E-01$ \\
\hline $\mathrm{Cr}$ & $\mathrm{mg} / \mathrm{L}$ & $4.94 \mathrm{E}+01$ & $4.95 \mathrm{E}+01$ & $5.00 \mathrm{E}+01$ \\
\hline $\mathrm{Cu}$ & $\mathrm{mg} / \mathrm{L}$ & $<5.83 E-01$ & $8.51 \mathrm{E}-01$ & $8.16 \mathrm{E}-01$ \\
\hline $\mathrm{Fe}$ & $\mathrm{mg} / \mathrm{L}$ & $4.06 \mathrm{E}+00$ & $2.99 \mathrm{E}+00$ & $2.16 \mathrm{E}+00$ \\
\hline $\mathrm{K}$ & $\mathrm{mg} / \mathrm{L}$ & $9.04 \mathrm{E}+01$ & $<1.19 E+01$ & $4.35 \mathrm{E}+01$ \\
\hline $\mathrm{Mg}$ & $\mathrm{mg} / \mathrm{L}$ & $1.57 \mathrm{E}-01$ & $<7.44 E-02$ & 7.98E-02 \\
\hline Mo & $\mathrm{mg} / \mathrm{L}$ & $5.53 \mathrm{E}+00$ & $5.45 \mathrm{E}+00$ & $5.23 \mathrm{E}+00$ \\
\hline $\mathrm{Na}$ & $\mathrm{mg} / \mathrm{L}$ & $4.12 \mathrm{E}+04$ & -- & $4.22 \mathrm{E}+04$ \\
\hline $\mathrm{Ni}$ & $\mathrm{mg} / \mathrm{L}$ & $<1.48 E+00$ & $<7.44 E-01$ & $<7.10 E-01$ \\
\hline $\mathrm{P}$ & $\mathrm{mg} / \mathrm{L}$ & $1.44 \mathrm{E}+01$ & $1.53 \mathrm{E}+01$ & $1.22 \mathrm{E}+01$ \\
\hline $\mathrm{S}$ & $\mathrm{mg} / \mathrm{L}$ & $4.08 \mathrm{E}+01$ & $<3.49 E+01$ & $<3.33 E+01$ \\
\hline $\mathrm{Si}$ & $\mathrm{mg} / \mathrm{L}$ & $4.58 \mathrm{E}+01$ & $4.83 \mathrm{E}+01$ & $4.74 \mathrm{E}+01$ \\
\hline $\mathrm{U}$ & $\mathrm{mg} / \mathrm{L}$ & $7.68 \mathrm{E}+01$ & $8.57 \mathrm{E}+01$ & $6.54 \mathrm{E}+01$ \\
\hline $\mathrm{Zn}$ & $\mathrm{mg} / \mathrm{L}$ & $2.69 \mathrm{E}+00$ & $2.56 \mathrm{E}+00$ & $2.19 \mathrm{E}+00$ \\
\hline $\mathrm{Zr}$ & $\mathrm{mg} / \mathrm{L}$ & 2.64E-01 & $2.05 \mathrm{E}-01$ & $<1.40 E+01$ \\
\hline $\mathrm{Hg}$ & $\mathrm{mg} / \mathrm{L}$ & -- & $2.75 \mathrm{E}+01$ & -- \\
\hline $\mathrm{m}-232(\mathrm{Th})$ & $\mathrm{mg} / \mathrm{L}$ & $<4.62 E-02$ & $<3.72 E-02$ & $<7.10 E-02$ \\
\hline m-233 (U) & $\mathrm{mg} / \mathrm{L}$ & $9.53 \mathrm{E}-02$ & $8.95 \mathrm{E}-02$ & 9.49E-02 \\
\hline m-234 (U) & $\mathrm{mg} / \mathrm{L}$ & $1.27 \mathrm{E}-01$ & $1.14 \mathrm{E}-01$ & $1.09 \mathrm{E}-01$ \\
\hline m-235 (U) & $\mathrm{mg} / \mathrm{L}$ & $1.38 \mathrm{E}+00$ & $1.38 \mathrm{E}+00$ & $1.09 \mathrm{E}+00$ \\
\hline m-236(U) & $\mathrm{mg} / \mathrm{L}$ & $1.79 \mathrm{E}-01$ & $1.25 \mathrm{E}-01$ & $1.22 \mathrm{E}-01$ \\
\hline m-237 (Np) & $\mathrm{mg} / \mathrm{L}$ & $<6.94 E-02$ & $<3.72 E-02$ & $<1.24 E-01$ \\
\hline m-238 (U,Pu) & $\mathrm{mg} / \mathrm{L}$ & 7.77E+01 & $6.99 \mathrm{E}+01$ & $6.41 \mathrm{E}+01$ \\
\hline $\mathrm{m}-239(\mathrm{Pu})$ & $\mathrm{mg} / \mathrm{L}$ & $1.01 \mathrm{E}-01$ & 6.91E-02 & $1.13 \mathrm{E}-01$ \\
\hline $\mathrm{Pu}-238$ & $\mathrm{dpm} / \mathrm{mL}$ & $3.55 \mathrm{E}+05$ & $3.11 \mathrm{E}+05$ & $3.25 \mathrm{E}+05$ \\
\hline $\mathrm{Pu}-239 / 240$ & $\mathrm{dpm} / \mathrm{mL}$ & $1.92 \mathrm{E}+04$ & $1.62 \mathrm{E}+04$ & $1.61 \mathrm{E}+04$ \\
\hline Sr-90 & $\mathrm{dpm} / \mathrm{mL}$ & -- & $8.57 \mathrm{E}+04$ & -- \\
\hline Cs -137 & $\mathrm{dpm} / \mathrm{mL}$ & -- & $7.18 \mathrm{E}+06$ & -- \\
\hline Am-241 & $\mathrm{dpm} / \mathrm{mL}$ & -- & $<1.74 E+02$ & -- \\
\hline $\mathrm{Cm}-244$ & $\mathrm{dpm} / \mathrm{mL}$ & -- & $9.05 \mathrm{E}+01$ & -- \\
\hline
\end{tabular}


Table 3-25: Soluble Components in the Deposition Tank for the Tank 12H ECC No-Light Test, Storage Condition 5: With Evaporation, With Sludge Heel, With pH Adjustment, T = $50{ }^{\circ} \mathrm{C}$.

\begin{tabular}{|c|c|c|c|c|}
\hline \multirow{2}{*}{ analyte } & \multirow{2}{*}{ units } & \multicolumn{3}{|c|}{ deposition tank holding time } \\
\hline & & 7 days & 21 days & 35 days \\
\hline $\mathrm{pH}$ & -- & -- & $>12$ & $>12$ \\
\hline ORP & $\mathrm{mV}$ & -- & 28.8 & 67.9 \\
\hline Al & $\mathrm{mg} / \mathrm{L}$ & $1.19 \mathrm{E}+03$ & $1.25 \mathrm{E}+03$ & $1.48 \mathrm{E}+03$ \\
\hline $\mathrm{Ba}$ & $\mathrm{mg} / \mathrm{L}$ & $<5.39 E-02$ & 7.69E-01 & 5.93E-02 \\
\hline $\mathrm{Ca}$ & $\mathrm{mg} / \mathrm{L}$ & $1.27 \mathrm{E}+00$ & $6.21 \mathrm{E}-01$ & 7.34E-01 \\
\hline $\mathrm{Cd}$ & $\mathrm{mg} / \mathrm{L}$ & $<2.78 E-01$ & $4.50 \mathrm{E}-01$ & $<1.46 E-01$ \\
\hline $\mathrm{Cr}$ & $\mathrm{mg} / \mathrm{L}$ & $4.52 \mathrm{E}+01$ & $4.06 \mathrm{E}+01$ & $4.52 \mathrm{E}+01$ \\
\hline $\mathrm{Cu}$ & $\mathrm{mg} / \mathrm{L}$ & $<5.65 E-01$ & 4.47E-01 & 4.83E-01 \\
\hline $\mathrm{Fe}$ & $\mathrm{mg} / \mathrm{L}$ & $2.04 \mathrm{E}+00$ & $2.43 \mathrm{E}+00$ & $1.70 \mathrm{E}+00$ \\
\hline $\mathrm{K}$ & $\mathrm{mg} / \mathrm{L}$ & $8.91 \mathrm{E}+01$ & $1.32 \mathrm{E}+01$ & $4.27 \mathrm{E}+01$ \\
\hline $\mathrm{Mg}$ & $\mathrm{mg} / \mathrm{L}$ & $<1.35 E-01$ & $<7.52 E-02$ & 7.30E-02 \\
\hline Mo & $\mathrm{mg} / \mathrm{L}$ & $4.64 \mathrm{E}+00$ & $4.19 \mathrm{E}+00$ & $4.72 \mathrm{E}+00$ \\
\hline $\mathrm{Na}$ & $\mathrm{mg} / \mathrm{L}$ & $3.76 \mathrm{E}+04$ & -- & $3.59 \mathrm{E}+04$ \\
\hline $\mathrm{Ni}$ & $\mathrm{mg} / \mathrm{L}$ & $<1.44 E+00$ & $<7.52 E-01$ & $<7.30 E-01$ \\
\hline $\mathrm{P}$ & $\mathrm{mg} / \mathrm{L}$ & $1.37 \mathrm{E}+01$ & $1.35 \mathrm{E}+01$ & $1.24 \mathrm{E}+01$ \\
\hline S & $\mathrm{mg} / \mathrm{L}$ & $3.84 \mathrm{E}+01$ & $<3.53 E+01$ & $5.22 \mathrm{E}+01$ \\
\hline $\mathrm{Si}$ & $\mathrm{mg} / \mathrm{L}$ & $3.99 \mathrm{E}+01$ & $3.85 \mathrm{E}+01$ & $4.08 \mathrm{E}+01$ \\
\hline $\mathrm{U}$ & $\mathrm{mg} / \mathrm{L}$ & $7.43 \mathrm{E}+01$ & $7.28 \mathrm{E}+01$ & $6.40 \mathrm{E}+01$ \\
\hline $\mathrm{Zn}$ & $\mathrm{mg} / \mathrm{L}$ & $2.21 \mathrm{E}+00$ & $2.06 \mathrm{E}+00$ & $1.69 \mathrm{E}+00$ \\
\hline $\mathrm{Zr}$ & $\mathrm{mg} / \mathrm{L}$ & $1.88 \mathrm{E}-01$ & $1.65 \mathrm{E}-01$ & $<1.44 E+01$ \\
\hline $\mathrm{Hg}$ & $\mathrm{mg} / \mathrm{L}$ & -- & $2.68 \mathrm{E}+01$ & -- \\
\hline $\mathrm{m}-232(\mathrm{Th})$ & $\mathrm{mg} / \mathrm{L}$ & $<4.49 E-02$ & $<3.76 E-02$ & $<7.30 E-02$ \\
\hline m-233 (U) & $\mathrm{mg} / \mathrm{L}$ & $1.25 \mathrm{E}-01$ & 7.89E-02 & $1.05 \mathrm{E}-01$ \\
\hline m-234 (U) & $\mathrm{mg} / \mathrm{L}$ & $1.51 \mathrm{E}-01$ & $1.52 \mathrm{E}-01$ & $1.08 \mathrm{E}-01$ \\
\hline m-235 (U) & $\mathrm{mg} / \mathrm{L}$ & $1.35 \mathrm{E}+00$ & $1.28 \mathrm{E}+00$ & $1.23 \mathrm{E}+00$ \\
\hline m-236 (U) & $\mathrm{mg} / \mathrm{L}$ & $1.50 \mathrm{E}-01$ & $1.48 \mathrm{E}-01$ & $2.02 \mathrm{E}-01$ \\
\hline $\mathrm{m}-237(\mathrm{~Np})$ & $\mathrm{mg} / \mathrm{L}$ & 7.23E-02 & 4.85E-02 & $<1.28 E-01$ \\
\hline m-238 (U,Pu) & $\mathrm{mg} / \mathrm{L}$ & $7.78 \mathrm{E}+01$ & $6.79 \mathrm{E}+01$ & $6.27 \mathrm{E}+01$ \\
\hline $\mathrm{m}-239(\mathrm{Pu})$ & $\mathrm{mg} / \mathrm{L}$ & $1.31 \mathrm{E}-01$ & $1.36 \mathrm{E}-01$ & 8.74E-02 \\
\hline $\mathrm{Pu}-238$ & $\mathrm{dpm} / \mathrm{mL}$ & $3.44 \mathrm{E}+05$ & $2.91 \mathrm{E}+05$ & $2.52 \mathrm{E}+05$ \\
\hline $\mathrm{Pu}-239 / 240$ & $\mathrm{dpm} / \mathrm{mL}$ & $1.95 \mathrm{E}+04$ & $1.51 \mathrm{E}+04$ & $9.76 \mathrm{E}+03$ \\
\hline Sr-90 & $\mathrm{dpm} / \mathrm{mL}$ & -- & $8.10 \mathrm{E}+04$ & -- \\
\hline Cs-137 & $\mathrm{dpm} / \mathrm{mL}$ & -- & $6.96 \mathrm{E}+06$ & -- \\
\hline Am-241 & $\mathrm{dpm} / \mathrm{mL}$ & -- & $<8.02 E+01$ & -- \\
\hline $\mathrm{Cm}-244$ & $\mathrm{dpm} / \mathrm{mL}$ & -- & $3.94 \mathrm{E}+02$ & -- \\
\hline
\end{tabular}


Table 3-26: Soluble Components in the Deposition Tank for the Tank 12H ECC No-Light Test, Storage Condition 6: With Evaporation, Without Sludge Heel, Without pH Adjustment, $\mathrm{T}=50^{\circ} \mathrm{C}$.

\begin{tabular}{|c|c|c|c|c|}
\hline \multirow{2}{*}{ analyte } & \multirow{2}{*}{ units } & \multicolumn{3}{|c|}{ deposition tank holding time } \\
\hline & & 7 days & 21 days & 35 days \\
\hline $\mathrm{pH}$ & -- & -- & 9.77 & 9.68 \\
\hline ORP & $\mathrm{mV}$ & -- & 143 & 223 \\
\hline $\mathrm{Al}$ & $\mathrm{mg} / \mathrm{L}$ & $9.56 \mathrm{E}+00$ & $7.83 \mathrm{E}+00$ & $7.63 \mathrm{E}+00$ \\
\hline $\mathrm{Ba}$ & $\mathrm{mg} / \mathrm{L}$ & $<5.80 E-02$ & 8.94E-01 & $5.95 \mathrm{E}-02$ \\
\hline $\mathrm{Ca}$ & $\mathrm{mg} / \mathrm{L}$ & $2.53 \mathrm{E}+00$ & $2.10 \mathrm{E}+00$ & $2.51 \mathrm{E}+00$ \\
\hline $\mathrm{Cd}$ & $\mathrm{mg} / \mathrm{L}$ & $<3.00 E-01$ & $5.10 \mathrm{E}-01$ & $<7.14 E-02$ \\
\hline $\mathrm{Cr}$ & $\mathrm{mg} / \mathrm{L}$ & $5.90 \mathrm{E}+01$ & $5.81 \mathrm{E}+01$ & $6.17 \mathrm{E}+01$ \\
\hline $\mathrm{Cu}$ & $\mathrm{mg} / \mathrm{L}$ & $<6.09 E-01$ & $<3.41 E-01$ & $<1.55 E-01$ \\
\hline $\mathrm{Fe}$ & $\mathrm{mg} / \mathrm{L}$ & $<9.28 E-01$ & $8.70 \mathrm{E}-01$ & $2.67 \mathrm{E}-01$ \\
\hline $\mathrm{K}$ & $\mathrm{mg} / \mathrm{L}$ & $8.75 \mathrm{E}+01$ & $2.77 \mathrm{E}+01$ & $4.84 \mathrm{E}+01$ \\
\hline $\mathrm{Mg}$ & $\mathrm{mg} / \mathrm{L}$ & $1.84 \mathrm{E}-01$ & $1.55 \mathrm{E}-01$ & $2.38 \mathrm{E}-01$ \\
\hline Mo & $\mathrm{mg} / \mathrm{L}$ & $3.98 \mathrm{E}+00$ & $4.24 \mathrm{E}+00$ & $4.59 \mathrm{E}+00$ \\
\hline $\mathrm{Na}$ & $\mathrm{mg} / \mathrm{L}$ & $2.38 \mathrm{E}+03$ & -- & $2.39 \mathrm{E}+03$ \\
\hline $\mathrm{Ni}$ & $\mathrm{mg} / \mathrm{L}$ & $<1.55 E+00$ & $<8.54 E-01$ & $<3.81 E-01$ \\
\hline $\mathrm{P}$ & $\mathrm{mg} / \mathrm{L}$ & $<7.93 E+00$ & $<8.75 E+00$ & $<3.90 E+00$ \\
\hline $\mathrm{S}$ & $\mathrm{mg} / \mathrm{L}$ & $5.26 \mathrm{E}+01$ & $4.08 \mathrm{E}+01$ & $4.15 \mathrm{E}+01$ \\
\hline $\mathrm{Si}$ & $\mathrm{mg} / \mathrm{L}$ & $<4.73 E+00$ & $<2.35 E+00$ & $1.37 \mathrm{E}+00$ \\
\hline $\mathrm{U}$ & $\mathrm{mg} / \mathrm{L}$ & $1.84 \mathrm{E}+02$ & $2.01 \mathrm{E}+02$ & $2.11 \mathrm{E}+02$ \\
\hline $\mathrm{Zn}$ & $\mathrm{mg} / \mathrm{L}$ & $<4.45 E-01$ & $<3.52 E-01$ & $<2.14 E-01$ \\
\hline $\mathrm{Zr}$ & $\mathrm{mg} / \mathrm{L}$ & $<1.26 E-01$ & $<1.39 E-01$ & $<7.55 E+00$ \\
\hline $\mathrm{Hg}$ & $\mathrm{mg} / \mathrm{L}$ & -- & $1.59 \mathrm{E}+01$ & -- \\
\hline $\mathrm{m}-232(\mathrm{Th})$ & $\mathrm{mg} / \mathrm{L}$ & $<4.83 E-02$ & $<1.07 E-01$ & $<2.38 E-01$ \\
\hline $\mathrm{m}-233(\mathrm{U})$ & $\mathrm{mg} / \mathrm{L}$ & $1.52 \mathrm{E}-01$ & $2.52 \mathrm{E}-01$ & $2.95 \mathrm{E}-01$ \\
\hline $\mathrm{m}-234(\mathrm{U})$ & $\mathrm{mg} / \mathrm{L}$ & $1.93 \mathrm{E}-01$ & 2.73E-01 & $3.15 \mathrm{E}-01$ \\
\hline $\mathrm{m}-235(\mathrm{U})$ & $\mathrm{mg} / \mathrm{L}$ & $1.45 \mathrm{E}+00$ & $3.52 \mathrm{E}+00$ & $3.36 \mathrm{E}+00$ \\
\hline $\mathrm{m}-236(\mathrm{U})$ & $\mathrm{mg} / \mathrm{L}$ & $1.85 \mathrm{E}-01$ & 4.09E-01 & 4.04E-01 \\
\hline $\mathrm{m}-237(\mathrm{~Np})$ & $\mathrm{mg} / \mathrm{L}$ & $<7.25 E-02$ & $<1.07 E-01$ & $<4.17 E-01$ \\
\hline $\mathrm{m}-238(\mathrm{U}, \mathrm{Pu})$ & $\mathrm{mg} / \mathrm{L}$ & $8.25 \mathrm{E}+01$ & $1.94 \mathrm{E}+02$ & $1.88 \mathrm{E}+02$ \\
\hline $\mathrm{m}-239(\mathrm{Pu})$ & $\mathrm{mg} / \mathrm{L}$ & $1.13 \mathrm{E}-01$ & 4.62E-01 & $4.73 \mathrm{E}-01$ \\
\hline $\mathrm{Pu}-238$ & $\mathrm{dpm} / \mathrm{mL}$ & $1.35 \mathrm{E}+06$ & $1.33 \mathrm{E}+06$ & $1.86 \mathrm{E}+06$ \\
\hline $\mathrm{Pu}-239 / 240$ & $\mathrm{dpm} / \mathrm{mL}$ & $6.73 \mathrm{E}+04$ & $6.76 \mathrm{E}+04$ & $9.45 \mathrm{E}+04$ \\
\hline Sr-90 & $\mathrm{dpm} / \mathrm{mL}$ & -- & $9.78 \mathrm{E}+05$ & -- \\
\hline Cs -137 & $\mathrm{dpm} / \mathrm{mL}$ & -- & $1.06 \mathrm{E}+07$ & -- \\
\hline Am-241 & $\mathrm{dpm} / \mathrm{mL}$ & -- & $<4.34 E+02$ & -- \\
\hline $\mathrm{Cm}-244$ & $\mathrm{dpm} / \mathrm{mL}$ & -- & $<2.51 E+01$ & -- \\
\hline
\end{tabular}


Table 3-27: Soluble Components in the Deposition Tank for the Tank 12H ECC No-Light Test, Storage Condition 7: With Evaporation, With Sludge Heel, With pH Adjustment, T = $\sim 25^{\circ} \mathrm{C}$ (ambient).

\begin{tabular}{|c|c|c|c|c|}
\hline \multirow{2}{*}{ analyte } & \multirow{2}{*}{ units } & \multicolumn{3}{|c|}{ deposition tank holding time } \\
\hline & & 7 days & 21 days & 35 days \\
\hline $\mathrm{pH}$ & -- & -- & $>12$ & $>12$ \\
\hline ORP & $\mathrm{mV}$ & -- & 74 & 96 \\
\hline $\mathrm{Al}$ & $\mathrm{mg} / \mathrm{L}$ & $6.83 \mathrm{E}+02$ & $7.30 \mathrm{E}+02$ & $7.45 \mathrm{E}+02$ \\
\hline $\mathrm{Ba}$ & $\mathrm{mg} / \mathrm{L}$ & $<5.17 E-02$ & 8.27E-01 & 7.15E-02 \\
\hline $\mathrm{Ca}$ & $\mathrm{mg} / \mathrm{L}$ & $6.89 \mathrm{E}-01$ & 7.33E-01 & 7.68E-01 \\
\hline $\mathrm{Cd}$ & $\mathrm{mg} / \mathrm{L}$ & $<2.67 E-01$ & $4.60 \mathrm{E}-01$ & $<1.53 E-01$ \\
\hline $\mathrm{Cr}$ & $\mathrm{mg} / \mathrm{L}$ & $4.43 \mathrm{E}+01$ & $4.50 \mathrm{E}+01$ & $4.68 \mathrm{E}+01$ \\
\hline $\mathrm{Cu}$ & $\mathrm{mg} / \mathrm{L}$ & $<5.43 E-01$ & $<2.91 E-01$ & $<3.05 E-01$ \\
\hline $\mathrm{Fe}$ & $\mathrm{mg} / \mathrm{L}$ & $1.67 \mathrm{E}+00$ & $2.11 \mathrm{E}+00$ & $1.51 \mathrm{E}+00$ \\
\hline $\mathrm{K}$ & $\mathrm{mg} / \mathrm{L}$ & $8.79 \mathrm{E}+01$ & $<1.17 E+01$ & $4.12 \mathrm{E}+01$ \\
\hline $\mathrm{Mg}$ & $\mathrm{mg} / \mathrm{L}$ & $<1.29 E-01$ & $7.28 \mathrm{E}-02$ & $1.10 \mathrm{E}-01$ \\
\hline Mo & $\mathrm{mg} / \mathrm{L}$ & $3.37 \mathrm{E}+00$ & $3.42 \mathrm{E}+00$ & $3.36 \mathrm{E}+00$ \\
\hline $\mathrm{Na}$ & $\mathrm{mg} / \mathrm{L}$ & $3.64 \mathrm{E}+04$ & -- & $3.87 \mathrm{E}+04$ \\
\hline $\mathrm{Ni}$ & $\mathrm{mg} / \mathrm{L}$ & $<1.38 E+00$ & $<7.28 E-01$ & $<7.63 E-01$ \\
\hline $\mathrm{P}$ & $\mathrm{mg} / \mathrm{L}$ & $8.62 \mathrm{E}+00$ & $9.93 \mathrm{E}+00$ & $8.85 \mathrm{E}+00$ \\
\hline $\mathrm{S}$ & $\mathrm{mg} / \mathrm{L}$ & $4.73 \mathrm{E}+01$ & $<3.41 E+01$ & $<3.58 E+01$ \\
\hline $\mathrm{Si}$ & $\mathrm{mg} / \mathrm{L}$ & $2.34 \mathrm{E}+01$ & $2.58 \mathrm{E}+01$ & $2.63 \mathrm{E}+01$ \\
\hline $\mathrm{U}$ & $\mathrm{mg} / \mathrm{L}$ & $6.20 \mathrm{E}+01$ & $6.85 \mathrm{E}+01$ & $6.23 \mathrm{E}+01$ \\
\hline $\mathrm{Zn}$ & $\mathrm{mg} / \mathrm{L}$ & $1.22 \mathrm{E}+00$ & $1.23 \mathrm{E}+00$ & $9.06 \mathrm{E}-01$ \\
\hline $\mathrm{Zr}$ & $\mathrm{mg} / \mathrm{L}$ & $2.67 \mathrm{E}-01$ & $<1.18 E-01$ & $<1.51 E+01$ \\
\hline $\mathrm{Hg}$ & $\mathrm{mg} / \mathrm{L}$ & -- & $2.24 \mathrm{E}+01$ & -- \\
\hline m-232 (Th) & $\mathrm{mg} / \mathrm{L}$ & $<4.31 E-02$ & $<3.64 E-02$ & $<7.63 E-02$ \\
\hline m-233 (U) & $\mathrm{mg} / \mathrm{L}$ & $9.22 \mathrm{E}-02$ & $8.66 \mathrm{E}-02$ & $1.04 \mathrm{E}-01$ \\
\hline $\mathrm{m}-234(\mathrm{U})$ & $\mathrm{mg} / \mathrm{L}$ & $1.03 \mathrm{E}-01$ & $1.09 \mathrm{E}-01$ & $8.17 \mathrm{E}-02$ \\
\hline $\mathrm{m}-235(\mathrm{U})$ & $\mathrm{mg} / \mathrm{L}$ & $1.17 \mathrm{E}+00$ & $1.13 \mathrm{E}+00$ & $1.01 \mathrm{E}+00$ \\
\hline $\mathrm{m}-236(\mathrm{U})$ & $\mathrm{mg} / \mathrm{L}$ & $1.49 \mathrm{E}-01$ & $1.52 \mathrm{E}-01$ & $1.13 \mathrm{E}-01$ \\
\hline $\mathrm{m}-237(\mathrm{~Np})$ & $\mathrm{mg} / \mathrm{L}$ & $<6.46 E-02$ & $3.82 \mathrm{E}-02$ & $<1.34 E-01$ \\
\hline $\mathrm{m}-238(\mathrm{U}, \mathrm{Pu})$ & $\mathrm{mg} / \mathrm{L}$ & $6.36 \mathrm{E}+01$ & $6.03 \mathrm{E}+01$ & $5.75 \mathrm{E}+01$ \\
\hline $\mathrm{m}-239(\mathrm{Pu})$ & $\mathrm{mg} / \mathrm{L}$ & $8.96 \mathrm{E}-02$ & $6.53 \mathrm{E}-02$ & $8.69 \mathrm{E}-02$ \\
\hline $\mathrm{Pu}-238$ & $\mathrm{dpm} / \mathrm{mL}$ & $2.74 \mathrm{E}+05$ & $2.06 \mathrm{E}+05$ & $2.41 \mathrm{E}+05$ \\
\hline $\mathrm{Pu}-239 / 240$ & $\mathrm{dpm} / \mathrm{mL}$ & $1.61 \mathrm{E}+04$ & $1.28 \mathrm{E}+04$ & $1.24 \mathrm{E}+04$ \\
\hline Sr-90 & $\mathrm{dpm} / \mathrm{mL}$ & -- & $8.12 \mathrm{E}+04$ & -- \\
\hline Cs-137 & $\mathrm{dpm} / \mathrm{mL}$ & -- & $6.83 \mathrm{E}+06$ & -- \\
\hline Am-241 & $\mathrm{dpm} / \mathrm{mL}$ & -- & $<1.45 E+02$ & -- \\
\hline $\mathrm{Cm}-244$ & $\mathrm{dpm} / \mathrm{mL}$ & -- & $9.93 \mathrm{E}+01$ & -- \\
\hline
\end{tabular}


Table 3-28: Soluble Components in the Deposition Tank for Test 1 (Tank 12H No-Light), Storage Condition 8: With Evaporation, With Sludge Heel, With pH Adjustment, $\mathbf{T}=$ $70{ }^{\circ} \mathrm{C}$.

\begin{tabular}{|c|c|c|c|c|}
\hline \multirow{2}{*}{ analyte } & \multirow{2}{*}{ units } & \multicolumn{3}{|c|}{ deposition tank holding time } \\
\hline & & 7 days & 21 days & 35 days \\
\hline $\mathrm{pH}$ & -- & -- & $>12$ & $>12$ \\
\hline ORP & $\mathrm{mV}$ & -- & 25 & 70.6 \\
\hline Al & $\mathrm{mg} / \mathrm{L}$ & $1.46 \mathrm{E}+03$ & $1.58 \mathrm{E}+03$ & $1.95 \mathrm{E}+03$ \\
\hline $\mathrm{Ba}$ & $\mathrm{mg} / \mathrm{L}$ & $<5.68 E-02$ & $6.56 \mathrm{E}-01$ & 6.04E-02 \\
\hline $\mathrm{Ca}$ & $\mathrm{mg} / \mathrm{L}$ & $1.09 \mathrm{E}+00$ & $5.65 \mathrm{E}-01$ & $6.45 \mathrm{E}-01$ \\
\hline $\mathrm{Cd}$ & $\mathrm{mg} / \mathrm{L}$ & $<2.94 E-01$ & $3.29 \mathrm{E}-01$ & $<1.49 E-01$ \\
\hline $\mathrm{Cr}$ & $\mathrm{mg} / \mathrm{L}$ & $4.65 \mathrm{E}+01$ & $4.57 \mathrm{E}+01$ & $5.76 \mathrm{E}+01$ \\
\hline $\mathrm{Cu}$ & $\mathrm{mg} / \mathrm{L}$ & $<5.97 E-01$ & $6.76 \mathrm{E}-01$ & 7.38E-01 \\
\hline $\mathrm{Fe}$ & $\mathrm{mg} / \mathrm{L}$ & $2.44 \mathrm{E}+00$ & $1.92 \mathrm{E}+00$ & $1.62 \mathrm{E}+00$ \\
\hline $\mathrm{K}$ & $\mathrm{mg} / \mathrm{L}$ & $8.87 \mathrm{E}+01$ & $1.57 \mathrm{E}+01$ & $5.24 \mathrm{E}+01$ \\
\hline $\mathrm{Mg}$ & $\mathrm{mg} / \mathrm{L}$ & $<1.42 E-01$ & $<6.36 E-02$ & $<5.57 E-02$ \\
\hline Mo & $\mathrm{mg} / \mathrm{L}$ & $4.31 \mathrm{E}+00$ & $4.40 \mathrm{E}+00$ & $5.32 \mathrm{E}+00$ \\
\hline $\mathrm{Na}$ & $\mathrm{mg} / \mathrm{L}$ & $3.40 \mathrm{E}+04$ & -- & $4.14 \mathrm{E}+04$ \\
\hline $\mathrm{Ni}$ & $\mathrm{mg} / \mathrm{L}$ & $<1.52 E+00$ & $<6.36 E-01$ & $<7.43 E-01$ \\
\hline $\mathrm{P}$ & $\mathrm{mg} / \mathrm{L}$ & $1.25 \mathrm{E}+01$ & $9.62 \mathrm{E}+00$ & $1.24 \mathrm{E}+01$ \\
\hline $\mathrm{S}$ & $\mathrm{mg} / \mathrm{L}$ & $5.11 \mathrm{E}+01$ & $<2.98 E+01$ & $5.65 \mathrm{E}+01$ \\
\hline $\mathrm{Si}$ & $\mathrm{mg} / \mathrm{L}$ & $3.24 \mathrm{E}+01$ & $3.26 \mathrm{E}+01$ & $3.91 \mathrm{E}+01$ \\
\hline $\mathrm{U}$ & $\mathrm{mg} / \mathrm{L}$ & $5.62 \mathrm{E}+01$ & $5.39 \mathrm{E}+01$ & $4.35 \mathrm{E}+01$ \\
\hline $\mathrm{Zn}$ & $\mathrm{mg} / \mathrm{L}$ & $1.66 \mathrm{E}+00$ & $1.28 \mathrm{E}+00$ & $1.38 \mathrm{E}+00$ \\
\hline $\mathrm{Zr}$ & $\mathrm{mg} / \mathrm{L}$ & $1.23 \mathrm{E}-01$ & 1.19E-01 & $<1.47 E+01$ \\
\hline $\mathrm{Hg}$ & $\mathrm{mg} / \mathrm{L}$ & -- & $2.72 \mathrm{E}+01$ & -- \\
\hline $\mathrm{m}-232(\mathrm{Th})$ & $\mathrm{mg} / \mathrm{L}$ & $<4.74 E-02$ & $<3.18 E-02$ & $<7.43 E-02$ \\
\hline m-233 (U) & $\mathrm{mg} / \mathrm{L}$ & $9.57 \mathrm{E}-02$ & 7.27E-02 & 7.62E-02 \\
\hline m-234(U) & $\mathrm{mg} / \mathrm{L}$ & $9.23 \mathrm{E}-02$ & 8.91E-02 & 6.97E-02 \\
\hline m-235 (U) & $\mathrm{mg} / \mathrm{L}$ & $1.07 \mathrm{E}+00$ & $8.83 \mathrm{E}-01$ & $8.21 \mathrm{E}-01$ \\
\hline m-236(U) & $\mathrm{mg} / \mathrm{L}$ & $1.50 \mathrm{E}-01$ & $1.46 \mathrm{E}-01$ & $9.84 \mathrm{E}-02$ \\
\hline $\mathrm{m}-237(\mathrm{~Np})$ & $\mathrm{mg} / \mathrm{L}$ & $<7.10 E-02$ & $<3.18 E-02$ & $<1.30 E-01$ \\
\hline m-238 (U,Pu) & $\mathrm{mg} / \mathrm{L}$ & $6.01 \mathrm{E}+01$ & $4.55 \mathrm{E}+01$ & $4.45 \mathrm{E}+01$ \\
\hline $\mathrm{m}-239(\mathrm{Pu})$ & $\mathrm{mg} / \mathrm{L}$ & $<9.47 E-02$ & 4.83E-02 & $5.78 \mathrm{E}-02$ \\
\hline $\mathrm{Pu}-238$ & $\mathrm{dpm} / \mathrm{mL}$ & $3.06 \mathrm{E}+05$ & $1.83 \mathrm{E}+05$ & $2.09 \mathrm{E}+05$ \\
\hline $\mathrm{Pu}-239 / 240$ & $\mathrm{dpm} / \mathrm{mL}$ & $1.07 \mathrm{E}+04$ & $<4.34 E+03$ & $1.00 \mathrm{E}+04$ \\
\hline Sr-90 & $\mathrm{dpm} / \mathrm{mL}$ & -- & $8.19 \mathrm{E}+04$ & -- \\
\hline Cs -137 & $\mathrm{dpm} / \mathrm{mL}$ & -- & $7.62 \mathrm{E}+06$ & -- \\
\hline Am-241 & $\mathrm{dpm} / \mathrm{mL}$ & -- & $<2.60 E+02$ & -- \\
\hline $\mathrm{Cm}-244$ & $\mathrm{dpm} / \mathrm{mL}$ & -- & $<2.04 E+01$ & -- \\
\hline
\end{tabular}


Table 3-29: Soluble Components in the Deposition Tank for the Tank 12H Control (without ECC Processing): Without Evaporation, With Sludge Heel, With pH Adjustment, $\mathbf{T}=50^{\circ} \mathrm{C}$.

\begin{tabular}{|c|c|c|c|c|}
\hline \multirow{2}{*}{ analyte } & \multirow{2}{*}{ units } & \multicolumn{3}{|c|}{ deposition tank holding time } \\
\hline & & 7 days & 21 days & 35 days \\
\hline $\mathrm{pH}$ & -- & -- & $>12$ & $>12$ \\
\hline ORP & $\mathrm{mV}$ & -- & 68 & 68.9 \\
\hline Al & $\mathrm{mg} / \mathrm{L}$ & $4.57 \mathrm{E}+02$ & $6.71 \mathrm{E}+02$ & $8.42 \mathrm{E}+02$ \\
\hline $\mathrm{Ba}$ & $\mathrm{mg} / \mathrm{L}$ & $<5.29 E-02$ & $7.45 \mathrm{E}-01$ & 5.27E-02 \\
\hline $\mathrm{Ca}$ & $\mathrm{mg} / \mathrm{L}$ & $1.30 \mathrm{E}+00$ & $1.04 \mathrm{E}+00$ & $1.04 \mathrm{E}+00$ \\
\hline $\mathrm{Cd}$ & $\mathrm{mg} / \mathrm{L}$ & $<2.73 E-01$ & 4.65E-01 & $<1.41 E-01$ \\
\hline $\mathrm{Cr}$ & $\mathrm{mg} / \mathrm{L}$ & $<8.99 E-01$ & $6.10 \mathrm{E}-01$ & 7.19E-01 \\
\hline $\mathrm{Cu}$ & $\mathrm{mg} / \mathrm{L}$ & $<5.55 E-01$ & 4.53E-01 & 3.69E-01 \\
\hline $\mathrm{Fe}$ & $\mathrm{mg} / \mathrm{L}$ & $1.03 \mathrm{E}+00$ & $1.78 \mathrm{E}+00$ & $1.17 \mathrm{E}+00$ \\
\hline $\mathrm{K}$ & $\mathrm{mg} / \mathrm{L}$ & $6.64 \mathrm{E}+01$ & $<1.20 E+01$ & $<1.32 E+01$ \\
\hline $\mathrm{Mg}$ & $\mathrm{mg} / \mathrm{L}$ & $<1.32 E-01$ & $<7.47 E-02$ & 6.59E-02 \\
\hline Mo & $\mathrm{mg} / \mathrm{L}$ & $<2.72 E+00$ & $<1.25 E+00$ & $<1.18 E+00$ \\
\hline $\mathrm{Na}$ & $\mathrm{mg} / \mathrm{L}$ & $3.41 \mathrm{E}+04$ & -- & $3.68 \mathrm{E}+04$ \\
\hline $\mathrm{Ni}$ & $\mathrm{mg} / \mathrm{L}$ & $<1.41 E+00$ & $<7.47 E-01$ & $<7.03 E-01$ \\
\hline $\mathrm{P}$ & $\mathrm{mg} / \mathrm{L}$ & $<7.23 E+00$ & $<7.66 E+00$ & $<7.21 E+00$ \\
\hline $\mathrm{S}$ & $\mathrm{mg} / \mathrm{L}$ & $<3.30 E+01$ & $<3.50 E+01$ & $3.59 \mathrm{E}+01$ \\
\hline $\mathrm{Si}$ & $\mathrm{mg} / \mathrm{L}$ & $1.50 \mathrm{E}+01$ & $1.40 \mathrm{E}+01$ & $1.42 \mathrm{E}+01$ \\
\hline $\mathrm{U}$ & $\mathrm{mg} / \mathrm{L}$ & $<3.56 E+01$ & $<1.52 E+01$ & $<1.43 E+01$ \\
\hline $\mathrm{Zn}$ & $\mathrm{mg} / \mathrm{L}$ & $3.50 \mathrm{E}-01$ & 3.17E-01 & $<4.04 E-01$ \\
\hline $\mathrm{Zr}$ & $\mathrm{mg} / \mathrm{L}$ & $<1.15 E-01$ & $<1.21 E-01$ & $<1.39 E+01$ \\
\hline $\mathrm{Hg}$ & $\mathrm{mg} / \mathrm{L}$ & -- & $4.50 \mathrm{E}+01$ & -- \\
\hline m-232 (Th) & $\mathrm{mg} / \mathrm{L}$ & $<4.41 E-02$ & $<3.73 E-02$ & $<7.03 E-02$ \\
\hline m-233 (U) & $\mathrm{mg} / \mathrm{L}$ & $<4.41 E-02$ & $<3.73 E-02$ & $<3.52 E-02$ \\
\hline m-234(U) & $\mathrm{mg} / \mathrm{L}$ & $<4.41 E-02$ & $<3.73 E-02$ & $<5.27 E-02$ \\
\hline m-235 (U) & $\mathrm{mg} / \mathrm{L}$ & $1.46 \mathrm{E}-01$ & $<1.31 E-01$ & $1.20 \mathrm{E}-01$ \\
\hline m-236(U) & $\mathrm{mg} / \mathrm{L}$ & $<4.41 E-02$ & $<5.60 E-02$ & $<7.03 E-02$ \\
\hline $\mathrm{m}-237(\mathrm{~Np})$ & $\mathrm{mg} / \mathrm{L}$ & $<6.61 E-02$ & $3.75 \mathrm{E}-02$ & $<1.23 E-01$ \\
\hline m-238 (U,Pu) & $\mathrm{mg} / \mathrm{L}$ & $1.40 \mathrm{E}+00$ & $1.67 \mathrm{E}+00$ & $1.81 \mathrm{E}+00$ \\
\hline $\mathrm{m}-239(\mathrm{Pu})$ & $\mathrm{mg} / \mathrm{L}$ & $<8.81 E-02$ & $<3.73 E-02$ & $<5.27 E-02$ \\
\hline $\mathrm{Pu}-238$ & $\mathrm{dpm} / \mathrm{mL}$ & $2.32 \mathrm{E}+04$ & $6.91 \mathrm{E}+03$ & $5.26 \mathrm{E}+03$ \\
\hline $\mathrm{Pu}-239 / 240$ & $\mathrm{dpm} / \mathrm{mL}$ & $3.11 \mathrm{E}+03$ & $<9.99 E+02$ & $5.92 \mathrm{E}+02$ \\
\hline Sr-90 & $\mathrm{dpm} / \mathrm{mL}$ & -- & $4.71 \mathrm{E}+05$ & -- \\
\hline Cs -137 & $\mathrm{dpm} / \mathrm{mL}$ & -- & $1.59 \mathrm{E}+06$ & -- \\
\hline Am-241 & $\mathrm{dpm} / \mathrm{mL}$ & -- & $<1.33 E+03$ & -- \\
\hline $\mathrm{Cm}-244$ & $\mathrm{dpm} / \mathrm{mL}$ & -- & $<1.23 E+02$ & -- \\
\hline
\end{tabular}


Table 3-30: Soluble Components in the Deposition Tank for Test 2 (Tank 5F No-Light), Storage Condition 1: Without Evaporation, Without Sludge Heel, With pH Adjustment, T $=50{ }^{\circ} \mathrm{C}$.

\begin{tabular}{|c|c|c|c|c|}
\hline \multirow{2}{*}{ analyte } & \multirow{2}{*}{ units } & \multicolumn{3}{|c|}{ deposition tank holding time } \\
\hline & & 1 day & 8 days & 15 days \\
\hline $\mathrm{pH}$ & -- & $>12$ & -- & $>12$ \\
\hline ORP & $\mathrm{mV}$ & 69 & -- & 48.2 \\
\hline Al & $\mathrm{mg} / \mathrm{L}$ & $1.03 \mathrm{E}+02$ & $1.16 \mathrm{E}+02$ & $8.69 \mathrm{E}+01$ \\
\hline $\mathrm{Ba}$ & $\mathrm{mg} / \mathrm{L}$ & 7.78E-01 & $8.25 \mathrm{E}-01$ & $<3.04 E-01$ \\
\hline $\mathrm{Ca}$ & $\mathrm{mg} / \mathrm{L}$ & $5.08 \mathrm{E}-01$ & $4.36 \mathrm{E}-01$ & $1.11 \mathrm{E}+00$ \\
\hline $\mathrm{Cd}$ & $\mathrm{mg} / \mathrm{L}$ & 4.32E-01 & 4.44E-01 & $<1.52 E-01$ \\
\hline $\mathrm{Cr}$ & $\mathrm{mg} / \mathrm{L}$ & $5.65 \mathrm{E}+00$ & $5.82 \mathrm{E}+00$ & $4.21 \mathrm{E}+00$ \\
\hline $\mathrm{Fe}$ & $\mathrm{mg} / \mathrm{L}$ & $1.59 \mathrm{E}+00$ & $1.57 \mathrm{E}+00$ & $2.11 \mathrm{E}+00$ \\
\hline $\mathrm{K}$ & $\mathrm{mg} / \mathrm{L}$ & $<1.42 E+01$ & $1.35 \mathrm{E}+01$ & $1.49 \mathrm{E}+01$ \\
\hline $\mathrm{Li}$ & $\mathrm{mg} / \mathrm{L}$ & $1.45 \mathrm{E}+00$ & $1.50 \mathrm{E}+00$ & $1.21 \mathrm{E}+00$ \\
\hline $\mathrm{Mg}$ & $\mathrm{mg} / \mathrm{L}$ & $<5.70 E-02$ & $<5.75 E-02$ & $1.28 \mathrm{E}-01$ \\
\hline $\mathrm{Mn}$ & $\mathrm{mg} / \mathrm{L}$ & $<3.80 E-02$ & $<1.05 E-01$ & $<1.04 E-01$ \\
\hline Mo & $\mathrm{mg} / \mathrm{L}$ & $<1.27 E+00$ & $<1.28 E+00$ & $<1.27 E+00$ \\
\hline $\mathrm{Na}$ & $\mathrm{mg} / \mathrm{L}$ & $2.28 \mathrm{E}+04$ & $2.29 \mathrm{E}+04$ & $1.80 \mathrm{E}+04$ \\
\hline $\mathrm{Ni}$ & $\mathrm{mg} / \mathrm{L}$ & $<7.59 E-01$ & $<7.67 E-01$ & $<7.59 E-01$ \\
\hline $\mathrm{P}$ & $\mathrm{mg} / \mathrm{L}$ & $<7.78 E+00$ & $<7.86 E+00$ & $<7.78 E+00$ \\
\hline $\mathrm{S}$ & $\mathrm{mg} / \mathrm{L}$ & $<3.56 E+01$ & $<3.60 E+01$ & $<3.56 E+01$ \\
\hline $\mathrm{Si}$ & $\mathrm{mg} / \mathrm{L}$ & $2.77 \mathrm{E}+01$ & $2.85 \mathrm{E}+01$ & $2.19 \mathrm{E}+01$ \\
\hline $\mathrm{Sr}$ & $\mathrm{mg} / \mathrm{L}$ & $<2.85 E-02$ & $<2.88 E-02$ & $<2.85 E-02$ \\
\hline $\mathrm{U}$ & $\mathrm{mg} / \mathrm{L}$ & $1.22 \mathrm{E}+02$ & $7.27 \mathrm{E}+01$ & $4.42 \mathrm{E}+01$ \\
\hline $\mathrm{Zr}$ & $\mathrm{mg} / \mathrm{L}$ & $3.51 \mathrm{E}-01$ & $1.63 \mathrm{E}-01$ & $<1.23 E-01$ \\
\hline $\mathrm{Hg}$ & $\mathrm{mg} / \mathrm{L}$ & -- & -- & $7.40 \mathrm{E}-01$ \\
\hline Oxalate & $\mathrm{mg} / \mathrm{L}$ & -- & -- & $1.42 \mathrm{E}+02$ \\
\hline m-234 (U) & $\mathrm{mg} / \mathrm{L}$ & $<1.78 E-01$ & $<4.79 E-02$ & $<1.52 E-01$ \\
\hline m-235 (U) & $\mathrm{mg} / \mathrm{L}$ & $9.31 \mathrm{E}-01$ & $4.51 \mathrm{E}-01$ & $3.29 \mathrm{E}-01$ \\
\hline m-236(U) & $\mathrm{mg} / \mathrm{L}$ & $<1.19 E-01$ & $<4.79 E-02$ & $<9.49 E-02$ \\
\hline m-237 (Np) & $\mathrm{mg} / \mathrm{L}$ & $<1.19 E-01$ & $<2.64 E-01$ & $<5.69 E-02$ \\
\hline $\mathrm{m}-238(\mathrm{U}, \mathrm{Pu})$ & $\mathrm{mg} / \mathrm{L}$ & $1.22 \mathrm{E}+02$ & $7.46 \mathrm{E}+01$ & $4.15 \mathrm{E}+01$ \\
\hline $\mathrm{m}-239(\mathrm{Pu})$ & $\mathrm{mg} / \mathrm{L}$ & $<1.78 E-01$ & $<7.19 E-02$ & $<1.14 E-01$ \\
\hline $\mathrm{Pu}-238$ & $\mathrm{dpm} / \mathrm{mL}$ & $8.35 \mathrm{E}+02$ & $8.12 \mathrm{E}+02$ & $1.20 \mathrm{E}+02$ \\
\hline $\mathrm{Pu}-239 / 240$ & $\mathrm{dpm} / \mathrm{mL}$ & $<2.33 E+03$ & $7.02 \mathrm{E}+02$ & $9.96 \mathrm{E}+01$ \\
\hline Sr-90 & $\mathrm{dpm} / \mathrm{mL}$ & -- & -- & $<1.57 E+05$ \\
\hline Cs -137 & $\mathrm{dpm} / \mathrm{mL}$ & -- & -- & $4.02 \mathrm{E}+06$ \\
\hline Am-241 & $\mathrm{dpm} / \mathrm{mL}$ & -- & -- & $<9.35 E+02$ \\
\hline $\mathrm{Cm}-244$ & $\mathrm{dpm} / \mathrm{mL}$ & -- & -- & $<8.93 E+01$ \\
\hline
\end{tabular}


Table 3-31: Soluble Components in the Deposition Tank for Test 2 (Tank 5F No-Light), Storage Condition 2: Without Evaporation, With Sludge Heel, With pH Adjustment, $\mathbf{T}=$ $50{ }^{\circ} \mathrm{C}$.

\begin{tabular}{|c|c|c|c|c|}
\hline \multirow{2}{*}{ analyte } & \multirow{2}{*}{ units } & \multicolumn{3}{|c|}{ deposition tank holding time } \\
\hline & & 1 day & 8 days & 15 days \\
\hline $\mathrm{pH}$ & -- & $>12$ & -- & $>12$ \\
\hline ORP & $\mathrm{mV}$ & 44 & -- & 44 \\
\hline Al & $\mathrm{mg} / \mathrm{L}$ & $1.89 \mathrm{E}+02$ & $2.01 \mathrm{E}+02$ & $2.08 \mathrm{E}+02$ \\
\hline $\mathrm{Ba}$ & $\mathrm{mg} / \mathrm{L}$ & $1.04 \mathrm{E}+00$ & $7.51 \mathrm{E}-01$ & $<3.27 E-01$ \\
\hline $\mathrm{Ca}$ & $\mathrm{mg} / \mathrm{L}$ & 7.75E-01 & 3.73E-01 & $1.25 \mathrm{E}+00$ \\
\hline $\mathrm{Cd}$ & $\mathrm{mg} / \mathrm{L}$ & 5.82E-01 & 3.38E-01 & $<1.63 E-01$ \\
\hline $\mathrm{Cr}$ & $\mathrm{mg} / \mathrm{L}$ & $5.92 \mathrm{E}+00$ & $5.79 \mathrm{E}+00$ & $6.01 \mathrm{E}+00$ \\
\hline $\mathrm{Fe}$ & $\mathrm{mg} / \mathrm{L}$ & $1.89 \mathrm{E}+00$ & $1.59 \mathrm{E}+00$ & $1.12 \mathrm{E}+00$ \\
\hline $\mathrm{K}$ & $\mathrm{mg} / \mathrm{L}$ & $<1.76 E+01$ & $1.43 \mathrm{E}+01$ & $1.63 \mathrm{E}+01$ \\
\hline $\mathrm{Li}$ & $\mathrm{mg} / \mathrm{L}$ & $1.47 \mathrm{E}+00$ & $1.48 \mathrm{E}+00$ & $1.65 \mathrm{E}+00$ \\
\hline $\mathrm{Mg}$ & $\mathrm{mg} / \mathrm{L}$ & $<7.05 E-02$ & $<5.33 E-02$ & $1.28 \mathrm{E}-01$ \\
\hline $\mathrm{Mn}$ & $\mathrm{mg} / \mathrm{L}$ & $<4.70 E-02$ & $<9.77 E-02$ & $<1.12 E-01$ \\
\hline Mo & $\mathrm{mg} / \mathrm{L}$ & $<1.57 E+00$ & $<1.19 E+00$ & $<1.37 E+00$ \\
\hline $\mathrm{Na}$ & $\mathrm{mg} / \mathrm{L}$ & $2.31 \mathrm{E}+04$ & $2.44 \mathrm{E}+04$ & $2.68 \mathrm{E}+04$ \\
\hline $\mathrm{Ni}$ & $\mathrm{mg} / \mathrm{L}$ & $<9.40 E-01$ & $<7.11 E-01$ & $<8.17 E-01$ \\
\hline $\mathrm{P}$ & $\mathrm{mg} / \mathrm{L}$ & $<9.63 E+00$ & $<7.29 E+00$ & $<8.38 E+00$ \\
\hline $\mathrm{S}$ & $\mathrm{mg} / \mathrm{L}$ & $<4.41 E+01$ & $<3.33 E+01$ & $<3.83 E+01$ \\
\hline $\mathrm{Si}$ & $\mathrm{mg} / \mathrm{L}$ & $3.13 \mathrm{E}+01$ & $3.20 \mathrm{E}+01$ & $3.37 \mathrm{E}+01$ \\
\hline $\mathrm{Sr}$ & $\mathrm{mg} / \mathrm{L}$ & $<3.52 E-02$ & $<2.67 E-02$ & $<3.06 E-02$ \\
\hline $\mathrm{U}$ & $\mathrm{mg} / \mathrm{L}$ & $1.13 \mathrm{E}+02$ & $7.25 \mathrm{E}+01$ & $5.83 \mathrm{E}+01$ \\
\hline $\mathrm{Zr}$ & $\mathrm{mg} / \mathrm{L}$ & $3.41 \mathrm{E}-01$ & $1.73 \mathrm{E}-01$ & $1.28 \mathrm{E}-01$ \\
\hline $\mathrm{Hg}$ & $\mathrm{mg} / \mathrm{L}$ & -- & -- & $1.61 \mathrm{E}+00$ \\
\hline Oxalate & $\mathrm{mg} / \mathrm{L}$ & -- & -- & $2.04 \mathrm{E}+02$ \\
\hline m-234 (U) & $\mathrm{mg} / \mathrm{L}$ & $<2.21 E-01$ & $<4.44 E-02$ & $<1.63 E-01$ \\
\hline m-235 (U) & $\mathrm{mg} / \mathrm{L}$ & 7.91E-01 & $5.11 \mathrm{E}-01$ & $3.95 \mathrm{E}-01$ \\
\hline m-236(U) & $\mathrm{mg} / \mathrm{L}$ & $<1.47 E-01$ & $<4.44 E-02$ & $<1.02 E-01$ \\
\hline $\mathrm{m}-237(\mathrm{~Np})$ & $\mathrm{mg} / \mathrm{L}$ & $<1.47 E-01$ & $<2.44 E-01$ & $<6.13 E-02$ \\
\hline m-238 (U,Pu) & $\mathrm{mg} / \mathrm{L}$ & $1.06 \mathrm{E}+02$ & $7.48 \mathrm{E}+01$ & $5.62 \mathrm{E}+01$ \\
\hline $\mathrm{m}-239(\mathrm{Pu})$ & $\mathrm{mg} / \mathrm{L}$ & $<2.21 E-01$ & $<6.66 E-02$ & $<1.23 E-01$ \\
\hline $\mathrm{Pu}-238$ & $\mathrm{dpm} / \mathrm{mL}$ & $6.99 \mathrm{E}+02$ & $3.54 \mathrm{E}+02$ & $7.68 \mathrm{E}+02$ \\
\hline $\mathrm{Pu}-239 / 240$ & $\mathrm{dpm} / \mathrm{mL}$ & $<1.34 E+03$ & $3.81 \mathrm{E}+02$ & $2.99 \mathrm{E}+02$ \\
\hline Sr-90 & $\mathrm{dpm} / \mathrm{mL}$ & -- & -- & $<3.04 E+05$ \\
\hline Cs -137 & $\mathrm{dpm} / \mathrm{mL}$ & -- & -- & $1.79 \mathrm{E}+07$ \\
\hline Am-241 & $\mathrm{dpm} / \mathrm{mL}$ & -- & -- & $<4.06 E+02$ \\
\hline $\mathrm{Cm}-244$ & $\mathrm{dpm} / \mathrm{mL}$ & -- & -- & $3.35 \mathrm{E}+02$ \\
\hline
\end{tabular}


Table 3-32: Soluble Components in the Deposition Tank for Test 2 (Tank 5F No-Light), Storage Condition 3: Without Evaporation, Without Sludge Heel, Without pH Adjustment, $\mathbf{T}=50^{\circ} \mathbf{C}$.

\begin{tabular}{|c|c|c|c|c|}
\hline \multirow{2}{*}{ analyte } & \multirow{2}{*}{ units } & \multicolumn{3}{|c|}{ deposition tank holding time } \\
\hline & & 1 day & 8 days & 15 days \\
\hline $\mathrm{pH}$ & -- & 7.52 & -- & 7.51 \\
\hline ORP & $\mathrm{mV}$ & 232 & -- & 260 \\
\hline $\mathrm{Al}$ & $\mathrm{mg} / \mathrm{L}$ & $6.92 \mathrm{E}-01$ & $1.65 \mathrm{E}+00$ & 7.47E-01 \\
\hline $\mathrm{Ba}$ & $\mathrm{mg} / \mathrm{L}$ & $9.27 \mathrm{E}-01$ & $8.22 \mathrm{E}-01$ & $<1.30 E-01$ \\
\hline $\mathrm{Ca}$ & $\mathrm{mg} / \mathrm{L}$ & $2.37 \mathrm{E}+00$ & $1.82 \mathrm{E}+00$ & $2.20 \mathrm{E}+00$ \\
\hline $\mathrm{Cd}$ & $\mathrm{mg} / \mathrm{L}$ & $4.58 \mathrm{E}-01$ & 3.95E-01 & $<6.02 E-02$ \\
\hline $\mathrm{Cr}$ & $\mathrm{mg} / \mathrm{L}$ & $5.05 \mathrm{E}+00$ & $4.79 \mathrm{E}+00$ & $4.42 \mathrm{E}+00$ \\
\hline $\mathrm{Fe}$ & $\mathrm{mg} / \mathrm{L}$ & $2.07 \mathrm{E}+00$ & $2.15 \mathrm{E}+00$ & $8.67 \mathrm{E}-01$ \\
\hline K & $\mathrm{mg} / \mathrm{L}$ & $1.25 \mathrm{E}+01$ & $<1.20 E+01$ & $1.28 \mathrm{E}+01$ \\
\hline $\mathrm{Li}$ & $\mathrm{mg} / \mathrm{L}$ & $2.68 \mathrm{E}+00$ & $2.37 \mathrm{E}+00$ & $2.40 \mathrm{E}+00$ \\
\hline $\mathrm{Mg}$ & $\mathrm{mg} / \mathrm{L}$ & $1.58 \mathrm{E}+00$ & $1.48 \mathrm{E}+00$ & $1.40 \mathrm{E}+00$ \\
\hline $\mathrm{Mn}$ & $\mathrm{mg} / \mathrm{L}$ & 8.08E-01 & 7.59E-01 & 4.70E-01 \\
\hline Mo & $\mathrm{mg} / \mathrm{L}$ & $<5.28 E-01$ & $<1.25 E+00$ & $<5.42 E-01$ \\
\hline $\mathrm{Na}$ & $\mathrm{mg} / \mathrm{L}$ & $3.20 \mathrm{E}+02$ & $<6.89 E+02$ & $2.76 \mathrm{E}+02$ \\
\hline $\mathrm{Ni}$ & $\mathrm{mg} / \mathrm{L}$ & $2.99 \mathrm{E}+00$ & $2.02 \mathrm{E}+00$ & $1.30 \mathrm{E}+00$ \\
\hline $\mathrm{P}$ & $\mathrm{mg} / \mathrm{L}$ & $<3.21 E+00$ & $<7.66 E+00$ & $<3.29 E+00$ \\
\hline $\mathrm{S}$ & $\mathrm{mg} / \mathrm{L}$ & $1.88 \mathrm{E}+01$ & $<3.50 E+01$ & $<1.51 E+01$ \\
\hline $\mathrm{Si}$ & $\mathrm{mg} / \mathrm{L}$ & $4.61 \mathrm{E}+00$ & $4.81 \mathrm{E}+00$ & $4.30 \mathrm{E}+00$ \\
\hline $\mathrm{Sr}$ & $\mathrm{mg} / \mathrm{L}$ & $1.53 \mathrm{E}-01$ & $1.21 \mathrm{E}-01$ & $1.13 \mathrm{E}-01$ \\
\hline $\mathrm{U}$ & $\mathrm{mg} / \mathrm{L}$ & $1.07 \mathrm{E}+02$ & $9.31 \mathrm{E}+01$ & $8.14 \mathrm{E}+01$ \\
\hline $\mathrm{Zr}$ & $\mathrm{mg} / \mathrm{L}$ & $<4.89 E-02$ & $<1.21 E-01$ & $<5.02 E-02$ \\
\hline $\mathrm{Hg}$ & $\mathrm{mg} / \mathrm{L}$ & -- & -- & $<5.02 E-01$ \\
\hline Oxalate & $\mathrm{mg} / \mathrm{L}$ & -- & -- & $2.21 \mathrm{E}+02$ \\
\hline $\mathrm{m}-234(\mathrm{U})$ & $\mathrm{mg} / \mathrm{L}$ & $<1.84 E-01$ & $<4.67 E-02$ & $<1.61 E-01$ \\
\hline $\mathrm{m}-235(\mathrm{U})$ & $\mathrm{mg} / \mathrm{L}$ & $6.79 \mathrm{E}-01$ & $6.25 \mathrm{E}-01$ & 5.83E-01 \\
\hline $\mathrm{m}-236(\mathrm{U})$ & $\mathrm{mg} / \mathrm{L}$ & $<1.22 E-01$ & $<4.67 E-02$ & $<1.00 E-01$ \\
\hline $\mathrm{m}-237(\mathrm{~Np})$ & $\mathrm{mg} / \mathrm{L}$ & $<1.22 E-01$ & $<2.57 E-01$ & $6.82 \mathrm{E}-02$ \\
\hline m-238 (U,Pu) & $\mathrm{mg} / \mathrm{L}$ & $9.87 \mathrm{E}+01$ & $9.62 \mathrm{E}+01$ & $8.35 \mathrm{E}+01$ \\
\hline $\mathrm{m}-239(\mathrm{Pu})$ & $\mathrm{mg} / \mathrm{L}$ & $<1.84 E-01$ & 8.87E-02 & $<1.20 E-01$ \\
\hline $\mathrm{Pu}-238$ & $\mathrm{dpm} / \mathrm{mL}$ & $4.20 \mathrm{E}+02$ & $5.24 \mathrm{E}+02$ & $1.48 \mathrm{E}+02$ \\
\hline $\mathrm{Pu}-239 / 240$ & $\mathrm{dpm} / \mathrm{mL}$ & $<6.46 E+02$ & $1.49 \mathrm{E}+02$ & $<7.29 E+01$ \\
\hline Sr-90 & $\mathrm{dpm} / \mathrm{mL}$ & -- & -- & $1.06 \mathrm{E}+07$ \\
\hline Cs-137 & $\mathrm{dpm} / \mathrm{mL}$ & -- & -- & $1.30 \mathrm{E}+07$ \\
\hline Am-241 & $\mathrm{dpm} / \mathrm{mL}$ & -- & -- & $<1.39 E+03$ \\
\hline $\mathrm{Cm}-244$ & $\mathrm{dpm} / \mathrm{mL}$ & -- & -- & $7.38 \mathrm{E}+02$ \\
\hline
\end{tabular}


Table 3-33: Soluble Components in the Deposition Tank for Test 2 (Tank 5F No-Light), Storage Condition 4: With Evaporation, Without Sludge Heel, With pH Adjustment, $\mathbf{T}=$ $50{ }^{\circ} \mathrm{C}$.

\begin{tabular}{|c|c|c|c|c|}
\hline \multirow{2}{*}{ analyte } & \multirow{2}{*}{ units } & \multicolumn{3}{|c|}{ deposition tank holding time } \\
\hline & & 1 day & 8 days & 15 days \\
\hline $\mathrm{pH}$ & -- & $>12$ & -- & $>12$ \\
\hline ORP & $\mathrm{mV}$ & 54 & -- & 63 \\
\hline Al & $\mathrm{mg} / \mathrm{L}$ & $4.26 \mathrm{E}+02$ & $4.77 \mathrm{E}+02$ & $5.03 \mathrm{E}+02$ \\
\hline $\mathrm{Ba}$ & $\mathrm{mg} / \mathrm{L}$ & 8.38E-01 & $8.02 \mathrm{E}-01$ & $<3.23 E-01$ \\
\hline $\mathrm{Ca}$ & $\mathrm{mg} / \mathrm{L}$ & 5.99E-01 & 3.94E-01 & $1.35 \mathrm{E}+00$ \\
\hline $\mathrm{Cd}$ & $\mathrm{mg} / \mathrm{L}$ & 4.06E-01 & 3.92E-01 & $<1.62 E-01$ \\
\hline $\mathrm{Cr}$ & $\mathrm{mg} / \mathrm{L}$ & $3.95 \mathrm{E}+01$ & $4.05 \mathrm{E}+01$ & $4.33 \mathrm{E}+01$ \\
\hline $\mathrm{Fe}$ & $\mathrm{mg} / \mathrm{L}$ & $1.84 \mathrm{E}+00$ & $1.99 \mathrm{E}+00$ & $1.63 \mathrm{E}+00$ \\
\hline $\mathrm{K}$ & $\mathrm{mg} / \mathrm{L}$ & $8.73 E+01$ & $9.44 \mathrm{E}+01$ & $9.64 \mathrm{E}+01$ \\
\hline $\mathrm{Li}$ & $\mathrm{mg} / \mathrm{L}$ & $6.84 \mathrm{E}+00$ & $7.23 \mathrm{E}+00$ & $7.47 \mathrm{E}+00$ \\
\hline $\mathrm{Mg}$ & $\mathrm{mg} / \mathrm{L}$ & $<6.10 E-02$ & $<5.70 E-02$ & $1.31 \mathrm{E}-01$ \\
\hline Mn & $\mathrm{mg} / \mathrm{L}$ & $<4.06 E-02$ & $<1.04 E-01$ & $<1.11 E-01$ \\
\hline Mo & $\mathrm{mg} / \mathrm{L}$ & $2.91 \mathrm{E}+00$ & $2.29 \mathrm{E}+00$ & $2.74 \mathrm{E}+00$ \\
\hline $\mathrm{Na}$ & $\mathrm{mg} / \mathrm{L}$ & $2.99 \mathrm{E}+04$ & $2.85 \mathrm{E}+04$ & $3.01 \mathrm{E}+04$ \\
\hline $\mathrm{Ni}$ & $\mathrm{mg} / \mathrm{L}$ & $<8.13 E-01$ & $<7.59 E-01$ & $<8.09 E-01$ \\
\hline $\mathrm{P}$ & $\mathrm{mg} / \mathrm{L}$ & $1.31 \mathrm{E}+01$ & $1.46 \mathrm{E}+01$ & $1.49 \mathrm{E}+01$ \\
\hline S & $\mathrm{mg} / \mathrm{L}$ & $4.34 \mathrm{E}+01$ & $<3.56 E+01$ & $<3.79 E+01$ \\
\hline $\mathrm{Si}$ & $\mathrm{mg} / \mathrm{L}$ & $6.58 \mathrm{E}+01$ & $6.78 \mathrm{E}+01$ & $7.33 \mathrm{E}+01$ \\
\hline $\mathrm{Sr}$ & $\mathrm{mg} / \mathrm{L}$ & $<3.05 E-02$ & $<2.85 E-02$ & $<3.03 E-02$ \\
\hline $\mathrm{U}$ & $\mathrm{mg} / \mathrm{L}$ & $1.50 \mathrm{E}+02$ & $7.08 \mathrm{E}+01$ & $6.01 \mathrm{E}+01$ \\
\hline $\mathrm{Zr}$ & $\mathrm{mg} / \mathrm{L}$ & 2.44E-01 & $1.61 \mathrm{E}-01$ & $<1.31 E-01$ \\
\hline $\mathrm{Hg}$ & $\mathrm{mg} / \mathrm{L}$ & -- & -- & $2.89 \mathrm{E}+00$ \\
\hline Oxalate & $\mathrm{mg} / \mathrm{L}$ & -- & -- & $1.14 \mathrm{E}+03$ \\
\hline m-234(U) & $\mathrm{mg} / \mathrm{L}$ & $<1.91 E-01$ & $<4.75 E-02$ & $<1.62 E-01$ \\
\hline m-235 (U) & $\mathrm{mg} / \mathrm{L}$ & 9.70E-01 & 4.32E-01 & 3.39E-01 \\
\hline m-236(U) & $\mathrm{mg} / \mathrm{L}$ & $<1.27 E-01$ & $<4.75 E-02$ & $<1.01 E-01$ \\
\hline $\mathrm{m}-237(\mathrm{~Np})$ & $\mathrm{mg} / \mathrm{L}$ & $<1.27 E-01$ & $<2.61 E-01$ & $<6.06 E-02$ \\
\hline m-238 (U,Pu) & $\mathrm{mg} / \mathrm{L}$ & $1.50 \mathrm{E}+02$ & $7.50 \mathrm{E}+01$ & $5.53 \mathrm{E}+01$ \\
\hline $\mathrm{m}-239(\mathrm{Pu})$ & $\mathrm{mg} / \mathrm{L}$ & $<1.91 E-01$ & 8.42E-02 & $<1.21 E-01$ \\
\hline $\mathrm{Pu}-238$ & $\mathrm{dpm} / \mathrm{mL}$ & $2.60 \mathrm{E}+02$ & $2.61 \mathrm{E}+02$ & $7.83 \mathrm{E}+02$ \\
\hline $\mathrm{Pu}-239 / 240$ & $\mathrm{dpm} / \mathrm{mL}$ & $<3.90 E+02$ & $6.01 \mathrm{E}+02$ & $5.26 \mathrm{E}+02$ \\
\hline Sr-90 & $\mathrm{dpm} / \mathrm{mL}$ & -- & -- & $<2.77 E+05$ \\
\hline Cs-137 & $\mathrm{dpm} / \mathrm{mL}$ & -- & -- & $1.91 \mathrm{E}+08$ \\
\hline Am-241 & $\mathrm{dpm} / \mathrm{mL}$ & -- & -- & $<3.12 E+02$ \\
\hline $\mathrm{Cm}-244$ & $\mathrm{dpm} / \mathrm{mL}$ & -- & -- & $8.79 \mathrm{E}+01$ \\
\hline
\end{tabular}


Table 3-34: Soluble Components in the Deposition Tank for Test 2 (Tank 5F No-Light), Storage Condition 5: With Evaporation, With Sludge Heel, With pH Adjustment, $\mathbf{T}=$ $50{ }^{\circ} \mathrm{C}$.

\begin{tabular}{|c|c|c|c|c|}
\hline \multirow{2}{*}{ analyte } & \multirow{2}{*}{ units } & \multicolumn{3}{|c|}{ deposition tank holding time } \\
\hline & & 1 day & 8 days & 15 days \\
\hline $\mathrm{pH}$ & -- & $>12$ & -- & $>12$ \\
\hline ORP & $\mathrm{mV}$ & 54 & -- & 64 \\
\hline Al & $\mathrm{mg} / \mathrm{L}$ & $4.72 \mathrm{E}+02$ & $5.03 \mathrm{E}+02$ & $5.32 \mathrm{E}+02$ \\
\hline $\mathrm{Ba}$ & $\mathrm{mg} / \mathrm{L}$ & 7.64E-01 & 7.30E-01 & $<3.14 E-01$ \\
\hline $\mathrm{Ca}$ & $\mathrm{mg} / \mathrm{L}$ & 4.75E-01 & 3.54E-01 & $1.33 \mathrm{E}+00$ \\
\hline $\mathrm{Cd}$ & $\mathrm{mg} / \mathrm{L}$ & 4.13E-01 & 4.22E-01 & $<1.57 E-01$ \\
\hline $\mathrm{Cr}$ & $\mathrm{mg} / \mathrm{L}$ & $4.01 \mathrm{E}+01$ & $3.91 \mathrm{E}+01$ & $4.17 \mathrm{E}+01$ \\
\hline $\mathrm{Fe}$ & $\mathrm{mg} / \mathrm{L}$ & $2.26 \mathrm{E}+00$ & $2.21 \mathrm{E}+00$ & $1.78 \mathrm{E}+00$ \\
\hline $\mathrm{K}$ & $\mathrm{mg} / \mathrm{L}$ & $8.63 \mathrm{E}+01$ & $8.72 \mathrm{E}+01$ & $9.08 \mathrm{E}+01$ \\
\hline $\mathrm{Li}$ & $\mathrm{mg} / \mathrm{L}$ & $7.90 \mathrm{E}+00$ & $7.69 \mathrm{E}+00$ & $8.10 \mathrm{E}+00$ \\
\hline $\mathrm{Mg}$ & $\mathrm{mg} / \mathrm{L}$ & $<5.33 E-02$ & $<5.18 E-02$ & 2.11E-01 \\
\hline $\mathrm{Mn}$ & $\mathrm{mg} / \mathrm{L}$ & $<3.55 E-02$ & $<9.50 E-02$ & $1.42 \mathrm{E}-01$ \\
\hline Mo & $\mathrm{mg} / \mathrm{L}$ & $2.47 \mathrm{E}+00$ & $2.13 \mathrm{E}+00$ & $2.31 \mathrm{E}+00$ \\
\hline $\mathrm{Na}$ & $\mathrm{mg} / \mathrm{L}$ & $3.28 \mathrm{E}+04$ & $3.27 \mathrm{E}+04$ & $3.24 \mathrm{E}+04$ \\
\hline $\mathrm{Ni}$ & $\mathrm{mg} / \mathrm{L}$ & $<7.10 E-01$ & $<6.91 E-01$ & $<7.84 E-01$ \\
\hline $\mathrm{P}$ & $\mathrm{mg} / \mathrm{L}$ & $1.31 \mathrm{E}+01$ & $1.19 \mathrm{E}+01$ & $1.27 \mathrm{E}+01$ \\
\hline $\mathrm{S}$ & $\mathrm{mg} / \mathrm{L}$ & $3.47 \mathrm{E}+01$ & $<3.24 E+01$ & $3.85 \mathrm{E}+01$ \\
\hline $\mathrm{Si}$ & $\mathrm{mg} / \mathrm{L}$ & $6.83 \mathrm{E}+01$ & $6.76 \mathrm{E}+01$ & $7.29 \mathrm{E}+01$ \\
\hline $\mathrm{Sr}$ & $\mathrm{mg} / \mathrm{L}$ & $<2.66 E-02$ & $<2.59 E-02$ & $<2.94 E-02$ \\
\hline $\mathrm{U}$ & $\mathrm{mg} / \mathrm{L}$ & $1.60 \mathrm{E}+02$ & $7.33 \mathrm{E}+01$ & $6.14 \mathrm{E}+01$ \\
\hline $\mathrm{Zr}$ & $\mathrm{mg} / \mathrm{L}$ & $3.86 \mathrm{E}-01$ & $2.76 \mathrm{E}-01$ & $2.50 \mathrm{E}-01$ \\
\hline $\mathrm{Hg}$ & $\mathrm{mg} / \mathrm{L}$ & -- & -- & $5.11 \mathrm{E}+00$ \\
\hline Oxalate & $\mathrm{mg} / \mathrm{L}$ & -- & -- & $1.21 \mathrm{E}+03$ \\
\hline m-234 (U) & $\mathrm{mg} / \mathrm{L}$ & $<1.67 E-01$ & $<4.32 E-02$ & $<1.57 E-01$ \\
\hline m-235 (U) & $\mathrm{mg} / \mathrm{L}$ & $1.04 \mathrm{E}+00$ & 4.79E-01 & $4.08 \mathrm{E}-01$ \\
\hline m-236(U) & $\mathrm{mg} / \mathrm{L}$ & $<1.11 E-01$ & $<4.32 E-02$ & $<9.80 E-02$ \\
\hline m-237 (Np) & $\mathrm{mg} / \mathrm{L}$ & $<1.11 E-01$ & $<2.38 E-01$ & $<5.88 E-02$ \\
\hline m-238 (U,Pu) & $\mathrm{mg} / \mathrm{L}$ & $1.52 \mathrm{E}+02$ & $7.46 \mathrm{E}+01$ & $5.80 \mathrm{E}+01$ \\
\hline $\mathrm{m}-239(\mathrm{Pu})$ & $\mathrm{mg} / \mathrm{L}$ & $<1.67 E-01$ & 8.98E-02 & $<1.18 E-01$ \\
\hline $\mathrm{Pu}-238$ & $\mathrm{dpm} / \mathrm{mL}$ & $1.07 \mathrm{E}+03$ & $1.12 \mathrm{E}+03$ & $1.76 \mathrm{E}+03$ \\
\hline $\mathrm{Pu}-239 / 240$ & $\mathrm{dpm} / \mathrm{mL}$ & $<6.13 E+02$ & $4.91 \mathrm{E}+02$ & $1.08 \mathrm{E}+03$ \\
\hline Sr-90 & $\mathrm{dpm} / \mathrm{mL}$ & -- & -- & $<2.95 E+05$ \\
\hline Cs-137 & $\mathrm{dpm} / \mathrm{mL}$ & -- & -- & $9.72 \mathrm{E}+07$ \\
\hline Am-241 & $\mathrm{dpm} / \mathrm{mL}$ & -- & -- & $<4.79 E+01$ \\
\hline $\mathrm{Cm}-244$ & $\mathrm{dpm} / \mathrm{mL}$ & -- & -- & $3.54 \mathrm{E}+01$ \\
\hline
\end{tabular}


Table 3-35: Soluble Components in the Deposition Tank for Test 2 (Tank 5F No-Light), Storage Condition 6: With Evaporation, Without Sludge Heel, Without pH Adjustment, T $=50{ }^{\circ} \mathrm{C}$.

\begin{tabular}{|c|c|c|c|c|}
\hline \multirow{2}{*}{ analyte } & \multirow{2}{*}{ units } & \multicolumn{3}{|c|}{ deposition tank holding time } \\
\hline & & 1 day & 8 days & 15 days \\
\hline $\mathrm{pH}$ & -- & 9.27 & - & 9.02 \\
\hline ORP & $\mathrm{mV}$ & 144 & -- & 51.1 \\
\hline $\mathrm{Al}$ & $\mathrm{mg} / \mathrm{L}$ & $<1.57 E+01$ & $2.45 \mathrm{E}+01$ & $2.49 \mathrm{E}+01$ \\
\hline $\mathrm{Ba}$ & $\mathrm{mg} / \mathrm{L}$ & $6.90 \mathrm{E}-01$ & 7.61E-01 & $<3.77 E-01$ \\
\hline $\mathrm{Ca}$ & $\mathrm{mg} / \mathrm{L}$ & $5.75 \mathrm{E}+00$ & $5.98 \mathrm{E}+00$ & $6.80 \mathrm{E}+00$ \\
\hline $\mathrm{Cd}$ & $\mathrm{mg} / \mathrm{L}$ & $4.66 \mathrm{E}-01$ & 4.11E-01 & $<1.89 E-01$ \\
\hline $\mathrm{Cr}$ & $\mathrm{mg} / \mathrm{L}$ & $5.31 \mathrm{E}+01$ & $5.16 \mathrm{E}+01$ & $5.27 \mathrm{E}+01$ \\
\hline $\mathrm{Fe}$ & $\mathrm{mg} / \mathrm{L}$ & $1.04 \mathrm{E}+00$ & $1.23 \mathrm{E}+00$ & $8.90 \mathrm{E}-01$ \\
\hline $\mathrm{K}$ & $\mathrm{mg} / \mathrm{L}$ & $8.48 \mathrm{E}+01$ & $8.10 \mathrm{E}+01$ & $7.51 \mathrm{E}+01$ \\
\hline $\mathrm{Li}$ & $\mathrm{mg} / \mathrm{L}$ & $1.29 \mathrm{E}+01$ & $1.10 \mathrm{E}+01$ & $1.04 \mathrm{E}+01$ \\
\hline $\mathrm{Mg}$ & $\mathrm{mg} / \mathrm{L}$ & $6.47 \mathrm{E}-01$ & $4.26 \mathrm{E}-01$ & $5.24 \mathrm{E}-01$ \\
\hline $\mathrm{Mn}$ & $\mathrm{mg} / \mathrm{L}$ & $4.45 \mathrm{E}-01$ & $6.81 \mathrm{E}-01$ & 7.78E-01 \\
\hline Mo & $\mathrm{mg} / \mathrm{L}$ & $4.14 \mathrm{E}+00$ & $2.45 \mathrm{E}+00$ & $4.22 \mathrm{E}+00$ \\
\hline $\mathrm{Na}$ & $\mathrm{mg} / \mathrm{L}$ & $3.06 \mathrm{E}+03$ & $2.75 \mathrm{E}+03$ & $2.78 \mathrm{E}+03$ \\
\hline $\mathrm{Ni}$ & $\mathrm{mg} / \mathrm{L}$ & $1.15 \mathrm{E}+00$ & $<8.01 E-01$ & $<9.43 E-01$ \\
\hline $\mathrm{P}$ & $\mathrm{mg} / \mathrm{L}$ & $<2.93 E+00$ & $<8.21 E+00$ & $<9.66 E+00$ \\
\hline $\mathrm{S}$ & $\mathrm{mg} / \mathrm{L}$ & $2.38 \mathrm{E}+01$ & $<3.76 E+01$ & $<4.42 E+01$ \\
\hline $\mathrm{Si}$ & $\mathrm{mg} / \mathrm{L}$ & $4.45 \mathrm{E}+00$ & $<2.20 E+00$ & $<2.59 E+00$ \\
\hline $\mathrm{Sr}$ & $\mathrm{mg} / \mathrm{L}$ & $3.41 \mathrm{E}-01$ & $2.75 \mathrm{E}-01$ & $3.10 \mathrm{E}-01$ \\
\hline $\mathrm{U}$ & $\mathrm{mg} / \mathrm{L}$ & $2.50 \mathrm{E}+03$ & $2.34 \mathrm{E}+03$ & $2.46 \mathrm{E}+03$ \\
\hline $\mathrm{Zr}$ & $\mathrm{mg} / \mathrm{L}$ & $<2.23 E+00$ & $<1.30 E-01$ & $<1.53 E-01$ \\
\hline $\mathrm{Hg}$ & $\mathrm{mg} / \mathrm{L}$ & -- & -- & $9.49 \mathrm{E}-01$ \\
\hline Oxalate & $\mathrm{mg} / \mathrm{L}$ & -- & -- & $1.27 \mathrm{E}+03$ \\
\hline $\mathrm{m}-234(\mathrm{U})$ & $\mathrm{mg} / \mathrm{L}$ & $2.23 \mathrm{E}-01$ & $<1.00 E+00$ & $<4.71 E+00$ \\
\hline $\mathrm{m}-235(\mathrm{U})$ & $\mathrm{mg} / \mathrm{L}$ & $1.64 \mathrm{E}+01$ & $1.49 \mathrm{E}+01$ & $1.50 \mathrm{E}+01$ \\
\hline $\mathrm{m}-236(\mathrm{U})$ & $\mathrm{mg} / \mathrm{L}$ & $1.34 \mathrm{E}+00$ & $<1.00 E+00$ & $<2.95 E+00$ \\
\hline $\mathrm{m}-237(\mathrm{~Np})$ & $\mathrm{mg} / \mathrm{L}$ & $<1.12 E-01$ & $<5.51 E+00$ & $<1.77 E+00$ \\
\hline $\mathrm{m}-238(\mathrm{U}, \mathrm{Pu})$ & $\mathrm{mg} / \mathrm{L}$ & $2.33 \mathrm{E}+03$ & $2.44 \mathrm{E}+03$ & $2.36 \mathrm{E}+03$ \\
\hline $\mathrm{m}-239(\mathrm{Pu})$ & $\mathrm{mg} / \mathrm{L}$ & $1.74 \mathrm{E}-01$ & $1.96 \mathrm{E}+00$ & $<3.53 E+00$ \\
\hline $\mathrm{Pu}-238$ & $\mathrm{dpm} / \mathrm{mL}$ & $1.59 \mathrm{E}+02$ & $1.60 \mathrm{E}+02$ & $1.89 \mathrm{E}+02$ \\
\hline $\mathrm{Pu}-239 / 240$ & $\mathrm{dpm} / \mathrm{mL}$ & $<3.21 E+02$ & $<3.99 E+02$ & $8.70 \mathrm{E}+01$ \\
\hline Sr-90 & $\mathrm{dpm} / \mathrm{mL}$ & -- & -- & $1.87 \mathrm{E}+07$ \\
\hline Cs -137 & $\mathrm{dpm} / \mathrm{mL}$ & -- & -- & $9.28 \mathrm{E}+07$ \\
\hline Am-241 & $\mathrm{dpm} / \mathrm{mL}$ & -- & -- & -- \\
\hline $\mathrm{Cm}-244$ & $\mathrm{dpm} / \mathrm{mL}$ & -- & -- & -- \\
\hline
\end{tabular}


Table 3-36: Soluble Components in the Deposition Tank for Test 2 (Tank 5F No-Light), Storage Condition 7: Without Evaporation, With Sludge Heel, With pH Adjustment, $\mathbf{T}=$ $\sim 25^{\circ} \mathrm{C}$ (ambient).

\begin{tabular}{|c|c|c|c|c|}
\hline \multirow{2}{*}{ analyte } & \multirow{2}{*}{ units } & \multicolumn{3}{|c|}{ deposition tank holding time } \\
\hline & & 1 day & 8 days & 15 days \\
\hline $\mathrm{pH}$ & -- & $>12$ & -- & $>12$ \\
\hline ORP & $\mathrm{mV}$ & 56 & -- & 63.5 \\
\hline Al & $\mathrm{mg} / \mathrm{L}$ & $1.38 \mathrm{E}+02$ & $1.55 \mathrm{E}+02$ & $1.54 \mathrm{E}+02$ \\
\hline $\mathrm{Ba}$ & $\mathrm{mg} / \mathrm{L}$ & $7.78 \mathrm{E}-01$ & 7.30E-01 & $<3.19 E-01$ \\
\hline $\mathrm{Ca}$ & $\mathrm{mg} / \mathrm{L}$ & 4.77E-01 & $3.61 \mathrm{E}-01$ & $1.26 \mathrm{E}+00$ \\
\hline $\mathrm{Cd}$ & $\mathrm{mg} / \mathrm{L}$ & $3.68 \mathrm{E}-01$ & 3.59E-01 & $<1.60 E-01$ \\
\hline $\mathrm{Cr}$ & $\mathrm{mg} / \mathrm{L}$ & $5.31 \mathrm{E}+00$ & $5.49 \mathrm{E}+00$ & $5.67 \mathrm{E}+00$ \\
\hline $\mathrm{Fe}$ & $\mathrm{mg} / \mathrm{L}$ & $1.68 \mathrm{E}+00$ & $1.61 \mathrm{E}+00$ & $2.08 \mathrm{E}+00$ \\
\hline $\mathrm{K}$ & $\mathrm{mg} / \mathrm{L}$ & $<1.43 E+01$ & $1.43 \mathrm{E}+01$ & $1.62 \mathrm{E}+01$ \\
\hline $\mathrm{Li}$ & $\mathrm{mg} / \mathrm{L}$ & $1.54 \mathrm{E}+00$ & $1.68 \mathrm{E}+00$ & $1.71 \mathrm{E}+00$ \\
\hline $\mathrm{Mg}$ & $\mathrm{mg} / \mathrm{L}$ & $<5.73 E-02$ & $<5.15 E-02$ & $1.75 \mathrm{E}-01$ \\
\hline $\mathrm{Mn}$ & $\mathrm{mg} / \mathrm{L}$ & $<3.82 E-02$ & $<9.44 E-02$ & $1.20 \mathrm{E}-01$ \\
\hline Mo & $\mathrm{mg} / \mathrm{L}$ & $<1.28 E+00$ & $<1.15 E+00$ & $<1.34 E+00$ \\
\hline $\mathrm{Na}$ & $\mathrm{mg} / \mathrm{L}$ & $2.62 \mathrm{E}+04$ & $2.51 \mathrm{E}+04$ & $2.81 \mathrm{E}+04$ \\
\hline $\mathrm{Ni}$ & $\mathrm{mg} / \mathrm{L}$ & $<7.64 E-01$ & $<6.87 E-01$ & $<7.98 E-01$ \\
\hline $\mathrm{P}$ & $\mathrm{mg} / \mathrm{L}$ & $<7.83 E+00$ & $<7.04 E+00$ & $<8.18 E+00$ \\
\hline $\mathrm{S}$ & $\mathrm{mg} / \mathrm{L}$ & $4.35 \mathrm{E}+01$ & $<3.22 E+01$ & $<3.74 E+01$ \\
\hline $\mathrm{Si}$ & $\mathrm{mg} / \mathrm{L}$ & $2.39 \mathrm{E}+01$ & $2.59 \mathrm{E}+01$ & $2.73 \mathrm{E}+01$ \\
\hline $\mathrm{Sr}$ & $\mathrm{mg} / \mathrm{L}$ & $<2.86 E-02$ & $<2.58 E-02$ & $<2.99 E-02$ \\
\hline $\mathrm{U}$ & $\mathrm{mg} / \mathrm{L}$ & $1.30 \mathrm{E}+02$ & $1.03 \mathrm{E}+02$ & $8.87 \mathrm{E}+01$ \\
\hline $\mathrm{Zr}$ & $\mathrm{mg} / \mathrm{L}$ & 4.87E-01 & $3.05 \mathrm{E}-01$ & 2.64E-01 \\
\hline $\mathrm{Hg}$ & $\mathrm{mg} / \mathrm{L}$ & -- & -- & $6.58 \mathrm{E}-01$ \\
\hline Oxalate & $\mathrm{mg} / \mathrm{L}$ & -- & -- & $1.10 \mathrm{E}+02$ \\
\hline m-234 (U) & $\mathrm{mg} / \mathrm{L}$ & $<1.79 E-01$ & 4.43E-02 & $<1.60 E-01$ \\
\hline m-235 (U) & $\mathrm{mg} / \mathrm{L}$ & $1.04 \mathrm{E}+00$ & $6.71 \mathrm{E}-01$ & $6.08 \mathrm{E}-01$ \\
\hline m-236(U) & $\mathrm{mg} / \mathrm{L}$ & $<1.19 E-01$ & $<4.29 E-02$ & $<9.97 E-02$ \\
\hline $\mathrm{m}-237(\mathrm{~Np})$ & $\mathrm{mg} / \mathrm{L}$ & $<1.19 E-01$ & $<2.36 E-01$ & $<5.98 E-02$ \\
\hline m-238 (U,Pu) & $\mathrm{mg} / \mathrm{L}$ & $1.33 \mathrm{E}+02$ & $1.05 \mathrm{E}+02$ & $8.55 \mathrm{E}+01$ \\
\hline $\mathrm{m}-239(\mathrm{Pu})$ & $\mathrm{mg} / \mathrm{L}$ & $<1.79 E-01$ & 8.84E-02 & $<1.20 E-01$ \\
\hline $\mathrm{Pu}-238$ & $\mathrm{dpm} / \mathrm{mL}$ & $8.55 \mathrm{E}+02$ & $5.66 \mathrm{E}+02$ & $1.07 \mathrm{E}+03$ \\
\hline $\mathrm{Pu}-239 / 240$ & $\mathrm{dpm} / \mathrm{mL}$ & $<1.08 E+03$ & $3.71 \mathrm{E}+02$ & $3.40 \mathrm{E}+02$ \\
\hline Sr-90 & $\mathrm{dpm} / \mathrm{mL}$ & -- & -- & $<3.13 E+05$ \\
\hline Cs -137 & $\mathrm{dpm} / \mathrm{mL}$ & -- & -- & $1.56 \mathrm{E}+07$ \\
\hline Am-241 & $\mathrm{dpm} / \mathrm{mL}$ & -- & -- & $<2.66 E+02$ \\
\hline $\mathrm{Cm}-244$ & $\mathrm{dpm} / \mathrm{mL}$ & -- & -- & $6.60 \mathrm{E}+01$ \\
\hline
\end{tabular}


Table 3-37: Soluble Components in the Deposition Tank for Test 2 (Tank 5F No-Light), Storage Condition 8: Without Evaporation, With Sludge Heel, With pH Adjustment, $\mathbf{T}=$ $70^{\circ} \mathrm{C}$.

\begin{tabular}{|c|c|c|c|c|}
\hline \multirow{2}{*}{ analyte } & \multirow{2}{*}{ units } & \multicolumn{3}{|c|}{ deposition tank holding time } \\
\hline & & 1 day & 8 days & 15 days \\
\hline $\mathrm{pH}$ & -- & $>12$ & -- & $>12$ \\
\hline ORP & $\mathrm{mV}$ & 58 & -- & 65.5 \\
\hline $\mathrm{Al}$ & $\mathrm{mg} / \mathrm{L}$ & $1.67 \mathrm{E}+02$ & $1.77 \mathrm{E}+02$ & $1.89 \mathrm{E}+02$ \\
\hline $\mathrm{Ba}$ & $\mathrm{mg} / \mathrm{L}$ & 7.43E-01 & 7.59E-01 & $<3.08 E-01$ \\
\hline $\mathrm{Ca}$ & $\mathrm{mg} / \mathrm{L}$ & $5.68 \mathrm{E}-01$ & $5.21 \mathrm{E}-01$ & $1.50 \mathrm{E}+00$ \\
\hline $\mathrm{Cd}$ & $\mathrm{mg} / \mathrm{L}$ & 4.07E-01 & 3.58E-01 & $<1.54 E-01$ \\
\hline $\mathrm{Cr}$ & $\mathrm{mg} / \mathrm{L}$ & $5.60 \mathrm{E}+00$ & $5.55 \mathrm{E}+00$ & $6.08 \mathrm{E}+00$ \\
\hline $\mathrm{Fe}$ & $\mathrm{mg} / \mathrm{L}$ & $2.54 \mathrm{E}+00$ & $1.86 \mathrm{E}+00$ & $1.90 \mathrm{E}+00$ \\
\hline K & $\mathrm{mg} / \mathrm{L}$ & $1.29 \mathrm{E}+01$ & $1.36 \mathrm{E}+01$ & $1.75 \mathrm{E}+01$ \\
\hline $\mathrm{Li}$ & $\mathrm{mg} / \mathrm{L}$ & $1.79 \mathrm{E}+00$ & $1.77 \mathrm{E}+00$ & $1.99 \mathrm{E}+00$ \\
\hline $\mathrm{Mg}$ & $\mathrm{mg} / \mathrm{L}$ & $<5.25 E-02$ & $8.54 \mathrm{E}-02$ & $1.73 \mathrm{E}-01$ \\
\hline $\mathrm{Mn}$ & $\mathrm{mg} / \mathrm{L}$ & $<3.50 E-02$ & $<9.89 E-02$ & $1.25 \mathrm{E}-01$ \\
\hline Mo & $\mathrm{mg} / \mathrm{L}$ & $<1.17 E+00$ & $<1.20 E+00$ & $<1.29 E+00$ \\
\hline $\mathrm{Na}$ & $\mathrm{mg} / \mathrm{L}$ & $2.53 \mathrm{E}+04$ & $2.50 \mathrm{E}+04$ & $2.55 \mathrm{E}+04$ \\
\hline $\mathrm{Ni}$ & $\mathrm{mg} / \mathrm{L}$ & $<7.00 E-01$ & $<7.19 E-01$ & $<7.70 E-01$ \\
\hline $\mathrm{P}$ & $\mathrm{mg} / \mathrm{L}$ & $<7.17 E+00$ & $<7.37 E+00$ & $<7.89 E+00$ \\
\hline $\mathrm{S}$ & $\mathrm{mg} / \mathrm{L}$ & $<3.28 E+01$ & $<3.37 E+01$ & $<3.61 E+01$ \\
\hline $\mathrm{Si}$ & $\mathrm{mg} / \mathrm{L}$ & $2.69 \mathrm{E}+01$ & $2.73 \mathrm{E}+01$ & $3.09 \mathrm{E}+01$ \\
\hline $\mathrm{Sr}$ & $\mathrm{mg} / \mathrm{L}$ & $<2.62 E-02$ & $<2.70 E-02$ & $<2.89 E-02$ \\
\hline $\mathrm{U}$ & $\mathrm{mg} / \mathrm{L}$ & $7.27 \mathrm{E}+01$ & $4.24 \mathrm{E}+01$ & $3.93 \mathrm{E}+01$ \\
\hline $\mathrm{Zr}$ & $\mathrm{mg} / \mathrm{L}$ & $3.28 \mathrm{E}-01$ & $1.39 \mathrm{E}-01$ & $<1.25 E-01$ \\
\hline $\mathrm{Hg}$ & $\mathrm{mg} / \mathrm{L}$ & -- & -- & $<4.81 E-01$ \\
\hline Oxalate & $\mathrm{mg} / \mathrm{L}$ & -- & -- & $1.73 \mathrm{E}+02$ \\
\hline $\mathrm{m}-234(\mathrm{U})$ & $\mathrm{mg} / \mathrm{L}$ & $<1.64 E-01$ & $<1.80 E-02$ & $<1.54 E-01$ \\
\hline $\mathrm{m}-235(\mathrm{U})$ & $\mathrm{mg} / \mathrm{L}$ & 4.50E-01 & $2.88 \mathrm{E}-01$ & $2.34 \mathrm{E}-01$ \\
\hline $\mathrm{m}-236(\mathrm{U})$ & $\mathrm{mg} / \mathrm{L}$ & $<1.09 E-01$ & $<1.80 E-02$ & $<9.63 E-02$ \\
\hline $\mathrm{m}-237(\mathrm{~Np})$ & $\mathrm{mg} / \mathrm{L}$ & $<1.09 E-01$ & $<9.89 E-02$ & $<5.78 E-02$ \\
\hline m-238 (U,Pu) & $\mathrm{mg} / \mathrm{L}$ & $6.85 \mathrm{E}+01$ & $4.45 \mathrm{E}+01$ & $3.36 \mathrm{E}+01$ \\
\hline $\mathrm{m}-239(\mathrm{Pu})$ & $\mathrm{mg} / \mathrm{L}$ & $<1.64 E-01$ & $3.87 \mathrm{E}-02$ & $<1.16 E-01$ \\
\hline $\mathrm{Pu}-238$ & $\mathrm{dpm} / \mathrm{mL}$ & $1.98 \mathrm{E}+03$ & $<6.10 E+02$ & $9.11 \mathrm{E}+02$ \\
\hline $\mathrm{Pu}-239 / 240$ & $\mathrm{dpm} / \mathrm{mL}$ & $<2.81 E+03$ & $1.14 \mathrm{E}+03$ & $5.15 \mathrm{E}+02$ \\
\hline Sr-90 & $\mathrm{dpm} / \mathrm{mL}$ & -- & -- & $<2.99 E+05$ \\
\hline Cs-137 & $\mathrm{dpm} / \mathrm{mL}$ & -- & -- & $1.52 \mathrm{E}+07$ \\
\hline Am-241 & $\mathrm{dpm} / \mathrm{mL}$ & -- & -- & $<2.36 E+02$ \\
\hline $\mathrm{Cm}-244$ & $\mathrm{dpm} / \mathrm{mL}$ & -- & -- & $3.09 \mathrm{E}+01$ \\
\hline
\end{tabular}


Table 3-38: Soluble Components in the Deposition Tank for the Tank 5F Control (without ECC Processing): Without Evaporation, With Sludge Heel, With pH Adjustment, T = 50

${ }^{\circ} \mathrm{C}$.

\begin{tabular}{|c|c|c|c|c|}
\hline \multirow{2}{*}{ analyte } & \multirow{2}{*}{ units } & \multicolumn{3}{|c|}{ deposition tank holding time } \\
\hline & & 1 day & 8 days & 15 days \\
\hline $\mathrm{pH}$ & -- & $>12$ & -- & $>12$ \\
\hline ORP & $\mathrm{mV}$ & 59 & -- & 54.2 \\
\hline $\mathrm{Al}$ & $\mathrm{mg} / \mathrm{L}$ & $3.52 \mathrm{E}+02$ & $3.80 \mathrm{E}+02$ & $3.79 \mathrm{E}+02$ \\
\hline $\mathrm{Ba}$ & $\mathrm{mg} / \mathrm{L}$ & $8.65 \mathrm{E}-01$ & 7.57E-01 & $<2.93 E-01$ \\
\hline $\mathrm{Ca}$ & $\mathrm{mg} / \mathrm{L}$ & $6.86 \mathrm{E}-01$ & 4.38E-01 & $1.16 \mathrm{E}+00$ \\
\hline $\mathrm{Cd}$ & $\mathrm{mg} / \mathrm{L}$ & 4.65E-01 & 4.16E-01 & $<1.46 E-01$ \\
\hline $\mathrm{Cr}$ & $\mathrm{mg} / \mathrm{L}$ & $1.08 \mathrm{E}+00$ & $1.06 \mathrm{E}+00$ & $1.35 \mathrm{E}+00$ \\
\hline $\mathrm{Fe}$ & $\mathrm{mg} / \mathrm{L}$ & $1.43 \mathrm{E}+00$ & $1.34 \mathrm{E}+00$ & $2.28 \mathrm{E}+00$ \\
\hline $\mathrm{K}$ & $\mathrm{mg} / \mathrm{L}$ & $2.15 \mathrm{E}+01$ & $1.73 \mathrm{E}+01$ & $1.89 \mathrm{E}+01$ \\
\hline $\mathrm{Li}$ & $\mathrm{mg} / \mathrm{L}$ & $<6.86 E-01$ & $<6.46 E-01$ & $<6.68 E-01$ \\
\hline $\mathrm{Mg}$ & $\mathrm{mg} / \mathrm{L}$ & $<5.64 E-02$ & $<5.31 E-02$ & $1.83 \mathrm{E}-01$ \\
\hline $\mathrm{Mn}$ & $\mathrm{mg} / \mathrm{L}$ & $<3.76 E-02$ & $<9.74 E-02$ & $2.15 \mathrm{E}-01$ \\
\hline Mo & $\mathrm{mg} / \mathrm{L}$ & $<1.26 E+00$ & $<1.19 E+00$ & $<1.23 E+00$ \\
\hline $\mathrm{Na}$ & $\mathrm{mg} / \mathrm{L}$ & $4.68 \mathrm{E}+04$ & $4.20 \mathrm{E}+04$ & $4.35 \mathrm{E}+04$ \\
\hline $\mathrm{Ni}$ & $\mathrm{mg} / \mathrm{L}$ & $<7.52 E-01$ & $<7.08 E-01$ & $<7.32 E-01$ \\
\hline $\mathrm{P}$ & $\mathrm{mg} / \mathrm{L}$ & $<7.71 E+00$ & $6.73 \mathrm{E}+00$ & $<7.50 E+00$ \\
\hline $\mathrm{S}$ & $\mathrm{mg} / \mathrm{L}$ & $3.84 \mathrm{E}+01$ & $<3.32 E+01$ & $3.87 \mathrm{E}+01$ \\
\hline $\mathrm{Si}$ & $\mathrm{mg} / \mathrm{L}$ & $4.41 \mathrm{E}+01$ & $6.02 \mathrm{E}+01$ & $6.36 \mathrm{E}+01$ \\
\hline $\mathrm{Sr}$ & $\mathrm{mg} / \mathrm{L}$ & $<2.82 E-02$ & $<2.66 E-02$ & $<2.74 E-02$ \\
\hline $\mathrm{U}$ & $\mathrm{mg} / \mathrm{L}$ & $<1.53 E+01$ & $<1.44 E+01$ & $<1.49 E+01$ \\
\hline $\mathrm{Zr}$ & $\mathrm{mg} / \mathrm{L}$ & $<1.22 E-01$ & $<1.15 E-01$ & $<1.19 E-01$ \\
\hline $\mathrm{Hg}$ & $\mathrm{mg} / \mathrm{L}$ & -- & -- & $2.33 \mathrm{E}+00$ \\
\hline Oxalate & $\mathrm{mg} / \mathrm{L}$ & -- & -- & $<9.14 E+01$ \\
\hline $\mathrm{m}-234(\mathrm{U})$ & $\mathrm{mg} / \mathrm{L}$ & $<1.41 E-02$ & $<4.43 E-03$ & $<1.46 E-01$ \\
\hline $\mathrm{m}-235(\mathrm{U})$ & $\mathrm{mg} / \mathrm{L}$ & 4.12E-02 & 4.74E-02 & $6.76 \mathrm{E}-02$ \\
\hline $\mathrm{m}-236(\mathrm{U})$ & $\mathrm{mg} / \mathrm{L}$ & $<9.40 E-03$ & $<4.43 E-03$ & $<9.14 E-02$ \\
\hline $\mathrm{m}-237(\mathrm{~Np})$ & $\mathrm{mg} / \mathrm{L}$ & $<9.40 E-03$ & $<2.43 E-02$ & $<5.49 E-02$ \\
\hline $\mathrm{m}-238(\mathrm{U}, \mathrm{Pu})$ & $\mathrm{mg} / \mathrm{L}$ & $5.91 \mathrm{E}+00$ & $6.87 \mathrm{E}+00$ & $6.70 \mathrm{E}+00$ \\
\hline $\mathrm{m}-239(\mathrm{Pu})$ & $\mathrm{mg} / \mathrm{L}$ & $<1.41 E-02$ & $8.71 \mathrm{E}-03$ & $<1.10 E-01$ \\
\hline $\mathrm{Pu}-238$ & $\mathrm{dpm} / \mathrm{mL}$ & $<8.96 E+02$ & $<7.71 E+02$ & $7.38 \mathrm{E}+02$ \\
\hline $\mathrm{Pu}-239 / 240$ & $\mathrm{dpm} / \mathrm{mL}$ & $<1.17 E+03$ & $9.56 \mathrm{E}+02$ & $1.28 \mathrm{E}+02$ \\
\hline Sr-90 & $\mathrm{dpm} / \mathrm{mL}$ & -- & -- & $<2.69 E+05$ \\
\hline Cs -137 & $\mathrm{dpm} / \mathrm{mL}$ & -- & -- & $2.49 \mathrm{E}+07$ \\
\hline Am-241 & $\mathrm{dpm} / \mathrm{mL}$ & -- & -- & $<9.69 E+01$ \\
\hline $\mathrm{Cm}-244$ & $\mathrm{dpm} / \mathrm{mL}$ & -- & -- & $2.62 \mathrm{E}+02$ \\
\hline
\end{tabular}


SRNL-STI-2011-00360

Revision 0

Table 3-39: Deposition Tank Storage Condition Summary for Soluble U-238 (mg/L)

\begin{tabular}{|c|c|c|c|c|c|c|c|c|c|c|}
\hline 苛 & $\underbrace{0}_{0}$ & $\approx$ & & & & & U-23 & ng/L) & & \\
\hline : & 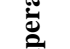 & 苛 & : & $e_{00}^{\circ}$ & & 1 (Tank & & & t 2 (Tank & \\
\hline & 离 & 童 & 空 & $\stackrel{\Xi}{\omega}$ & 7 days & 21 days & 35 days & 1 day & 8 days & 15 days \\
\hline 1 & 50 & No & Yes & No & $2.59 \mathrm{E}+01$ & $2.53 \mathrm{E}+01$ & $2.56 \mathrm{E}+01$ & $1.22 \mathrm{E}+02$ & $7.46 \mathrm{E}+01$ & $4.15 \mathrm{E}+01$ \\
\hline 2 & 50 & No & Yes & Yes & $3.95 \mathrm{E}+01$ & $3.88 \mathrm{E}+01$ & $3.85 \mathrm{E}+01$ & $1.06 \mathrm{E}+02$ & $7.48 \mathrm{E}+01$ & $5.62 \mathrm{E}+01$ \\
\hline 3 & 50 & No & No & No & $5.19 \mathrm{E}+00$ & $5.32 \mathrm{E}+00$ & $5.31 \mathrm{E}+00$ & $9.87 \mathrm{E}+01$ & $9.62 \mathrm{E}+01$ & $8.35 \mathrm{E}+01$ \\
\hline 4 & 50 & Yes & Yes & No & $7.77 \mathrm{E}+01$ & $6.99 \mathrm{E}+01$ & $6.41 \mathrm{E}+01$ & $1.50 \mathrm{E}+02$ & $7.50 \mathrm{E}+01$ & $5.53 \mathrm{E}+01$ \\
\hline 5 & 50 & Yes & Yes & Yes & $7.78 \mathrm{E}+01$ & $6.79 \mathrm{E}+01$ & $6.27 \mathrm{E}+01$ & $1.52 \mathrm{E}+02$ & $7.46 \mathrm{E}+01$ & $5.80 \mathrm{E}+01$ \\
\hline 6 & 50 & Yes & No & No & $8.25 \mathrm{E}+01$ & $1.94 \mathrm{E}+02$ & $1.88 \mathrm{E}+02$ & $2.33 \mathrm{E}+03$ & $2.44 \mathrm{E}+03$ & $2.36 \mathrm{E}+03$ \\
\hline 7 & $\sim 25$ & $*$ & Yes & Yes & $6.36 \mathrm{E}+01$ & $6.03 \mathrm{E}+01$ & $5.75 \mathrm{E}+01$ & $1.33 \mathrm{E}+02$ & $1.05 \mathrm{E}+02$ & $8.55 \mathrm{E}+01$ \\
\hline 8 & 70 & $*$ & Yes & Yes & $6.01 \mathrm{E}+01$ & $4.55 \mathrm{E}+01$ & 4. $45 \mathrm{E}+01$ & $6.85 \mathrm{E}+01$ & $4.45 \mathrm{E}+01$ & $3.36 \mathrm{E}+01$ \\
\hline 9 & 50 & No & Yes & Yes & $1.40 \mathrm{E}+00$ & $1.67 \mathrm{E}+00$ & $1.81 \mathrm{E}+00$ & $5.91 \mathrm{E}+00$ & $6.87 \mathrm{E}+00$ & $6.70 \mathrm{E}+00$ \\
\hline
\end{tabular}

* With evaporation for Test 1, Without evaporation for Test 2

Table 3-40: Deposition Tank Storage Condition Summary for Soluble Pu-238 (dpm/mL)

\begin{tabular}{|c|c|c|c|c|c|c|c|c|c|c|}
\hline 苛 & $\underbrace{0}_{0}$ & $\approx$ & & & & & Pu-238 & $\mathrm{dpm} / \mathrm{mL}$ ) & & \\
\hline 品 & 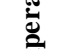 & 苛 & 孛 & $D_{0.0}^{\circ}$ & & t 1 (Tank 1 & & & est 2 (Tank 5 & \\
\hline & تِّ & 童 & & $\stackrel{\Xi}{\omega}$ & 7 days & 21 days & 35 days & 1 day & 8 days & 15 days \\
\hline 1 & 50 & No & Yes & No & $2.24 \mathrm{E}+05$ & $2.33 \mathrm{E}+05$ & $2.78 \mathrm{E}+05$ & $8.35 \mathrm{E}+02$ & $8.12 \mathrm{E}+02$ & $1.20 \mathrm{E}+02$ \\
\hline 2 & 50 & No & Yes & Yes & $4.62 \mathrm{E}+05$ & $4.97 \mathrm{E}+05$ & $5.34 \mathrm{E}+05$ & $6.99 \mathrm{E}+02$ & $3.54 \mathrm{E}+02$ & $7.68 \mathrm{E}+02$ \\
\hline 3 & 50 & No & No & No & $1.87 \mathrm{E}+05$ & $4.05 \mathrm{E}+04$ & $1.93 \mathrm{E}+04$ & $4.20 \mathrm{E}+02$ & $5.24 \mathrm{E}+02$ & $1.48 \mathrm{E}+02$ \\
\hline 4 & 50 & Yes & Yes & No & $3.55 \mathrm{E}+05$ & $3.11 \mathrm{E}+05$ & $3.25 \mathrm{E}+05$ & $2.60 \mathrm{E}+02$ & $2.61 \mathrm{E}+02$ & $7.83 \mathrm{E}+02$ \\
\hline 5 & 50 & Yes & Yes & Yes & $3.44 \mathrm{E}+05$ & $2.91 \mathrm{E}+05$ & $2.52 \mathrm{E}+05$ & $1.07 \mathrm{E}+03$ & $1.12 \mathrm{E}+03$ & $1.76 \mathrm{E}+03$ \\
\hline 6 & 50 & Yes & No & No & $1.35 \mathrm{E}+06$ & $1.33 \mathrm{E}+06$ & $1.86 \mathrm{E}+06$ & $1.59 \mathrm{E}+02$ & $1.60 \mathrm{E}+02$ & $1.89 \mathrm{E}+02$ \\
\hline 7 & $\sim 25$ & $*$ & Yes & Yes & $2.74 \mathrm{E}+05$ & $2.06 \mathrm{E}+05$ & $2.41 \mathrm{E}+05$ & $8.55 \mathrm{E}+02$ & $5.66 \mathrm{E}+02$ & $1.07 \mathrm{E}+03$ \\
\hline 8 & 70 & $*$ & Yes & Yes & $3.06 \mathrm{E}+05$ & $1.83 \mathrm{E}+05$ & $2.09 \mathrm{E}+05$ & $1.98 \mathrm{E}+03$ & $<6.10 E+02$ & $9.11 \mathrm{E}+02$ \\
\hline 9 & 50 & No & Yes & Yes & $2.32 \mathrm{E}+04$ & $6.91 \mathrm{E}+03$ & $5.26 \mathrm{E}+03$ & $<8.96 E+02$ & $<7.71 E+02$ & $7.38 \mathrm{E}+02$ \\
\hline
\end{tabular}

* With evaporation for Test 1 , Without evaporation for Test 2 
Table 3-41: Deposition Tank Storage Condition Summary for Soluble Iron (mg/L)

\begin{tabular}{|c|c|c|c|c|c|c|c|c|c|c|}
\hline 苛 & $\underbrace{0}_{0}$ & & & & & & & s/L) & & \\
\hline$\underset{8}{0}$ & 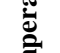 & 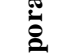 & 宗 & 范 & & t 1 (Tank & & & t 2 (Tank & \\
\hline & 离 & 童 & T & के & 7 days & 21 days & 35 days & 1 day & 8 days & 15 days \\
\hline 1 & 50 & No & Yes & No & $1.08 \mathrm{E}+00$ & $1.42 \mathrm{E}+00$ & $1.50 \mathrm{E}+00$ & $1.59 \mathrm{E}+00$ & $1.57 \mathrm{E}+00$ & $2.11 \mathrm{E}+00$ \\
\hline 2 & 50 & No & Yes & Yes & $3.82 \mathrm{E}+00$ & $1.56 \mathrm{E}+00$ & $4.62 \mathrm{E}+00$ & $1.89 \mathrm{E}+00$ & $1.59 \mathrm{E}+00$ & $1.12 \mathrm{E}+00$ \\
\hline 3 & 50 & No & No & No & $<9.00 E-01$ & $8.65 \mathrm{E}-01$ & 2.72E-01 & $2.07 \mathrm{E}+00$ & $2.15 \mathrm{E}+00$ & 8.67E-01 \\
\hline 4 & 50 & Yes & Yes & No & $4.06 \mathrm{E}+00$ & $2.99 \mathrm{E}+00$ & $2.16 \mathrm{E}+00$ & $1.84 \mathrm{E}+00$ & $1.99 \mathrm{E}+00$ & $1.63 \mathrm{E}+00$ \\
\hline 5 & 50 & Yes & Yes & Yes & $2.04 \mathrm{E}+00$ & $2.43 \mathrm{E}+00$ & $1.70 \mathrm{E}+00$ & $2.26 \mathrm{E}+00$ & $2.21 \mathrm{E}+00$ & $1.78 \mathrm{E}+00$ \\
\hline 6 & 50 & Yes & No & No & $<9.28 E-01$ & $8.70 \mathrm{E}-01$ & 2.67E-01 & $1.04 \mathrm{E}+00$ & $1.23 \mathrm{E}+00$ & 8.90E-01 \\
\hline 7 & $\sim 25$ & $*$ & Yes & Yes & $1.67 \mathrm{E}+00$ & $2.11 \mathrm{E}+00$ & $1.51 \mathrm{E}+00$ & $1.68 \mathrm{E}+00$ & $1.61 \mathrm{E}+00$ & $2.08 \mathrm{E}+00$ \\
\hline 8 & 70 & * & Yes & Yes & $2.44 \mathrm{E}+00$ & $1.92 \mathrm{E}+00$ & $1.62 \mathrm{E}+00$ & $2.54 \mathrm{E}+00$ & $1.86 \mathrm{E}+00$ & $1.90 \mathrm{E}+00$ \\
\hline 9 & 50 & No & Yes & Yes & $1.03 \mathrm{E}+00$ & $1.78 \mathrm{E}+00$ & $1.17 \mathrm{E}+00$ & $1.43 \mathrm{E}+00$ & $1.34 \mathrm{E}+00$ & $2.28 \mathrm{E}+00$ \\
\hline
\end{tabular}

* With evaporation for Test 1, Without evaporation for Test 2

Table 3-42: Deposition Tank Storage Condition Summary for Soluble Aluminum (mg/L)

\begin{tabular}{|c|c|c|c|c|c|c|c|c|c|c|}
\hline \multirow{3}{*}{ 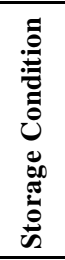 } & \multirow{3}{*}{ 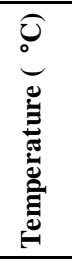 } & \multirow{3}{*}{ 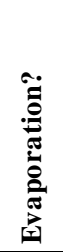 } & \multirow{3}{*}{ 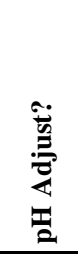 } & \multirow{3}{*}{ 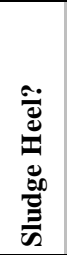 } & \multicolumn{6}{|c|}{$\mathrm{Al}(\mathrm{mg} / \mathrm{L})$} \\
\hline & & & & & \multicolumn{3}{|c|}{ Test 1 (Tank 12H) } & \multicolumn{3}{|c|}{ Test 2 (Tank 5F) } \\
\hline & & & & & 7 days & 21 days & 35 days & 1 day & 8 days & 15 days \\
\hline 1 & 50 & No & Yes & No & $2.16 \mathrm{E}+02$ & $2.99 \mathrm{E}+02$ & $3.33 \mathrm{E}+02$ & $1.03 \mathrm{E}+02$ & $1.16 \mathrm{E}+02$ & $8.69 \mathrm{E}+01$ \\
\hline 2 & 50 & No & Yes & Yes & $4.67 \mathrm{E}+02$ & $5.62 \mathrm{E}+02$ & $7.05 \mathrm{E}+02$ & $1.89 \mathrm{E}+02$ & $2.01 \mathrm{E}+02$ & $2.08 \mathrm{E}+02$ \\
\hline 3 & 50 & No & No & No & $<3.11 E+00$ & $<1.58 E+00$ & $1.08 \mathrm{E}+00$ & $6.92 \mathrm{E}-01$ & $1.65 \mathrm{E}+00$ & 7.47E-01 \\
\hline 4 & 50 & Yes & Yes & No & $1.25 \mathrm{E}+03$ & $1.46 \mathrm{E}+03$ & $1.58 \mathrm{E}+03$ & $4.26 \mathrm{E}+02$ & $4.77 \mathrm{E}+02$ & $5.03 \mathrm{E}+02$ \\
\hline 5 & 50 & Yes & Yes & Yes & $1.19 \mathrm{E}+03$ & $1.25 \mathrm{E}+03$ & $1.48 \mathrm{E}+03$ & $4.72 \mathrm{E}+02$ & $5.03 \mathrm{E}+02$ & $5.32 \mathrm{E}+02$ \\
\hline 6 & 50 & Yes & No & No & $9.56 \mathrm{E}+00$ & $7.83 \mathrm{E}+00$ & $7.63 \mathrm{E}+00$ & $<1.57 E+01$ & $2.45 \mathrm{E}+01$ & $2.49 \mathrm{E}+01$ \\
\hline 7 & $\sim 25$ & * & Yes & Yes & $6.83 \mathrm{E}+02$ & $7.30 \mathrm{E}+02$ & $7.45 \mathrm{E}+02$ & $1.38 \mathrm{E}+02$ & $1.55 \mathrm{E}+02$ & $1.54 \mathrm{E}+02$ \\
\hline 8 & 70 & $*$ & Yes & Yes & $1.46 \mathrm{E}+03$ & $1.58 \mathrm{E}+03$ & $1.95 \mathrm{E}+03$ & $1.67 \mathrm{E}+02$ & $1.77 \mathrm{E}+02$ & $1.89 \mathrm{E}+02$ \\
\hline 9 & 50 & No & Yes & Yes & $4.57 \mathrm{E}+02$ & $6.71 \mathrm{E}+02$ & $8.42 \mathrm{E}+02$ & $3.52 \mathrm{E}+02$ & $3.80 \mathrm{E}+02$ & $3.79 \mathrm{E}+02$ \\
\hline
\end{tabular}

* With evaporation for Test 1 , Without evaporation for Test 2 
Table 3-43: Analysis of Test 1 Deposition Tank Solids, Wet Basis

\begin{tabular}{|c|c|c|c|c|c|c|c|c|c|c|}
\hline \multicolumn{2}{|c|}{ Storage Condition: } & \multirow{2}{*}{$\frac{1}{<1.43 E+01}$} & \multirow{2}{*}{$\frac{2}{<1.40 E+01}$} & \multirow{2}{*}{$\frac{3}{<1.39 E+01}$} & \multirow{2}{*}{$\frac{4}{<1.40 E+01}$} & \multirow{2}{*}{$\frac{\mathbf{5}}{<1.43 E+01}$} & \multirow{2}{*}{$\frac{\mathbf{6}}{<1.38 E+01}$} & \multirow{2}{*}{$\frac{7}{<1.43 E+01}$} & \multirow{2}{*}{\begin{tabular}{|c|}
$\mathbf{8}$ \\
$<1.40 E+01$
\end{tabular}} & \multirow{2}{*}{$\frac{9}{<1.43 E+01}$} \\
\hline $\mathrm{Ag}$ & $\mathrm{ng} / \mathrm{kg}$ & & & & & & & & & \\
\hline Al & & $1.40 \mathrm{E}+03$ & $3.66 \mathrm{E}+03$ & $1.19 \mathrm{E}+04$ & $6.12 \mathrm{E}+03$ & $4.89 \mathrm{E}+03$ & $2.01 \mathrm{E}+04$ & $6.62 \mathrm{E}+03$ & $2.14 \mathrm{E}+03$ & $5.30 \mathrm{E}+03$ \\
\hline B & & $.98 E+01$ & $1.93 E+01$ & $1.93 E+01$ & $1.93 E+01$ & $1.98 E+01$ & $1.91 E+01$ & $<1.98 E+01$ & $<1.93 E+01$ & $<1.98 E+01$ \\
\hline $\mathrm{Ba}$ & & $3.80 \mathrm{E}+01$ & $6.30 \mathrm{E}+01$ & $6.33 \mathrm{E}+01$ & $7.32 \mathrm{E}+01$ & $5.71 \mathrm{E}+01$ & $5 \mathrm{E}+01$ & $5.30 \mathrm{E}+01$ & $2.52 \mathrm{E}+01$ & $4.95 \mathrm{E}+01$ \\
\hline $\mathrm{Be}$ & $\mathrm{g} / \mathrm{kg}$ & $<7.86 E-01$ & $<7.65 E-01$ & $<7.63 E-01$ & $<7.65 E-01$ & $<7.84 E-01$ & $<7.56 E-01$ & $<7.84 E-01$ & $<7.65 E-01$ & $<7.84 E-01$ \\
\hline $\mathrm{Ca}$ & $\mathrm{g} / \mathrm{kg}$ & $2.75 \mathrm{E}+02$ & $4.62 \mathrm{E}+02$ & $4.06 \mathrm{E}+02$ & $5.17 \mathrm{E}+02$ & $5.67 \mathrm{E}+02$ & $6.01 \mathrm{E}+02$ & $4.62 \mathrm{E}+02$ & $2.18 \mathrm{E}+02$ & $4.38 \mathrm{E}+02$ \\
\hline $\mathrm{Cd}$ & $\mathrm{mg} / \mathrm{kg}$ & $3.54 \mathrm{E}+00$ & $7.74 \mathrm{E}+00$ & $6.01 \mathrm{E}+00$ & $6.88 \mathrm{E}+00$ & $7.65 \mathrm{E}+00$ & $7.65 \mathrm{E}+00$ & $8.82 \mathrm{E}+00$ & $4.59 \mathrm{E}+00$ & $1.14 \mathrm{E}+01$ \\
\hline $\mathrm{Ce}$ & $\mathrm{mg} / \mathrm{kg}$ & $6.95 E+01$ & $<6.76 E+01$ & $<6.74 E+01$ & $<6.76 E+01$ & $1.14 \mathrm{E}+03$ & $<6.68 E+01$ & $1.19 \mathrm{E}+02$ & $<6.76 E+01$ & $<6.93 E+01$ \\
\hline $\mathrm{Cr}$ & $\mathrm{g} / \mathrm{kg}$ & $1.03 \mathrm{E}+01$ & $1.14 \mathrm{E}+01$ & $1.15 \mathrm{E}+01$ & $5.98 \mathrm{E}+01$ & $4.73 \mathrm{E}+01$ & $6.34 \mathrm{E}+01$ & $5.39 \mathrm{E}+01$ & $6.50 \mathrm{E}+01$ & $1.56 \mathrm{E}+01$ \\
\hline $\mathrm{Cu}$ & $\mathrm{mg} / \mathrm{kg}$ & $5.16 \mathrm{E}+01$ & $7.93 \mathrm{E}+01$ & $8.03 \mathrm{E}+01$ & $1.06 \mathrm{E}+02$ & $7.40 \mathrm{E}+01$ & $1.12 \mathrm{E}+02$ & $7.33 \mathrm{E}+01$ & $3.71 \mathrm{E}+01$ & $6.64 \mathrm{E}+01$ \\
\hline $\mathrm{Fe}$ & $\mathrm{g} / \mathrm{kg}$ & $4.41 \mathrm{E}+03$ & $7.23 \mathrm{E}+03$ & $7.25 \mathrm{E}+03$ & $9.08 \mathrm{E}+03$ & $7.11 \mathrm{E}+03$ & $1.04 \mathrm{E}+04$ & $6.87 \mathrm{E}+03$ & $3.26 \mathrm{E}+03$ & $5.38 \mathrm{E}+03$ \\
\hline Gd & $\mathrm{hg} / \mathrm{kg}$ & $2.11 E+01$ & $<2.06 E+01$ & $2.05 E+01$ & $2.06 E+01$ & $<2.11 E+01$ & $<2.03 E+01$ & $<2.11 E+01$ & $<2.06 E+01$ & $<2.11 E+01$ \\
\hline $\mathrm{K}$ & $\mathrm{mg} / \mathrm{kg}$ & $2.96 E+02$ & $<2.88 E+02$ & $<2.87 E+02$ & $<2.88 E+02$ & $<2.95 E+02$ & $<2.84 E+02$ & $<2.95 E+02$ & $<2.88 E+02$ & $<2.95 E+02$ \\
\hline $\mathrm{La}$ & $\mathrm{mg} / \mathrm{kg}$ & $1.88 \mathrm{E}+01$ & $3.10 \mathrm{E}+01$ & $3.13 \mathrm{E}+01$ & $3.59 \mathrm{E}+01$ & $3.09 \mathrm{E}+01$ & $4.20 \mathrm{E}+01$ & $2.73 \mathrm{E}+01$ & $1.34 \mathrm{E}+01$ & $5.01 \mathrm{E}+01$ \\
\hline $\mathrm{Li}$ & $\mathrm{mg} / \mathrm{kg}$ & $6.58 E+00$ & $7.17 \mathrm{E}+00$ & $<6.39 E+00$ & $7.36 \mathrm{E}+00$ & $<6.57 E+00$ & $8.60 \mathrm{E}+00$ & $<6.57 E+00$ & $<6.41 E+00$ & $<6.57 E+00$ \\
\hline $\mathrm{Mg}$ & $\mathrm{mg} / \mathrm{kg}$ & $1.15 \mathrm{E}+02$ & $2.01 \mathrm{E}+02$ & $1.83 \mathrm{E}+02$ & $2.15 \mathrm{E}+02$ & $1.89 \mathrm{E}+02$ & $2.58 \mathrm{E}+02$ & $1.89 \mathrm{E}+02$ & $8.67 \mathrm{E}+01$ & $1.78 \mathrm{E}+02$ \\
\hline $\mathrm{Mn}$ & $\mathrm{mg} / \mathrm{kg}$ & $2.48 \mathrm{E}+03$ & $4.09 \mathrm{E}+03$ & $4.26 \mathrm{E}+03$ & $4.70 \mathrm{E}+03$ & $3.54 \mathrm{E}+03$ & $5.85 \mathrm{E}+03$ & $3.21 \mathrm{E}+03$ & $1.55 \mathrm{E}+03$ & $2.48 \mathrm{E}+03$ \\
\hline Mo & $\mathrm{mg} / \mathrm{kg}$ & $2.21 E+01$ & $2.15 E+01$ & $2.15 E+01$ & $2.15 E+01$ & $<2.21 E+01$ & $<2.13 E+01$ & $<2.21 E+01$ & $<2.15 E+01$ & $<2.21 E+01$ \\
\hline $\mathrm{Na}$ & $\mathrm{ng} / \mathrm{kg}$ & $2.52 \mathrm{E}+04$ & $2.43 \mathrm{E}+04$ & $<7.04 E+02$ & $4.09 \mathrm{E}+04$ & $3.35 \mathrm{E}+04$ & $2.72 \mathrm{E}+03$ & $3.49 \mathrm{E}+04$ & $4.06 \mathrm{E}+04$ & $3.45 \mathrm{E}+04$ \\
\hline $\mathrm{Ni}$ & $\mathrm{mg} / \mathrm{kg}$ & $3.05 \mathrm{E}+02$ & $4.65 \mathrm{E}+02$ & $4.63 \mathrm{E}+02$ & $7.30 \mathrm{E}+02$ & $5.46 \mathrm{E}+02$ & $7.15 \mathrm{E}+02$ & $5.73 \mathrm{E}+02$ & $2.73 \mathrm{E}+02$ & $5.16 \mathrm{E}+02$ \\
\hline $\mathrm{P}$ & $\mathrm{mg} / \mathrm{kg}$ & $1.61 E+02$ & $<1.57 E+02$ & $1.56 E+02$ & $<1.57 E+02$ & $<1.61 E+02$ & $<1.55 E+02$ & $<1.61 E+02$ & $<1.57 E+02$ & $<1.61 E+02$ \\
\hline S & $\mathrm{mg} / \mathrm{kg}$ & $7.37 E+02$ & $<7.17 E+02$ & $<7.16 E+02$ & $<7.17 E+02$ & $<7.35 E+02$ & $<7.09 E+02$ & $<7.35 E+02$ & $<7.17 E+02$ & $<7.35 E+02$ \\
\hline $\mathrm{Sb}$ & $\mathrm{mg} / \mathrm{kg}$ & $1.05 E+02$ & $1.02 E+02$ & $<1.02 E+02$ & $1.02 E+02$ & $<1.05 E+02$ & $<1.01 E+02$ & $<1.05 E+02$ & $<1.02 E+02$ & $<1.05 E+02$ \\
\hline $\mathrm{Si}$ & $\mathrm{mg} / \mathrm{kg}$ & $1.03 \mathrm{E}+02$ & $2.12 \mathrm{E}+02$ & $4.70 \mathrm{E}+02$ & $2.28 \mathrm{E}+02$ & $2.39 \mathrm{E}+02$ & $7.09 \mathrm{E}+02$ & $2.16 \mathrm{E}+02$ & $1.47 \mathrm{E}+02$ & $9.48 \mathrm{E}+01$ \\
\hline $\mathrm{Sn}$ & $\mathrm{mg} / \mathrm{kg}$ & $5.51 E+01$ & $<5.36 E+01$ & $<5.35 E+01$ & $5.36 E+01$ & $<5.50 E+01$ & $<5.30 E+01$ & $<5.50 E+01$ & $<5.36 E+01$ & $<5.50 E+01$ \\
\hline $\mathrm{Sr}$ & $\mathrm{mg} / \mathrm{kg}$ & $1.88 \mathrm{E}+01$ & $3.04 \mathrm{E}+01$ & $3.00 \mathrm{E}+01$ & $3.62 \mathrm{E}+01$ & $2.79 \mathrm{E}+01$ & $4.22 \mathrm{E}+01$ & $2.63 \mathrm{E}+01$ & $1.26 \mathrm{E}+01$ & $2.42 \mathrm{E}+01$ \\
\hline Th & $\mathrm{mg} / \mathrm{kg}$ & $5.69 \mathrm{E}+02$ & $8.93 \mathrm{E}+02$ & $9.48 \mathrm{E}+02$ & $1.14 \mathrm{E}+03$ & $8.11 \mathrm{E}+02$ & $1.30 \mathrm{E}+03$ & $7.33 \mathrm{E}+02$ & $3.57 \mathrm{E}+02$ & $1.28 \mathrm{E}+03$ \\
\hline $\mathrm{Ti}$ & $\mathrm{mg} / \mathrm{kg}$ & $1.97 \mathrm{E}+01$ & $3.28 \mathrm{E}+01$ & $2.30 \mathrm{E}+01$ & $3.98 \mathrm{E}+01$ & $2.85 \mathrm{E}+01$ & $4.75 \mathrm{E}+01$ & $2.30 \mathrm{E}+01$ & $1.01 \mathrm{E}+01$ & $1.24 \mathrm{E}+01$ \\
\hline $\mathrm{U}$ & $\mathrm{mg} / \mathrm{kg}$ & $2.94 E+02$ & $2.87 E+02$ & $4.25 \mathrm{E}+02$ & $8.22 \mathrm{E}+02$ & $<2.94 E+02$ & $4.42 \mathrm{E}+02$ & $6.68 \mathrm{E}+02$ & $4.56 \mathrm{E}+02$ & $6.61 \mathrm{E}+02$ \\
\hline $\mathrm{V}$ & $\mathrm{mg} / \mathrm{kg}$ & $4.62 E+00$ & $<4.49 E+00$ & $<4.48 E+00$ & $<4.49 E+00$ & $<4.61 E+00$ & $<4.44 E+00$ & $<4.61 E+00$ & $<4.49 E+00$ & $<4.61 E+00$ \\
\hline $\mathrm{Zn}$ & $\mathrm{mg} / \mathrm{kg}$ & $1.47 \mathrm{E}+01$ & $2.71 \mathrm{E}+01$ & $3.50 \mathrm{E}+01$ & $3.40 \mathrm{E}+01$ & $2.81 \mathrm{E}+01$ & $5.18 \mathrm{E}+01$ & $2.62 \mathrm{E}+01$ & $1.56 \mathrm{E}+01$ & $3.30 \mathrm{E}+01$ \\
\hline $\mathrm{Zr}$ & $\mathrm{mg} / \mathrm{kg}$ & $1.35 \mathrm{E}+02$ & $2.28 \mathrm{E}+02$ & $2.34 \mathrm{E}+02$ & $2.68 \mathrm{E}+02$ & $2.01 \mathrm{E}+02$ & $2.32 \mathrm{E}+02$ & $1.86 \mathrm{E}+02$ & $9.01 \mathrm{E}+01$ & $1.72 \mathrm{E}+02$ \\
\hline Co & $\mathrm{mg} / \mathrm{kg}$ & $5.06 \mathrm{E}+00$ & $4.78 E+00$ & $<4.77 E+00$ & $5.69 \mathrm{E}+00$ & $5.17 \mathrm{E}+00$ & $6.58 \mathrm{E}+00$ & $<4.90 E+00$ & $<4.78 E+00$ & $<4.90 E+00$ \\
\hline $\mathrm{Hg}$ & $\mathrm{g} / \mathrm{kg}$ & $2.33 \mathrm{E}+02$ & $6.66 \mathrm{E}+02$ & $1.11 \mathrm{E}+03$ & $1.02 \mathrm{E}+03$ & $9.66 \mathrm{E}+02$ & $1.42 \mathrm{E}+03$ & $9.72 \mathrm{E}+02$ & $4.10 \mathrm{E}+02$ & $2.22 \mathrm{E}+03$ \\
\hline $\mathrm{Pb}$ & $\mathrm{mg} / \mathrm{kg}$ & $9.82 E+00$ & $1.37 \mathrm{E}+01$ & $<1.02 E+01$ & $1.48 \mathrm{E}+01$ & $1.12 \mathrm{E}+01$ & $<9.94 E+00$ & $<1.11 E+01$ & $2.03 \mathrm{E}+01$ & $1.48 \mathrm{E}+01$ \\
\hline $\mathrm{m}-230(\mathrm{Tl}$ & $\mathrm{mg} / \mathrm{kg}$ & $2.95 E+00$ & $<2.87 E+00$ & $<2.86 E+00$ & $<2.87 E+00$ & $<2.94 E+00$ & $<2.84 E+00$ & $<2.94 E+00$ & $<2.87 E+00$ & $<2.94 E+00$ \\
\hline $\mathrm{m}-232(\mathrm{Th})$ & $\mathrm{mg} / \mathrm{kg}$ & $4.73 E+02$ & $6.59 \mathrm{E}+02$ & & & & & & & \\
\hline $\mathrm{m}-233(\mathrm{U})$ & $\mathrm{mg} / \mathrm{kg}$ & $1.96 E+00$ & $1.91 E+00$ & $<1.91 E+00$ & $1.96 \mathrm{E}+00$ & $<1.96 E+00$ & $<1.89 E+00$ & $<1.96 E+00$ & $<1.91 E+00$ & $<1.96 E+00$ \\
\hline $\mathrm{m}-234(\mathrm{U})$ & $\mathrm{mg} / \mathrm{kg}$ & $4.91 E+00$ & $<4.78 E+00$ & $<4.77 E+00$ & $<4.78 E+00$ & $<4.90 E+00$ & $<4.73 E+00$ & $<4.90 E+00$ & $<4.78 E+00$ & $<4.90 E+00$ \\
\hline & $\mathrm{mg} / \mathrm{kg}$ & $4.91 E+00$ & $4.78 E+00$ & $9.82 \mathrm{E}+00$ & & & $9.01 \mathrm{E}+00$ & $1.44 \mathrm{E}+01$ & $1.09 \mathrm{E}+01$ & $8.62 \mathrm{E}+00$ \\
\hline $\mathrm{m}-236(\mathrm{U})$ & $\mathrm{mg} / \mathrm{kg}$ & $<1.96 E+00$ & $<1.91 E+00$ & $2.06 \mathrm{E}+00$ & $<1.91 E+00$ & $<1.96 E+00$ & $<1.89 E+00$ & $<1.96 E+00$ & $<1.91 E+00$ & $<1.96 E+00$ \\
\hline $\mathrm{m}-237(\mathrm{~Np})$ & $\mathrm{mg} / \mathrm{kg}$ & $1.96 E+00$ & $<1.91 E+00$ & $<1.91 E+00$ & $<1.91 E+00$ & $<1.96 E+00$ & $2.61 \mathrm{E}+00$ & $<1.96 E+00$ & $<1.91 E+00$ & $<1.96 E+00$ \\
\hline m-238 (U,Pu) & $\mathrm{mg} / \mathrm{kg}$ & $3.63 \mathrm{E}+01$ & $1.64 \mathrm{E}+02$ & $5.51 \mathrm{E}+02$ & $8.12 \mathrm{E}+02$ & $6.53 \mathrm{E}+02$ & $5.53 \mathrm{E}+02$ & $7.87 \mathrm{E}+02$ & $4.86 \mathrm{E}+02$ & $8.05 \mathrm{E}+02$ \\
\hline $\mathrm{m}-239(\mathrm{Pu})$ & $\mathrm{mg} / \mathrm{kg}$ & $1.13 \mathrm{E}+01$ & $1.28 \mathrm{E}+01$ & $1.70 \mathrm{E}+01$ & $2.27 \mathrm{E}+01$ & $1.92 \mathrm{E}+01$ & $2.66 \mathrm{E}+01$ & $1.88 \mathrm{E}+01$ & $1.22 \mathrm{E}+01$ & $1.79 \mathrm{E}+01$ \\
\hline $\mathrm{m}-240(\mathrm{Pu})$ & $\mathrm{mg} / \mathrm{kg}$ & $<1.96 E+00$ & $<1.91 E+00$ & $2.04 \mathrm{E}+00$ & $3.69 \mathrm{E}+00$ & $2.38 \mathrm{E}+00$ & $2.59 \mathrm{E}+00$ & $2.86 \mathrm{E}+00$ & $<1.91 E+00$ & $<1.96 E+00$ \\
\hline $\mathrm{m}-241(\mathrm{Am}, \mathrm{Pu})$ & $\mathrm{mg} / \mathrm{kg}$ & $<1.96 E+00$ & $<1.91 E+00$ & $<1.91 E+00$ & $<1.91 E+00$ & $<1.96 E+00$ & $<1.89 E+00$ & $<1.96 E+00$ & $<1.91 E+00$ & $<1.96 E+00$ \\
\hline $\mathrm{Pu}-238$ & $\mathrm{dpm} / \mathrm{mL}$ & $4.54 \mathrm{E}+07$ & $5.91 \mathrm{E}+07$ & $7.49 \mathrm{E}+07$ & $9.56 \mathrm{E}+07$ & $8.40 \mathrm{E}+07$ & $1.04 \mathrm{E}+08$ & $8.65 \mathrm{E}+07$ & $4.21 \mathrm{E}+07$ & $8.25 \mathrm{E}+07$ \\
\hline $\mathrm{Pu}-239 / 240$ & $\mathrm{dpm} / \mathrm{mL}$ & $2.32 \mathrm{E}+06$ & $3.28 \mathrm{E}+06$ & $3.68 \mathrm{E}+06$ & $4.37 \mathrm{E}+06$ & $4.11 \mathrm{E}+06$ & $5.05 \mathrm{E}+06$ & $3.93 \mathrm{E}+06$ & $2.03 \mathrm{E}+06$ & $3.58 \mathrm{E}+06$ \\
\hline Sr-90 & $\mathrm{dpm} / \mathrm{mL}$ & $1.47 \mathrm{E}+09$ & $2.85 \mathrm{E}+09$ & $2.65 \mathrm{E}+09$ & $2.54 \mathrm{E}+09$ & $2.28 \mathrm{E}+09$ & $3.15 \mathrm{E}+09$ & $1.90 \mathrm{E}+09^{r}$ & $1.07 \mathrm{E}+09$ & $1.80 \mathrm{E}+09$ \\
\hline Cs-137 & $\mathrm{dpm} / \mathrm{mL}$ & $1.03 \mathrm{E}+06$ & $2.68 \mathrm{E}+06$ & $4.44 \mathrm{E}+06$ & $7.48 \mathrm{E}+06$ & $9.39 \mathrm{E}+06$ & $2.01 \mathrm{E}+07$ & $9.73 \mathrm{E}+06$ & $1.07 \mathrm{E}+07$ & $2.32 \mathrm{E}+06$ \\
\hline $\mathrm{Eu}-154$ & $\mathrm{dpm} / \mathrm{mL}$ & $<2.02 E+06$ & $2.04 \mathrm{E}+06$ & $2.03 \mathrm{E}+06$ & $1.52 \mathrm{E}+06$ & $2.37 \mathrm{E}+06$ & $2.51 \mathrm{E}+06$ & $2.29 \mathrm{E}+06$ & $<9.20 E+05$ & $3.60 \mathrm{E}+06$ \\
\hline
\end{tabular}


Table 3-44: Analysis of Test 2 Deposition Tank Solids, Wet Basis

\begin{tabular}{|c|c|c|c|c|c|c|c|c|c|c|}
\hline \multicolumn{2}{|c|}{ Storage Condition: } & \multirow{2}{*}{$\frac{1}{<1.44 E+01}$} & \multirow{2}{*}{$\frac{2}{2.38 \mathrm{E}+01}$} & \multirow{2}{*}{$\frac{3}{2.80 \mathrm{E}+01}$} & \multirow{2}{*}{$\frac{\mathbf{4}}{3.93 \mathrm{E}+01}$} & \multirow{2}{*}{$\frac{\mathbf{5}}{3.80 \mathrm{E}+01}$} & \multirow{2}{*}{$\frac{\mathbf{6}}{8.07 \mathrm{E}+01}$} & \multirow{2}{*}{$\frac{7}{<1.41 E+01}$} & \multirow{2}{*}{\begin{tabular}{|c|}
$\mathbf{8}$ \\
$<1.41 E+01$
\end{tabular}} & \multirow{2}{*}{$\frac{9}{4.74 \mathrm{E}+01}$} \\
\hline $\mathrm{Ag}$ & $\mathrm{mg} / \mathrm{kg}$ & & & & & & & & & \\
\hline $\mathrm{A} 1$ & $\mathrm{mg} / \mathrm{kg}$ & $3.65 \mathrm{E}+02$ & $8.42 \mathrm{E}+02$ & $2.89 \mathrm{E}+03$ & $1.43 \mathrm{E}+03$ & $1.76 \mathrm{E}+03$ & $8.88 \mathrm{E}+03$ & $7.89 \mathrm{E}+02$ & $3.70 \mathrm{E}+02$ & $1.86 \mathrm{E}+03$ \\
\hline $\mathrm{B}$ & $\mathrm{mg} / \mathrm{kg}$ & $<1.99 E+01$ & $<2.00 E+01$ & $<1.97 E+01$ & $<1.96 E+01$ & $<1.95 E+01$ & $<2.00 E+01$ & $<1.95 E+01$ & $<1.95 E+01$ & $<1.96 E+01$ \\
\hline $\mathrm{Ba}$ & $\mathrm{mg} / \mathrm{kg}$ & $1.46 \mathrm{E}+02$ & $2.68 \mathrm{E}+02$ & $2.78 \mathrm{E}+02$ & $3.14 \mathrm{E}+02$ & $2.76 \mathrm{E}+02$ & $7.16 \mathrm{E}+02$ & $1.21 \mathrm{E}+02$ & $1.33 \mathrm{E}+02$ & $1.58 \mathrm{E}+02$ \\
\hline $\mathrm{Be}$ & $\mathrm{mg} / \mathrm{kg}$ & $<7.88 E-01$ & $<7.90 E-01$ & $<7.78 E-01$ & $<7.77 E-01$ & $<7.74 E-01$ & 7.92E-01 & $<7.72 E-01$ & $<7.71 E-01$ & $<7.77 E-01$ \\
\hline $\mathrm{Ca}$ & $\mathrm{mg} / \mathrm{kg}$ & $3.13 \mathrm{E}+02$ & $5.93 \mathrm{E}+02$ & $2.40 \mathrm{E}+02$ & $7.68 \mathrm{E}+02$ & $7.96 \mathrm{E}+02$ & $1.59 \mathrm{E}+03$ & $3.35 \mathrm{E}+02$ & $4.00 \mathrm{E}+02$ & $5.38 \mathrm{E}+02$ \\
\hline $\mathrm{Cd}$ & $\mathrm{mg} / \mathrm{kg}$ & $6.99 \mathrm{E}+00$ & $1.43 \mathrm{E}+01$ & $9.83 \mathrm{E}+00$ & $1.48 \mathrm{E}+01$ & $1.60 \mathrm{E}+01$ & $2.81 \mathrm{E}+01$ & $7.43 E+00$ & $9.44 \mathrm{E}+00$ & $1.63 \mathrm{E}+01$ \\
\hline $\mathrm{Ce}$ & $\mathrm{mg} / \mathrm{kg}$ & $<6.96 E+01$ & $<6.99 E+01$ & $<6.88 E+01$ & $<6.86 E+01$ & $<6.84 E+01$ & $<7.00 E+01$ & $<6.83 E+01$ & $<6.81 E+01$ & $8.80 \mathrm{E}+01$ \\
\hline $\mathrm{Cr}$ & $\mathrm{mg} / \mathrm{kg}$ & $1.49 \mathrm{E}+01$ & $2.08 \mathrm{E}+01$ & $4.18 \mathrm{E}+01$ & $5.99 \mathrm{E}+01$ & $6.11 \mathrm{E}+01$ & $1.08 \mathrm{E}+02$ & $1.55 \mathrm{E}+01$ & $1.58 \mathrm{E}+01$ & $5.11 \mathrm{E}+01$ \\
\hline $\mathrm{Cu}$ & $\mathrm{mg} / \mathrm{kg}$ & $4.34 \mathrm{E}+01$ & $7.26 \mathrm{E}+01$ & $4.99 \mathrm{E}+01$ & $1.28 \mathrm{E}+02$ & $1.35 \mathrm{E}+02$ & $1.56 \mathrm{E}+02$ & $3.98 \mathrm{E}+01$ & $4.85 \mathrm{E}+01$ & $7.71 \mathrm{E}+01$ \\
\hline $\mathrm{Fe}$ & $\mathrm{mg} / \mathrm{kg}$ & $1.74 \mathrm{E}+04$ & $2.65 E+04$ & $1.87 \mathrm{E}+04$ & $4.61 \mathrm{E}+04$ & $4.47 \mathrm{E}+04$ & $8.04 \mathrm{E}+04$ & $1.40 \mathrm{E}+04$ & $1.64 \mathrm{E}+04$ & $1.87 \mathrm{E}+04$ \\
\hline $\mathrm{Gd}$ & $\mathrm{mg} / \mathrm{kg}$ & $<2.12 E+01$ & $3.45 \mathrm{E}+01$ & $2.41 \mathrm{E}+01$ & $6.73 \mathrm{E}+01$ & $7.00 \mathrm{E}+01$ & $7.93 \mathrm{E}+01$ & $<2.08 E+01$ & $2.22 \mathrm{E}+01$ & $2.45 \mathrm{E}+01$ \\
\hline $\mathrm{K}$ & $\mathrm{mg} / \mathrm{kg}$ & $<2.96 E+02$ & $<2.97 E+02$ & $<2.93 E+02$ & $<2.92 E+02$ & $<2.91 E+02$ & $4.40 \mathrm{E}+02$ & $<2.90 E+02$ & $<2.90 E+02$ & $<2.92 E+02$ \\
\hline $\mathrm{La}$ & $\mathrm{mg} / \mathrm{kg}$ & $2.96 \mathrm{E}+01$ & $4.39 \mathrm{E}+01$ & $3.64 \mathrm{E}+01$ & $6.76 \mathrm{E}+01$ & $6.72 \mathrm{E}+01$ & $1.18 \mathrm{E}+02$ & $2.42 \mathrm{E}+01$ & $2.84 \mathrm{E}+01$ & $1.23 \mathrm{E}+02$ \\
\hline $\mathrm{Li}$ & $\mathrm{mg} / \mathrm{kg}$ & $2.21 \mathrm{E}+01$ & $4.01 \mathrm{E}+01$ & $2.49 \mathrm{E}+01$ & $8.87 \mathrm{E}+01$ & $8.51 \mathrm{E}+01$ & $1.53 \mathrm{E}+02$ & $2.20 \mathrm{E}+01$ & $2.11 \mathrm{E}+01$ & $3.75 \mathrm{E}+01$ \\
\hline $\mathrm{Mg}$ & $\mathrm{mg} / \mathrm{kg}$ & $4.24 \mathrm{E}+01$ & $1.04 \mathrm{E}+02$ & $4.87 \mathrm{E}+01$ & $1.20 \mathrm{E}+02$ & $1.49 \mathrm{E}+02$ & $2.05 \mathrm{E}+02$ & $5.99 \mathrm{E}+01$ & $7.20 \mathrm{E}+01$ & $1.65 \mathrm{E}+02$ \\
\hline $\mathrm{Mn}$ & $\mathrm{mg} / \mathrm{kg}$ & $4.93 \mathrm{E}+03$ & $9.06 \mathrm{E}+03$ & $9.54 \mathrm{E}+03$ & $1.01 \mathrm{E}+04$ & $8.64 \mathrm{E}+03$ & $2.45 \mathrm{E}+04$ & $4.01 \mathrm{E}+03$ & $4.35 \mathrm{E}+03$ & $6.61 E+03$ \\
\hline Mo & $\mathrm{mg} / \mathrm{kg}$ & $<2.22 E+01$ & $<2.22 E+01$ & $<2.19 E+01$ & $<2.18 E+01$ & $<2.18 E+01$ & $<2.23 E+01$ & $<2.17 E+01$ & $<2.17 E+01$ & $<2.18 E+01$ \\
\hline $\mathrm{Na}$ & $\mathrm{mg} / \mathrm{kg}$ & $2.34 \mathrm{E}+04$ & $2.48 \mathrm{E}+04$ & $<7.18 E+02$ & $2.91 \mathrm{E}+04$ & $3.28 \mathrm{E}+04$ & $6.30 \mathrm{E}+03$ & $2.52 \mathrm{E}+04$ & $2.45 \mathrm{E}+04$ & $4.42 \mathrm{E}+04$ \\
\hline $\mathrm{Ni}$ & $\mathrm{mg} / \mathrm{kg}$ & $6.24 \mathrm{E}+02$ & $9.30 \mathrm{E}+02$ & $8.24 \mathrm{E}+02$ & $1.38 \mathrm{E}+03$ & $1.39 \mathrm{E}+03$ & $2.40 \mathrm{E}+03$ & $4.73 \mathrm{E}+02$ & $5.47 \mathrm{E}+02$ & $3.55 \mathrm{E}+03$ \\
\hline $\mathrm{P}$ & $\mathrm{mg} / \mathrm{kg}$ & $<1.61 E+02$ & $<1.62 E+02$ & $<1.60 E+02$ & $<1.59 E+02$ & $<1.59 E+02$ & $<1.62 E+02$ & $<1.58 E+02$ & $<1.58 E+02$ & $<1.59 E+02$ \\
\hline $\mathrm{S}$ & $\mathrm{mg} / \mathrm{kg}$ & $<7.38 E+02$ & $<7.41 E+02$ & $<7.30 E+02$ & $<7.28 E+02$ & $<7.25 E+02$ & $<7.43 E+02$ & $<7.24 E+02$ & $<7.23 E+02$ & $<7.28 E+02$ \\
\hline $\mathrm{Sb}$ & $\mathrm{mg} / \mathrm{kg}$ & $<1.05 E+02$ & $<1.05 E+02$ & $<1.04 E+02$ & $1.19 \mathrm{E}+02$ & $1.19 \mathrm{E}+02$ & $2.34 \mathrm{E}+02$ & $<1.03 E+02$ & $<1.03 E+02$ & $<1.04 E+02$ \\
\hline $\mathrm{Si}$ & $\mathrm{mg} / \mathrm{kg}$ & $4.85 \mathrm{E}+02$ & $7.12 \mathrm{E}+02$ & $7.57 \mathrm{E}+02$ & $1.59 \mathrm{E}+03$ & $1.52 \mathrm{E}+03$ & $1.07 \mathrm{E}+03$ & $3.55 \mathrm{E}+02$ & $3.96 \mathrm{E}+02$ & $4.57 \mathrm{E}+02$ \\
\hline $\mathrm{Sn}$ & $\mathrm{mg} / \mathrm{kg}$ & $<5.52 E+01$ & $<5.54 E+01$ & $<5.46 E+01$ & $<5.45 E+01$ & $<5.42 E+01$ & $<5.55 E+01$ & $<5.42 E+01$ & $<5.41 E+01$ & $<5.45 E+01$ \\
\hline $\mathrm{Sr}$ & $\mathrm{mg} / \mathrm{kg}$ & $6.33 \mathrm{E}+01$ & $1.14 \mathrm{E}+02$ & $1.02 \mathrm{E}+02$ & $1.54 \mathrm{E}+02$ & $1.37 \mathrm{E}+02$ & $3.42 \mathrm{E}+02$ & $5.33 \mathrm{E}+01$ & $6.05 \mathrm{E}+01$ & $6.80 \mathrm{E}+01$ \\
\hline Th & $\mathrm{mg} / \mathrm{kg}$ & $5.50 \mathrm{E}+01$ & $8.48 \mathrm{E}+01$ & $<5.21 E+01$ & $1.40 \mathrm{E}+02$ & $1.36 \mathrm{E}+02$ & $2.75 \mathrm{E}+02$ & $<5.17 E+01$ & $<5.15 E+01$ & $<5.19 E+01$ \\
\hline $\mathrm{Ti}$ & $\mathrm{mg} / \mathrm{kg}$ & $1.09 \mathrm{E}+01$ & $1.57 \mathrm{E}+01$ & $<3.70 E+00$ & $2.92 \mathrm{E}+01$ & $3.10 \mathrm{E}+01$ & $4.67 \mathrm{E}+01$ & $1.00 \mathrm{E}+01$ & $1.09 \mathrm{E}+01$ & $1.17 \mathrm{E}+01$ \\
\hline $\mathrm{U}$ & $\mathrm{mg} / \mathrm{kg}$ & $6.54 \mathrm{E}+03$ & $1.11 \mathrm{E}+04$ & $8.09 \mathrm{E}+03$ & $2.19 \mathrm{E}+04$ & $2.26 \mathrm{E}+04$ & $2.78 \mathrm{E}+04$ & $5.40 \mathrm{E}+03$ & $7.07 \mathrm{E}+03$ & $7.44 \mathrm{E}+03$ \\
\hline $\mathrm{V}$ & $\mathrm{mg} / \mathrm{kg}$ & $<4.63 E+00$ & $<4.64 E+00$ & $<4.57 E+00$ & $<4.56 E+00$ & $<4.54 E+00$ & $<4.65 E+00$ & $<4.54 E+00$ & $<4.53 E+00$ & $<4.56 E+00$ \\
\hline $\mathrm{Zn}$ & $\mathrm{mg} / \mathrm{kg}$ & $7.58 \mathrm{E}+00$ & $1.38 \mathrm{E}+01$ & $6.71 \mathrm{E}+00$ & $2.04 \mathrm{E}+01$ & $2.43 \mathrm{E}+01$ & $2.70 \mathrm{E}+01$ & $8.88 \mathrm{E}+00$ & $9.83 \mathrm{E}+00$ & $4.17 \mathrm{E}+01$ \\
\hline $\mathrm{Zr}$ & $\mathrm{mg} / \mathrm{kg}$ & $2.45 \mathrm{E}+02$ & $3.77 \mathrm{E}+02$ & $1.91 \mathrm{E}+02$ & $8.10 \mathrm{E}+02$ & $7.63 \mathrm{E}+02$ & $1.61 \mathrm{E}+03$ & $2.06 \mathrm{E}+02$ & $2.44 \mathrm{E}+02$ & $3.31 \mathrm{E}+02$ \\
\hline Co & $\mathrm{mg} / \mathrm{kg}$ & $<3.94 E+00$ & $6.81 \mathrm{E}+00$ & $9.24 \mathrm{E}+00$ & $8.38 \mathrm{E}+00$ & $9.48 \mathrm{E}+00$ & $1.13 \mathrm{E}+01$ & $<3.86 E+00$ & $<3.85 E+00$ & $1.88 \mathrm{E}+01$ \\
\hline $\mathrm{Hg}$ & $\mathrm{mg} / \mathrm{kg}$ & $7.95 \mathrm{E}+01$ & $4.48 \mathrm{E}+02$ & $1.65 \mathrm{E}+02$ & $3.04 \mathrm{E}+02$ & $6.15 \mathrm{E}+02$ & $5.88 \mathrm{E}+02$ & $2.99 \mathrm{E}+02$ & $2.47 \mathrm{E}+02$ & $1.03 E+03$ \\
\hline $\mathrm{Pb}$ & $\mathrm{mg} / \mathrm{kg}$ & $<2.42 E+01$ & $4.22 \mathrm{E}+01$ & $<3.14 E+01$ & $6.40 \mathrm{E}+01$ & $6.90 \mathrm{E}+01$ & $1.04 \mathrm{E}+02$ & $<2.32 E+01$ & $<2.56 E+01$ & $4.46 \mathrm{E}+01$ \\
\hline $\mathrm{m}-230(\mathrm{Th})$ & $\mathrm{mg} / \mathrm{kg}$ & $<7.87 E+00$ & $<7.91 E+00$ & $<7.78 E+00$ & $<7.77 E+00$ & $<7.74 E+00$ & $<7.92 E+00$ & $<7.72 E+00$ & $<7.71 E+00$ & $<7.77 E+00$ \\
\hline $\mathrm{m}-232(\mathrm{Th})$ & $\mathrm{mg} / \mathrm{kg}$ & $3.93 \mathrm{E}+01$ & $6.74 \mathrm{E}+01$ & $3.59 \mathrm{E}+01$ & $1.21 \mathrm{E}+02$ & $1.24 \mathrm{E}+02$ & $2.06 \mathrm{E}+02$ & $3.68 \mathrm{E}+01$ & $4.95 \mathrm{E}+01$ & $2.35 \mathrm{E}+01$ \\
\hline $\mathrm{m}-233(\mathrm{U})$ & $\mathrm{mg} / \mathrm{kg}$ & $<7.87 E+00$ & $<7.91 E+00$ & $<7.78 E+00$ & $<7.77 E+00$ & $<7.74 E+00$ & $<7.92 E+00$ & $<7.72 E+00$ & $<7.71 E+00$ & $<7.77 E+00$ \\
\hline $\mathrm{m}-234(\mathrm{U})$ & $\mathrm{mg} / \mathrm{kg}$ & $<5.91 E+00$ & $<5.93 E+00$ & $<5.84 E+00$ & $<5.83 E+00$ & $<5.80 E+00$ & $<5.94 E+00$ & $<5.79 E+00$ & $<5.78 E+00$ & $<5.83 E+00$ \\
\hline $\mathrm{m}-235(\mathrm{U})$ & $\mathrm{mg} / \mathrm{kg}$ & $4.04 \mathrm{E}+01$ & $7.96 \mathrm{E}+01$ & $5.25 \mathrm{E}+01$ & $1.48 \mathrm{E}+02$ & $1.55 \mathrm{E}+02$ & $1.74 \mathrm{E}+02$ & $3.46 \mathrm{E}+01$ & $4.85 \mathrm{E}+01$ & $4.75 \mathrm{E}+01$ \\
\hline $\mathrm{m}-236(\mathrm{U})$ & $\mathrm{mg} / \mathrm{kg}$ & $<3.94 E+00$ & $4.67 \mathrm{E}+00$ & $<3.89 E+00$ & $7.34 \mathrm{E}+00$ & $7.58 \mathrm{E}+00$ & $7.27 \mathrm{E}+00$ & $<3.86 E+00$ & $<3.85 E+00$ & $<3.88 E+00$ \\
\hline m-237 (Np) & $\mathrm{mg} / \mathrm{kg}$ & $<7.87 E+00$ & $<7.91 E+00$ & $<7.78 E+00$ & $<7.77 E+00$ & $<7.74 E+00$ & $1.17 \mathrm{E}+01$ & $<7.72 E+00$ & $<7.71 E+00$ & $<7.77 E+00$ \\
\hline $\mathrm{m}-238(\mathrm{U}, \mathrm{Pu})$ & $\mathrm{mg} / \mathrm{kg}$ & $6.33 \mathrm{E}+03$ & $1.18 \mathrm{E}+04$ & $7.58 \mathrm{E}+03$ & $2.31 \mathrm{E}+04$ & $2.38 \mathrm{E}+04$ & $2.73 \mathrm{E}+04$ & $5.12 \mathrm{E}+03$ & $7.15 \mathrm{E}+03$ & $7.07 \mathrm{E}+03$ \\
\hline $\mathrm{m}-239(\mathrm{Pu})$ & $\mathrm{mg} / \mathrm{kg}$ & $7.17 \mathrm{E}+00$ & $1.01 \mathrm{E}+01$ & $7.32 \mathrm{E}+00$ & $1.34 \mathrm{E}+01$ & $1.48 \mathrm{E}+01$ & $2.69 \mathrm{E}+01$ & $6.99 \mathrm{E}+00$ & $5.79 \mathrm{E}+00$ & $1.27 \mathrm{E}+01$ \\
\hline $\mathrm{m}-240(\mathrm{Pu})$ & $\mathrm{mg} / \mathrm{kg}$ & $<3.94 E+00$ & $<3.95 E+00$ & $<3.89 E+00$ & $<3.88 E+00$ & $<3.87 E+00$ & $<3.96 E+00$ & $<3.86 E+00$ & $<3.85 E+00$ & $<3.88 E+00$ \\
\hline $\mathrm{m}-241(\mathrm{Am}, \mathrm{Pu})$ & $\mathrm{mg} / \mathrm{kg}$ & $<3.94 E+00$ & $<3.95 E+00$ & $<3.89 E+00$ & $<3.88 E+00$ & $<3.87 E+00$ & $<3.96 E+00$ & $<3.86 E+00$ & $<3.85 E+00$ & $<3.88 E+00$ \\
\hline $\mathrm{Pu}-238$ & $\mathrm{dpm} / \mathrm{mL}$ & $1.33 \mathrm{E}+08$ & $8.54 \mathrm{E}+08$ & $1.36 \mathrm{E}+08$ & $3.47 \mathrm{E}+08$ & $1.02 \mathrm{E}+09$ & $5.81 \mathrm{E}+08$ & $5.66 \mathrm{E}+08$ & $7.32 \mathrm{E}+08$ & $2.87 \mathrm{E}+09$ \\
\hline $\mathrm{Pu}-239 / 240$ & $\mathrm{dpm} / \mathrm{mL}$ & $7.07 \mathrm{E}+07$ & $1.45 \mathrm{E}+08$ & $8.58 \mathrm{E}+07$ & $2.17 \mathrm{E}+08$ & $2.34 \mathrm{E}+08$ & $4.11 \mathrm{E}+08$ & $7.37 \mathrm{E}+07$ & $9.77 \mathrm{E}+07$ & $2.25 \mathrm{E}+08$ \\
\hline Sr-90 & $\mathrm{dpm} / \mathrm{mL}$ & $<4.72 E+09$ & $<8.10 E+09$ & $<1.01 E+10$ & $<9.71 E+09$ & $<1.04 E+10$ & $<2.43 E+10$ & $<4.51 E+09$ & $<4.77 E+09$ & $<4.74 E+09$ \\
\hline Co-60 & $\mathrm{dpm} / \mathrm{mL}$ & $<6.06 E+05$ & $3.93 \mathrm{E}+05$ & $3.50 \mathrm{E}+05$ & $4.08 \mathrm{E}+05$ & $4.79 \mathrm{E}+05$ & $9.83 \mathrm{E}+05$ & $<4.88 E+05$ & $<5.99 E+05$ & $1.10 \mathrm{E}+06$ \\
\hline Cs-137 & $\mathrm{dpm} / \mathrm{mL}$ & $1.82 \mathrm{E}+07$ & $2.18 \mathrm{E}+07$ & $1.40 \mathrm{E}+08$ & $7.02 \mathrm{E}+07$ & $9.80 \mathrm{E}+07$ & $8.14 \mathrm{E}+08$ & $1.66 \mathrm{E}+07$ & $1.72 \mathrm{E}+07$ & $6.28 \mathrm{E}+07$ \\
\hline Eu-154 & $\mathrm{dpm} / \mathrm{mL}$ & -- & -- & -- & -- & -- & -- & -- & -- & $5.05 \mathrm{E}+06$ \\
\hline Am-241 & $\mathrm{dpm} / \mathrm{mL}$ & -- & -- & -- & -- & -- & -- & -- & -- & $7.83 \mathrm{E}+06$ \\
\hline
\end{tabular}




\subsection{Treatment Tank Sludge Physical and Flow Properties}

Measurement of the flow properties and behavior of the solids during and after the dissolution process in the Treatment Tank contributes to the understanding of how the solids may be transferred out of the tank. As a background for the ECC process, SRR has theorized that over the course of sludge heel chemical cleaning by multiple batches of heated OA, the particle size distribution shifts toward smaller particle sizes and the rheology becomes more favorable for the suspension and transfer of undissolved solids out of the tank.

\subsubsection{Rheology}

Baseline, midpoint, and endpoint Tank $5 \mathrm{~F}$ and $12 \mathrm{H}$ slurry samples were prepared and characterized as described in detail in the Experimental Section. Baseline samples correspond to the original sludge materials. Rheology on the baseline samples was conducted after exposure of the sludge to $2 \mathrm{wt} \% \mathrm{OA}$ at acid:sludge mass phase ratios of approximately 3 and 7 (correspond to sludge solids of of 13 and $26 \mathrm{wt} \%$, respectively). Midpoint and endpoint sludge samples were intended to represent sludge in the treatment tank near the middle and end (respectively) of the ECC process. These samples were generated by exposing the sludge samples to two successive contacts with 2 wt. \% OA using an acid:sludge mass ratio of approximately 50:1 for each acid contact. Acid contacts were conducted without agitation at $70{ }^{\circ} \mathrm{C}$. Based on previous testing, it was expected that these conditions would result in partial dissolution of the sludge samples while leaving sufficient residual materials for characterization. Seven days was used for the first OA contact (midpoint) and 3 days was used for the second OA contact (endpoint). For many samples, rheology testing was conducted at two solids loading levels referred to as dilute and concentrated. Rheology testing for the midpoint and endpoint samples was conducted in the spent OA solutions used for the dissolution batch contacts. Weight percent solids and particle size measurements were conducted on sub-samples of each sample type in order to aid in the interpretation of the rheology data.

For comparison, rheology measurements were conducted using the same equipment on $2 \mathrm{wt} \%$ OA $(0.224 \mathrm{M})$ solution containing no dissolved sludge materials across the temperature range of interest. The results are provided in Table 3-45. As expected, $2 \mathrm{wt} \%$ OA solution is thin and exhibits ideal Newtonian behavior with no yield stress. Yield stress values within $\pm 0.5 \mathrm{~Pa}$ of zero should be treated as zero values for this instrument. As the solution temperature was increased from 30 to $50{ }^{\circ} \mathrm{C}$, the viscosity decreased by approximately $19 \%$ from 1.79 to $1.45 \mathrm{cP}$. A viscosity approaching $2 \mathrm{cP}$ at $30{ }^{\circ} \mathrm{C}$ is significantly higher than expected for $2 \mathrm{wt} \% \mathrm{OA}$. At 20 ${ }^{\circ} \mathrm{C}$ the viscosity of $2 \mathrm{wt} \% \mathrm{OA}$ is reported to be $1.04 \mathrm{cP} .{ }^{18}$ Based on this result and the previously discussed issues with adjusting the instrumental zero point (Section 2.3), this instrument and experimental methodology are not believed to be highly accurate for the measurement of low viscosity fluids. For comparison, the viscosity of water decreases from 0.80 to $0.65 \mathrm{cP}$ as the temperature is increased from 30 to $50{ }^{\circ} \mathrm{C} .{ }^{18}$

Rheology data for the baseline, midpoint, and endpoint Tank 5F/OA slurries is provided in Table 3-46. For all Tank 5F treatment tank samples, most of the solids settled quickly (within 5 minutes) and the slurries were not well suited for rheological measurement. Nonetheless, rheology data was collected on the baseline material at two solids loadings and three temperatures. Although the dilute and concentrated Tank 5F baseline slurry samples were known 
to contain 15 and $28 \mathrm{wt} \%$ total solids, respectively, the suspendable solids were determined to be $<5 \mathrm{wt} \%$ for both samples (see Table 3-43). Furthermore, the measured viscosities for both the dilute and concentrated samples of 1.6 to $2.1 \mathrm{cP}$ were only slightly higher than was observed for $\mathrm{OA}$ acid at the same temperatures and the yield stress values were all near zero. The results are consistent with visual observations regarding particle settling and indicate that the rheology of the baseline material was representative of the liquid phase alone rather than a solid suspension. Presumably, the Tank 5F baseline slurry characteristics were dominated by iron oxide and oxyhydroxide phases, which are known to be primary chemical constituents of this sludge material. It appears based on these results that the removal of these dense and rapidly settling phases from the tanks will be best accomplished by dissolution rather than suspension. Fortunately, the iron phases tend to dissolve well in OA. The settling characteristics of the midpoint and endpoint samples were similar to the baseline material. As a result, rheology was only conducted on these materials at a single solids loading and temperature. The viscosities of both samples were in the range observed for the baseline material.

Rheology data for the Tank 12H/OA slurries is provided in Table 3-47. The initial baseline Tank $12 \mathrm{H}$ slurry samples were observed to contain particles which settled quickly as well as particles which remained suspended. Sub-samples of the dilute and concentrated slurries collected within one minute after sample agitation were determined to contain 17 and $25 \mathrm{wt}$ \% total solids, respectively. All of the dilute baseline Tank $12 \mathrm{H}$ slurry samples exhibited Newtonian behavior with essentially no yield stress. However, later samples analyzed after aging for an additional day (see second set of results for Baseline Dilute material at 30 and $40{ }^{\circ} \mathrm{C}$ ) showed signs of thickening and better particle suspension. Similarly, the concentrated baseline slurry was observed to exhibit non-Newtonian behavior characteristic of a Bingham Plastic material in some later measurements $\left(\mathrm{T}=40\right.$ and $\left.50^{\circ} \mathrm{C}\right)$. Since this behavior appeared to be increasing with time, the baseline concentrated slurry sample was left over the weekend to allow time for sample stabilization. Subsequent rheology data collected at $30^{\circ} \mathrm{C}$ on the concentrated sample after 4 days of aging revealed that the viscosity $(6.3 \mathrm{cP})$ and yield stress $(4.9 \mathrm{~Pa})$ had increased considerably relative to initial measurements at this temperature.

Rheology data was also collected for the Tank $12 \mathrm{H}$ midpoint and endpoint slurries at three temperatures and two solids loadings. Due to sample size limitations, it was not possible to prepare slurries at total solids loading levels greater than 10 weight percent. The midpoint concentrate sample contained the highest solids loading of any sample (9.6 wt. \%) and exhibited the highest viscosity and yield stress. The stress versus strain curve for one rheology measurement at $40{ }^{\circ} \mathrm{C}$ on the midpoint concentrate sample is provided in Figure 3-10. The rheological characteristics of this sample were typical of a Bingham Plastic material and the average viscosity and yield stress values for duplicate measurements were $6.6 \mathrm{cP}$ and $5.5 \mathrm{~Pa}$, respectively. Temperature variations had minimal effects on the rheological properties across the range of interest $\left(30-50{ }^{\circ} \mathrm{C}\right)$. The rheological properties were primarily affected by the solids loading. At total solids loadings $<5 \mathrm{wt} . \%$ the Tank $12 \mathrm{H}$ slurries also behaved as Bingham Plastic materials. The stress versus strain curve for the Endpoint Concentrate sample is provided in Figure 3-11. The midpoint dilute and endpoint concentrate samples serendipitously contained similar weight percent total solids (5-6 wt. \%) and had similar viscosities (2-3 cP) and yield stress (0.6-0.8 Pa) values. These results indicate that the rheological properties of the Tank $12 \mathrm{H}$ slurry changed little between the midpoint and endpoint samples. In contrast, very high weight percent solids levels in the baseline materials only resulted in moderate viscosity and yield stress values. This characteristic of the baseline slurry may have been associated with the fact that the sludge sample had been dried prior to testing. The rheological properties of the Tank $12 \mathrm{H}$ slurries are presumably dominated by aluminum phases which are known to be the primary chemical constituents. In contrast to the solids in the Tank 5F slurry, the Tank $12 \mathrm{H}$ solids form 
suspensions which are somewhat stable over the timescale of rheology measurements. These solids are therefore expected to be more suitable for suspension within and transfer from the waste tanks.

Table 3-45: Rheology Data for 2 wt\% OA Solution at Various Temperatures

\begin{tabular}{c|c|c}
\hline Temperature $\left({ }^{\circ} \mathbf{C}\right)$ & Viscosity $(\mathbf{c P})$ & Yield Stress (Pa) \\
\hline 30 & 1.79 & -0.25 \\
\hline 40 & 1.61 & -0.25 \\
50 & 1.45 & -0.25 \\
\hline
\end{tabular}

Table 3-46: Rheology Data for Tank 5F Sludge/OA (2 wt\%) Slurry

\begin{tabular}{c|c|c|c|c}
\hline Sample $^{\mathbf{a}}$ & $\begin{array}{c}\text { Total Solids } \\
\left(\mathbf{W t} \mathbf{\%} \mathbf{b}^{\mathbf{b}}\right.\end{array}$ & $\begin{array}{c}\text { Temperature } \\
(\mathbf{(} \mathbf{C})\end{array}$ & $\begin{array}{c}\text { Viscosity } \\
\mathbf{( c P )}\end{array}$ & $\begin{array}{c}\text { Yield Stress } \\
\mathbf{( P a})\end{array}$ \\
\hline Baseline Dilute $^{\mathrm{c}}$ & 2.8 & 30 & 2.10 & -0.31 \\
\hline Baseline Dilute $^{\mathrm{c}}$ & 2.8 & 40 & 1.89 & -0.28 \\
\hline Baseline Dilute $^{\mathrm{c}}$ & 2.8 & 50 & 1.62 & -0.27 \\
\hline Baseline Concentrate $^{\mathrm{c}}$ & 4.2 & 30 & 2.16 & -0.30 \\
\hline Baseline Concentrate $^{\mathrm{c}}$ & 4.2 & 40 & 2.04 & -0.26 \\
\hline Baseline Concentrate $^{\mathrm{c}}$ & 4.2 & 50 & 1.81 & -0.26 \\
\hline Midpoint & $\mathrm{NA}$ & 30 & 1.76 & -0.30 \\
\hline Endpoint & $\mathrm{NA}$ & 30 & 2.13 & -0.30 \\
\hline
\end{tabular}

${ }^{a}$ significant settling observed on analysis timescale for all samples

${ }^{\mathrm{b}}$ represents total suspendable and dissolved solids; as-prepared Baseline dilute and concentrate samples contained 15 and 28 wt. \% total solids, respectively, based on added sludge and accounting for dissolved oxalic acid

${ }^{\mathrm{c}}$ solution contained $3.6 \mathrm{wt}$. \% soluble solids 
SRNL-STI-2011-00360

Revision 0

Table 3-47: Rheology Data for Tank 12H Sludge/OA (2 wt\%) Slurry

\begin{tabular}{|c|c|c|c|c|}
\hline Sample & $\begin{array}{l}\text { Total Solids }^{\mathrm{a}} \\
\text { (Wt. \%) }\end{array}$ & $\begin{array}{c}\text { Temperature } \\
\left({ }^{\circ} \mathrm{C}\right)\end{array}$ & $\begin{array}{l}\text { Viscosity } \\
\quad \text { (cP) }\end{array}$ & $\begin{array}{c}\text { Yield Stress } \\
\text { (Pa) }\end{array}$ \\
\hline Baseline Dilute $^{\mathrm{b}}$ & 16.8 & 30 & 2.26 & -0.31 \\
\hline Baseline Dilute $^{b}$ & 16.8 & 40 & 2.26 & -0.31 \\
\hline Baseline Dilute $^{\mathrm{b}}$ & 16.8 & 50 & 2.38 & -0.20 \\
\hline Baseline Dilute $^{\mathrm{c}}$ & 16.8 & 30 & 2.45 & 0.07 \\
\hline Baseline Dilute $^{\mathrm{c}}$ & 16.8 & 40 & 2.16 & 0.19 \\
\hline Baseline Concentrate $^{b}$ & 25.2 & 30 & 2.56 & 0.42 \\
\hline Baseline Concentrate $^{b}$ & 25.2 & 40 & 2.77 & 0.85 \\
\hline Baseline Concentrate $^{b}$ & 25.2 & 50 & 3.16 & 1.37 \\
\hline Baseline Concentrate $^{\mathrm{d}}$ & 25.2 & 30 & 6.27 & 4.93 \\
\hline Midpoint Dilute & 5.7 & 30 & 2.68 & 0.67 \\
\hline Midpoint Dilute $^{\mathrm{e}}$ & 5.7 & 40 & 2.34 & 0.78 \\
\hline Midpoint Dilute & 5.7 & 50 & 2.33 & 0.77 \\
\hline Midpoint Concentrate & 9.6 & 30 & 6.52 & 5.37 \\
\hline Midpoint Concentrate & 9.6 & 40 & 6.58 & 5.52 \\
\hline Midpoint Concentrate & 9.6 & 50 & 6.90 & 6.20 \\
\hline Endpoint Dilute & 3.3 & 30 & 2.16 & 0.01 \\
\hline Endpoint Dilute & 3.3 & 40 & 1.95 & 0.07 \\
\hline Endpoint Dilute & 3.3 & 50 & 1.97 & 0.10 \\
\hline Endpoint Concentrate & 5.0 & 30 & 2.65 & 0.61 \\
\hline Endpoint Concentrate & 5.0 & 40 & 2.43 & 0.67 \\
\hline Endpoint Concentrate & 5.0 & 50 & 2.26 & 0.77 \\
\hline
\end{tabular}

${ }^{a}$ represents total suspendable and dissolved solids; as-prepared Baseline dilute and concentrate samples contained 15 and 28 wt. \% total solids, respectively, based on added sludge and accounting for dissolved oxalic acid

${ }^{\mathrm{b}}$ significant settling observed on analysis timescale

$\mathrm{c}$ after 1 day of storage at ambient temperature

d after 4 days storage at ambient temperature 


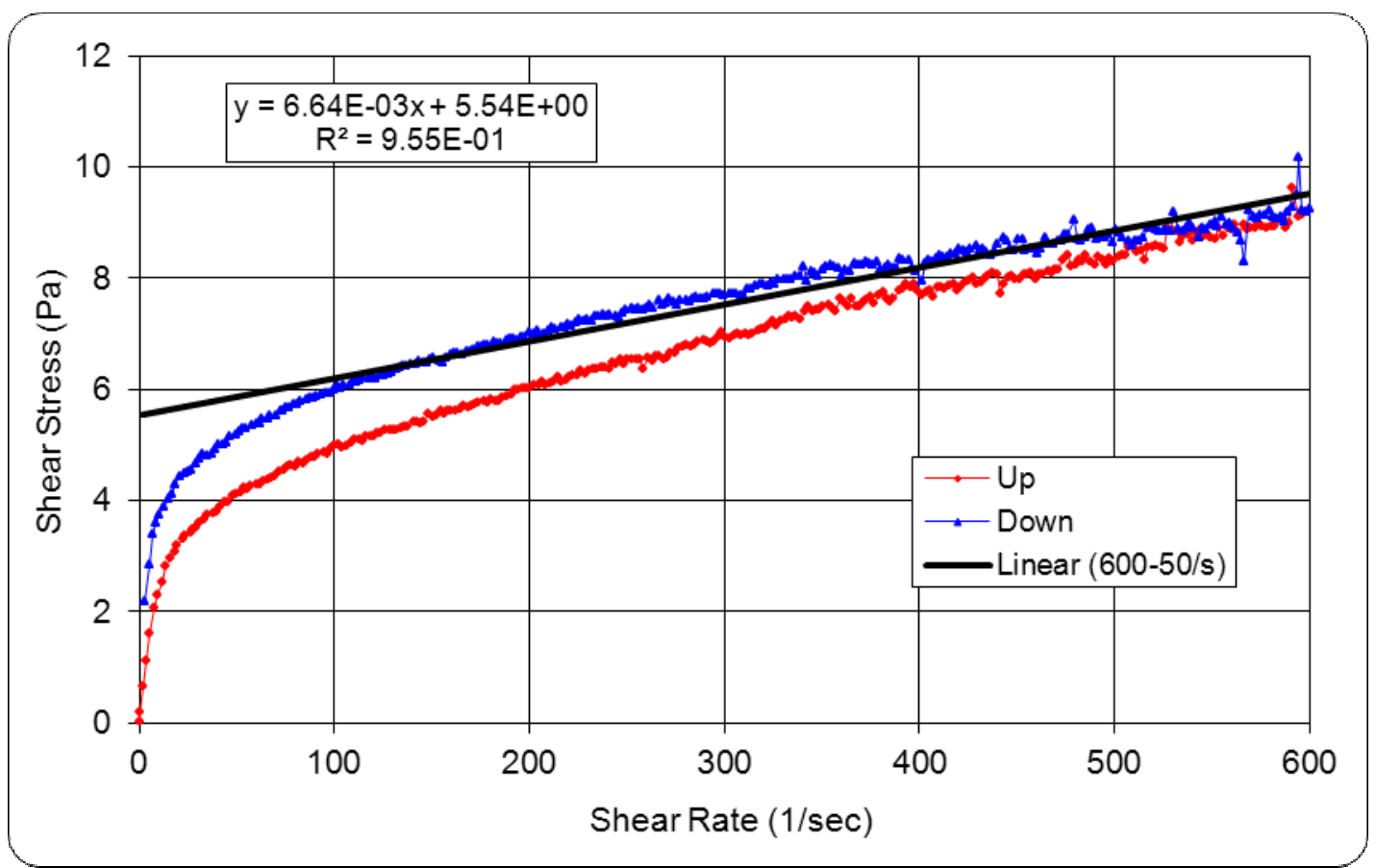

Figure 3-10: Rheology Results for the Concentrated Tank 12H Slurry Midpoint Sample at $40{ }^{\circ} \mathrm{C}$.

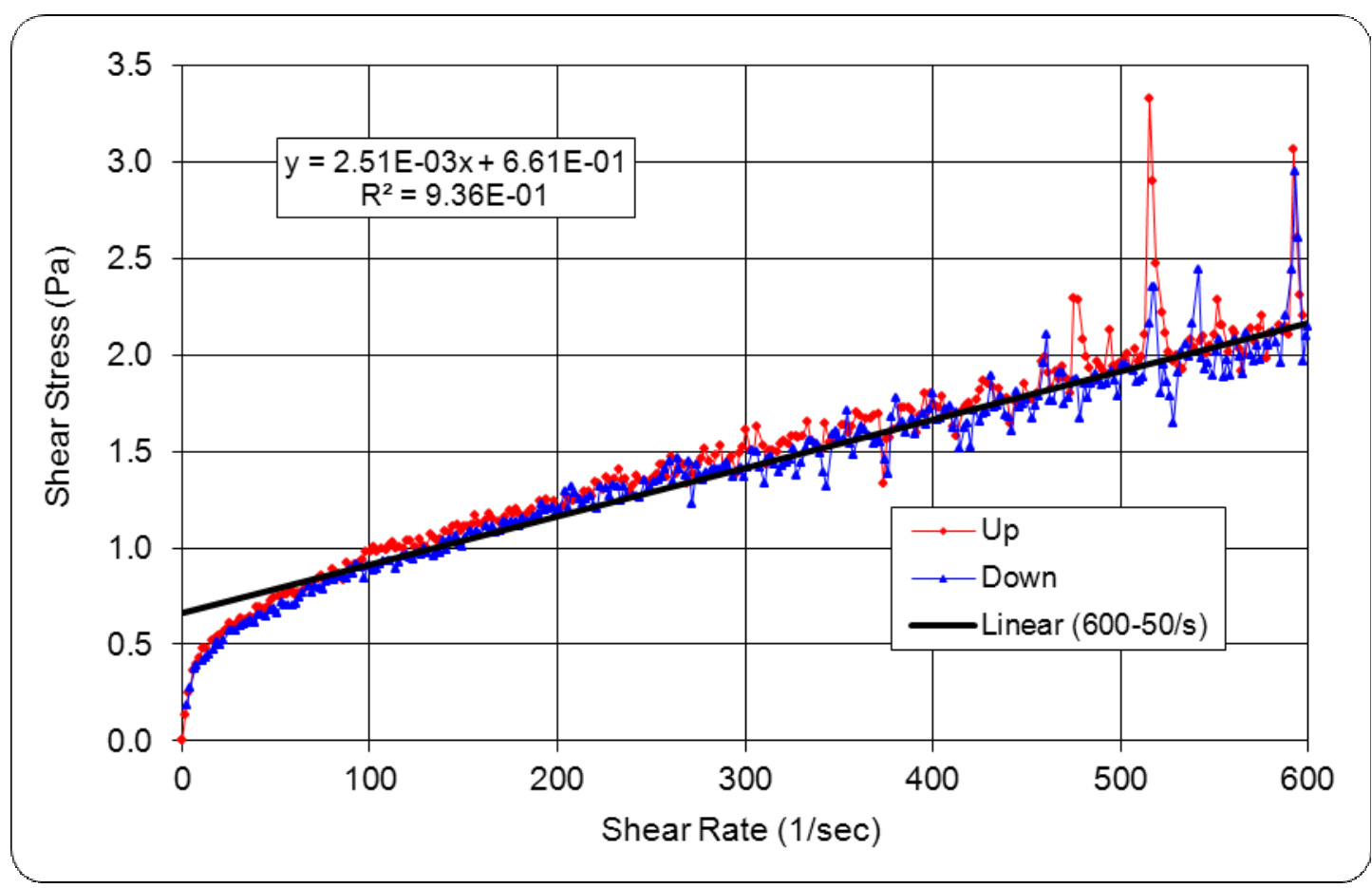

Figure 3-11: Rheology Results for the Concentrated Tank 12H Slurry Endpoint Sample at $40{ }^{\circ} \mathrm{C}$. 
SRNL-STI-2011-00360

Revision 0

\subsubsection{Particle size}

Individual sub-samples of the baseline, midpoint, and endpoint Tank $5 \mathrm{~F}$ and $12 \mathrm{H}$ materials were submitted for particle size analysis. Complete analysis results for each sample are provided in Appendix B (Figure B-75 through Figure B-80). The Tank 5F particles were larger (mean volume range: $37-118 \mu \mathrm{m}$ ) than the Tank $12 \mathrm{H}$ particles (mean volume range: $3.7-11.7 \mu \mathrm{m}$ ). The baseline Tank 5F material was bimodal with a significant population of particles in the $1-10 \mu \mathrm{m}$ range. The midpoint and endpoint Tank 5F samples contained a smaller fraction of particles below $10 \mu \mathrm{m}$. Trends in the distributions also indicate that the larger particles were dissolving in the Tank 5F slurry as a result of OA contact. The mean volume observed for the endpoint sample was below $40 \mu \mathrm{m}$ with few particles $(<10$ percentile) greater than $100 \mu \mathrm{m}$. Evidence for the dissolution of smaller particles was also observed for the Tank $12 \mathrm{H}$ sample. The baseline material contained a significant number of particles below $1 \mu \mathrm{m}$ ( 25 percentile), while the midpoint and endpoint samples contained smaller fractions of particles below $1 \mu \mathrm{m}$. Little difference was observed between the midpoint and endpoint Tank $12 \mathrm{H}$ samples, which is consistent with the fact that little sludge dissolution occurred during the second and third ECC process batches for Test 1 (Section 3-3).

\subsubsection{Settling}

As stated previously, Tank $5 \mathrm{~F}$ sludge showed quick settling both prior to and after partial dissolution with OA. A portion of the solids settled quickly enough that difficulty was encountered removing the solids from the dissolution apparatus. For example, when a bottle with the residual solids is agitated and poured, the solids are large enough that they do not stay suspended long enough to be poured out of the bottle. Figure 3-12 contains a picture of the material after the first OA dissolution for the Treatment Tank rheology test. On the left is a cylinder with the portion that was able to be poured from the bottle. Within 8 minutes, the major sludge level was at around $50 \%$ of the height of the liquid in the cylinder. The residual materials in the bottle on the right are a significant portion of the quickly settling solids that could not be poured from the bottle. 


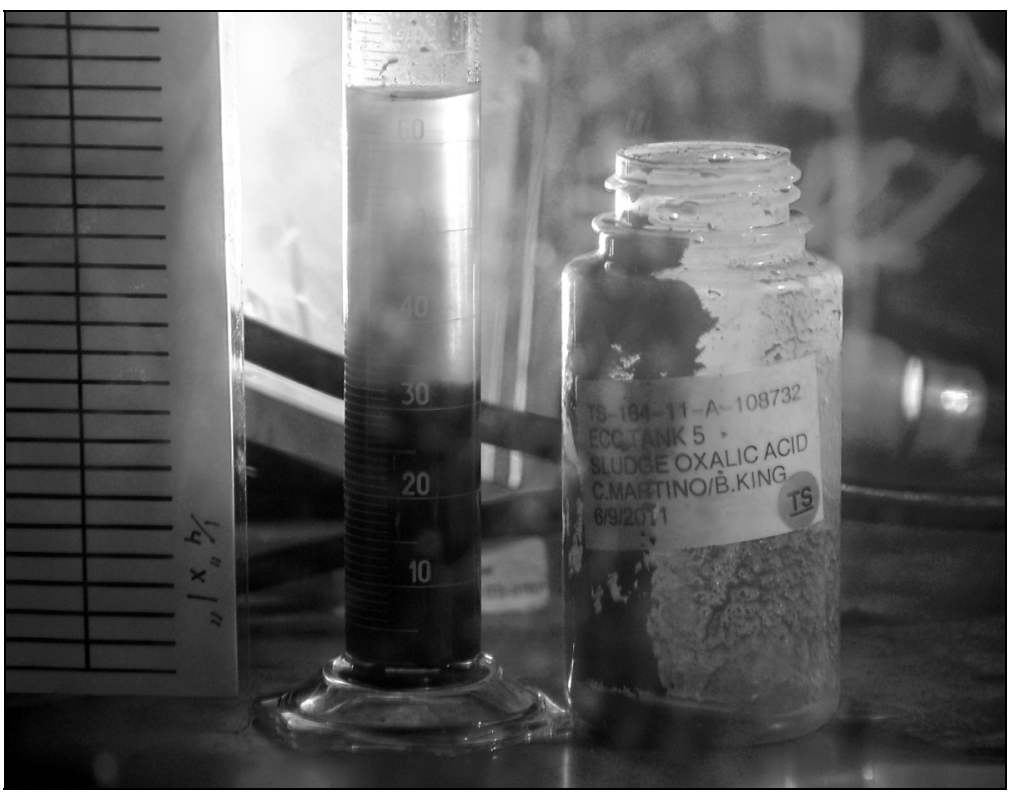

Figure 3-12: Tank 5F Sludge Intermediate Dissolution Sample, a Graduated Cylinder with the Fluid Portion After 8 Minutes of Settling (left) and the Fast-Settling Portion Not Removed From the Sample Bottle (right)

\subsection{ECC Product Sludge Physical and Flow Properties}

The following are the expectations held prior to investigating the physical properties of the products of the ECC process. The particle size of the recently precipitated sludge at nearly neutral $\mathrm{pH}$ might be expected to be very small in comparison with typical Tank Farm sludge, and thus may not settle as quickly as typical high-pH sludge. The solids concentration of the ECC reactor product slurry was low, and might be expected to have a relatively low viscosity and nearly Newtonian rheology. Slurries leaving the evaporator would have have higher solids concentrations than those prior to evaporation, so changes in flow behavior might be expected. However, it was expected that the rheology would still be favorable to pumping. After hydroxide adjustment, the particle size was expected to be large enough for the sludge solids to settle quickly so that outages would not be required to provide deposition tank settling time. The testing was designed to either confirm or contradict these expectations.

\subsubsection{Rheology}

Rheology data for the Tank 5F and 12H Deposition Tank samples are provided in Table 3-4. The rheology measurements were performed for Storage Conditions 3 and 6, which correspond to the unadjusted material from the ECC reactor without and with evaporation, respectively. Note that the rheology was measured only after the completion of the Deposition Tank testing, so some changes may have occurred during the several weeks of storage at $50^{\circ} \mathrm{C}$. The rheology was measured on the suspended slurry from the storage bottle with no additional solid/liquid separation. Weight percent solids measurements were not conducted on these samples, but it was known based on the sample preparation details that these samples contained low solids levels. Partial settling of the solids was observed during all rheology measurements for the Tank $5 \mathrm{~F}$ deposition samples and during one measurement with the Tank $12 \mathrm{H}$ samples. As expected based 
on the low solids levels in the samples, all measured viscosities were low (2-3 cP) and no samples exhibited a significant yield stress. The measured viscosities for these samples was only slightly higher than was observed for pure OA (Table 3-1). Deposition Tank storage conditions that included evaporation for removal of $85 \%$ of the water added during the ECC dissolution process did not result in slurries that exhibited significant yield stresses.

Table 3-4. Rheology Data for Tank 5F and 12H Deposition Tank Samples

\begin{tabular}{c|c|c|c}
\hline Sample & Temperature $\left({ }^{\circ} \mathbf{C}\right)$ & Viscosity (cP) & Yield Stress (Pa) \\
\hline Tank 12 No Evaporation (T1SC3) & 30 & 1.88 & -0.29 \\
\hline Tank 12 No Evaporation (T1SC3) $^{\mathrm{a}}$ & 45 & 1.65 & -0.24 \\
\hline Tank 12 Evaporated (T1SC6) & 30 & 1.88 & -0.29 \\
\hline Tank 12 Evaporated (T1SC6) & 45 & 2.26 & -0.30 \\
\hline Tank 5 No Evaporation (T2SC3) & 30 & 2.03 & -0.31 \\
\hline Tank 5 No Evaporation (T2SC3) $)^{\mathrm{a}}$ & 45 & 1.66 & -0.28 \\
\hline Tank 5 Evaporated (T2SC6) & 30 & 1.80 & -0.28 \\
\hline Tank 5 Evaporated (T2SC6) & 45 & 2.15 & -0.32 \\
\hline
\end{tabular}

${ }^{a}$ partial settling observed on analysis timescale

\subsubsection{Particle size}

Particle size measurements for Deposition Tank storage bottle samples are included in Appendix B. Note that the particle size analysis was performed only after the completion of the Deposition Tank testing, so some changes may have occurred during the several weeks of storage at $50^{\circ} \mathrm{C}$.

Figure B-75 and Figure B-76 show the particle size analyses for the Tank 5F and 12H sample material, respectively, at Treatment Tank conditions. From these results it is clear that the initial Tank $12 \mathrm{H}$ material has a narrower size range with the volume percentage centering around $2 \mu \mathrm{m}$, while the initial Tank 5F material has a very large particle size range centering around $80 \mu \mathrm{m}$. Figure B-84 and Figure B-88 contain the Deposition Tank control samples for Tank $12 \mathrm{H}$ and 5F materials, respectively, and are consistent with the Treatment Tank analysis with the addition of another solids source with a relatively large particle size (from the sludge heel addition). Figure B-81, Figure B-83, and Figure B-82 show the Tank 12H ECC Reactor product at positions B, C, and D of Figure 2-6, respectively. The Tank $12 \mathrm{H} \mathrm{ECC}$ product is consistently narrow in particle size range centering around $2 \mu \mathrm{m}$, with the added sludge heel evident in Figure B-82 as a completely separate narrow distribution centering around $\sim 200 \mu \mathrm{m}$. Figure B-86 and Figure B-87 show the Tank 5F ECC Reactor product at positions B and C of, respectively. However, Figure B-85 does not correspond directly to Figure 2-6 because the nominal F-Area Deposition Tank condition does not include evaporation. Unadjusted Tank $5 \mathrm{~F}$ ECC product that was not evaporated had a particle size distribution that centered below $2 \mu \mathrm{m}$, while the evaporated and the not evaporated $\mathrm{pH}$ adjusted products had particle size distributions that centered around $3 \mu \mathrm{m}$. Despite the relatively large particle size of the initial Tank $5 \mathrm{~F}$ sludge heel material, the Tank $5 \mathrm{~F}$ ECC Reactor product was comparable to that of Tank $12 \mathrm{H}$. 


\subsubsection{Settling}

A visual settling test was performed for the unadjusted ECC reactor products (Tests 1 and 2, Storage Condition 3), the nominally adjusted products (Test 1 Storage Condition 5 and Test 2 Storage Condition 2) and the adjusted controls (Tests 1 and 2, Storage Condition 9). Figure 3-13 shows an image of the storage bottles for these conditions after 8 hours of settling. A larger series of such photographs from 0 hours of settling through 150 hours of settling are included in Appendix B, Figure B-89 through Figure B-92.

By the one and two hour settling observations, layers of solids were evident for all bottles except for the unadjusted Test 2 ECC product (Test 2 Storage Condition 3). By the six and eight hour observations of the settling, all supernatant liquids showed significant transparency except for the unadjusted Test 2 ECC product (Test 2 Storage Condition 3). By comparing the adjusted ECC products with the adjusted control samples, settling behavior is comparable. Thus, settling of adjusted ECC products is not expected to differ from that of Tank 5F or Tank $12 \mathrm{H}$ sludge. However, unadjusted Tank 5F ECC product may require adjustment if normal sludge settling behavior is desired.

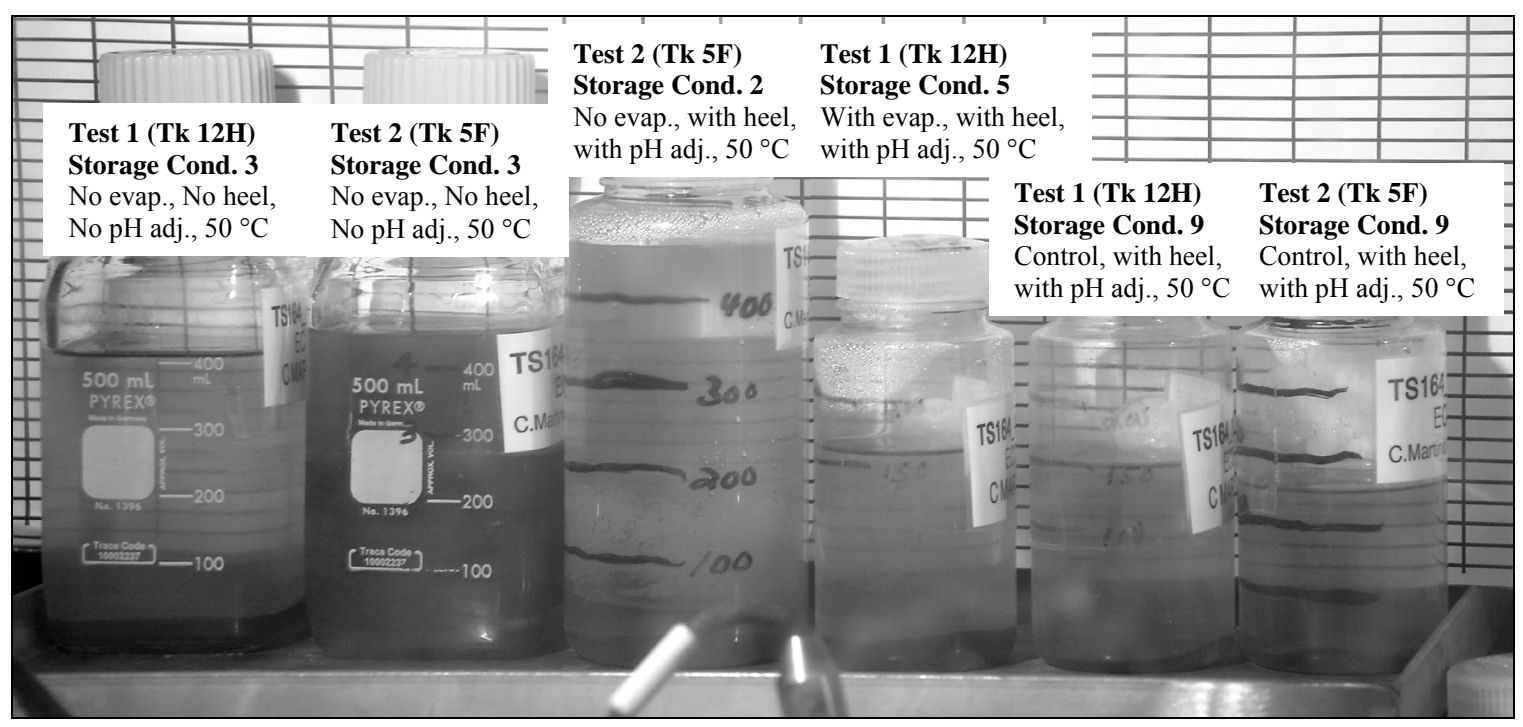

Figure 3-13: ECC Products after 8 Hours of Settling 
SRNL-STI-2011-00360

Revision 0

\subsection{Conclusions}

SRNL conducted two tests using actual SRS waste material from Tanks $5 \mathrm{~F}$ and $12 \mathrm{H}$. Testing involved sludge dissolution with an initial $2 \mathrm{wt} \% \mathrm{OA}$, the decomposition of the oxalates by ozonolysis, the evaporation of a portion of the material for water removal, and tracking the concentrations of key components in the Deposition Tank. The UV light was not utilized in the decomposition testing. The following are results of the ECC RWT:

- Using three OA batches per test, Test 1 showed an approximately 73\% removal of Tank $12 \mathrm{H}$ sludge and Test 2 showed an approximately 80\% removal of Tank $5 \mathrm{~F}$ sludge. Removal includes both dissolution of soluble components and partial transfer of insoluble solids. The dissolution process was constrained to eight hours per OA batch and thus did not precisely represent the in tank dissolution process.

- Residuals remaining in the dissolution vessel at the conclusion of Test 1 were primarily aluminum (accounts for over $90 \%$ of the mass assuming boehmite is the major form) with small amounts of iron and manganese. Residuals remaining in the dissolution vessel at the conclusion of Test 2 were primarily iron (accounts for $66 \%$ of the mass assuming hematite is the major form) with small amounts of manganese and nickel.

- Some minor metals were not removed effectively ( $<40 \%$ removal) from the dissolution vessel of the RWTE with $2 \mathrm{wt} \%$ OA. For Test 1, these metals included the lanthanides and actinides $\mathrm{Ce}, \mathrm{Gd}, \mathrm{La}, \mathrm{Th}, \mathrm{Eu}, \mathrm{Am}$, and possibly $\mathrm{Np}$. For Test 2, OA was more effective at removing the minor metals and only thorium was removed at a level below $40 \%$.

- Decomposition of $2 \mathrm{wt} \%$ OA to levels of $<100 \mathrm{ppm}$ using $2 \mathrm{~L} / \mathrm{min}$ of $5 \mathrm{wt} \%$ ozone at 70 ${ }^{\circ} \mathrm{C}$, a pressure of $8 \mathrm{psi}$, and a liquid recirculation rate of $1.8 \mathrm{gal} / \mathrm{min}$ required 8 to 14 hours for nominal 3.2L batches. The $\mathrm{pH}$ and oxidation/reduction potential (ORP) were tracked during decomposition testing.

- Sludge components were tracked during OA decomposition, showing that most components have the highest soluble levels in the initial dissolved sludge and early decomposition samples and exhibit lower soluble levels as OA decomposition progresses. Samples from the end of the decomposition process typically have the lowest soluble level of sludge components.

- Over the time period studied, the changes to the solubilities of important components in the Deposition Tank storage tests were insignificant. .

- Deposition Tank storage testing for Tank 12H ECC processing indicated higher solubilities for uranium and plutonium when compared with the control condition. Similar testing for Tank $5 \mathrm{~F}$ indicated higher solubilities for uranium when compared with the control condition. The solubilities of uranium and plutonium remained within the levels expected for Tank Farm high $\mathrm{pH}$ supernatant liquids, which are relatively low compared to the levels in the sludge solids. 
- Use of the ECC Evaporator generally led to higher soluble component concentrations in the Deposition Tank storage tests.

- Over the range of 25 to $70{ }^{\circ} \mathrm{C}$, the storage temperature was not a major factor affecting soluble concentrations during the Deposition Tank storage tests.

- Tank 5F sludge/OA slurries, believed to representative of ECC Treatment Tank materials, were not suitable for rheology measurements, due to the fact that most of the sludge particles settled rapidly. Chemical dissolution of these materials is likely necessary for the removal these materials from the treatment tank. Since iron oxide and hydroxide materials are primary chemical constituents for Tank $5 \mathrm{~F}$ sludge, chemical dissolution in OA should be effective.

- Tank 12H sludge/OA slurries, believed to representative of ECC Treatment Tank materials, formed suspensions which were suitable for rheology measurements. Stress versus strain curves observed for these materials revealed that the rheological properties were characteristic of Bingham Plastic materials. For sludge/acid slurries containing 10 weight percent total solids, the measured viscosity and yield stress values were $7 \mathrm{cP}$ and 6 $\mathrm{Pa}$, respectively. Only minor effects on the rheological properties were observed across the temperature range of 30 to $50^{\circ} \mathrm{C}$. Little difference was observed between the process midpoint and endpoint samples. Based on these results, the transfer of significant amounts of Tank $12 \mathrm{H}$ sludge materials from the treatment tank by suspension (rather than dissolution) appears possible.

- Rheology testing of the Tank 5F and 12H Deposition Tank samples revealed that these slurries were thin with little yield stress. Even the ECC product slurries that were evaporated to remove $\geq 85 \%$ of the water had low enough insoluble solids content as to not exhibit a significant yield stress.

- Settling of the adjusted Deposition Tank solids was comparable to the control and is fast enough as to not likely delay processing. Only the Tank $5 \mathrm{~F}$ product without $\mathrm{pH}$ adjustment exhibited settling significantly slower than the control sample of sludge that did not go through the ECC process.

\subsection{Recommendations, Path Forward or Future Work}

Testing of the Decomposition portion of the ECC process utilizing the UV light is in progress. The scope of the testing involves processing two dissolved sludge batches for each of the two sludge feeds through the Decomposition Module. Decomposition will be tracked in a manner consistent with Figure 3-2 through Figure 3-8. Sampling will be performed to provide information analogous to that of Table 3-11 through Table 3-16. Future testing using the UV light does not include additional Dissolution and Evaporation testing. Rheology, Settling, and Particle Size measurements are also not planned to be performed. 


\subsection{References}

${ }^{1}$ J. A Pike,. N. P. Badheka, E. T. Ketusky, "Flowsheet for SRS Waste Tank Heel Removal Using Oxalic Acid," WSRC-TR-2004-00317, Rev. 0, November 2004.

${ }^{2}$ K. Adu-Wasu, , M. J. Barnes, N. E. Bibler, J. R. Cantrell, F. F. Fondeur, B. A. Hamm, C. C. Herman, D. T. Hobbs, E. T. Ketusky, M. Singleton, M. E. Stallings, W. E. Stevens, and B. J. Wiersma, "Waste Tank Heel Chemical Cleaning Summary," WSRC-TR-2003-00401, Rev. 0, September 2003.

${ }^{3}$ E. T. Ketusky, "Determination of an Alternative Technology for HLW Tank Chemical Cleaning," WSRC-STI-2007-00587, Rev. 0, October 2007.

${ }^{4}$ M. S. Hay and D. C. Koopman, "Review of Alternative Enhanced Chemical Cleaning Options for SRS Waste Tanks," SRNL-STI-2009-00500, Rev. 0, August 27, 2009.

${ }^{5}$ C. J. Martino and W. D. King, "Task Technical and Quality Assurance Plan for Real Waste Testing to Support Enhanced Chemical Cleaning for Sludge Heel Removal," SRNL-RP-200800602, Rev. 2, March 21, 2011.

${ }^{6}$ A. G. Hansen, "Enhanced Chemical Cleaning (ECC) Real Waste Testing," HLE-TTR-2008-033, Rev. 3, December 13, 2010.

7 "ECC Real Waste Testing Equipment Process Flow Diagram," AREVA, 02-9124816D-005, February 4, 2011.

${ }^{8}$ W. E. Narrows, "Statement of Work: Enhanced Chemical Cleaning Real Waste Test Equipment," G-SOW-A-00049, Rev. 1, October 7, 2009.

9 "Real Waste Testing Technical Requirements Document and Conceptual Design Basis," AREVA, 51-9084868-000, July 22, 2008.

10 "Enhanced Chemical Cleaning Real Waste Test Equipment Project Plan," AREVA, PP3001988-000B, September 30, 2009.

11 "ECC-RWT Equipment Piping and Instrumentation Diagram," AREVA, 02-9125569D-005, January 5, 2011.

12 "Enhanced Chemical Cleaning Real Waste Test Equipment Operating Procedure," Manual L29, Procedure ITS-0170, Revision 2, June 21, 2011.

${ }^{13}$ M. S. Hay, K. P. Crapse, S. D. Fink, and J. M. Pareizs, "Characterization and Actual Waste Tests with Tank 5F Samples,” WSRC-STI-2007-00192, Rev. 1, August 30, 2007.

${ }^{14}$ M. S. Hay and D. J. McCabe, Characterization of Tank $11 \mathrm{H}$ and $51 \mathrm{H}$ Post Aluminum Dissolution Process Samples. WSRC-STI-2008-00227, Rev. 0, May 6, 2008.

${ }^{15}$ W. D. King and M. S. Hay, "Alternative Enhanced Chemical Cleaning: Basic Studies Results FY09" SRNL-STI-2009-00791, Rev. 0, February 2010.

${ }^{16}$ W. D. King and M. S. Hay, "Alternative and Enhanced Chemical Cleaning: Basic Studies Results FY10" SRNL-STI-2010-00541, Rev. 0, January 2011.

${ }^{17}$ W. T. Hix and S. E. Evans, "ECC-HST Task 1 and 2 Report: UV Light Evaluation, AREVA, 51-9159879-003, June 28, 2011.

${ }^{18}$ Weast, R. C. (editor), CRC Handbook of Chemistry and Physics, 67th Edition, CRC Press, Boca Raton, FL, 1986. 
SRNL-STI-2011-00360

Revision 0

Appendix A. Dissolution, Decomposition, and Evaporation Process Data 
SRNL-STI-2011-00360

Revision 0

Table A-1: Oxalic Acid Decomposition (without actual tank waste sludge)

\begin{tabular}{|c|c|c|c|c|c|c|}
\hline $\begin{array}{c}\text { Date/Time } \\
\text { (mm/dd/yy hh:mm) }\end{array}$ & $\begin{array}{l}\text { Run Time } \\
\text { (hr) }\end{array}$ & $\begin{array}{c}\text { Temperature } \\
\left({ }^{\circ} \mathrm{C}\right)\end{array}$ & $\begin{array}{c}\text { Pressure } \\
\text { (psi) }\end{array}$ & $\begin{array}{c}\text { Flow } \\
\text { (gal } / \text { min) }\end{array}$ & $\mathbf{p H}$ & $\begin{array}{l}\text { ORP } \\
(\mathrm{mV})\end{array}$ \\
\hline $04 / 19 / 1107: 45$ & 0 & 70.2 & 8.5 & 1.55 & 1.12 & -- \\
\hline 04/19/11 08:00 & 0.25 & 70.0 & 8.5 & 1.55 & 1.13 & -- \\
\hline 04/19/11 08:15 & 0.50 & 70.0 & 8.5 & 1.50 & 1.13 & 664 \\
\hline 04/19/11 08:30 & 0.75 & 70.0 & 8.5 & 1.50 & 1.13 & 662 \\
\hline 04/19/11 08:45 & 1.00 & 70.0 & 8.0 & 1.50 & 1.14 & 669 \\
\hline 04/19/11 09:00 & 1.25 & 70.0 & 8.0 & 1.50 & 1.15 & 653 \\
\hline 04/19/11 09:15 & 1.50 & 70.0 & 8.0 & 1.50 & 1.16 & 657 \\
\hline 04/19/11 09:30 & 1.75 & 70.0 & 8.0 & 1.50 & 1.17 & 668 \\
\hline 04/19/11 09:45 & 2.00 & 70.0 & 8.5 & 1.50 & 1.18 & 661 \\
\hline 04/19/11 10:00 & 2.25 & 70.0 & 8.5 & 1.50 & 1.19 & 647 \\
\hline $04 / 19 / 11 \quad 10: 15$ & 2.50 & 70.0 & 8.5 & 1.50 & 1.20 & 647 \\
\hline 04/19/11 10:30 & 2.75 & 70.0 & 8.0 & 1.50 & 1.21 & 648 \\
\hline $04 / 19 / 11 \quad 10: 45$ & 3.00 & 70.0 & 8.0 & 1.50 & 1.22 & 645 \\
\hline 04/19/11 11:00 & 3.25 & 70.0 & 8.0 & 1.50 & 1.23 & 639 \\
\hline $04 / 19 / 1111: 15$ & 3.50 & 69.9 & 7.5 & 1.50 & 1.23 & 642 \\
\hline 04/19/11 11:30 & 3.75 & 70.0 & 7.5 & 1.50 & 1.24 & 653 \\
\hline 04/19/11 11:45 & 4.00 & 70.0 & 7.5 & 1.50 & 1.25 & 661 \\
\hline $04 / 19 / 1112: 00$ & 4.25 & 70.0 & 7.3 & 1.50 & 1.26 & 640 \\
\hline $04 / 19 / 11 \quad 12: 15$ & 4.50 & 70.0 & 8.3 & 1.50 & 1.27 & 631 \\
\hline $04 / 19 / 11 \quad 12: 30$ & 4.75 & 70.0 & 8.3 & 1.49 & 1.28 & 653 \\
\hline $04 / 19 / 11 \quad 12: 45$ & 5.00 & 70.0 & 8.3 & 1.50 & 1.29 & 648 \\
\hline 04/19/11 13:00 & 5.25 & 70.0 & 8.3 & 1.50 & 1.31 & 650 \\
\hline $04 / 19 / 11$ 13:15 & 5.50 & 70.0 & 8.3 & 1.50 & 1.32 & 648 \\
\hline 04/19/11 13:30 & 5.75 & 70.0 & 8.3 & 1.50 & 1.33 & 642 \\
\hline 04/19/11 13:45 & 6.00 & 70.0 & 8.3 & 1.50 & 1.34 & 634 \\
\hline 04/19/11 14:00 & 6.25 & 70.0 & 8.3 & 1.50 & 1.35 & 622 \\
\hline $04 / 19 / 11 \quad 14: 15$ & 6.50 & 70.0 & 8.3 & 1.50 & 1.36 & 641 \\
\hline 04/19/11 14:30 & 6.75 & 70.0 & 8.0 & 1.50 & 1.37 & 620 \\
\hline $04 / 19 / 11 \quad 14: 45$ & 7.00 & 70.0 & 8.0 & 1.50 & 1.38 & 628 \\
\hline 04/19/11 15:00 & 7.25 & 70.0 & 7.5 & 1.50 & 1.40 & 634 \\
\hline 04/19/11 15:15 & 7.50 & 70.0 & 8.5 & 1.50 & 1.41 & 632 \\
\hline 04/19/11 15:30 & 7.75 & 70.0 & 8.5 & 1.50 & 1.43 & 633 \\
\hline 04/19/11 15:45 & 8.00 & 70.0 & 8.5 & 1.50 & 1.44 & 664 \\
\hline 04/19/11 16:00 & 8.25 & 70.0 & 8.5 & 1.50 & 1.45 & 644 \\
\hline
\end{tabular}


SRNL-STI-2011-00360

Revision 0

Table A-2: Oxalic Acid Decomposition (without actual tank waste sludge), continued

\begin{tabular}{|c|c|c|c|c|c|c|}
\hline $\begin{array}{c}\text { Date/Time } \\
\text { (mm/dd/yy hh:mm) }\end{array}$ & $\begin{array}{c}\text { Run Time } \\
\text { (hr) }\end{array}$ & $\begin{array}{c}\text { Temperature } \\
\left({ }^{\circ} \mathrm{C}\right)\end{array}$ & $\begin{array}{c}\text { Pressure } \\
\text { (psi) }\end{array}$ & $\begin{array}{c}\text { Flow } \\
\text { (gal/min) }\end{array}$ & pH & $\begin{array}{l}\text { ORP } \\
(\mathrm{mV})\end{array}$ \\
\hline $04 / 19 / 1116: 15$ & 8.50 & 70.0 & 8.5 & 1.50 & 1.47 & 618 \\
\hline $04 / 19 / 11 \quad 16: 30$ & 8.75 & 70.0 & 8.5 & 1.50 & 1.49 & 646 \\
\hline $04 / 19 / 11 \quad 16: 45$ & 9.00 & 70.0 & 8.0 & 1.50 & 1.51 & 640 \\
\hline 04/19/11 17:00 & 9.25 & 70.0 & 8.0 & 1.50 & 1.53 & 631 \\
\hline 04/19/11 17:15 & 9.50 & 70.0 & 8.0 & 1.50 & 1.55 & 645 \\
\hline 04/19/11 17:30 & 9.75 & 70.0 & 8.0 & 1.50 & 1.58 & 630 \\
\hline 04/19/11 17:45 & 10.00 & 70.0 & 8.0 & 1.50 & 1.60 & 640 \\
\hline 04/19/11 18:00 & 10.25 & 70.0 & 8.0 & 1.50 & 1.63 & 652 \\
\hline 04/19/11 18:15 & 10.50 & 70.0 & 8.0 & 1.50 & 1.67 & 650 \\
\hline 04/20/11 07:45 & 10.50 & 70.0 & 8.5 & 1.50 & 1.78 & 657 \\
\hline 04/20/11 08:00 & 10.75 & 70.0 & 8.5 & 1.50 & 1.82 & 649 \\
\hline 04/20/11 08:15 & 11.00 & 70.0 & 8.5 & 1.50 & 1.89 & 658 \\
\hline 04/20/11 08:45 & 11.50 & 69.9 & 8.5 & 1.50 & 2.07 & 676 \\
\hline 04/20/11 09:00 & 11.75 & 70.1 & 8.5 & 1.50 & 2.25 & 653 \\
\hline 04/20/11 09:15 & 12.00 & 70.0 & 9.0 & 1.50 & 2.93 & 709 \\
\hline 04/20/11 09:30 & 12.25 & 70.0 & 9.0 & 1.58 & 3.69 & 1068 \\
\hline 04/20/11 09:45 & 12.50 & 69.9 & 9.0 & 1.55 & 4.39 & 1020 \\
\hline 04/20/11 10:00 & 12.75 & 70.0 & 8.5 & 1.50 & 7.26 & 920 \\
\hline $04 / 20 / 11 \quad 10: 15$ & 13.00 & 70.0 & 8.5 & 1.50 & 7.69 & 883 \\
\hline $04 / 20 / 1110: 30$ & 13.25 & 70.0 & 8.5 & 1.50 & 7.79 & 892 \\
\hline $04 / 20 / 11 \quad 10: 45$ & 13.50 & 70.0 & 8.5 & 1.50 & 7.88 & 886 \\
\hline 04/20/11 11:00 & 13.75 & 70.0 & 8.5 & 1.50 & 7.94 & 889 \\
\hline $04 / 20 / 1111: 15$ & 14.00 & 70.0 & 8.5 & 1.50 & 7.97 & 887 \\
\hline
\end{tabular}


Table A-3: Test 1 (Tank 12H) OA Batch 1 Dissolution Module Data

\begin{tabular}{|c|c|c|c|c|}
\hline $\begin{array}{c}\text { Date/Time } \\
\text { (mm/dd/yy hh:mm) }\end{array}$ & $\begin{array}{c}\text { Run Time } \\
\text { (hr) }\end{array}$ & $\begin{array}{c}\text { Temperature } \\
\left({ }^{\circ} \mathrm{C}\right)\end{array}$ & $\begin{array}{c}\text { Flow } \\
\text { (gal/min) }\end{array}$ & pH \\
\hline 04/20/11 10:48 & 0.0 & 65.2 & 1.0 & 1.52 \\
\hline 04/20/11 11:02 & 0.2 & 70.1 & 1.0 & 1.86 \\
\hline 04/20/11 11:49 & 1.0 & 70.1 & 1.0 & 2.50 \\
\hline $04 / 20 / 1112: 49$ & 2.0 & 70.0 & 1.0 & 3.10 \\
\hline 04/20/11 13:49 & 3.0 & 70.0 & 1.0 & 3.40 \\
\hline 04/20/11 14:49 & 4.0 & 70.0 & 1.0 & 3.50 \\
\hline $04 / 20 / 1115: 49$ & 5.0 & 70.0 & 1.0 & 3.55 \\
\hline $04 / 20 / 1116: 49$ & 6.0 & 70.0 & 1.0 & 3.56 \\
\hline $04 / 20 / 1117: 49$ & 7.0 & 70.0 & 1.0 & 3.81 \\
\hline $04 / 20 / 1118: 55$ & 8.1 & 70.5 & 1.0 & 4.06 \\
\hline $04 / 25 / 1108: 30$ & 8.1 & 69.2 & 1.0 & 1.95 \\
\hline $04 / 25 / 1108: 46$ & 8.4 & 70.4 & 1.0 & 2.33 \\
\hline 04/25/11 09:01 & 8.6 & 69.9 & 1.0 & 2.45 \\
\hline 04/25/11 09:15 & 8.9 & 70.1 & 1.0 & 2.66 \\
\hline 04/25/11 09:33 & 9.2 & 70.0 & 1.0 & 2.82 \\
\hline $04 / 25 / 1109: 47$ & 9.4 & 70.1 & 1.0 & 3.03 \\
\hline $04 / 25 / 11 \quad 10: 02$ & 9.7 & 70.0 & 1.0 & 3.17 \\
\hline $04 / 25 / 11 \quad 10: 15$ & 9.9 & 70.0 & 1.0 & 3.34 \\
\hline $04 / 25 / 1110: 35$ & 10.2 & 70.1 & 1.0 & 3.57 \\
\hline 04/25/11 10:46 & 10.4 & 70.0 & 1.0 & 3.68 \\
\hline 04/25/11 11:00 & 10.6 & 70.6 & 1.0 & 3.41 \\
\hline
\end{tabular}


SRNL-STI-2011-00360

Revision 0

Table A-4: Test 1 (Tank 12H Sludge) OA Batch 1 Decomposition Module Data

\begin{tabular}{|c|c|c|c|c|c|c|}
\hline $\begin{array}{c}\text { Date/Time } \\
\text { (mm/dd/yy hh:mm) }\end{array}$ & $\begin{array}{l}\text { Run Time } \\
\text { (hr) }\end{array}$ & $\begin{array}{c}\text { Temperature } \\
\left({ }^{\circ} \mathrm{C}\right)\end{array}$ & $\begin{array}{l}\text { Pressure } \\
\text { (psi) }\end{array}$ & $\begin{array}{c}\text { Flow } \\
\text { (gal/min) }\end{array}$ & pH & $\begin{array}{l}\text { ORP } \\
(\mathrm{mV})\end{array}$ \\
\hline $04 / 25 / 1111: 03$ & -- & 40.3 & 0.0 & 1.50 & 1.42 & 438 \\
\hline 04/25/11 11:17 & -- & 54.2 & 0.0 & 1.50 & 1.69 & 454 \\
\hline $04 / 25 / 1111: 32$ & -- & 63.3 & 0.0 & 1.50 & 1.77 & 467 \\
\hline $04 / 25 / 1112: 00$ & -- & 70.7 & 0.0 & 1.50 & 1.82 & 464 \\
\hline $04 / 25 / 1112: 17$ & 0.00 & 70.2 & 9.0 & 1.60 & 2.34 & 690 \\
\hline $04 / 25 / 1112: 30$ & 0.22 & 70.0 & 8.5 & 1.50 & 2.58 & 654 \\
\hline 04/25/11 12:46 & 0.48 & 69.9 & 8.5 & 1.50 & 3.28 & 644 \\
\hline 04/25/11 13:02 & 0.75 & 70.0 & 8.3 & 1.54 & 3.39 & 638 \\
\hline $04 / 25 / 1113: 15$ & 0.97 & 70.0 & 8.3 & 1.53 & 3.60 & 635 \\
\hline $04 / 25 / 1113: 30$ & 1.22 & 70.0 & 8.3 & 1.53 & 3.48 & 632 \\
\hline $04 / 25 / 11 \quad 13: 45$ & 1.47 & 70.0 & 8.2 & 1.35 & 3.63 & 632 \\
\hline $04 / 25 / 11 \quad 14: 00$ & 1.72 & 70.0 & 8.2 & 1.50 & 3.51 & 635 \\
\hline $04 / 25 / 1114: 15$ & 1.97 & 70.0 & 8.2 & 1.50 & 3.71 & 656 \\
\hline $04 / 25 / 1114: 30$ & 2.22 & 70.0 & 8.2 & 1.50 & 3.97 & 767 \\
\hline $04 / 25 / 1114: 45$ & 2.47 & 70.0 & 8.1 & 1.50 & 5.08 & 615 \\
\hline 04/25/11 15:00 & 2.72 & 70.0 & 8.1 & 1.48 & 5.73 & 581 \\
\hline $04 / 25 / 1115: 15$ & 2.97 & 70.0 & 8.1 & 1.50 & 6.30 & 535 \\
\hline $04 / 25 / 1115: 30$ & 3.22 & 70.0 & 8.0 & 1.45 & 6.54 & 507 \\
\hline $04 / 25 / 11 \quad 15: 45$ & 3.47 & 70.0 & 8.0 & 1.45 & 6.65 & 595 \\
\hline 04/25/11 16:07 & 3.83 & 70.1 & 8.2 & 1.45 & 6.68 & 671 \\
\hline $04 / 25 / 11 \quad 16: 20$ & 4.05 & 70.0 & 8.0 & 1.45 & 6.93 & 668 \\
\hline 04/25/11 16:32 & 4.25 & 70.0 & 8.0 & 1.45 & 7.07 & 670 \\
\hline $04 / 25 / 1116: 45$ & 4.47 & 70.0 & 8.0 & 1.45 & 7.44 & 690 \\
\hline 04/25/11 17:00 & 4.72 & 70.0 & 8.0 & 1.45 & 7.34 & 694 \\
\hline 04/25/11 17:19 & 5.03 & 70.0 & 7.8 & 1.45 & 7.79 & 700 \\
\hline $04 / 25 / 1117: 35$ & 5.30 & 70.0 & 7.8 & 1.42 & 8.09 & 698 \\
\hline $04 / 25 / 11 \quad 17: 45$ & 5.47 & 70.0 & 7.8 & 1.40 & 7.85 & 694 \\
\hline $04 / 25 / 1118: 00$ & 5.72 & 70.0 & 7.8 & 1.40 & 8.31 & 693 \\
\hline $04 / 25 / 11 \quad 18: 16$ & 5.98 & 70.0 & 7.8 & 1.40 & 7.94 & 688 \\
\hline $04 / 25 / 1118: 30$ & 6.22 & 70.0 & 7.5 & 1.40 & 8.52 & 682 \\
\hline 04/25/11 19:00 & 6.72 & 70.0 & 7.5 & 1.40 & 7.99 & 686 \\
\hline 04/25/11 19:30 & 7.22 & 70.0 & 7.5 & 1.40 & 8.13 & 673 \\
\hline $04 / 25 / 1120: 04$ & 7.78 & 70.0 & 7.3 & 1.40 & 8.39 & 672 \\
\hline
\end{tabular}


Table A-5: Test 1 (Tank 12H) OA Batch 1 Evaporator Module Data

\begin{tabular}{c|c|c|c|c}
\hline $\begin{array}{c}\text { Date/Time } \\
(\mathbf{m m} / \mathbf{d d} / \mathbf{y y} \mathbf{h h}: \mathbf{m m})\end{array}$ & $\begin{array}{c}\text { Run Time } \\
(\mathbf{h r})\end{array}$ & $\begin{array}{c}\text { Temperature } \\
\mathbf{(} \mathbf{C})\end{array}$ & $\begin{array}{c}\text { Pressure } \\
(\mathbf{i n . ~ H g})\end{array}$ & $\begin{array}{c}\text { Condensate } \\
(\mathbf{m L})\end{array}$ \\
\hline $04 / 26 / 1109: 22$ & 0.0 & 24.3 & 23.0 & $<1000$ \\
\hline $04 / 26 / 1110: 19$ & 0.9 & 76.3 & 0.0 & $<1000$ \\
\hline $04 / 26 / 1110: 30$ & 1.1 & 72.3 & 18.0 & $<1000$ \\
\hline $04 / 26 / 1111: 30$ & 2.1 & 67.2 & 18.0 & $<1000$ \\
\hline $04 / 26 / 1112: 30$ & 3.1 & 67.3 & 22.0 & $<1000$ \\
\hline $04 / 26 / 1113: 33$ & 4.2 & 70.6 & 18.5 & $<1000$ \\
\hline $04 / 26 / 1114: 30$ & 5.1 & 71.6 & 18.5 & 1100 \\
\hline $04 / 26 / 1115: 30$ & 6.1 & 70.9 & 19.0 & 1100 \\
\hline $04 / 26 / 1117: 02$ & 7.7 & 70.0 & 18.0 & 1100 \\
\hline $04 / 26 / 1117: 30$ & 8.1 & 70.3 & 19.0 & 1100 \\
\hline $04 / 26 / 1118: 28$ & 9.1 & 70.3 & 18.0 & 1180 \\
\hline $04 / 26 / 1118: 47$ & 9.4 & 70.3 & 18.0 & 1180 \\
\hline $04 / 26 / 1120: 20$ & 9.4 & 30.7 & 24.5 & 1180 \\
\hline $04 / 26 / 1120: 46$ & 9.8 & 52.5 & 25.0 & 1220 \\
\hline $04 / 26 / 1120: 57$ & 10.0 & 57.6 & 23.5 & 1320 \\
\hline $04 / 26 / 1121: 21$ & 10.4 & 57.7 & 23.5 & 1460 \\
\hline & & & & \\
\hline
\end{tabular}

Table A-6: Test 1 (Tank 12H) OA Batch 2 Dissolution Module Data

\begin{tabular}{c|c|c|c|c}
\hline $\begin{array}{c}\text { Date/Time } \\
(\mathbf{m m} / \mathbf{d d} / \mathbf{y} \mathbf{h} \text { hh:mm) }\end{array}$ & $\begin{array}{c}\text { Run Time } \\
\mathbf{( h r})\end{array}$ & $\begin{array}{c}\text { Temperature } \\
\mathbf{(} \mathbf{C})\end{array}$ & $\begin{array}{c}\text { Flow } \\
(\mathbf{g a l} / \mathbf{m i n})\end{array}$ & $\mathbf{p H}$ \\
\hline $04 / 26 / 1105: 00$ & 0.0 & 69.7 & 1.04 & 1.61 \\
\hline $04 / 26 / 1106: 00$ & 1.0 & 70.0 & 1.04 & 1.66 \\
\hline $04 / 26 / 1107: 00$ & 2.0 & 70.0 & 1.04 & 1.65 \\
\hline $04 / 26 / 1108: 00$ & 3.0 & 70.0 & 1.04 & 1.64 \\
\hline $04 / 26 / 1109: 06$ & 4.1 & 70.0 & 1.04 & 1.64 \\
\hline $04 / 26 / 1110: 00$ & 5.0 & 70.0 & 1.04 & 1.64 \\
\hline $04 / 26 / 1111: 00$ & 6.0 & 70.0 & 1.04 & 1.64 \\
\hline $04 / 26 / 1112: 00$ & 7.0 & 70.0 & 1.02 & 1.64 \\
\hline $04 / 26 / 1113: 00$ & 8.0 & 70.0 & 1.02 & 1.64 \\
\hline $04 / 26 / 1114: 00$ & 9.0 & 59.3 & 0.00 & 1.62 \\
\hline & & & &
\end{tabular}


SRNL-STI-2011-00360

Revision 0

Table A-7: Test 1 (Tank 12H Sludge) OA Batch 2 Decomposition Module Data

\begin{tabular}{|c|c|c|c|c|c|c|}
\hline $\begin{array}{c}\text { Date/Time } \\
\text { (mm/dd/yy hh:mm) }\end{array}$ & $\begin{array}{l}\text { Run Time } \\
\text { (hr) }\end{array}$ & $\begin{array}{c}\text { Temperature } \\
\left({ }^{\circ} \mathrm{C}\right)\end{array}$ & $\begin{array}{l}\text { Pressure } \\
\text { (psi) }\end{array}$ & $\begin{array}{c}\text { Flow } \\
\text { (gal/min) }\end{array}$ & pH & $\begin{array}{l}\text { ORP } \\
(\mathrm{mV})\end{array}$ \\
\hline $04 / 26 / 1114: 45$ & -- & 70.2 & 0.0 & 1.50 & 1.99 & 461 \\
\hline $04 / 26 / 11$ 15:00 & -- & 70.2 & 0.0 & 1.52 & 1.99 & 458 \\
\hline $04 / 26 / 1115: 15$ & 0.00 & 69.9 & 8.0 & 1.55 & 2.01 & 655 \\
\hline $04 / 26 / 11 \quad 15: 30$ & 0.25 & 70.1 & 8.0 & 1.55 & 2.02 & 639 \\
\hline 04/26/11 15:46 & 0.52 & 70.0 & 8.0 & 1.55 & 2.03 & 631 \\
\hline $04 / 26 / 1116: 00$ & 0.75 & 70.0 & 8.0 & 1.55 & 2.04 & 627 \\
\hline $04 / 26 / 1116: 15$ & 1.00 & 70.0 & 8.0 & 1.55 & 2.06 & 625 \\
\hline $04 / 26 / 1116: 30$ & 1.25 & 70.0 & 8.0 & 1.55 & 2.07 & 622 \\
\hline $04 / 26 / 1116: 45$ & 1.50 & 69.9 & 8.2 & 1.55 & 2.09 & 621 \\
\hline $04 / 26 / 1117: 00$ & 1.75 & 70.0 & 8.2 & 1.55 & 2.10 & 621 \\
\hline $04 / 26 / 1117: 15$ & 2.00 & 70.0 & 8.2 & 1.55 & 2.12 & 619 \\
\hline $04 / 26 / 1117: 30$ & 2.25 & 70.0 & 8.2 & 1.55 & 2.14 & 617 \\
\hline $04 / 26 / 1117: 45$ & 2.50 & 70.0 & 8.2 & 1.55 & 2.16 & 618 \\
\hline 04/26/11 18:01 & 2.77 & 70.0 & 8.0 & 1.55 & 2.18 & 616 \\
\hline $04 / 26 / 1118: 18$ & 3.05 & 70.0 & 8.0 & 1.55 & 2.20 & 614 \\
\hline $04 / 26 / 1118: 30$ & 3.25 & 70.0 & 8.0 & 1.45 & 2.22 & 617 \\
\hline $04 / 26 / 1118: 45$ & 3.50 & 70.0 & 8.0 & 1.45 & 2.25 & 618 \\
\hline 04/26/11 19:00 & 3.75 & 70.0 & 8.0 & 1.45 & 2.28 & 620 \\
\hline 04/26/11 19:15 & 4.00 & 70.0 & 8.0 & 1.45 & 2.32 & 620 \\
\hline $04 / 26 / 11 \quad 19: 30$ & 4.25 & 70.0 & 7.5 & 1.45 & 2.37 & 623 \\
\hline $04 / 26 / 1119: 46$ & 4.52 & 70.0 & 7.5 & 1.45 & 2.44 & 630 \\
\hline $04 / 26 / 1120: 00$ & 4.75 & 70.0 & 7.5 & 1.45 & 2.52 & 640 \\
\hline $04 / 26 / 1120: 15$ & 5.00 & 70.0 & 7.5 & 1.45 & 2.68 & 693 \\
\hline $04 / 26 / 1120: 22$ & 5.12 & 69.9 & 7.5 & 1.45 & 2.82 & 742 \\
\hline 04/26/11 20:29 & 5.23 & 70.0 & 7.5 & 1.45 & 3.14 & 796 \\
\hline $04 / 26 / 1120: 33$ & 5.30 & 70.0 & -- & -- & 3.65 & 674 \\
\hline $04 / 26 / 1120: 35$ & 5.33 & 70.0 & -- & -- & 3.94 & 658 \\
\hline $04 / 26 / 1120: 40$ & 5.42 & 70.0 & -- & -- & 4.09 & 625 \\
\hline $04 / 26 / 1120: 45$ & 5.50 & 70.0 & 7.0 & 1.47 & 4.17 & 621 \\
\hline $04 / 26 / 1120: 50$ & 5.58 & 70.0 & -- & -- & 4.28 & 610 \\
\hline $04 / 26 / 1120: 55$ & 5.67 & 70.0 & 7.5 & 1.45 & 4.36 & 597 \\
\hline 04/26/11 21:00 & 5.75 & 69.9 & 7.0 & 1.45 & 4.39 & 578 \\
\hline 04/26/11 21:08 & 5.88 & 70.0 & 8.0 & 1.45 & 4.34 & 570 \\
\hline $04 / 26 / 1121: 15$ & 6.00 & 70.0 & 8.0 & 1.45 & 4.38 & 554 \\
\hline
\end{tabular}


SRNL-STI-2011-00360

Revision 0

Table A-8: Test 1 (Tank 12H Sludge) OA Batch 2 Decomposition Module Data, continued

\begin{tabular}{|c|c|c|c|c|c|c|}
\hline $\begin{array}{c}\text { Date/Time } \\
\text { (mm/dd/yy hh:mm) }\end{array}$ & $\begin{array}{c}\text { Run Time } \\
\text { (hr) }\end{array}$ & $\begin{array}{c}\text { Temperature } \\
\left({ }^{\circ} \mathrm{C}\right)\end{array}$ & $\begin{array}{l}\text { Pressure } \\
\text { (psi) }\end{array}$ & $\begin{array}{c}\text { Flow } \\
\text { (gal/min) }\end{array}$ & $\mathbf{p H}$ & $\begin{array}{l}\text { ORP } \\
(\mathrm{mV})\end{array}$ \\
\hline $04 / 26 / 1121: 22$ & 6.12 & 70.0 & -- & -- & 4.63 & 577 \\
\hline $04 / 26 / 1121: 30$ & 6.25 & 70.0 & 8.0 & 1.45 & 4.91 & 593 \\
\hline $04 / 26 / 1121: 35$ & 6.33 & 70.0 & -- & -- & 5.11 & 644 \\
\hline $04 / 26 / 1121: 40$ & 6.42 & 70.0 & -- & -- & 5.29 & 712 \\
\hline $04 / 26 / 1121: 45$ & 6.50 & 70.0 & 8.0 & 1.40 & 5.46 & 751 \\
\hline $04 / 26 / 1121: 50$ & 6.58 & 70.0 & -- & -- & 5.62 & 768 \\
\hline $04 / 26 / 1121: 55$ & 6.67 & 70.0 & -- & -- & 5.79 & 770 \\
\hline $04 / 26 / 1122: 00$ & 6.75 & 70.0 & 8.0 & 1.40 & 5.98 & 765 \\
\hline $04 / 26 / 1122: 07$ & 6.87 & 70.0 & -- & -- & 6.24 & 748 \\
\hline $04 / 26 / 1122: 11$ & 6.93 & 70.0 & -- & -- & 6.40 & 728 \\
\hline $04 / 26 / 1122: 15$ & 7.00 & 70.1 & 8.0 & 1.40 & 6.55 & 704 \\
\hline $04 / 26 / 1122: 20$ & 7.08 & 70.0 & -- & -- & 6.72 & 685 \\
\hline $04 / 26 / 1122: 25$ & 7.17 & 70.0 & -- & -- & 6.87 & 672 \\
\hline $04 / 26 / 1122: 31$ & 7.27 & 70.0 & 8.0 & 1.40 & 7.11 & 668 \\
\hline $04 / 26 / 1122: 35$ & 7.33 & 70.0 & -- & -- & 7.27 & 666 \\
\hline $04 / 26 / 1122: 40$ & 7.42 & 70.0 & -- & -- & 7.41 & 675 \\
\hline $04 / 26 / 1122: 50$ & 7.58 & 70.0 & 8.0 & 1.40 & 7.56 & 710 \\
\hline 04/26/11 23:00 & 7.75 & 70.0 & 7.5 & 1.40 & 7.68 & 719 \\
\hline $04 / 26 / 1123: 15$ & 8.00 & 70.0 & 7.5 & 1.40 & 7.91 & 723 \\
\hline $04 / 26 / 1123: 30$ & 8.25 & 70.0 & 7.5 & 1.40 & 8.19 & 728 \\
\hline $04 / 26 / 1123: 45$ & 8.50 & 70.0 & 7.5 & 1.40 & 8.37 & 733 \\
\hline 04/27/11 00:00 & 8.75 & 70.0 & 7.5 & 1.38 & 8.46 & 733 \\
\hline $04 / 27 / 1100: 15$ & 9.00 & 69.9 & 7.5 & 1.40 & 8.52 & 740 \\
\hline 04/27/11 00:30 & 9.25 & 70.0 & 7.5 & 1.40 & 8.55 & 741 \\
\hline 04/27/11 00:45 & 9.50 & 69.9 & 7.5 & 1.40 & 8.58 & 744 \\
\hline 04/27/11 01:00 & 9.75 & 70.0 & 7.5 & 1.40 & 8.60 & 738 \\
\hline $04 / 27 / 1101: 15$ & 10.00 & 69.9 & 7.5 & 1.38 & 8.66 & 740 \\
\hline 04/27/11 01:30 & 10.25 & 70.1 & 7.5 & 1.38 & 8.68 & 727 \\
\hline 04/27/11 01:45 & 10.50 & 69.9 & 7.0 & 1.38 & 8.69 & 731 \\
\hline 04/27/11 02:00 & 10.75 & 70.0 & 7.0 & 1.38 & 8.70 & 729 \\
\hline 04/27/11 02:07 & 10.87 & -- & -- & -- & -- & -- \\
\hline
\end{tabular}


Table A-9: Test 1 (Tank 12H) OA Batch 2 Evaporator Module Data

\begin{tabular}{c|c|c|c|c}
\hline $\begin{array}{c}\text { Date/Time } \\
(\mathbf{m m} / \mathbf{d d} / \mathbf{y y} \text { hh:mm) }\end{array}$ & $\begin{array}{c}\text { Run Time } \\
\mathbf{( h r})\end{array}$ & $\begin{array}{c}\text { Temperature } \\
\left.\mathbf{(}{ }^{\circ} \mathbf{C}\right)\end{array}$ & $\begin{array}{c}\text { Pressure } \\
(\mathbf{i n . ~ H g})\end{array}$ & $\begin{array}{c}\text { Condensate } \\
(\mathbf{m L})\end{array}$ \\
\hline $04 / 27 / 1113: 33$ & 0.0 & 26.2 & 23.5 & $<1000$ \\
\hline $04 / 27 / 1114: 05$ & 0.5 & 58.8 & 24.0 & $<1000$ \\
\hline $04 / 27 / 1114: 40$ & 1.1 & 70.0 & 21.0 & $<1000$ \\
\hline $04 / 27 / 1115: 01$ & 1.5 & 70.0 & 19.5 & $<1000$ \\
\hline $04 / 27 / 1116: 00$ & 2.4 & 70.0 & 19.0 & $<1000$ \\
\hline $04 / 27 / 1117: 00$ & 3.5 & 69.5 & 19.5 & 1200 \\
\hline $04 / 27 / 1118: 00$ & 4.5 & 69.8 & 19.5 & 1600 \\
\hline
\end{tabular}

Table A-10: Test 1 (Tank 12H) OA Batch 3 Dissolution Module Data

\begin{tabular}{c|c|c|c|c}
\hline $\begin{array}{c}\text { Date/Time } \\
\text { (mm/dd/yy hh:mm) }\end{array}$ & $\begin{array}{c}\text { Run Time } \\
\mathbf{( h r})\end{array}$ & $\begin{array}{c}\text { Temperature } \\
\left.\mathbf{(}{ }^{\circ} \mathbf{C}\right)\end{array}$ & $\begin{array}{c}\text { Flow } \\
\text { (gal/min) }\end{array}$ & pH \\
\hline $04 / 27 / 1117: 32$ & 0.0 & 65.8 & 1.0 & 2.16 \\
\hline $04 / 27 / 1118: 00$ & 0.5 & 70.1 & 1.0 & 2.16 \\
\hline $04 / 27 / 1119: 00$ & 1.5 & 70.0 & 1.0 & 2.10 \\
\hline $04 / 27 / 1120: 02$ & 2.5 & 70.0 & 1.05 & 2.04 \\
\hline $04 / 27 / 1121: 00$ & 3.5 & 70.0 & 1.05 & 2.02 \\
\hline $04 / 27 / 1122: 00$ & 4.5 & 70.0 & 1.0 & 2.01 \\
\hline $04 / 27 / 1123: 09$ & 5.6 & 70.0 & 1.0 & 2.00 \\
\hline $04 / 28 / 1100: 00$ & 6.5 & 70.0 & 1.0 & 2.00 \\
\hline $04 / 28 / 1101: 00$ & 7.5 & 70.0 & 1.0 & 2.01 \\
\hline $04 / 28 / 1102: 01$ & 8.5 & 70.0 & 1.0 & 2.00 \\
\hline $04 / 28 / 1102: 15$ & 8.7 & -- & -- & -- \\
\hline
\end{tabular}


SRNL-STI-2011-00360

Revision 0

Table A-11 Test 1 (Tank 12H Sludge) OA Batch 3 Decomposition Module Data

\begin{tabular}{|c|c|c|c|c|c|c|}
\hline $\begin{array}{c}\text { Date/Time } \\
\text { (mm/dd/yy hh:mm) }\end{array}$ & $\begin{array}{c}\text { Run Time } \\
\text { (hr) }\end{array}$ & $\begin{array}{c}\text { Temperature } \\
\left({ }^{\circ} \mathrm{C}\right)\end{array}$ & $\begin{array}{l}\text { Pressure } \\
\text { (psi) }\end{array}$ & $\begin{array}{c}\text { Flow } \\
\text { (gal/min) }\end{array}$ & $\mathbf{p H}$ & $\begin{array}{l}\text { ORP } \\
(\mathbf{m V})\end{array}$ \\
\hline $04 / 28 / 1102: 23$ & -- & 46.4 & 0.0 & 1.55 & 2.08 & 662 \\
\hline 04/28/11 03:02 & 0.00 & 67.1 & 8.0 & 1.50 & 1.90 & 615 \\
\hline 04/28/11 03:15 & 0.22 & 70.6 & 8.0 & 1.50 & 1.90 & 607 \\
\hline 04/28/11 03:30 & 0.47 & 70.0 & 8.0 & 1.50 & 1.93 & 605 \\
\hline 04/28/11 03:45 & 0.72 & 69.9 & 8.0 & 1.50 & 1.93 & 604 \\
\hline 04/28/11 04:05 & 1.05 & 70.0 & 8.0 & 1.50 & 1.95 & 604 \\
\hline 04/28/11 04:18 & 1.27 & 70.0 & 7.5 & 1.50 & 1.95 & 604 \\
\hline 04/28/11 04:32 & 1.50 & 70.0 & 7.0 & 1.50 & 1.96 & 604 \\
\hline 04/28/11 04:46 & 1.73 & 70.0 & 8.0 & 1.50 & 1.97 & 604 \\
\hline 04/28/11 05:02 & 2.00 & 70.0 & 8.0 & 1.50 & 1.98 & 604 \\
\hline 04/28/11 05:15 & 2.22 & 70.0 & 8.0 & 1.50 & 1.99 & 604 \\
\hline $04 / 28 / 1105: 33$ & 2.52 & 69.9 & 8.0 & 1.50 & 1.99 & 603 \\
\hline 04/28/11 05:45 & 2.72 & 69.9 & 8.2 & 1.50 & 2.00 & 603 \\
\hline 04/28/11 06:00 & 2.97 & 70.0 & 8.2 & 1.50 & 2.01 & 603 \\
\hline 04/28/11 06:15 & 3.22 & 70.0 & 8.2 & 1.50 & 2.02 & 603 \\
\hline $04 / 28 / 1106: 30$ & 3.47 & 70.0 & 7.5 & 1.50 & 2.03 & 602 \\
\hline 04/28/11 06:45 & 3.72 & 70.0 & 7.5 & 1.50 & 2.04 & 602 \\
\hline 04/28/11 07:00 & 3.97 & 70.0 & 7.5 & 1.50 & 2.05 & 602 \\
\hline 04/28/11 07:15 & 4.22 & 70.0 & 8.0 & 1.50 & 2.07 & 602 \\
\hline 04/28/11 07:30 & 4.47 & 70.0 & 8.0 & 1.35 & 2.08 & 601 \\
\hline $04 / 28 / 1107: 45$ & 4.72 & 70.0 & 8.0 & 1.50 & 2.09 & 601 \\
\hline 04/28/11 08:00 & 4.97 & 70.0 & 8.0 & 1.50 & 2.11 & 600 \\
\hline 04/28/11 08:15 & 5.22 & 70.0 & 8.0 & 1.50 & 2.12 & 600 \\
\hline 04/28/11 08:30 & 5.47 & 70.0 & 8.0 & 1.50 & 2.14 & 600 \\
\hline 04/28/11 08:45 & 5.72 & 70.0 & 8.3 & 1.50 & 2.16 & 599 \\
\hline 04/28/11 09:00 & 5.97 & 70.0 & 8.3 & 1.50 & 2.18 & 599 \\
\hline 04/28/11 09:15 & 6.22 & 69.9 & 8.3 & 1.50 & 2.20 & 599 \\
\hline 04/28/11 09:30 & 6.47 & 70.0 & 8.3 & 1.50 & 2.23 & 598 \\
\hline 04/28/11 09:45 & 6.72 & 70.0 & 8.3 & 1.50 & 2.26 & 599 \\
\hline $04 / 28 / 11$ 10:00 & 6.97 & 70.0 & 8.3 & 1.50 & 2.30 & 600 \\
\hline $04 / 28 / 11 \quad 10: 15$ & 7.22 & 70.0 & 7.5 & 1.50 & 2.34 & 601 \\
\hline $04 / 28 / 11 \quad 10: 30$ & 7.47 & 69.9 & 8.0 & 1.50 & 2.40 & 607 \\
\hline $04 / 28 / 11 \quad 10: 45$ & 7.72 & 70.1 & 8.0 & 1.50 & 2.50 & 617 \\
\hline 04/28/11 11:00 & 7.97 & 70.0 & 8.0 & 1.50 & 2.71 & 641 \\
\hline 04/28/11 11:05 & 8.05 & 69.9 & 8.0 & 1.50 & 2.92 & 645 \\
\hline $04 / 28 / 1111: 10$ & 8.13 & 70.0 & 7.9 & 1.50 & 3.42 & 616 \\
\hline $04 / 28 / 1111: 15$ & 8.22 & 70.0 & 7.9 & 1.50 & 3.74 & 584 \\
\hline
\end{tabular}


SRNL-STI-2011-00360

Revision 0

Table A-12: Test 1 (Tank12H Sludge) OA Batch 3 Decomposition Module Data, continued

\begin{tabular}{|c|c|c|c|c|c|c|}
\hline $\begin{array}{c}\text { Date/Time } \\
\text { (mm/dd/yy hh:mm) }\end{array}$ & $\begin{array}{c}\text { Run Time } \\
\text { (hr) }\end{array}$ & $\begin{array}{c}\text { Temperature } \\
\left({ }^{\circ} \mathrm{C}\right)\end{array}$ & $\begin{array}{l}\text { Pressure } \\
\text { (psi) }\end{array}$ & $\begin{array}{c}\text { Flow } \\
\text { (gal/min) }\end{array}$ & pH & $\begin{array}{l}\text { ORP } \\
(\mathrm{mV})\end{array}$ \\
\hline $04 / 28 / 1111: 20$ & 8.30 & 69.9 & 7.8 & 1.50 & 3.80 & 563 \\
\hline $04 / 28 / 1111: 25$ & 8.38 & 69.9 & 7.8 & 1.50 & 3.90 & 544 \\
\hline $04 / 28 / 1111: 30$ & 8.47 & 70.0 & 7.8 & 1.50 & 3.98 & 527 \\
\hline $04 / 28 / 1111: 35$ & 8.55 & 70.0 & 7.7 & 1.50 & 4.01 & 517 \\
\hline 04/28/11 11:40 & 8.63 & 70.0 & 7.8 & 1.50 & 4.03 & 524 \\
\hline 04/28/11 11:45 & 8.72 & 70.0 & 7.9 & 1.50 & 4.12 & 808 \\
\hline 04/28/11 11:50 & 8.80 & 70.0 & 7.8 & 1.50 & 4.26 & 861 \\
\hline 04/28/11 11:55 & 8.88 & 70.0 & 7.8 & 1.50 & 4.36 & 900 \\
\hline $04 / 28 / 1112: 00$ & 8.97 & 70.0 & 7.7 & 1.50 & 4.43 & 873 \\
\hline 04/28/11 12:05 & 9.05 & 70.0 & 7.6 & 1.50 & 4.48 & 866 \\
\hline $04 / 28 / 1112: 15$ & 9.22 & 70.0 & 8.5 & 1.50 & 4.55 & 982 \\
\hline 04/28/11 12:20 & 9.30 & 70.0 & 8.3 & 1.50 & 4.58 & 970 \\
\hline $04 / 28 / 1112: 30$ & 9.47 & 70.0 & 8.3 & 1.50 & 4.67 & 907 \\
\hline $04 / 28 / 11 \quad 12: 40$ & 9.63 & 70.0 & 8.2 & 1.50 & 4.78 & 826 \\
\hline $04 / 28 / 1112: 50$ & 9.80 & 70.0 & 8.2 & 1.50 & 4.95 & 803 \\
\hline 04/28/11 13:00 & 9.97 & 70.0 & 8.1 & 1.50 & 5.20 & 782 \\
\hline 04/28/11 13:05 & 10.05 & 70.0 & 8.1 & 1.50 & 5.42 & 755 \\
\hline 04/28/11 13:10 & 10.13 & 70.0 & 8.1 & 1.50 & 5.63 & 731 \\
\hline 04/28/11 13:15 & 10.22 & 70.0 & 8.1 & 1.50 & 5.89 & 709 \\
\hline 04/28/11 13:20 & 10.30 & 70.0 & 8.1 & 1.50 & 6.25 & 676 \\
\hline $04 / 28 / 1113: 25$ & 10.38 & 70.1 & 8.1 & 1.50 & 6.51 & 660 \\
\hline $04 / 28 / 1113: 30$ & 10.47 & 70.0 & 8.1 & 1.50 & 6.83 & 651 \\
\hline $04 / 28 / 1113: 35$ & 10.55 & 70.0 & 8.0 & 1.50 & 6.97 & 668 \\
\hline 04/28/11 13:40 & 10.63 & 70.0 & 8.0 & 1.50 & 7.09 & 696 \\
\hline $04 / 28 / 1113: 45$ & 10.72 & 70.0 & 8.0 & 1.50 & 7.18 & 718 \\
\hline $04 / 28 / 1113: 50$ & 10.80 & 70.0 & 8.0 & 1.50 & 7.25 & 724 \\
\hline 04/28/11 13:55 & 10.88 & 70.0 & 8.0 & 1.50 & 7.35 & 738 \\
\hline 04/28/11 14:00 & 10.97 & 70.0 & 8.0 & 1.48 & 7.46 & 743 \\
\hline 04/28/11 14:05 & 11.05 & 70.1 & 8.0 & 1.48 & 7.54 & 753 \\
\hline 04/28/11 14:10 & 11.13 & 70.0 & 8.0 & 1.47 & 7.66 & 751 \\
\hline $04 / 28 / 1114: 20$ & 11.30 & 70.0 & 8.0 & 1.45 & 7.76 & 738 \\
\hline $04 / 28 / 1114: 30$ & 11.47 & 69.9 & 8.0 & 1.45 & 7.86 & 702 \\
\hline $04 / 28 / 1114: 45$ & 11.72 & 70.1 & 8.0 & 1.45 & 7.98 & 730 \\
\hline 04/28/11 15:00 & 11.97 & 70.0 & 8.0 & 1.45 & 8.06 & 708 \\
\hline 04/28/11 15:15 & 12.22 & 69.9 & 8.0 & 1.45 & 8.09 & 677 \\
\hline $04 / 28 / 1115: 25$ & 12.38 & 70.0 & 8.0 & 1.45 & 8.11 & 649 \\
\hline 04/28/11 15:40 & 12.63 & 69.0 & 8.0 & 1.45 & 8.15 & 667 \\
\hline
\end{tabular}


Table A-13: Test 1 (Tank12H) OA Batch 3 Evaporator Module Data

\begin{tabular}{c|c|c|c|c}
\hline $\begin{array}{c}\text { Date/Time } \\
(\mathbf{m m} / \mathbf{d d} / \mathbf{y} \mathbf{h h : m m})\end{array}$ & $\begin{array}{c}\text { Run Time } \\
\mathbf{( h r})\end{array}$ & $\begin{array}{c}\text { Temperature } \\
\left.\mathbf{(}{ }^{\circ} \mathbf{C}\right)\end{array}$ & $\begin{array}{c}\text { Pressure } \\
(\mathbf{i n . ~ H g})\end{array}$ & $\begin{array}{c}\text { Condensate } \\
(\mathbf{m L})\end{array}$ \\
\hline $04 / 28 / 1118: 15$ & 0.0 & -- & 21.0 & $<1000$ \\
\hline $04 / 28 / 1118: 50$ & 0.6 & 68.8 & 20.0 & $<1000$ \\
\hline $04 / 28 / 1119: 07$ & 0.9 & 68.7 & 20.0 & $<1000$ \\
\hline $04 / 28 / 1119: 40$ & 1.4 & 69.9 & 19.5 & $<1000$ \\
\hline $04 / 28 / 1119: 55$ & 1.7 & 70.2 & 19.5 & $<1000$ \\
\hline $04 / 28 / 1120: 00$ & 1.8 & 70.0 & 20.0 & $<1000$ \\
\hline $04 / 28 / 1121: 00$ & 2.8 & 69.8 & 20.0 & $<1000$ \\
\hline $04 / 28 / 1122: 00$ & 3.8 & 68.8 & 20.0 & $<1000$ \\
\hline $04 / 28 / 1123: 00$ & 4.8 & 69.0 & 20.0 & 1300 \\
\hline $04 / 28 / 1123: 40$ & 5.4 & 68.9 & 20.0 & 1450 \\
\hline $04 / 29 / 1100: 02$ & 5.8 & 68.9 & 20.0 & 1570 \\
\hline $04 / 29 / 1100: 07$ & 5.9 & 68.9 & 20.0 & 1600 \\
\hline $04 / 29 / 1100: 17$ & 6.0 & 68.7 & 20.0 & $>1600$ \\
\hline
\end{tabular}

Table A-14: Test 2 (Tank 5F) OA Batch 1 Dissolution Module Data

\begin{tabular}{c|c|c|c|c}
\hline $\begin{array}{c}\text { Date/Time } \\
\text { (mm/dd/yy hh:mm) }\end{array}$ & $\begin{array}{c}\text { Run Time } \\
\mathbf{( h r})\end{array}$ & $\begin{array}{c}\text { Temperature } \\
\left.\mathbf{(}{ }^{\circ} \mathbf{C}\right)\end{array}$ & $\begin{array}{c}\text { Flow } \\
\text { (gal/min) }\end{array}$ & pH \\
\hline $05 / 05 / 1108: 28$ & 0.0 & 65.1 & 1.25 & 2.77 \\
\hline $05 / 05 / 1108: 43$ & 0.2 & 70.0 & 1.25 & 2.87 \\
\hline $05 / 05 / 1109: 43$ & 1.3 & 70.2 & 1.25 & 3.65 \\
\hline $05 / 05 / 1110: 43$ & 2.3 & 70.0 & 1.25 & 4.00 \\
\hline $05 / 05 / 1111: 43$ & 3.2 & 69.9 & 1.25 & 2.89 \\
\hline $05 / 05 / 1112: 48$ & 4.3 & 70.0 & 1.25 & 2.18 \\
\hline $05 / 05 / 1113: 48$ & 5.3 & 70.0 & 1.25 & 2.15 \\
\hline $05 / 05 / 1114: 48$ & 6.3 & 70.0 & 1.25 & 2.18 \\
\hline $05 / 05 / 1116: 51$ & 8.4 & 70.0 & 1.25 & 2.20 \\
\hline
\end{tabular}


SRNL-STI-2011-00360

Revision 0

Table A-15: Test 2 (Tank 5F Sludge) OA Batch 1 Decomposition Module Data

Note: the $\mathrm{pH}$ probe was malfunctioning during this data set

\begin{tabular}{|c|c|c|c|c|c|c|}
\hline $\begin{array}{c}\text { Date/Time } \\
\text { (mm/dd/yy hh:mm) }\end{array}$ & $\begin{array}{l}\text { Run Time } \\
\text { (hr) }\end{array}$ & $\begin{array}{c}\text { Temperature } \\
\left({ }^{\circ} \mathrm{C}\right)\end{array}$ & $\begin{array}{c}\text { Pressure } \\
\text { (psi) }\end{array}$ & $\begin{array}{c}\text { Flow } \\
\text { (gal } / \text { min) }\end{array}$ & $\mathbf{p H}$ & $\begin{array}{l}\text { ORP } \\
(\mathrm{mV})\end{array}$ \\
\hline 05/09/11 06:45 & -- & 32.3 & 0.0 & 1.60 & 2.09 & 499 \\
\hline 05/09/11 07:45 & 0.00 & 66.8 & 8.0 & 1.50 & 2.49 & 728 \\
\hline 05/09/11 08:00 & 0.25 & 69.4 & 8.0 & 1.50 & 2.37 & 730 \\
\hline 05/09/11 08:15 & 0.50 & 70.4 & 8.0 & 1.50 & 2.33 & 731 \\
\hline 05/09/11 08:30 & 0.75 & 70.0 & 8.0 & 1.50 & 2.36 & 746 \\
\hline 05/09/11 08:45 & 1.00 & 70.1 & 8.0 & 1.50 & 2.38 & 758 \\
\hline 05/09/11 09:00 & 1.25 & 70.1 & 8.0 & 1.50 & 2.07 & 767 \\
\hline 05/09/11 09:15 & 1.50 & 69.9 & 7.0 & 1.50 & 1.92 & 821 \\
\hline 05/09/11 09:30 & 1.75 & 70.1 & 8.0 & 1.50 & 2.15 & 797 \\
\hline 05/09/11 09:45 & 2.00 & 69.9 & 8.0 & 1.50 & 2.50 & 730 \\
\hline 05/09/11 09:50 & 2.08 & 70.0 & 8.0 & 1.50 & 2.62 & 717 \\
\hline 05/09/11 09:55 & 2.17 & 70.0 & 8.0 & 1.50 & 2.72 & 704 \\
\hline 05/09/11 10:00 & 2.25 & 70.0 & 8.0 & 1.40 & 2.84 & 679 \\
\hline 05/09/11 10:05 & 2.33 & 70.0 & 8.0 & 1.40 & 2.97 & 656 \\
\hline 05/09/11 10:10 & 2.42 & 70.0 & 8.0 & 1.50 & 3.14 & 618 \\
\hline 05/09/11 10:15 & 2.50 & 70.0 & 8.0 & 1.50 & 3.19 & 626 \\
\hline 05/09/11 10:20 & 2.58 & 70.0 & 8.0 & 1.50 & 3.26 & 615 \\
\hline $05 / 09 / 11 \quad 10: 25$ & 2.67 & 70.0 & 8.0 & 1.50 & 3.33 & 603 \\
\hline 05/09/11 10:30 & 2.75 & 69.9 & 8.0 & 1.50 & 3.38 & 591 \\
\hline 05/09/11 10:35 & 2.83 & 70.0 & 7.5 & 1.50 & 3.44 & 581 \\
\hline 05/09/11 10:40 & 2.92 & 70.0 & 7.5 & 1.47 & 3.49 & 573 \\
\hline 05/09/11 10:45 & 3.00 & 70.0 & 7.5 & 1.48 & 3.64 & 567 \\
\hline 05/09/11 10:50 & 3.08 & 70.0 & 7.5 & 1.48 & 3.77 & 561 \\
\hline 05/09/11 10:56 & 3.18 & 70.0 & 7.5 & 1.50 & 3.79 & 554 \\
\hline 05/09/11 11:00 & 3.25 & 70.0 & 7.5 & 1.50 & 3.81 & 550 \\
\hline 05/09/11 11:05 & 3.33 & 70.0 & 7.5 & 1.50 & 3.83 & 546 \\
\hline 05/09/11 11:10 & 3.42 & 70.0 & 7.5 & 1.50 & 3.84 & 542 \\
\hline 05/09/11 11:15 & 3.50 & 70.0 & 7.5 & 1.50 & 3.85 & 538 \\
\hline 05/09/11 11:20 & 3.58 & 70.0 & 7.5 & 1.50 & 3.88 & 530 \\
\hline 05/09/11 11:30 & 3.75 & 70.0 & 7.5 & 1.50 & 3.99 & 491 \\
\hline 05/09/11 11:45 & 4.00 & 69.9 & 7.5 & 1.50 & 4.06 & 452 \\
\hline 05/09/11 12:00 & 4.25 & 70.0 & 7.5 & 1.50 & 4.17 & 479 \\
\hline 05/09/11 12:15 & 4.50 & 70.0 & 7.5 & 1.50 & 4.25 & 570 \\
\hline 05/09/11 12:30 & 4.75 & 70.0 & 7.5 & 1.42 & 4.33 & 658 \\
\hline
\end{tabular}


Table A-16: Test 2 (Tank 5F Sludge) OA Batch 1 Decomposition Module Data, continued

Note: the $\mathrm{pH}$ probe was malfunctioning during this data set

\begin{tabular}{|c|c|c|c|c|c|c|}
\hline $\begin{array}{c}\text { Date/Time } \\
\text { (mm/dd/yy hh:mm) }\end{array}$ & $\begin{array}{l}\text { Run Time } \\
\text { (hr) }\end{array}$ & $\begin{array}{c}\text { Temperature } \\
\left({ }^{\circ} \mathrm{C}\right)\end{array}$ & $\begin{array}{c}\text { Pressure } \\
\text { (psi) }\end{array}$ & $\begin{array}{c}\text { Flow } \\
\text { (gal } / \text { min) }\end{array}$ & pH & $\begin{array}{l}\text { ORP } \\
(\mathrm{mV})\end{array}$ \\
\hline 05/09/11 12:45 & 5.00 & 70.0 & 7.5 & 1.42 & 4.28 & 739 \\
\hline 05/09/11 13:00 & 5.25 & 70.0 & 7.5 & 1.42 & 4.35 & 782 \\
\hline 05/09/11 13:15 & 5.50 & 70.0 & 7.5 & 1.42 & 4.25 & 788 \\
\hline 05/09/11 13:30 & 5.75 & 70.0 & 7.5 & 1.42 & 4.07 & 788 \\
\hline 05/09/11 13:45 & 6.00 & 70.0 & 7.5 & 1.40 & 4.16 & 787 \\
\hline 05/09/11 14:00 & 6.25 & 70.0 & 7.5 & 1.40 & 4.08 & 783 \\
\hline 05/09/11 14:15 & 6.50 & 70.0 & 7.2 & 1.41 & 4.10 & 778 \\
\hline 05/09/11 14:30 & 6.75 & 70.0 & 8.3 & 1.45 & 4.21 & 782 \\
\hline 05/09/11 14:45 & 7.00 & 70.1 & 8.3 & 1.45 & 4.30 & 779 \\
\hline 05/09/11 15:00 & 7.25 & 70.0 & 8.0 & 1.45 & 4.42 & 779 \\
\hline 05/09/11 15:15 & 7.50 & 70.1 & 8.3 & 1.45 & 4.50 & 777 \\
\hline 05/09/11 15:30 & 7.75 & 70.0 & 8.3 & 1.46 & 4.54 & 775 \\
\hline 05/09/11 15:45 & 8.00 & 70.0 & 8.3 & 1.46 & 4.59 & 772 \\
\hline 05/09/11 16:00 & 8.25 & 70.0 & 8.3 & 1.46 & 4.56 & 766 \\
\hline 05/09/11 16:15 & 8.50 & 70.0 & 8.3 & 1.46 & 4.60 & 767 \\
\hline 05/09/11 16:30 & 8.75 & 70.0 & 8.3 & 1.46 & 4.57 & 763 \\
\hline 05/09/11 16:45 & 9.00 & 70.0 & 8.5 & 1.48 & 4.54 & 760 \\
\hline 05/09/11 17:00 & 9.25 & 70.1 & 8.5 & 1.48 & 4.47 & 755 \\
\hline 05/09/11 17:15 & 9.50 & 70.0 & 8.5 & 1.48 & 4.39 & 751 \\
\hline 05/09/11 17:30 & 9.75 & 70.0 & 8.5 & 1.48 & 4.34 & 755 \\
\hline 05/09/11 17:45 & 10.00 & 70.0 & 8.5 & 1.48 & 4.33 & 754 \\
\hline 05/09/11 18:00 & 10.25 & 70.0 & 8.5 & 1.48 & 4.33 & 755 \\
\hline 05/09/11 18:18 & 10.55 & 70.0 & 8.5 & 1.48 & 4.34 & 746 \\
\hline 05/09/11 18:30 & 10.75 & 70.0 & 8.5 & 1.48 & 4.31 & 745 \\
\hline 05/09/11 18:45 & 11.00 & 69.9 & 8.5 & 1.48 & 4.27 & 746 \\
\hline 05/09/11 19:00 & 11.25 & 70.0 & 8.5 & 1.48 & 4.19 & 750 \\
\hline
\end{tabular}


Table A-17: Test 2 (Tank 5F) OA Batch 1 Evaporator Module Data

\begin{tabular}{c|c|c|c|c}
\hline $\begin{array}{c}\text { Date/Time } \\
(\mathbf{m m} / \mathbf{d d} / \mathbf{y} \mathbf{y} \text { hh:mm) }\end{array}$ & $\begin{array}{c}\text { Run Time } \\
\mathbf{( h r})\end{array}$ & $\begin{array}{c}\text { Temperature } \\
\left({ }^{\circ} \mathbf{C}\right)\end{array}$ & $\begin{array}{c}\text { Pressure } \\
(\mathbf{i n . ~ H g})\end{array}$ & $\begin{array}{c}\text { Condensate } \\
(\mathbf{m L})\end{array}$ \\
\hline $05 / 11 / 1106: 29$ & 0.0 & -- & 15.5 & $<1000$ \\
\hline $05 / 11 / 1107: 00$ & 0.5 & 69.1 & 20.0 & $<1000$ \\
\hline $05 / 11 / 1108: 00$ & 1.5 & 70.0 & 20.0 & $<1000$ \\
\hline $05 / 11 / 1109: 00$ & 2.5 & 69.3 & 20.0 & $<1000$ \\
\hline $05 / 11 / 1110: 00$ & 3.5 & 68.9 & 20.0 & 1200 \\
\hline $05 / 11 / 1111: 00$ & 4.5 & 68.7 & 20.0 & 1580 \\
\hline $05 / 11 / 1111: 20$ & 4.8 & 68.7 & 20.0 & $>1600$ \\
\hline
\end{tabular}

Table A-18: Test 2 (Tank 5F) OA Batch 2 Dissolution Module Data

\begin{tabular}{|c|c|c|c|c|}
\hline $\begin{array}{c}\text { Date/Time } \\
(\mathrm{mm} / \mathrm{dd} / \mathrm{yy} \text { hh:mm) }\end{array}$ & $\begin{array}{c}\text { Run Time } \\
\text { (hr) }\end{array}$ & $\begin{array}{c}\text { Temperature } \\
\left({ }^{\circ} \mathrm{C}\right)\end{array}$ & $\begin{array}{c}\text { Flow } \\
\text { (gal/min) }\end{array}$ & $\mathbf{p H}^{*}$ \\
\hline $05 / 11 / 1107: 17$ & 0.0 & 67.1 & 1.03 & 1.89 \\
\hline 05/11/11 08:00 & 0.7 & 70.0 & 1.03 & $3.05^{*}$ \\
\hline 05/11/11 08:11 & 0.9 & 70.0 & 1.03 & $3.30^{*}$ \\
\hline 05/11/11 09:00 & 1.7 & 70.0 & 1.03 & $3.73^{*}$ \\
\hline 05/11/11 10:00 & 2.7 & 70.0 & 1.03 & $4.07^{*}$ \\
\hline 05/11/11 11:00 & 3.7 & 70.0 & 1.03 & $4.35^{*}$ \\
\hline $05 / 11 / 1112: 00$ & 4.7 & 70.0 & 1.03 & $4.48^{*}$ \\
\hline 05/11/11 13:00 & 5.7 & 70.0 & 1.03 & $4.52 *$ \\
\hline 05/11/11 15:15 & 8.0 & 70.0 & 1.03 & $4.55^{*}$ \\
\hline
\end{tabular}

* $\mathrm{pH}$ behavior indicates probe not in contact with Dis solution Module fluid. 
SRNL-STI-2011-00360

Revision 0

Table A-19: Test 2 (Tank 5F Sludge) OA Batch 2 Decomposition Module Data

\begin{tabular}{|c|c|c|c|c|c|c|}
\hline $\begin{array}{c}\text { Date/Time } \\
\text { (mm/dd/yy hh:mm) }\end{array}$ & $\begin{array}{c}\text { Run Time } \\
\text { (hr) }\end{array}$ & $\begin{array}{c}\text { Temperature } \\
\left({ }^{\circ} \mathrm{C}\right)\end{array}$ & $\begin{array}{l}\text { Pressure } \\
\text { (psi) }\end{array}$ & $\begin{array}{c}\text { Flow } \\
\text { (gal/min) }\end{array}$ & $\mathbf{p H}$ & $\begin{array}{l}\text { ORP } \\
(\mathrm{mV})\end{array}$ \\
\hline $05 / 12 / 1106: 10$ & -- & 22.0 & 0.0 & 1.50 & -- & -- \\
\hline 05/12/11 07:01 & 0.00 & 65.5 & 8.0 & 1.50 & 1.27 & 812 \\
\hline 05/12/11 07:15 & 0.23 & 69.6 & 8.3 & 1.50 & 1.31 & 879 \\
\hline $05 / 12 / 1107: 30$ & 0.48 & 70.4 & 8.0 & 1.50 & 1.37 & 884 \\
\hline $05 / 12 / 1107: 45$ & 0.73 & 70.0 & 8.0 & 1.50 & 1.40 & 899 \\
\hline 05/12/11 08:00 & 0.98 & 70.0 & 8.0 & 1.50 & 1.45 & 912 \\
\hline 05/12/11 08:15 & 1.23 & 70.0 & 8.0 & 1.50 & 1.48 & 919 \\
\hline 05/12/11 08:30 & 1.48 & 70.0 & 8.0 & 1.50 & 1.52 & 930 \\
\hline $05 / 12 / 1108: 45$ & 1.73 & 70.0 & 8.0 & 1.50 & 1.56 & 947 \\
\hline 05/12/11 09:00 & 1.98 & 70.0 & 8.5 & 1.50 & 1.61 & 977 \\
\hline $05 / 12 / 1109: 15$ & 2.23 & 70.0 & 8.5 & 1.50 & 1.70 & 981 \\
\hline 05/12/11 09:30 & 2.48 & 70.0 & 8.5 & 1.50 & 1.87 & 960 \\
\hline $05 / 12 / 1109: 45$ & 2.73 & 70.0 & 8.5 & 1.50 & 2.56 & 921 \\
\hline $05 / 12 / 1109: 50$ & 2.82 & 69.9 & 8.5 & 1.48 & 2.65 & 938 \\
\hline $05 / 12 / 1109: 55$ & 2.90 & 70.0 & 8.0 & 1.35 & 2.69 & 944 \\
\hline 05/12/11 10:00 & 2.98 & 70.0 & 8.5 & 1.35 & 2.74 & 932 \\
\hline 05/12/11 10:05 & 3.07 & 70.0 & 8.0 & 1.35 & 2.79 & 930 \\
\hline $05 / 12 / 1110: 10$ & 3.15 & 70.0 & 8.0 & 1.35 & 2.87 & 929 \\
\hline $05 / 12 / 1110: 15$ & 3.23 & 70.0 & 8.0 & 1.35 & 2.94 & 929 \\
\hline 05/12/11 10:20 & 3.32 & 70.0 & 8.0 & 1.35 & 3.02 & 905 \\
\hline $05 / 12 / 11 \quad 10: 25$ & 3.40 & 70.0 & 8.0 & 1.35 & 3.12 & 919 \\
\hline $05 / 12 / 1110: 30$ & 3.48 & 70.0 & 8.0 & 1.35 & 3.20 & 899 \\
\hline $05 / 12 / 1110: 35$ & 3.57 & 70.0 & 8.0 & 1.35 & 3.32 & 902 \\
\hline 05/12/11 10:40 & 3.65 & 70.0 & 8.0 & 1.23 & 3.45 & 884 \\
\hline $05 / 12 / 1110: 45$ & 3.73 & 70.0 & 8.0 & 1.23 & 3.56 & 885 \\
\hline $05 / 12 / 1110: 50$ & 3.82 & 69.9 & 7.5 & 1.23 & 3.68 & 860 \\
\hline $05 / 12 / 1110: 55$ & 3.90 & 69.9 & 8.0 & 1.23 & 3.78 & 892 \\
\hline $05 / 12 / 1111: 00$ & 3.98 & 70.0 & 8.0 & 1.23 & 3.87 & 887 \\
\hline $05 / 12 / 1111: 05$ & 4.07 & 70.0 & 8.0 & 1.22 & 3.97 & 879 \\
\hline $05 / 12 / 1111: 10$ & 4.15 & 70.0 & 8.0 & 1.22 & 4.05 & 877 \\
\hline $05 / 12 / 1111: 15$ & 4.23 & 70.0 & 8.0 & 1.22 & 4.13 & 871 \\
\hline $05 / 12 / 1111: 20$ & 4.32 & 70.0 & 8.0 & 1.20 & 4.23 & 888 \\
\hline $05 / 12 / 1111: 25$ & 4.40 & 70.1 & 7.5 & 1.20 & 4.33 & 909 \\
\hline $05 / 12 / 1111: 30$ & 4.48 & 70.0 & 8.0 & 1.20 & 4.61 & 928 \\
\hline
\end{tabular}


SRNL-STI-2011-00360

Revision 0

Table A-20: Test 2 (Tank 5F Sludge) OA Batch 2 Decomposition Module Data, continued

\begin{tabular}{|c|c|c|c|c|c|c|}
\hline $\begin{array}{c}\text { Date/Time } \\
\text { (mm/dd/yy hh:mm) }\end{array}$ & $\begin{array}{c}\text { Run Time } \\
\text { (hr) }\end{array}$ & $\begin{array}{c}\text { Temperature } \\
\left({ }^{\circ} \mathrm{C}\right)\end{array}$ & $\begin{array}{l}\text { Pressure } \\
\text { (psi) }\end{array}$ & $\begin{array}{c}\text { Flow } \\
\text { (gal/min) }\end{array}$ & pH & $\begin{array}{l}\text { ORP } \\
(\mathrm{mV})\end{array}$ \\
\hline $05 / 12 / 1111: 35$ & 4.57 & 70.0 & 7.5 & 1.20 & 4.95 & 924 \\
\hline 05/12/11 11:40 & 4.65 & 70.0 & 8.0 & 1.20 & 5.30 & 922 \\
\hline $05 / 12 / 1111: 45$ & 4.73 & 70.0 & 8.0 & 1.20 & 5.62 & 923 \\
\hline 05/12/11 11:50 & 4.82 & 70.0 & 7.5 & 1.20 & 5.93 & 907 \\
\hline 05/12/11 11:55 & 4.90 & 70.0 & 7.5 & 1.20 & 6.17 & 911 \\
\hline 05/12/11 12:00 & 4.98 & 70.0 & 7.5 & 1.20 & 6.38 & 901 \\
\hline 05/12/11 12:06 & 5.08 & 70.0 & 7.5 & 1.20 & 6.60 & 887 \\
\hline $05 / 12 / 1112: 10$ & 5.15 & 70.0 & 7.5 & 1.20 & 6.76 & 897 \\
\hline $05 / 12 / 1112: 15$ & 5.23 & 70.0 & 8.0 & 1.20 & 6.85 & 894 \\
\hline $05 / 12 / 1112: 20$ & 5.32 & 70.0 & 8.0 & 1.20 & 7.04 & 900 \\
\hline $05 / 12 / 1112: 25$ & 5.40 & 70.0 & 8.0 & 1.20 & 7.18 & 898 \\
\hline $05 / 12 / 1112: 30$ & 5.48 & 70.0 & 8.0 & 1.20 & 7.31 & 896 \\
\hline $05 / 12 / 1112: 35$ & 5.57 & 70.0 & 8.0 & 1.20 & 7.51 & 894 \\
\hline $05 / 12 / 1112: 40$ & 5.65 & 70.0 & 8.0 & 1.20 & 7.57 & 905 \\
\hline $05 / 12 / 1112: 45$ & 5.73 & 70.0 & 8.0 & 1.20 & 7.78 & 901 \\
\hline 05/12/11 12:50 & 5.82 & 70.0 & 8.0 & 1.20 & 7.88 & 907 \\
\hline 05/12/11 12:55 & 5.90 & 70.0 & 8.0 & 1.20 & 7.94 & 912 \\
\hline 05/12/11 13:00 & 5.98 & 70.0 & 8.0 & 1.20 & 8.02 & 920 \\
\hline $05 / 12 / 1113: 15$ & 6.23 & 70.0 & 8.0 & 1.20 & 8.15 & 917 \\
\hline 05/12/11 13:30 & 6.48 & 70.1 & 8.0 & 1.20 & 8.24 & 916 \\
\hline $05 / 12 / 1113: 45$ & 6.73 & 70.0 & 8.0 & 1.20 & 8.30 & 917 \\
\hline 05/12/11 14:00 & 6.98 & 70.0 & 8.0 & 1.20 & 8.35 & 918 \\
\hline 05/12/11 14:15 & 7.23 & 70.0 & 7.5 & 1.08 & 8.42 & 930 \\
\hline $05 / 12 / 1114: 30$ & 7.48 & 70.0 & 7.5 & 1.08 & 8.49 & 955 \\
\hline $05 / 12 / 11 \quad 14: 42$ & 7.68 & 70.0 & 7.5 & 1.20 & 8.52 & 956 \\
\hline
\end{tabular}


Table A-21: Test 2 (Tank 5F) OA Batch 2 Evaporator Module Data

\begin{tabular}{c|c|c|c|c}
\hline $\begin{array}{c}\text { Date/Time } \\
(\mathbf{m m} / \mathbf{d d} / \mathbf{y} \mathbf{~ h h : m m})\end{array}$ & $\begin{array}{c}\text { Run Time } \\
\mathbf{( h r})\end{array}$ & $\begin{array}{c}\text { Temperature } \\
\left.\mathbf{(}{ }^{\circ} \mathbf{C}\right)\end{array}$ & $\begin{array}{c}\text { Pressure } \\
(\mathbf{i n . ~ H g})\end{array}$ & $\begin{array}{c}\text { Condensate } \\
(\mathbf{m L})\end{array}$ \\
\hline $05 / 16 / 1110: 58$ & 0.0 & 21.0 & 19.5 & $<1000$ \\
\hline $05 / 16 / 1111: 33$ & 0.6 & 70.1 & 19.5 & $<1000$ \\
\hline $05 / 16 / 1112: 30$ & 1.5 & 69.3 & 19.5 & $<1000$ \\
\hline $05 / 16 / 1113: 00$ & 2.0 & 69.0 & 19.5 & $<1000$ \\
\hline $05 / 16 / 1114: 00$ & 3.0 & 69.6 & 19.5 & $<1000$ \\
\hline $05 / 16 / 1114: 40$ & 3.7 & 69.1 & 19.5 & 1200 \\
\hline $05 / 16 / 1115: 00$ & 4.0 & 69.2 & 19.5 & 1300 \\
\hline
\end{tabular}

Table A-22: Test 2 (Tank 5F) OA Batch 3 Dissolution Module Data

\begin{tabular}{c|c|c|c|c}
\hline $\begin{array}{c}\text { Date/Time } \\
\mathbf{( m m / d d / y y ~ h h : m m ) ~}\end{array}$ & $\begin{array}{c}\text { Run Time } \\
\mathbf{( h r})\end{array}$ & $\begin{array}{c}\text { Temperature } \\
\left.\mathbf{(}{ }^{\circ} \mathbf{C}\right)\end{array}$ & $\begin{array}{c}\text { Flow } \\
\text { (gal/min) }\end{array}$ & pH \\
\hline $05 / 16 / 1108: 19$ & 0.0 & 65.3 & 1.05 & 1.25 \\
\hline $05 / 16 / 1108: 30$ & 0.2 & 70.0 & 1.05 & 1.24 \\
\hline $05 / 16 / 1109: 30$ & 1.2 & 70.0 & 1.05 & 1.21 \\
\hline $05 / 16 / 1110: 30$ & 2.2 & 70.1 & 1.05 & 1.11 \\
\hline $05 / 16 / 1111: 30$ & 3.2 & 70.0 & 1.05 & 1.09 \\
\hline $05 / 16 / 1112: 30$ & 4.2 & 70.0 & 1.05 & 1.08 \\
\hline $05 / 16 / 1113: 30$ & 5.2 & 70.0 & 1.05 & 1.08 \\
\hline $05 / 16 / 1114: 30$ & 6.2 & 70.0 & 1.05 & 1.07 \\
\hline $05 / 16 / 1115: 30$ & 7.2 & 70.0 & 1.05 & 1.07 \\
\hline $05 / 16 / 1116: 29$ & 8.2 & 70.0 & 1.10 & 1.06 \\
\hline
\end{tabular}


SRNL-STI-2011-00360

Revision 0

Table A-23: Test 2 (Tank 5F Sludge) OA Batch 3 Decomposition Module Data

\begin{tabular}{|c|c|c|c|c|c|c|}
\hline $\begin{array}{c}\text { Date/Time } \\
\text { (mm/dd/yy hh:mm) }\end{array}$ & $\begin{array}{l}\text { Run Time } \\
\text { (hr) }\end{array}$ & $\begin{array}{c}\text { Temperature } \\
\left({ }^{\circ} \mathrm{C}\right)\end{array}$ & $\begin{array}{l}\text { Pressure } \\
\text { (psi) }\end{array}$ & $\begin{array}{c}\text { Flow } \\
\text { (gal/min) }\end{array}$ & $\mathbf{p H}$ & $\begin{array}{l}\text { ORP } \\
(\mathrm{mV})\end{array}$ \\
\hline 05/17/11 06:20 & & 18.6 & 0.0 & 1.50 & 1.22 & 624 \\
\hline 05/17/11 07:32 & & 64.1 & 8.0 & 1.50 & 1.20 & 679 \\
\hline 05/17/11 07:45 & 0.00 & 66.8 & 8.0 & 1.58 & 1.20 & 701 \\
\hline 05/17/11 08:00 & 0.25 & 69.8 & 8.0 & 1.58 & 1.22 & 692 \\
\hline 05/17/11 08:15 & 0.50 & 70.3 & 7.7 & 1.58 & 1.24 & 694 \\
\hline 05/17/11 08:30 & 0.75 & 69.9 & 8.0 & 1.58 & 1.26 & 692 \\
\hline 05/17/11 08:45 & 1.00 & 70.0 & 8.0 & 1.57 & 1.27 & 695 \\
\hline 05/17/11 09:00 & 1.25 & 70.0 & 8.0 & 1.57 & 1.29 & 695 \\
\hline 05/17/11 09:15 & 1.50 & 70.0 & 8.0 & 1.57 & 1.31 & 698 \\
\hline 05/17/11 09:30 & 1.75 & 70.1 & 8.0 & 1.57 & 1.33 & 698 \\
\hline 05/17/11 09:45 & 2.00 & 70.1 & 8.5 & 1.57 & 1.36 & 699 \\
\hline 05/17/11 10:00 & 2.25 & 70.0 & 8.5 & 1.57 & 1.38 & 701 \\
\hline 05/17/11 10:15 & 2.50 & 69.9 & 8.5 & 1.57 & 1.40 & 704 \\
\hline 05/17/11 10:30 & 2.75 & 69.9 & 8.8 & 1.57 & 1.41 & 707 \\
\hline 05/17/11 10:45 & 3.00 & 70.0 & 8.8 & 1.57 & 1.43 & 710 \\
\hline 05/17/11 11:00 & 3.25 & 70.0 & 9.0 & 1.58 & 1.46 & 713 \\
\hline 05/17/11 11:15 & 3.50 & 70.0 & 9.0 & 1.57 & 1.48 & 718 \\
\hline 05/17/11 11:30 & 3.75 & 70.0 & 8.5 & 1.47 & 1.52 & 717 \\
\hline 05/17/11 11:45 & 4.00 & 70.0 & 8.5 & 1.45 & 1.55 & 720 \\
\hline 05/17/11 12:00 & 4.25 & 70.0 & 8.5 & 1.45 & 1.59 & 723 \\
\hline $05 / 17 / 11 \quad 12: 15$ & 4.50 & 70.0 & 8.5 & 1.45 & 1.66 & 715 \\
\hline 05/17/11 12:30 & 4.75 & 70.0 & 8.5 & 1.42 & 1.77 & 698 \\
\hline $05 / 17 / 11 \quad 12: 45$ & 5.00 & 70.0 & 8.5 & 1.40 & 2.13 & 759 \\
\hline $05 / 17 / 1112: 55$ & 5.17 & 70.0 & 8.5 & 1.40 & 2.31 & 752 \\
\hline 05/17/11 13:00 & 5.25 & 70.0 & 8.5 & 1.40 & 2.33 & 740 \\
\hline 05/17/11 13:05 & 5.33 & 70.0 & 8.5 & 1.38 & 2.36 & 731 \\
\hline 05/17/11 13:10 & 5.42 & 70.0 & 8.5 & 1.38 & 2.40 & 726 \\
\hline 05/17/11 13:15 & 5.50 & 70.0 & 8.5 & 1.38 & 2.44 & 722 \\
\hline 05/17/11 13:20 & 5.58 & 70.0 & 8.5 & 1.38 & 2.49 & 718 \\
\hline 05/17/11 13:25 & 5.67 & 70.0 & 8.5 & 1.38 & 2.55 & 714 \\
\hline 05/17/11 13:30 & 5.75 & 70.0 & 8.5 & 1.38 & 2.62 & 710 \\
\hline 05/17/11 13:35 & 5.83 & 70.1 & 8.5 & 1.38 & 2.71 & 702 \\
\hline 05/17/11 13:40 & 5.92 & 70.0 & 8.2 & 1.38 & 2.78 & 696 \\
\hline 05/17/11 13:45 & 6.00 & 70.0 & 8.5 & 1.38 & 2.88 & 688 \\
\hline
\end{tabular}


SRNL-STI-2011-00360

Revision 0

Table A-24: Test 2 (Tank 5F Sludge) OA Batch 3 Decomposition Module Data, continued

\begin{tabular}{|c|c|c|c|c|c|c|}
\hline $\begin{array}{c}\text { Date/Time } \\
(\mathrm{mm} / \mathrm{dd} / \mathrm{yy} \text { hh:mm) }\end{array}$ & $\begin{array}{c}\text { Run Time } \\
\text { (hr) }\end{array}$ & $\begin{array}{c}\text { Temperature } \\
\left({ }^{\circ} \mathrm{C}\right)\end{array}$ & $\begin{array}{l}\text { Pressure } \\
\text { (psi) }\end{array}$ & $\begin{array}{c}\text { Flow } \\
\text { (gal/min) }\end{array}$ & pH & $\begin{array}{l}\text { ORP } \\
(\mathrm{mV})\end{array}$ \\
\hline 05/17/11 13:50 & 6.08 & 70.0 & 8.5 & 1.38 & 3.01 & 679 \\
\hline 05/17/11 13:55 & 6.17 & 70.0 & 8.5 & 1.38 & 3.14 & 673 \\
\hline 05/17/11 14:00 & 6.25 & 70.0 & 8.5 & 1.38 & 3.29 & 667 \\
\hline 05/17/11 14:05 & 6.33 & 70.0 & 8.5 & 1.38 & 3.44 & 661 \\
\hline 05/17/11 14:10 & 6.42 & 70.0 & 8.5 & 1.38 & 3.55 & 661 \\
\hline 05/17/11 14:15 & 6.50 & 70.0 & 8.5 & 1.38 & 3.64 & 644 \\
\hline $05 / 17 / 1114: 20$ & 6.58 & 70.0 & 8.5 & 1.38 & 3.68 & 644 \\
\hline $05 / 17 / 1114: 25$ & 6.67 & 70.0 & 8.5 & 1.38 & 3.72 & 638 \\
\hline 05/17/11 14:30 & 6.75 & 70.1 & 8.5 & 1.38 & 4.05 & 622 \\
\hline 05/17/11 14:35 & 6.83 & 70.0 & 8.5 & 1.38 & 4.52 & 611 \\
\hline 05/17/11 14:40 & 6.92 & 70.0 & 8.5 & 1.38 & 5.00 & 594 \\
\hline $05 / 17 / 1114: 45$ & 7.00 & 70.0 & 8.5 & 1.38 & 5.36 & 594 \\
\hline 05/17/11 14:50 & 7.08 & 70.0 & 8.5 & 1.38 & 5.72 & 612 \\
\hline 05/17/11 14:55 & 7.17 & 69.9 & 8.5 & 1.38 & 5.98 & 643 \\
\hline 05/17/11 15:02 & 7.28 & 70.0 & 8.5 & 1.35 & 6.45 & 681 \\
\hline 05/17/11 15:05 & 7.33 & 70.0 & 8.5 & 1.35 & 6.57 & 684 \\
\hline 05/17/11 15:10 & 7.42 & 70.0 & 8.5 & 1.35 & 6.75 & 700 \\
\hline 05/17/11 15:16 & 7.52 & 70.0 & 8.5 & 1.32 & 6.97 & 709 \\
\hline 05/17/11 15:20 & 7.58 & 69.9 & 8.5 & 1.32 & 7.12 & 714 \\
\hline $05 / 17 / 1115: 25$ & 7.67 & 70.0 & 8.5 & 1.32 & 7.25 & 714 \\
\hline 05/17/11 15:30 & 7.75 & 70.0 & 8.5 & 1.32 & 7.33 & 709 \\
\hline 05/17/11 15:35 & 7.83 & 70.0 & 8.5 & 1.32 & 7.41 & 709 \\
\hline 05/17/11 15:40 & 7.92 & 70.0 & 8.4 & 1.32 & 7.49 & 708 \\
\hline 05/17/11 15:45 & 8.00 & 70.1 & 8.4 & 1.32 & 7.52 & 706 \\
\hline 05/17/11 15:50 & 8.08 & 70.0 & 8.5 & 1.32 & 7.58 & 708 \\
\hline 05/17/11 15:55 & 8.17 & 70.0 & 8.5 & 1.30 & 7.67 & 704 \\
\hline 05/17/11 16:00 & 8.25 & 70.0 & 8.5 & 1.35 & 7.72 & 704 \\
\hline 05/17/11 16:05 & 8.33 & 70.0 & 8.3 & 1.32 & 7.72 & 701 \\
\hline 05/17/11 16:10 & 8.42 & 70.0 & 8.3 & 1.31 & 7.77 & 698 \\
\hline 05/17/11 16:20 & 8.58 & 69.9 & 8.2 & 1.31 & 7.84 & 698 \\
\hline 05/17/11 16:30 & 8.75 & 70.0 & 8.2 & 1.31 & 7.90 & 690 \\
\hline
\end{tabular}


Table A-25: Test 2 (Tank 5F) OA Batch 3 Evaporator Module Data

\begin{tabular}{c|c|c|c|c}
\hline $\begin{array}{c}\text { Date/Time } \\
(\mathbf{m m} / \mathbf{d d} / \mathbf{y} \mathbf{~ h h : m m})\end{array}$ & $\begin{array}{c}\text { Run Time } \\
(\mathbf{h r})\end{array}$ & $\begin{array}{c}\text { Temperature } \\
\mathbf{(} \mathbf{C} \mathbf{)}\end{array}$ & $\begin{array}{c}\text { Pressure } \\
(\mathbf{i n . ~ H g})\end{array}$ & $\begin{array}{c}\text { Condensate } \\
(\mathbf{m L})\end{array}$ \\
\hline $05 / 19 / 1108: 26$ & 0.0 & 18.2 & 18.0 & $<1000$ \\
\hline $05 / 19 / 1109: 02$ & 0.6 & 70.5 & 18.0 & $<1000$ \\
\hline $05 / 19 / 1110: 00$ & 1.6 & 70.4 & 20.0 & $<1000$ \\
\hline $05 / 19 / 1111: 00$ & 2.6 & 70.3 & 20.0 & $<1000$ \\
\hline $05 / 19 / 1112: 00$ & 3.6 & 69.5 & 20.0 & $<1000$ \\
\hline $05 / 19 / 1113: 00$ & 4.6 & 69.5 & 20.0 & 1200 \\
\hline $05 / 19 / 1114: 00$ & 5.6 & 69.6 & 20.0 & 1520 \\
\hline $05 / 19 / 1114: 20$ & 5.9 & 69.6 & 20.0 & $>1600$ \\
\hline
\end{tabular}


SRNL-STI-2011-00360

Revision 0

Appendix B. Particle Size, Rheology, and Settling Data

B-1 


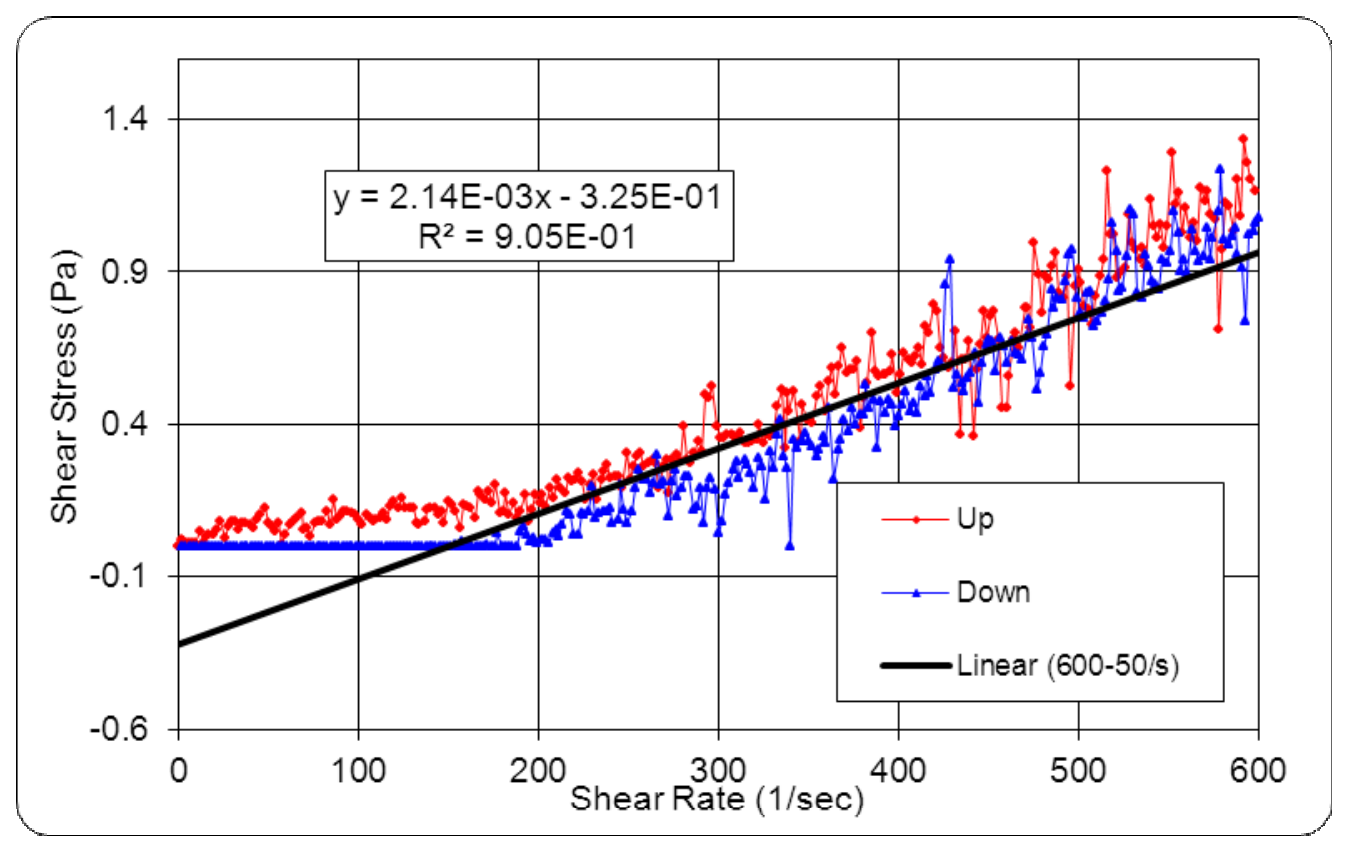

Figure B-1: Stress Versus Strain Curve for Tank 5F Baseline Dilute at $30^{\circ} \mathrm{C}$ (First Replicate).

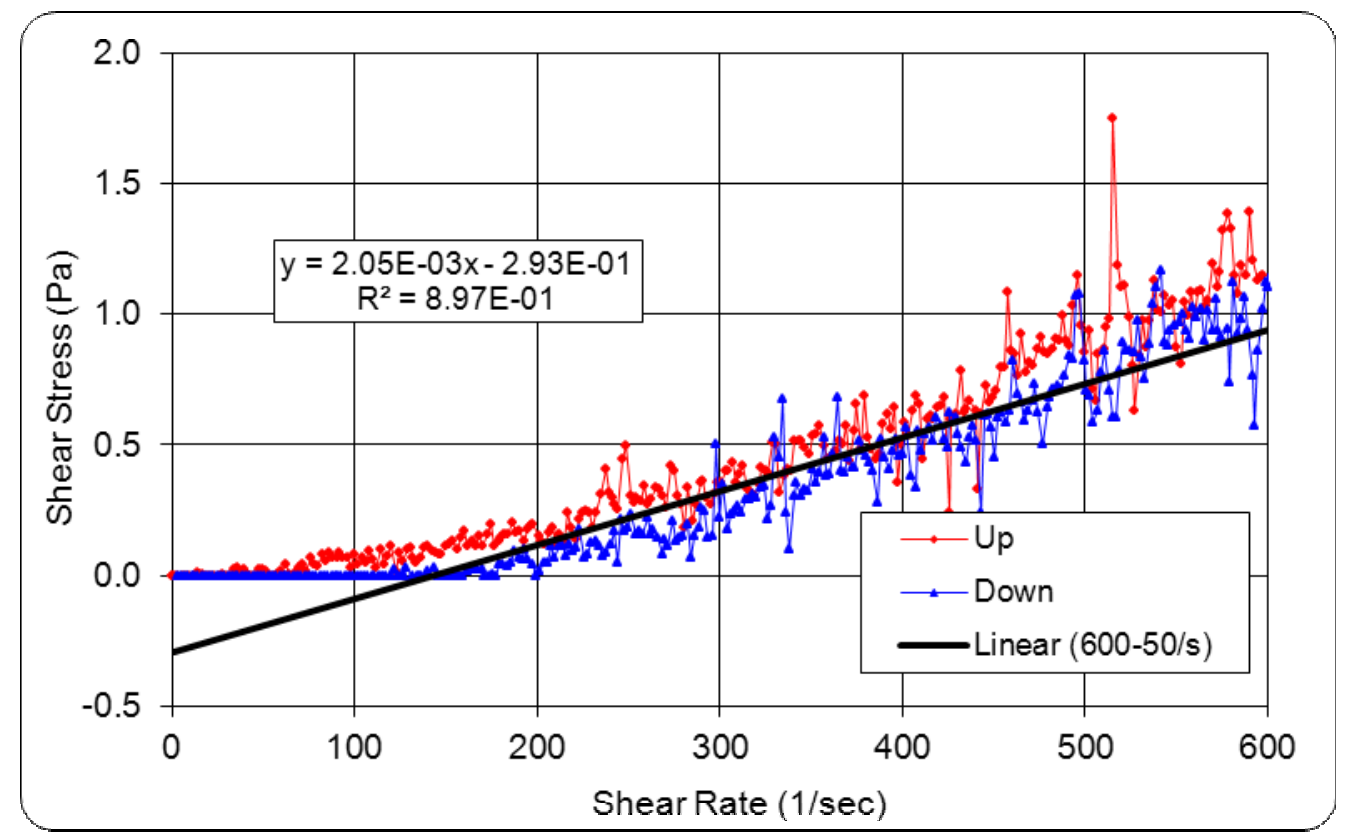

Figure B-2: Stress Versus Strain Curve for Tank 5F Baseline Dilute at $30^{\circ} \mathrm{C}$ (Second Replicate). 


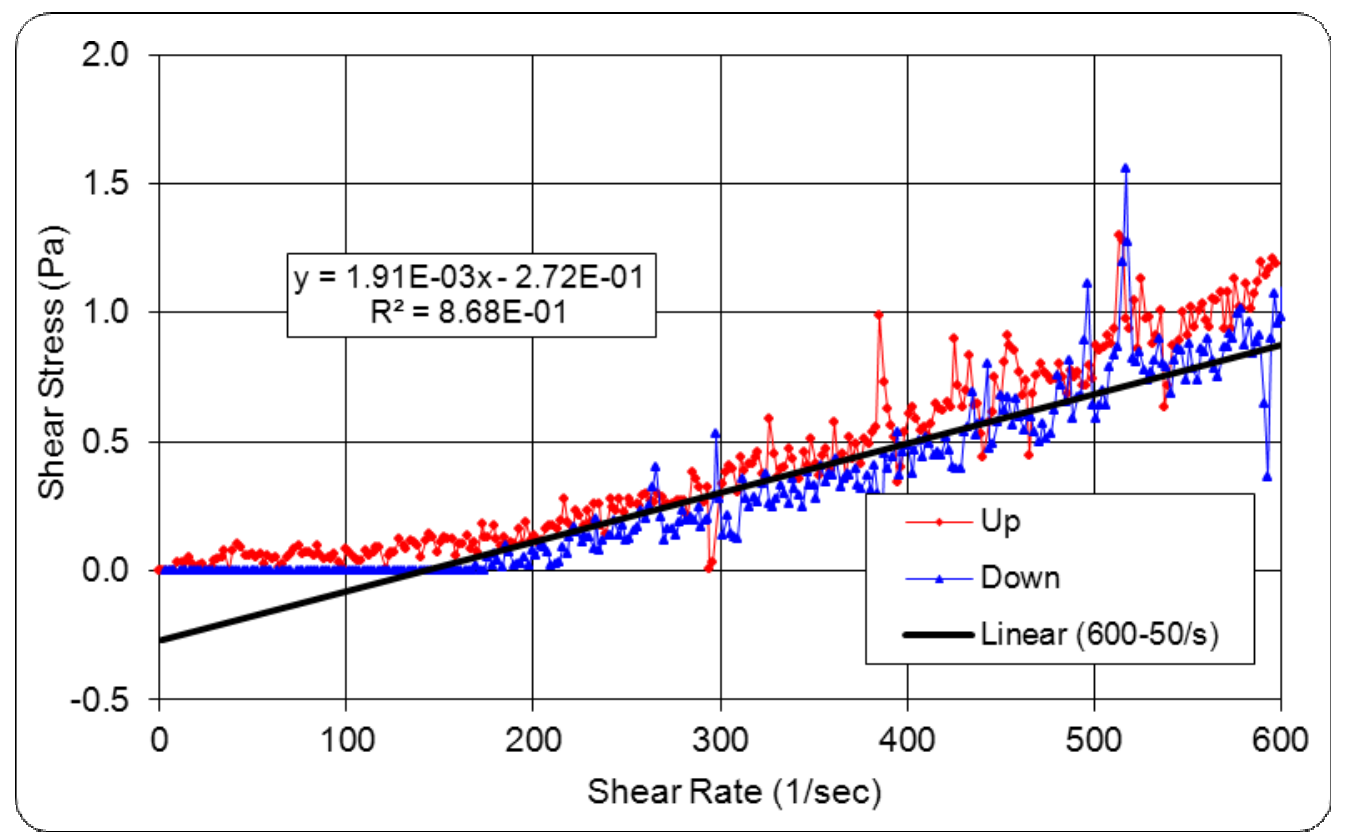

Figure B-3: Stress Versus Strain Curve for Tank 5F Baseline Dilute at $40{ }^{\circ} \mathrm{C}$ (First Replicate).

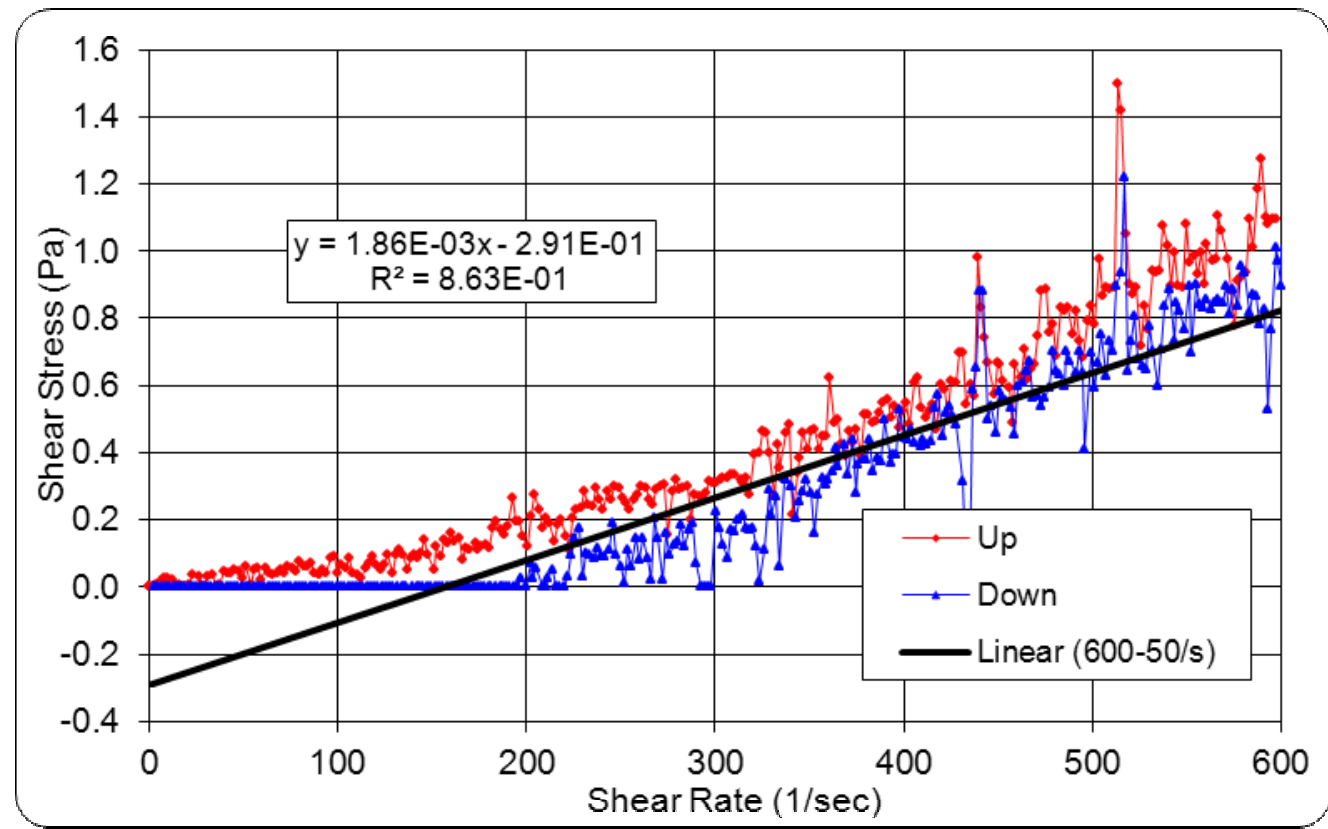

Figure B-4: Stress Versus Strain Curve for Tank 5F Baseline Dilute at $40{ }^{\circ} \mathrm{C}$ (Second Replicate). 


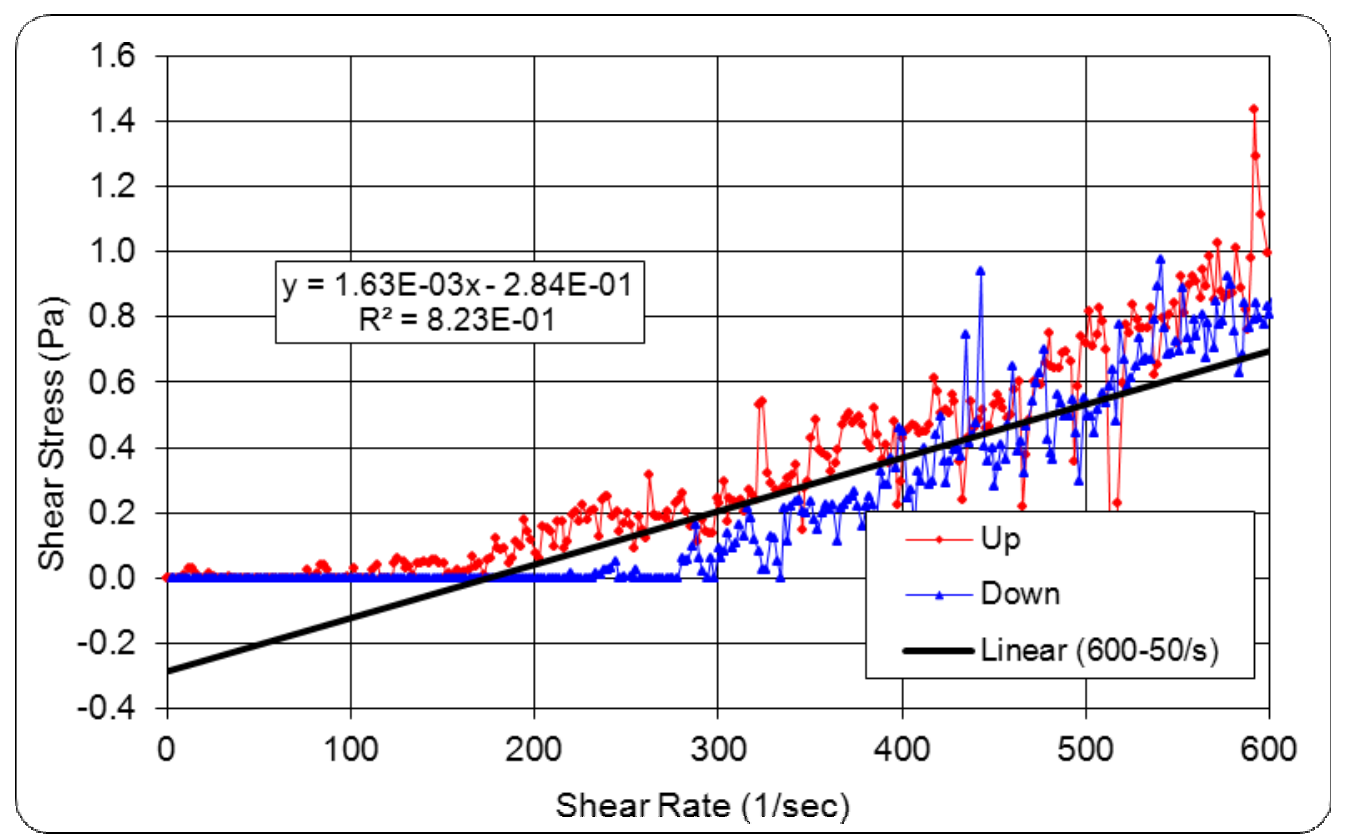

Figure B-5: Stress Versus Strain Curve for Tank 5F Baseline Dilute at $50{ }^{\circ} \mathrm{C}$ (First Replicate).

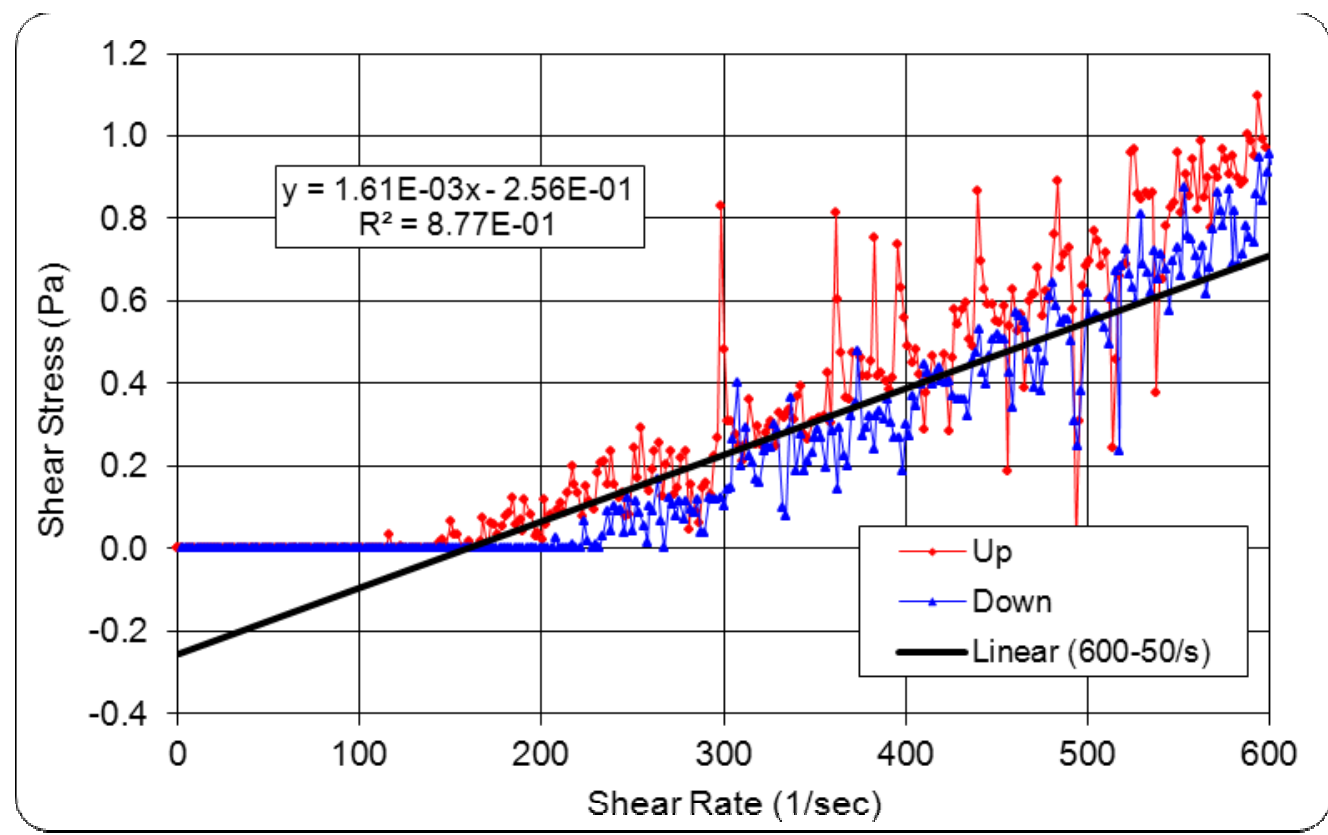

Figure B-6: Stress Versus Strain Curve for Tank 5F Baseline Dilute at $50{ }^{\circ} \mathrm{C}$ (Second Replicate). 


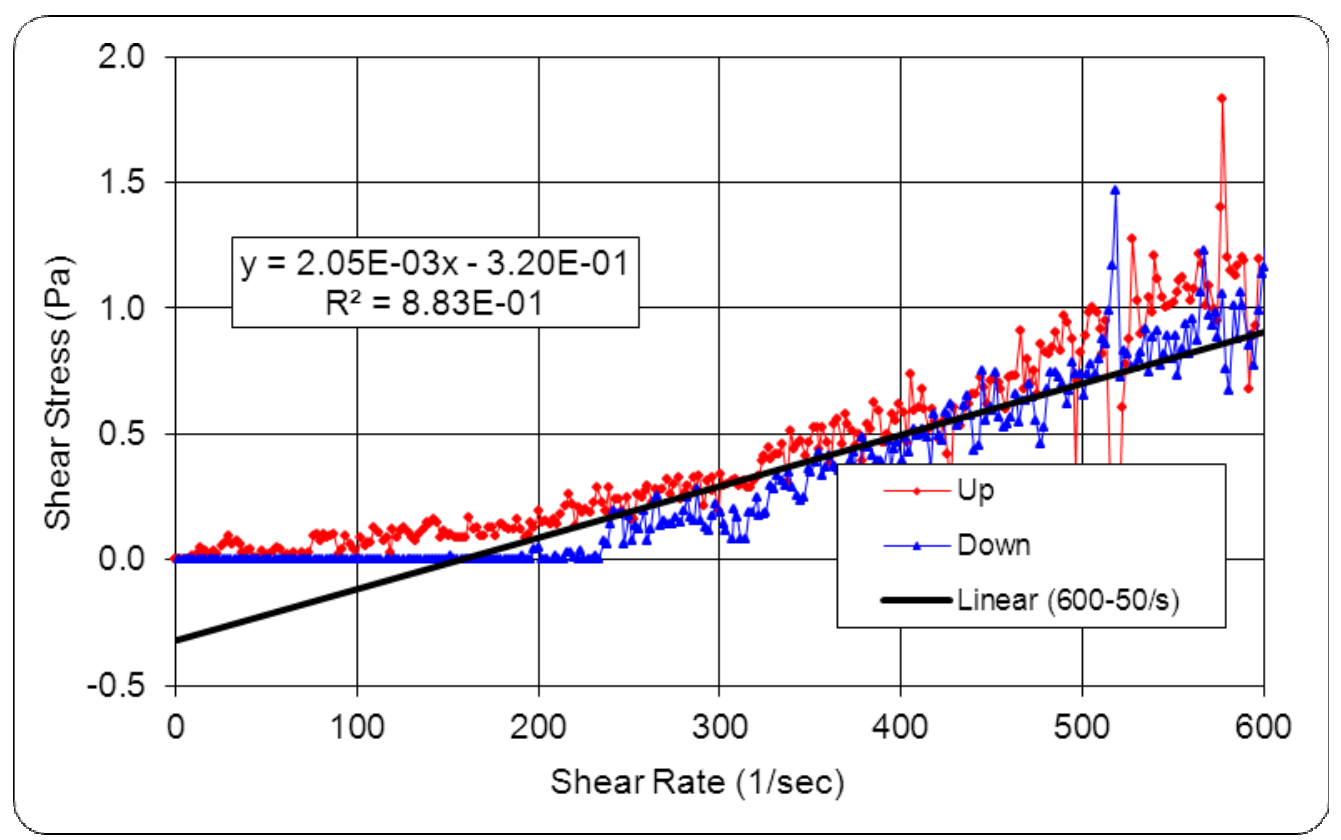

Figure B-7: Stress Versus Strain Curve for Tank 5F Baseline Concentrate at $30^{\circ} \mathrm{C}$ (First Replicate).

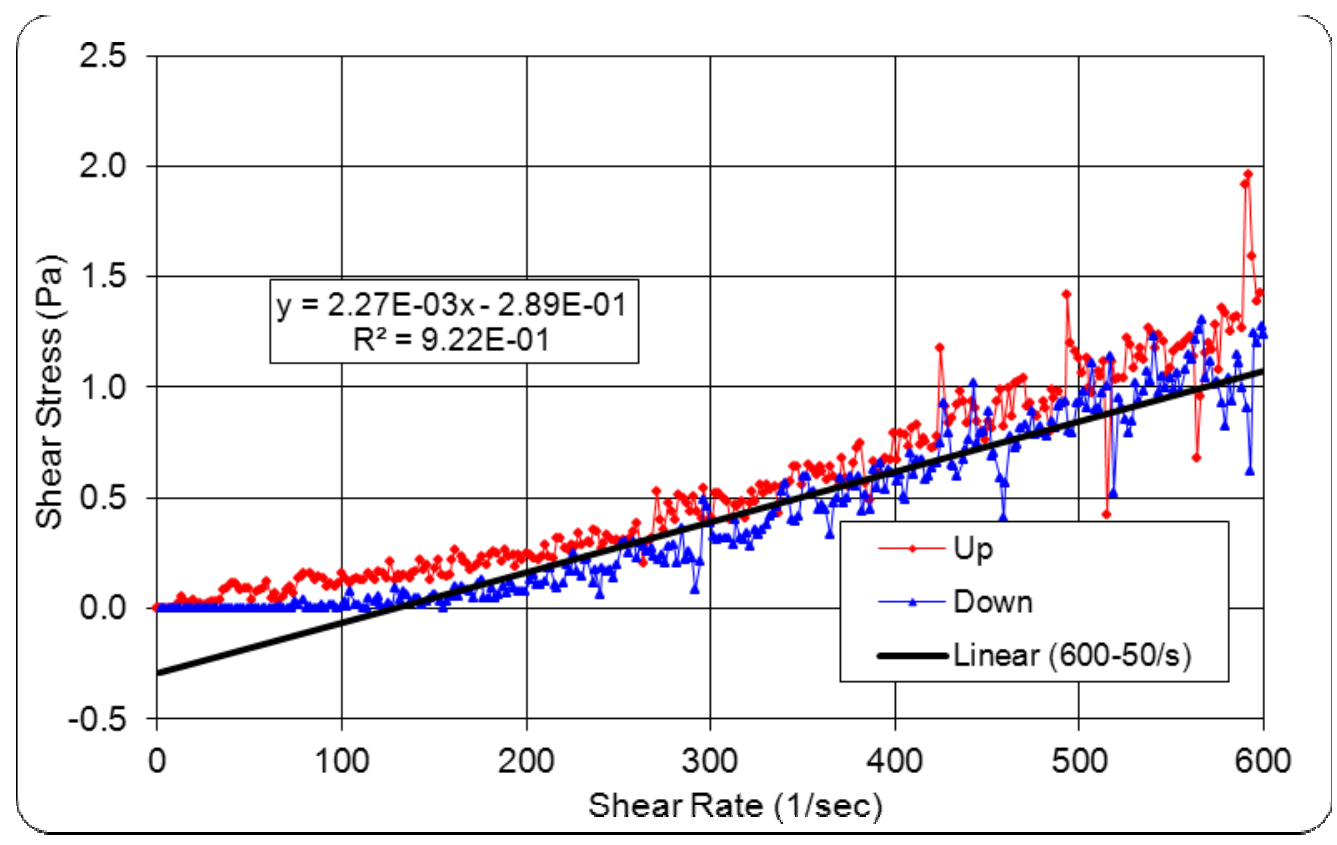

Figure B-8: Stress Versus Strain Curve for Tank 5F Baseline Concentrate at $30^{\circ} \mathrm{C}$ (Second Replicate). 


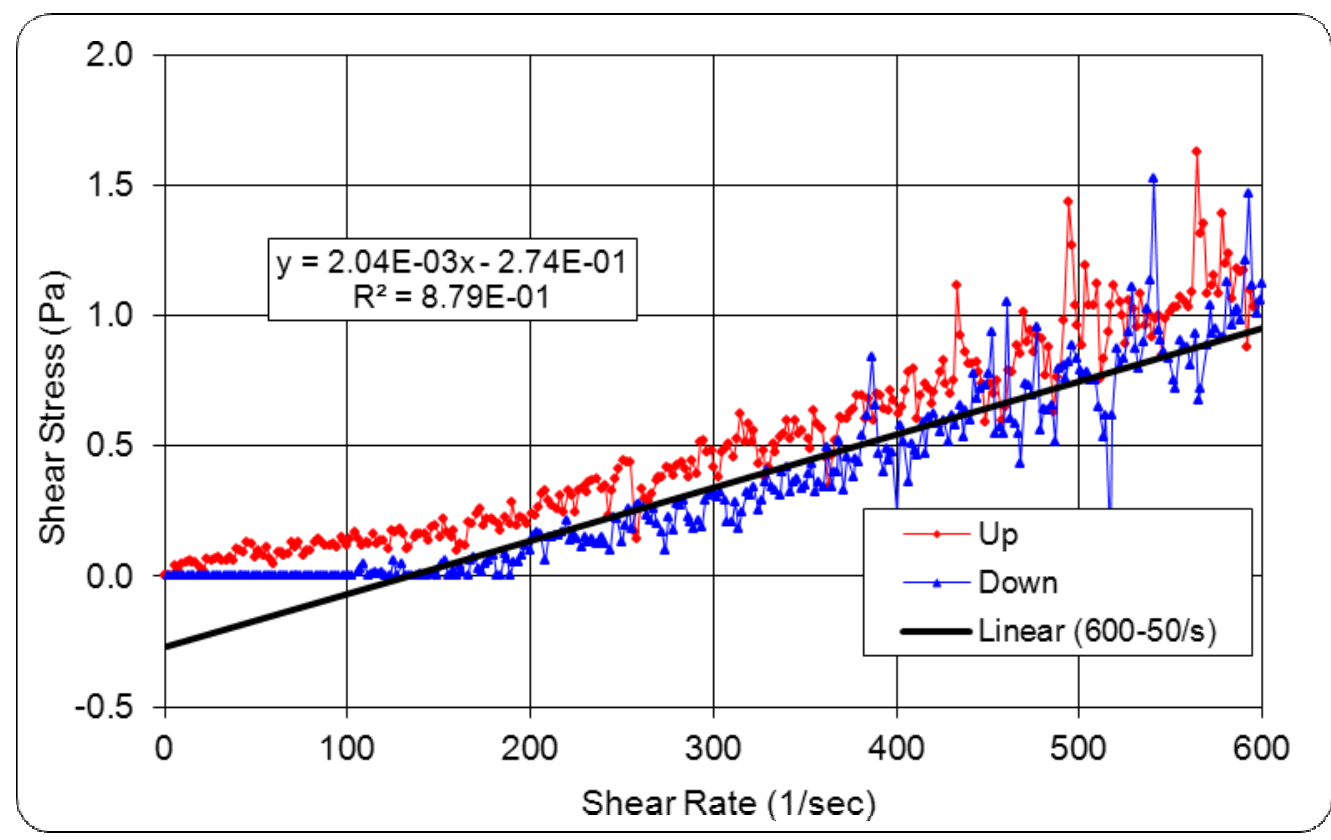

Figure B-9: Stress Versus Strain Curve for Tank 5F Baseline Concentrate at $40{ }^{\circ} \mathrm{C}$ (First Replicate).

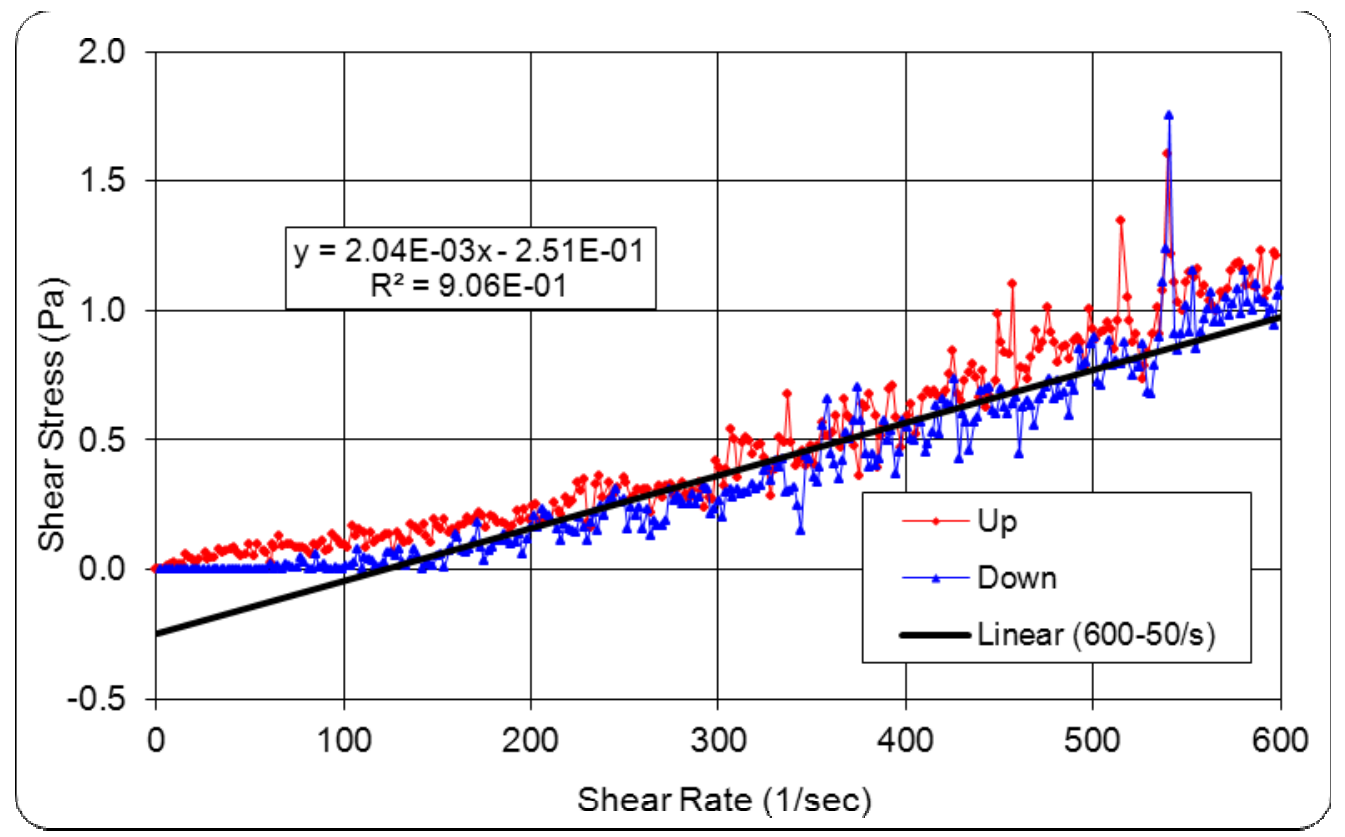

Figure B-10: Stress Versus Strain Curve for Tank 5F Baseline Concentrate at $40{ }^{\circ} \mathrm{C}$ (Second Replicate). 


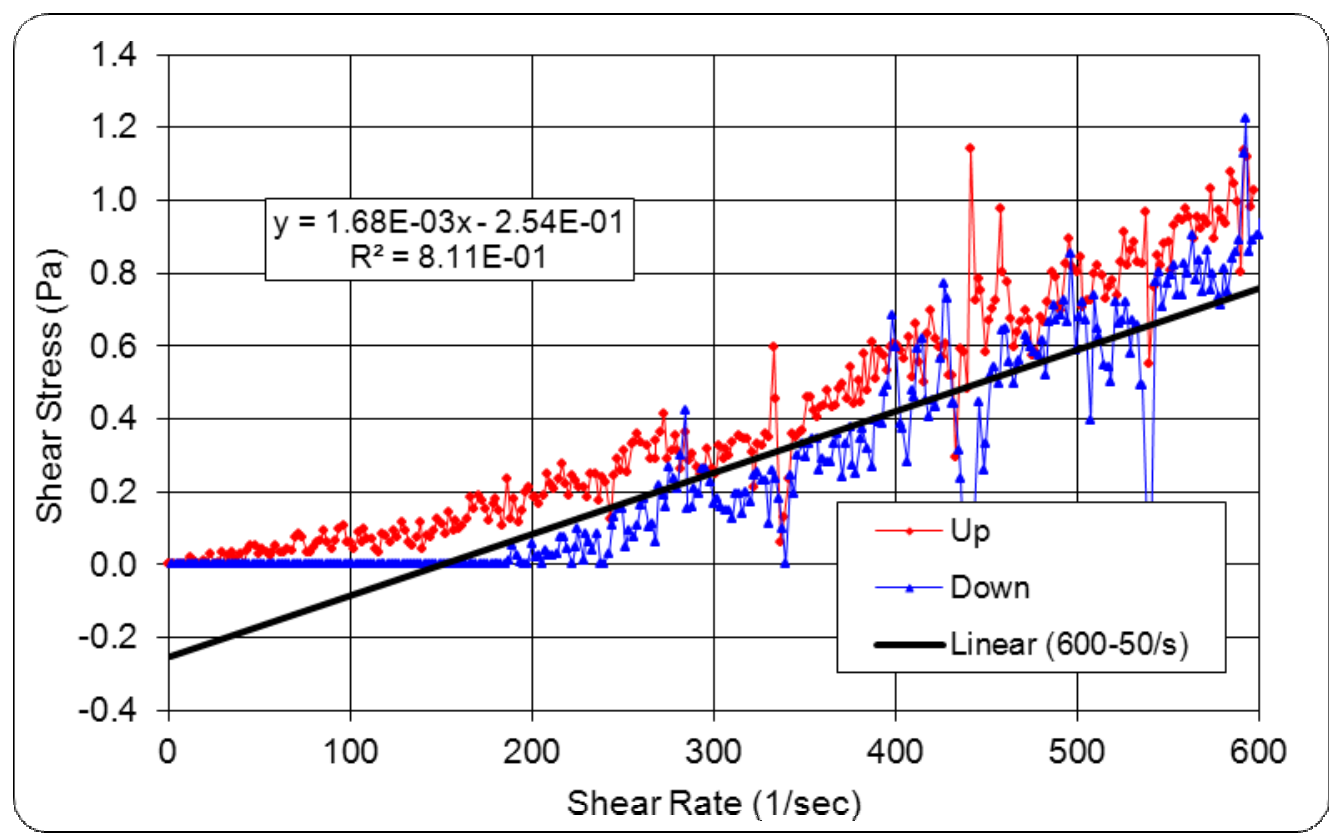

Figure B-11: Stress Versus Strain Curve for Tank 5F Baseline Concentrate at $50{ }^{\circ} \mathrm{C}$ (First Replicate).

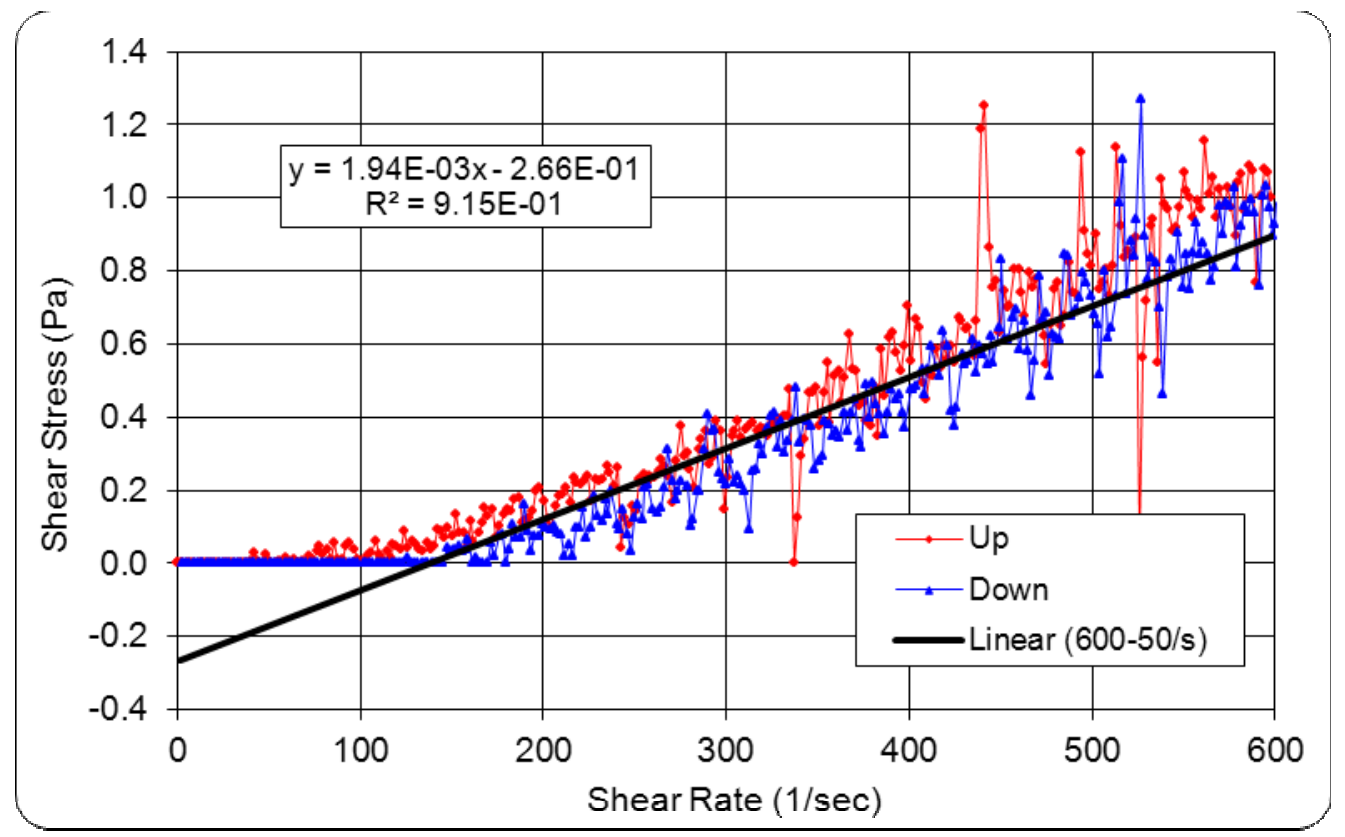

Figure B-12: Stress Versus Strain Curve for Tank 5F Baseline Concentrate at $50{ }^{\circ} \mathrm{C}$ (Second Replicate). 


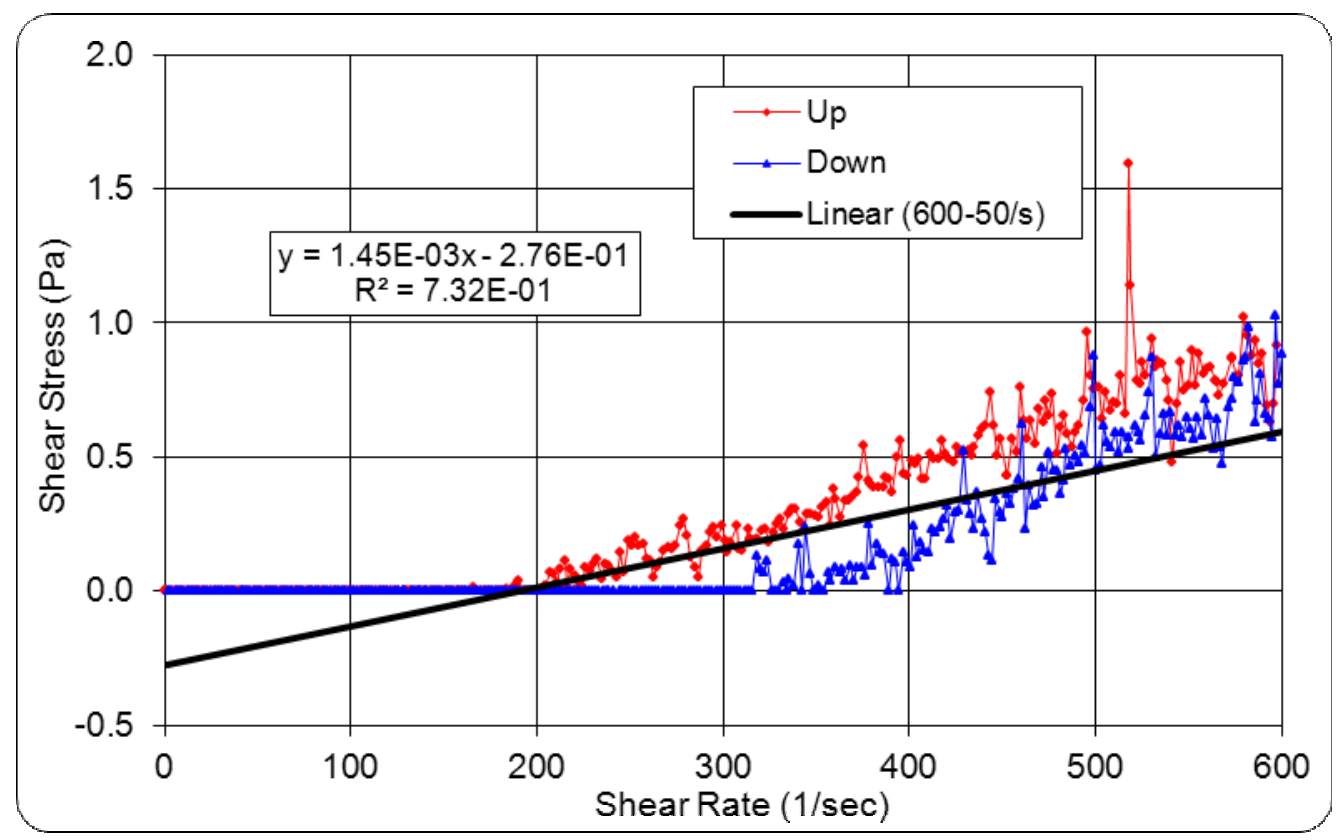

Figure B-13: Stress Versus Strain Curve for Tank 5F Midpoint Concentrate at $30^{\circ} \mathrm{C}$ (First Replicate).

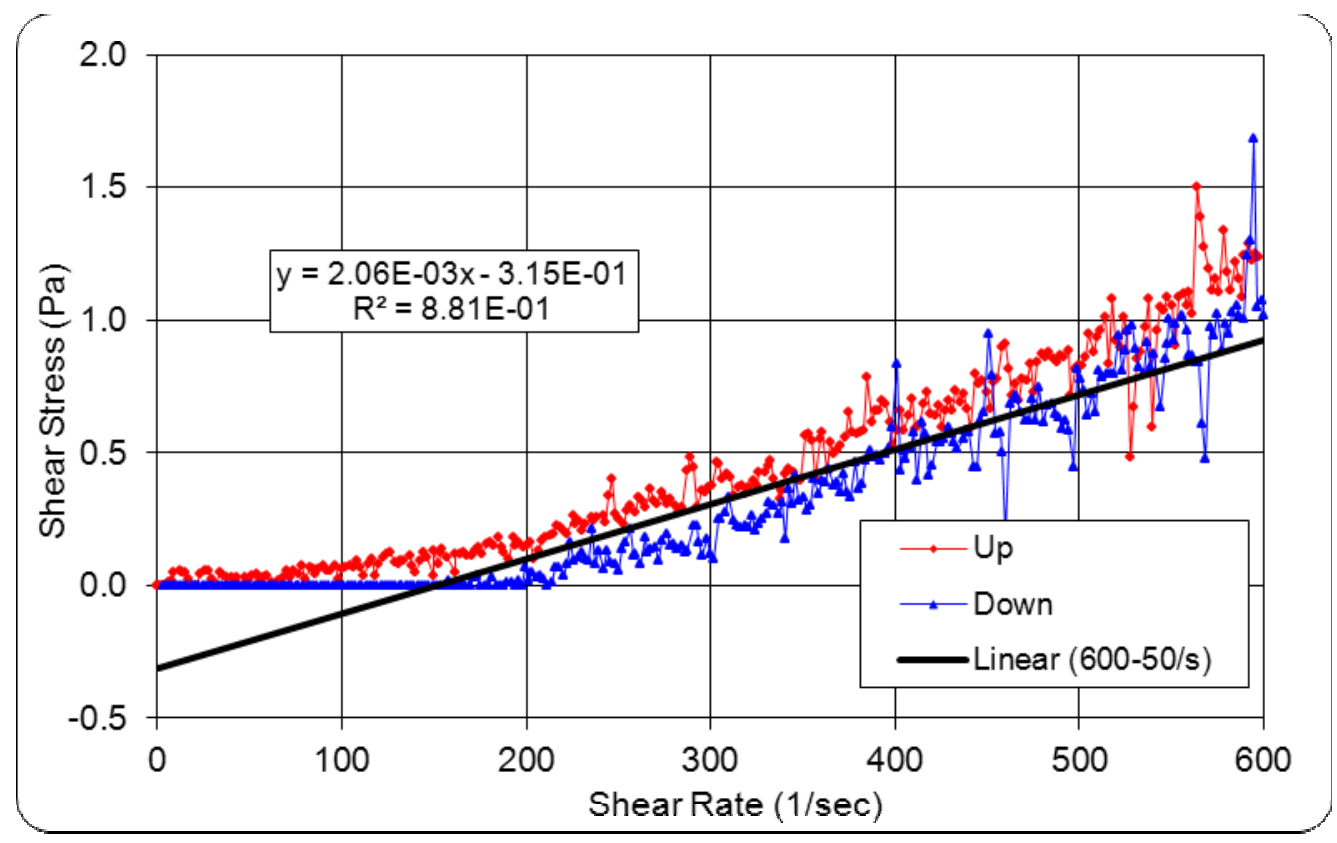

Figure B-14: Stress Versus Strain Curve for Tank 5F Midpoint Concentrate at $30{ }^{\circ} \mathrm{C}$ (Second Replicate). 


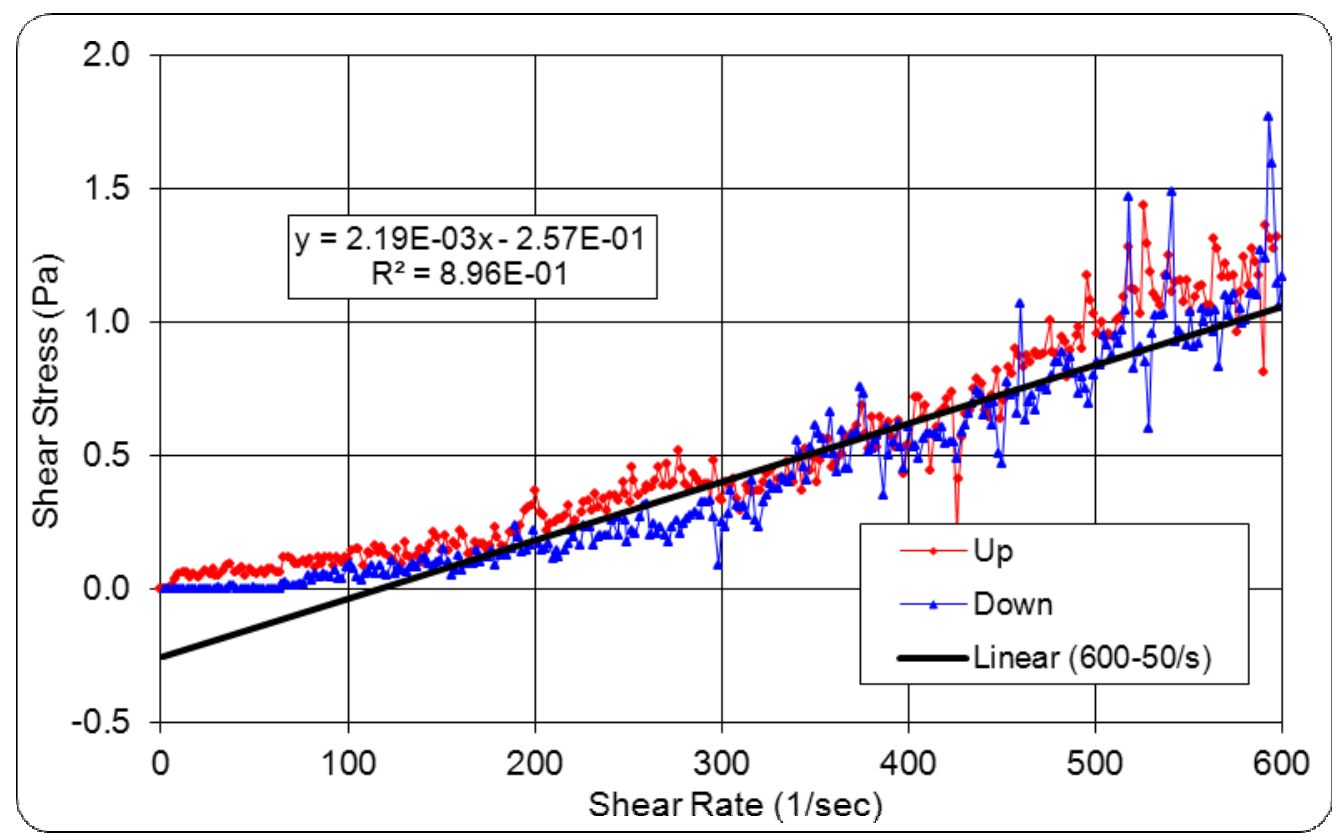

Figure B-15: Stress Versus Strain Curve for Tank 5F Endpoint Concentrate at $30^{\circ} \mathrm{C}$ (First Replicate).

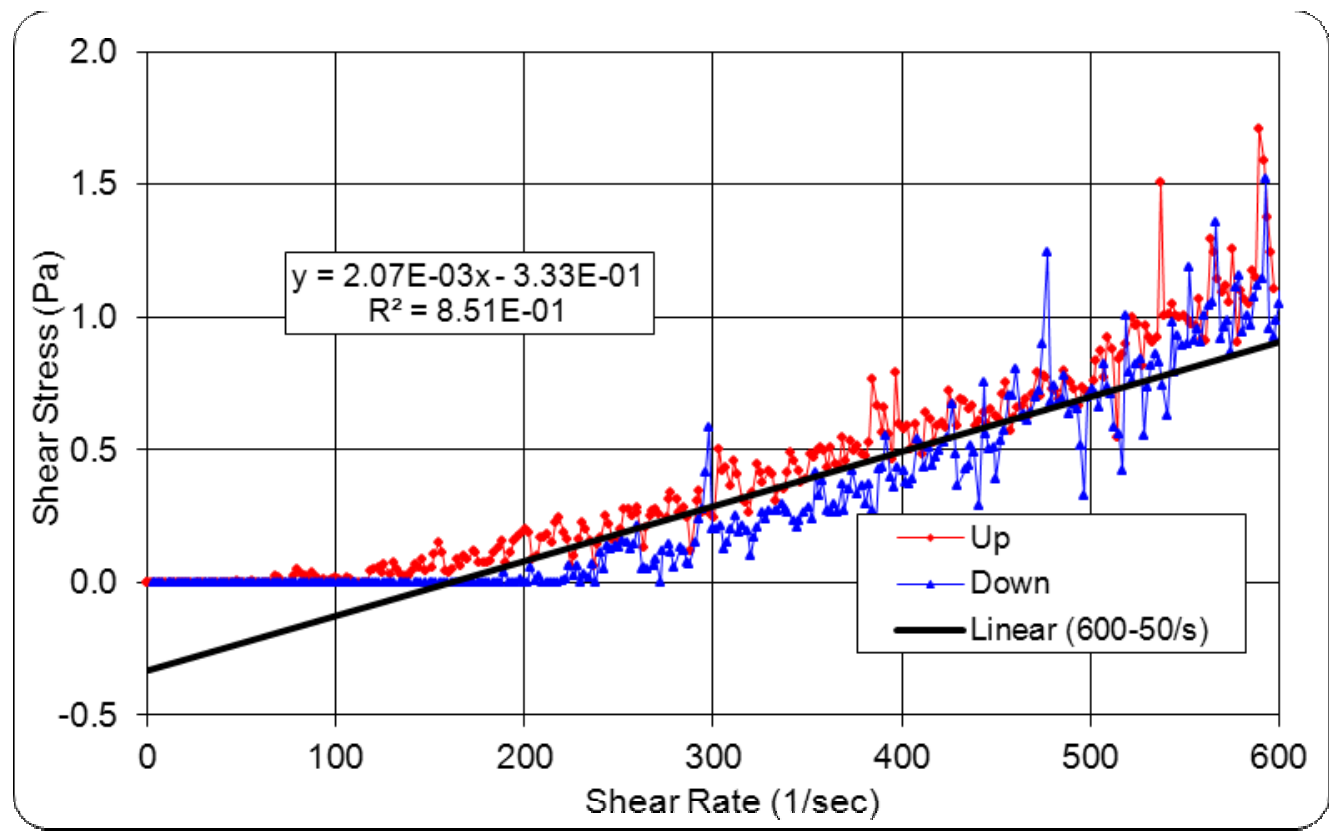

Figure B-16: Stress Versus Strain Curve for Tank 5F Endpoint Concentrate at $30{ }^{\circ} \mathrm{C}$ (Second Replicate). 


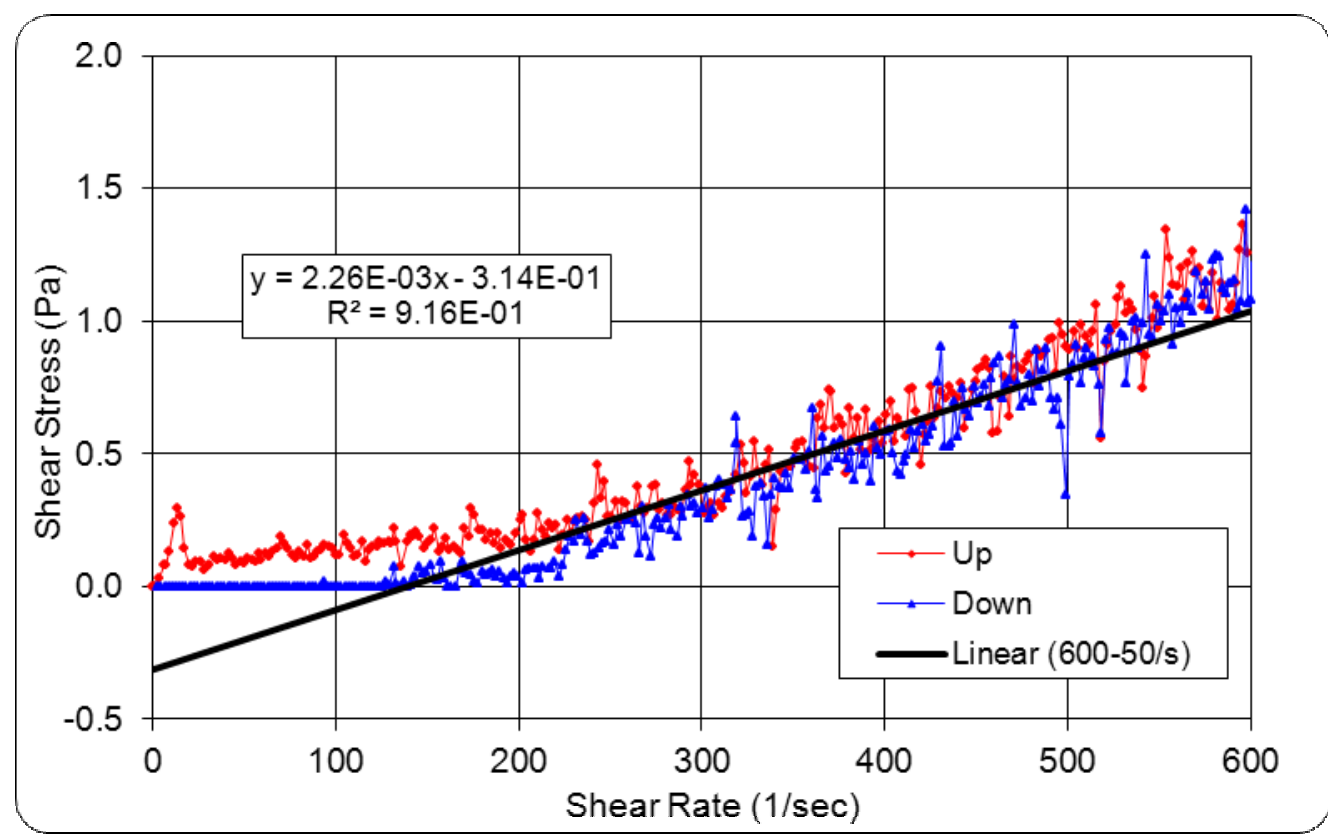

Figure B-17: Stress Versus Strain Curve for Tank $12 \mathrm{H}$ Baseline Dilute at $30^{\circ} \mathrm{C}$ (First Replicate)

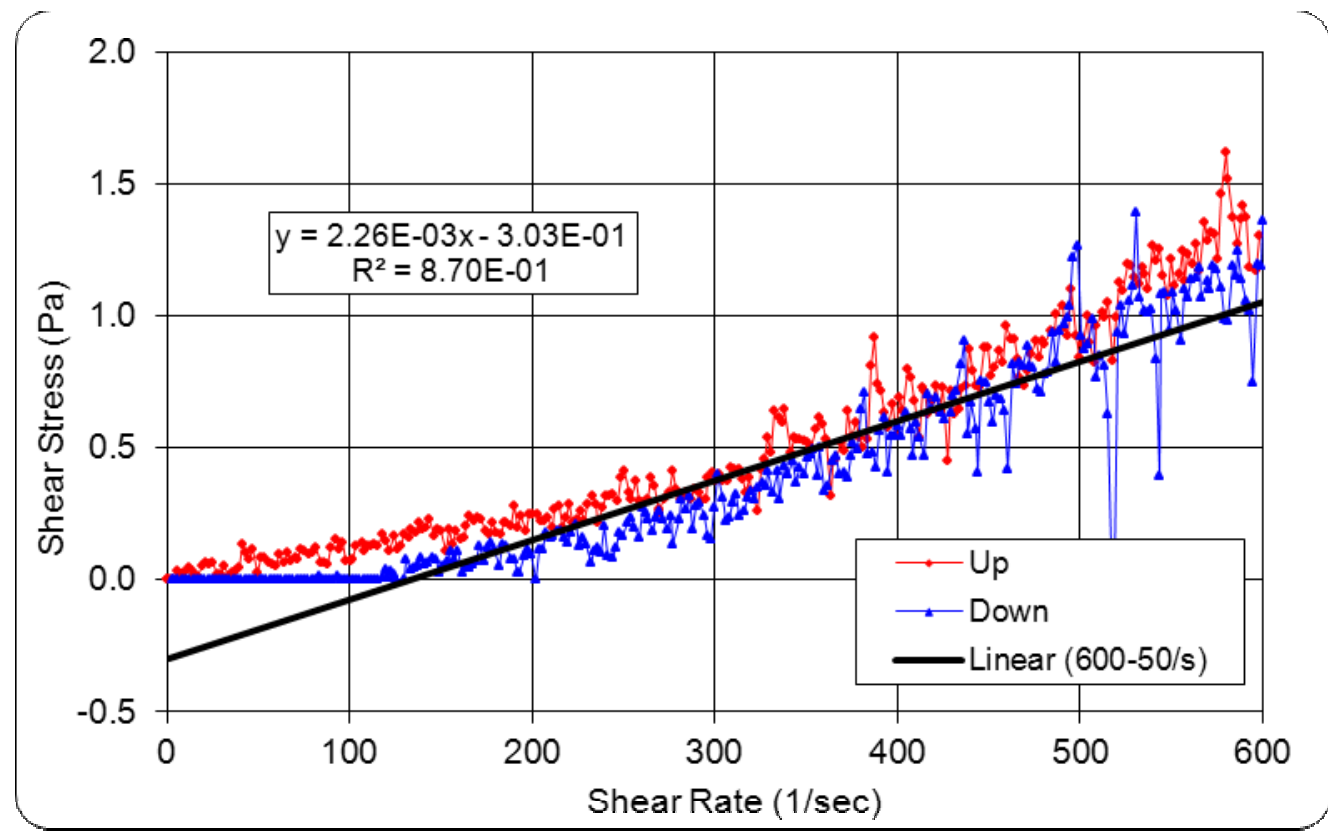

Figure B-18: Stress Versus Strain Curve for Tank $12 \mathrm{H}$ Baseline Dilute at $30^{\circ} \mathrm{C}$ (Second Replicate) 


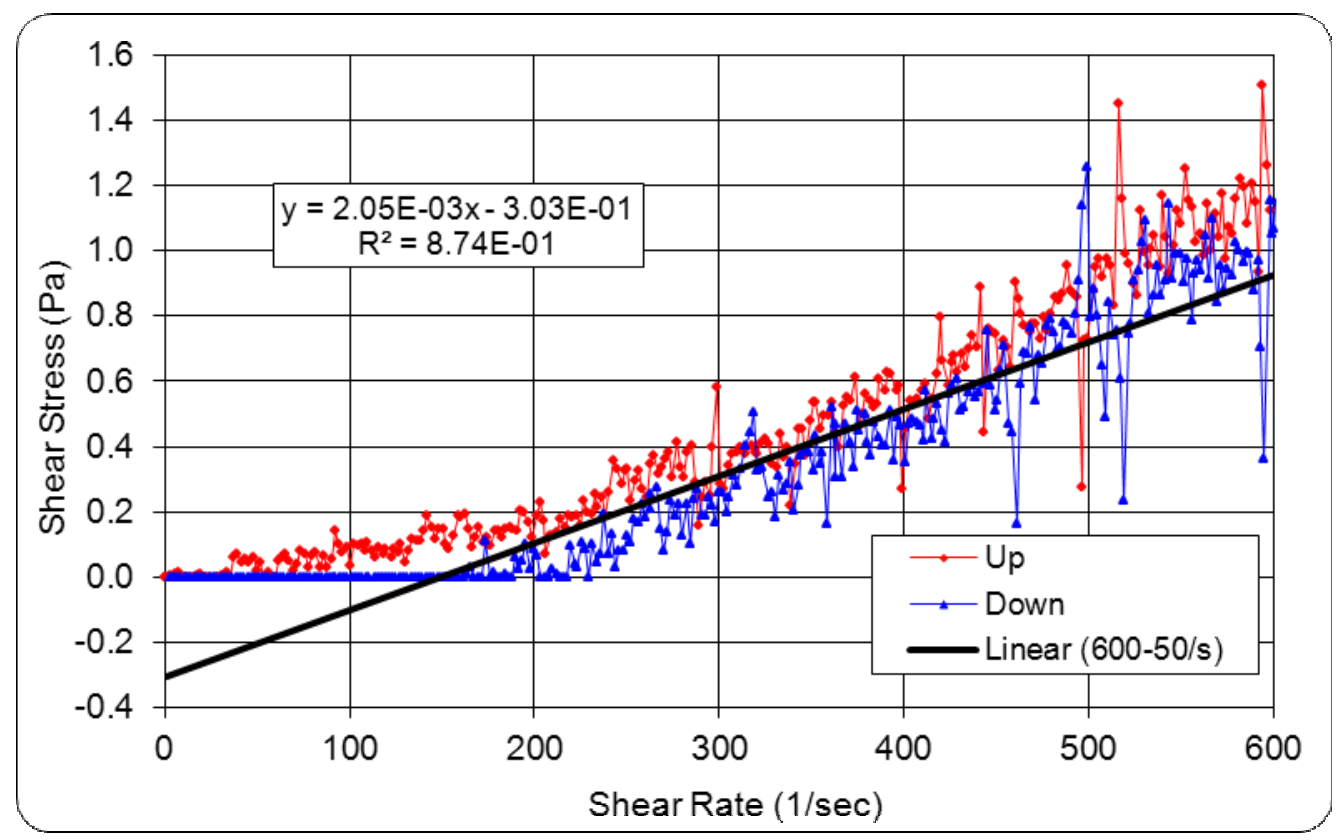

Figure B-19: Stress Versus Strain Curve for Tank 12H Baseline Dilute at $40{ }^{\circ} \mathrm{C}$ (First Replicate)

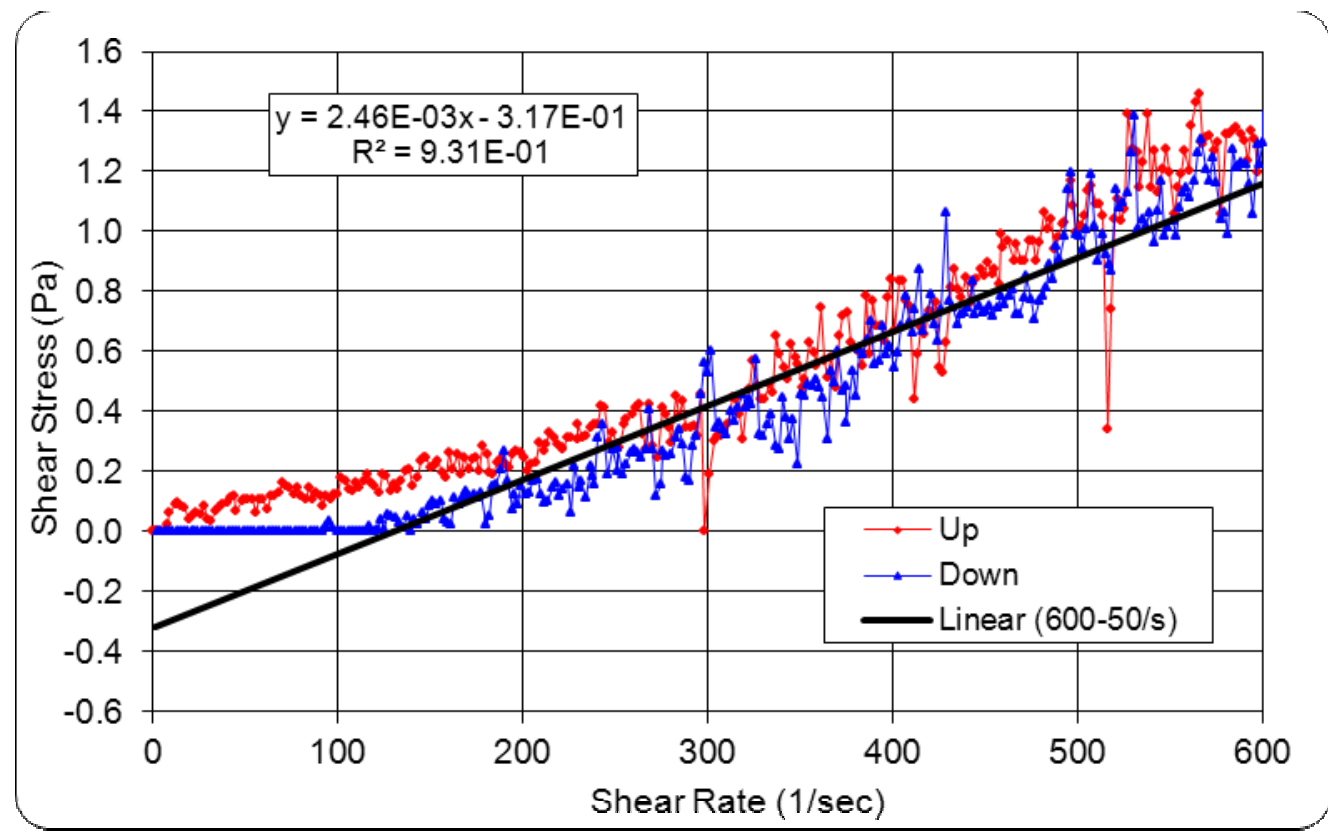

Figure B-20: Stress Versus Strain Curve for Tank $12 \mathrm{H}$ Baseline Dilute at $40{ }^{\circ} \mathrm{C}$ (Second Replicate) 


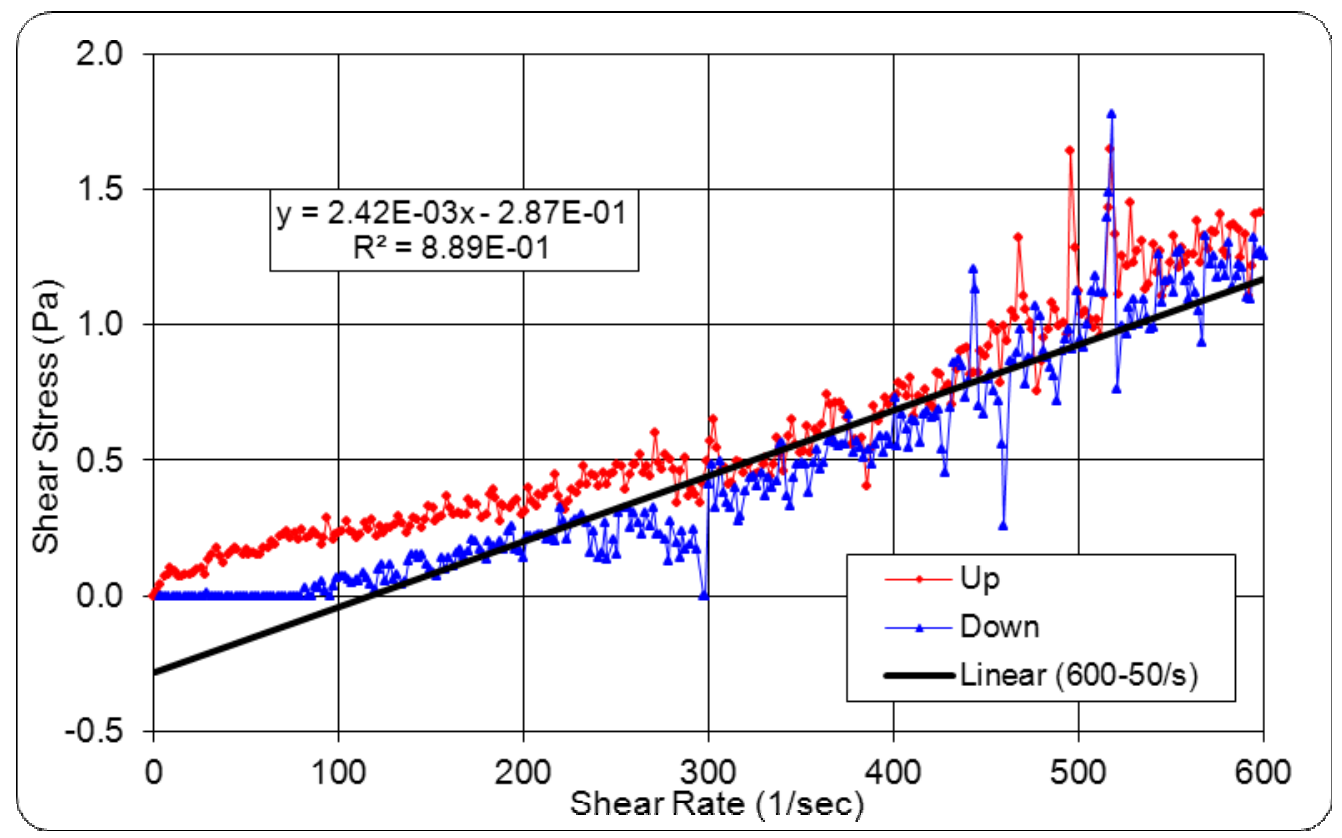

Figure B-21: Stress Versus Strain Curve for Tank $12 \mathrm{H}$ Baseline Dilute at $50{ }^{\circ} \mathrm{C}$ (First Replicate)

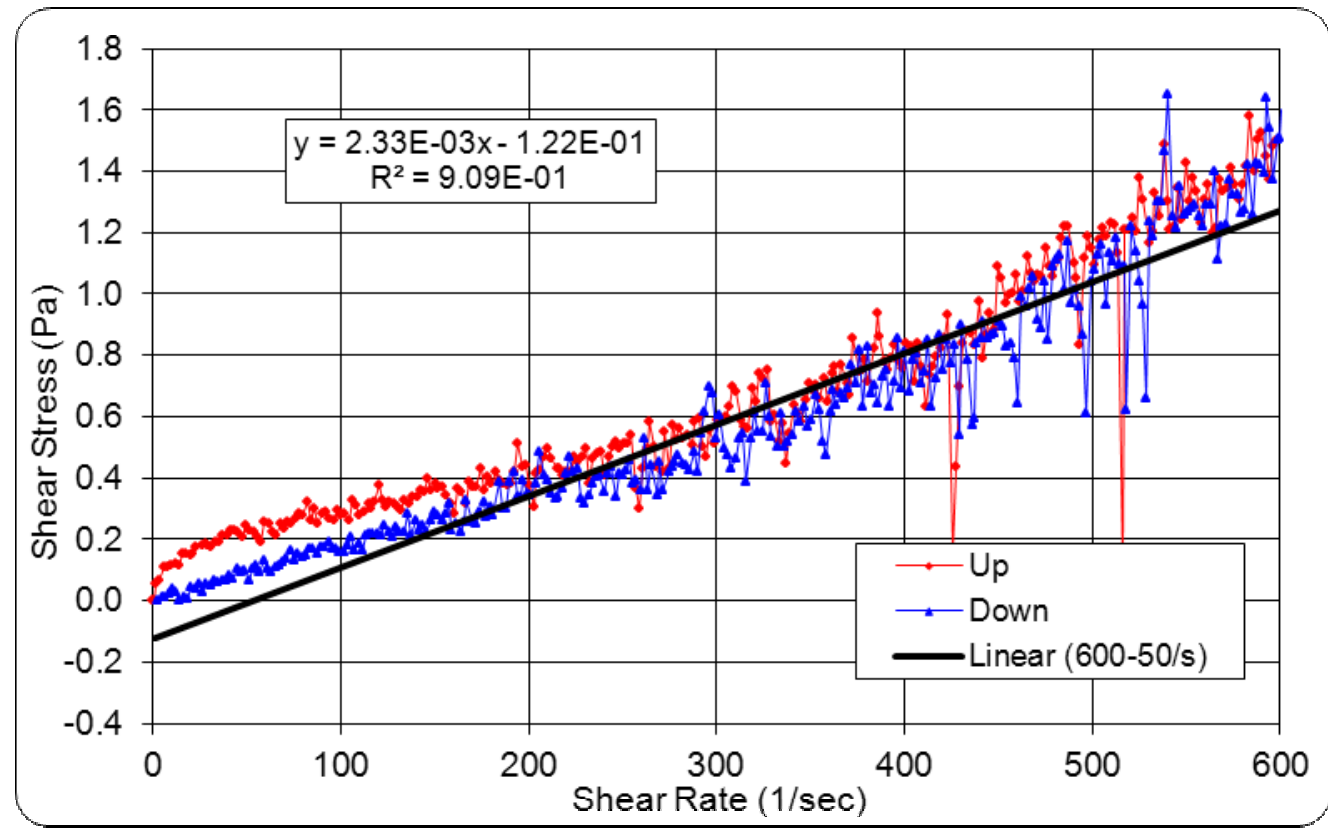

Figure B-22: Stress Versus Strain Curve for Tank $12 \mathrm{H}$ Baseline Dilute at $50{ }^{\circ} \mathrm{C}$ (Second Replicate) 


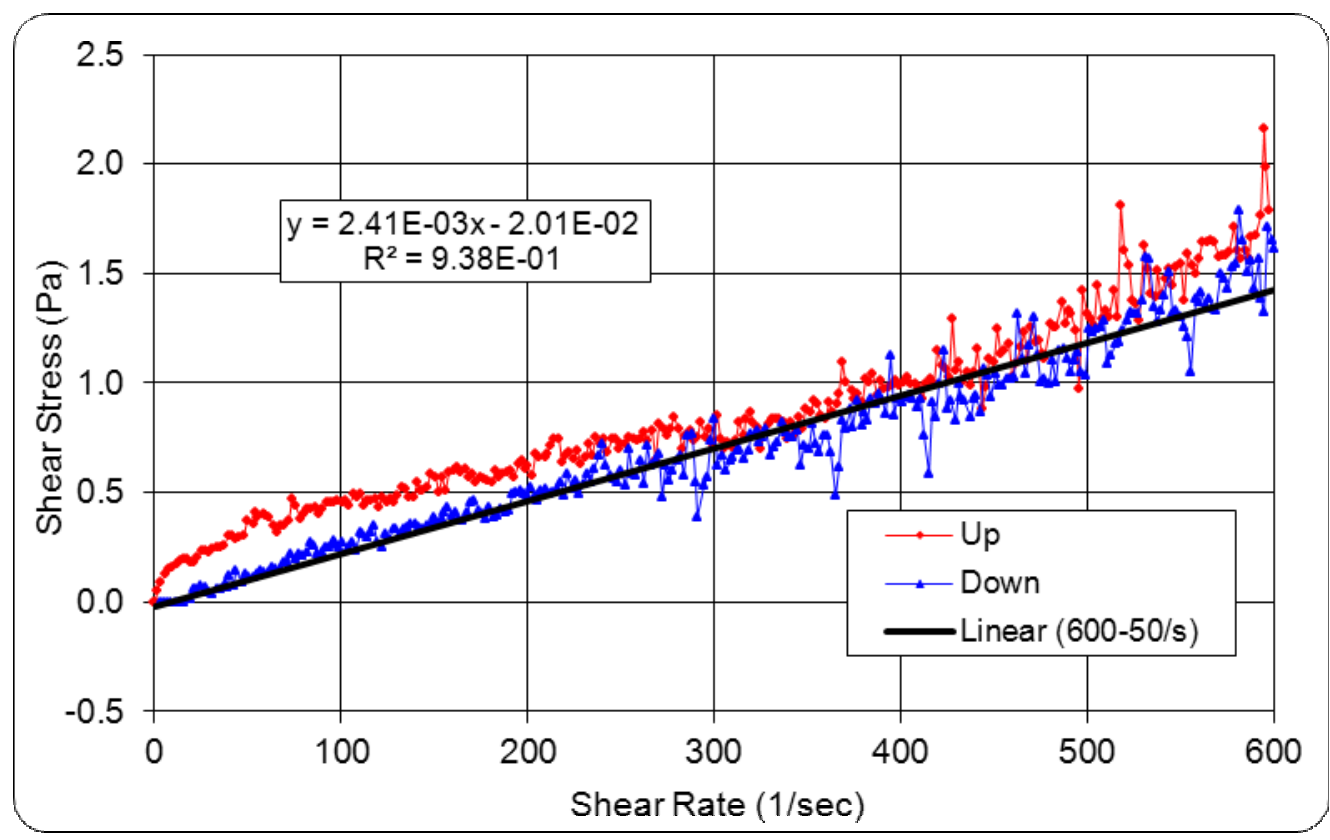

Figure B-23: Stress Versus Strain Curve for Tank $12 \mathrm{H}$ Baseline Dilute at $30{ }^{\circ} \mathrm{C}$ after 1 Day (First Replicate)

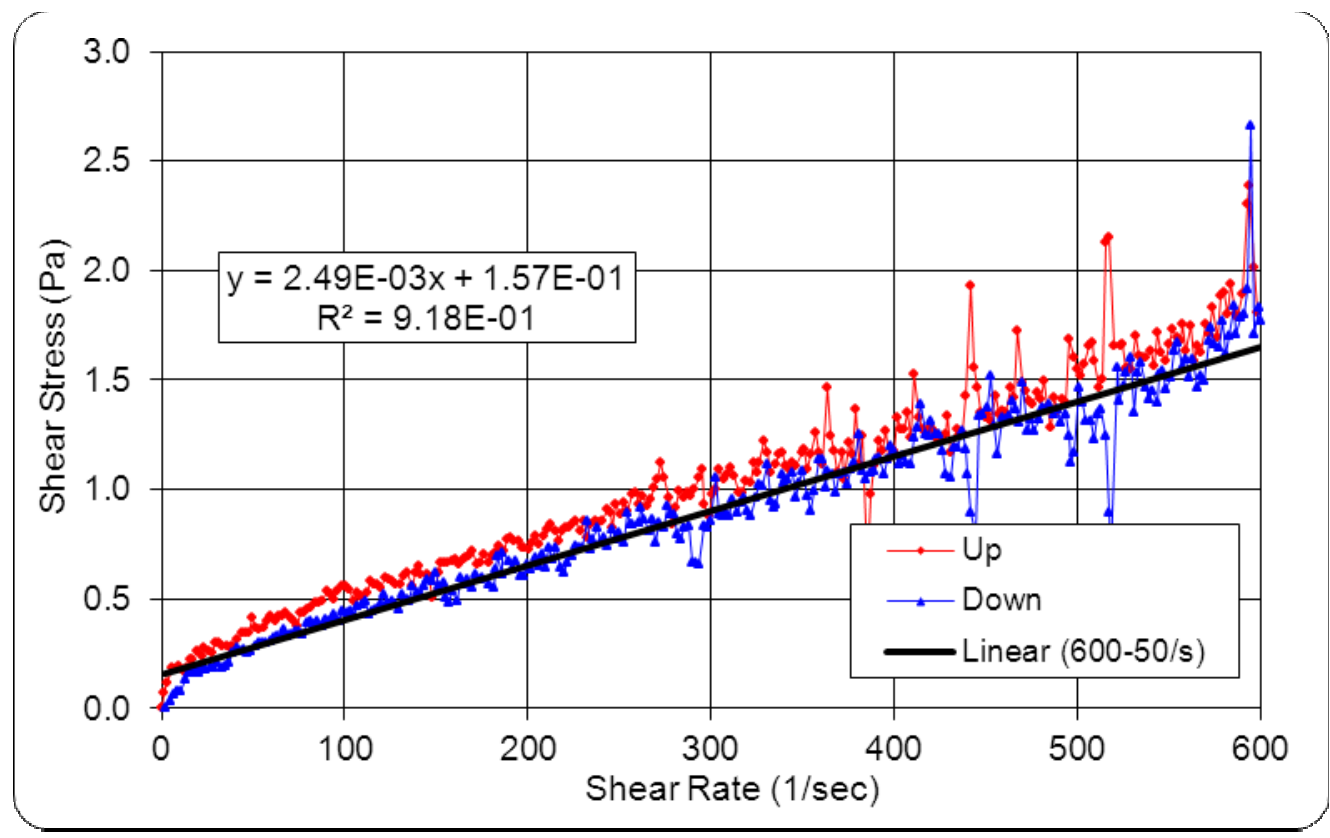

Figure B-24: Stress Versus Strain Curve for Tank $12 \mathrm{H}$ Baseline Dilute at $30^{\circ} \mathrm{C}$ after 1 Day (Second Replicate) 


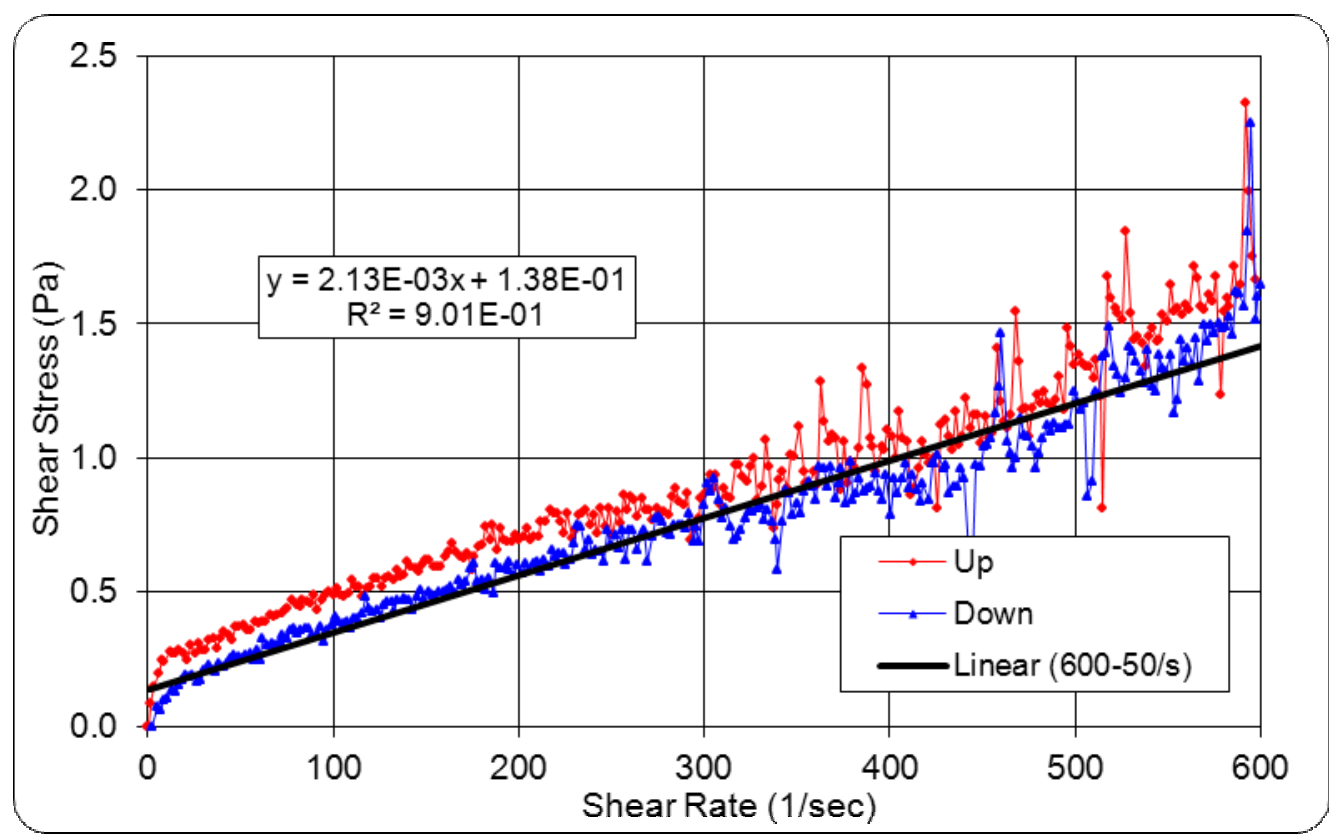

Figure B-25: Stress Versus Strain Curve for Tank $12 \mathrm{H}$ Baseline Dilute at $40{ }^{\circ} \mathrm{C}$ after 1 Day (First Replicate)

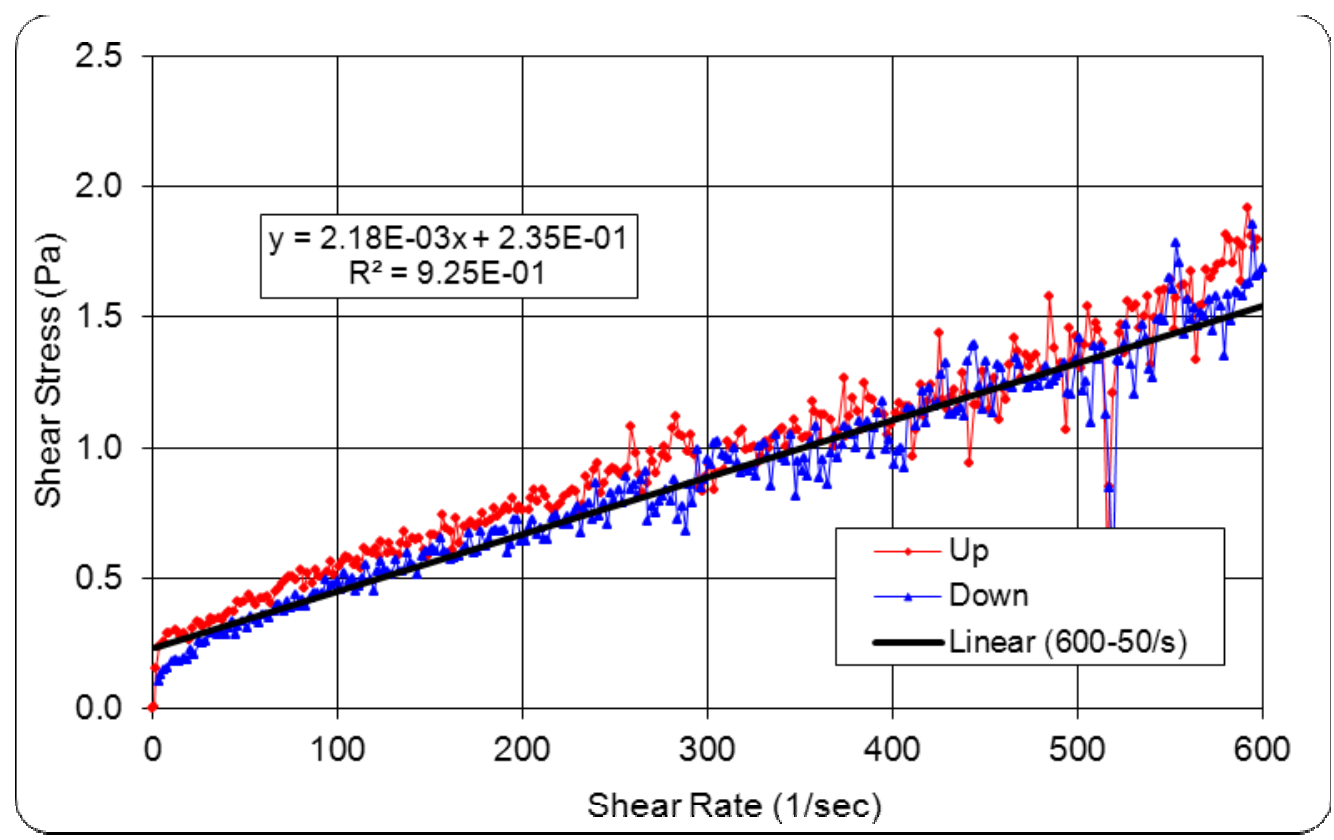

Figure B-26: Stress Versus Strain Curve for Tank 12H Baseline Dilute at $40{ }^{\circ} \mathrm{C}$ after 1 Day (Second Replicate) 


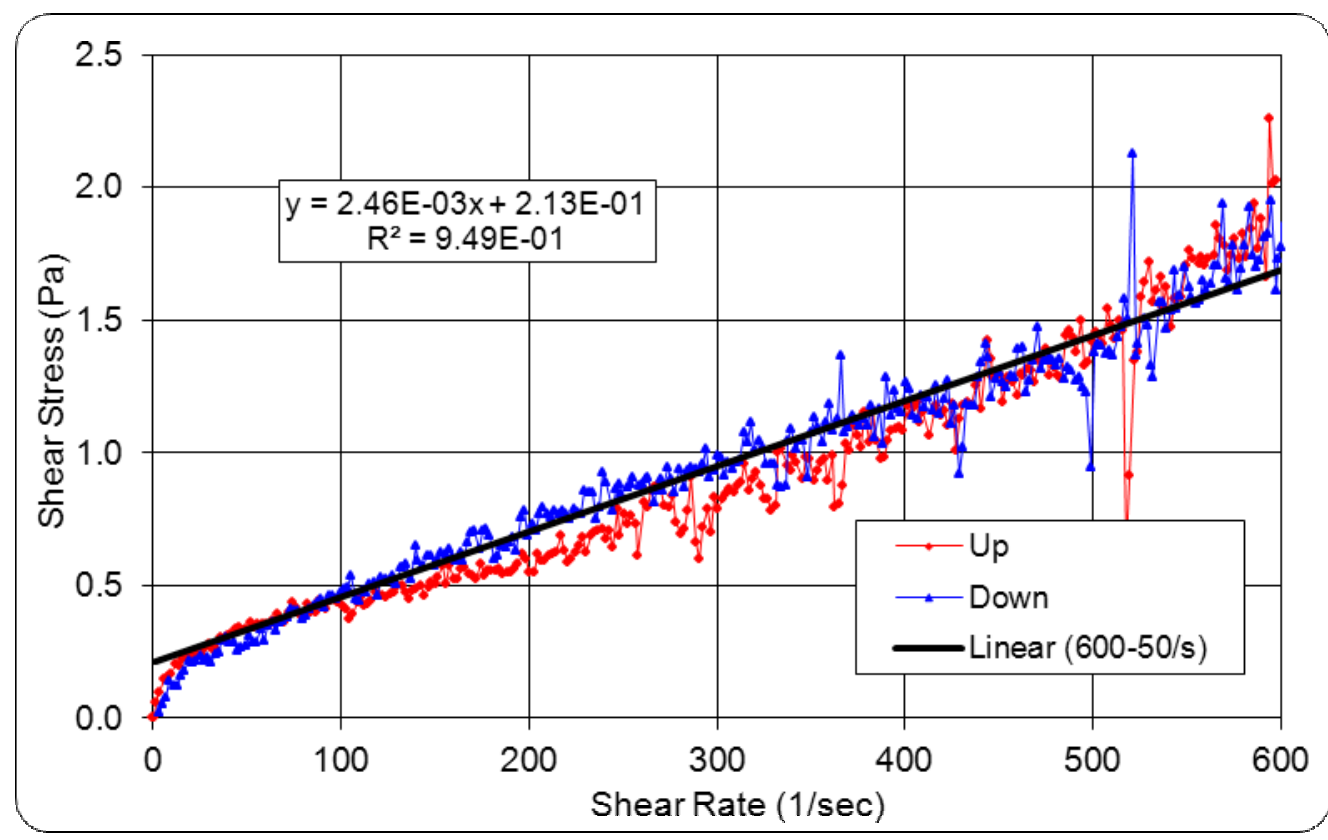

Figure B-27: Stress Versus Strain Curve for Tank 12H Baseline Concentrate at $30^{\circ} \mathrm{C}$ (First Replicate)

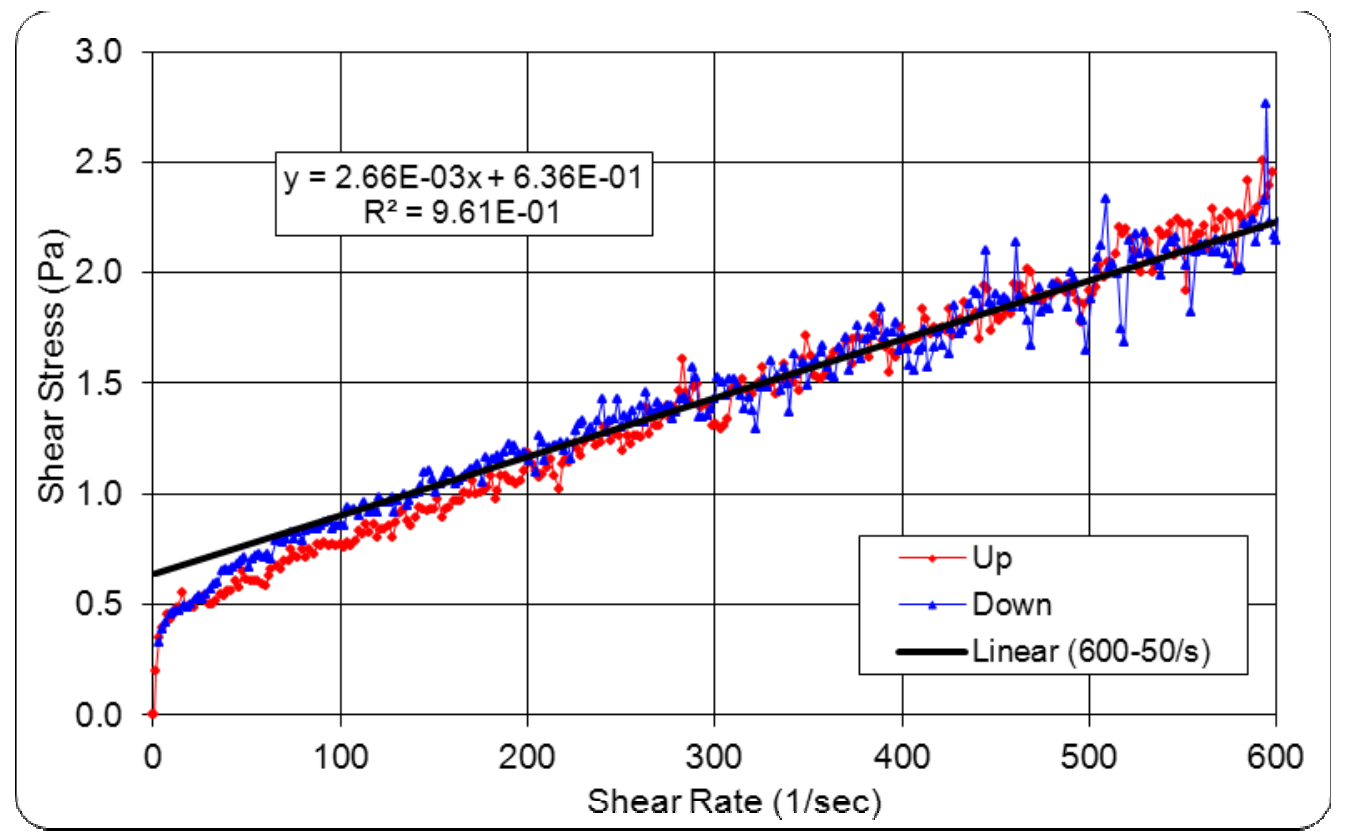

Figure B-28: Stress Versus Strain Curve for Tank $12 \mathrm{H}$ Baseline Concentrate at $30{ }^{\circ} \mathrm{C}$ (Second Replicate) 


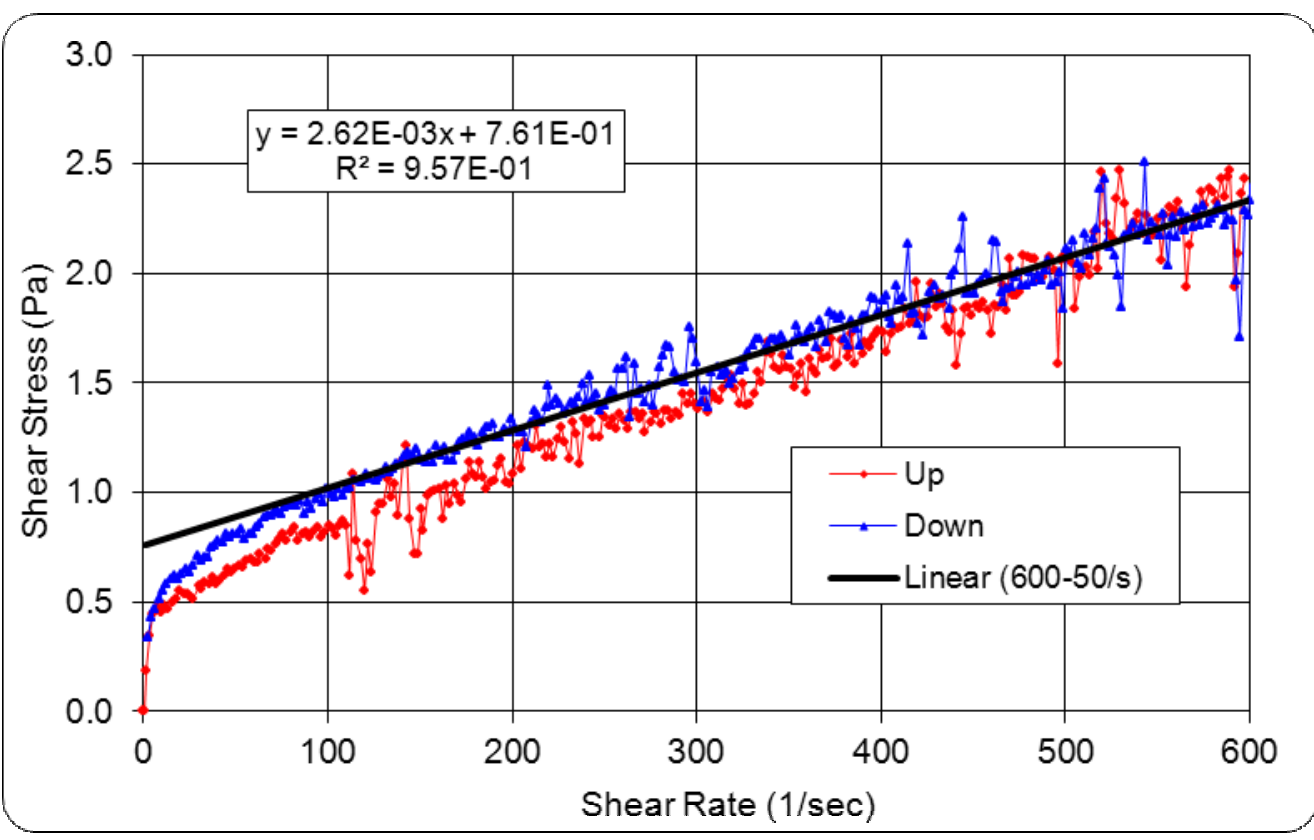

Figure B-29: Stress Versus Strain Curve for Tank 12H Baseline Concentrate at $40{ }^{\circ} \mathrm{C}$ (First Replicate)

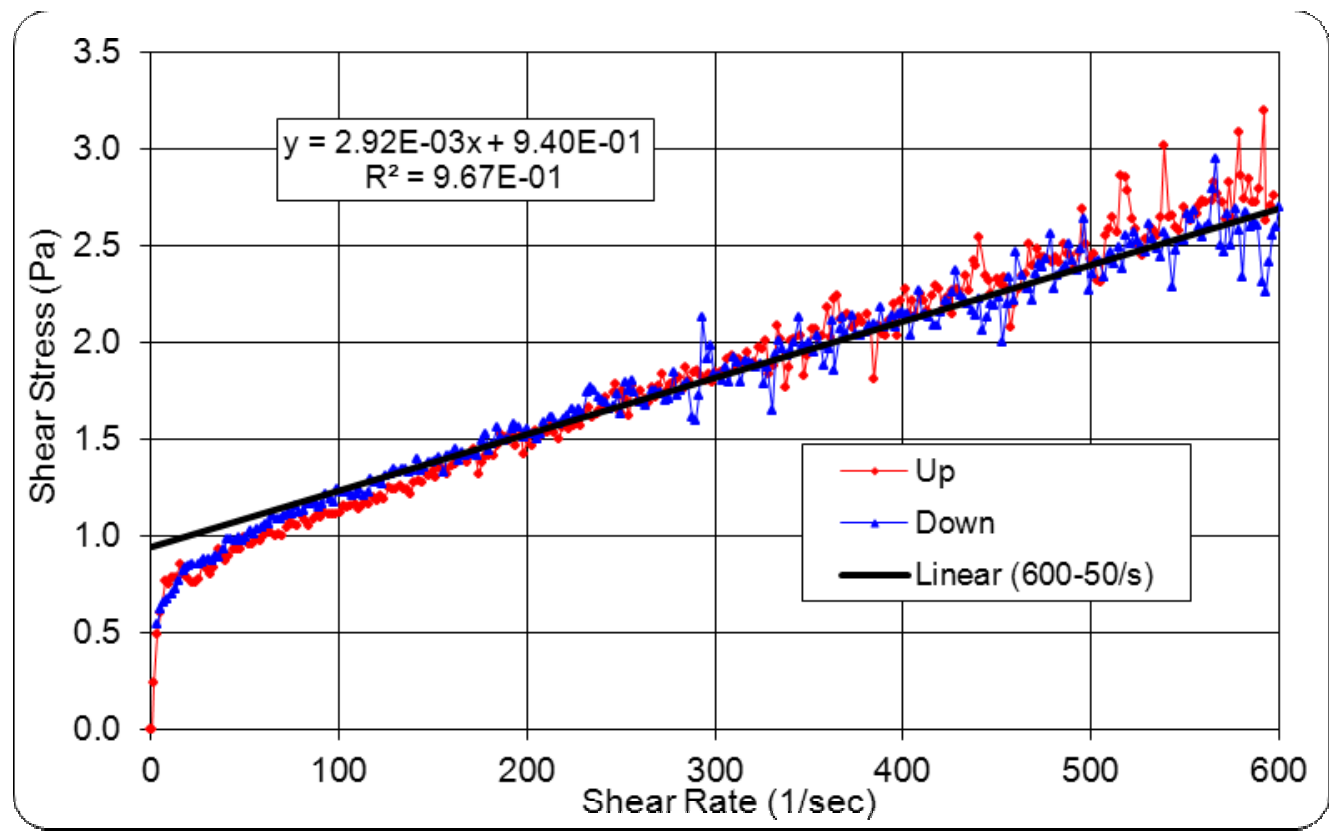

Figure B-30: Stress Versus Strain Curve for Tank 12H Baseline Concentrate at $40{ }^{\circ} \mathrm{C}$ (Second Replicate) 


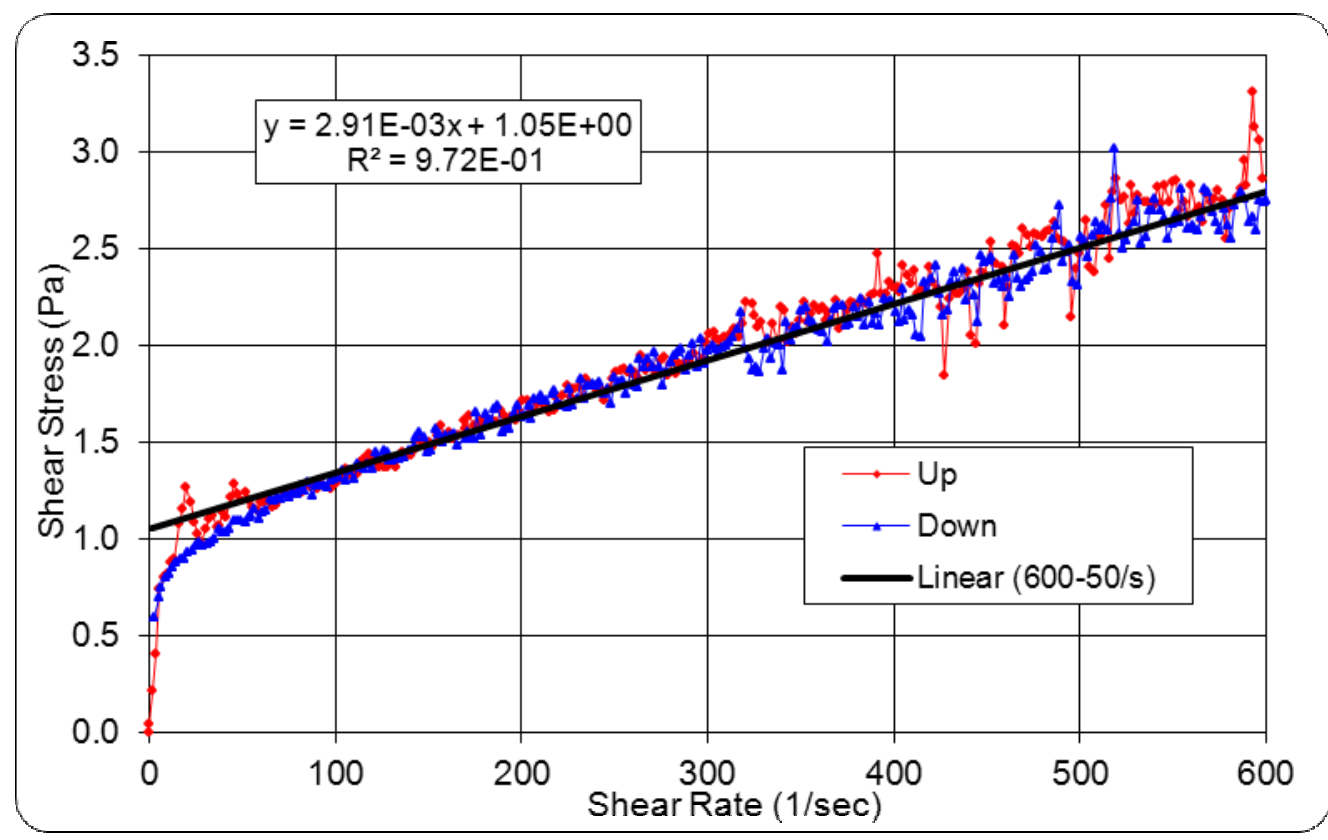

Figure B-31: Stress Versus Strain Curve for Tank 12H Baseline Concentrate at $50{ }^{\circ} \mathrm{C}$ (First Replicate)

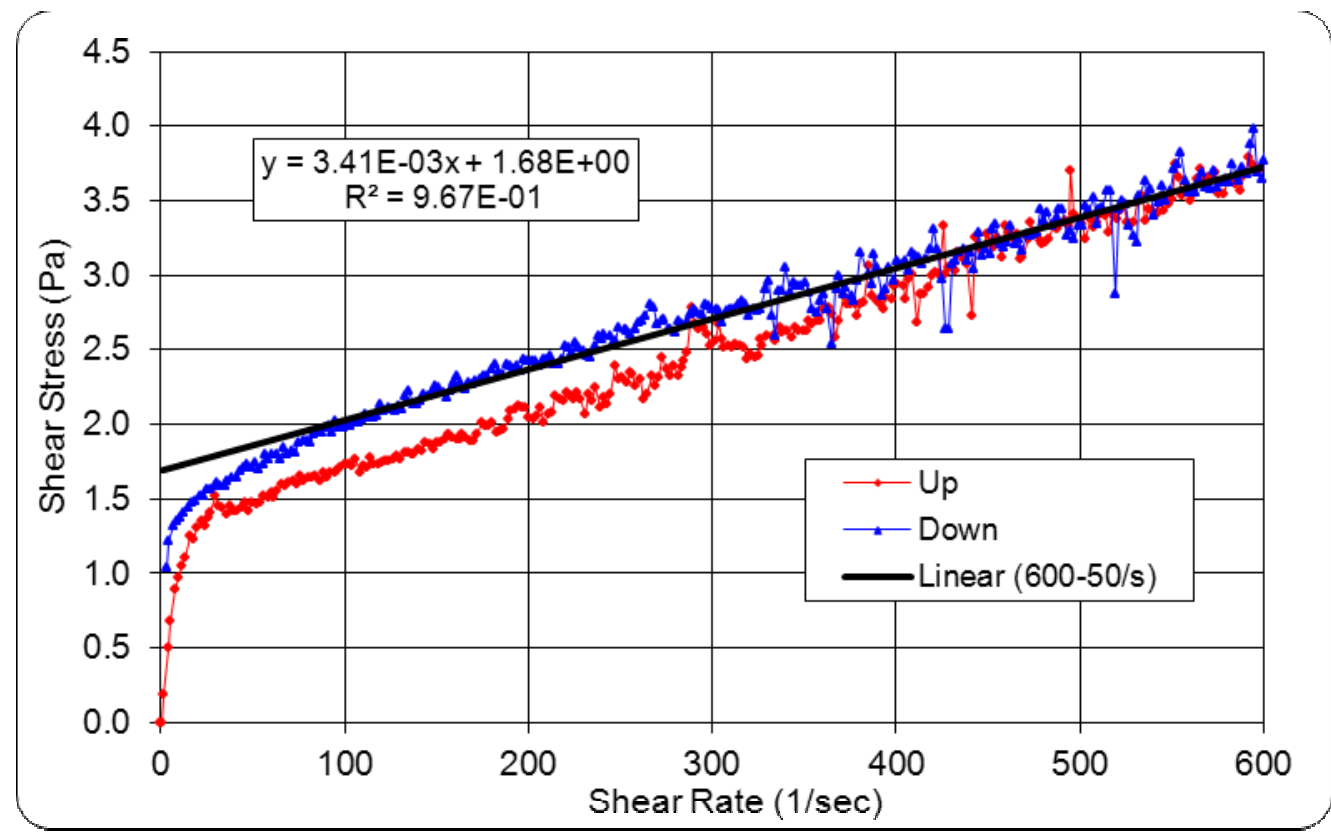

Figure B-32: Stress Versus Strain Curve for Tank 12H Baseline Concentrate at $50{ }^{\circ} \mathrm{C}$ (Second Replicate) 


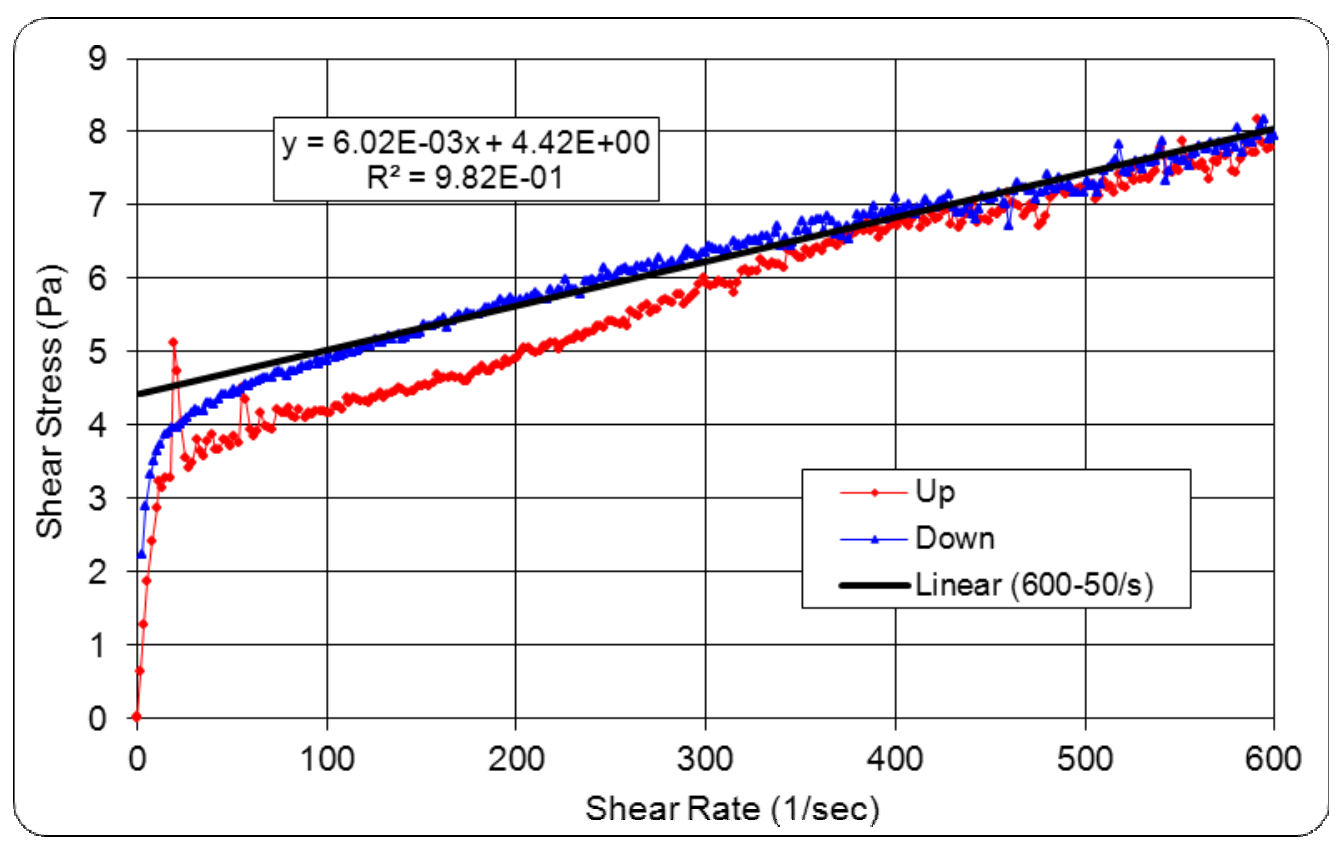

Figure B-33: Stress Versus Strain Curve for Tank $12 \mathrm{H}$ Baseline Concentrate at $30{ }^{\circ} \mathrm{C}$ after 4 Days (First Replicate)

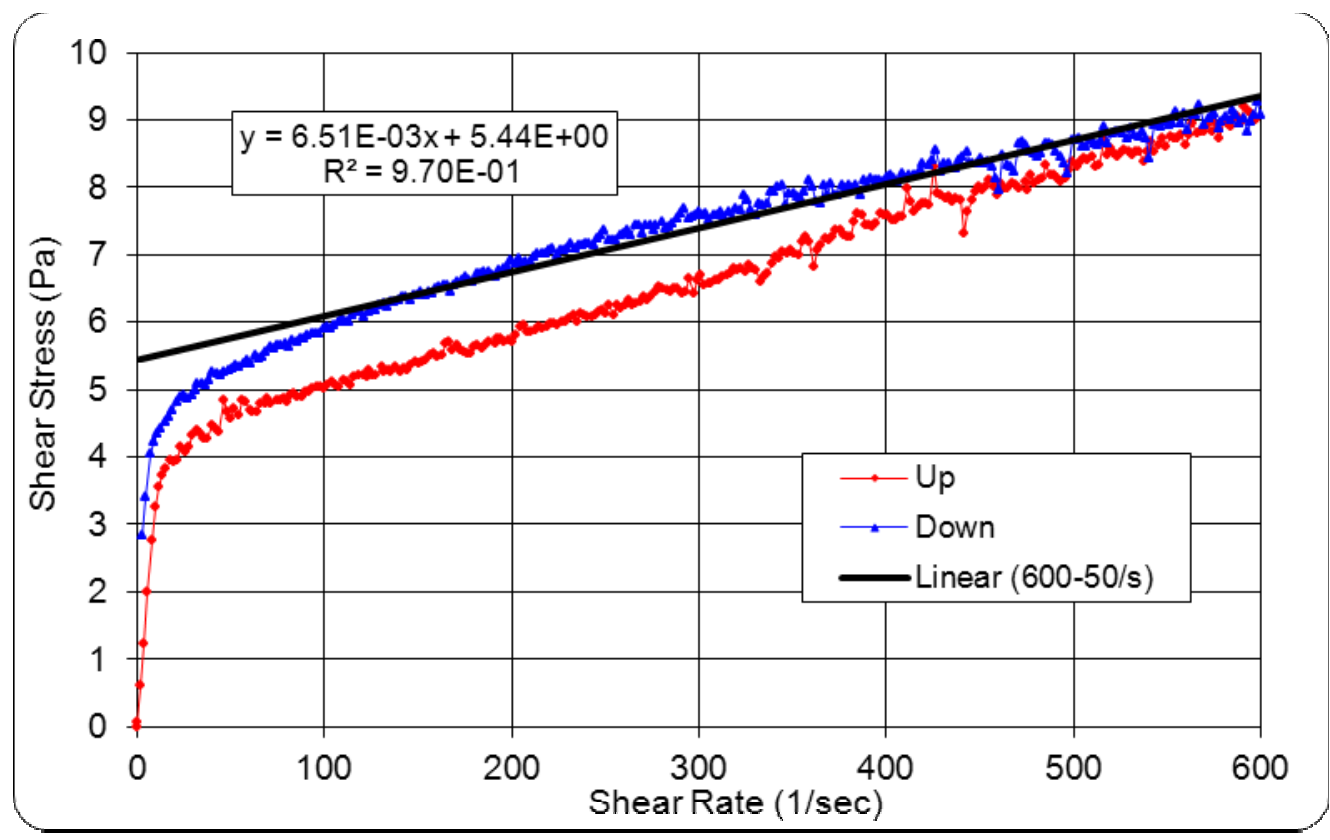

Figure B-34: Stress Versus Strain Curve for Tank $12 \mathrm{H}$ Baseline Concentrate at $30{ }^{\circ} \mathrm{C}$ after 4 Days (Second Replicate) 


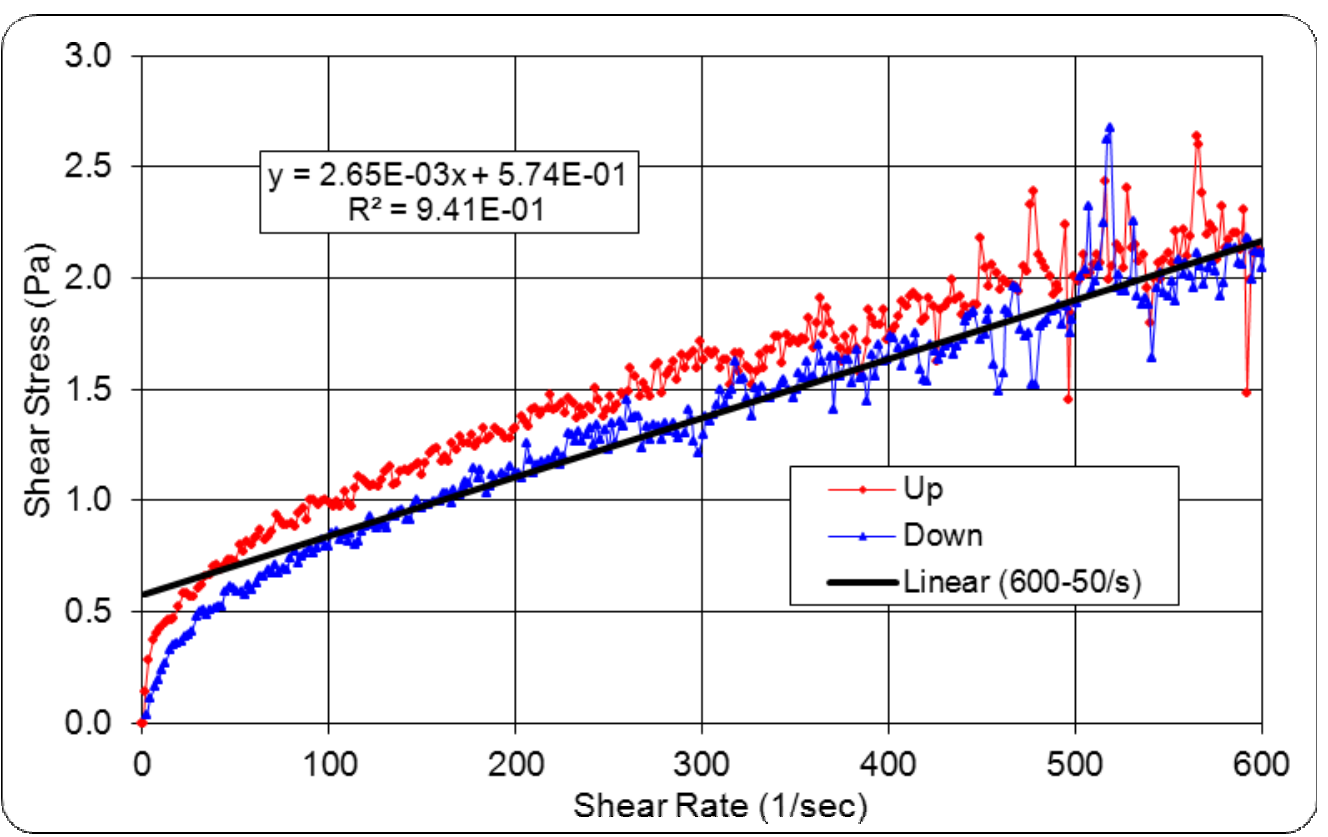

Figure B-35: Stress Versus Strain Curve for Tank $12 \mathrm{H}$ Midpoint Dilute at $30^{\circ} \mathrm{C}$ (First Replicate)

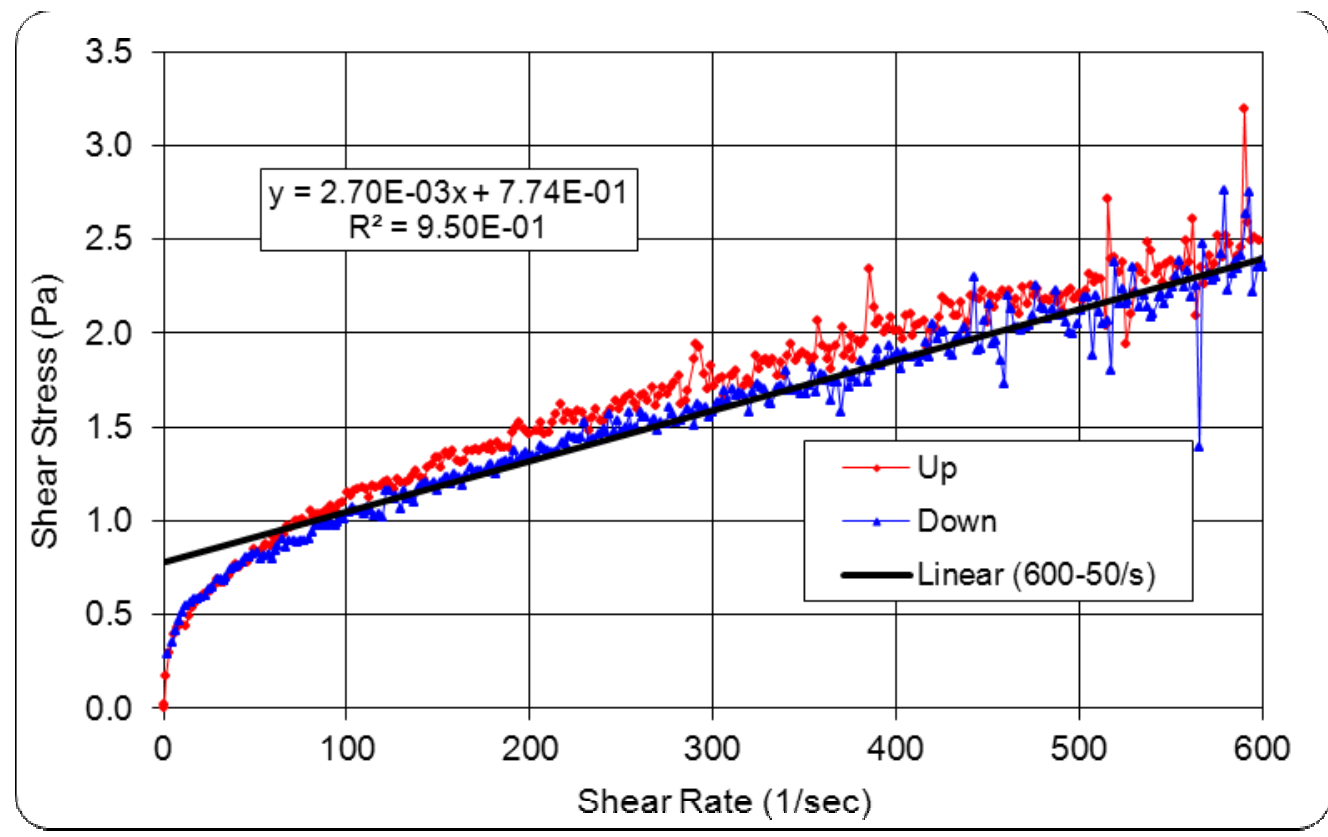

Figure B-36: Stress Versus Strain Curve for Tank $12 \mathrm{H}$ Midpoint Dilute at $30^{\circ} \mathrm{C}$ (Second Replicate) 


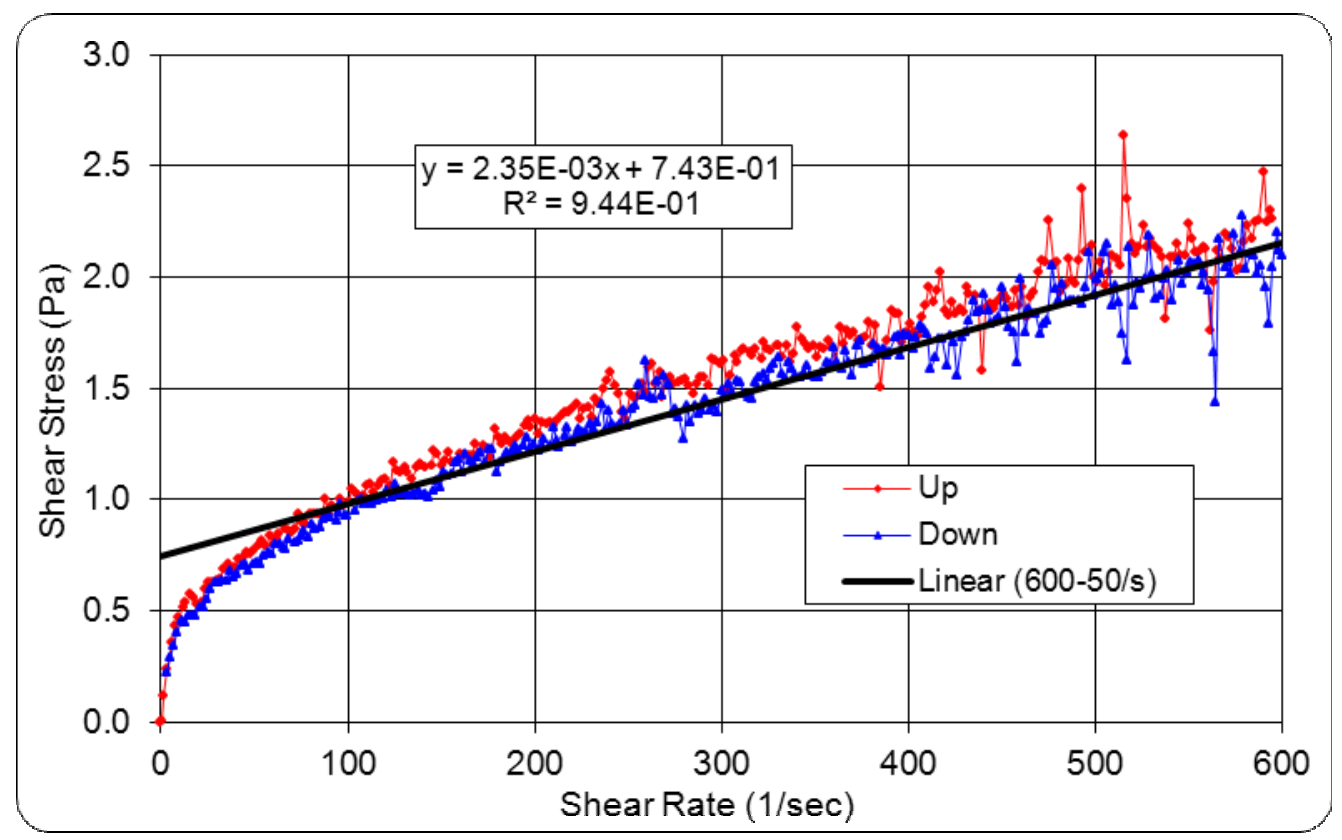

Figure B-37: Stress Versus Strain Curve for Tank 12H Midpoint Dilute at $40^{\circ} \mathrm{C}$ (First Replicate)

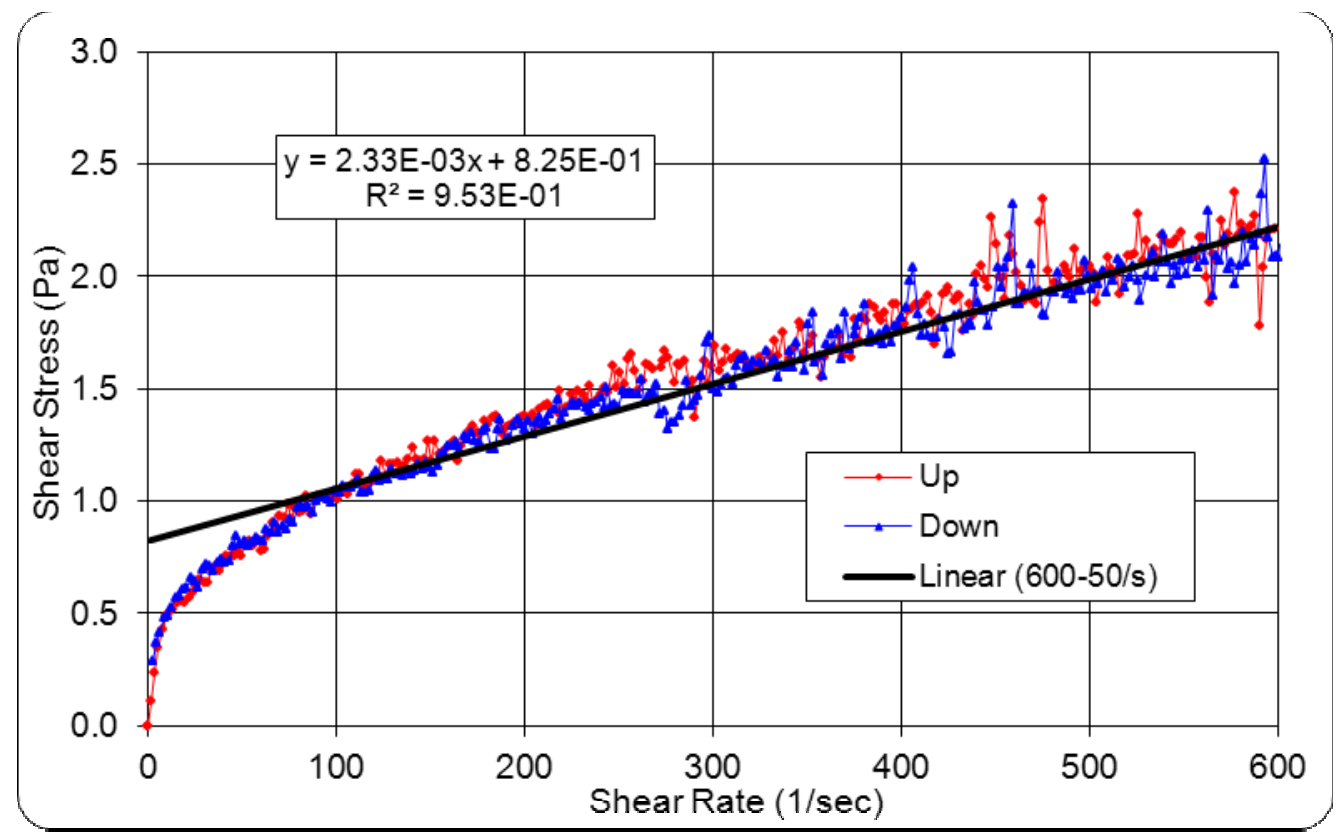

Figure B-38: Stress Versus Strain Curve for Tank $12 \mathrm{H}$ Midpoint Dilute at $40^{\circ} \mathrm{C}$ (Second Replicate) 


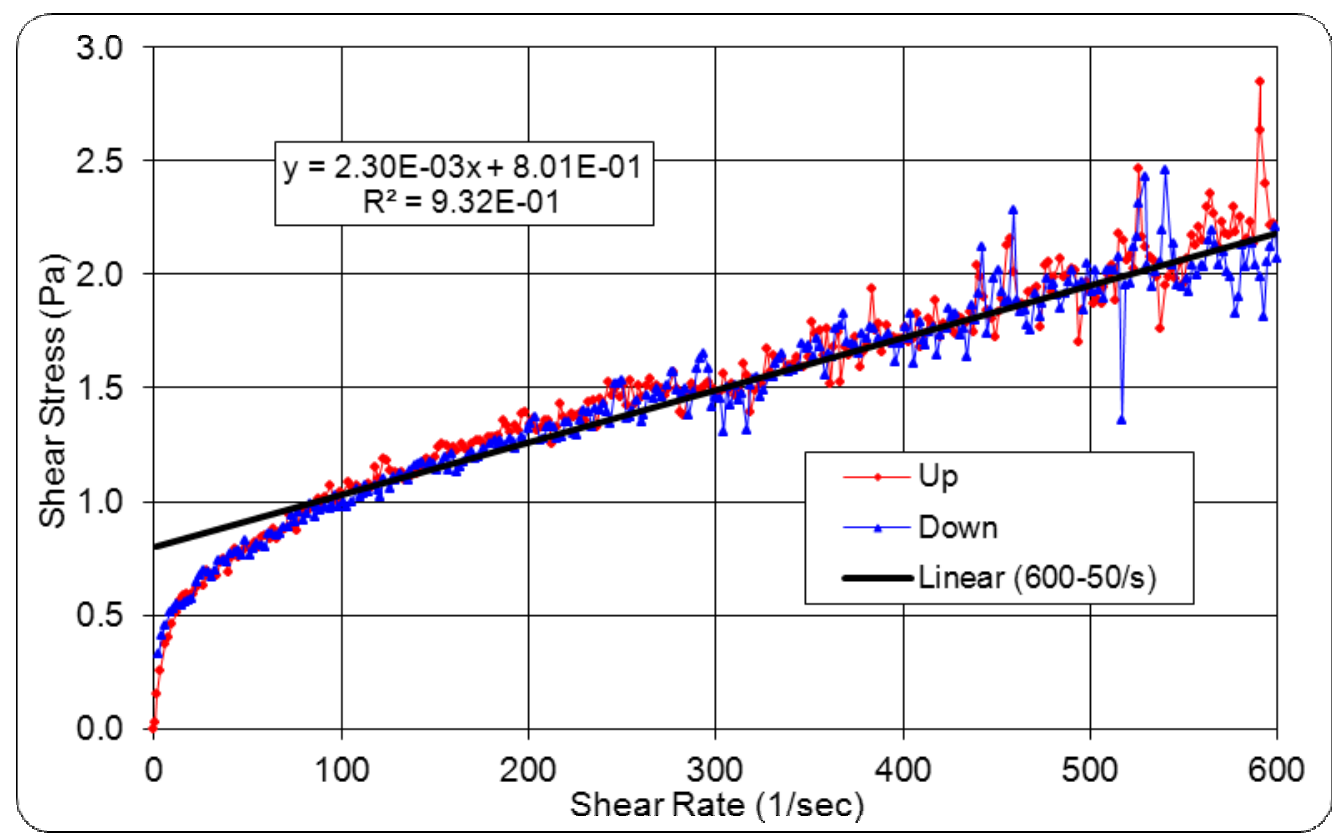

Figure B-39: Stress Versus Strain Curve for Tank 12H Midpoint Dilute at $50{ }^{\circ} \mathrm{C}$ (First Replicate)

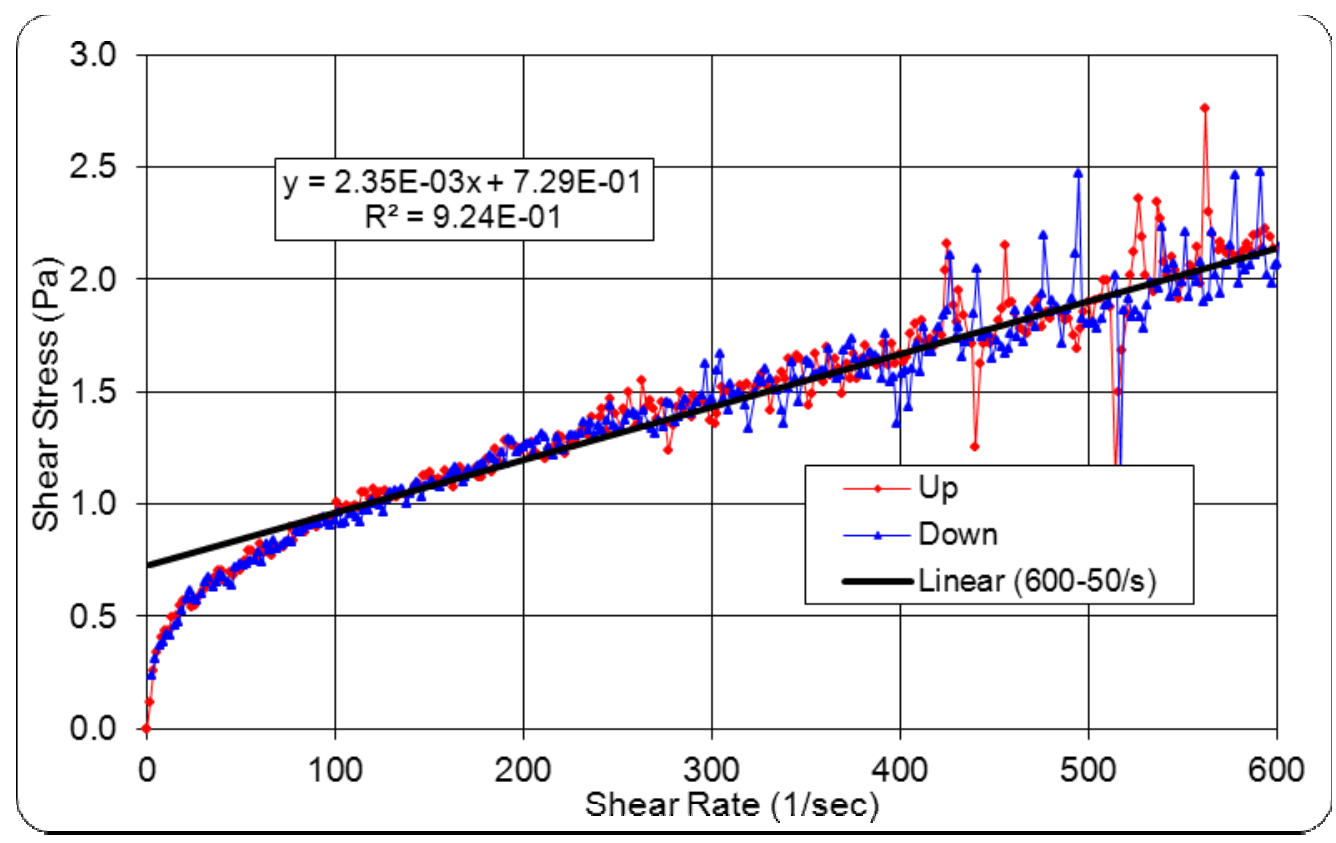

Figure B-40: Stress Versus Strain Curve for Tank $12 \mathrm{H}$ Midpoint Dilute at $50{ }^{\circ} \mathrm{C}$ (Second Replicate) 


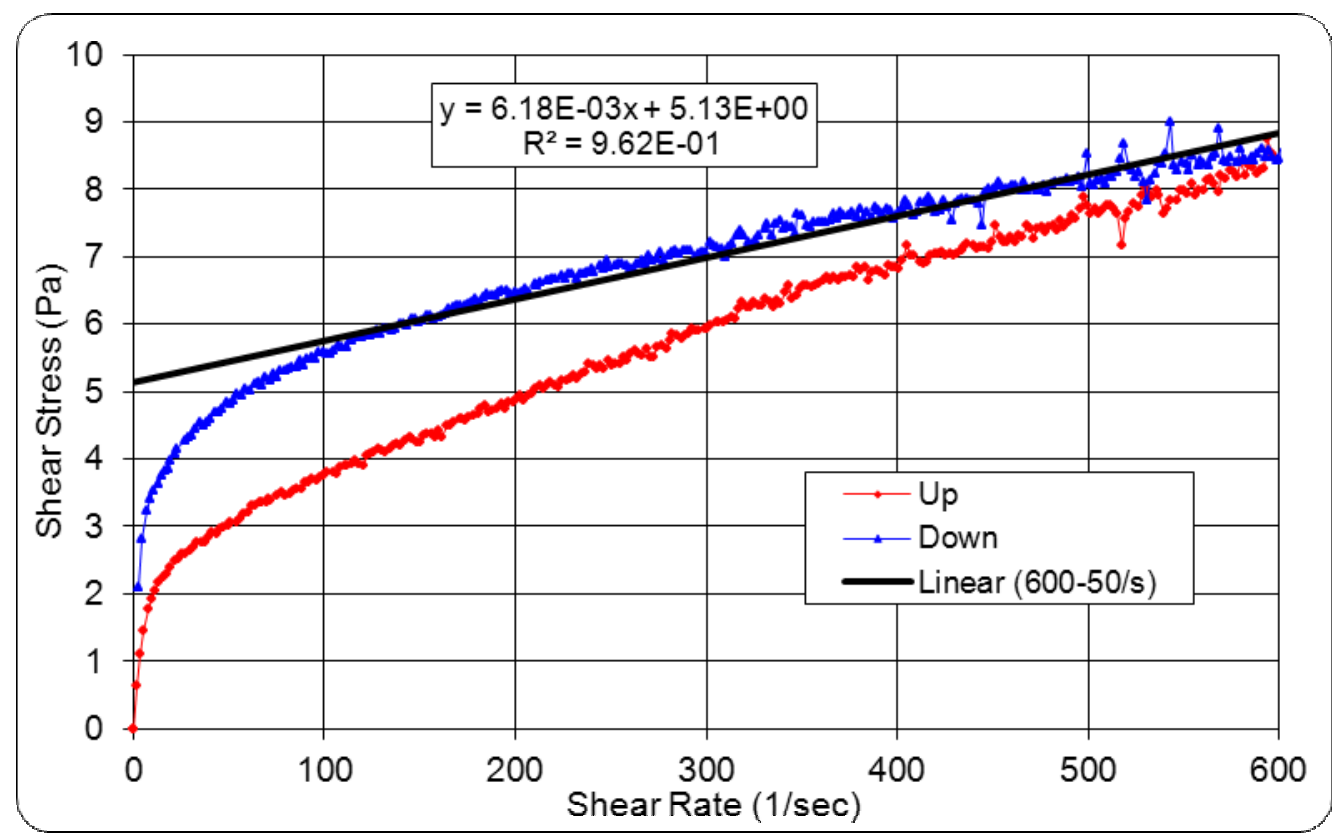

Figure B-41: Stress Versus Strain Curve for Tank $12 \mathrm{H}$ Midpoint Concentrate at $30^{\circ} \mathrm{C}$ (First Replicate)

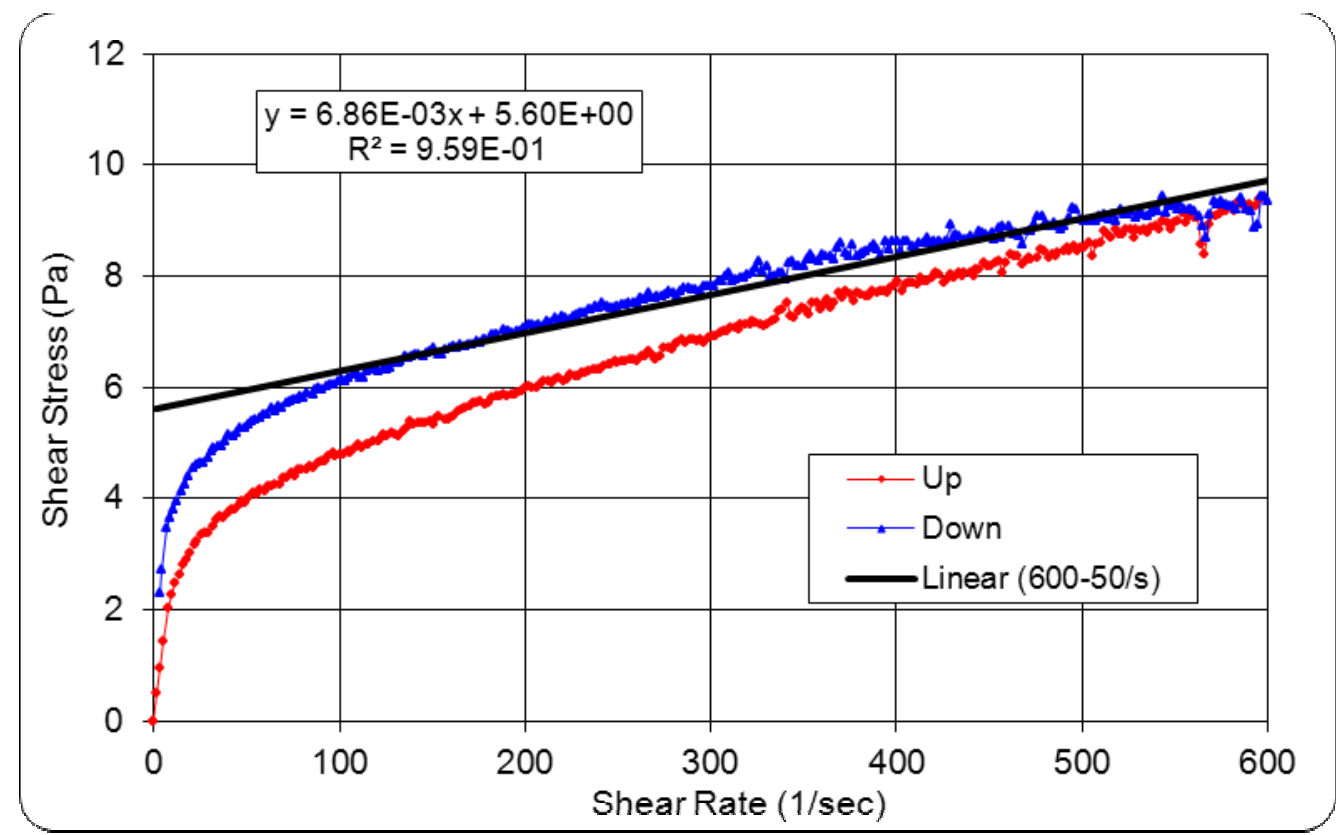

Figure B-42: Stress Versus Strain Curve for Tank $12 \mathrm{H}$ Midpoint Concentrate at $30^{\circ} \mathrm{C}$ (Second Replicate) 


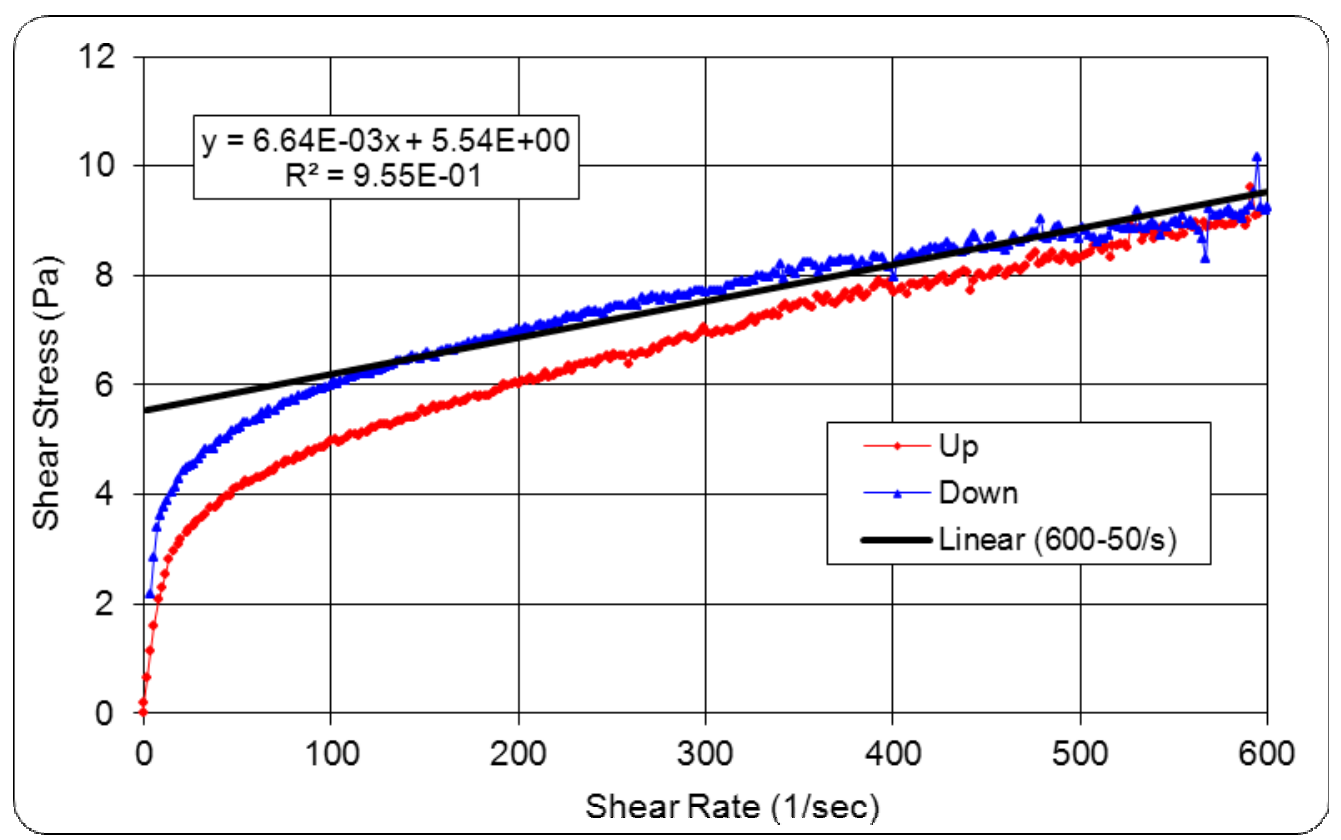

Figure B-43: Stress Versus Strain Curve for Tank 12H Midpoint Concentrate at $40{ }^{\circ} \mathrm{C}$ (First Replicate)

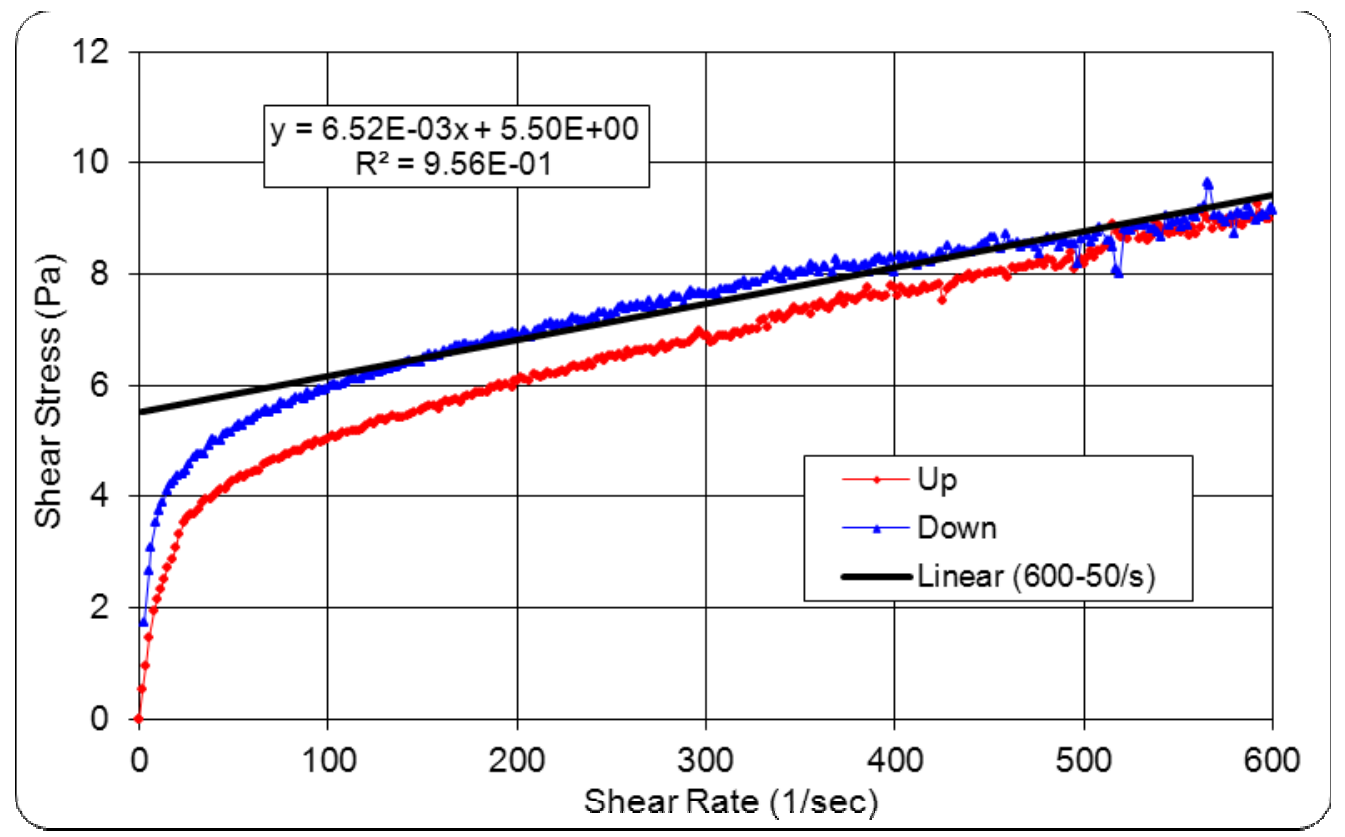

Figure B-44: Stress Versus Strain Curve for Tank 12H Midpoint Concentrate at $40{ }^{\circ} \mathrm{C}$ (Second Replicate) 


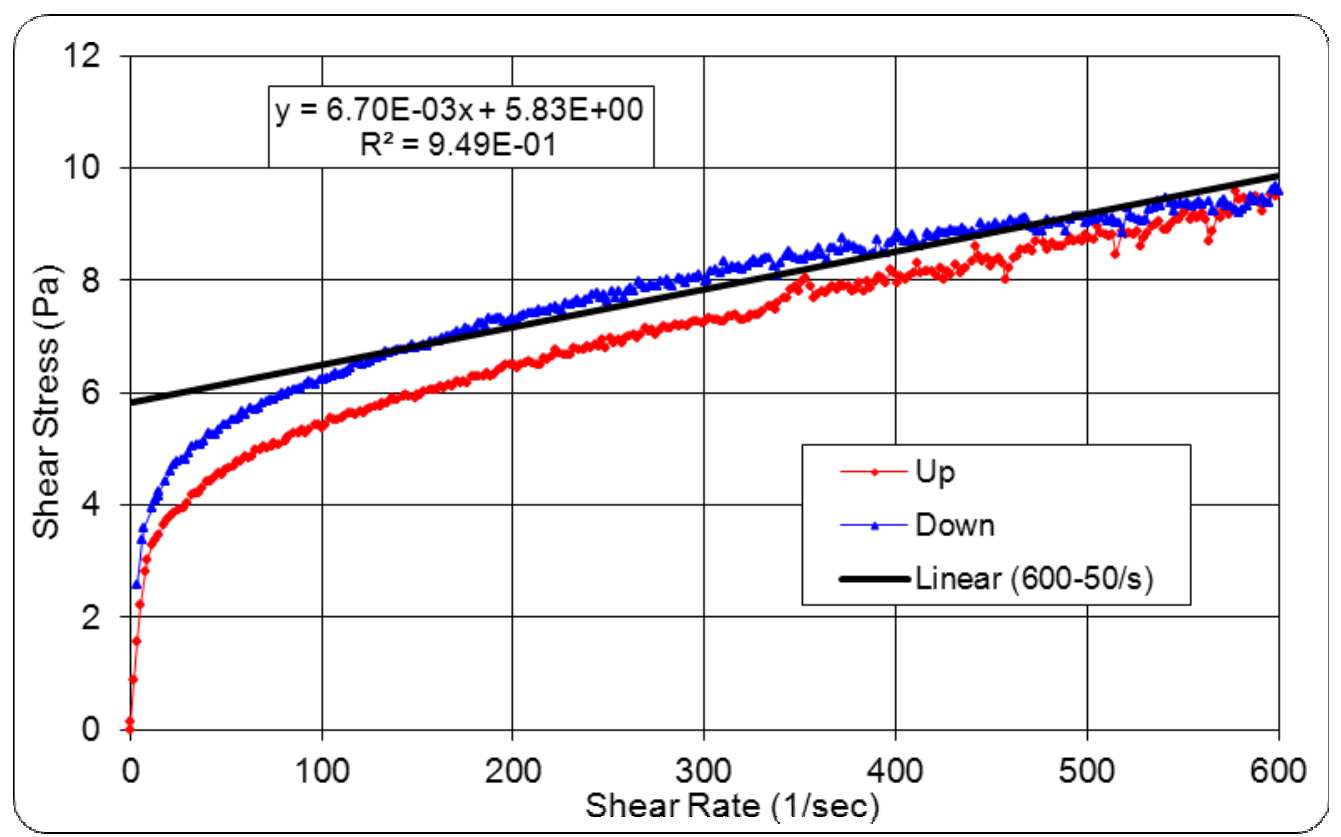

Figure B-45: Stress Versus Strain Curve for Tank $12 \mathrm{H}$ Midpoint Concentrate at $50{ }^{\circ} \mathrm{C}$ (First Replicate)

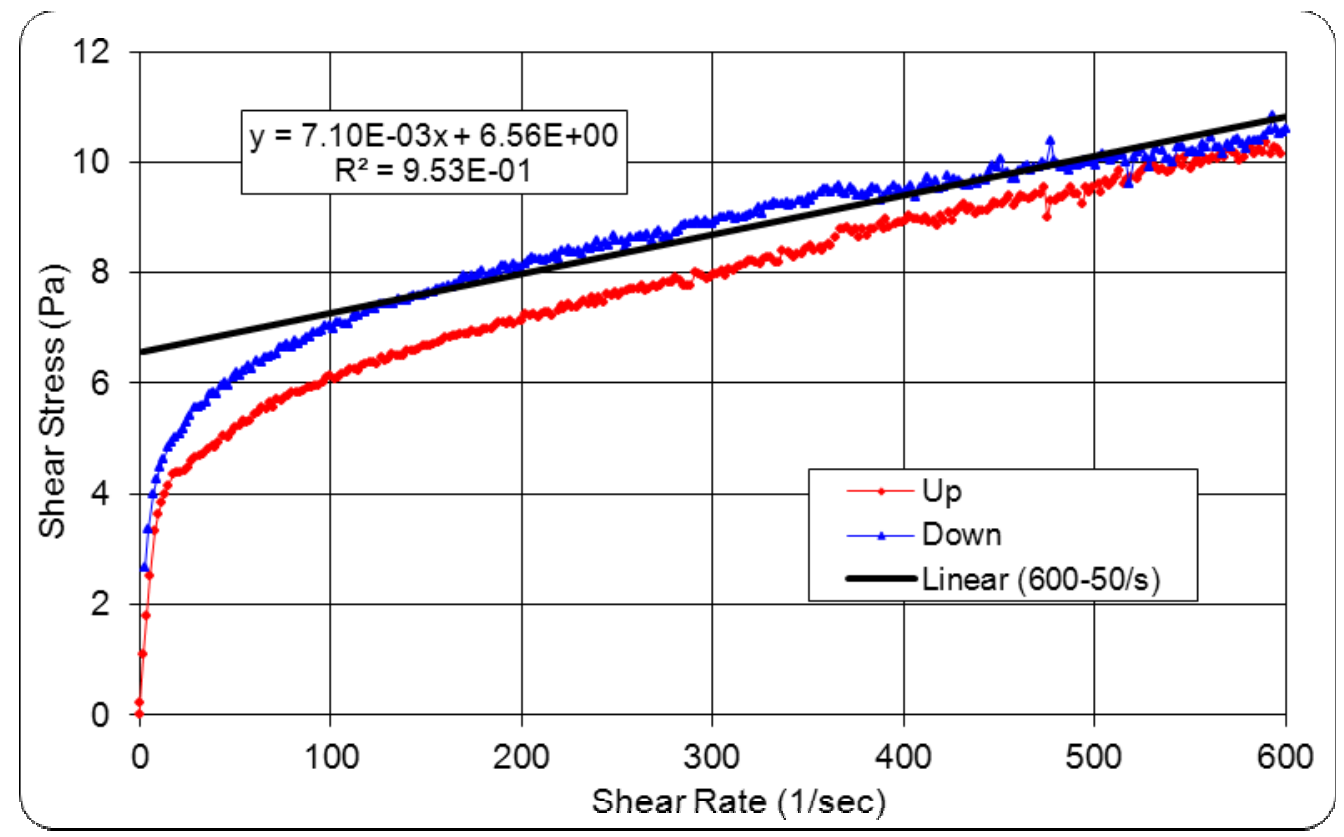

Figure B-46: Stress Versus Strain Curve for Tank $12 \mathrm{H}$ Midpoint Concentrate at $50{ }^{\circ} \mathrm{C}$ (Second Replicate) 


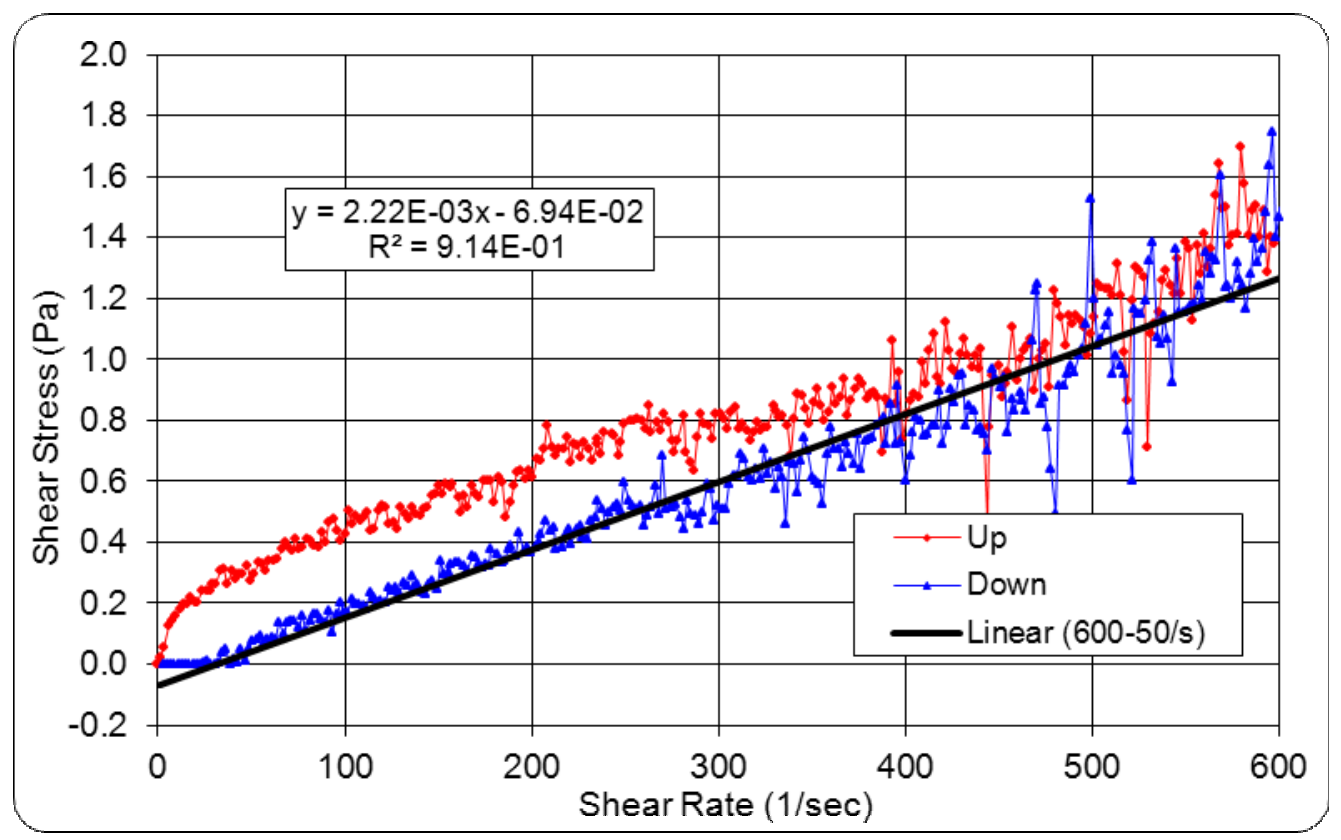

Figure B-47: Stress Versus Strain Curve for Tank $12 \mathrm{H}$ Endpoint Dilute at $30^{\circ} \mathrm{C}$ (First Replicate)

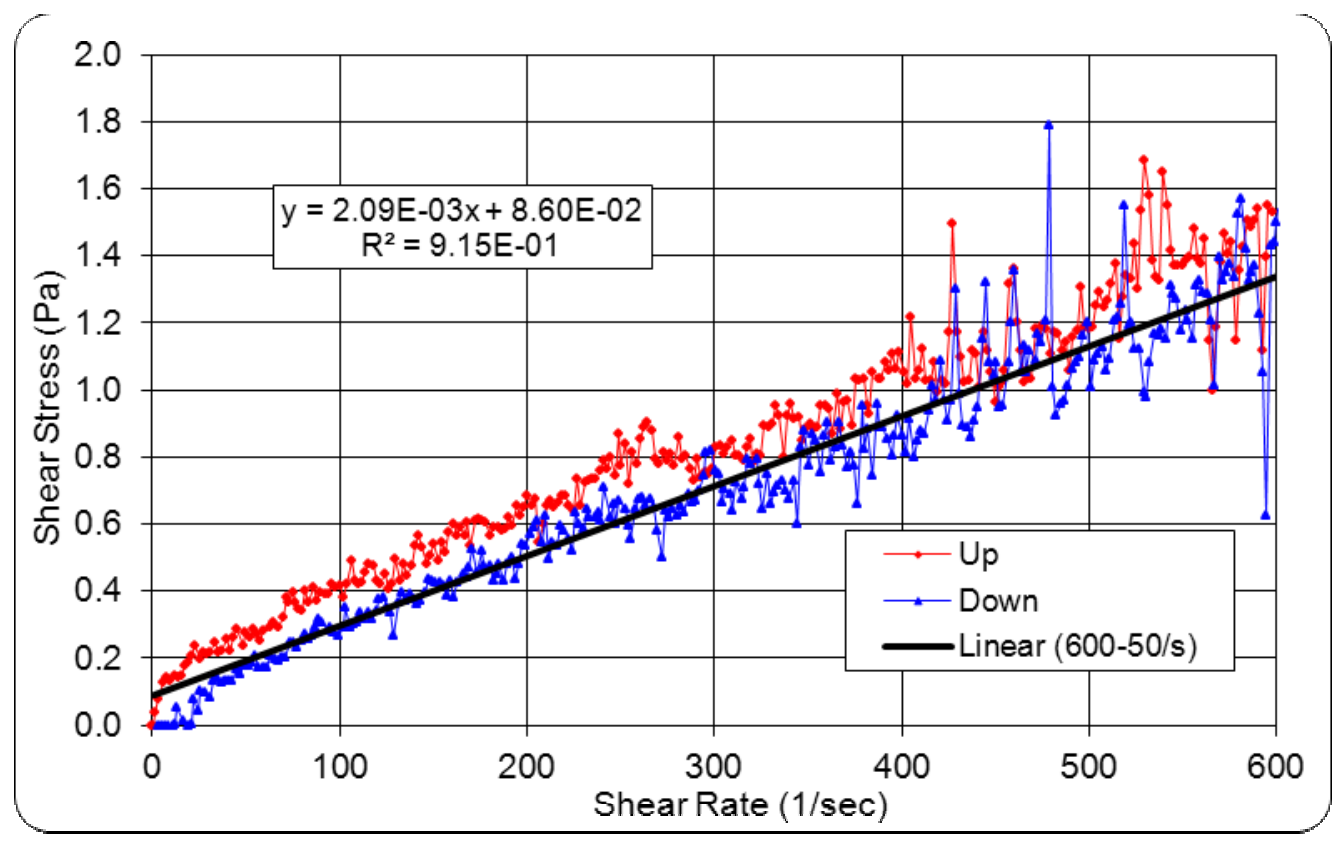

Figure B-48: Stress Versus Strain Curve for Tank $12 \mathrm{H}$ Endpoint Dilute at $30^{\circ} \mathrm{C}$ (Second Replicate) 


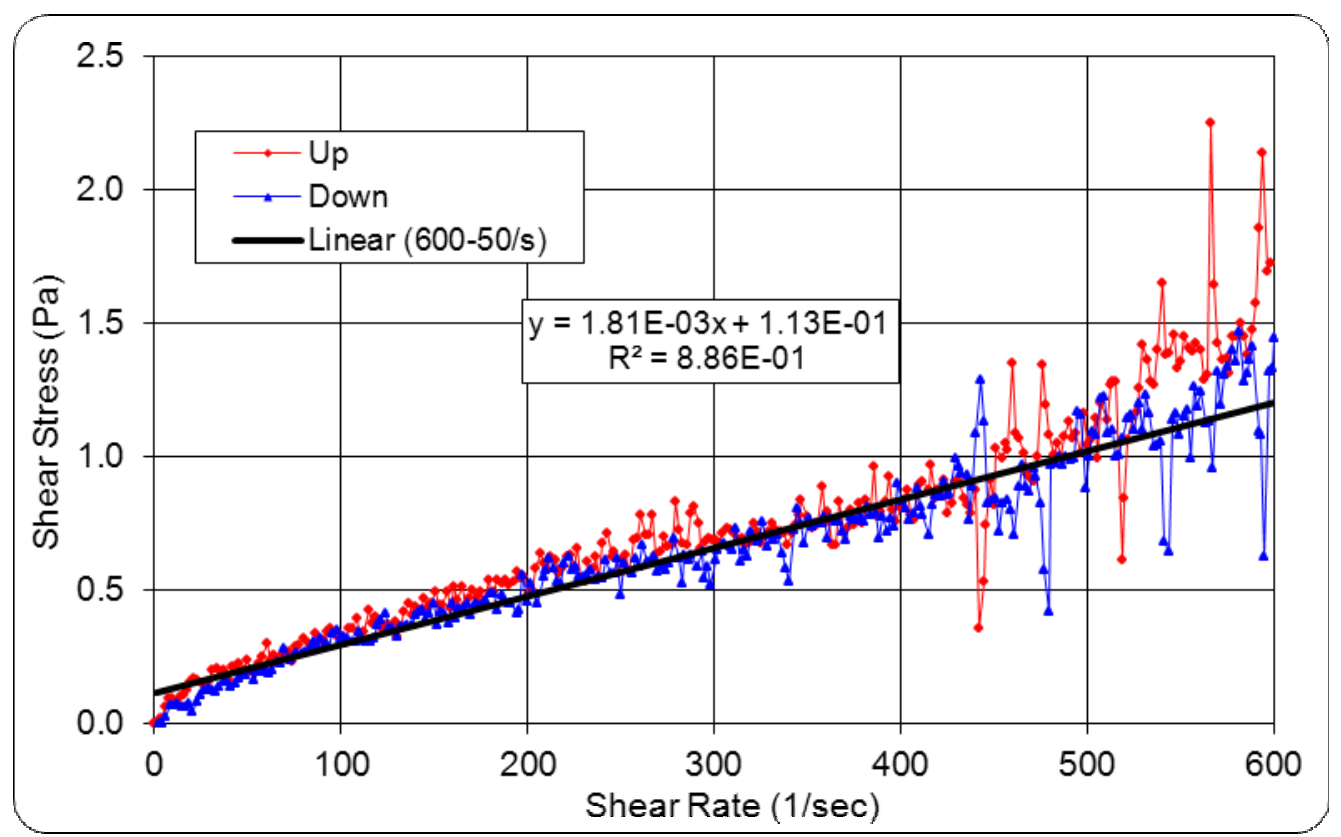

Figure B-49: Stress Versus Strain Curve for Tank 12H Endpoint Dilute at $40{ }^{\circ} \mathrm{C}$ (First Replicate)

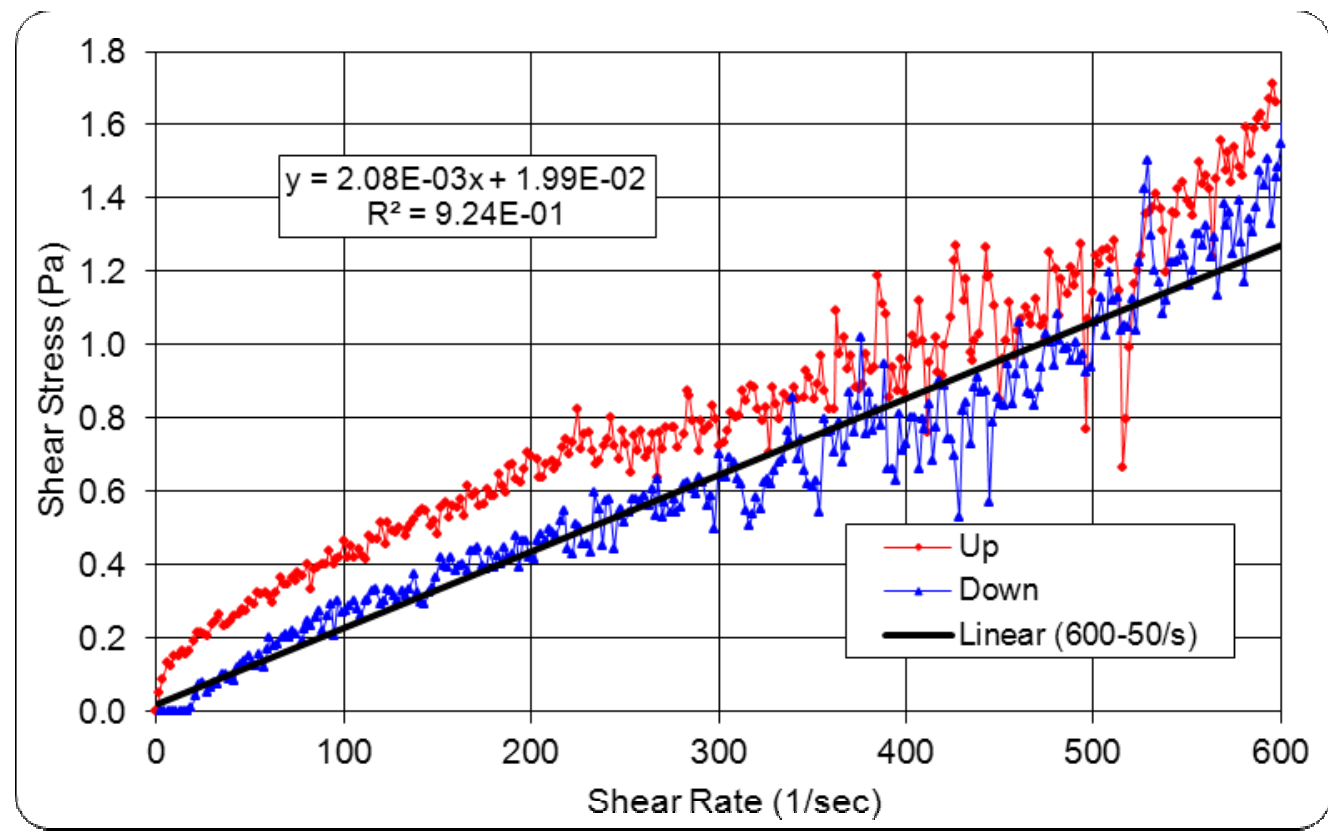

Figure B-50: Stress Versus Strain Curve for Tank 12H Endpoint Dilute at $40^{\circ} \mathrm{C}$ (Second Replicate) 


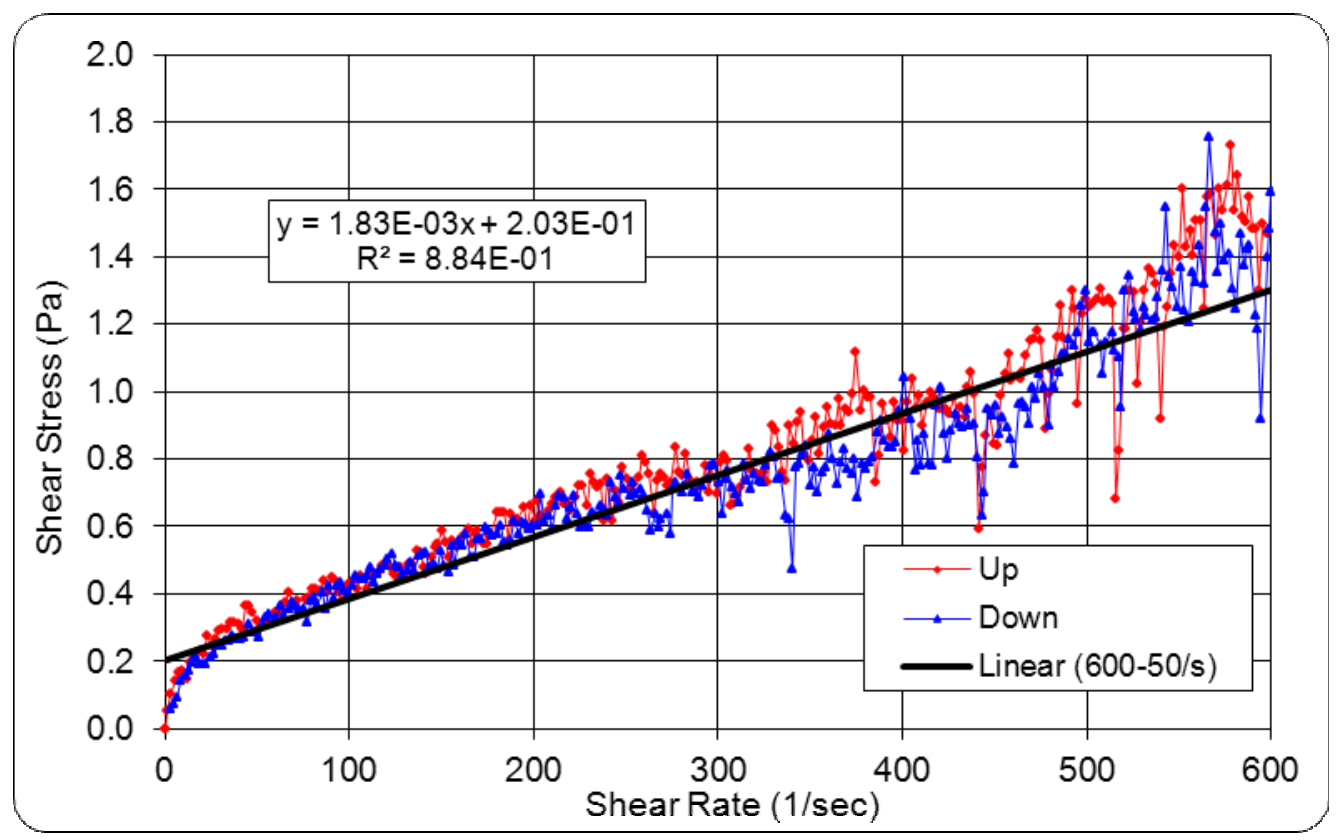

Figure B-51: Stress Versus Strain Curve for Tank 12H Endpoint Dilute at $50{ }^{\circ} \mathrm{C}$ (First Replicate)

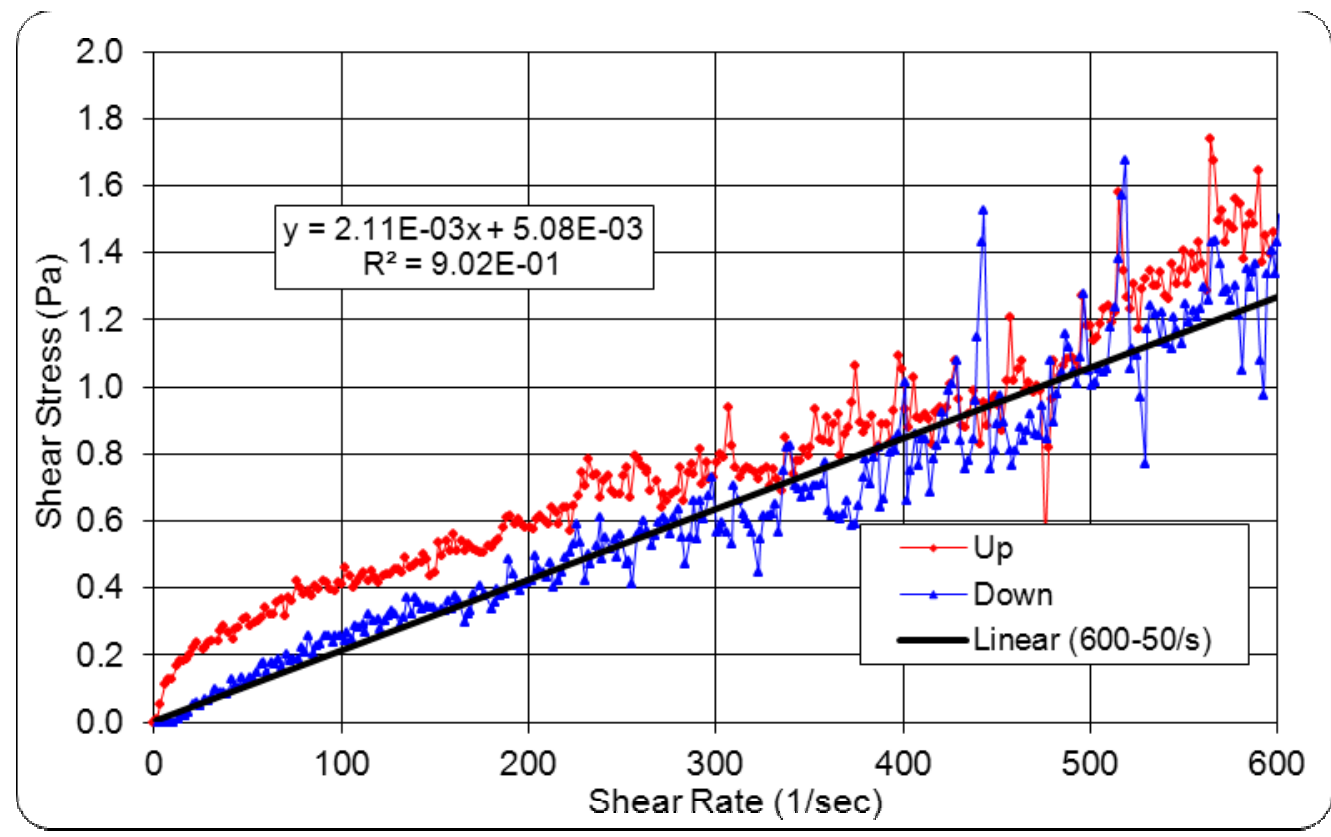

Figure B-52: Stress Versus Strain Curve for Tank $12 \mathrm{H}$ Endpoint Dilute at $50^{\circ} \mathrm{C}$ (Second Replicate) 


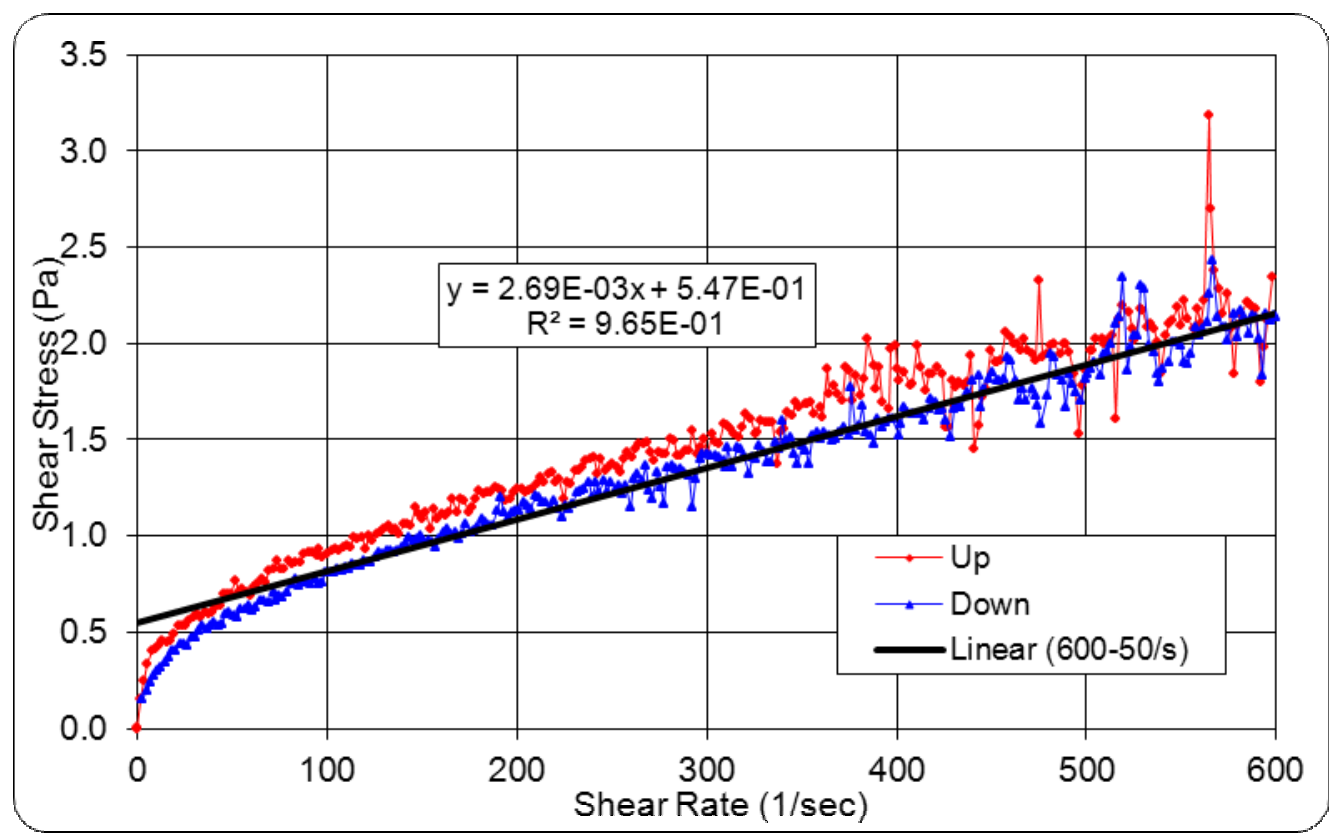

Figure B-53: Stress Versus Strain Curve for Tank 12H Endpoint Concentrate at $30{ }^{\circ} \mathrm{C}$ (First Replicate)

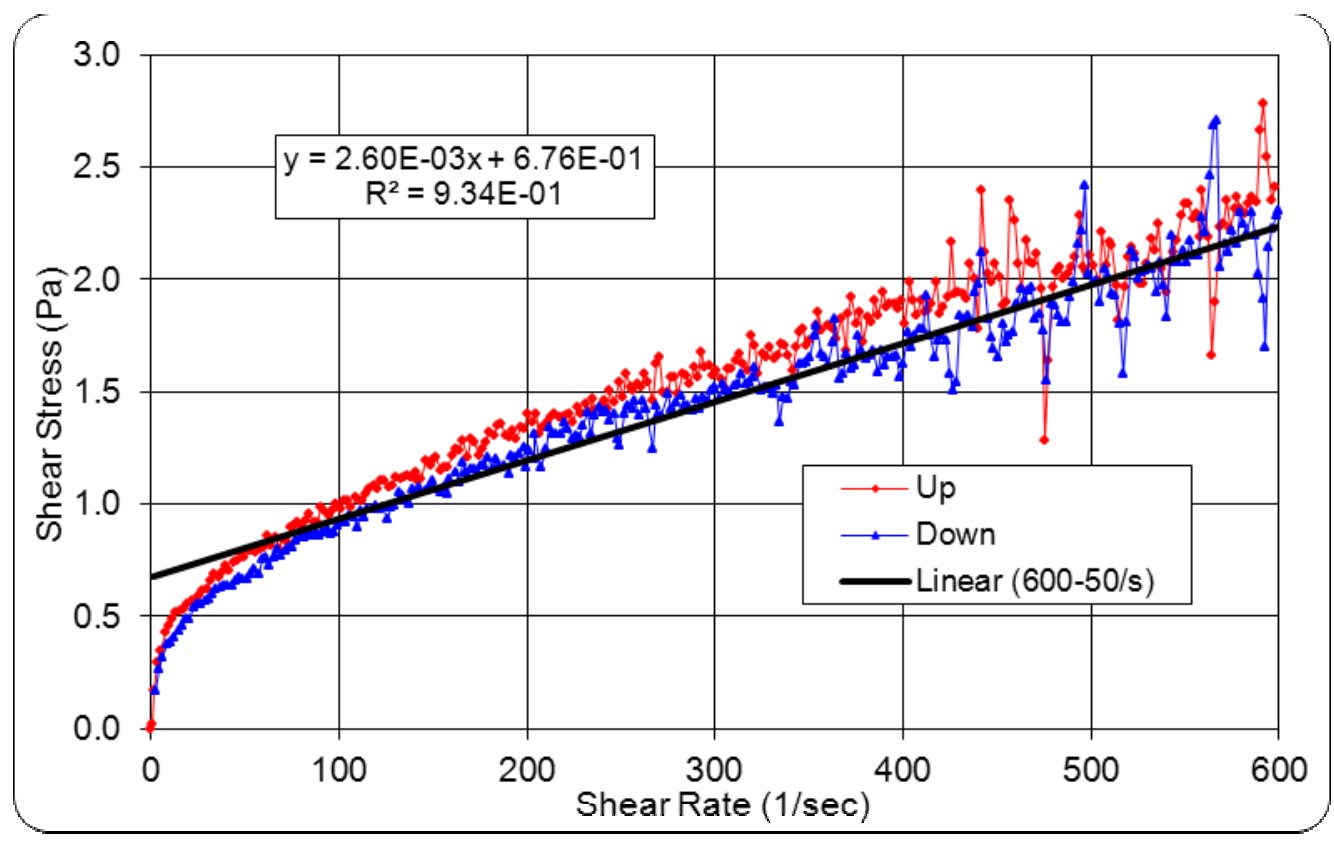

Figure B-54: Stress Versus Strain Curve for Tank $12 \mathrm{H}$ Endpoint Concentrate at $30^{\circ} \mathrm{C}$ (Second Replicate) 


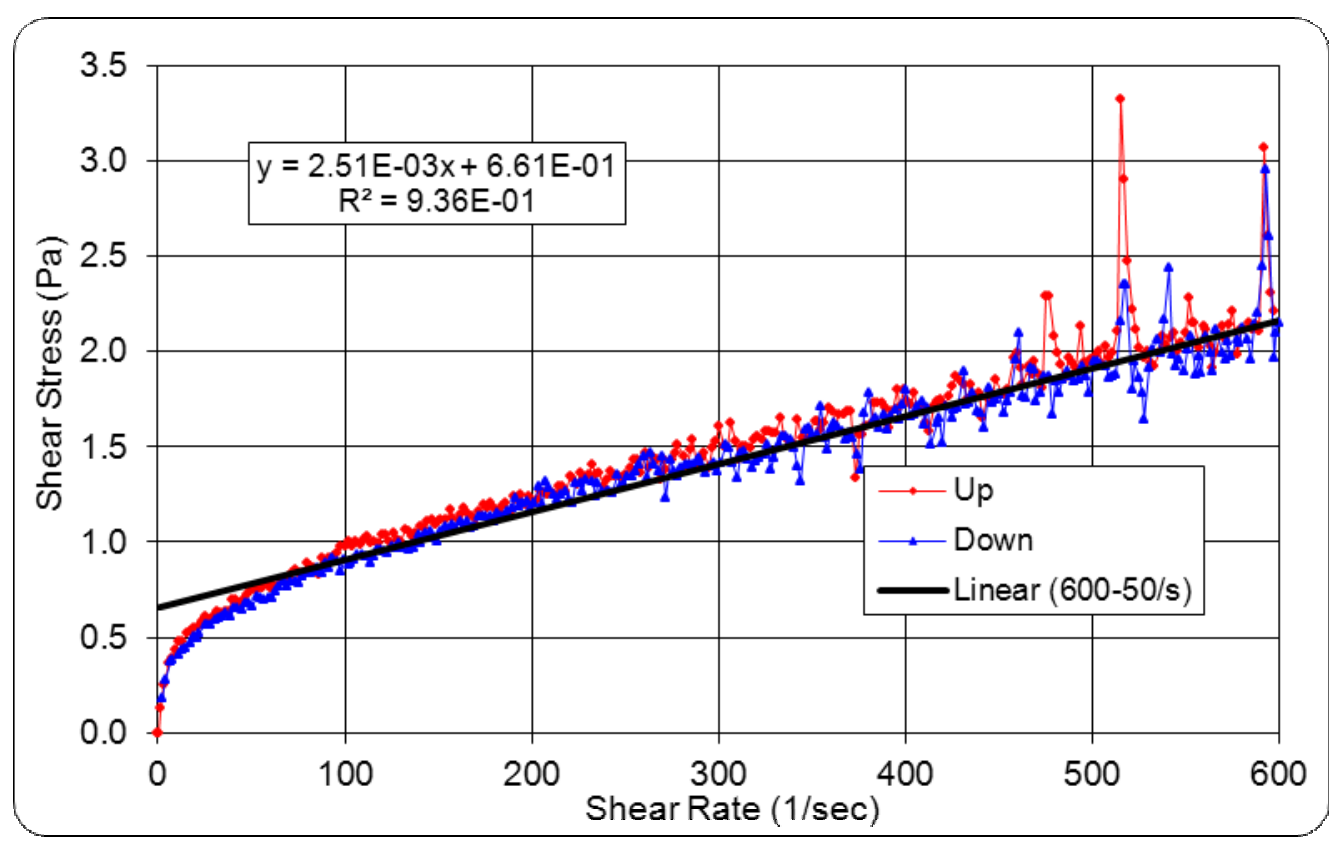

Figure B-55: Stress Versus Strain Curve for Tank 12H Endpoint Concentrate at $40{ }^{\circ} \mathrm{C}$ (First Replicate)

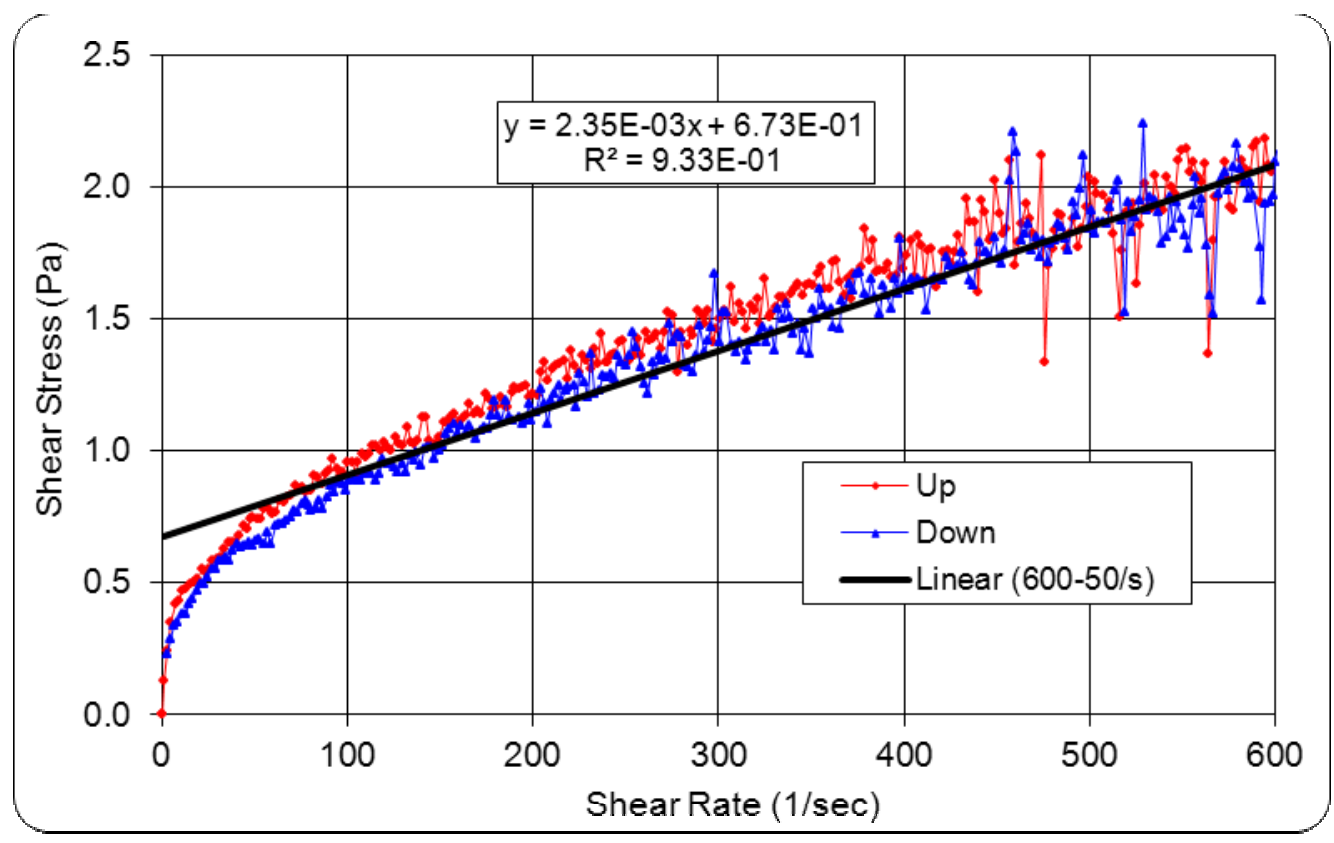

Figure B-56: Stress Versus Strain Curve for Tank 12H Endpoint Concentrate at $40{ }^{\circ} \mathrm{C}$ (Second Replicate) 


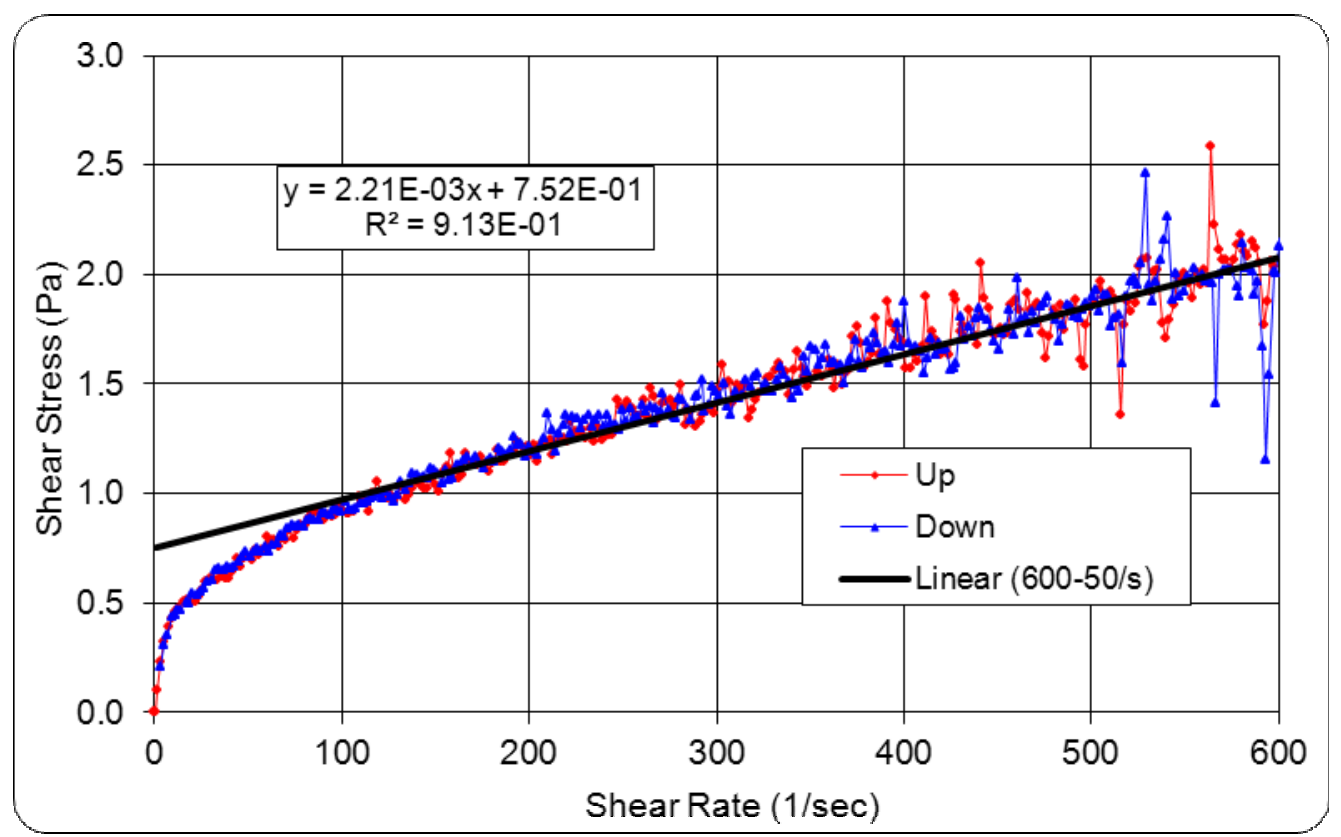

Figure B-57: Stress Versus Strain Curve for Tank 12H Endpoint Concentrate at $50{ }^{\circ} \mathrm{C}$ (First Replicate)

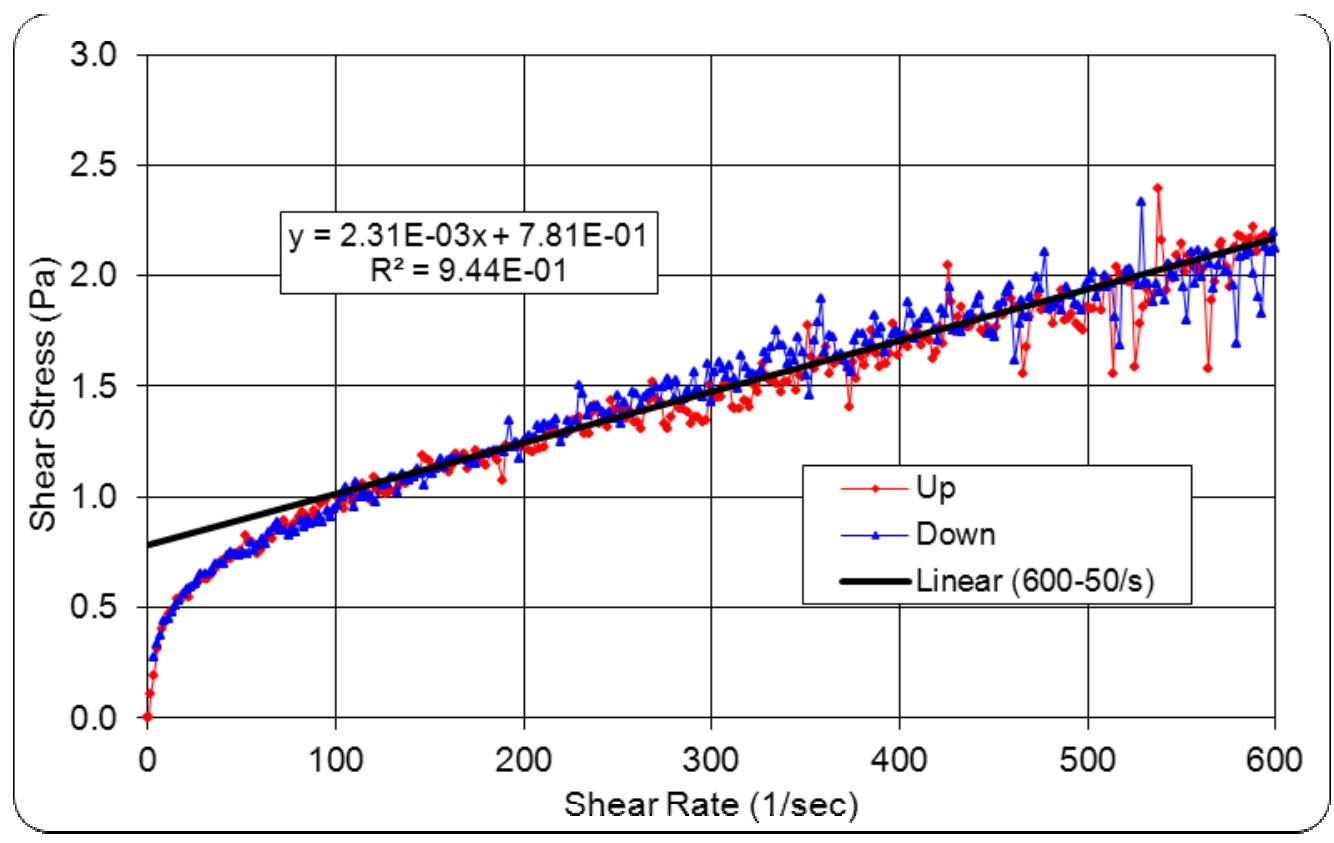

Figure B-58: Stress Versus Strain Curve for Tank $12 \mathrm{H}$ Endpoint Concentrate at $50{ }^{\circ} \mathrm{C}$ (Second Replicate) 


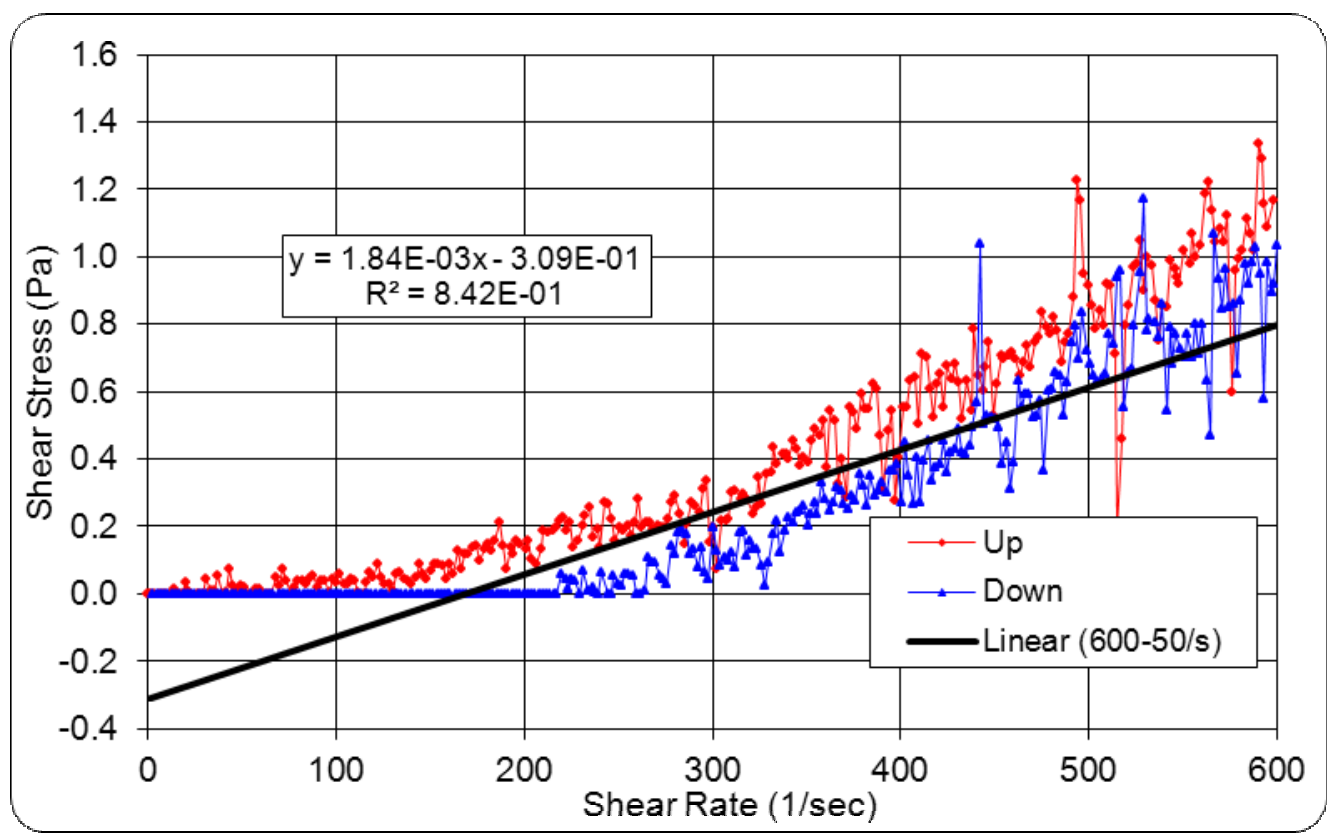

Figure B-59: Stress Versus Strain Curve for Tank 5F No Evaporation Deposition Sample at $30{ }^{\circ} \mathrm{C}$ (First Replicate)

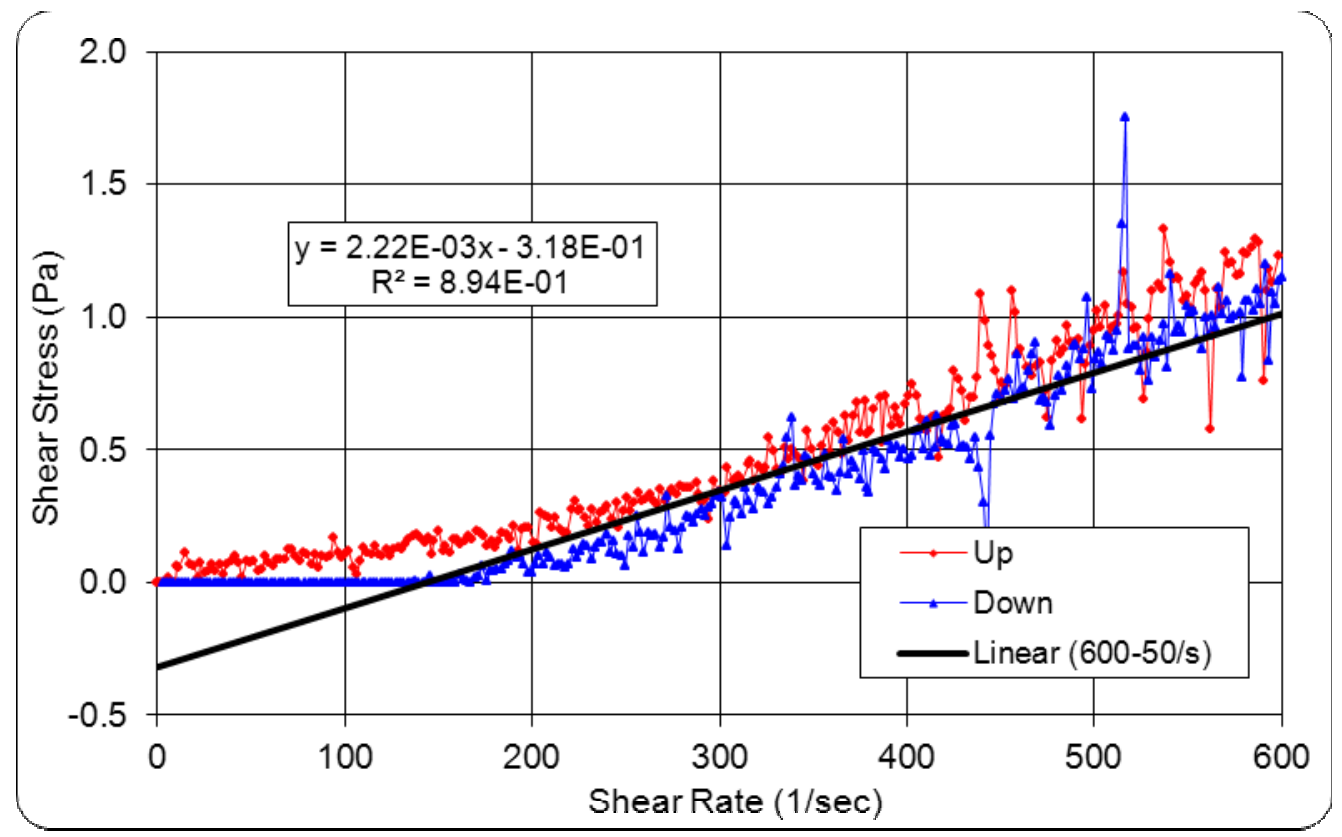

Figure B-60: Stress Versus Strain Curve for Tank 5F No Evaporation Deposition Sample at $30^{\circ} \mathrm{C}$ (Second Replicate) 


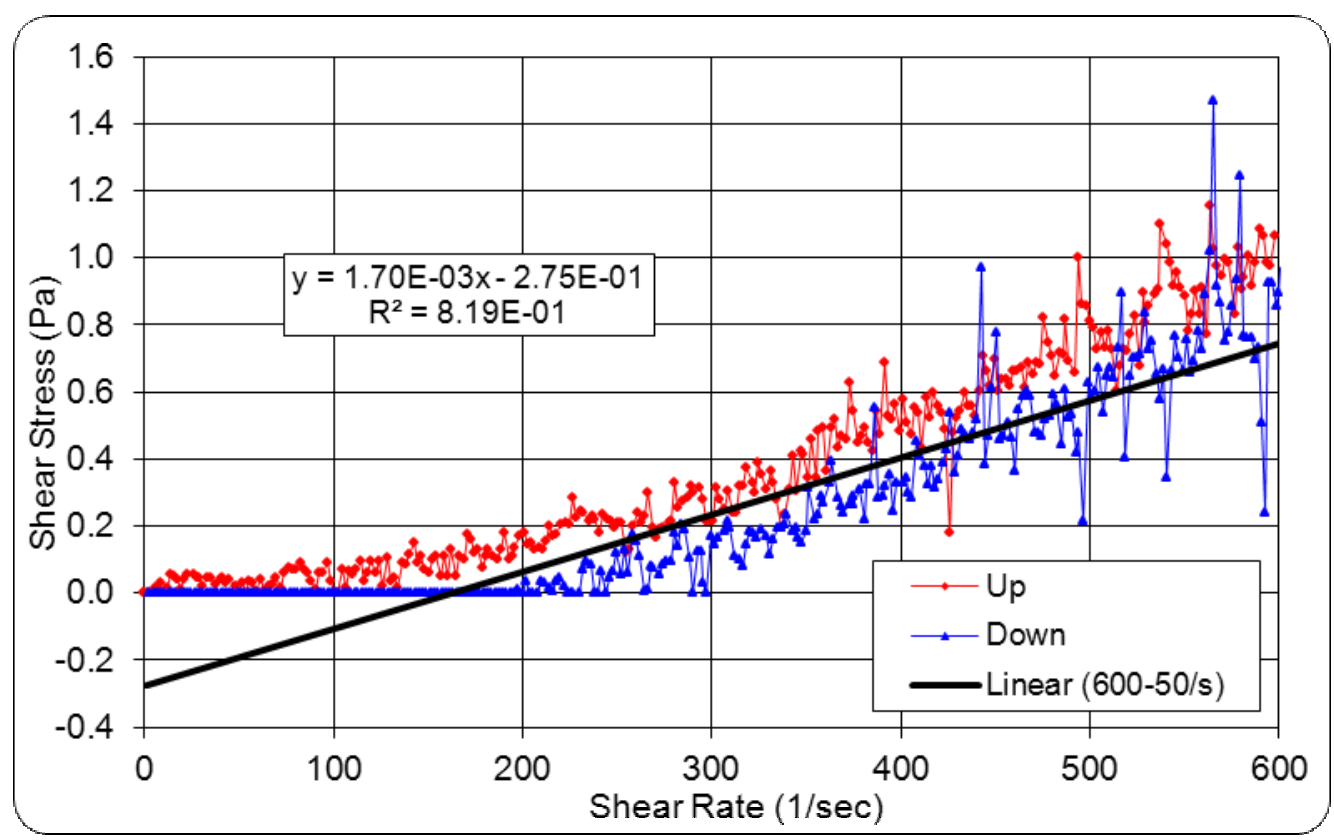

Figure B-61: Stress Versus Strain Curve for Tank 5F No Evaporation Deposition Sample at $45^{\circ} \mathrm{C}$ (First Replicate)

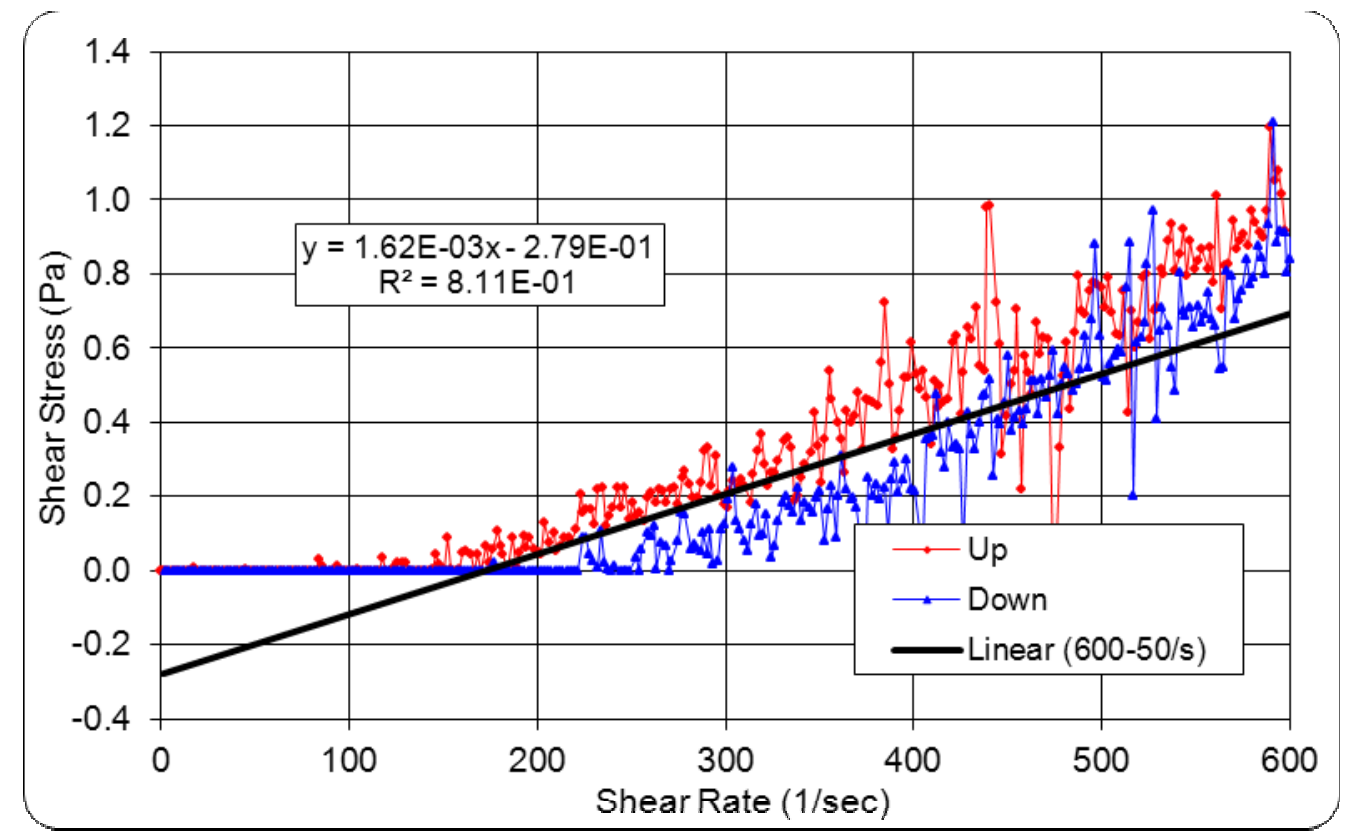

Figure B-62: Stress Versus Strain Curve for Tank 5F No Evaporation Deposition Sample at $45^{\circ} \mathrm{C}$ (Second Replicate) 


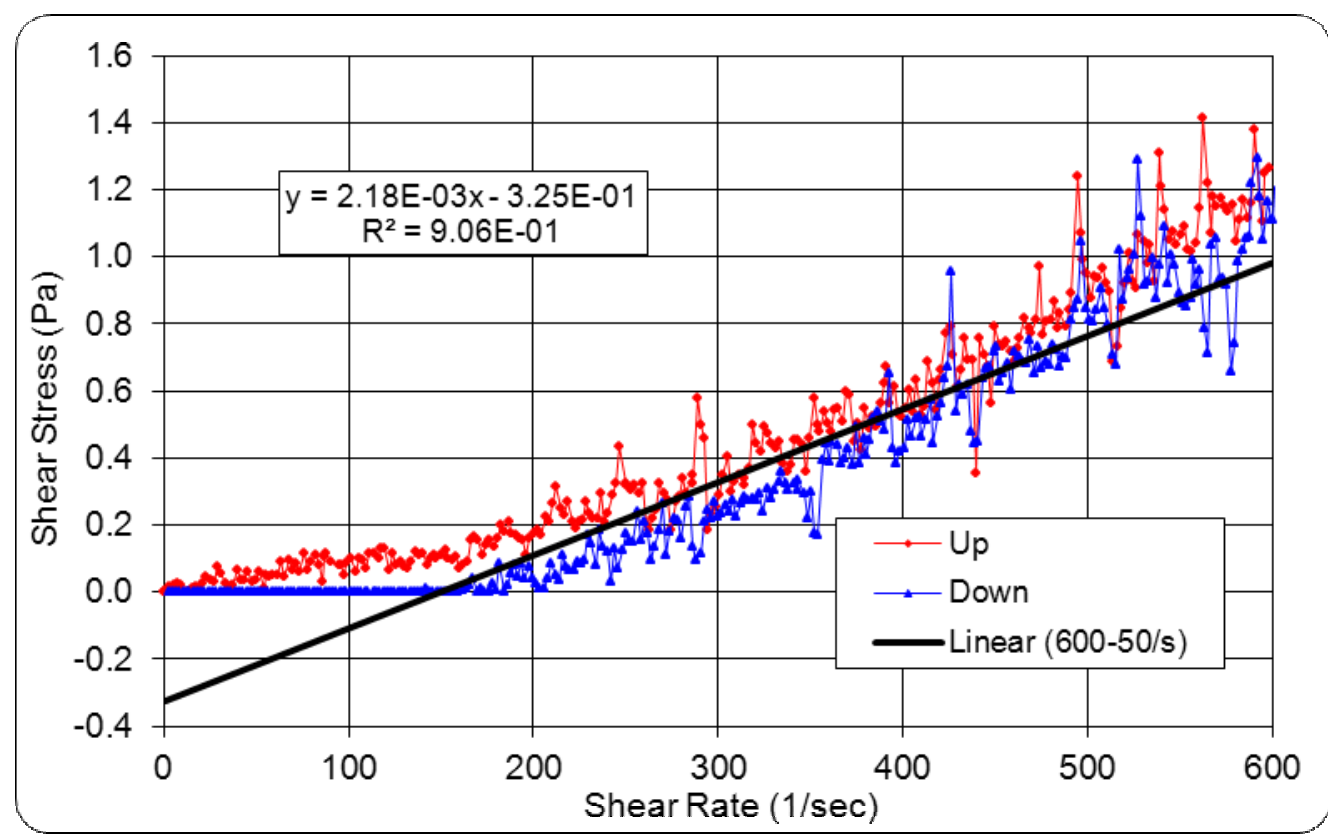

Figure B-63: Stress Versus Strain Curve for Tank 5F Evaporated Deposition Sample at 30 ${ }^{\circ} \mathrm{C}$ (First Replicate)

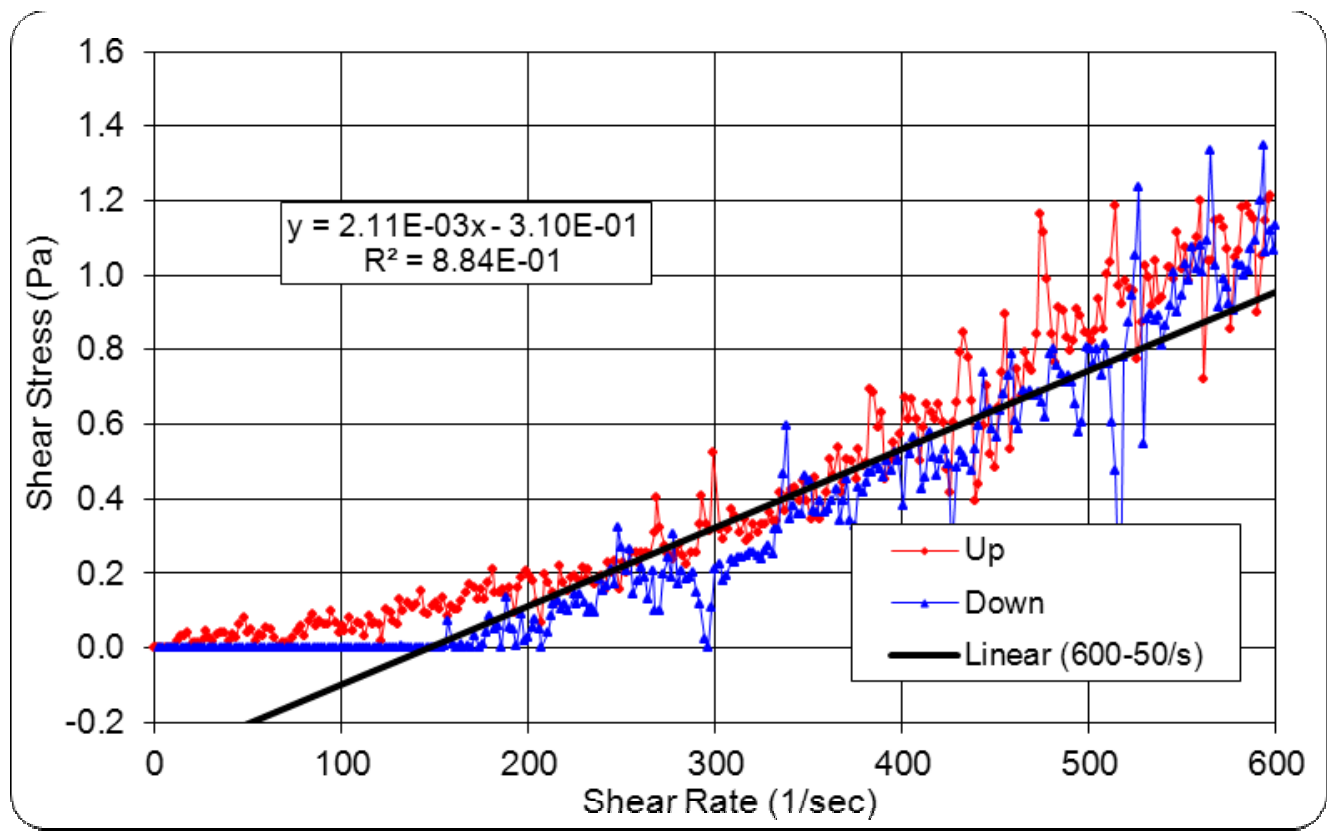

Figure B-64: Stress Versus Strain Curve for Tank 5F Evaporated Deposition Sample at 30 ${ }^{\circ} \mathrm{C}$ (Second Replicate) 


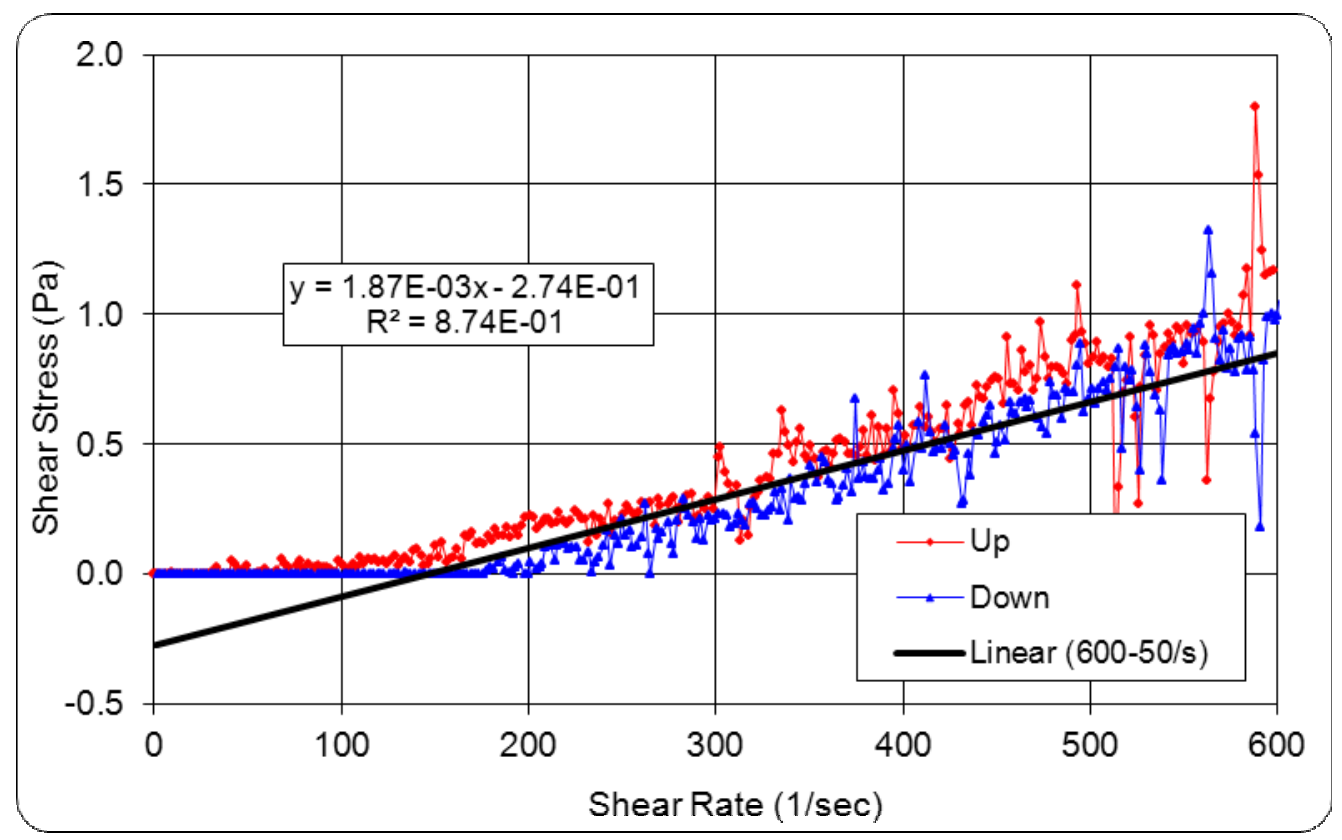

Figure B-65: Stress Versus Strain Curve for Tank 5F Evaporated Deposition Sample at 45 ${ }^{\circ} \mathrm{C}$ (First Replicate)

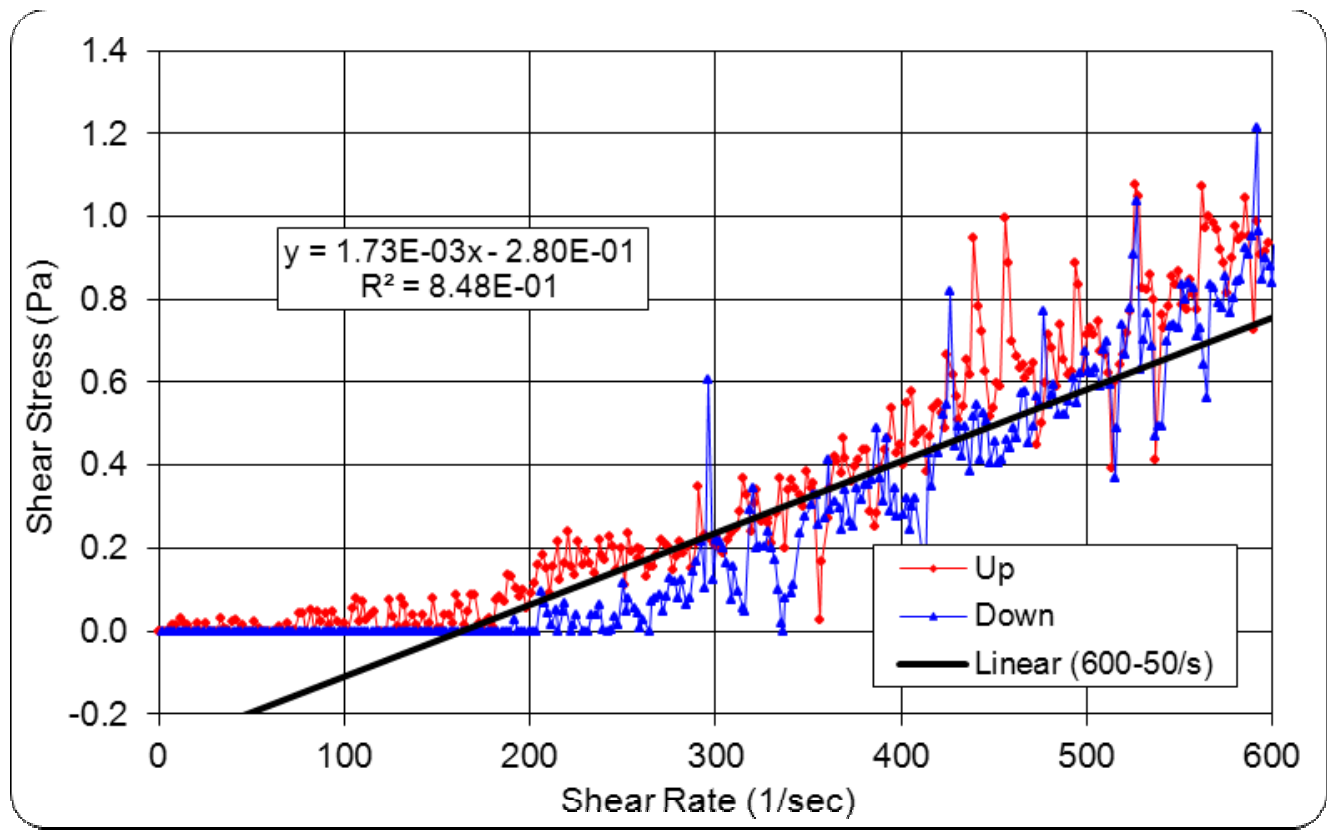

Figure B-66: Stress Versus Strain Curve for Tank 5F Evaporated Deposition Sample at 45 ${ }^{\circ} \mathrm{C}$ (Second Replicate) 


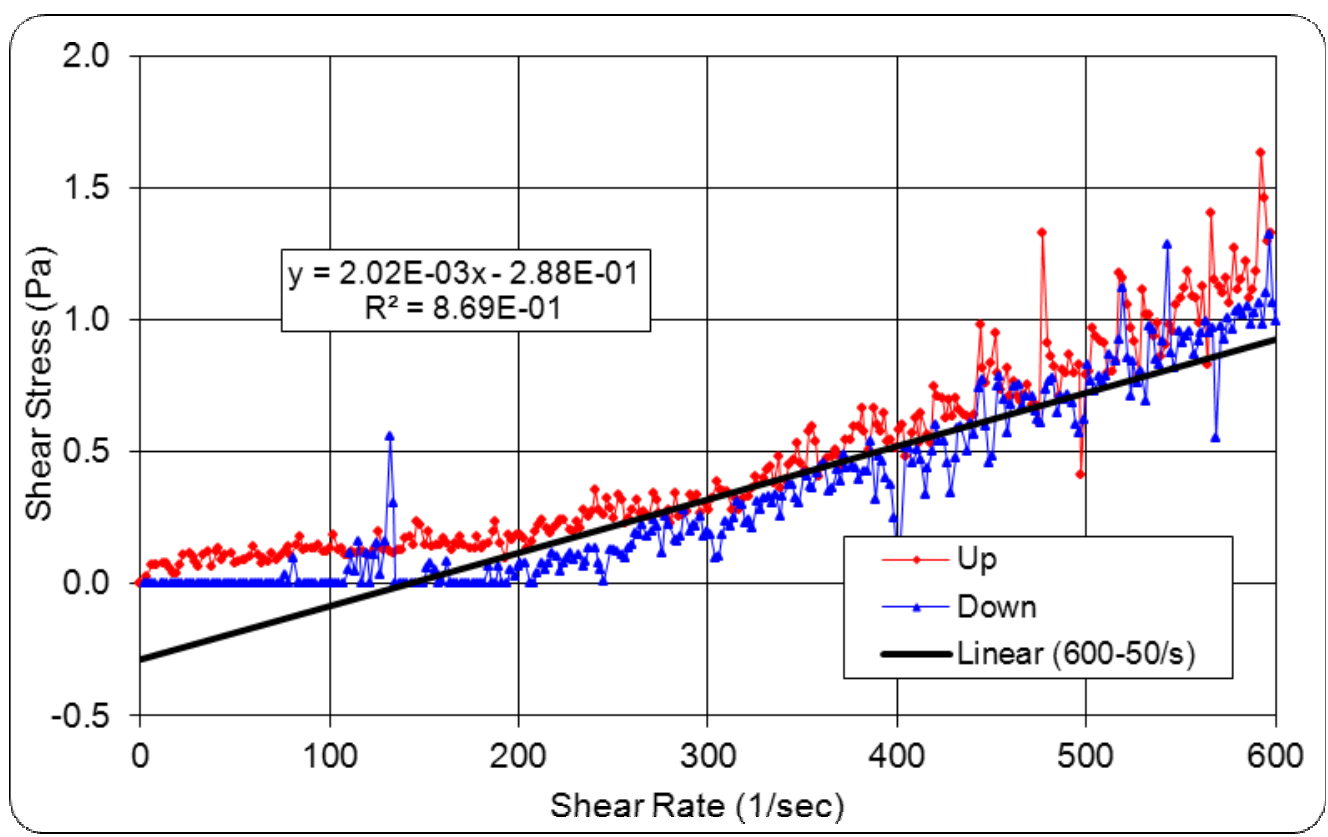

Figure B-67: Stress Versus Strain Curve for Tank 12H No Evaporation Deposition Sample at $30{ }^{\circ} \mathrm{C}$ (First Replicate)

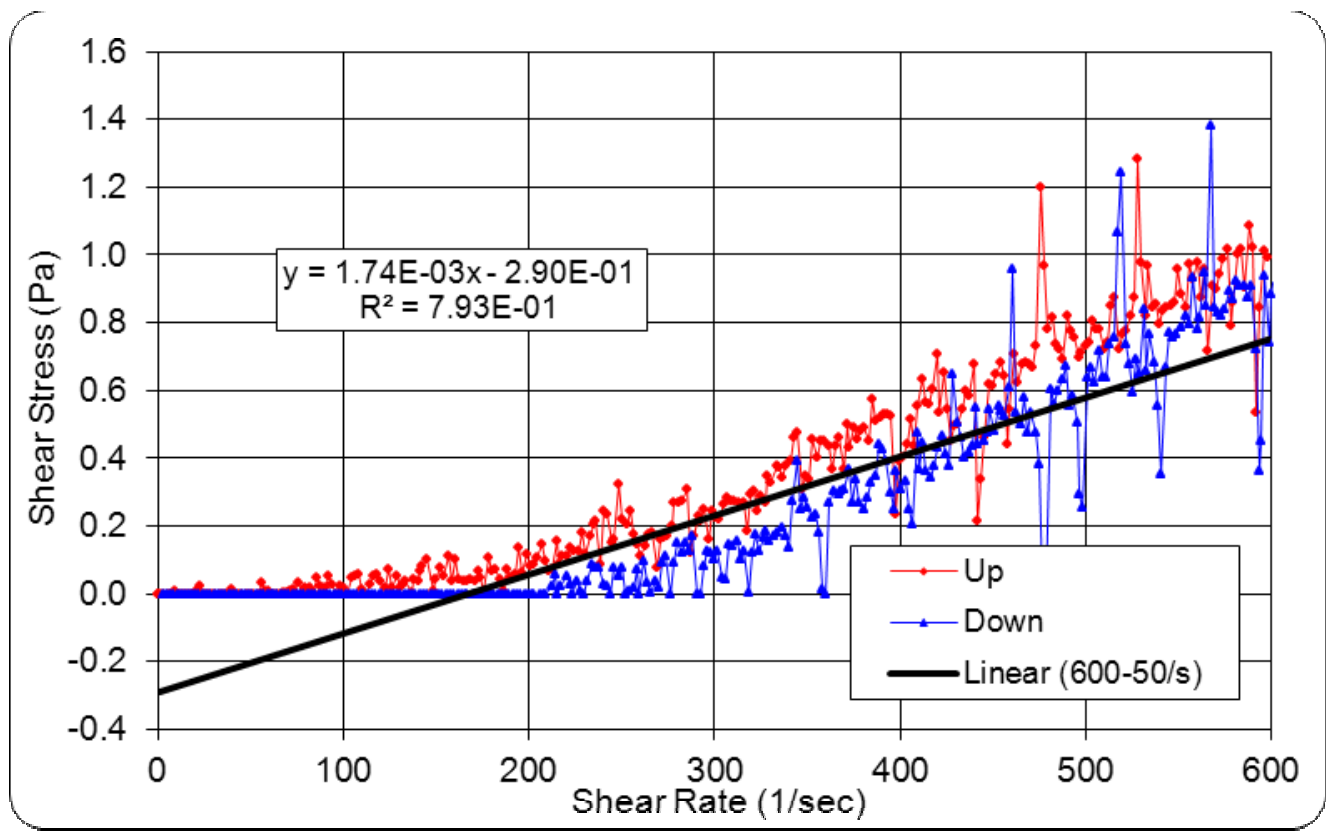

Figure B-68: Stress Versus Strain Curve for Tank 12H No Evaporation Deposition Sample at $30{ }^{\circ} \mathrm{C}$ (Second Replicate) 


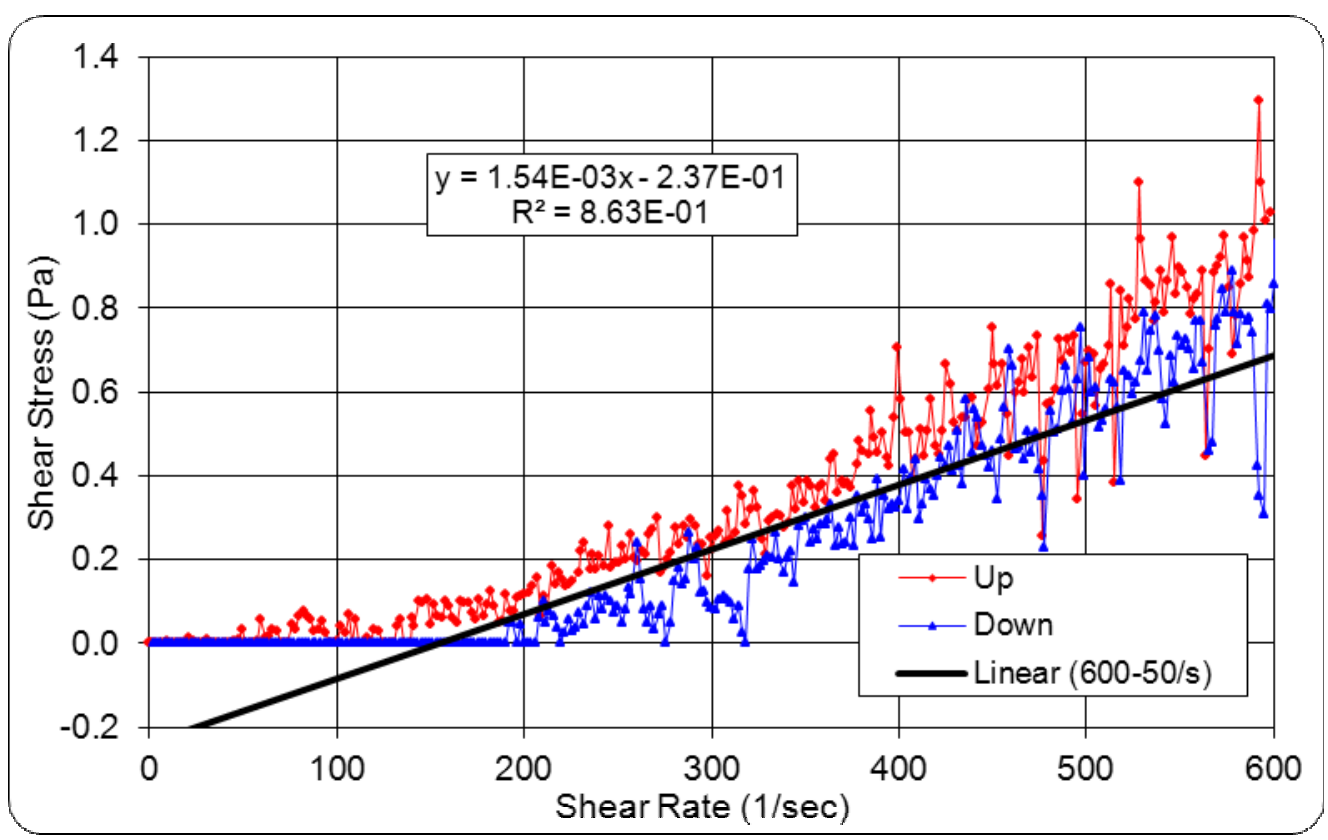

Figure B-69: Stress Versus Strain Curve for Tank 12H No Evaporation Deposition Sample at $45{ }^{\circ} \mathrm{C}$ (First Replicate)

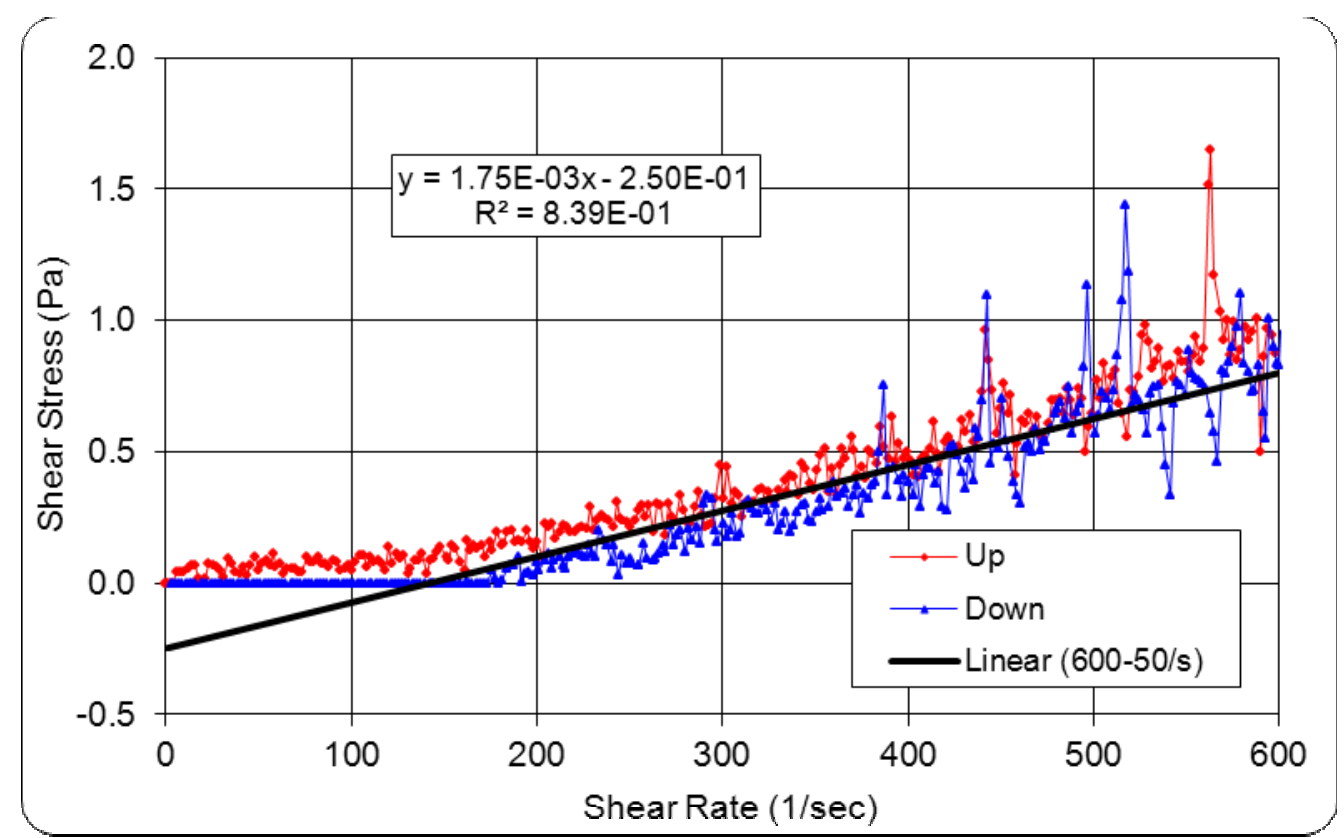

Figure B-70: Stress Versus Strain Curve for Tank 12H No Evaporation Deposition Sample at $45^{\circ} \mathrm{C}$ (Second Replicate) 


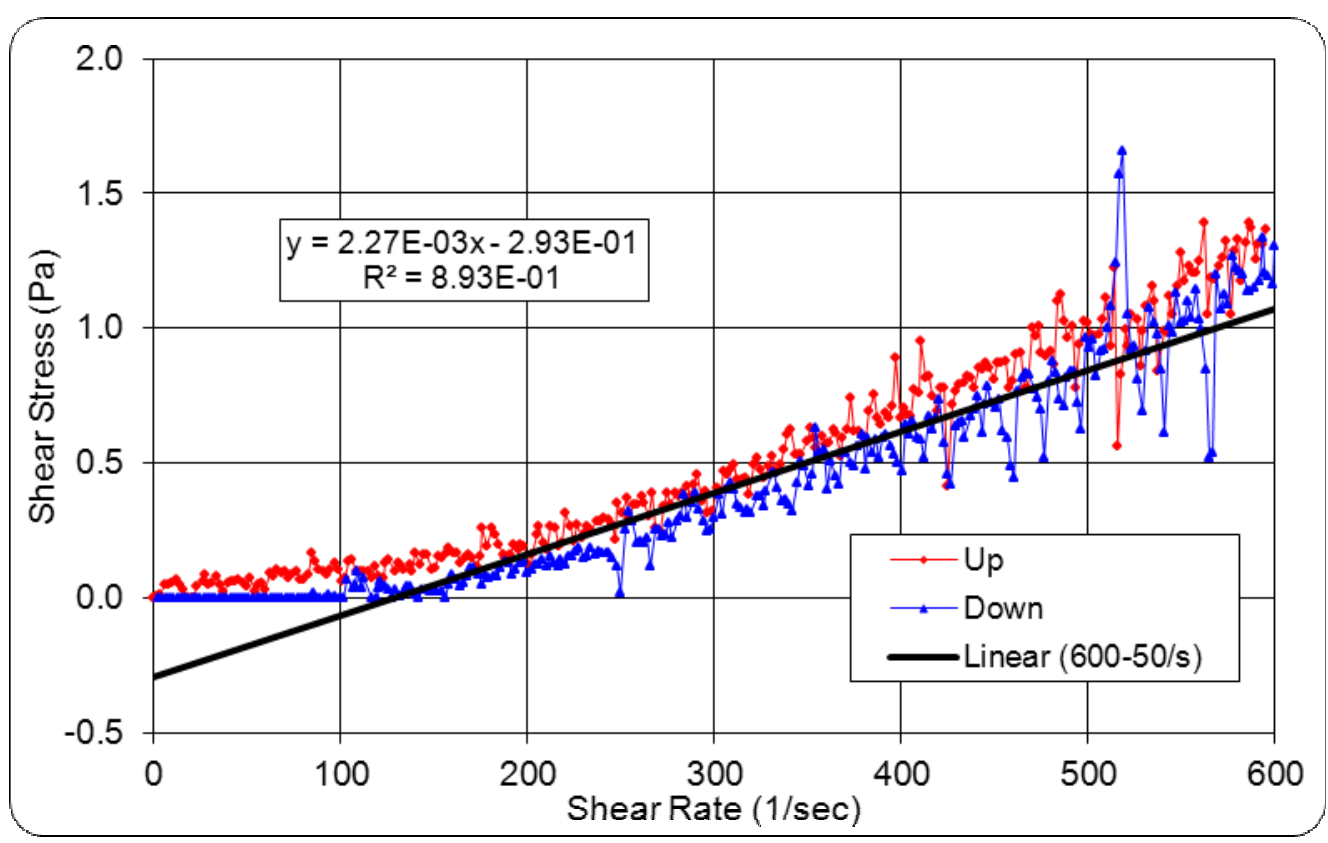

Figure B-71: Stress Versus Strain Curve for Tank 12H Evaporated Deposition Sample at $30{ }^{\circ} \mathrm{C}$ (First Replicate)

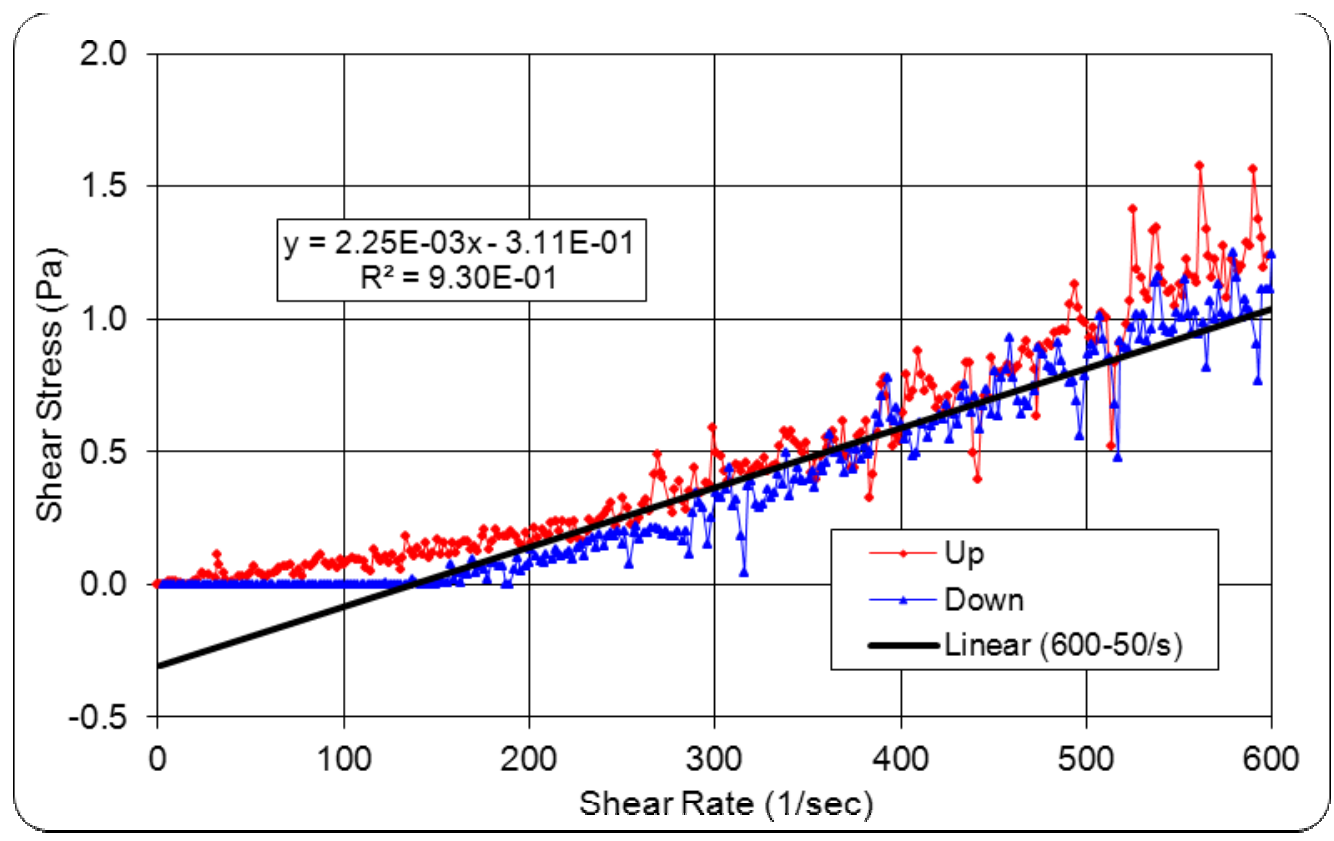

Figure B-72: Stress Versus Strain Curve for Tank 12H Evaporated Deposition Sample at $30{ }^{\circ} \mathrm{C}$ (Second Replicate) 


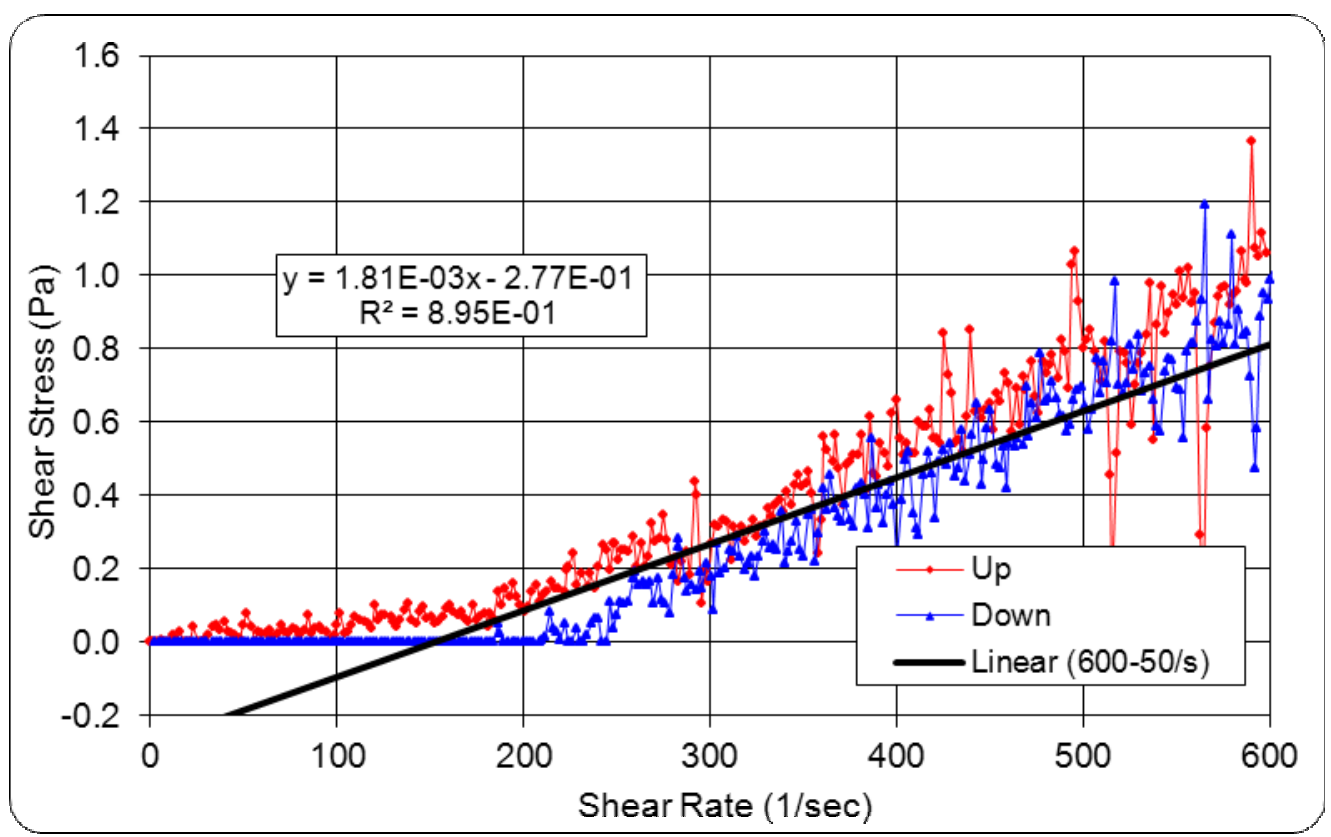

Figure B-73: Stress Versus Strain Curve for Tank 12H Evaporated Deposition Sample at $45^{\circ} \mathrm{C}$ (First Replicate)

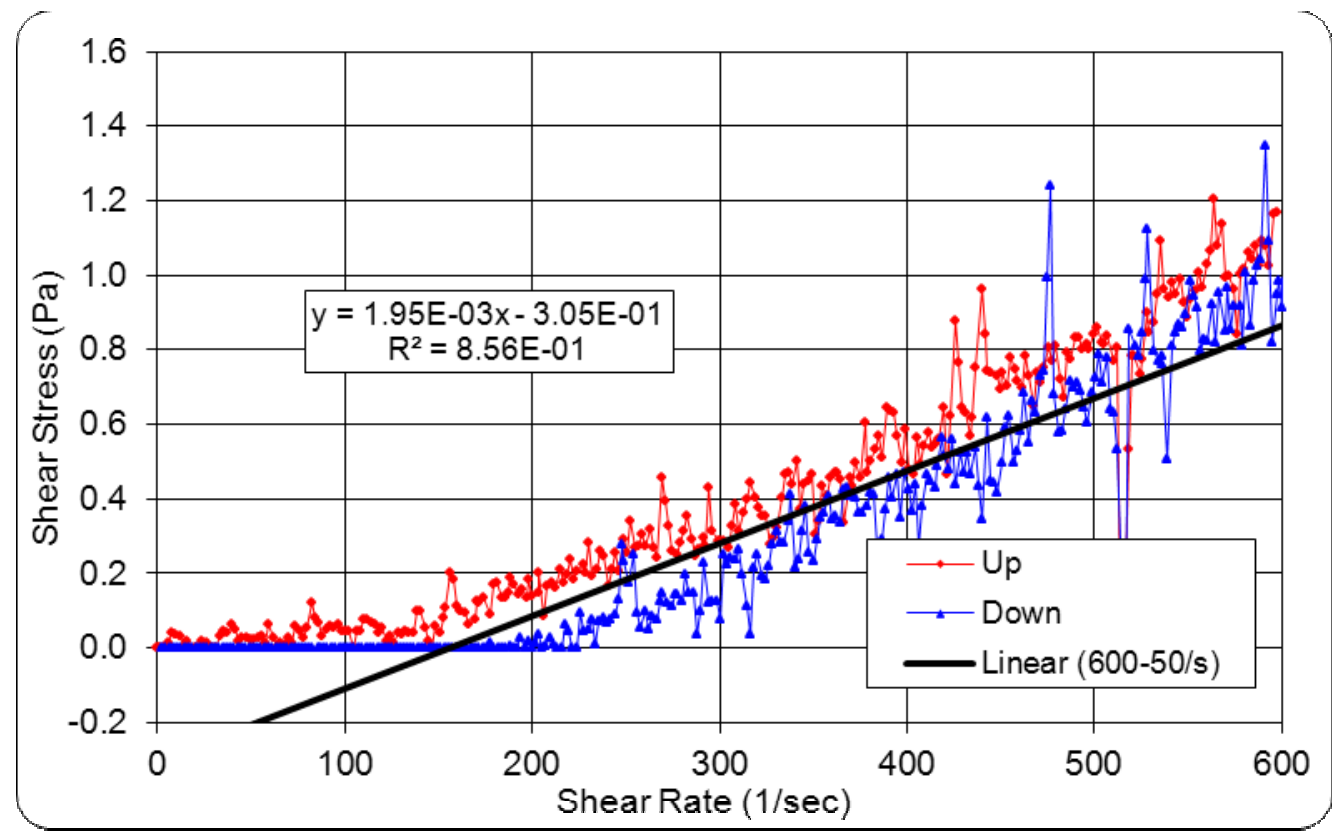

Figure B-74: Stress Versus Strain Curve for Tank 12H Evaporated Deposition Sample at $45^{\circ} \mathrm{C}$ (Second Replicate) 
Figure B-75: Particle Size Analysis for Tank 5F Sludge Prior to Contact with OA

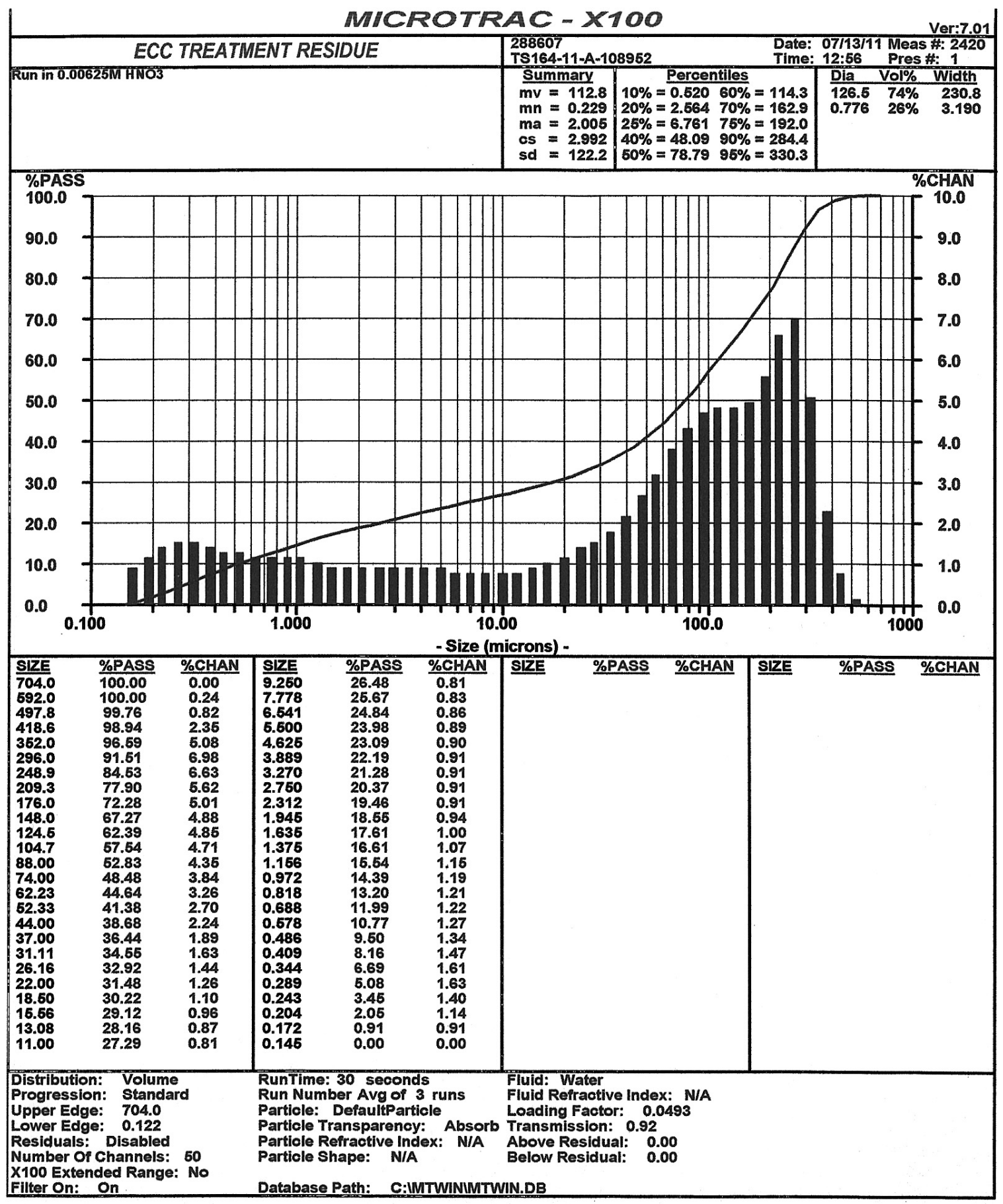


Figure B-76: Particle Size Analysis for Tank 12H Sludge Prior to Contact with OA

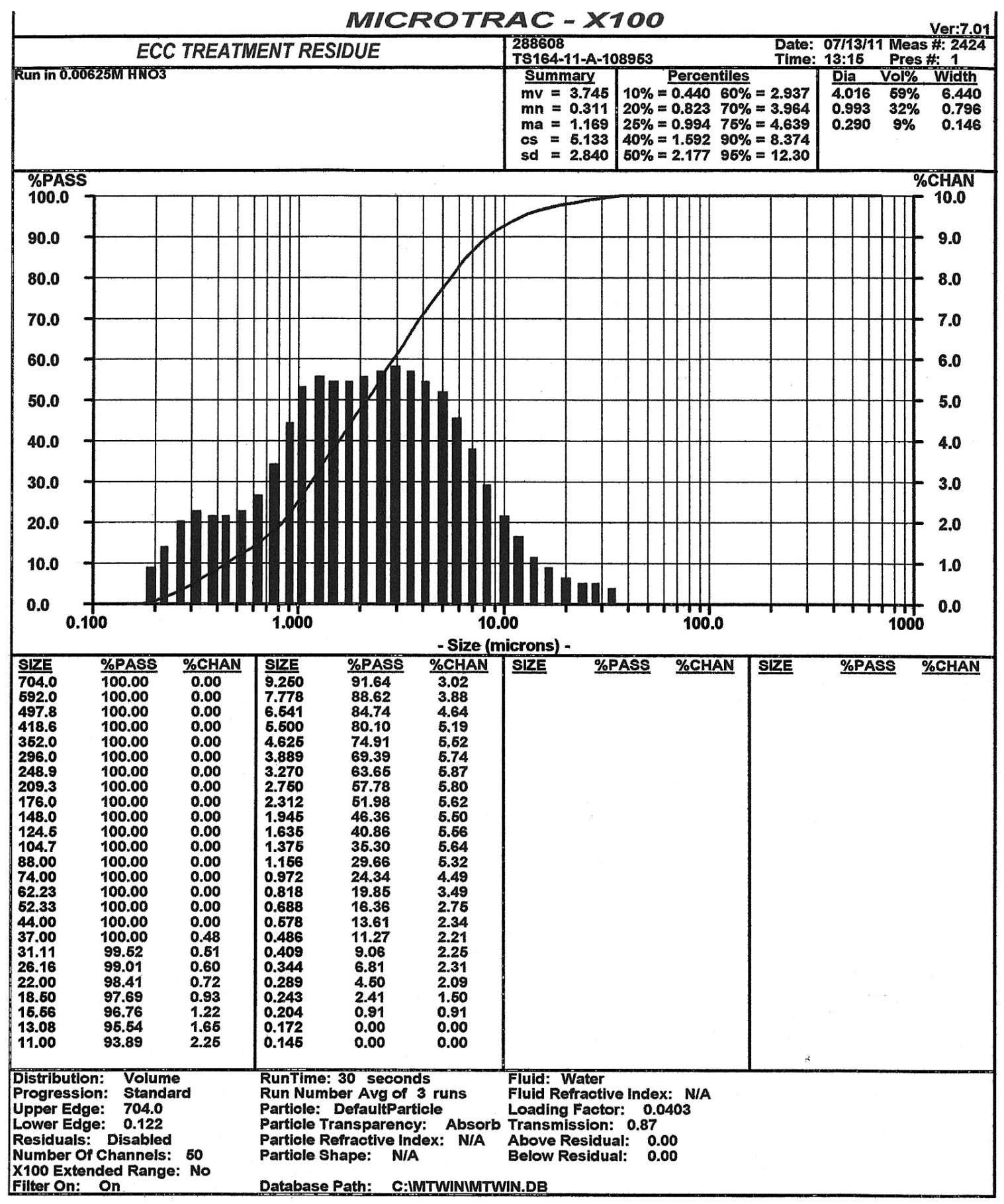


Figure B-77: Particle Size Analysis of Intermediate Treatment Tank Heel after Dissolution of Tank 5F Sludge with One OA Batch

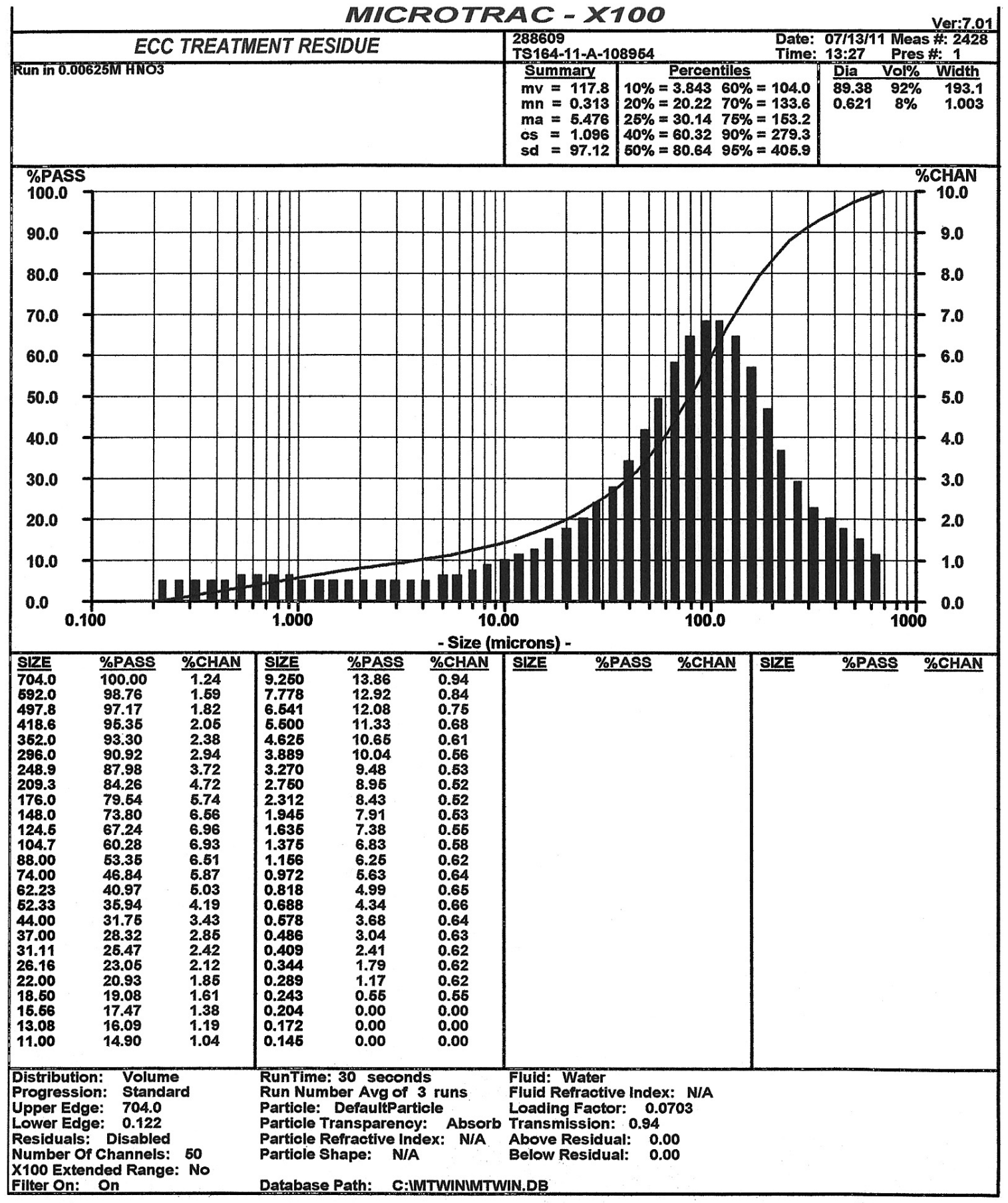


Figure B-78: Particle Size Analysis of Intermediate Treatment Tank Heel after Dissolution of Tank 12H Sludge with One OA Batch

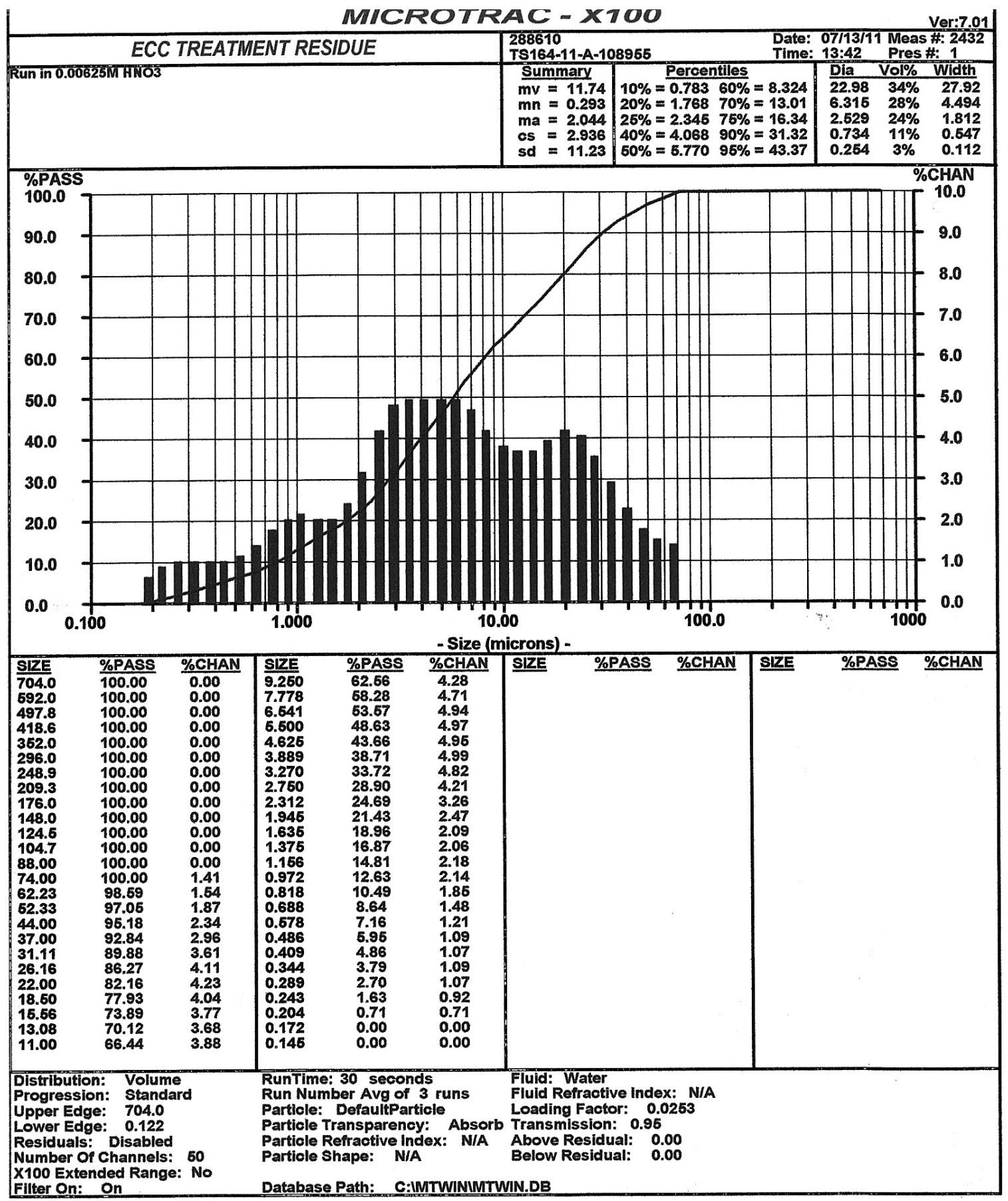


Figure B-79: Particle Size Analysis of Final Treatment Tank Heel after Dissolution of Tank 5F Sludge with Two OA Batches

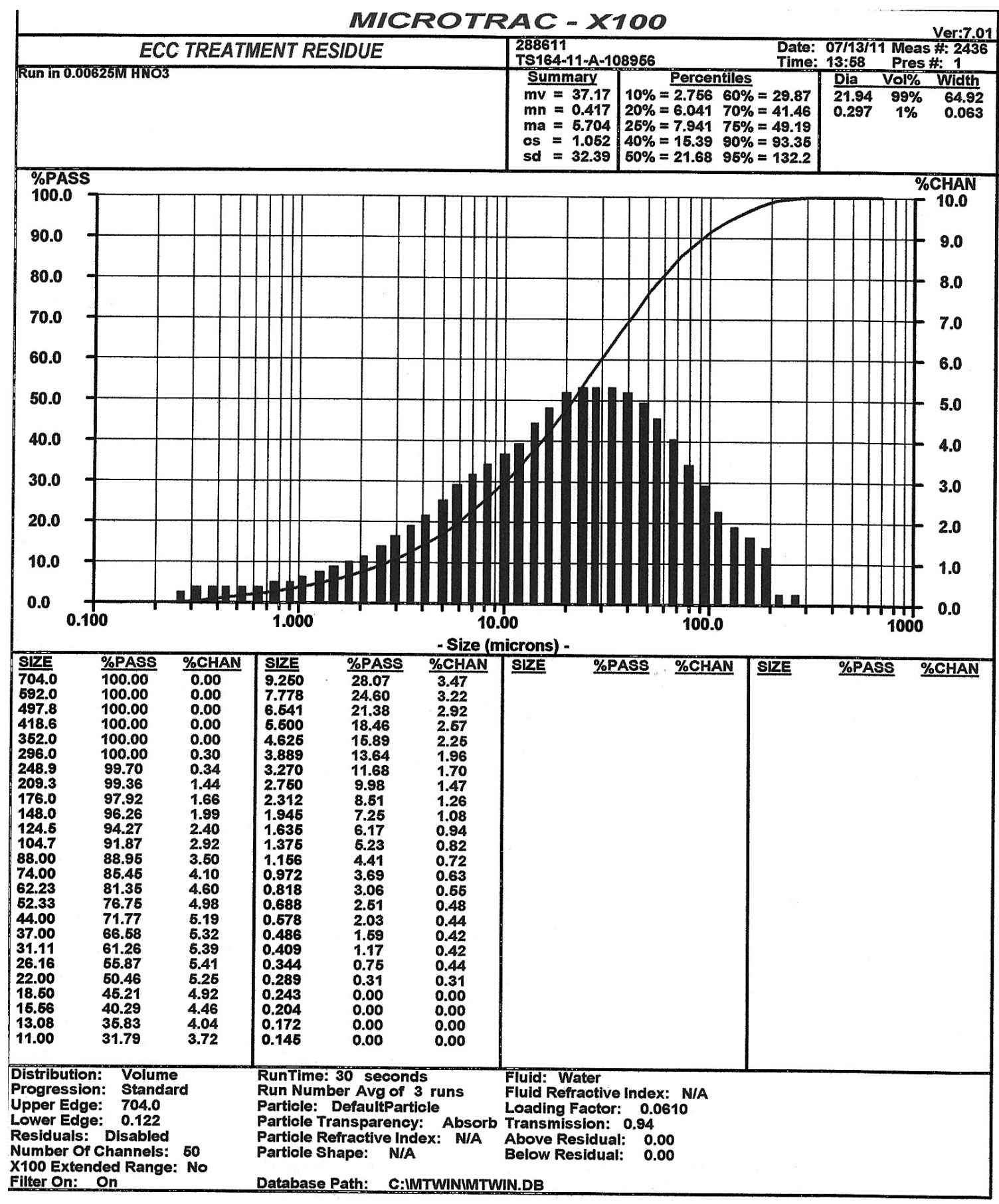


Figure B-80: Particle Size Analysis of Final Treatment Tank Heel after Dissolution of Tank 12H Sludge with Two OA Batches

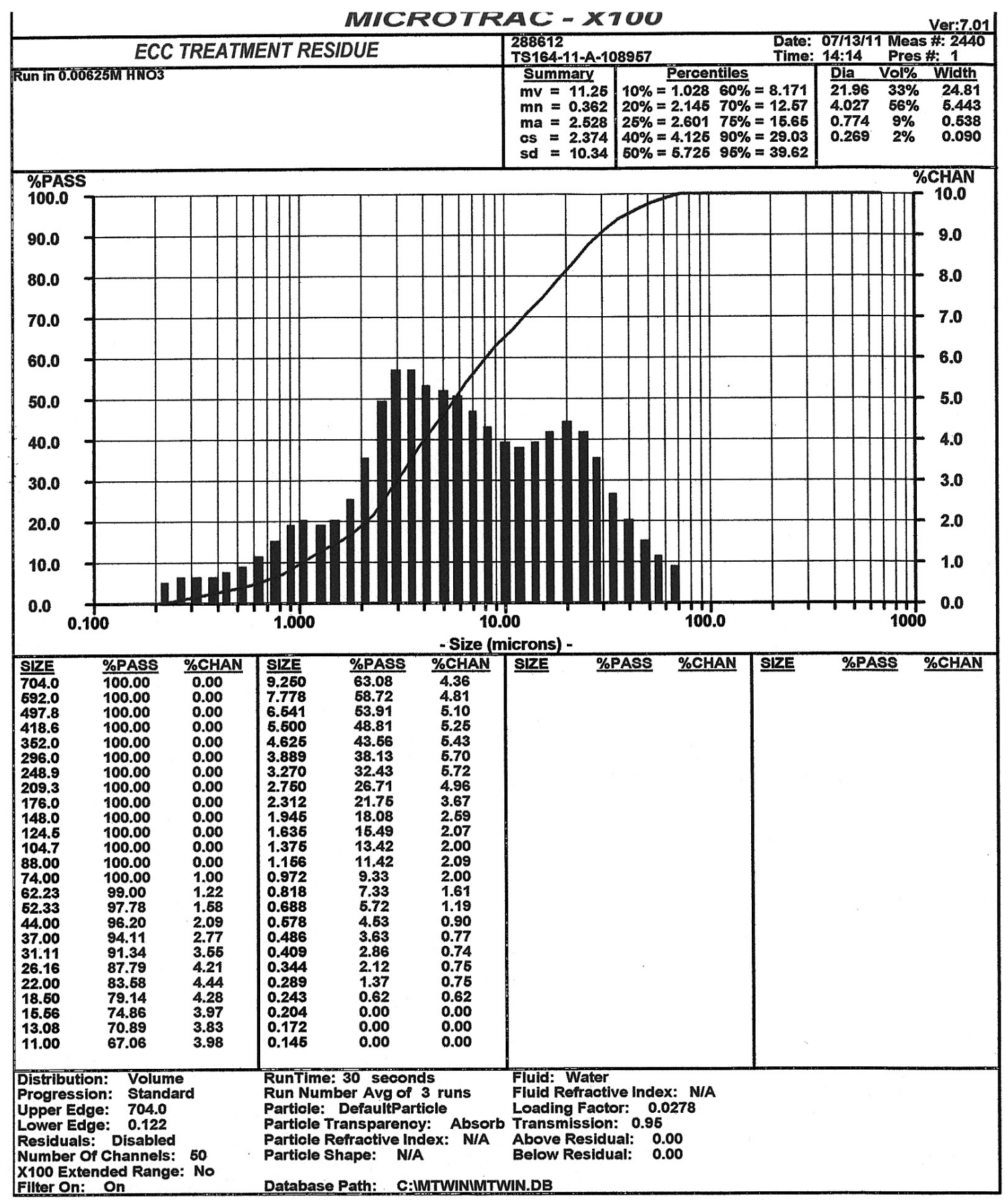


Figure B-81: Particle Size Analysis of Test 1 (Tank 12H) Storage Condition 3 (without evaporation, without $\mathrm{pH}$ adjustment, and without sludge heel), representing ECC product prior to $\mathrm{pH}$ adjustment and evaporation.

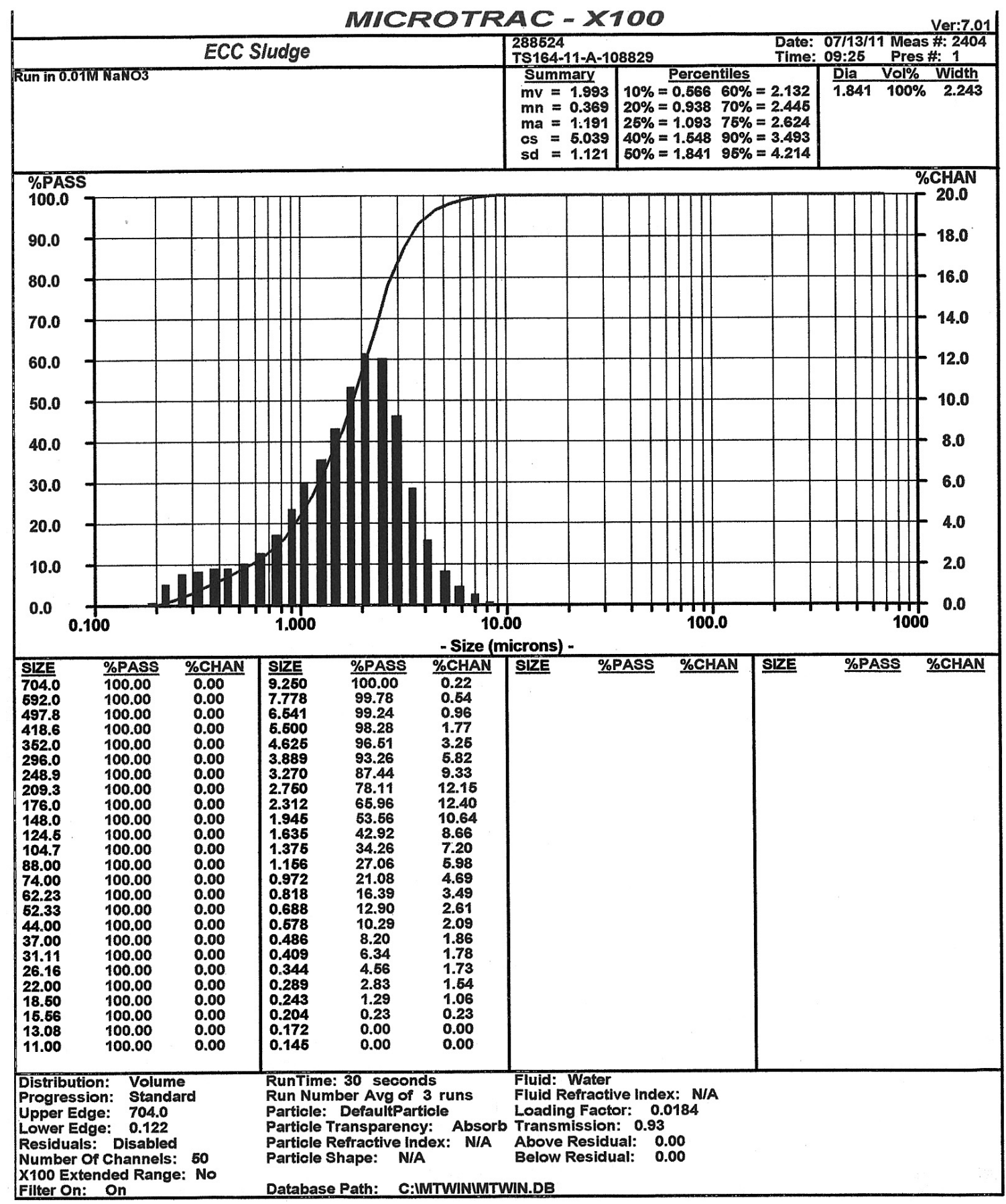


Figure B-82: Particle Size Analysis of Test 1 (Tank 12H) Storage Condition 5 (with evaporation, with pH adjustment, and with sludge heel), representing ECC product after evaporation ( $\mathrm{H}$-area baseline) and at storage tank conditions.

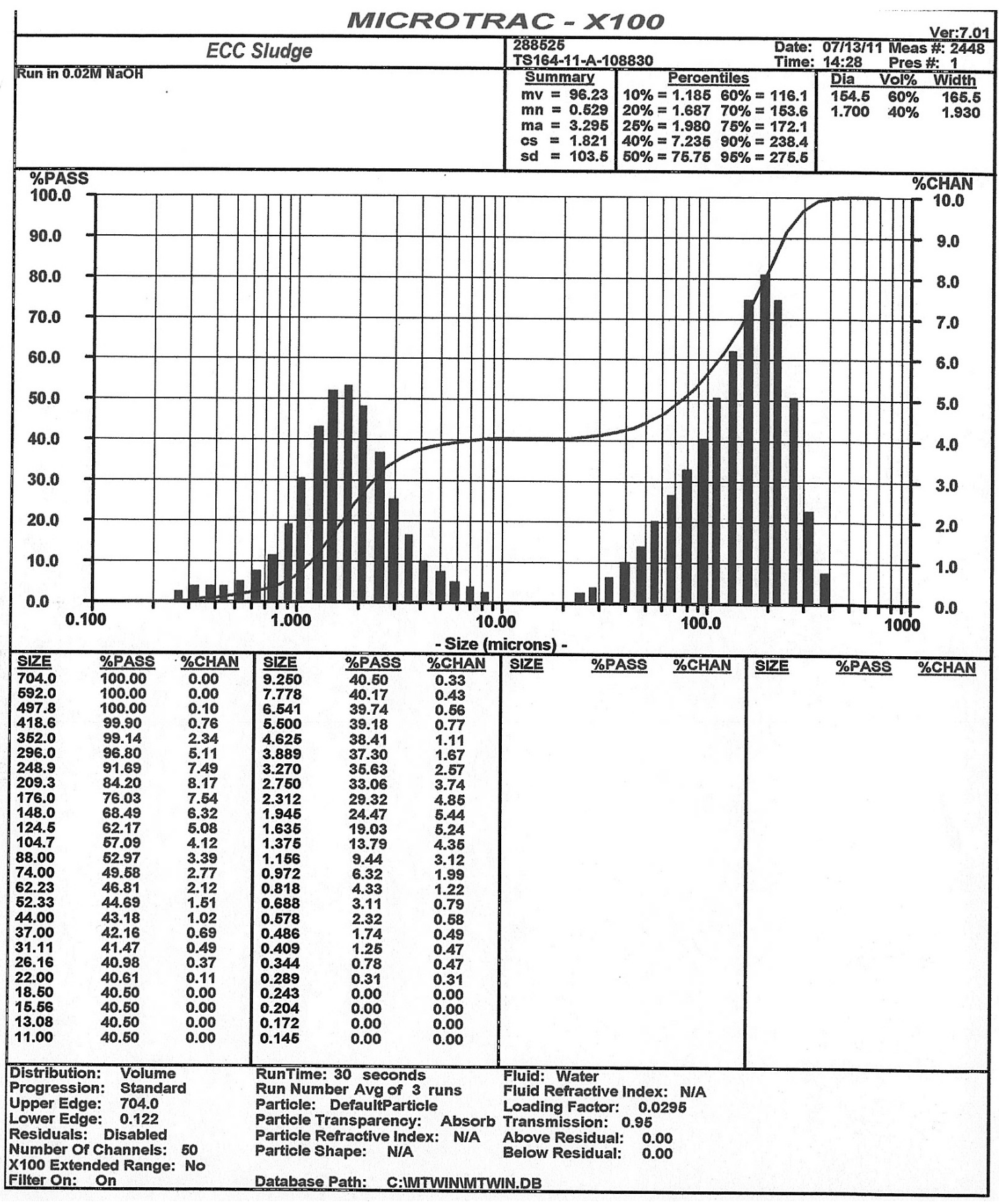


Figure B-83: Particle Size Analysis of Test 1 (Tank 12H) Storage Condition 6 (with evaporation, without $\mathrm{pH}$ adjustment, and without sludge heel), representing ECC product just after evaporation and prior to $\mathrm{pH}$ adjustment.

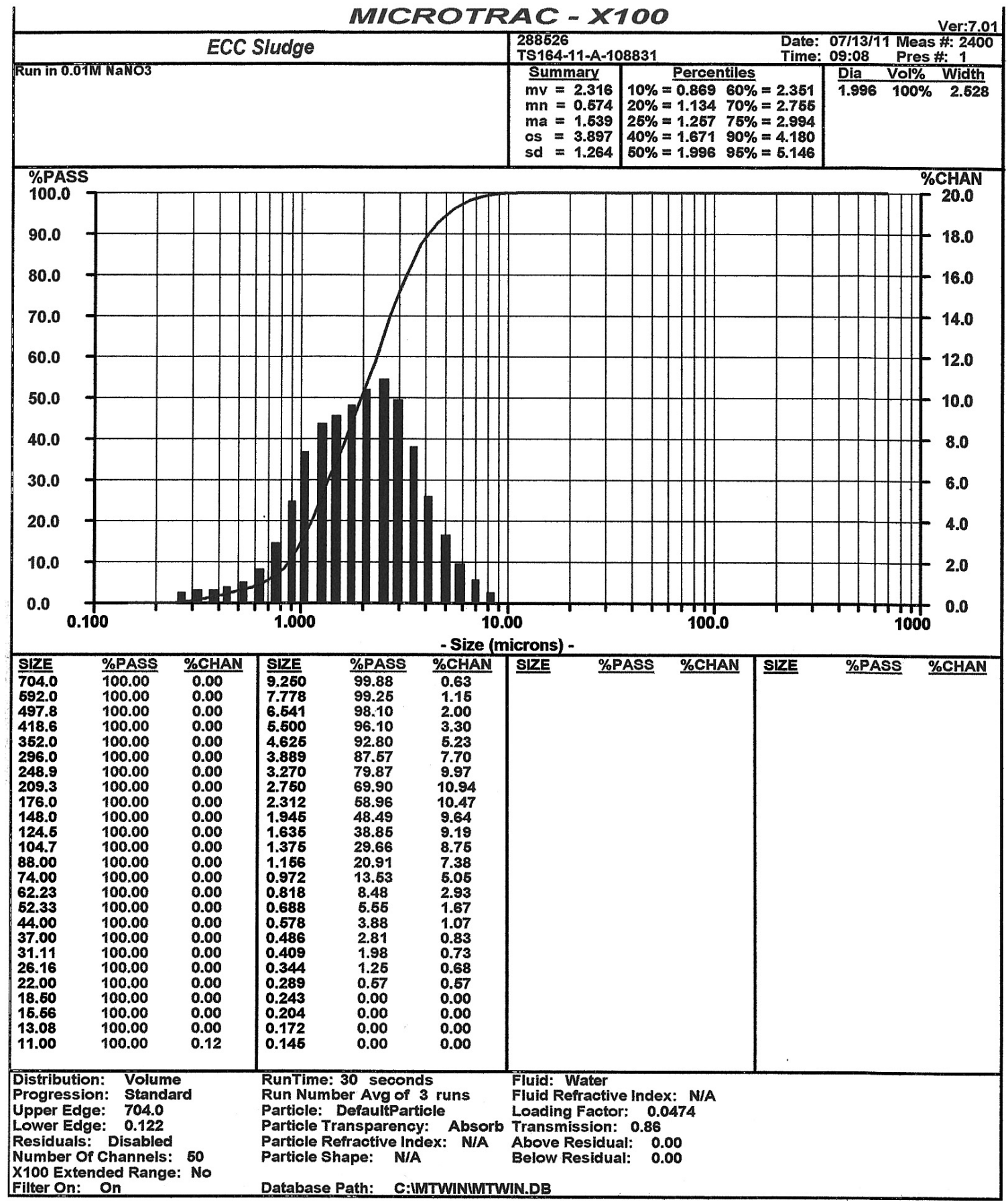


Figure B-84: Particle Size Analysis of Test 1 (Tank 12H) Control (Storage Condition 9) (without evaporation, with $\mathrm{pH}$ adjustment, and with sludge heel), representing the original Tank 12H sludge with pH adjustment and heel in deposition tank.

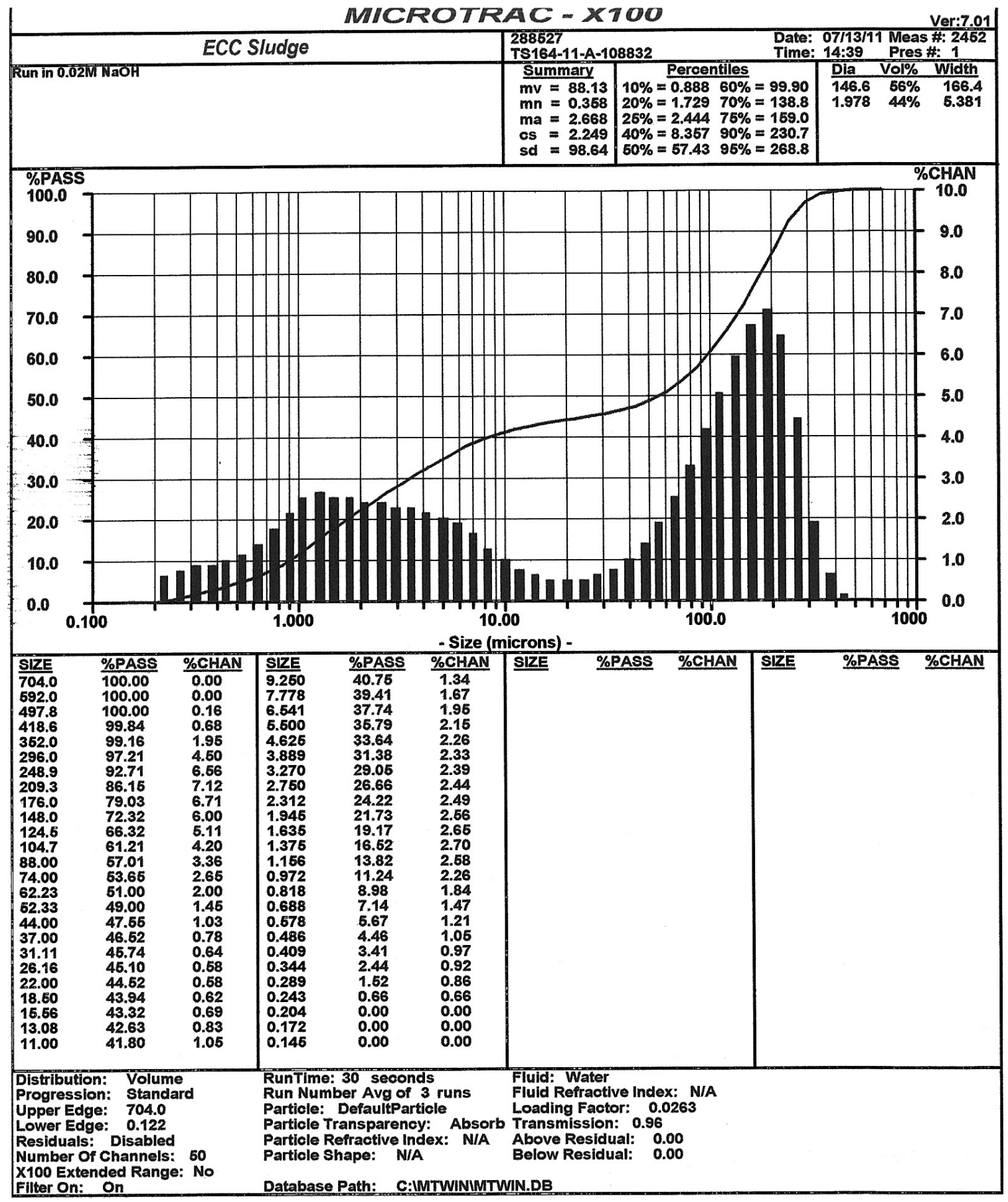


Figure B-85: Particle Size Analysis of Test 2 (Tank 5F) Storage Condition 2 (without evaporation, with pH adjustment, and with sludge heel), representing ECC product without evaporation (F-area baseline) and at storage tank conditions.

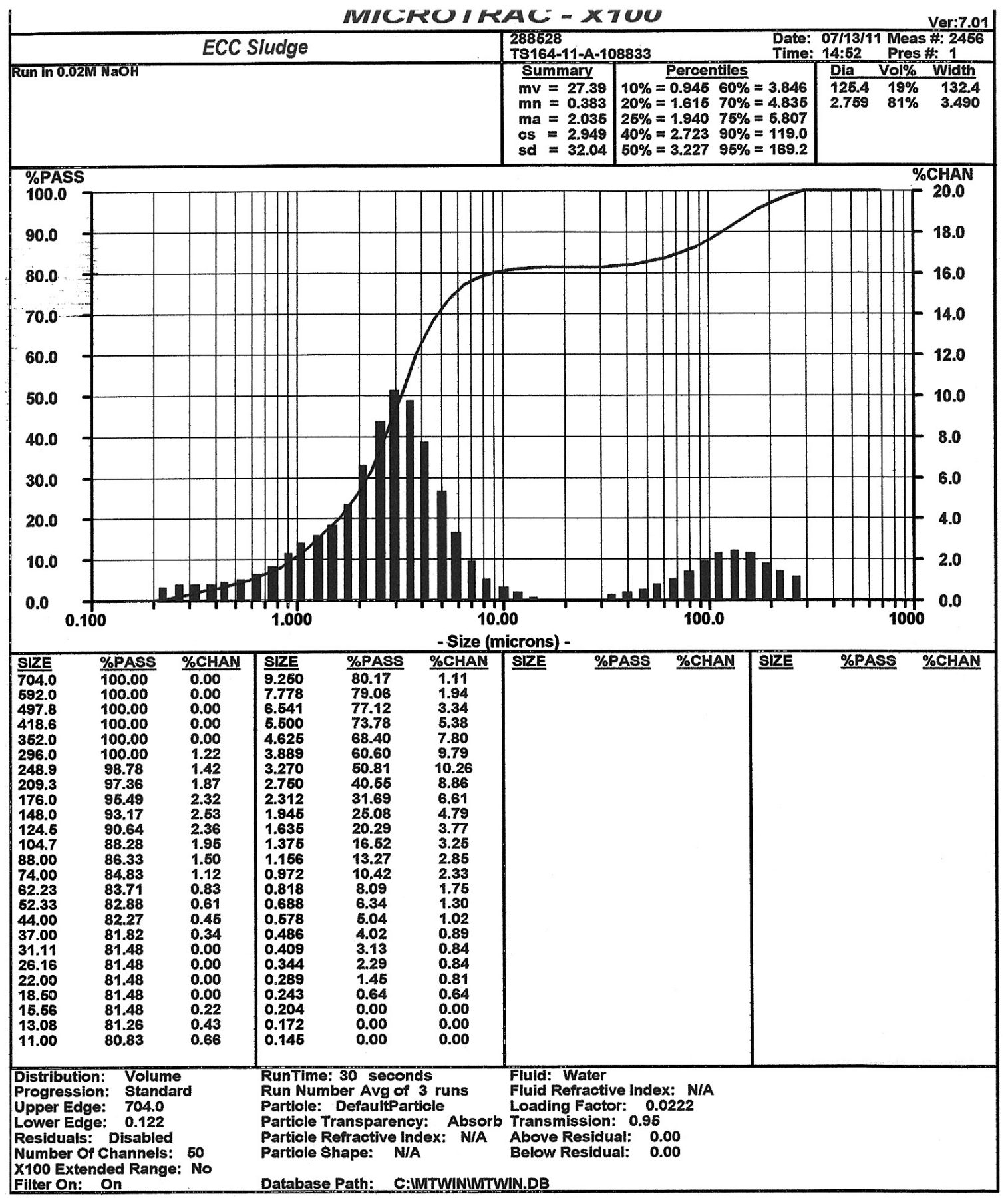


Figure B-86: Particle Size Analysis of Test 2 (Tank 5F) Storage Condition 3 (without evaporation, without $\mathrm{pH}$ adjustment, and without sludge heel), representing ECC product prior to $\mathrm{pH}$ adjustment and evaporation.

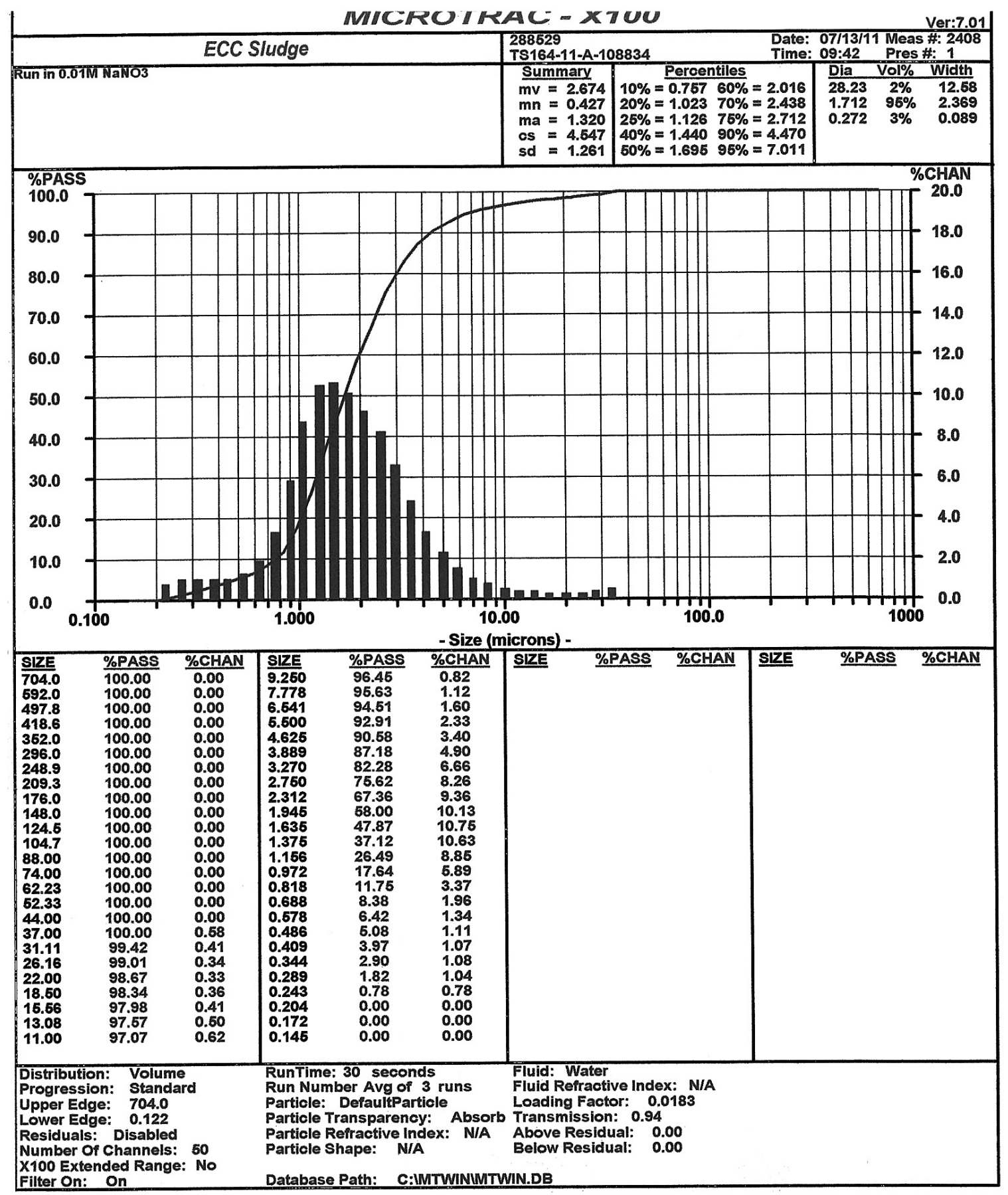


Figure B-87: Particle Size Analysis of Test 2 (Tank 5F) Storage Condition 6 (with evaporation, without $\mathrm{pH}$ adjustment, and without sludge heel), representing ECC product just after evaporation and prior to $\mathrm{pH}$ adjustment.

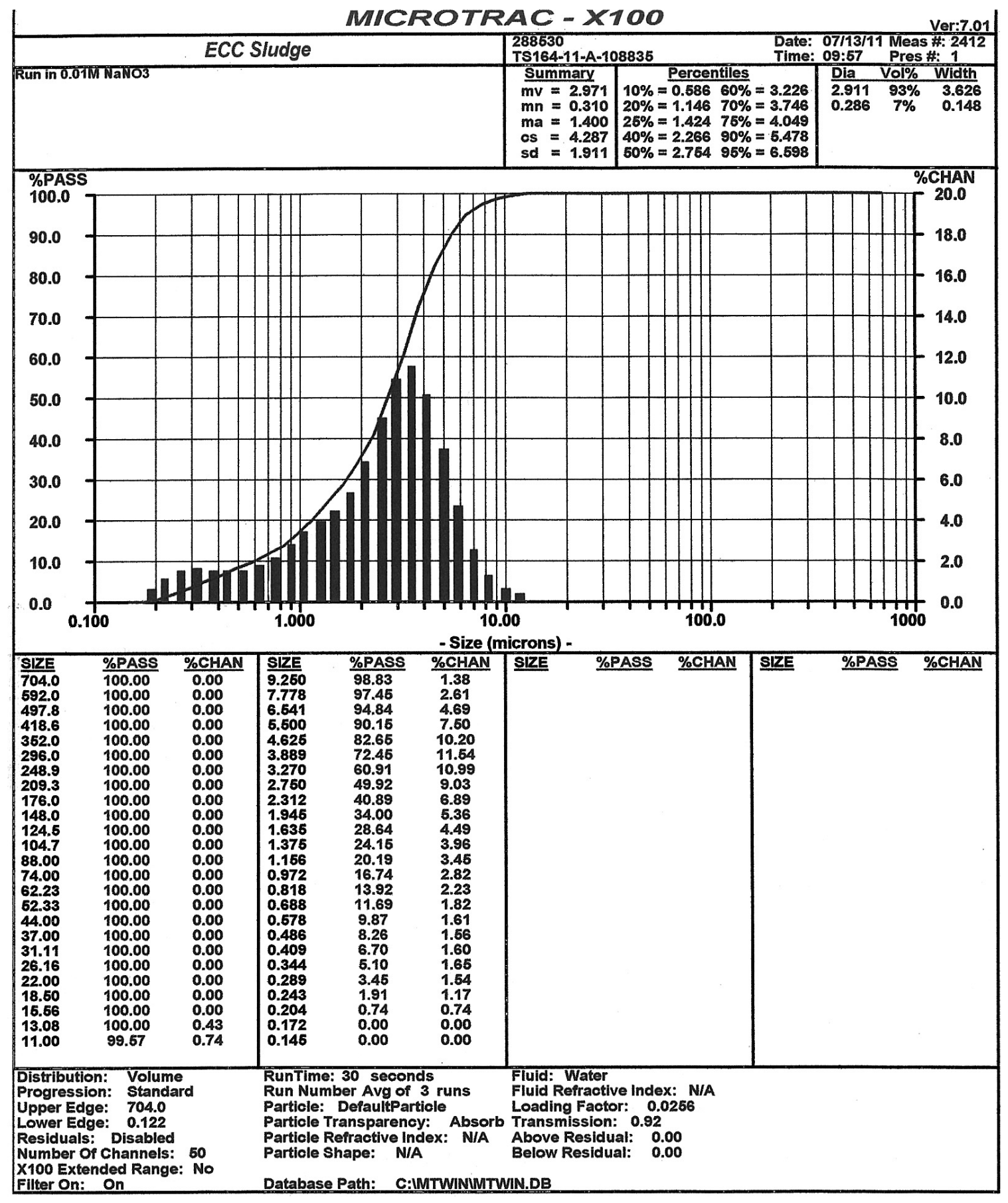


Figure B-88: Particle Size Analysis of Test 2 (Tank 5F) Control (Storage Condition 9) (without evaporation, with $\mathrm{pH}$ adjustment, and with sludge heel), representing the original Tank 12H sludge with pH adjustment and heel in deposition tank.

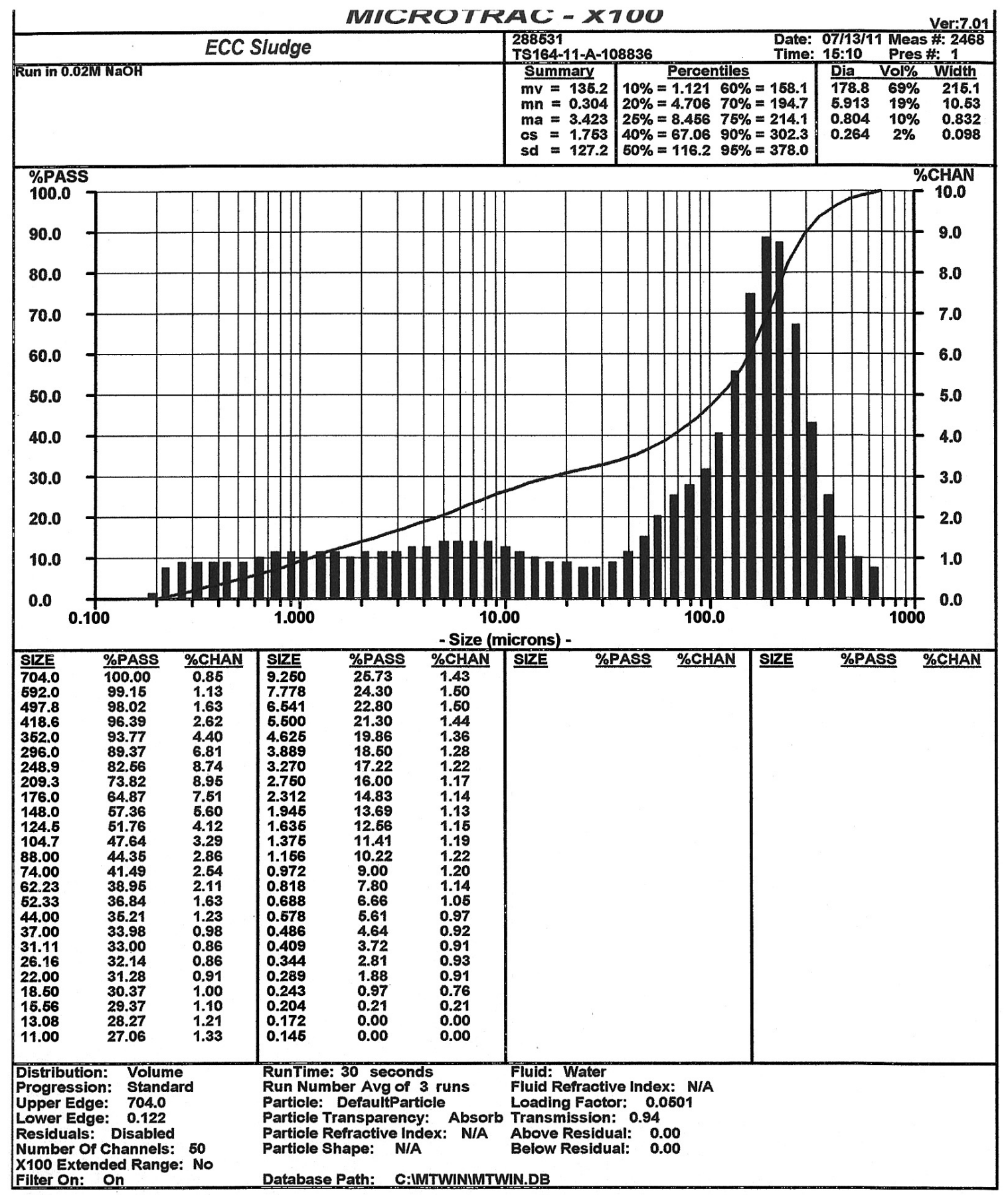



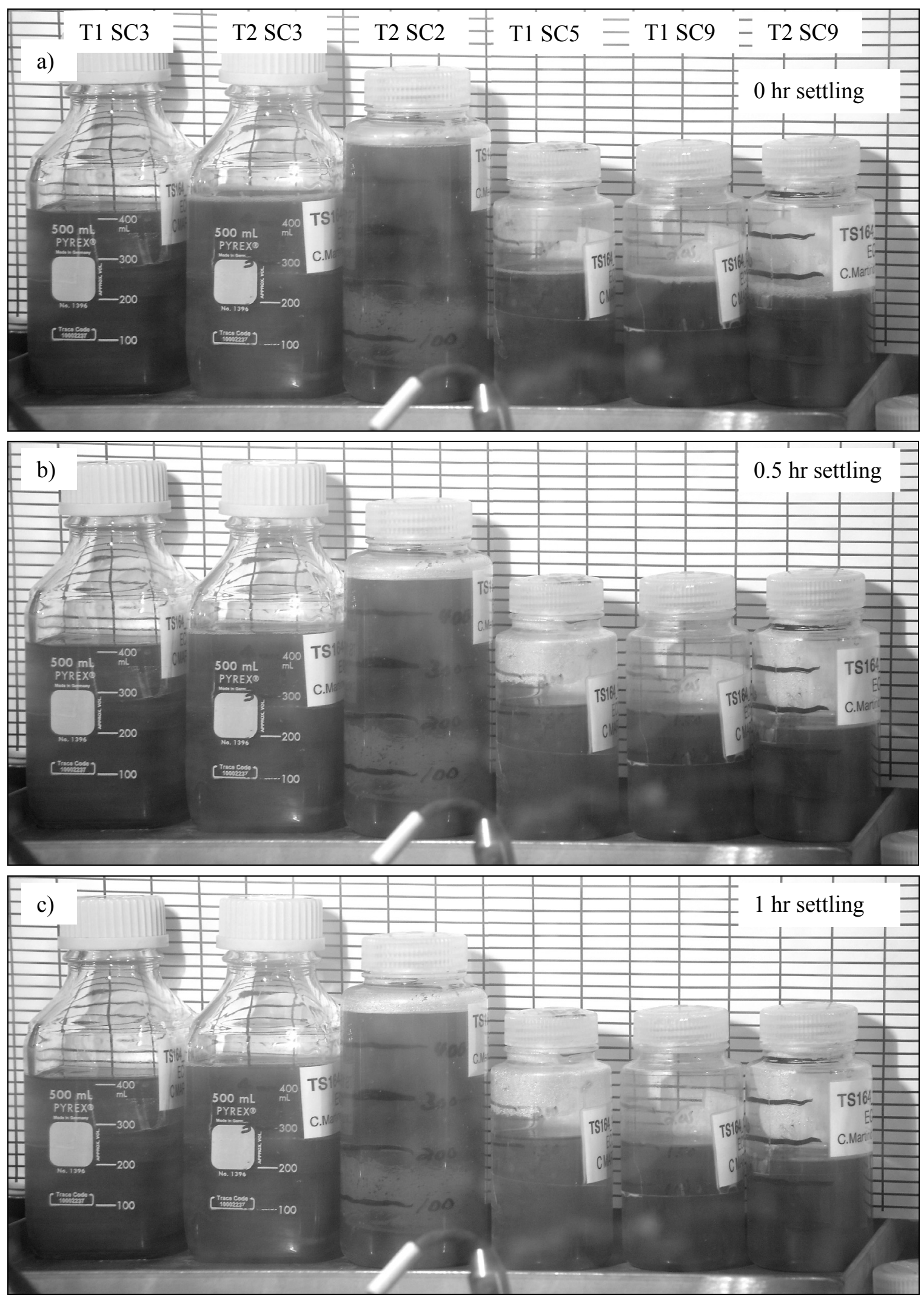

Figure B-89: ECC Product Settling (part 1 of 4)

B-53 

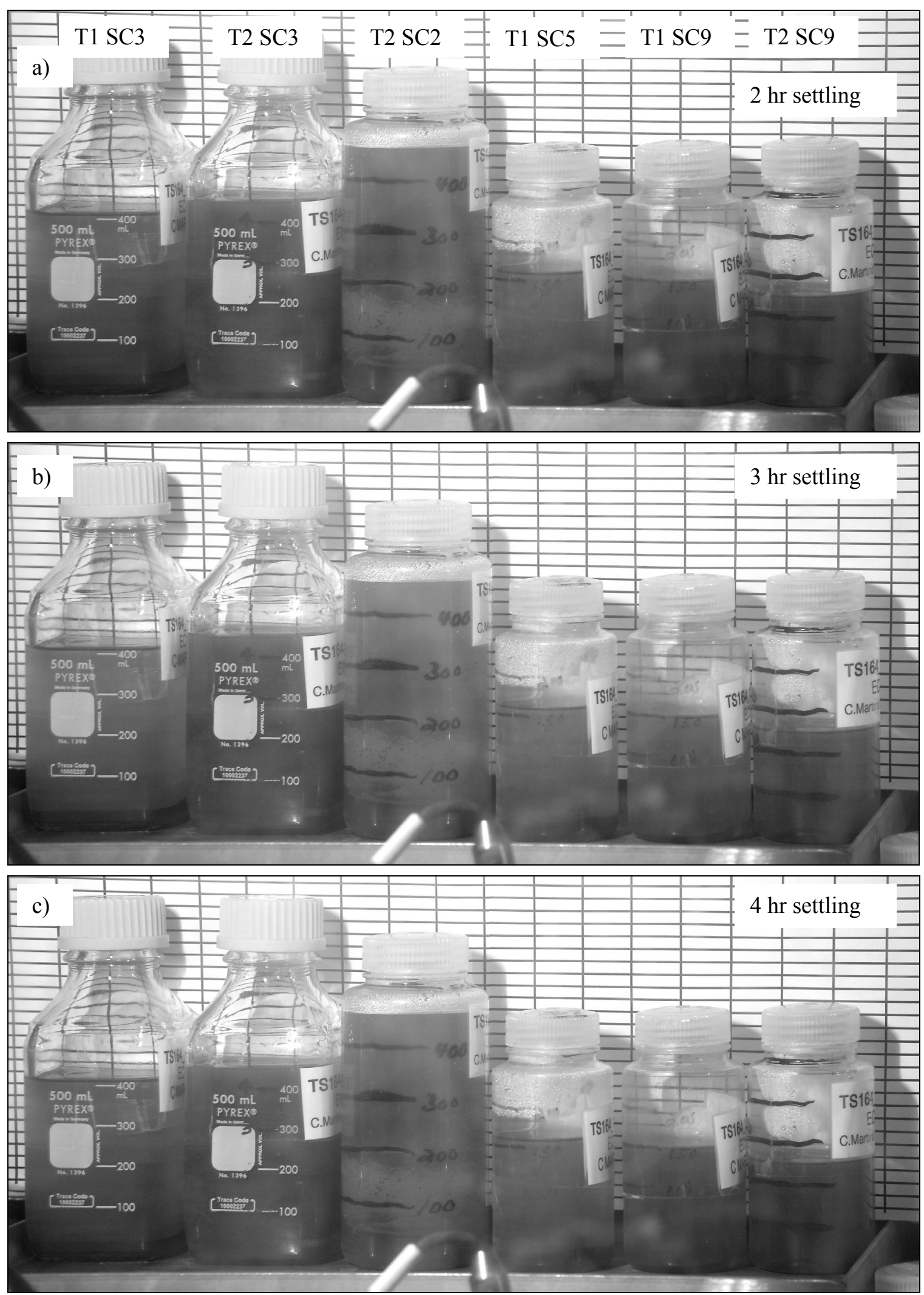

Figure B-90: ECC Product Settling (part 2 of 4) 

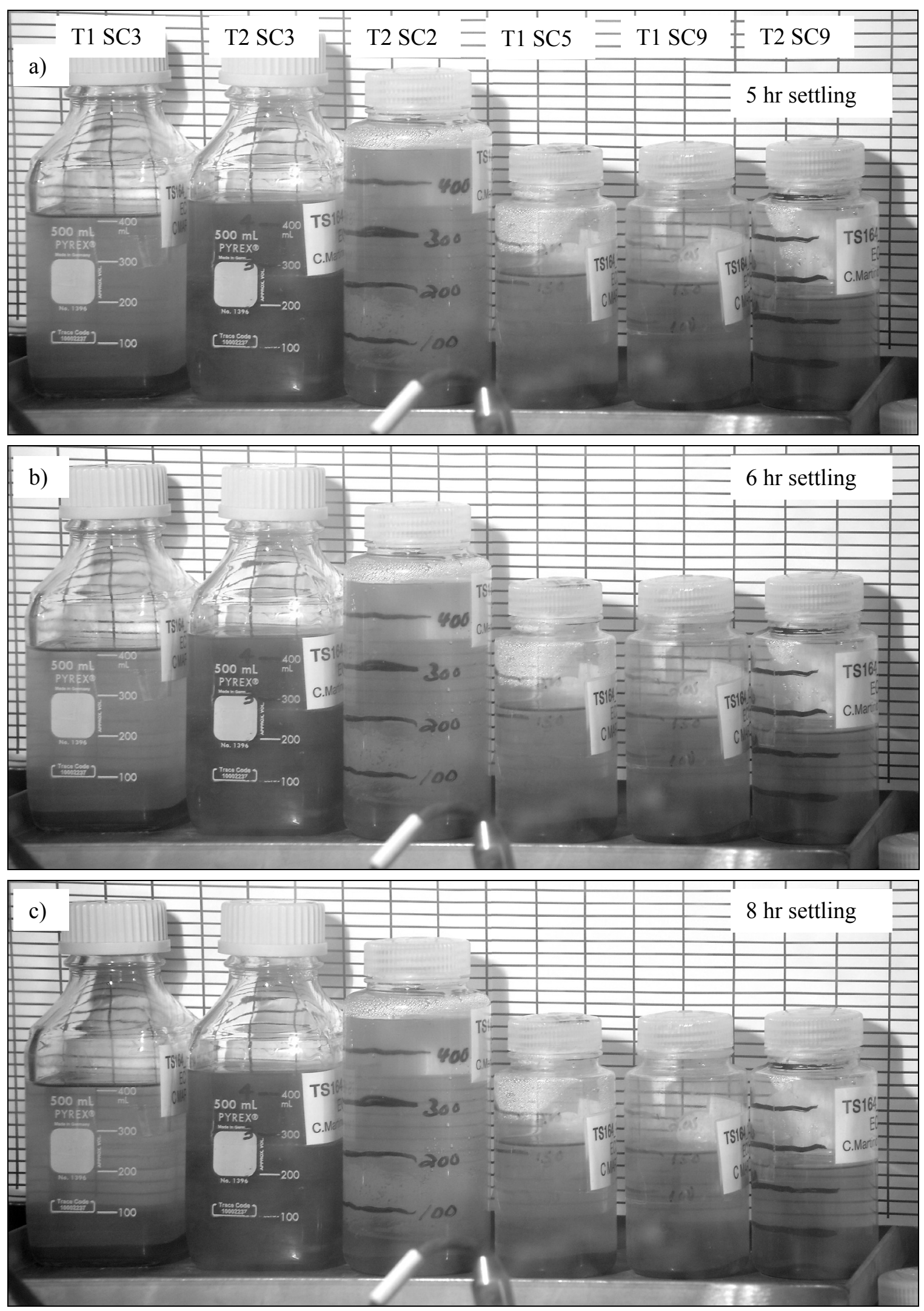

Figure B-91: ECC Product Settling (part 3 of 4)

B-55 

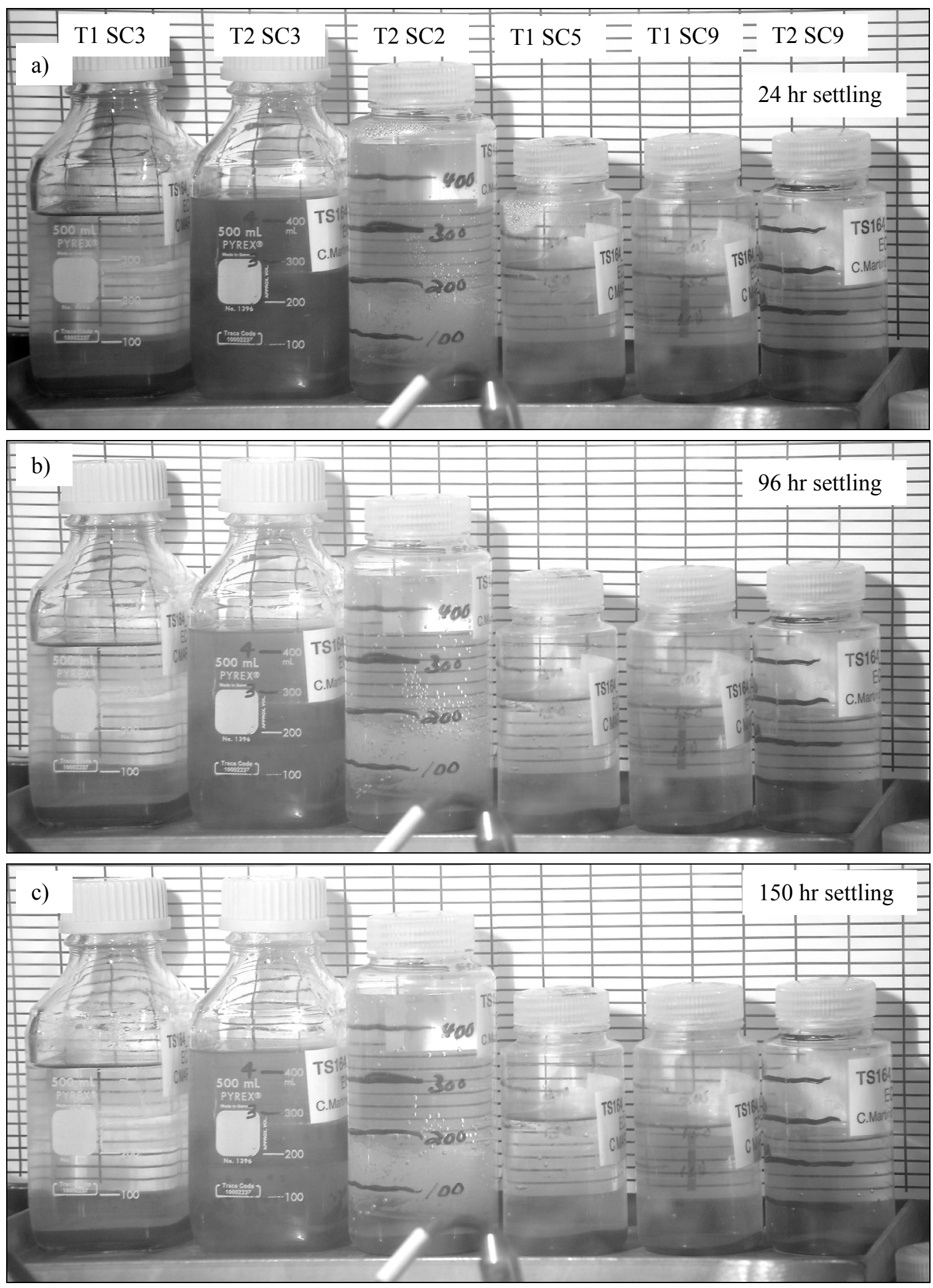

Figure B-92: ECC Product Settling (part 4 of 4) 


\section{Distribution:}

R. H. Spires, 704-26F

T. M. Punch, 704-71F

C. B. Sherburne, 704-71F

P. E. Carroll, 704-71F

E. T. Ketusky, 704-70F

J. R. Vitali, 704-71F

D. C. Wood, 704-26F

B. J. Rabe, $704-26 \mathrm{~F}$

N. R. Davis, 704-26F

W. L. Isom, 704-26F

A. J. Tisler, 704-26F

L. Carey, 742-7G

J. R. Gregory (AREVA), 704-S

C. J. Martino, 773-42A

F. M. Pennebaker, 773-42A

W. D. King, 773-42A

M. S. Hay, 773-42A

B. J. Wiersma, 773-A

J. I. Mickalonis, 773-A

S. H. Reboul, 773-A

A. B. Barnes, 999-W

D. A. Crowley, 773-43A

A. P. Fellinger, 773-41A

S. D. Fink, 773-A

B. J. Giddings, 786-5A

C. C. Herman, 999-W

S. L. Marra, 773-A

A. M. Murray, 773-A

W. R. Wilmarth, 773-A

P. R. Jackson, DOE-SR, 703-46A

K. H. Subramanian, 766-H 